\title{
Alveolar Macrophages
}

The bone marrow origin of the alveolar macrophages as well as the other cells of the mononuclear phagocyte system in the peritoneal and pleural cavities, the liver (Kupffer cells), the kidney, and other organs including the central nervous system (microglial cells) has been shown in a large number of chimera studies (for review see van FURTH 1980). The kinetics of mononuclear phagocytes during an acute inflammation have been studied in great detail in animal models, the inflammatory stimulus consisting of an intraperitoneal or an intravenous injection of new-born calf serum, polystyrene particles, or silica and/or other dust particles. Compared with experiments on alveolar macrophages gained by bronchopulmonary lavage these methods avoid bacterial or fungal contaminations. Thus Munder and Modolell (1987) used only bone macrophages to elevate the damaging capacity of reactive oxygen species produced during the interaction of airborne dust particles with phagocytic cells. BRÜCKNER-NIEDER et al. (1992) developed a human cell model using HL-60 cells, a promyelocytic line. These cells can be differentiated by phorbol myristate acetate into non-proliferating macrophage-like cells (HL-60-M). Sikron F600 quartz particles were engulfed. At the ultrastructural level, the quartz was seen inside phagolysosomes, but the morphology of the cell remained normal. During the differentiation of HL-60 cells, both an optimal concentration of calcitriol $\left(10^{-8} \mathrm{M}\right.$ to $\left.10^{-7} \mathrm{M}\right)$ and of ethanol is necessary (Zoller et al. 1996, Zoller and ZeLLER 2000). Differentiation of HL-60 cells in the presence of $0.1 \%(\mathrm{v} / \mathrm{v})$ ethanol significantly reduced their ability to produce $\mathrm{O}_{2}{ }^{--}$; an ethanol concentration of $2 \%(\mathrm{v} / \mathrm{v})$, on the other hand, was toxic. HL-60-G cells exposed to a high concentration of calcitriol ( $400 \mathrm{nM})$ slowly developed the ability to reduce nitroblue tetrazolium and did not proliferate (STUDZINSKI et al. 1997).

\section{1 Electron Microscopy}

All 48 alveolar macrophages that were seen in the serially sectioned human alveoli fixed in the inflated state were found within or bordering on alveolar junction zones (PARRA et al. 1986). A computer-reconstruction showed the predilection of alveolar macrophages and type II pneumocytes located in septal junction zones. Superposing alveolar macrophages and type II cells in such a reconstruction obliterated almost all gaps in the basement membranes, except for those produced by pores of Kohn when these were free of cells.

The size of the alveolar macrophage varies from $15 \mu \mathrm{m}$ to $30 \mu \mathrm{m}$ (BowDEN 1971). The cytoplasm is faintly basophilic. The nuclei of the smaller cells are round but in the larger cells they may be deeply indented. Their chromatin is much finer than that of the lymphocytes. Nucleoli are inconspicuous.

The basic ultrastructure of macrophages obtained from lung, lymph nodes, liver and spleen is similar (KARRER 1958). The structural heterogeneity of the alveolar macrophages largely depends on the phase of cellular activity and, in particular, on the nature and quantity of material engulfed (Fig. 108). The cytoplasma contains numerous mitochondria of the crista type, plenty of endoplasmic reticulum, many ribosomes, and polyribosomes (Fig. 122). Primary granules, which are generally small and round or very elongated (Fig. 107) are without a halo. The dense bodies, which are bounded by a single unit membrane, do not contain any recognisable structures. Myelin and lattice forms were phagocytosed from the alveolar lumen (Kissler 1983). According to Schaffner et al. (1967) they are surfactant, but ADAMson and BowDEN (1970) objected that the saturated phospholipids of surfactant cannot be demonstrated by conventional staining methods.

Rat alveolar macrophages obtained from unstimulated lungs by endobronchial lavage showed heterogeneity with respect to cell size $(88-20 \mu \mathrm{m}$ diameter), surface morphology and cytochemistry 
(Holt et al. 1982): the largest cells exhibited surface characteristics typical to stimulated macrophages (spreading, prominent peripheral lamellopodia with short, blunt filopodia protrudes to the substratum) while the smallest cells more closely resembled monocytes (poorly spread). Morphometry of pulmonary alveolar macrophages in situ and lavaged macrophages revealed significant differences in their volume fractions of nucleus, cytoplasm, ectoplasm, mitochondria, lysosome-like structures, lipid droplets, vacuoles and phagosomes/autophagosomes (Lum et al. 1983).

Microinjection and transfection studies have demonstrated that $\mathrm{Cdc} 42$ induces the formation of filopodia in several mammalian cell types, including fibroblasts and macrophages (ALLEN et al. 1997, TAPON and HALL 1997).

Lamellipodia are plasma membrane protrusions containing a meshwork of actin filaments, and extend over the substratum to form new adhesive contacts known as focal complexes (WeLCH et al. 1997). They are commonly found at the leading edge of migrating cells, driving the forward extension of cells. On adherent cells, membrane ruffles are similar in structure to lamellipodia, but protrude upwards from the dorsal surface of the cell. Both lamellipodium extension and membrane ruffling involve active actin polymerisation occurring adjacent to the plasma membrane.

Bovine alveolar macrophages cultivated for $20 \mathrm{~h}$ were heterogeneous, too (WILCZEK 1991). While freshly isolated cells prepared for electron microscopy varied in size from $8.5 \mu \mathrm{m}$ to $20 \mu \mathrm{m}$ with a mean of $13 \mu \mathrm{m}$, in culture cell diameters were from $12 \mu \mathrm{m}$ to $40 \mu \mathrm{m}$ with a mean of $22 \mu \mathrm{m}$ (Fox 1973).
Bielefeldt Ohman and Babiuk (1984) first used bovine alveolar macrophages for the in vitro generation of superoxide anion and hydrogen peroxide. The spontaneous superoxide anion release was very high immediately after lavage and became almost nil after $20 \mathrm{~h}$, as measured by cytochrome $c$ reduction; $20 \mathrm{~h}$ after lavage, the electrophoretic profiles obtained 3 or 4 subpopulation (PoLzer et al. 1991).

\subsection{1 \\ Lysosomes}

For an organelle to be interpreted as a lysosome, at least one structural requirement must be met: it must have a single limiting membrane with the relative large dimensions of the exoplasmic space membranes (DE DUVE 1969). Furthermore, the limiting membrane is commonly separated from the matrix by a clear halo (DAEMs et al. 1969, NoviKOFF 1973).

\subsubsection{1 \\ Primary Lysosomes}

With certain exceptions, primary lysosomes are more difficult to identify than secondary lysosomes. In most cells the primary lysosomes take the form of small vesicles deriving from the Golgi region (Novikoff 1973, Whaley 1975). The only types of cells in which primary lysosomes are found in a virgin condition are the granulocytes and monocytes. It is of interest to mention here that even the group of primary lysosomes is heterogeneous in the sense that - in monocytes - various types of primary lysosomes can be distinguished,
Fig. 107. Elongated primary granule in an alveolar macrophage from $\mathrm{a} \sim 250 \mathrm{~g}$ female white rat (breeder: Winkelmann, BorchenKirchborchen). Fixed under methitural anaesthesia by intratracheal instillation of $2.5 \%$ glutaraldehyde in phosphate buffer $(\mathrm{pH}$ 7.4) before opening the thorax. Postfixation with $1 \%$ osmium tetroxide in phosphate buffer $(\mathrm{pH}$ 7.4). Contrasted en bloc for $12 \mathrm{~h}$ with $0.5 \%$ uranyl acetate in $70 \%$ ethanol. Embedded in a $2: 8$ mixture of methyl and butyl methacrylate. Sectioned at $50 \mathrm{~nm}$. Lead citrate after REYNOLDS (1963). Plates 13/11 and $13 / 12$

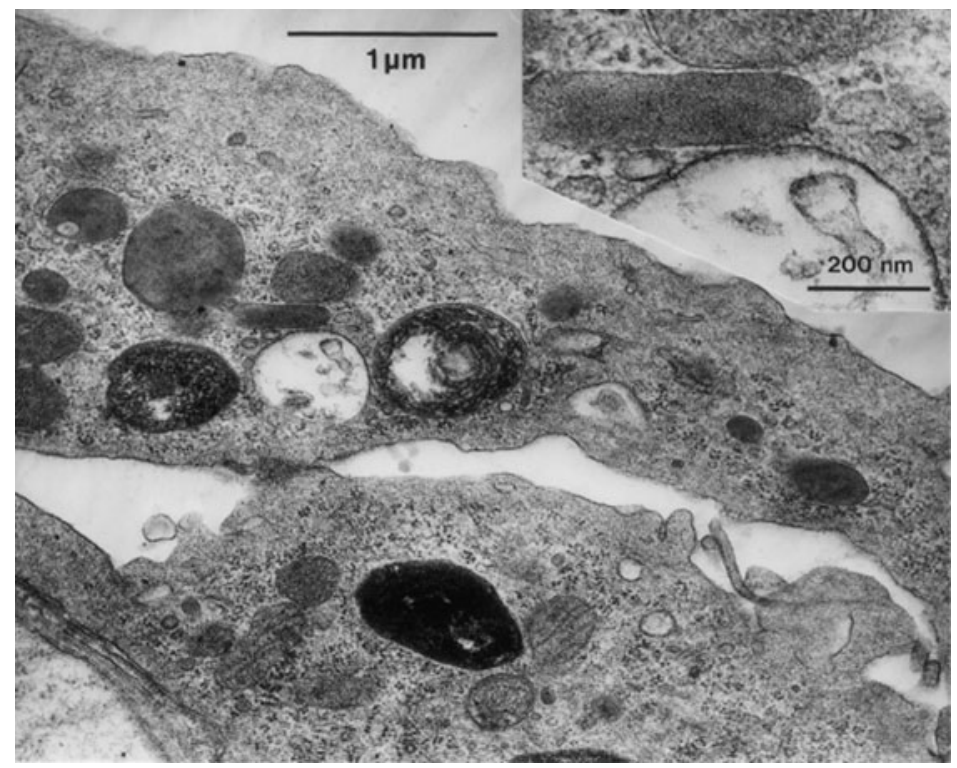


not only morphologically and cytochemically, but also on a functional bases (DAEMS and Brederoo 1973, DAEms et al. 1973, 1975, Nichols and BAINTON 1975).

The fate of most, if not all, of the primary granules is fusion with phagosomes to form a secondary lysosome.

\subsubsection{2 \\ Secondary Lysosomes}

\subsubsection{1}

Ingestion of Particles

Alveolar macrophages ingest and remove inhaled particulates from the lung (BRAIN 1986). The uptake can be seen easily under the phase contrast microscope and documented by time-lapse cinematography (SCHILlER 1954). Holm $(1972,1974)$ and Holm and Hammarström (1973) devised an in vitro system in which haemolysis and phagocytosis by human peripheral blood monocytes were quantitated by the release of radioactivity from red blood corpuscles labelled with ${ }^{51} \mathrm{Cr}$. GoldsMith et al. (1997) found a dose-dependent increase in alveolar macrophage-associated right angle light scatter after uptake of residual oil fly ash or concentrated ambient air particulates.

Actin polymerisation is necessary for phagocytosis (Allen and ANDERem 1996), and Rac and Cdc42, two Rho-related proteins, are required for Fc $\gamma$ receptor mediated phagocytosis (Cox et al. 1997). It is not known precisely which stage of phagocytosis involves Rac and Cdc42, although it is intriguing that phosphoinositide 3-kinase has been shown to play a role in the final membrane fusion step of phagocytosis (ARAKI et al. 1996). Given that phosphoinositide 3-kinase can act upstream of Rac (PARKER 1995), it is possible that Rac also plays a role at this stage. In contrast to Rac and Cdc42, Rho is required during phagocytosis for the initial clustering of $F c \gamma$ receptors on macrophages (HACKAM et al. 1997).

Rac has been shown to regulate the activity of a membrane-associated NADPH oxidase complex (SEgAL and Аво 1993; Вокосн 1995). This multiprotein complex derives electrons from NADPH on its cytosolic face and pumps them into the lumen of phagosomes where they consequently serve to generate superoxide anion $\left(\mathrm{O}_{2}{ }^{--}\right)$and subsequently hydrogen peroxide $\left(\mathrm{H}_{2} \mathrm{O}_{2}\right)$ and hydroxyl radicals $\left(\mathrm{HO}^{\circ}\right)$ as well as hypochlorous acid ( $\left.\mathrm{HOCl}\right)$. The latter compounds represent the killing agents of the cell in the fight against phagocytosed material. The basic $\mathrm{e}^{-}$transport function of the complex resides in the unusual cytochrome $b_{558}$, consisting of two subunits $\mathrm{gp} 91^{\text {phox }}$ and $\mathrm{p} 22^{\text {phox }}$, which are ingested into the plasma membrane. Proper regulation of the complex requires two additional soluble proteins, $\mathrm{p} 47^{\text {phox }}$ and $\mathrm{p} 67^{\text {phox }}$, which, upon activation, are tethered to the membrane components of the complex.

In search of a specific effector molecule of Rac in the oxidase complex, Diekmann et al. (1994) revealed $\mathrm{p} 67^{\text {phox }}$ as a Rac interacting protein. SEASTONE et al. (1998) have shown that a phorbol esterbinding protein is important for RacC-mediated phagocytosis. Phorbol 12-myristate 13-acetate stimulated phagocytosis of recombinant Sindbis viruses encoding Rab5:WT and increased the number and the size of endocytic vesicles, even in the presence of Rab5:S34N, a dominant negative mutant of the rate-limiting regulator of endosome fusion in BHK-21 cell monolayers (ABALlay et al. 1999). Zinc depletion with $N, N, N^{\prime}, N^{\prime}$-tetrakis-(2-pyridylmethyl) ethylenediamine and addition of calphostin $\mathrm{C}$, an inhibitor of protein kinase $C$ that interacts with zinc and phorbol ester binding motifs, inhibited both basal and Rab5-stimulated fluid phase endocytosis. These two reagents also inhibited the size and number of endocytotic vesicles promoted by Rab5.

\section{Phagocytosis and Membranes}

The best-documented way of endocytosis is receptor-mediated uptake of ligands via clathrincoated vesicles (reviewed in ScHMid 1997). Receptors are recruited and concentrated into clathrincoated pits at the plasma membrane. After coated vesicle formation, the clathrin coat is removed by the concerted action of auxilin and heat shock protein 70 (UngewiCKELl et al. 1995). The uncoated vesicles fuse with early endosomes in a rab5regulated manner (RUBino et al. 2000).

In the Chinese hamster ovary cell line ts20, containing a thermosensitive ubiquitin-activating enzyme, E1, SACHSE et al. (2002) showed that this coat is predominantly present on early endosomes and has a characteristic bilayered appearance in the electron microscope. The coat contains clathrin heavy as well as light chain, but lacks the adaptor complexes AP1, AP2, and AP3, by which it differs from clathrin coats on endocytic vesicles and recycling endosomes. The coat is insensitive to short incubations with brefeldin A, but disappears in the presence of the phosphatidylinositol 3-kinase inhibitor wortmannin.

A role for scavenger-type receptors in alveolar macrophage uptake of components of residual oil fly ash or concentrated ambient air particulates was identified by marked inhibition of right angle light scatter increases in alveolar macrophages pretreated with the specific scavenger-type receptor inhibitor polyinosinic acid (Goldsmith et al. 1997). 


\section{Phagocytosis and the Golgi Apparatus}

Golgi-specific agents (brefeldin, $\mathrm{AlF}_{4}^{-}$, monensin) which induce vesiculation all enhanced phagocytosis of liposomes by rat alveolar macrophages (Perry et al. 1996).

\section{Phagocytosis and Cyclic AMP}

The level of intracellular cyclic nucleotides is a regulatory factor in a variety of immune processes. Increases in intracellular cyclic AMP (cAMP) and/ or cyclic GMP (cGMP) concentration by the inhibition of phosphodieserase have been shown to modulate the inflammatory response.

Phagocytosis of polystyrene latex beads $(0.481$ $\mu \mathrm{m}$ diameter) by rabbit alveolar macrophages was accompanied by an increase of cAMP, which preceded or coincided with the onset of other metabolic events (SCHMIDT-GAYK et al. 1975). Dibutyryl cAMP, which mimics several action of cAMP, stimulated oxygen consumption and hexose monophosphate activity in phagocytosing cells, whereas in resting cells no significant change was observed.

Adenosine triphosphatase (ATPase) was localised in the plasmalemma of guinea pig peritoneal macrophages (NoRTH 1966). Enzymatic activity could be removed from the cells by trypsin. While scanning electron microscopy of endothelial cells monolayers harvested using $0.25 \%$ trypsin or $0.125 \%$ trypsin $+0.01 \%$ EDTA failed to reveal any distinctive differences in their surface morphology (KIRKPATRICK et al. 1986), bovine alveolar macrophages showed blebbing (BREHM 1996).

Intracellular dissolution of a large variety of inhaled inorganic particles not readily soluble in the pulmonary epithelial lining fluid constitutes an important long-term clearance mechanism of the lungs. Fluorescence microscope photometry and dual laser flow cytometry of intraphagolysosomal $\mathrm{pH}$ in canine and rat alveolar macrophages were compared using fluoresceinisothiocyanate labelled, amorphous silica particles (Nyberg et al. 1989, Heilmann et al. 1992). The higher intraphagolysosomal $\mathrm{pH}$ in rat alveolar macrophages correlated with the smaller intracellular particle dissolution rate in Wistar rat alveolar macrophages when compared with the data in Beagle dogs (KREYLING et al. 1992).

\subsubsection{2 \\ Phagolysosomes}

The distinction between phagosomes and secondary lysosomes cannot always be made with certainty. Special difficulties are encountered when a distinction of this kind is essential for the understanding of the course of a given process, e.g. an intracellular parasitism (see DrAPER and D'ARCY HART 1975).

\section{Destruction of the Lysosomal Membranes by $\mathrm{SiO}_{4}$ Tetrahedra}

Late cytotoxicity of silica is evidently due to the capacity of silica particles after proteins and other protective biological material have been removed by digestion to interact with, and to make permeable, the membranes surrounding secondary lysosomes (Allison et al. 1966).

Like other indigestible polymers, polyvinylpyridine- $N$-oxide accumulates in secondary lysosomes, where it can interact with silanol groups of enclosed particles, preventing them from damaging the lysosomal membrane.

\subsubsection{3 \\ Autophagous Vacuoles}

Autophagy is a widespread phenomenon in the degradation of cellular components (DUNN 1994). Depending on the mechanism involved, different forms of autophagic degradation have been described: crinophagy (Fig. 266) for degradation of secretory proteins after fusion of secretory granules with lysosomes (SMith and FARQUHAR 1966, LENK et al. 1991), carrier-mediated proteolysis for the degradation of cytosolic protein bearing KFERQ sequence as a signal for a lysosomal receptor/transporter protein (OLson 1989), microautophagy where cytosolic domains including ribosomes or glycogen are internalised into lysosomes via an endocytosis-like process (MARzELla and GlaUMANN 1987), and macroautophagy for degradation of cytosolic domains and intracellular membrane compartments or specialised regions of the plasma membrane (Kovacs and Reith 1982, ElsäsSER et al. 1993). Autophagic vacuoles are dynamic structures which can be induced by several experimental regimen. In experimental silicosis research, BRUCH (1970) was the first to describe membraneorganelles in pulmonary histiocytes that were phagocytizing DQ12 quartz particles. SCHILLER (1980, 1993) found such structures both in unmedicated rats (Figs. 109, 110) and macrophages stimulated with particulate matter or methylcellulose (Figs. 161, 162) or activated with complete Freund's adjuvant.

Structure, function and turnover of autophagic vacuoles have been studied biochemically after enrichment of the organelles by cell fractionation 
techniques (Marzella et al. 1982, Fellinger and RÉz 1990), or by ultrastructural methods (FURUNo et al. 1990, Toоze et al. 1990). Үокота et al. (1995) described the formation of autophagosomes during the degradation of excess peroxisomes induced by di-(2-ethylhexyl)-phthalate, a peroxisome proliferator.

Within $24 \mathrm{~h}$ of exposure to cholesterol oxidation products $(28$ and $56 \mu \mathrm{M})$, J774 cells suffered lysosomal destabilisation, release to the cytosol of the lysosomal marker-enzyme cathepsin D (EC 3.4.23.5), apoptosis, and postapoptotic necrosis (YUAN et al. 2000). Enhanced autophagocytosis and chromatin margination was found $12 \mathrm{~h}$ after the exposure to cholesterol oxidation products, whereas apoptosis and postapoptotic necrosis were pronounced 24 and $48 \mathrm{~h}$ after the exposure. Some lysosomal vacuoles were then filled with degraded cellular organelles, indicating phagocytosis of apoptotic bodies by surviving cells. Because caspase-3 activation was detected in the cells exposed to cholesterol oxidation products, lysosomal destabilisation may associate with the leakage of lysosomal

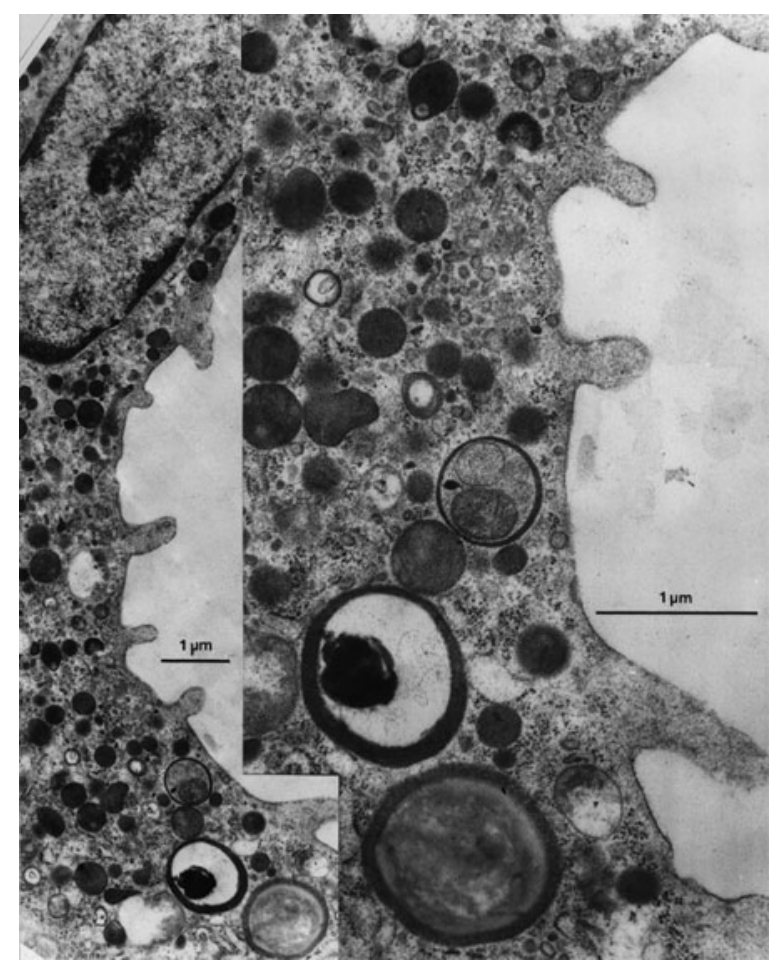

Fig. 108. Cytosegresomes in an alveolar macrophage (block 4424) from an unmedicated $235 \mathrm{~g}$ Sprague-Dawley rat (Charles River, France). On July 25, 1978 under pentobarbital anaesthesia $(30 \mathrm{mg} / \mathrm{kg})$, the animal was perfused from the abdominal aorta with $2.5 \%$ glutaraldehyde in $0.1 \mathrm{M}$ sodium cacodylate buffer ( $\mathrm{pH} 7.4)$. Postfixation with $1 \%$ osmium tetroxide in sodium cacodylate buffer. Embedded in Epon 812 and sectioned at $50 \mathrm{~nm}$. Lead citrate and uranyl acetate. Plates 4199 and 4200

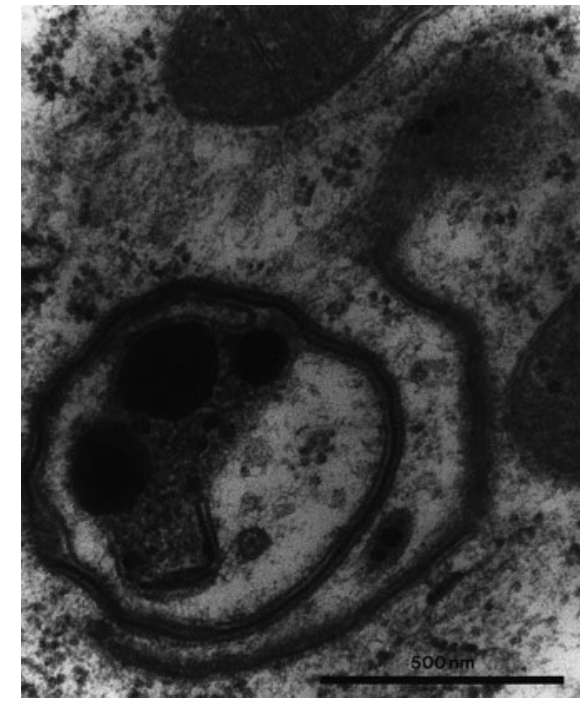

Fig. 109. Phagophore in a mesenteric lymph node macrophage (block 4421) from an unmedicated $240 \mathrm{~g}$ SpragueDawley rat (Charles River, France). Under pentobarbital anaesthesia $(30 \mathrm{mg} / \mathrm{kg})$, the animal was perfused from the abdominal aorta with $2.5 \%$ glutaraldehyde in $0.1 \mathrm{M}$ sodium cacodylate buffer ( $\mathrm{pH}$ 7.4). Postfixation with $1 \%$ osmium tetroxide in sodium cacodylate buffer. Embedded in Epon 812 and sectioned at $50 \mathrm{~nm}$. Stained with lead citrate and uranyl acetate. Plate 4197

enzymes, and activation of the caspase cascade. Manganese superoxide dismutase mRNA levels were markedly increased after $24 \mathrm{~h}$ of exposure to cholesterol oxidation products, suggesting associate induction of mitochondrial protection or turnover.

BARTH et al. (2001) identified AUT10 as a novel gene required for both the cytoplasm to vacuole targeting of proaminopeptidase I and starvationinduced autophagy.

\subsection{2 \\ Peroxisomes}

Peroxisomes are ubiquitous, spherical organelles bounded by a single membrane and having a diameter of $100 \mathrm{~nm}$ to $1 \mu \mathrm{m}$. Drath et al. (1982) found catalase activity in similar amounts in both supernatants and granule-rich fractions of rat pulmonary macrophages isolated by exhaustive pulmonary lavage and postlavage homogenisation techniques.

\subsection{3 Cytoskeleton}

One of the characteristic features of mononuclear cell activation is the deformation of their cytoskeleton. While Phaire-Washington et al. (1980) determined the spreading of peritoneal macrophages stimulated by phorbol myristate acetate by draw- 


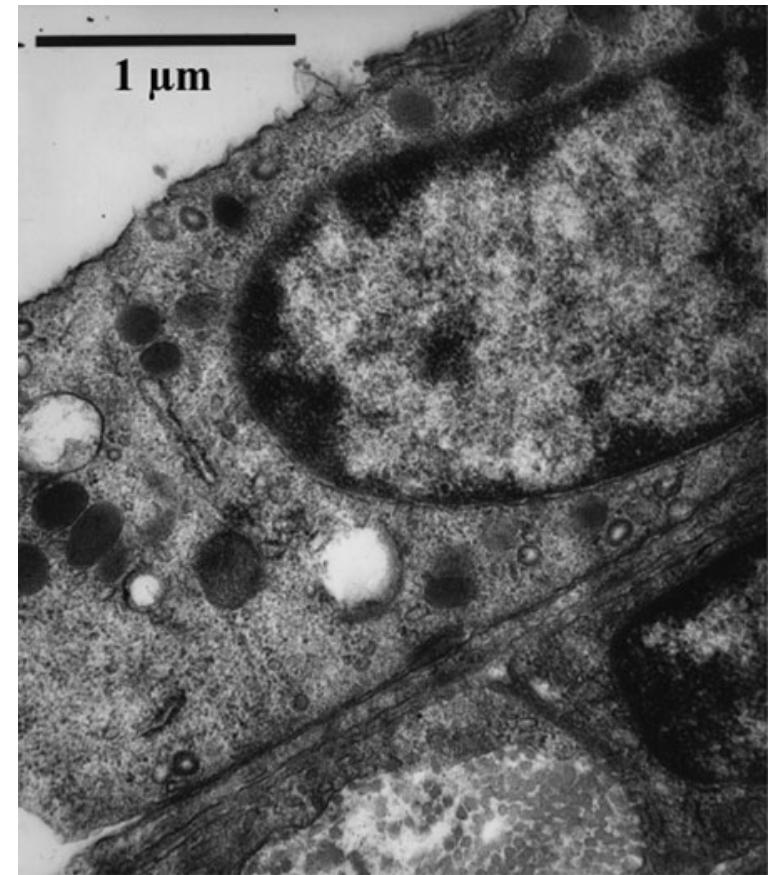

Fig. 110. Peroxisomes in an alveolar macrophage adjacent to a type I pneumocyte of a female rat (breeder: Winkelmann, Borchen-Kirchborchen) which inhaled micronized deptropine citrate and isoprenaline hydrochloride ãa from a suspension type self-propelled aerosol (Medihaler ${ }^{\circledR}$ ) containg a mixture of $28 \mu \mathrm{g}$ hexadecylpyridinium chloride/puff + sorbitan trioleate for surfactants. $12 \mathrm{Puff} / \mathrm{min}$ were dispersed into a $164.5 \mathrm{l}$ box where the animals stayed for $15 \mathrm{~min}$. $1 \mathrm{~h}$ later under methitural anaesthesia, the lung was fixed by intratracheal instillation of $2.5 \%$ glutaraldehyde in phosphate buffer (pH 7.4) before opening the thorax. Postfixation with $1 \%$ osmium tetroxide in phosphate buffer ( $\mathrm{pH} 7.4)$. Contrasted en bloc for $12 \mathrm{~h}$ with $0.5 \%$ uranyl acetate in $70 \%$ ethanol. Embedded in a 2:8 mixture of methyl and butyl methacrylate. Sectioned at $50 \mathrm{~nm}$. Lead citrate after REY NOLDs (1963). Plate 20/02. (from SCHILLER 1971)

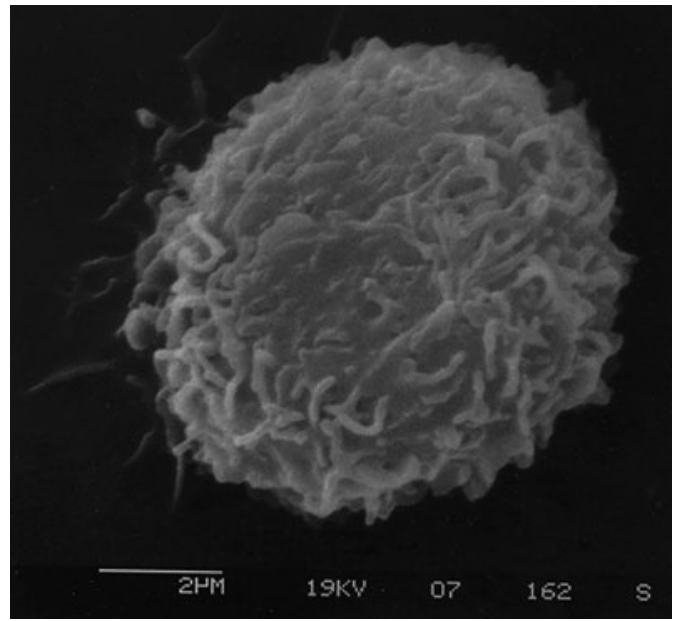

Fig. 112. Ruffled coat of a peritoneal macrophages from a SPF-NMRI mouse $48 \mathrm{~h}$ after a single intraperitoneal injection of $1 \mathrm{ml} \mathrm{NaCl}$. The cells harvested with the ascites were centrifuged for $10 \mathrm{~min}$ at 1000 r.p.m. and incubated in TCM $199+$ $20 \%$ fetal calf serum on a plastic cover slip for $2 \mathrm{~h}$, rinsed with phosphate buffered saline after Dulbecco and fixed in $1 \%$ glutaraldehyde in $0.07 \mathrm{M}$ phosphate buffer ( $\mathrm{pH}$ 7.4). Ethanol, amyl acetate. Critical point drying. Gold coating. Cambridge Stereoscan 150 operated at $19 \mathrm{kV}$. APh-R. 854/80, negative 07 162. (from ScHILler 1982)

ing, cutting out, and weighing (cf. Romeis 1968, $\$ 916)$, ScHOEVAERT et al. (1983) measured the increase of the surface area induced by immunomodulators with a 720 lines Plumbicon camera and analysed it using a Quantimet 720 interfaced with a PDP $11 / 34$.

The microtubules, microfilaments, and intermediate filaments of the cytoskeleton influence the shape of free cells such as macrophages, but also
Fig. 111. Peritoneal macrophages from a SPFNMRI mouse $48 \mathrm{~h}$ after a single intraperitoneal injection of $1 \mathrm{ml} \mathrm{NaCl}$. The cells harvested with the ascites were centrifuged for $10 \mathrm{~min}$ at 1000 r.p.m. and incubated in TCM $199+20 \%$ fetal calf serum on a plastic cover slip for $2 \mathrm{~h}$, rinsed with phosphate buffered saline after Dulbecco and fixed in $1 \%$ glutaraldehyde in $0.07 \mathrm{M}$ phosphate buffer (pH 7.4). Ethanol, amyl acetate. Critical point drying. Gold coating. Cambridge Stereoscan 150 operated at $19 \mathrm{kV}$. APh-R. 102/80, negative 03 798. (from Schiller 1982)

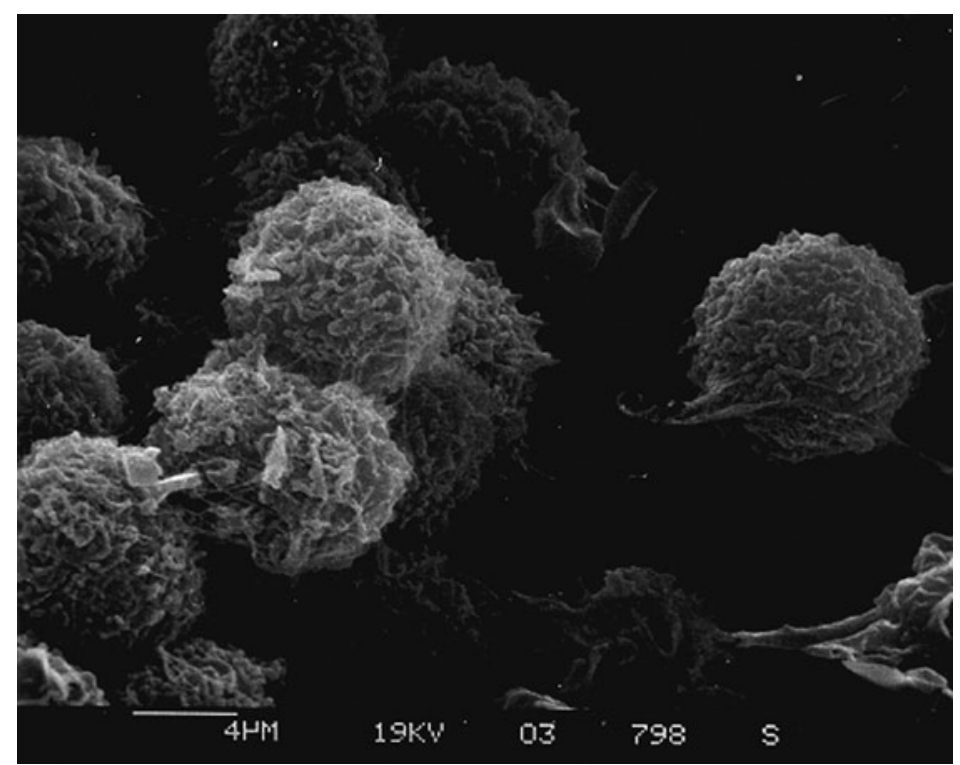


Fig. 113. Both rounded and spread peritoneal macrophages from a SPF-NMRI mouse, the former showing surface ridges and ruffles attaching themselves to the underlying substrate by means of thin veils of cytoplasm spreading beneath the dome-shaped nuclear pole. The cells were harvested $48 \mathrm{~h}$ after a single intraperitoneal injection of $1 \mathrm{ml}$ saline by centrifugation of the ascites for $10 \mathrm{~min}$ at 1000 r.p.m. and incubated in TCM $199+$ $20 \%$ foetal calf serum on a plastic cover slip for $2 \mathrm{~h}$, rinsed with phosphate buffered saline after Dulbecco and fixed in $1 \%$ glutaraldehyde in $0.07 \mathrm{M}$ phosphate buffer ( $\mathrm{pH}$ 7.4). Ethanol, amyl acetate. Critical point drying. Gold coating. Cambridge Stereoscan 150 operated at $19 \mathrm{kV}$. APh-R. 102/80, negative 03842

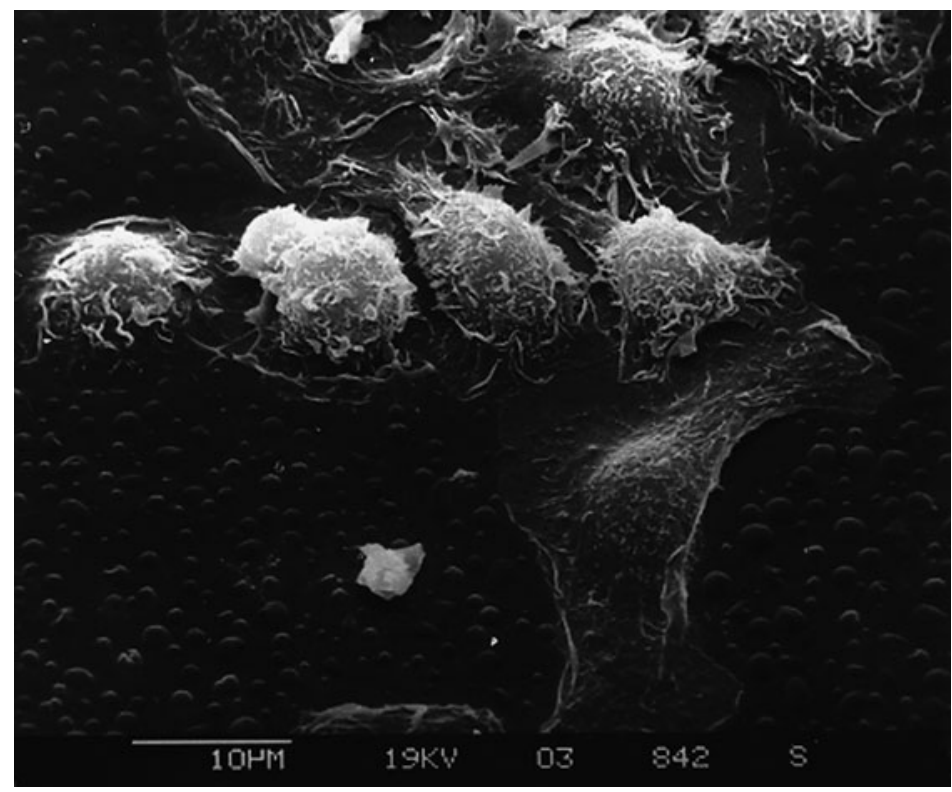

the locomotion of the cell organelles such as phagosomes, lysosomes, phagolysosomes and secretory granules, and the process of ciliogenesis. The outer surface of macrophages under the influence of saline (Figs.111-114) or isotactic polyacrylic acid (Figs. 160-165) depends on the cytoskeleton.

Oxidant injury produces dramatic changes in cytoskeletal organisation and cell shape as shown in the $\mathrm{P}_{388 D_{1}}$ cell line by Hinshaw et al. (1988).

\subsubsection{1}

\section{Actin and Microfilaments}

At least six different isoforms of actin have been identified in vertebrate tissues, each encoded by a different gene (VANDERKerckHove and Weber 1978, REDDy et al. 1990). Non-muscle cells contain two general cytoplasmic actins, $\beta$ and $\gamma$. They are very similar to each other (only 4 amino acid substitutions) (VANDEKERCKHOVE and Weber 1979). Ultrastructural evidence that calponin binds selectively to "cytoskeletal" actin has led some investiga-
Fig. 114. Rounded peritoneal macrophages from a SPF-NMRI mouse, showing surface ridges and ruffles attaching itself to the underlying substrate by means of thin veils of cytoplasm spreading beneath the domeshaped nuclear pole. The cell was harvested $48 \mathrm{~h}$ after a single intraperitoneal injection of $1 \mathrm{ml}$ saline by centrifugation of the ascites for $10 \mathrm{~min}$ at 1000 r.p.m. and incubated in TCM $199+20 \%$ foetal calf serum on a plastic cover slip for $2 \mathrm{~h}$, rinsed with phosphate buffered saline after Dulbecco and fixed in $1 \%$ glutaraldehyde in $0.07 \mathrm{M}$ phosphate buffer (pH 7.4). Ethanol, amyl acetate. Critical point drying. Gold coating. Cambridge Stereoscan 150 operated at $19 \mathrm{kV}$. APhR. 102/80, negative 03 806. (from SCHILlER 1982)

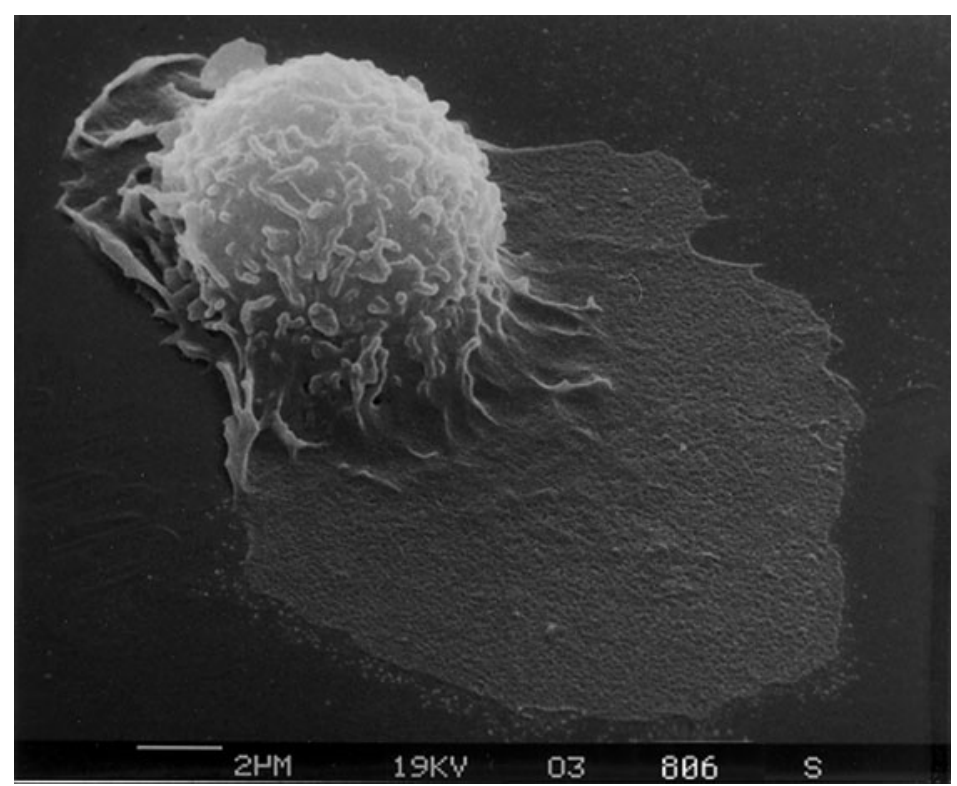


tors to propose that the function of calponin in smooth muscle may be structural rather than regulatory. Based on evidence that calponin binds to desmin as well as $\beta$ actin, MABUCHI et al. (1997) proposed that calponin may function as a bridging protein between actin and intermediate filament networks at dense bodies. Ultrastructural analysis of thin filaments by Hodgkinson et al. (1997) indicated that the location of calponin on F-actin is similar to that of the actin cross-linking protein fimbrin, as well as to that of $\alpha$-actinin and gelsolin. They suggested that a possible function of calponin may be the competitive inhibition of the binding of these or other actin-binding proteins to the actin filament. This could serve to regulate the building or remodelling of the actin cytoskeleton and thereby affect its mechanical properties.

Monomeric actin is stable in distilled water only.

Actin has been isolated from alveolar macrophages by HARTwig and STOSSEL $(1975,1982)$, StosSEL and Hartwig $(1975,1976)$, and Hartwig et al. (1977). Under the electron microscope, Allison et al. (1971) and Sende et al. (1975) saw arrow heads typical for actin microfilaments with heavy meromyosin.

The reversible assembly of cytoplasmic actin can be regulated at different levels (KoRN 1982, STOSSEL et al. 1985, Pollard 1986). Dissociation of the actin monomer-binding protein, profilin (CARLSSON et al. 1977) of profilin-actin complexes would increase the local concentration of free actin monomers available for nucleation or to polymerise onto nuclei formed through another process. A second class of regulatory proteins, the filament-severing proteins bind to the barbed end of actin filaments and block the binding of actin monomers to that end, such as gelsolin (YIN and STOSSEL 1979). These molecules may function to cap filament ends in resting cells and dissociate as a consequence of agonist binding. Once dissociated, actin monomers could access to the filament ends leading to filament elongation. This mechanism would increase filament length, but not increase the number of filaments in a region of cytoplasm. Net filament growth could also occur by the formation of new nuclei, either by fragmentation of existing filaments or by the activation of proteins expressing sites equivalent to the barbed end of filaments. Nuclei serve as templates for the assembly of new filaments. Nucleation differs from uncapping in that the number of filaments in a region of a cell increases not just the length of pre-existing filaments.

Hartwig and JANMEY (1989) found that phorbol 12-myristate 13-acetate would increase the nucleation activity of macrophages washed out of the lungs of New Zealand white rabbits given complete
Freund's adjuvant intravenously. This increment was completely cytochalasin-sensitive, indicating that exposure to PMA leads to formation of free barbed ends.

Xanthine oxidase increased the rate of actin polymerisation and accelerated the conversion of F(ATP)actin into F(ADP,Pi)actin (LANZARA et al. 1988).

$\mathrm{HOCl}$ induced a rapidly increasing yield of carbonyl groups in rabbit skeletal muscle actin (Dalle-Donne et al. 2001). However, when carbonylation became evident, some cysteine and methionine residues had been already oxidised. HOClmediated oxidation induced the progressive disruption of actin filaments and the inhibition of F-actin formation. The molar ratios of $\mathrm{HOCl}$ to actin that lead to inhibition of actin polymerisation seemed to have effect only on cysteines and methionines.

In human intestinal (Caco-2) monolayers exposed to reactive oxygen metabolites $\left(\mathrm{H}_{2} \mathrm{O}_{2} ; \mathrm{HOCl}\right)$, preincubation with OPC (Otsuka America Pharmaceutical Company) compounds 12759 or rebamipide $\{[2-(4$-chlorobenzoylamino $)-3-[2-(1 \mathrm{H})$-quinolinon-4-yl] propionic acid $\}$ and 6535 \{6-[2-(3,4diethoxyphenyl)thiazol-4-yl]pyridine-2-carboxylic acid \} pr stable F-actin (BANAN et al. 2001).

In the $\mathrm{P}_{338 \mathrm{D}_{1}}$ murine cell line $5 \mathrm{mM} \mathrm{H} \mathrm{H}_{2} \mathrm{O}_{2}$ induced side-to-side aggregates or bundles of microfilaments (HINsHAw et al. 1988) suggesting that declining levels of ATP either from metabolic inhibition or $\mathrm{H}_{2} \mathrm{O}_{2}$ injury are correlated with the fragmentation and shortening of microfilaments into aggregates. No net change in monomeric or polymeric actin was necessary for this to occur. However, at later time points after $\mathrm{H}_{2} \mathrm{O}_{2}$ exposure some actin assembly did occur.

$\mathrm{N}$-Formyl-methionyl-leucyl-phenylalanine-induced neutrophil activation (actin reorganisation and chemotaxis) was inhibited by the nitric oxide donor, 1,2,3,4-oxatriazolium,5-amino-3-(3,4-dichlorophenyl)-chloride (WARD et al. 2000).

The ability of lipopolysaccharide to cause altered phosphate labelling of $\beta / \gamma$-actin suggests a participation of the microfilament network in lipopolysaccharide-induced monocyte activation (HAUsCHILDT et al. 1997).

Nitric oxide has been reported to be involved in the regulation of pseudopodia formation (JUN et al. 1996). KE et al. (2001) separated the globular and filamentous actins from quiescent or "NOstimulated macrophage-like cell line RAW 264.7 cells. Predominant G-actin coexisted with Triton X100-insoluble filamentous (TIF) and Triton X-100soluble filamentous actin in resting RAW 264.7 cells. The exogenous "NO produced by $( \pm)-(E)$ 2-[(E)-hydroxyimino]-6-methoxy-4-methyl-5-nitro- 
hexenamide (NOR1), the endogenous ${ }^{\circ} \mathrm{NO}$ induced by lipopolysaccharide plus interferon- $\gamma$, and dibutyryl-cGMP increased the contents of TIF-actin in dose- and time-dependent manners and altered its morphology. The increase in the TIF-actin contents induced by NOR1 or lipopolysaccharide plus interferon- $\gamma$ was efficiently blocked by the radical scavenger 2-(4-carboxyphenyl)-4,4,5,5-tetramethylimidazoline-1-oxyl 3-oxide and the soluble guanylate cyclase inhibitor $1 H$-[1,2,4] oxodiazolo[4,3- $a$ ] quinoxalin-1-one or the arginine analogue $N^{\mathrm{G}}$ monomethyl-L-arginine acetate, respectively. Preincubation with the calmodulin antagonist $\mathrm{W}-7$ almost completely blocked the ${ }^{N}$ NO-induced TIFactin increase and morphological change. On the other hand, preincubation with C3 transferase, an inhibitor of Rho protein, efficiently prevented the change in cell morphology, but had no effect on the TIF-actin increase.

In human intestinal (Caco-2) monolayers exposed to reactive oxygen metabolites $\left(\mathrm{H}_{2} \mathrm{O}_{2} ; \mathrm{HOCl}\right)$, preincubation with OPC (Otsuka America Pharmaceutical Company) compounds 12759 or rebamipide $\{[2-(4-$ chlorobenzoylamino $)-3-[2-(1 \mathrm{H})$-quinolinon-4-yl] propionic acid $\}$ and $6535\{6-[2-(3,4-$ diethoxyphenyl)thiazol-4-yl]pyridine-2-carboxylic acid \} prevented actin oxidation, decreased depolymerized G-actin, and enhanced the stable F-actin (BANAN et al. 2001).

Nitric oxide has been reported to be involved in the regulation of pseudopodia formation (Jun et al. 1996). Ke et al. (2001) separated the globular and filamentous actins from quiescent or "NOstimulated macrophage-like cell line RAW 264.7 cells. Predominant G-actin coexisted with Triton X100-insoluble filamentous (TIF) and Triton X-100soluble filamentous actin in resting RAW 264.7 cells. The exogenous 'NO produced by $( \pm)-(E)$ 2-[(E)-hydroxyimino]-6-methoxy-4-methyl-5-nitrohexenamide (NOR1), the endogenous ${ }^{\circ} \mathrm{NO}$ induced by lipopolysaccharide plus interferon- $\gamma$, and dibutyryl-cGMP increased the contents of TIF-actin in dose- and time-dependent manners and altered its morphology. The increase in the TIF-actin contents induced by NOR1 or lipopolysaccharide plus interferon- $\gamma$ was efficiently blocked by the radical scavenger 2-(4-carboxyphenyl)-4,4,5,5-tetramethylimidazoline-1-oxyl 3-oxide and the soluble guanylate cyclase inhibitor $1 H$-[1,2,4] oxodiazolo[4,3a] quinoxalin-1-one or the arginine analogue $N^{\mathrm{G}}$ monomethyl-L-arginine acetate, respectively. Preincubation with the calmodulin antagonist W-7 almost completely blocked the ${ }^{N}$ NO-induced TIFactin increase and morphological change. On the other hand, preincubation with C3 transferase, an inhibitor of Rho protein, efficiently prevented the change in cell morphology, but had no effect on the TIF-actin increase.

Rho family proteins control actin organisation (Ridley and Hall 1992). Like Ras, most members of the Rho family cycle between an inactive GDPbound form and an active GTP-bound form. Three major regulators control their activity: RhoGDIs interact with the geranyl-geranylated form of the proteins to keep them in a "resting" cytosolic complex (Zalcman et al. 1996, 1999).

Paraquat known to induce production of $\mathrm{O}_{2}{ }^{--}$ through ist reduction/oxidation (formula [158]) affected actin cytoskeleton by an increase of the filamentous pool of actin and a parallel decrease of the monomeric actin (CAPpelletti et al. 1996). Paraquat induced an increase of de novo synthesis of actin, but did not affect the actin degradation rate.

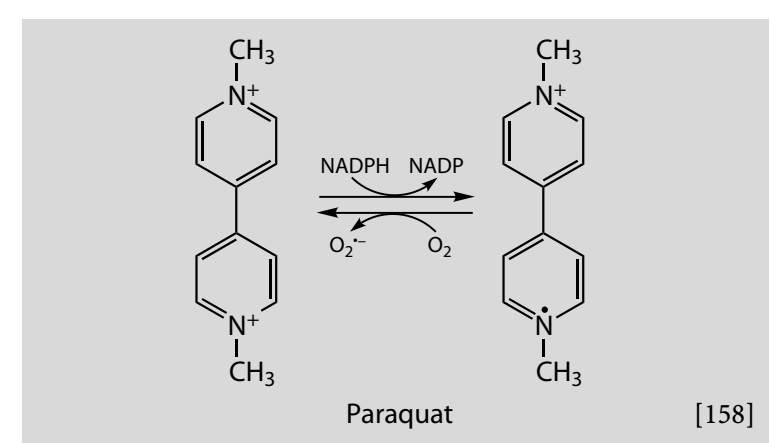

The copper complex tetraanhydroaminobenzaldehyde (16 atoms in a macrocyclic ring, four donor nitrogens associated with $\mathrm{Cu}^{2+}$ ) as to its functional similarity to superoxide dismutase is able to catalyse superoxide to molecular oxygen and paraquat interacted with the actin cytoskeleton in a competitive-like manner (UrbANCíková and KoRYTÁR 1999).

The dynamics of the actin cytoskeleton are controlled by a collection of actin-binding proteins.

Severin is a member of a family of actin-binding proteins which consist of a tandem arrangement of homologous domains which have developed slightly modified activities. Severin has three such domains, as does fragmin, while gelsolin and villin have six. Although the first domain of severin can cap the ends of actin filaments the second domain is required to sever them. It is therefore assumed that the second domain binds to side of the actin filament while the first is forced into the filament disrupting it. Calcium has been implicated in the regulation of the activity and calcium-induced changes in the NMR spectrum of severin has identified residues which may bind calcium ions.

Myosin from vertebrate macrophages is composed of two heavy chains of $M_{\mathrm{r}} 200,000$, two light 


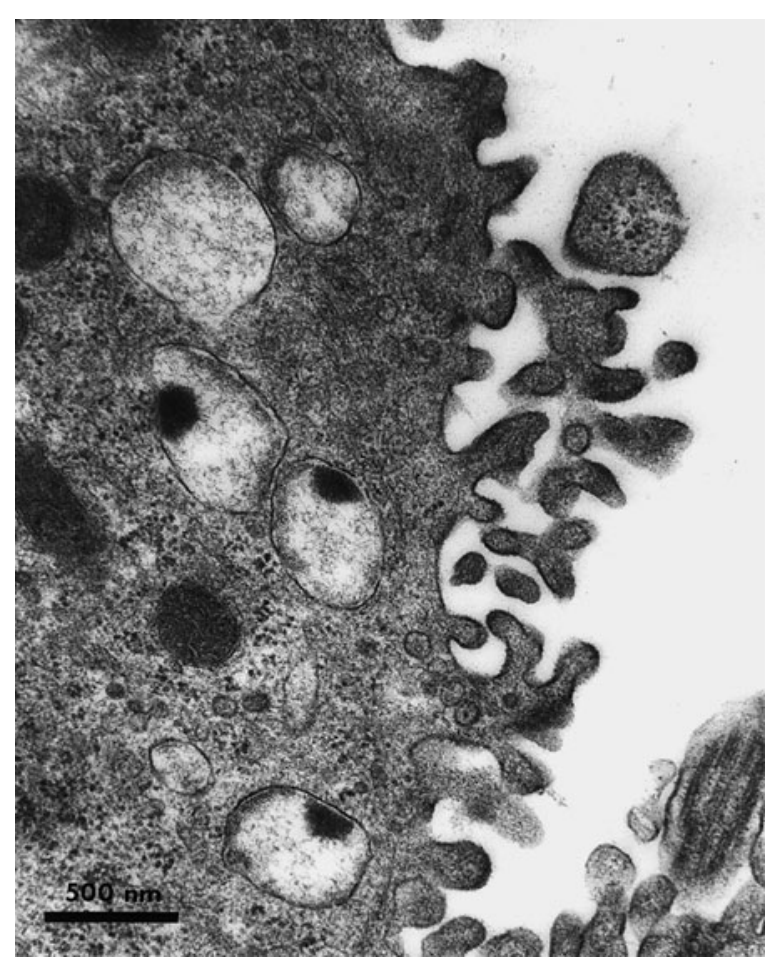

Fig. 115. Numerous subplasmalemmal microfilaments in an alveolar macrophage eliminated via the ciliated epithelium. Lung (block S2) of a female rat (breeder Winkelmann, Borchen-Kirchborchen) exposed to $20 \mathrm{mg}$ quartz-free shale dust $/ \mathrm{m}^{3}$ (particles less than $5 \mu \mathrm{m}$ in size; origin: seem Erda, Fürst Leopold-Baldur mine at Harvest-Dorsten) in Polley's $(1963,1965)$ dust channel $4 \mathrm{~h}$ per day, 5 days per week. Fixed under methitural anaesthesia by intratracheal instillation of $2.5 \%$ glutaraldehyde in phosphate buffer ( $\mathrm{pH} 7.4)$ before opening the thorax. Postfixation with $1 \%$ osmium tetroxide in phosphate buffer ( $\mathrm{pH}$ 7.4). Contrasted en bloc for $12 \mathrm{~h}$ in $0.5 \%$ uranyl acetate in $70 \%$ ethanol. Embedded in methylmethacrylate:butylmethacrylate $2: 8$. Sectioned at $50 \mathrm{~nm}$. Lead citrate after Rey nolds (1963). Plate 3594 (from SchiLLER 1982)

Table 27. Actin-associated proteins
Myosin
Tropomyosin
Fimbrin
Alpha-Actinin
Gelsolin
Vinculin
Talin
Villin
Synaptopodin
Myopodin

chains of $M_{\mathrm{r}} 15,000$ and two light chains of $M_{\mathrm{r}}$ 20,000 (Hartwig and Stossel 1975, Trotter and ADELSTEIN 1979). It structure is, by analogy with other vertebrate myosins (WEEDS and LOWEY 1971, BURRIDGE 1979), likely to be characterised by two head regions, each of which is composed of a part of the heavy chain in association with one of each of the two classes of light chain. The $20 \mathrm{kDa}$ light chain plays a regulatory role: it must be phosphorylated in order for the myosin to be activated by actin (Trotter and Adelstein 1979). Myosin from rabbit alveolar macrophages is heterogeneous with respect to its $20 \mathrm{kDa}$ light chain in a ratio of $2: 1$ (Trotter et al. 1983).

The multiple non-muscle-type myosin heavy chain isoforms can be grouped into two myosin heavy chain A and B types, the latter being predominantly expressed in brain (SUN and CHANTLER 1992, TAKAHASHI et al. 1992) and the former essentially in muscle and in non-muscle-non-brain tissues (MuraKami et al. 1993).

Tropomyosin may be involved in the regulatory subunits that mediate cooperativity within thin filament (reviewed by El-SALEH et al. 1986). Head-totail polymerisation of tropomyosin within the thin filament (MAK and SMILLIE 1981) could provide a means for co-operative interaction between functional groups. In a test of this idea WALSH et al. (1984) found that the response of regulated actinactivated myosin ATPase to $\mathrm{Ca}^{2+}$ was unaffected by removal of regions of overlap between adjacent tropomyosin molecules. However, PAN et al. (1989) found that removal of head-to-tail overlap of tropomyosin molecules reduced the cooperativity of S1ADP binding to reconstitutes thin filaments, and they concluded that overlap of adjacent tropmyosin is necessary for near-neighbour interactions. The difference in results between the two studies may be due to the low ionic strength of the assay system (approximately $20 \mathrm{mM}$ ) used by WALSH et al., and their low rations $(1: 100)$ of myosin relative to actin. Thus far, the role of tropomyosin overlap in the apparent cooperativity of tension development has not been studies directly since it as not been feasible to modify the tropomyosin content of skinned fibres.

The expression of nonmuscle tropmyosin was restored to pretransformation levels in ouabainselected revertants of v-Ki-ras transformed $\mathrm{NIH}$ / 3T3 cells (BAssin and NodA 1987).

Alpha-Actinin is a rod-like $(3-4 \mathrm{~nm} \times 30-40 \mathrm{~nm})$ cytoskeletal protein belonging to the same family as spectrin, dystrophin and utrophin. $\alpha$-Actinin is a homodimer with a subunit molecular weight of $94-103 \mathrm{kDa}$ in which the subunits are antiparallel in orientation. The molecule can be devised into three domains, an N-terminal actin binding domain (approximately residues 1-245), four internal 120 residue repeats, and a C-terminal region containing two EF-hand $\mathrm{Ca}^{2+}$-binding motifs (BARoN et al. 1987, BLANCHARD et al. 1989). Apart from actin, $\alpha$-actinin has been reported to bind to the cytoskel- 
etal protein vinculin (WACHSstock et al. 1987), nebulin (NAve et al. 1990), clathrin (Merisko et al. 1988 ), and to the cytoplasmic domain of the $\beta 1$ family of integrins, receptors for extracellular matrix proteins (ОTEY et al. 1990).

Gelsolin is the most potent actin filament severing protein identified to date (SUN et al. 1999). Severing is the weakening of enough non-covalent bonds between actin molecules within a filament to break the filament in two. Membrane ruffling is a functional readout for a co-ordinated series of membrane and cytoskeletal events, and it is activated by the small GTPase, Rac. Gelsolin null fibroblasts have increased Rac expression (Azuma et al. 1998), and Rac-GTP dissociates gelsolin-actin complexes (equivalent to uncapping) in cell extracts but not purified gelsolin-actin complexes (ARCARo 1998).

The rapid depletion of plasma gelsolin following major trauma in patients who subsequently develop respiratory distress suggested that this actinscavenging protein might protect against delayed pulmonary complications. CHRISTOFIDOU-SOLOMIDou et al. (2002) measured gelsolin levels in three murine models of oxidant injury: immunotargeting of pulmonary endothelium with an $\mathrm{H}_{2} \mathrm{O}_{2}$-generating enzyme; continuous exposure to $>95 \% \mathrm{O}_{2}$; and single high-dose thoracic irradiation. The degree of lung injury was inversely related to gelsolin levels in mice treated with glucose oxidase-conjugated antibodies against platelet endothelial cell adhesion molecule-1 $(P<0.0001)$. By $60-72 \mathrm{~h}$ of hyperoxic exposure, gelsolin levels had dropped precipitously in all mice who sustained major lung damage $(P$ $<0001$ ), establishing a quantitative association between gelsolin concentration and hyperoxic lung injury ( $\mathrm{r}=-0.72 ; 95 \%$ confidence interval: -0.81 to -0.59). Gelsolin levels modestly but progressively fell in irradiated mice over the 3 days following treatment $(P=0.012)$ despite the development of only microscopic lung damage during this timeframe.

Gelsolin is expressed at low levels in most cancer cell lines and is up-regulated during in vitro differentiation induced by agents such as phorbol esters and histone acetylase inhibitors in leukaemic (KwIATKOWSKI 1988) and epithelial cell lines (JARRARD et al. 1998). Gelsolin has also been shown to have decreased expression in lung cancers compared with histologically normal surrounding lung (DosAKA-AkiтA et al. 1998). Ciglitizone treatment resulted in a prominent induction of gelsolin in multiple cell lines, although 15 -deoxy- $\Delta^{12,14}$-prostaglandin $\mathrm{J}_{2}$ had a lesser effect in the same cell line (Chang and Szabo 2000).

Vinculin and talin are major components of focal contacts that interact with each other and that are thought to be involved in linking actin filaments to integral membrane proteins. Microinjection studies with anti-vinculin antibody (NucKolls et al. 1992) indicated that vinculin is a key protein in the microfilament-membrane linkage and that talin is essential for the development of focal contacts. In cultured chick embryo fibroblasts, a fraction $(2-5 \%)$ of the newly synthesised vinculin and talin reached maximal levels in the cytoskeleton in 30-45 min (LeE and Отто 1997). Both proteins had 2-3 times shorter half-lives in the cytoskeletal pool $\left(t_{1 / 2}=6-7 \mathrm{~h}\right)$ than in the cytosolic pool $\left(t_{1 / 2}=\right.$ 14-15 h), which suggested that the incorporation of cytosolic vinculin and talin into the cytoskeleton does not involve a simple equilibrium between the two pools.

Talin is a large molecule $(270 \mathrm{kDa})$. Below $0.7 \mathrm{mg} / \mathrm{ml}$ it exists as a monomer. Above this concentration it begins to self-associate to form dimers (Molony et al. 1987). In many types of cultured cells, talin in concentrated in focal adhesions (BuRRIDGE and CoRNELl 1983), regions where bundles of actin filaments attach to the cytoplasmic face of the membrane and where the external face of the membrane adheres most tightly to the underlying substratum. Talin is also found in ruffling membranes and subjacent the bundles of extracellular matrix on the cell surface (Collier et al. 1982).

Talin interacts with the $\beta 1$ integrin subunit on the one hand and vinculin and actin on the other hand (HoRwitz et al. 1986). These proteins recognise distinct binding sites on talin (HoRwitz et al. 1986) and, as they are colocative to adhesion sites on the plasma membrane (BURRIDGE et al. 1990), it is likely that talin plays a pivotal role in associating matrix recognising integrins with the cytoskeleton.

BAss et al. (1999) defined the three vinculinbinding sites in talin to residues $607-636,852-876$ and 1944-1969; alignment of these sequences shows $59 \%$ similarity, although there are only two identical residues. Predictions of secondary structure indicate that this vinculin-binding motif forms an amphipathic $\alpha$-helix. The hydrophobic face of helix 607-637 contains three aligned leucines (residues 608,615 and 622), which show conservative substitution in the other two site.

Phosphorylation of talin is prompted by the exposure of $1,25(\mathrm{OH})_{2}$ vitamin $\mathrm{D}_{3}$ pre-treated CSF-1 dependent macrophage-like BAC $1.25 \mathrm{~F} 5$ cells to phorbol 12-myristate 13-acetate in place of CSF-1 (Meenakshi et al. 1993).

Binding of calmodulin to the microfilament network correlates with induction of a macrophage tumoricidal response (МесHAM et al. 1985).

Calponin (TAKAHASHi et al. 1988, El-MezGUELDi 1996) and caldesmon (SobUe and Sellers 
1991) are two thin filament associated proteins that bind to F-actin, tropomyosin and calmodulin. Interaction of $34 \mathrm{kDa}$ calponin with F-actin and tropomyosin occurs in a $\mathrm{Ca}^{2+}$-independent manner, whereas that with calmodulin is regulated in a $\mathrm{Ca}^{2+}-$ dependent manner. Despite their apparent functional similarity, sequence analysis indicated that calponin and caldesmon are not related proteins. They act by different mechanisms of inhibition and bind to distinct thin filament populations in smooth muscle cells (Sobue and Sellers 1991, TAKAHASHI and NADAL-Ginard 1991, North et al. 1994).

Ultrastructural evidence that calponin binds selectively to "cytoskeletal" actin has led some investigators to propose that the function of calponin in smooth muscle may be structural rather than regulatory. Based on evidence that calponin binds to desmin as well as $\beta$ actin, MABUCHI et al. 1997) proposed that calponin may function as a bridging protein between actin and intermediate filament networks at dense bodies. Ultrastructural analysis of thin filaments by Hodg KINson et al. (1997) indicated that the location of calponin on F-actin is similar to that of the actin cross-linking protein fimbrin, as well as to that of $\alpha$-actinin and gelsolin.

Profilins are small, highly abundant cytoplasmic proteins that bind actin, poly-L-proline (TANAKA and Shibata 1985), and polyphosphoinositides, raising the possibility that they are second messengers to carry informations between the polyphosphoinositide signalling pathway and the cytoskeleton. From knockout mutants of profilins, such as generated in mice, Dictyostelium and yeast, it became evident that profilins are essential components of the microfilament system (SCHLÜTER et al. 1997). The soluble actin-binding protein profilin binds up to five molecules of phosphatidylinositol4,5-biphosphate, but not phosphatidylcholine, phosphatidylserine or phosphatidyl ethanolamine, with reasonably high affinity; this binding inhibits both the interaction between profilin and actin and the hydrolysis of phosphatidylinositol-4,5-biphosphate by soluble phosphoinositidase C. GolDSCHMIDT-CLERMONT et al. (1991) have shown that in the presence of profilin, phospholipase $C \gamma_{1}$ activity is dependent upon phosphorylation state; i.e. phosphorylation of phospholipase $C \gamma_{1}$ by the epidermal growth factor receptor relieves the tonic inhibition of phospholipase $\mathrm{C} \gamma_{1}$ activity by profilin. The considerable increases in a highly charged lipid such as phosphatidylinositol-3,4,5-triphosphate (STEPHENS et al. 1991) are likely to exert profound effects upon the inner leaflet of the plasmalemma and might be expected for this reason alone to modify cytoskeleton-membrane interactions.
Ezrin, moesin and radixin (ERM), three actinbinding proteins, which link actin filaments to the plasma membrane (Tsukita et al. 1997), are primary located in microvilli on the apical surface of epithelial cells, although they have also been observed at intercellular boundaries (BRETSCHER et al. 1997). Rho can colocalise to the plasma membrane with ERM proteins, and Rho inactivation prevents the localisation of ERM proteins and vinculin to the plasma membrane (KoTANi et al. 1997). RhoGDI apparently binds in a complex with CD44 and ERM proteins, and could therefore act as an intermediary to target Rho to ERM proteins, or alternatively ERM proteins could increase Rho activity by titrating out RhoGDI (TAKAHASHI et al. 1997). ERM protein interaction with actin is required for Rho and Rac to induce actin reorganisation and focal complex assembly in permeabilized fibroblasts (MACKAY et al. 1997). A Cdc42-interacting protein, CIP4, shows sequence homology to a small region of the ERM proteins and may act as a transducer to the actin cytoskeleton as it induces actin reorganisation when overexpressed in Swiss 3T3 cells (AsPENSTRÖM 1997).

Synaptopodin (formerly called pp44), a member of a family of actin-associated proteins rich in proline, was found in telencephalic dendrites and renal podocytes (Mundel et al. 1997). The cytoskeletal rearrangements of mouse podocyte clones are accompanied by the onset of synaptopodin synthesis.

Myopodin, another member of this family, is associated with the $\mathrm{Z}$ disc of human skeletal muscle (MUNDEL et al. 1999).

\subsubsection{2 \\ Tubulin and Microtubuli}

Microtubules are proteinaceous organelles, present in nearly all eukaryotic cells, made of subunits of tubulin molecules assembled into long tubular structures, with an average exterior diameter of $24 \mathrm{~nm}$, capable of changes of length by assembly or disassembly of their subunits. Macrotubules with a diameter of 31 to $52 \mathrm{~nm}$, which are related to the paracrystalline tubulin assemblies produced by Vinca alkaloids, result from a helical winding of protofilaments (Tilney and Porter 1967). Their formation is an indication of changes in the lateral links of tubulin protofilaments. The protein tubulin is acidic, combined with two molecules of guanine nucleotides.

The $\alpha \beta$ tubulin heterodimer is the structural subunit of microtubules. Each tubulin monomer binds a guanine nucleotide, which is non-exchangeable when it is bound to the $\alpha$ subunit, or $\mathrm{N}$ site, and exchangeable when bound in the $\beta$ subunit, or 
E-site. The $\alpha$ - and $\beta$-tubulins share $40 \%$ aminoacid sequence identity, both exist in several isotype forms, and both undergo a variety of posttranslational modifications (LUDVEÑA 1998). Limited sequence homology has been found with the proteins FtsZ (MuKheRJEe and LutKenhaus 1994) and Misato (GABOR MikLos et al. 1997), which are involved in cell division in bacteria and Drosophila, respectively. Nogales et al. (1998) presented an atomic model of the $\alpha \beta$ tubulin dimer fitted to a 3.7- $\varnothing$ density map obtained by electron crystallography of zinc-induced tubulin sheets. The structures of $\alpha$ - and $\beta$-tubulin are basically identical: each monomer is formed by a core of two $\beta$-sheets surrounded by $\alpha$-helices. The monomer structure is very compact, but can be devided into three functional domains: the amino-terminal domain containing the nucleotide-binding region, an intermediate domain containing the taxol-binding site, and the carboxyl-terminal domain, which probably constitutes the binding surface for motor proteins.

Fresh human blood mononuclear cells contained an average of 26 microtubules per cell which significantly increased to 31 microtubules per cell following a 30 -min exposure to LPS $(P<0.001)$. Using a nocodazole-based assay of microtubule dynamic instability, the half-life of fresh unstimulated monocyte microtubules was approximately $18 \mathrm{~s}$ and extended to $26 \mathrm{~s}$ following a $30-\mathrm{min}$ exposure to LPS (Allen et al. 1997). Endotoxin caused a rapid alteration in monocyte microtubule stability (ALLEN et al. 1997).

The direct interaction of peroxisomes with the microtubular network was visualised by SCHRADER et al. (1994, 1996), showing a specific binding of isolated peroxisomes to microtubules. In vitro, the binding of peroxisomes to the microtubular network was sensitive to pre-treatment of the organelles with high concentration of $\mathrm{KCl}$ or proteases.

Synaptosomal microtubules, contrary to axon microtubules (MATUs et al. 1981), are associated in rat brain with high molecular weight microtubule associated proteins which are susceptible to an endogenous $\mathrm{Ca}^{2+}$-dependent protease, proteolysis being essentially complete in $5 \mathrm{~min}$ at $37^{\circ} \mathrm{C}$. This could explain the difficulty in demonstrating the microtubule associated proteins by histochemistry (Burgoyne and Cumming 1982).

In tumour cells, the 3-D reconstruction gives a clear image of the spatial arrangement of tubulin fibres in relation to cell shape and position of other cellular organelles, particularly the nucleus (STrohmaier et al. 2000). The tubulin forms an intricate network of fibres of variable thickness. The highest tubulin concentrations appear in the cell periphery and particularly in pseudopodia/invado- podia. This is indicative of an enhanced transport of intracellular material facilitating cell movement and lysis of the extracellular matrix.

Melatonin antagonised the action of colchicine on melanocytes (MALAW ISTA 1965). It inhibited the regeneration of cilia in Stentor, and this effect was antagonised by colchicine (BANERJEe et al. 1972). On the other hand, the two drugs inhibited synergistically the regeneration of the oral band, suggesting that both bound to microtubule protein. In HeLa and KB cells preincubation for $1 \mathrm{~h}$ in $10^{-5} \mathrm{M}$ melatonin protected the microtubuli of the spindle against the action $10^{-7} \mathrm{M}$ colchicine (FitzGerald and Veal 1976). High concentrations of melatonin prevented ${ }^{3} \mathrm{H}$-colchicine binding to brain tubulin (Winston et al. 1974), a result contradicted by the findings of Poffenbarger and Fuller (1976) that melatonin does not affect in vitro assembly of bovine brain tubulin nor the mitosis of Chinese hamster ovary cells. In retinal ganglion cells of male New Zealand albino rabbits $1.5 \mu \mathrm{g}$ or $15 \mu \mathrm{g}$ melatonin injected 90 min before ${ }^{3} \mathrm{H}$-leucine inhibited fast axoplasmic flow by $71.9 \pm 8.4 \%$ and $87.2 \pm 4.6 \%$, respectively (Cardinali and Freire 1975). Under these conditions melatonin tended to increase ${ }^{3} \mathrm{H}$ leucine incorporation into retinal proteins in vitro (not significant).

Halothane $(20 \mathrm{mM})$ significantly $(P=0.001)$ prevented repolymerisation of microtubules in the sciatic nerve of the rat, when depolymerization was induced in vitro by incubation at $0^{\circ} \mathrm{C}$ for $30 \mathrm{~min}$ (Livingston and Vergara 1979).

There is increasing evidence that the microtubules are an important component in the interaction between the cytoskeleton and specific mRNAs. Microtubule preparations have been found to contain ribosomes and polysomes (HAMILL et al. 1994) and inhibitor studies showed that mRNA localisation in oocytes is sensitive to colchicine (YISRAELI et al. 1990, Pokrywka and Stephenson 1994). A cytoskeletal fraction from Drosophila oocytes is enriched in bicoid mRNA, and this mRNA is released by microtubule-disrupting agent colchicine (РоKRYWKA and STEPHENSON 1994).

Chlorpromazine arrests cultured cells in mitosis and disorganises the organised microtubule structure produced by cyclic adenosine monophosphate (Poffenbarger and Fuller 1977). It causes a reduction in the number of microtubules in spinal ganglion cells (EDSTRÖm et al. 1973, ThYBERG et al. 1977) and neuroblastoma cells (EDSTRöm et al. 1975) in vitro. The micellar form of chlorpromazine interacts preferentially with one site on brain tubulin (CANN et al. 1981). Chlorpromazine has been shown to bind reversibly to tubulin prepared from mouse brain via two well-resolved processes (HIN- 
Table 28. Microtubule-associated proteins

MAP 1 Protein

MAP 2 Proteins

$\tau$-Proteins

Ankyrin

Dyneins

MAP 1 and MAP 2 are basic proteins (ERICKSON and VOTER 1976).

MAN and CANN 1976). One molecule binds strongly compared with 8-9 molecules that bind weakly.

Aluminium induced nonenzymatic phospho-incorporation into human $\boldsymbol{\tau}$-proteins (ABDEL-GHANY et al. 1993). While $500 \mu \mathrm{M} \mathrm{Fe}^{3}+$ induced $\left[\gamma^{3}{ }^{32} \mathrm{P}\right] \mathrm{ATP}-$ incorporation only weakly, $500 \mu \mathrm{M} \mathrm{Sc}^{3+}$ were twice more effective than $500 \mu \mathrm{M} \mathrm{Al}^{3+}$.

Dyneins are proteins with adenosine triphosphatase activity (Gibions and Rowe 1965). These multipeptide complexes have molecular masses between $5.4 \times 10^{6}$ and $6 \times 10^{5} \mathrm{Da}$. They have been fractionated by proteolysis into fragments of $135 \mathrm{kDa}$ to $400 \mathrm{kDa}$ (WARNER and Mitchell 1980). WARNER and McIlvain (1982) analysed the binding properties of Tetrahymena 21S dynein to doublet A and B subfiber microtubules by both a turbidimetric assay $(\triangle \mathrm{A} 350 \mathrm{~nm})$ and electron microscopy. KClextracted, sucrose-gradient, purified $21 \mathrm{~S}$ dynein bound to each of the two kinds of axonemal microtubules in both ATP-insensitive and ATP-sensitive modes, even though only a single type of binding occurred to each of the subfibers in situ. REED et al. (2000) characterised airway epithelial expression of a gene identified by two human expressed sequence tags that encoded peptides with sequence similarity to an invertebrate ciliary dynein heavy chain. Molecular analyses showed that the gene has a very large RNA transcript that encodes a very high molecular weight polypeptide with biochemical properties that are characteristic of a dynein heavy chain. Expression of the gene transcript correlated with the presence of ciliated cells in tissues, and immunohistochemical localisation of the gene product confirmed its presence in the cilia of mature airway epithelium.

In addition to axonemal dyneins, there are cytoplasmic dyneins, distinct isoforms that transport molecular cargoes along cytoplasmic microtubules and participate in aspects of cell division (KARKI and Holzbaur 1999).

\subsubsection{3 \\ Intermediate Filaments}

Structure, dynamics, function, and disease of intermediate filaments were reviewed by FuCHS and WEBER (1994).
Table 29. Molecular types of intermediate filaments

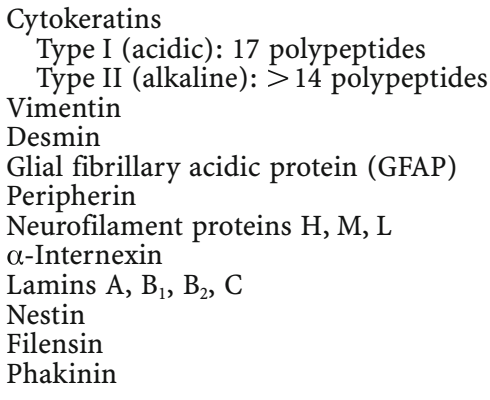

\section{Intermediate Filaments of the Vimentin Type}

Intermediate filaments of 7-11 nm diameter (WEBER and OSBORN 1982) of the vimentin type (FrANKe et al. 1979) are arranged immediately around the cell nucleus, while the remaining cytoplasm reveals only small amounts of $10 \mathrm{~nm}$ filaments, which usually do not extend up to the outer membrane of the mononuclear phagocyte (CAIN et al. 1982, 1983). With increasing differentiation of monocytes into mature macrophages and epithelioid cell equivalents, a loosening up of the perinuclear vimentin filament network was observed. This development was associated with a straightening of the filaments, which could now be followed into the ectoplasm and into the cytoplasmic processes.

Western blotting analyses of the extract from thioglycollate-stimulated mouse peritoneal macrophages incubated with $1 \mu \mathrm{M}$ showed selective deimination of vimentin without detectable degradation (AsAgA et al. 1998). Double immunofluorescence staining of deiminated proteins and vimentin suggested localisation of deiminated vimentin around the periphery of the round-shaped nucleus, which was thought to be an early morphological sign of apoptosis.

The application of both crocidolite asbestos $(10 \mu \mathrm{g} / \mathrm{ml})$ and silicon carbide $(50 \mu \mathrm{g} / \mathrm{ml})$ affected the vimentin system of the Syrian hamster epithelial cell line (M3E3/C3) derived from the lung of a foetus on day 15 of gestation in a time-dependent manner (AUFDERHEIDE et al. 1994). The vimentin network, which normally appears as a filigree-like pattern throughout the cytoplasm, after exposure to asbestos for $38 \mathrm{~h}$ concentrated in bundles. Exposure to silicon carbide induced a concentration of vimentin filaments within the cells at the expense of the normally anastomosing network.

Human KD fibroblasts on per cell volume basis contained $151.6 \mathrm{ng}$ vimentin/ $\mu$ l (LAI et al. 1993). Protein phosphorylation was augmented by treatment of $600 \mathrm{nM}$ okadaic acid for $1 \mathrm{~h}$ in these cells. 


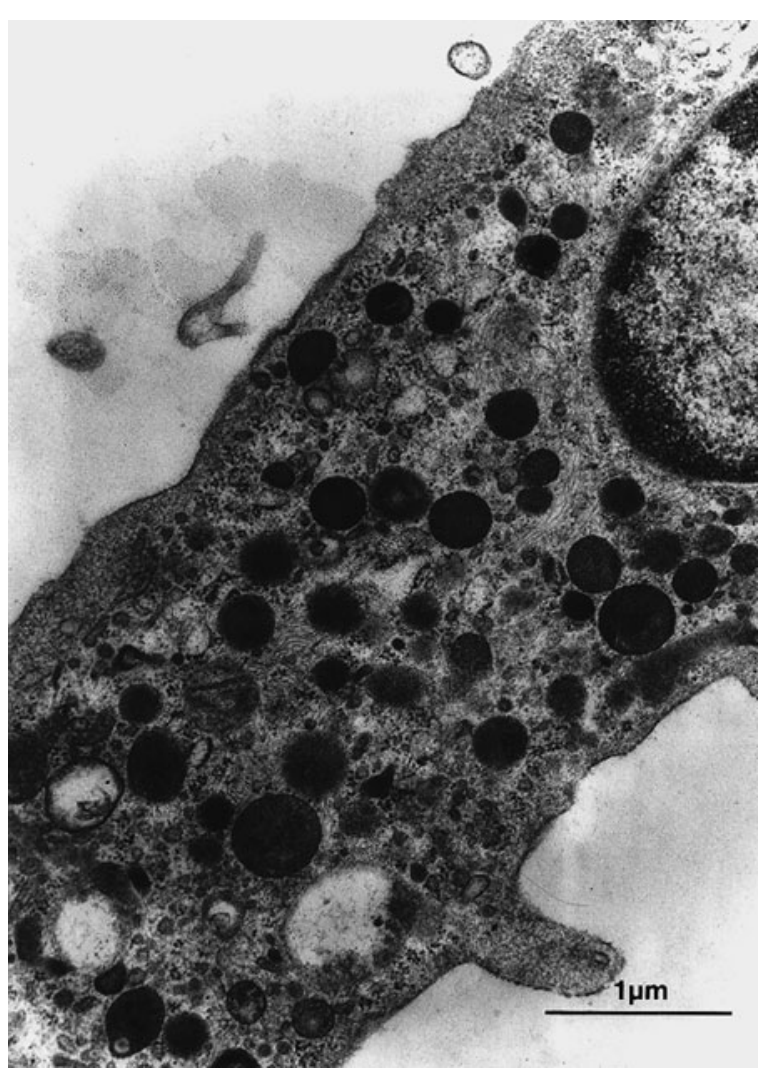

Fig. 116. Numerous intermediate filaments immediately around the cell nucleus in an alveolar macrophage (block 4424) from an unmedicated $235 \mathrm{~g}$ Sprague-Dawley rat (Charles River, France). On July 25, 1978, under pentobarbital anaesthesia $(30 \mathrm{mg} / \mathrm{kg})$, the animal was perfused from the abdominal aorta with $2.5 \%$ glutaraldehyde in $0.1 \mathrm{M}$ sodium cacodylate buffer ( $\mathrm{pH} 7.4$ ). Postfixation with $1 \%$ osmium tetroxide in sodium cacodylate buffer. Embedded in Epon 812 and sectioned at $50 \mathrm{~nm}$. Stained with lead citrate and uranyl acetate. Plate 4201

During the apparent activation of protein kinases, vimentin became hyperphosphorylated and the phosphorylation level of other nonvimentin phosphoproteins was relatively little affected in KD cells.

Glial fibrillary acidic protein (GFAP) immunostaining as a marker of astrocyte density increased in the oriens layer of the hippocampus of untreated bilateral carotid artery-occluded rats as compared to sham occluded animals (DE LA ToRRE et al. 1998).

\section{2 \\ Histochemistry}

\subsection{1}

\section{Glutathione}

Using the GSH-specific enzymatic assay with glyoxalase I, SibILLe et al. (1984) observed a GSH content of $5.96 \pm 0.61 \mu \mathrm{M} / 10^{6}$ human alveolar macrophages. The GSH content of freshly isolated rabbit lung macrophhages was $3.12 \pm 0.62 \mathrm{nmol} / 10^{6}$ cells; it was depleted to $1.63 \pm 0.32 \mathrm{nmol} / 10^{6}$ cells by incubating with $500 \mu \mathrm{M}$ diethyl maleate for $20 \mathrm{~min}$ (HorTon et al. 1987). Cysteine was best able to support resynthesis of GSH. There was no evidence for participation of a cystathionine pathway for glutathione synthesis.

\subsection{2 \\ Immunophenotypes}

The polyclonal anti pan-cathedrin antibody selectively decorated alveolar macrophages, type I pneumocytes, and endothelial cells of large blood vessels (KASPER et al. 1996).

\subsection{3 \\ Enzymes}

\subsubsection{1 \\ Oxidoreductases}

While interference with electron transmission and uncoupling of oxidative phosphorylation have no effect on the phagocytic function of polymorphonuclear leucocytes or monocytes they impair the activity of the alveolar macrophage (KARNOVSKY et al. 1966).

\section{Oxidoreductases with $\mathrm{NAD}^{+}$or $\mathrm{NADP}^{+}$ as Acceptor}

\section{Lactate Dehydrogenase (EC 1.1.1.27)}

\section{Lactate dehydrogenase (EC 1.1.1.27)}

$\mathrm{S}$-Lactate $+\mathrm{NAD}^{+} \rightarrow$ Pyruvate $+\mathrm{NADH}$

Rat alveolar macrophages contain five lactate dehydrogenase isoenzymes, $\mathrm{LDH}_{5}$ being the most prominent (BANSAL and KaW 1981). Silica-exposed macrophages liberated $\mathrm{LDH}_{5}$ in the supernatant culture medium. This LDH isoenzyme also increased in the serum of silicotic rats in the early stages of the development of the disease.

\subsubsection{2 \\ Oxidoreductases with Oxygen as Acceptor}

\section{Xanthine Oxidase (EC 1.1.3.22)}

Bovine lung macrophages produced 386 pmol uric $\mathrm{acid} / \mathrm{min} \times \mathrm{mg}$ protein (BRUDER et al. 1983).

Tubaro et al. (1980) reported a marked increase in the xanthine oxidase activity of macrophages obtained from infected animals compared with those obtained from normal mice. 


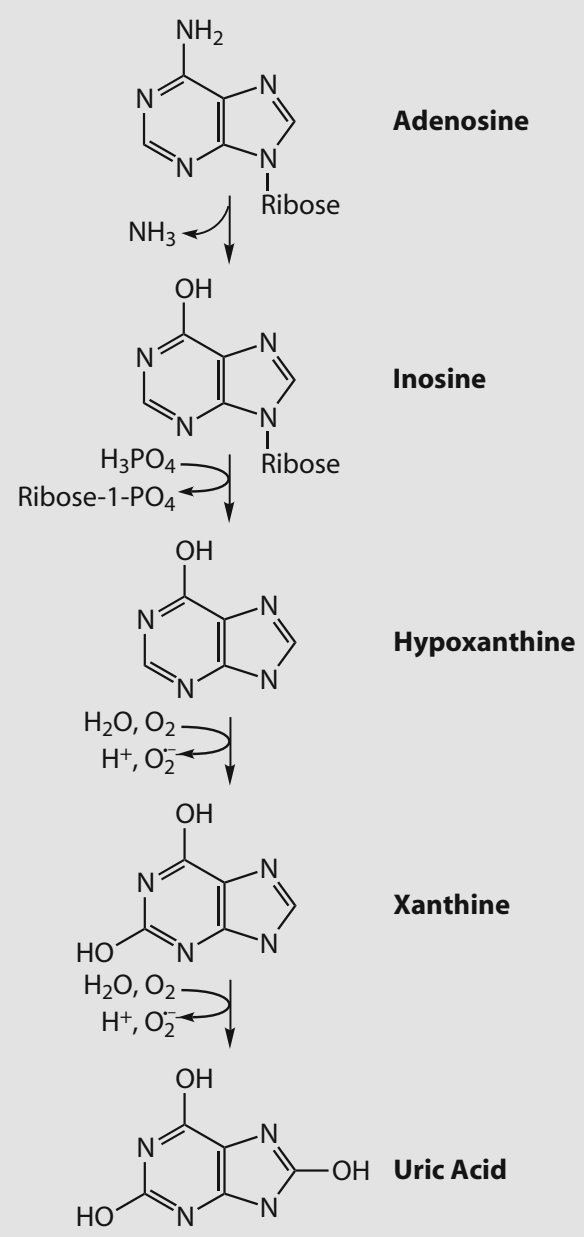

Substrates of xanthine oxidase

\subsubsection{3}

\section{Oxidoreductases with Reduced NAD or NADP as Donators}

Increased oxidative stress in the RAW 264.7 macrophage cell line is partially mediated via the $S$ nitrosothiol-induced inhibition of glutathione reductase (EC 1.6.4.2) (BUTZER et al. 1999). Fujil et al. (2000) isolated a cDNA for rat glutathione reductase and constructed a baculovirus system to produce recombinant glutathione reductase on a large scale. NO donors ( $S$-nitrosoglutathione, SIN-1, and $S$-nitroso- $N$-acetyl-D, L-penicillamine) inhibited the enzymatic activities of purified glutathione reductase.

\subsubsection{4 \\ Oxidoreductases with Hydrogen Peroxide as Donator}

Catalase (EC 1.11.1.6)

Detoxification of hydrogen peroxide by catalase (EC 1.11.1.6)

$$
2 \mathrm{H}_{2} \mathrm{O}_{2} \longrightarrow 2 \mathrm{H}_{2} \mathrm{O}+\mathrm{O}_{2}
$$

Salicylic acid may bind to and inactivate catalase, thus affecting $\mathrm{H}_{2} \mathrm{O}_{2}$ (SUNDARESAN et al. 1995).

\section{Peroxidase (EC 1.11.1.7)}

In HL-60 cells, the peroxidase reaction was observed in the nuclear envelope, primary granules, endoplasmic reticulum and Golgi body (MiKAMI et al. 1998).

\subsubsection{5 \\ Oxidoreductases Acting on Single Donors with Incorporation of Molecular Oxygen (0xygenases)}

Lipoxygenase (EC 1.13.11.12) catalyses the stereospecific dioxygenation of polyunsaturated fatty acids containing a 1,4-cis,cis-pentadiene system to a pentadienyl radical intermediate which reacts with molecular oxygen to yield cis,trans-conjugated diene hydroperoxides (WISEMAN et al. 1988).

5-Lipoxygenase (EC 1.13.11.12) and 5-lipoxygenase-activating protein are key proteins in leukotriene formation. In both unstimulated normal and ARDS human alveolar macrophages the two proteins were found by immonoelectronmicroscopy in the cytoplasm followed by the cell surface and nuclear membranes (CHI et al. 1996). After lipopolysaccharide treatment, approximately twofold increases in the number of immunogold particles/unit area were observed in the cytoplasmic and nuclear membrane compartments. In activated cells, both 5-lipoxygenase and 5-lipoxygenase-activating protein distributed primarily to the cytoplasmic filament network and secondarily to the endoplasmic reticulum.

On stimulation of rat alveolar macrophages with the calcium ionophore A-23187 ( $1 \mu \mathrm{M}$ for $30 \mathrm{~min})$, synthesis of leukotriene $\mathrm{B}_{4}$ increased with the degree of maturation, although it was diminished in the oldest subpopulation (Covin et al. 1998). This maturation-dependent upregulation was not explained by increases in arachidonic acid release but was associated with increased expression of 5lipoxygenase protein as determined by immunoblot analysis. Whereas 5-lipoxygenase is primarily cyto- 
solic in monocytes, it is known to be primarily intranuclear in unfractionated alveolar macrophages. Covin et al. (1998) investigating the localisation of 5-lipoxygenase by immunofluorescence microscopy found it predominantly nuclear in all alveolar macrophage subpopulations. By contrast, the protein was cytosolic in interstitial macrophages isolated by mechanical and enzymatic lung digestion.

\subsubsection{6 \\ Oxidoreductases Acting on Paired Donors with Incorporation of Molecular Oxygen}

Prolyl 4-hydroxylase (EC 1.14.11.2) is a key enzyme required for the posttranslational hydroxylation of proline residues in collagen. The enzyme consists of a tetramer composed of two pairs of nonidentical subunits $\left(\alpha_{2} \beta_{2}\right)$ of $\approx 60 \mathrm{kDa}$ each (BERG et al. 1979). Peptide mapping has demonstrated that the $\alpha$ and $\beta$ subunits are products of different genes (BERG et al. 1979). The $\beta$ subunit has been shown (PinlaJANIEMI et al. 1987) to be virtually identical with the enzyme protein disulphide isomerase (EC 5.3.4.1).

Alveolar macrophages obtained by pulmonary lavage from normal male New Zealand white rabbits and humans evaluated for their lung disease contained significant amounts of prolyl hydroxylase (KeLleHER et al. 1977).

When freshly isolated rat peritoneal macrophages were incubated in suspension with $\left[{ }^{14} \mathrm{C}\right]$ proline, they synthesised a small but significant amount of non-diffusible hydroxy $\left[{ }^{14} \mathrm{C}\right]$ proline (MYllylä and SEPPÄ 1979).

Nitric oxide synthases (EC 1.14.13.39), the inducible form of which has been obtained from the murine RAW 264.7 macrophage cell line (Hevel et al. 1991, STUEHr et al. 1991) was found by Liu et al. (1996) in the normal Lewis rat alveolar interstitium by immunostaining, whereas alveolar macrophages were iNOS negative. Interferon $\gamma$ maximally stimulated NO production by alveolar macrophages.

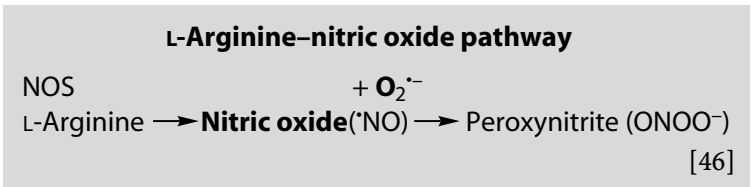

iNOS mRNA of bovine alveolar macrophages obtained by bronchoalveolar lavage immediately after isolation was below the detection limit (HöCKELE et al. 1997). Two or four h later increasing mRNA levels could be observed in both control and LPS stimulated cells. After $24 \mathrm{~h}$ treatment with LPS high levels of iNOS mRNA were induced while in control macrophages iNOS mRNA had declined to a low but detectable level.

Upon lipopolysaccharide stimulation $(1 \mu \mathrm{g} / \mathrm{ml})$, PrABHU et al. (2002) found significantly higher iNOS transcript and protein expression levels with an increase in NO production in selenium-deficient RAW 264.7 cells than in the Se-complemented cells. Electrophoretic mobility-shift assays, nuclear factor- $\varkappa \mathrm{B}$-luciferase reporter assays and Western blot analyses indicated that the increased expression of iNOS in Se deficiency could be due to an increased activation and consequent nuclear localization of the redox-sensitive transcription factor NF$x \mathrm{~B}$.

Co-cultivation of mouse RAW264.7 macrophages stimulated with interferon- $\gamma+$ lipopolysaccharide with human lymphoblastoid TK6 and Chinese hamster ovary AA8 cells resulted in a significant increase in mutant fraction in the endogenous genes of target cells and in the macrophages themselves, accompanied by a substantial decrease in cell viability (ZHUANG et al. 2002). Addition of $N$-methyl-Larginine abrogated much of the cytotoxicity and genotoxicity in both target and macrophage cells, verifying the role of ${ }^{\circ} \mathrm{NO}$ in the induction of these responses.

The human iNOS gene, containing 26 exons, excodes a protein of $131 \mathrm{kDa}$. Alternative messenger splicing from a single transcript allows for the generation of various forms of mRNA that can be translated into different protein products. These may have distinct functions and regulatory properties. Eissa et al. (1996) identified four sites of alternative splicing which included deletion of: i) exon 5 ; ii) exons 8 and 9; iii) exons 9, 10 and 11; and iv) exons 15 and 16. Deletion of exon 5 (149 bases) leads to a translational frame shift and a premature stop codon in exon 6 predicting a truncated protein.

iNOS cDNA was cloned and characterised from CD-1 mice 4 days after an intraperitoneal injection of thioglycollate broth after incubation of isolated peritoneal macrophages for $16 \mathrm{~h}$ with IFN- $\gamma(10 \mathrm{ng} /$ $\mathrm{ml}$ ) and lipopolysaccharide $(1 \mu \mathrm{g} / \mathrm{ml}$ ) (XIE et al. 1992).

Activation of the peroxisome proliferator-activated receptor- $\gamma$ reduced proinflammatory cytokine and iNOS expression in macrophages (LEMBERGER et al. 1996, Colville-Nash et al. 1998, Ricote et al. 1998), microglial cells (Petrova et al. 1999), and monocytes (JiANG et al. 1998, Combs et al. 2000).

The amino acid sequence of mouse macrophage iNOS is only $51 \%$ identical to the deduced sequence of rat cerebellar constitutive nitric oxide synthase (cNOS) (BREDT et al. 1991).

Constitutive nitric oxide (cNOS) was produced by naive unstimulated rat alveolar macrophages 
(Miles et al. 1998). Using antibodies against two known types of cNOS, i.e., eNOS and bNOS, and an antibody against iNOS, positive results were obtained with the anti-eNOs antibody only. The amount of ${ }^{\circ} \mathrm{NO}$ formed was much less than that produced by eNOS in other cells, i.e., alveolar type II cells and endothelial cells. Some properties of the alveolar macrophage eNOS are similar to and some are different from the eNOS in these other cell types. Alveolar macrophage ${ }^{\circ} \mathrm{NO}$ levels do not seem to be related to cellular metabolism. "NO production was increased approximately threefold in the presence of dipalmitoyl phosphatidylcholine vesicles or pulmonary surfactant.

Type III (endothelial) NOS was detected by immunoperoxidase staining of bronchoalveolar lavage cytospins and normal human and rat lung tissue cryostat sections (КовzIK et al. 1996). In $\geq 90 \%$ of alveolar macrophages positive labelling was found by two separate monoclonal anti-type III NOS antibodies. Using cytofluometry to measure the effects of NOS substrate, L-arginine (1-5 mM), on intracellular basal or phorbol 12-myristate 13-acetate $\left(2 \times 10^{-7} \mathrm{M}\right)$-stimulated oxidative metabolism, Larginine, but not its inactive stereoisomer, Darginine caused a substantial, dose-dependent decrease in intracellular oxidation of dichlorofluorescin. Since nitric oxide can reversibly inhibit mitochondrial respiration, KовzIK et al. (1996) hypothesised that type III NOS in alveolar macrophages may function to modulate mitochondrial-derived reactive oxygen species production and redoxbased signalling.

NOS enzymes are multidomain proteins consisting of an $\mathrm{NH}_{2}$-terminal oxygenase domain that contains the active site, a $\mathrm{COOH}$-terminal reductase domain that shuttles electrons from NADPH to the heme iron (Sheta et al. 1994, StUehr 1997), and a central calmodulin domain that governs electron flow between the two domains (ABU-Soud and StUehr 1993). The NOS reductase domain shares sequence homology with the flavoenzyme, NADPHcytochrome P450 oxidoreductase (EC 1.6.2.4), the activating enzyme of the cytochrome $\mathrm{P} 450$ monooxygenase complex (BREDT et al. 1991).

Apigenin and kaempferol were markedly active inhibitors of transcriptional activation of iNOS in the mouse macrophage cell line RAW 264.7, with IC $_{50}<15 \mu \mathrm{M}$ (LIANG et al. 1999). In murine J774macrophages cyclosporin A (but not tacrolimus [FK506] at $1 \mu \mathrm{M}$ was inhibitory when co-incubated with the inducing agent but not when the cells were treated with the immunosuppressant before or after the inducer (Dusting et al. 1999). Cyclosporin A suppressed expression of mRNA for the inducible NO synthase 2 in a concentration dependent man- ner when co-incubated with lipopolysaccharide ( $E$. coli serotype 0111:B4) as shown in RNA extracted from J774-macrophages and subjected to reverse transcription-polymerase chain reaction.

For L-arginine supply to nitric oxide synthases by cationic amino acid transporters (CATs), Closs et al. (2000) studied mouse J774A1 macrophages and human EA.hy926 endothelial cells. CAT-1 was expressed in both cell types, whereas CAT-2B was only expressed in activated macrophages. Apparent $K_{\mathrm{M}}$ values for transport of L-arginine in both cell types were consistent with the expression of the system $\mathrm{y}^{+}$carriers CAT-1 (and CAT-2B in macrophages). L-Arginine transport was $\mathrm{Na}^{+}$independent and sensitive to trans-stimulation. At $2 \mathrm{~h}$ preincubation of activated macrophages in $2 \mathrm{mM} \mathrm{L}$ lysine (which is exchanged for $\mathrm{L}$-arginine by the CATs) reduced the intracellular L-arginine concentration from $2 \mathrm{mM}$ to $160 \mu \mathrm{M}$. At the same time, NOS II activity was completely abolished. NOS II activity could be restored with extracellular Larginine.

\section{Cytochrome P450 (CYP; EC 1.14.14.1)}

CYPs (EC 1.14.14.1) are a multiple family of constitutive and inducible enzymes that play a control role in the metabolic activation and detoxification of various xenobiotics, including small molecular weight compounds that cause allergic reactions. BARON et al. 1998) demonstrated that human blood monocytes and monocyte-derived macrophages constitutively express $\mathrm{P} 450$ isoenzymes. Of particular interest is the strong and constitutive expression of CYP1B1 in monocytes and macrophages demonstrated both on the mRNA and protein levels. CYP1B1 is the only gene of the CYP1B gene family which has been mapped in the human chromosome 2p21-22, which contains three exons and two introns, and is present especially in extrahepatic tissues (SutTer et al. 1994, TANG et al. 1996).

Macrophage subtype 27E10, which has been shown to possess pro-inflammatory activity (ZWADLo et al. 1986, BHARDWAJ et al. 1992), expressed 1B1, 2E1, and 2B6/7 (Baron et al. 1998). on the other hand, in the anti-inflammatory macrophage subtype $\mathrm{RM} 3 / 1$, predominantly $1 \mathrm{~B} 1$ and to some extent $2 \mathrm{~B} 6 / 7$ were found. Treatment with cyclosporin A, phenobarbital, benzanthracene or ethanol resulted in induction of the expression of $3 \mathrm{~A} 3 / 4$. Cyp 1B1 was the predominant isoenzyme in all monocytes and macrophages. In monocytes purified by adherence or induced by benzanthracene, lipopolysaccharide or 12-O-tetradecanoyl-phorbol13-acetate, 1A1 was also expressed. 


\section{Prostaglandin H Synthase (EC 1.14.99.1)}

Prostaglandin $\mathrm{H}$ synthases, also called cyclooxygenases, catalyse the two first steps in the biosynthesis of prostanoids such as prostaglandin and thromboxane. These membrane-linked enzymes exhibit heme-dependent cyclooxygenase and peroxidase activities which are responsible for the regio- and stereospecific synthesis of prostaglandin hydroxyendoperoxide $\mathrm{PGH}_{2}$ from arachidonic acid. As with ribonucleotide reductase and photosystem II, prostaglandin $\mathrm{H}$ synthases proceed via radical chemistry. The enzymatic reaction begins with the removal of the 13-pro $\mathrm{S}$ hydrogen atom of arachidonic acid to create a carbon-centred radical. A free radical chain transfer reaction then allows the addition of a first molecule of dioxygen on $\mathrm{C}_{11}$. A subsequent intramolecular cyclisation leads to a cyclic endoperoxide. Incorporation of a second molecule of dioxygen in $\mathrm{C}_{15}$ followed by the transfer of one hydrogen atom back to the substrate results in the formation of the cyclic hydroperoxyendoperoxide $\mathrm{PGG}_{2}$. The second step of the enzymatic reaction performed by the peroxidase activity involves a two-electron reduction of $\mathrm{PGG}_{2}$ to the cyclic hydroxyendoperoxide $\mathrm{PGH}_{2}$.

The tyrosyl radical has been shown to be involved in the initiation of the cyclooxygenase reaction, since it is able to generate an arachidonyl radical from arachidonic acid under anaerobic conditions (Tsai et al. 1995, 1998). Addition of dioxygen to this substrate-radical-bearing enzyme resulted in the regeneration of the tyrosyl radical, completing cyclooxygenase turnover. The crucial role of $\mathrm{Tyr}_{385}$ in prostaglandin $\mathrm{H}$ synthase- 1 ( $\mathrm{Tyr}_{371}$ in prostaglandin $\mathrm{H}$ synthase-2) in the catalytic mechanism was demonstrated using chemical modifications of tyrosine residues with tetranitromethane, peptide mapping and also by site-directed mutagenesis experiments (ShimokaVA et al. 1990, Tsai et al. 1998).

Vitamin E (30 ppm) inhibited cyclooxygenase activity in macrophages from old mice by reducing peroxynitrite production (BEHARKA et al. 2002). Increasing ${ }^{\circ} \mathrm{NO}$ levels alone using $S$-nitroso- $\mathrm{N}$-acetylpenicillamine or $\mathrm{O}_{2}{ }^{--}$levels, using xanthine/xanthine oxidase, had no effect; however, increasing peroxynitrite levels using SIN-1 or xanthine/xanthine oxidase $+S$-nitroso- $N$-acetyl-penicillamine significantly increased cyclooxygenase activity in macrophages from old mice fed 500, but not those fed $30 \mathrm{ppm}$ vitamin $\mathrm{E}$.

Desferrioxamine, an iron chelator, upregulated cyclooxygenase-2 expression and prostaglandin production in a human macrophage cell line, U937 (TANJI et al. 2001).

Aqueous cigarette tar extracts increased prostaglandin $\mathrm{H}$ synthase activity in isolated rat pul- monary alveolar macrophages 3-fold above the initial activity within $2 \mathrm{~h}$ of incubation and gradually decreased it below the initial activity after $8 \mathrm{~h}$ of incubation (HwANg et al. 1999).

Procainamide induced prostaglandin $\mathrm{H}$ synthase-2 in mouse peritoneal macrophages (GoEBEL et al. 1999).

In RAW 264.7 macrophages, lipopolysaccharideinduced cyclooxygenase-2-dependent synthesis of prostaglandin $\mathrm{E}_{2}$ was suppressed by aspirin $\left(\mathrm{IC}_{50}=\right.$ $5.35 \mu \mathrm{M}$ ), whereas no significant inhibition was observed in the presence of sodium salicylate and the salicylate metabolite salicyluric acid at concentrations up to $100 \mu \mathrm{M}$ (Hinz et al. 2000). However, the salicylate metabolite gentisic acid (2,5-dihydroxybenzoic acid; $10-100 \mu \mathrm{M})$ and salicyl-coenzyme A $(100 \mu \mathrm{M})$, the intermediate product in the formation of salicyluric acid from salicylic acid, significantly suppressed LPS-induced $\mathrm{PGE}_{2}$ production. In contrast, $\gamma$-resorcylic acid (2,6-dihydroxybenzoic acid) as well as unconjugated coenzyme $A$ failed to affect prostanoid synthesis, implying that the parasubstitution of hydroxyl groups and the activated coenzyme A thioester are important for cyclooxygenase-2 inhibition.

\subsubsection{7 \\ Oxidoreductases with Superoxide Radical as Acceptor}

The superoxide dismutases are a family of enzymes responsible for metabolising superoxide free radical $\left(\mathrm{O}_{2}{ }^{\circ-}\right)$ to produce hydrogen peroxide and water. Three types of SOD are found in mammalian systems: mitochondrial Mn-SOD, cytosolic CuZnSOD, and extracellular SOD (MARKLUND 1984, ZeLKo et al. 2002).

$$
\begin{gathered}
\text { Superoxide dismutase (EC 1.15.1.1) } \\
\qquad 2 \mathrm{O}_{2}^{--}+2 \mathrm{H}^{+} \longrightarrow \mathrm{H}_{2} \mathrm{O}_{2}+{ }^{3} \Sigma_{g}^{-} \mathrm{O}_{2}
\end{gathered}
$$

The ability of the CuZn-SOD to stimulate oxidation of substrates in the presence of $\mathrm{H}_{2} \mathrm{O}_{2}$ was first reported by Hodgson and Fridovitch (1975). The formation of carbonate (Goss et al. 1999) and nitro radicals (ZHANG et al. 1999) which carry the oxidising power out of the active site, accounts for the "peroxidase' activity of the dismutase. The formation of the copper oxidant at the active site has been examined by JEWETT et al. (1999) in terms of the sequence of the events leading to inactivation through 2-oxohistidine formation, copper(I) loss, sitespecific and random peptide fragmentation, and inactivation: 


$$
\begin{aligned}
& \left.2 \mathrm{Cu}^{+}+\mathrm{H}_{2} \mathrm{O}_{2} \leftrightarrow 2 \mathrm{Cu}^{+}+\mathrm{O}_{2}+2 \mathrm{HH}^{+}\right] \\
& \mathrm{Cu}^{+}+\mathrm{H}_{2} \mathrm{O}_{2} \longrightarrow(\mathrm{CuOH})^{2+}+\mathrm{OH}^{-}
\end{aligned}
$$

$$
(\mathrm{CuOH})^{2+} \mathrm{HCO}_{3}{ }^{-} \longrightarrow \mathrm{HCO}_{3}+\mathrm{H}_{2} \mathrm{O}
$$

CuZn-SOD is an iron-regulated protein in Aspergillus nidulans and A.fumigatus (OBEREgGer et al. 2000). After $24 \mathrm{~h}$ growth under iron deplete conditions, this $16-\mathrm{kDA}$ protein was approximately 5 -fold upregulated.

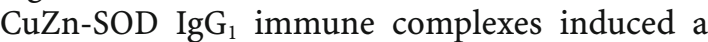
Fc $\gamma$-dependent intracellular delivery of the antioxidant enzyme in IFN- $\gamma$-activated murine J774 macrophages (Vouldoukis et al. 2000). The concomitant stimulation of the $\mathrm{Fc} \gamma$-receptor and the translocation of the SOD1 in the cytoplasm of IFN$\gamma$-activated macrophages not only reduced the production of superoxide anion but also induced the expression of iNOS and the related ${ }^{\circ} \mathrm{NO}$ production. This inducing effect in the absence of superoxide anion production reduced mitochondrial damage and cell death by apoptosis and promoted the intracellular antioxidant armature.

At constant peroxide, nitrite and azide only partially protect the enzyme against loss of copper(I) and activation up to one anion per copper (JEWETT et al. 2000).

Peroxynitrite reacts with CuZn-SOD to form a complex that selectively nitrates other proteins (IsCHIRopoulos et al. 1992). The Fe-SOD and MnSOD also catalyse nitration, but are slowly inactivated by peroxynitrite. The CuZn-SOD is not appreciably inactivated by the reaction with peroxynitrite. While the copper was necessary for nitration, the active site of superoxide dismutase could by modified to reduce the superoxide scavenging activity, but not significantly affecting the nitrating activity. For example, the essential amino acid arginine 141 which hydrogen bonds to the superoxide in the active site can be covalently modified with phenylglyoxyl with a $90 \%$ loss in superoxide scavenging activity (BEYER et al. 1987).

Mutation in CuZn-SOD causes $25 \%$ of familial amyotrophic lateral sclerosis cases. LiU et al. (2000) examined one such mutant, His46Arg, which has no superoxide dismutase activity yet presumably retains the gain-of-function activity that leads to disease. They demonstrated that $\mathrm{Cu}^{2+}$ does not bind to the copper-specific catalytic site of His46Arg CuZnSOD and that $\mathrm{Cu}^{2+}$ competes with other metals for the zinc binding site. Most importantly, $\mathrm{Cu}^{2+}$ was found to bind strongly to a surface residue near the dimer interface of His46Arg CuZn-SOD. Cysteine was identified as the new binding site on the basis of multiple criteria including UV-visible spectros- copy, resonance Raman spectroscopy and chemical derivatization. Cysteine 111 was pinpointed as the position of reactive ligand by tryptic digestion of the modified protein and by mutational analysis.

Extracellular superoxide dismutase mRNA expression was demonstrated in the alveolar macrophages of four patients undergoing surgical resections for carcinoma and carcinoid tumour, respectively (Su et al. 1997).

Analysis of a series of chimeric and point mutated extracellular SODs showed that the $N$-terminal region contributes to the oligomeric state of the extracellular SODs, and that a single amino acid, a valine (human amino acid position 24), is essential for the tetramerization (CARLSson et al. 1996). This residue is replaced by an aspartate in the rat. Rat extracellular SOD carrying an Asp $\rightarrow$ Val mutation is tetrameric and has a high heparin affinity, while mouse extracellular SOD with a Val $\rightarrow$ Asp mutation is dimeric and has lost its high heparin affinity.

\subsubsection{2 \\ Transferases}

Histamine $\boldsymbol{O}$-methyltransferase (EC 2.1.1.8) is present in human monocytes at a mean concentration of $10.08 \mathrm{nM} / \mathrm{h} / \mathrm{mg}$ protein (ZEIGER et al. 1976).

$O^{6}$-Alkylguanine-DNA-alkyltransferase (EC 2.1.1.63) activity of alveolar macrophages isolated from male New Zealand White rabbits was 15.81 $\mathrm{fM} / 10^{6}$ cells (Deilhaug et al. 1985).

$\boldsymbol{\gamma}$-Glutamyl transferase (EC 2.3.2.2), a glycoprotein enzyme widely distributed on cell surfaces, catalyses the conversion of leukotriene $\mathrm{C}_{4}$ to leukotriene $\mathrm{D}_{4}$. Since $\mathrm{LTD}_{4}$ is biologically much more potent than $\mathrm{LTC}_{4}$ (Lewis and AUSTen 1984, Piper 1984), the partial degradation of the glutathione moiety to the cysteinylglycine derivative $\mathrm{LTD}_{4}$ may be considered a biosynthetic reaction generating the ligand for the $\mathrm{LTD}_{4} / \mathrm{LTE}_{4}$ receptor.

\section{Radical scavenging effects of leukotrienes$$
\mathrm{LTD}_{4}>\mathrm{LTC}_{4}>\mathrm{LTB}_{4}
$$

$\gamma$-Glutamyltransferase was found by OHLsson et al. (1982) in canine alveolar macrophages gained by broncho-alveolar lavage and cultured in medium 199. The activity of the enzyme was $\mathrm{Ca}^{2+}$-dependent and did not increase after preincubation with thrombin. The incorporation of $\left[{ }^{14} \mathrm{C}\right]$ putrescine into casein catalysed by this transferase was partially inhibited by small concentrations of monodansylthiacadaverine.

R-Glutaminyl-peptide:amine $\boldsymbol{\gamma}$-glutamyltransferase (EC 2.3.2.13) activity increased during the 
course of differentiation of immature human myeloblastoid $\mathrm{M}^{-}$to mature $\mathrm{M}^{+}$cells more than 10 times (Kannagi et al. 1982).

\section{Glutathione S-transferase (EC 2.5.1.18)}

Glutathione S-transferase (EC 2.5.1.18) $\mathrm{RX}+$ Glutathione $\rightarrow \mathrm{HX}+\mathrm{R}-\mathrm{S}-\mathrm{G}$

The glutathione $S$-transferases are a group of enzymes, which catalyse the nucleophilic attack of GSH to many kinds of electrophiles (MANNERVIK and DANIELSON 1988). Most of glutathione $S$-transferase isoenzymes localize in the cytoplasm and are classified into 3 groups: class $\alpha, \mu$ and $\pi$. Human glutathione $S$-transferase $\pi$ can be selectively reduced by hydrogen peroxide (TAMAI et al. 1990). Rat class $\mu$ enzymes were activated by the xanthinexanthine oxidase system (Murata et al. 1990).

Glutathione $S$-transferase activity in pulmonary alveolar macrophages obtained from 11 patients by bronchoalveolar lavage was $0.22 \pm 0.12 \mathrm{nmol} / \mathrm{min}$ $\times$ mg protein (Petruzzelli et al. 1988). Glutathione $S$-transferase activity was $0.31 \pm 0.12 \mathrm{nmol} / \mathrm{min}$ $\times$ mg protein in non-smokers and $0.14 \pm 0.07 \mathrm{nmol} /$ min $\times$ mg protein in smokers $(P<0.001)$.

Adenosine kinase (ATP : adenosine 5'-phosphotransferase, EC 2.7.1.20) activity in 2-week human pulmonary alveolar macrophage cultures obtained from cigarette smokers (one to three packs per day) exhibited significant substrate inhibition with increasing adenosine concentrations (ZUCKERMAN and Douglas 1980).

Phosphofructokinase (EC 2.7.1.30) catalyses the phosphorylation of fructose-6-phosphate to fructose-1,6-biphosphate as the key regulatory enzyme of glycolysis. Inhibition of phosphofructokinase by adenosine triphosphate and its activation by adenosine monophosphate and inorganic phosphate is held responsible for the induction of the Pasteur effect (for review see Ramaiah 1974).

\subsubsection{3}

\section{Hydrolases}

Phospholipases $\mathrm{A}_{2}$ (EC 3.1.1.4) hydrolyse the fatty acyl ester bond at the $s n-2$ position of membrane phospholipids to yield free fatty acids such as arachidonic acid. Recognised isoforms include: i) low molecular weight, $14 \mathrm{kDa}$ proteins that are inhibited by reducing agents, require calcium for activation and exhibit no preference for the hydrolysis of arachidonic acid vs. other fatty acids; ii) cytosolic, an $85 \mathrm{kDa}$ protein that is resistant to reducing agents, calcium-requiring and preferentially hydrolysed arachidonic acid; and iii) calcium-indepen- dent phospholipase $A_{2}$, which is non-reducible and exhibits no preference for arachidonic acid hydrolysis, Rat alveolar macrophages obtained by bronchoalveolar lavage, disrupted by dounce homogenization and fractioned to yield 4 subcellular fractions (cytosolic, non-nuclear particulate, nuclear particulate and nuclear soluble). The $85 \mathrm{kDa}$ protein was found only in the cytosolic and nonnuclear particulate fractions, whereas the $14 \mathrm{kDa}$ protein was demonstrated in non-nuclear particulate and nuclear particulare fractions and the nonarachidonic acid selective calcium-independent isoenzyme activity was found only in the nonnuclear particulate fraction (Ojo and PetersGOLDEN 1996).

Freund's adjuvant $\left(1.4-140 \mu \mathrm{l} / 25 \times 10^{6}\right.$ peritoneal exudate cells of mice) induced a significant activation of phospholipase A (Munder et al. 1973).

Triacylglyceroprotein acylhydrolase (EC 3.1.1.34) was secreted by human blood monocytes and New Zealand White rabbit alveolar macrophages cultivated in vitro (MAHONEY et al. 1982).

Alkaline phosphatase (EC 3.1.3.1) was visualised (Ca-Co method of Gomori 1939) in guinea pig peritoneal macrophages cultivated in vitro (RILKE and Kessel 1963). In human skin window macrophages, however, LEDER and Nicolas (1963) and Nicolas and Leder 1965) were unable to detect this enzyme using Kaplow's (1955) technique.

Acid phosphatase (EC 3.1.3.2) is the classic lysosomal enzyme. The lead substitution method has localised acid phosphatase in the phagosomes of macrophages (СоHN and Wiener 1963) and can be used for electron microscopy.

Tartrate-resistant acid phosphatase is characterised for activated macrophages (EFSTRATIADIS and Moss 1985). Tartrate-resistant acid phosphatase contains a binuclear iron centre that has been shown to generate reactive oxygen species. RäIs ÄNEN et al. (2001) showed that the murine macrophage-like cell line RAW-264 overexpressing tartrate-resistant acid phosphatase produced elevated levels of hydroxyl radicals compared to parental cells.

5'-Nucleotidase (EC 3.1.3.5) hydrolyses the phosphoester linkage in 5'-mononucleotides, liberating a nucleoside and inorganic phosphate. It has been identified as an ecto enzyme (EDELSON 1980) in mouse alveolar macrophages (JAMES and EDELson).

Alkaline phosphodiesterase I (EC 3.1.4.1) hydrolyses polyribonucleotides or oligodeoxyribonucleotides which have a free 3'-OH group, sequentially liberating 5'-nucleoside monophosphate (KHORANA 1961). This enzyme has been recognised as a component of the plasma membrane of rabbit alveolar 
macrophages (WANG et al. 1976). In mouse peritoneal macrophages the specific activity of the enzyme was from two to fourfold higher in thioglycollate-stimulated cells than it was in any of the other macrophage varieties examined. Alkaline phosphodiesterase I levels per square micrometer of cell surface area were increased in certain fractions of line 66 vs. those of lines 168 and 67 of mouse mammary tumour-associated macrophages (MAHONEY et al. 1983).

Cyclic 3',5'-Adenosine Monophosphate Phosphodiesterase (EC 3.1.4.17)

Two of the seven cyclic nucleotide phosphodiesterase gene families PDE3 and PDE4, have a high affinity of cAMP and are specifically inhibited by cilostamide and rolipram, respectively. Using these inhibitors in assays with $0.1 \mu \mathrm{M}\left[{ }^{3} \mathrm{H}\right] \mathrm{cAMP}$ as substrate, GAO et al. (1996) found PDE3 activity $>$ PDE4 in primary blood macrophage cultures and in a human monocyte cell line THP-1, whereas in cultured U937 mononuclear cells, PDE4 >> PDE3.

Using reverse transcription and polymerase chain reaction, GAo et al. (1997) identified mRNAs of PDE3 and PDE4 subtypes in human inflammatory cells, including T lymphocytes, NK lymphocytes, B lymphocytes, human peripheral monocytes and macrophages, Jurkat $\mathrm{T}$ cells and cultured $h u$ man mononuclear cells U937 and THP-1. PDE3B, but not PDE3A, mRNA was expressed in all these cells except in B lymphocytes and U937 cells, which also exhibited low PDE3 activity (assays with $0.1 \mu \mathrm{M}$ $\left[{ }^{3} \mathrm{H}\right]$ cAMP as substrate. In fresh peripheral elutriated monocytes, PDE4 > PDE3, whereas PDE3 $>$ PDE4 in macrophages. mRNA for all PDE4 isoenzymes were present in U937 and T lymphocytes.

Arylsulphatase (EC 3.1.6.1) from a $96 \mathrm{~h}$ rabbit peritoneal exudate macrophage was present in segments of the rough endoplasmic reticulum, and perinuclear cisterna as well as within numerous small vesicles in the Golgi region, probably corresponding to secondary lysosomes (Nichols et al. 1971, DAVIEs and BonNeY 1980).

Glucosaminidase (EC 3.2.1.17): Lysozyme

Lysozyme (EC 3.2.1.17, mucopeptide $N$-acetylmuramoyl hydrolase or muramidase) catalyses hydrolysis of a $\beta(1 \rightarrow 4)$-glycosidic linkages of polysaccharide component of the peptidoglycan (mucopolymer of the bacterial cell wall), which is composed of alternating $\mathrm{N}$-acetylmuramic acid and $\mathrm{N}$-acetylglucosamine residues with alternating $\beta(1 \rightarrow 4)$ and $\beta(1 \rightarrow 6)$ linkages, cross-linked with peptide chains. The hydrolytic action of lysozyme gives rise to disaccharide units attached to the peptide chains called muropeptides.

High concentrations of lysozyme were found in monocytes (SCHMALZL and BraUnSTEINER 1970,
GORDON et al. 1974) and alveolar macrophages (Myrvik et al. 1961, Cohn and Wiener 1963, Heise and Myrvik 1967, Leake and Myrvik 1968, McClelland and van Furth 1975). Lysozyme is considered to be one of the constitutive enzymes of the macrophages (GoRdon et al. 1974, GoRdon 1978). It is the major secretory product and forms about $25 \%$ of the extracellularly secreted protein of macrophages.

Hen and turkey egg-white lysozymes differ by seven amino acids but have very similar tertiary structures (SARMA and BотT 1977). Azide radicals $\left(\mathrm{N}_{3}{ }^{\circ}\right)$ react first only with tryptophan residues (Prütz and LAND 1979, Prütz et al. 1980), giving an indolyl type radical. Tyrosine residues are then oxidised by a long-range intramolecular electron transfer to tryptophan, followed by dimerization. Using $\mathrm{N}_{3}{ }^{\bullet}$ free radicals, the initial yields of dimerization are equal to $(8.6 \pm 0.7) \times 10^{-9} \mathrm{~mol} \mathrm{~J}^{-1}$ for both proteins (AUDETTE et al. 2000). Using $\mathrm{HO}^{\circ}$ free radicals, they become equal to $(1.23 \pm 0.1) \times 10^{-8}$ and $(4.42 \pm 0.1) \times 10^{-8} \mathrm{~mol} \mathrm{~J}^{-1}$ for hen and turkey eggwhite lysozymes, respectively ( $\gamma$ radiolysis).

\section{$\boldsymbol{\beta}$-Glucuronidase (EC 3.2.1.31)}

Epoxide hydrolase (EC 3.3.2.3) activity in pulmonary alveolar macrophages obtained from 11 patients by bronchoalveolar lavage was $0.24 \pm 0.10 \mathrm{nmol} /$ min $\times$ mg protein (Petruzzelli et al. 1988). Epoxide hydroxylase activity was $0.16 \pm 0.02 \mathrm{nmol} / \mathrm{min}$ $\times$ mg protein in non-smokers and $0.31 \pm 0.08 \mathrm{nmol} /$ min $\times$ mg protein in smokers $(P<0.001)$.

Leucine aminopeptidase (EC 3.4.1.2) is a plasma membrane-bound enzyme present on macrophages (WACHSMuth and Stoye 1977).

Angiotensin converting enzyme (EC 3.4.15.1) is a membrane-bound ectoenzyme of human blood monocytes augmented by dexamethasone in a biphasic dose-dependent manner with maximum effect after 6 days in culture at $10^{-8} \mathrm{M}$ concentration (VUK-PAVLOVIC et al. 1989). In a pure population of human alveolar macrophages obtained by centrifugal elutriation of bronchoalveolar lavage fluid from 7 sarcoid patients the distribution pattern of angiotensin converting enzyme closely resembled that of NADPH-cytochrome-c-reductase and sialyltransferase, markers of the endoplasmic reticulum and the Golgi complex, respectively, indicating a common localisation (EKLUND et al. 1987).

Cathepsin B (EC 3.4.22.1), a lysosomal cysteine endopeptidase was localised in human alveolar macrophages by the indirect peroxidaseantiperoxidase technique (BURNETT et al. 1983). 


\section{Gelatinase B (EC 3.4.24.35)}

This $92-\mathrm{kDa}$ gelatinase (= matrix metalloproteinase 9 = type $\mathrm{V}$ collagenase $=92-\mathrm{kDa}$ type IV collagenase $=$ macrophage collagenase $)$ is similar to collagenase A, but possesses a further domain. It is expressed in macrophages and eosinophils. Stimulated neonatal alveolar macrophages secreted four to five times more gelatinase than stimulated adult alveolar macrophages (Delacourt et al. 1995). After stimulation by phorbol 12-myristate 13 -acetate, DELACOURT et al. (1997) observed a dose-dependent increase in gelatinase secretion that was significantly more marked in alveolar macrophages from 6-day-old rats than in alveolar macrophages from adult rats and that was inhibited by the protein kinase C inhibitor calphostin C. Adenosine 3',5'cyclo-monophosphate mimetics or concanavalin A failed to induce an increase in gelatinase secretion by alveolar macrophages.

Characterisation of matrix metalloproteinase production by isolated lung fibroblasts, endothelial cells, type II epithelial cells, and alveolar macrophages revealed that only the macrophage has the same spectrum for matrix metalloproteinase activity as seen in the bronchoalveolar lavage fluids in lipopolysaccharide-induced acute lung injury in Long-Evans rats (GiBBs et al. 1999).

Concomitant treatment with catalase (EC 1.11.1.6) greatly inhibited metalloproteinase 9 production by rat alveolar macrophages in response to immune complexes, but this treatment had little effect on basal production of either metalloproteinase 9 or metalloproteinase 2 by macrophage (WARNER et al. 2000).

Arginase (EC 3.5.3.1) catalyses the thermodynamically favoured hydrolysis of L-arginine to Lornithine and urea, whereas nitric oxide synthase (EC 1.14.13.39) catalyses the oxidation of L-arginine to L-citrulline and $\mathrm{NO}$ with formation of the intermediate $N^{\omega}$-hydroxyl-L-arginine (KERWIN et al. 1995). At least two distinct arginase genes are coding for immunologically distinct isoforms (Gотон et al. 1996, Vockley et al. 1996, Jenkinson et al. 1997, Perozich et al. 1998). In cells possessing an inducible nitric oxide synthase in addition to an active arginase, $N^{\omega}$-hydroxy-nor-L-arginine appears as an attractive pharmacological tool to study possible interactions between these two pathways.

Arginase was induced in resident mouse peritoneal macrophages of male $\mathrm{B}_{6} \mathrm{D}_{2} \mathrm{~F}_{1}$ mice in vitro by two nondialyzable factors contained in sera from man, rat, mouse and calf (JAKWAY et al. 1980).

The incubation of resident peritoneal macrophages with bacterial lipopolysaccharide induced high arginase activity as judged by the consumption of
${ }^{14} \mathrm{C}(\mathrm{U})$-L-arginine and the release of labelled ornithine into the cell supernatant (KRIEGBAUM and DRÖGE 1985).

The simultaneous presence of iNOS and arginase may play an important regulatory role, through the control of "NO production, in lipopolysaccharideactivated macrophages (CHANG et al. 1998). RouZAUT et al. (1999) reported that monocytes, entering a process of activation and differentiation, responded with a differential expression of mRNAs corresponding to iNOS and arginase II and showed different arginase and NOS enzyme activities. An increase in arginase II expression seemed to rescue monocytes from apoptosis, whereas the downregulation of arginase II, concomitant with an upregulation of iNOS, led to apoptosis.

$N^{\omega}$-Hydroxy-nor-L-arginine is much more potent than $N^{\omega}$-hydroxy-L-arginine to inhibit murine macrophage arginase and neither induces nor inhibits NOS (Tenu et al. 1999).

In cultured DBA/2 mouse peritoneal macrophages pre-treated with supernatants of concanavalin A-stimulated spleen cells containing immune interferon (IFN- $\gamma$ ) activity, the acquisition of an intrinsic restriction to herpes simplex virus replication correlated with the generation of appreciably elevated levels of arginase in supernatant (SETHI 1983),

Adenosine deaminase (adenosine aminohydrolase; EC 3.5.4.4) catalyses the deamination of adenosine and deoxyadenosine. Thioglycollatestimulated C57BL/6 mouse peritoneal macrophages contained high levels of adenosine deaminase activity (CHAN 1979). In the presence of deoxycoformycin $(1 \mu \mathrm{g} / \mathrm{ml})$, a potent inhibitor of adenosine deaminase (AgARWAL et al. 1977), thioglycollatestimulated mouse peritoneal macrophages excreted deoxyadenosine (CHAN 1979).

$\mathrm{Ca}^{2+}$-dependent adenosine triphosphatase (EC 3.6.1.3) of a plasma membrane fraction isolated from lysates of Bacillus Calmette-Guérin-induced rabbit alveolar macrophages was markedly stimulated by concentrations of $\mathrm{Ca}^{2+}$ from $6 \times 10^{-8}$ to $1 \times 10^{-5} \mathrm{M}$, with an apparent $K_{\mathrm{m}}\left(\mathrm{Ca}^{2+}\right)$ of $1 \times 10^{-6} \mathrm{M}$ (Gennaro et al. 1979). ATPase activity and phosphorylation by $\left[\gamma^{32} \mathrm{P}\right]$ ATP of isolated plasma membrane of alveolar macrophages were stimulated in a parallel fashion by physiologic concentrations of $\mathrm{Ca}^{2+}$, with half-maximal activating effect of this ion at (3-7) $\times 10^{-7} \mathrm{M}$ (SCHNeider et al. 1979). For various membrane preparations, a direct proportionality existed between $\mathrm{Ca}^{2+}$-dependent ATPase activity and amount of ${ }^{32} \mathrm{P}$ incorporated.

$\mathrm{Mg}^{2+}$-dependent adenosine triphosphatase (EC 3.6.1.3) activity of the rabbit alveolar macrophage actomyosin plus cofactor, whether assembled from 
purified components or studied in a complex collected from crude macrophage extracts, was not influenced by the presence or absence of calcium ions (Stossel and Hartwig 1975).

\subsubsection{4}

Lyases

Guanylate cyclase (EC 4.6.1.2) obtained from the cytosol of murine bone marrow macrophages which were cultured for 7 days in Teflon bags and seeded on culture plates was activated in a concentrationdependent manner and synergistic mode by Larginine and NADPH (MüLSCH et al. 1991). Activation was not observed with $\mathrm{D}$-arginine or $\mathrm{NADH}$, in accordance with the specific requirements for the oxidative L-arginine pathway (MARLETTA et al. 1988). -NO synthesis in murine peritoneal macrophages (MARletta et al. 1988) and bone marrow macrophages (MüLSCH et al. 1991) was $\mathrm{Ca}^{2+}$-independent.

Rat growth hormone in vitro enhanced soluble guanylate cyclase activity in the lungs of 15-day-old Sprague-Dawley rats; in $10 \mathrm{~min}$ of incubation the production of $\left[\alpha^{-32} \mathrm{P}\right] \mathrm{cGMP}$ per mg protein significantly $(P<0.001)$ increased from $1,760 \pm 12 \mathrm{pmol}$ in the control to $3,470 \pm 15 \mathrm{pmol}$ and $3,608 \pm 13$ under $10 \mathrm{nM}$ and $1 \mu \mathrm{M}$ growth hormone, respectively (VESELY 1981). Heat-treated growth hormone caused no enhancement of guanylate cyclase activity.

The guanylate cyclase inhibitor LY83583 inhibited human alveolar macrophage spreading and staining for cGMP-dependent protein kinase at the cell periphery (PrYZWANSKY et al. 1995).

\subsection{4}

\section{Lipids}

The total lipid and protein content per cell after the differentiation of human blood monocytes to macrophages due to the increase in cell size increased by approximately four-fold (VIsioli et al. 2000). The percentage of docosapentaenoic acid $(22: 5 n-3)$ increased from $1.47 \pm 0.23 \%$ to $2.93 \pm 0.32 \%(P<0.05)$ and that of docosahexaenoic $(22: 6 n-3)$ acid from $2.48 \pm 1.02 \%$ to $5.96 \pm 1.21 \%(P<0.05)$, while linoleic acid decreased from $7.91 \pm 1.54 \pm$ to $3.24 \pm$ $1.23 \%(P<0.05)$.

Rat alveolar macrophages contained only neutral fats but no complex lipids (CAULET et al. 1968).

\subsection{5 \\ Ascorbic Acid}

Ascorbic acid is able to scavenge reactive oxygen species as $\mathrm{O}_{2}{ }^{--}$(Nishikimi 1975), "HO (BIELSKi et al. 1975) and ${ }^{1} \mathrm{O}_{2}$ (Bodannes and Chan 1979). On the other hand ascorbic acid can reduce $\mathrm{Fe}^{3+}$ to $\mathrm{Fe}^{2+}$ and thus give rise to Fenton catalysis of $\mathrm{H}_{2} \mathrm{O}_{2}$ to form $\mathrm{HO}^{\circ}$.

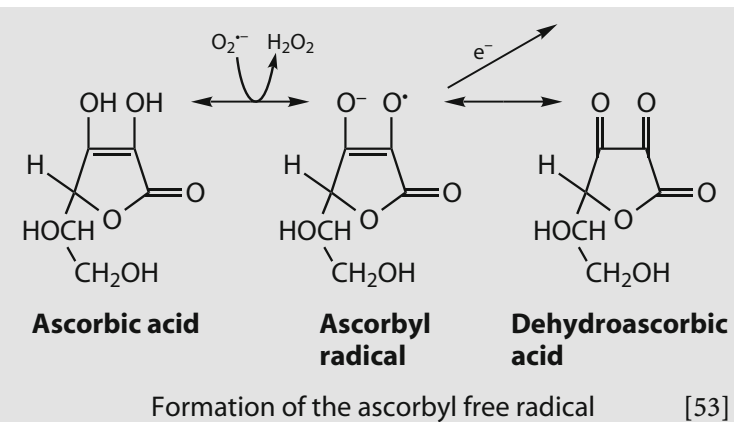

Dehydroascorbic acid, the two-electron oxidised form of the vitamin, is taken up on the glucose transporter and reduced to ascorbate to a much greater extent than ascorbate itself is accumulated in human monocytic U-937 cells (MAY et al. 1999). In contrast to dehydroascorbic acid, ascorbate enters the cells in a sodium- and energy-dependent transporter.

Matzner (1938) detected ascorbic acid within the "detached alveolar phagocytes", but not within the sessile alveolar wall cells. He supposed the density of vitamin $C$ load to be an indicator of the instantaneous performance of the cell. Occasionally the vitamin was located at the Golgi apparatus. The load with "real" granules varies extremely (ToEPFER 1961, p. 92). About $50 \%$ of the ascorbic acid content of sliced rat lungs was removed in 2 min by washing with Krebs-phosphate solution suggesting that it was not intracellular (WILLIS and KRATZING 1974a). Ascorbic acid removed in this way was not present in alveolar macrophages since it remained in solution when these cells were removed by centrifugation. Since $30 \%$ of the ascorbic acid of an intact lung could be removed by washing the air spaces it was concluded that about half the ascorbic acid in lung is present in the fluid lining the respiratory epithelium. Fresh mouse lung contained $368 \mu \mathrm{g} / \mathrm{g}$ of total (oxidised plus reduced) ascorbic acid of which $75 \mu \mathrm{g} / \mathrm{g}$ was oxidised (WILLIS and KRATZING 1974c).

Dehydroascorbic acid, the two-electron oxidised form of the vitamin, was taken up by human monocytic U-937 cells on the glucose transporter and reduced to ascorbate to a much greater extent than ascorbate itself was accumulated in the cells (MAY et al. 1999). In contrast to dehydroascorbic acid, ascorbate entered the cells on a sodium- and energydependent transporter. Intracellular ascorbate enhanced the transfer of electrons across the cell membrane to extracellular ferricyanide. Rates of ascorbate-dependent ferricyanide reduction was 
saturable, fivefold greater than basal rates, and facilitated by intracellular recycling of ascorbate. Whereas reduction of dehydroascorbic acid concentrations above $400 \mu \mathrm{M}$ consumed reduced glutathione (GSH), even severe GSH depletion by 1chloro-2,4-dinitrobenzene was without effect on the ability of the cells to reduce concentrations of dehydroascorbic acid likely to be in the physiologic range $(<200 \mu \mathrm{M})$. Dialysed cytosolic fractions from U-937 cells reduced dehydroascorbic acid to ascorbate in a NADPH-dependent manner that appeared due to thioredoxin reductase. However, thioredoxin reductase did not account for the bulk of dehydroascorbic acid reduction, since the activity was also decreased by treatment of intact cells with 1-chloro-2,4-dinitrobenzene.

\subsection{6}

\section{Pantothenol}

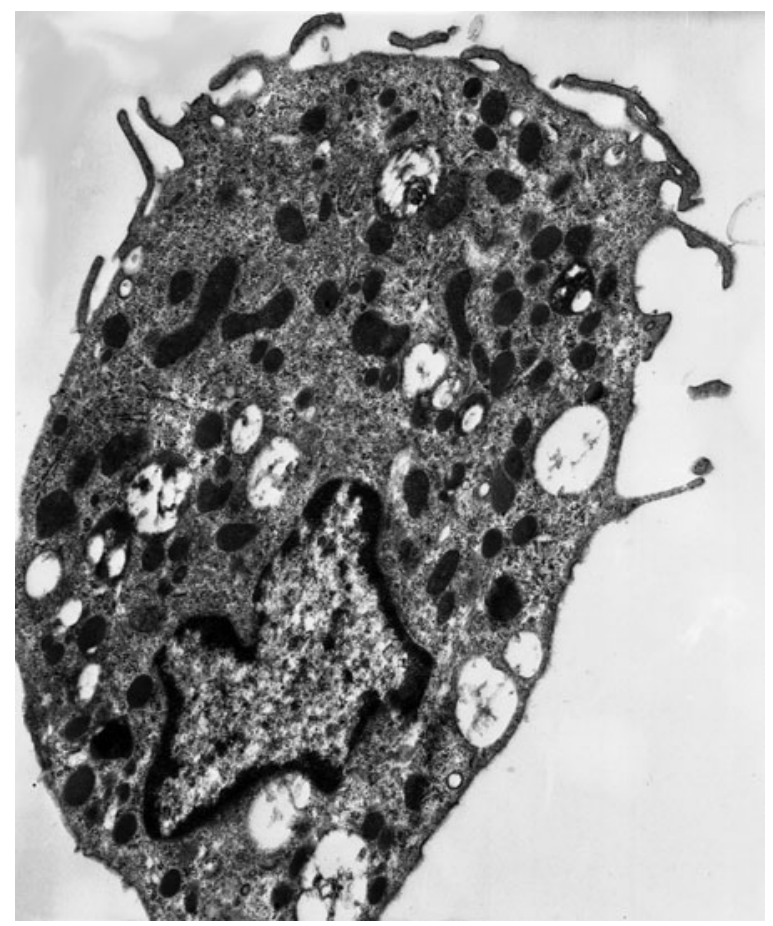

Fig. 117. Plenty of mitochondria and free ribosomes in an alveolar macrophage from a female white rat (breeder: Winkelmann, Borchen-Kirchborchen) medicated with $125 \mathrm{mg}$ dexpanthenol per ( $\mathrm{kg}$ body weight $\times$ day) in a $5 \%$ aqueous solution during a 5-day work week. Oral application took place from April 17 to August 14, 1967 for a total of 82 days. Fixed on August 14, 1967 under methitural anaesthesia by intratracheal instillation of $2.5 \%$ glutaraldehyde in phosphate buffer ( $\mathrm{pH}$ 7.4) before opening the thorax. Postfixation with $1 \%$ osmium tetroxide in phosphate buffer ( $\mathrm{pH}$ 7.4). Contrasted en bloc for $12 \mathrm{~h}$ with $0.5 \%$ uranyl acetate in $70 \%$ ethanol. Embedded in a 2:8 mixture of methyl and butyl methacrylate. Sectioned at $50 \mathrm{~nm}$. Lead citrate after Rey nolds (1963). Plate $6 / 08$

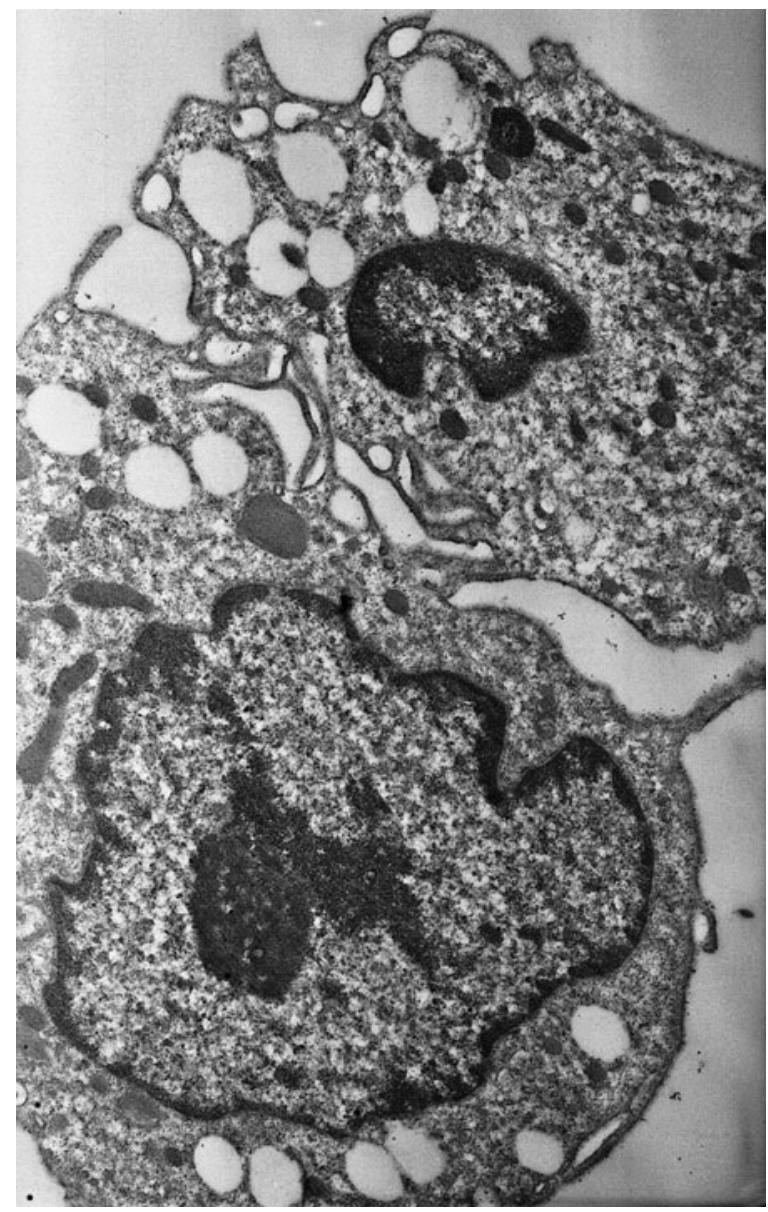

Fig. 118. Beginning of the formation of a multinucleate giant cell by fusion of mononuclear alveolar phagocytes. Female white rat (breeder: Winkelmann, Borchen-Kirchborchen) medicated with $125 \mathrm{mg}$ dexpanthenol in a $5 \%$ aqueous solution per $\mathrm{kg}$ body weight $\times$ day during a 5-day work week. Oral application took place from April 17 to August 14, 1967 for a total of 82 days. Fixed on August 14, 1967 under methitural anaesthesia by intratracheal instillation of $2.5 \%$ glutaraldehyde in phosphate buffer ( $\mathrm{pH}$ 7.4) before opening the thorax. Postfixation with $1 \%$ osmium tetroxide in phosphate buffer ( $\mathrm{pH} 7.4$ ). Contrasted en bloc for $12 \mathrm{~h}$ with $0.5 \%$ uranyl acetate in $70 \%$ ethanol. Embedded in a $2: 8$ mixture of methyl and butyl methacrylate. Sectioned at $50 \mathrm{~nm}$. Lead citrate after ReynolDs (1963). Plate 5/05 


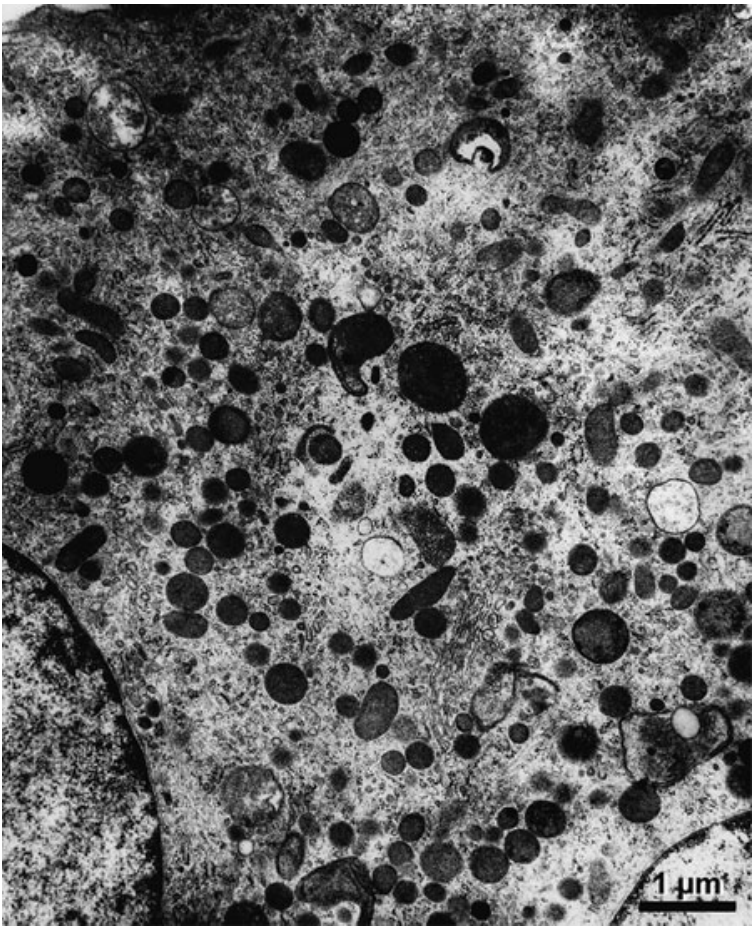

Fig. 119. Numerous autophagosomes and autophagolysosomes, respectively, in an alveolar macrophage polykaryon (block B22) from a ca. $235 \mathrm{~g}$ female white rat (breeder: Winkelmann, Borchen-Kirchborchen) medicated with $125 \mathrm{mg}$ dexpanthenol in a $5 \%$ aqueous solution per $\mathrm{kg}$ body weight $x$ day during a 5-day work week. Oral application took place from April 17 to August 14, 1967 for a total of 82 days. Fixed on August 14, 1967 under methitural anaesthesia by intratracheal instillation of $2.5 \%$ glutaraldehyde in phosphate buffer ( $\mathrm{pH}$ 7.4) before opening the thorax. Postfixation with $1 \%$ osmium tetroxide in phosphate buffer ( $\mathrm{pH}$ 7.4). Contrasted en bloc for $12 \mathrm{~h}$ with $0.5 \%$ uranyl acetate in $70 \%$ ethanol. Embedded in a 2:8 mixture of methyl and butyl methacrylate. Sectioned at $50 \mathrm{~nm}$. Lead citrate after REY NOLDs (1963). Plate 3606

\subsection{7 Retinoids}

The human promyelocytic leucaemia cell line, HL60 , can be induced to differentiate into granulocyte like cells when cultured in the presence of $10^{-6} \mathrm{M}$ all-trans retinoic acid for several days (LADoux et al. 1986). About $80 \%$ of the viable cells ( $70 \%$ of the total number of cells) reduced nitroblue tetrazolium.

Retinoids failed to activate NADPH oxidase in dibutyryl cAMP differentiated HL-60 cells (SEIFERT and Schächtele 1988). In HL-60 cells retinoids have been found to potentiate formyl-methionylleucyl-phenylalanine-induced and phorbol myristate acetate-induced $\mathrm{O}_{2}{ }^{--}$formation (SEIFERT and SCHÄCHTELE 1988). Using receptor specific retinoid analogues it was demonstrated that retinoid $\mathrm{X}$ receptor-retinoic acid receptor heterodimers mediate retinoid-induced differentiation of HL60 cells, while retinoid $\mathrm{X}$ receptor-retinoid $\mathrm{X}$ receptor homodimers mediate subsequent retinoid-mediated apoptosis (NAGY et al. 1995, KIzAKI et al. 1996).

In untreated U937 (human monoblastic leucaemia cell line) cells retinoid receptor- $\alpha$ mRNA levels increased after induction of differentiation via phorbol myristate acetate (Brown et al. 1997). Using plasmids containing sense or antisense retinoid receptor- $\alpha$ sequences under the control of an inducible promoter, Brown et al. generated stable transfected cell lines which expressed either increased or decreased levels of retinoid X receptor- $\alpha$, respectively. The sense cell lines showed an increased sensitivity to 9-cis retinoid acid, while the antisense cell lines presented decreased sensitivity. Combined 9-cis retinoic acid and 1 1,25 -dihydroxy
Fig. 120. Alveolar macrophage from a female rat (breeder: Winkelmann, Borchen-Kirchborchen) which received daily intragastric applications of $82 \mathrm{mg}$ retinol palmitate per $\mathrm{kg}$ body weight $\times$ day, 5 days per week from April 17 to June 20, 1967 for a total of 42 days. Fixed on June 20, 1967 under methitural anaesthesia by intratracheal instillation of $2.5 \%$ glutaraldehyde in phosphate buffer ( $\mathrm{pH}$ 7.4) before opening the thorax. After washing in phosphate buffer the tissue was postfixed with $1 \%$ osmium tetroxide in phosphate buffer for $2 \mathrm{~h}$. Contrasted en bloc for $12 \mathrm{~h}$ with $0.5 \%$ uranyl acetate in $70 \%$ ethanol. Embedded in a 2:8 mixture of methyl and butyl methacrylate. Sectioned at $50 \mathrm{~nm}$. Lead citrate after REYNOLDs (1963). Film 92671

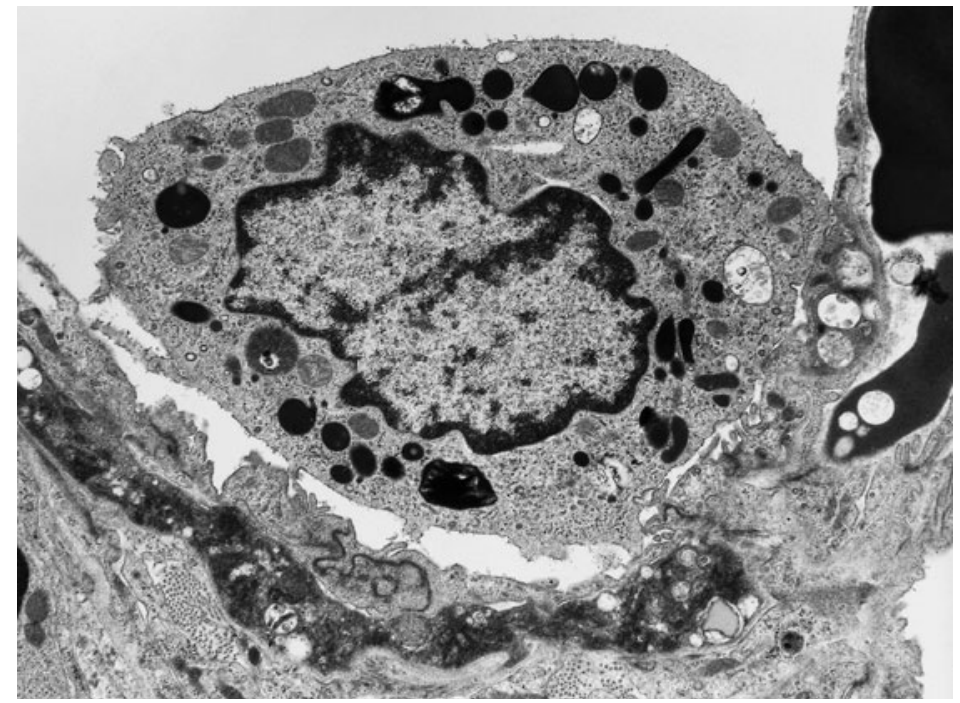


vitamin $\mathrm{D}_{3}$ exhibited significantly higher levels of phagocytosis of antibody-coated ovine erythrocytes $(61 \pm 7 \mathrm{cpm})$ compared to MEP cells (U937 cells harbouring the pMEP4 expression plasmid without an insert; Hewison et al. 1994) (35 $\pm 4 \mathrm{cpm})$.

\subsection{8}

\section{Tocopherol}

As opposed to an initial human blood monocytic vitamin $\mathrm{E}$ content of $4.75 \mathrm{pmol} / 10^{6}$ cells, macrophagic vitamin E levels were undetectable (VIsIOLI et al. 2000).

Six hours after an intravenous application of $30 \mathrm{mg}$ of $\left[{ }^{3} \mathrm{H}\right]$ tocopheryl nicotinate radioactivity was detected in rat alveolar macrophages (GALLOTorres 1980).

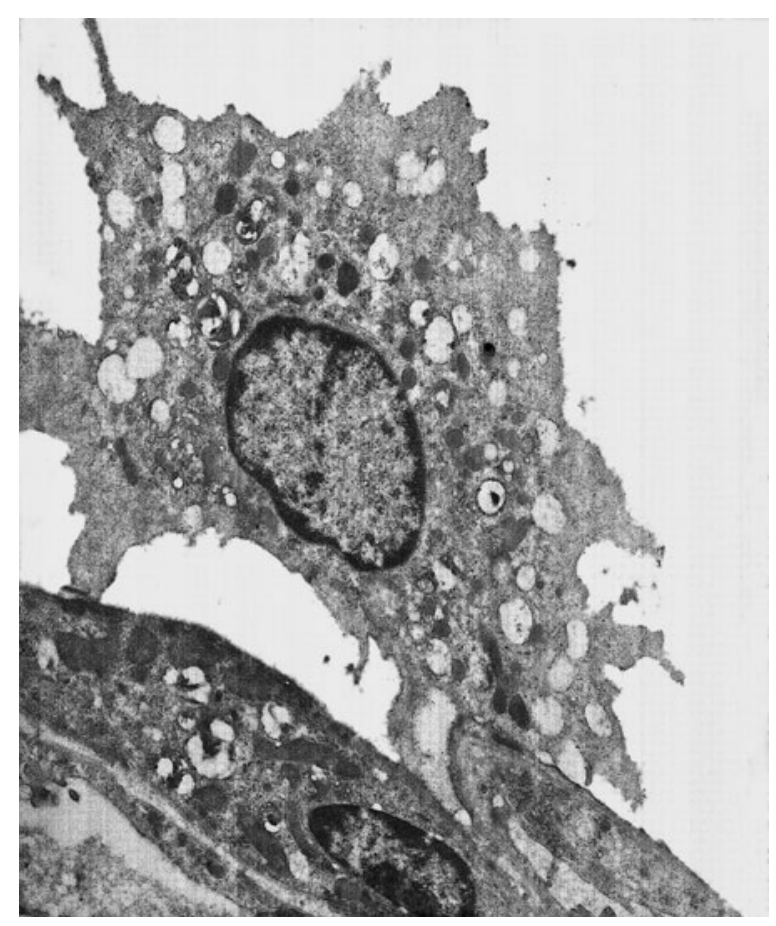

Fig. 121. Alveolar macrophage from a female rat (breeder: Winkelmann, Borchen-Kirchborchen), which received intramuscular injection of $150 \mathrm{mg}$ DL- $\alpha$-tocopherol acetate in a colloidal solution (Ephynal ${ }^{\circledR}$ ) per kg body weight $\times$ day, 5 days per week from April 12 to June 12, 1967 for a total of 40 days. Fixed on June 12, 1967 under methitural anaesthesia by intratracheal instillation of $2.5 \%$ glutaraldehyde in phosphate buffer ( $\mathrm{pH}$ 7.4) before opening the thorax. Postfixation with $1 \%$ osmium tetroxide in phosphate buffer ( $\mathrm{pH} 7.4)$. Contrasted en bloc for $12 \mathrm{~h}$ with $0.5 \%$ uranyl acetate in $70 \%$ ethanol. Embedded in a 2:8 mixture of methyl and butyl methacrylate. Sectioned at $50 \mathrm{~nm}$. Lead citrate after REYNOLDS (1963). Plate 275/03

\section{3 Origin of Alveolar Macrophages}

In the preterm Macaca nemestrina (130-156 days of gestation), the number of alveolar macrophages is low and increases rapidly after birth (JАСовs et al. 1985). In foetal rabbits, macrophage appearance coincides with maturation of the lung near term and in the first days after birth (ZeLIGs et al. 1977, SIEGER 1978).

While monoblasts pinocytose very infrequently (Goud et al. 1975) promonoxytes (van FurTh 1978) and monocytes/macrophages (COHN and Benson 1965, Cohn and Parks 1967, Ehrenreich and Cohn 1968, Bowden 1971, Edelson et al. 1975, KAPLAN and Nielsen 1978, van Furth 1978, 1981, Von Melchner and Hilgard 1979, Kaplan and Keogh 1981, Papadimitriou and Van BrugGen 1982, Burgert and Thilo 1983, Pratten and Lloyd 1983, AbRASs 1988) do pinocytose avidly.

\section{4 \\ Secretion}

Macrophages, originally considered as mere scavenger cells, are now respectfully regarded as one of the most productive cell population. First CARrel (1922) and Carrel and Ebeling (1922) in the chicken found that extracts of leukocytes secrete substances that determine the multiplication of fibroblasts.

From about 15 known activities in 1976 (UNANUE 1976) the list comprised approximately fifty substances in 1984 (TAKEMURA and WebB 1984) and about one hundred products in 1987 (NATHAN 1987). Many substances formerly defined according to their biological activities, could now be presented with accurate molecular definitions.

In an attempt to identify novel molecules selectively expressed by alternatively activated macrophages, Kodelja et al. (1998) applied subtractive cloning methods and differential hybridisation and, finally, cloned a novel human CC-chemokine, alternative macrophage activation-associated CC-chemokine-1 (AMAC-1). The AMAC-1 gene consists of three exons (GoERDT et al. 1999). Several putative regulatory sequences for interleukin 4- and interferon- $\gamma$-dependent transcriptional pathways were found including STAT6 and STAT1 binding sites as well as several AP-1 and C/EBP elements. Interestingly, a combined STAT6/STAT1 binding element is located in the direct vicinity of the first putative transcription start point. Competitive binding of IL-4-induced STAT6 versus interferon- $\gamma$-induced STAT1 to this site may explain the antagonistic effects these cytokines exert on AMAC-1 expression. 


\subsection{1 \\ Polypeptide Hormones}

\subsubsection{1}

\section{Interleukin 1}

The biological basis for interleukin-1 was review by DINARELLO (1996) giving 586 references.

Three members of the interleukin-1 family have been cloned (Dinarello 1996). All bind both types of receptors but only IL- $1 \alpha$ and IL- $1 \beta$ (encoded on chromosome 2) are agonists. The third is the interleukin 1 receptor antagonist protein (IRAP). All share $20-25 \%$ amino acid homology. Mature IL- $1 \alpha$ and IL- $1 \beta$ have similar three-dimensional open barrel structures of $\beta$ sheets.

Interleukin- $1 \beta$ is a potent immunomodulatory cytokine that is produced in large quantities by mononuclear phagocytes, and by other cells present in essentially all tissues. It affects cell targets by binding with high affinity to well-characterised cell-surface receptors. Murine IL-1 $\beta$ is initially synthesised as a $33-\mathrm{kDa}$ precursor protein that has no measurable affinity for the IL-1 receptor and that carries no biological signal. This precursor protein is activated by proteolytic cleavage between $\mathrm{Asp}^{117}$ and $\mathrm{Val}^{118}$. The C-terminal 17-kDa fragment represents the mature IL- $1 \beta$ protein with high receptor affinity.

Although several different proteinases can cleave the IL- $1 \beta$ precursor in vitro, the natural cleavage of the IL- $1 \beta$ precursor in macrophages and monocytes occurs via the action of a novel cysteine proteinase designated the IL-1 $\beta$-converting enzyme.

There are two IL-1 receptors, the type I receptor transduces a signal, whereas the type II receptor binds IL-1 but does not transduce a signal. In fact, IL-1 receptor II acts as a sink for IL- $1 \beta$ and has been termed a "decoy" receptor, which is somewhat unique to cytokine biology (СоцоттA et al. 1994). When IL-1 binds to IL-1 receptor I, a complex is formed that then binds to the IL-1 receptor accessory protein, resulting in high-affinity binding (GREENFEDER et al. 1995).

An IL-1 receptor antagonist (IRAP) has been isolated and purified from activated monocytes. IRAP inhibits IL-1 bioactivity by binding competitively at the IL-1 receptor (EISENBERG et al. 1990, HANNUM et al. 1990, MAZzeI et al. 1990). In vitro IRAP can attenuate IL-1-dependent fibroblast proliferation, collagenase synthesis, and prostaglandin $\mathrm{E}_{2}$ release (HANNum et al. 1990). IRAP suppressed IL-1-induced monocyte production of IL-1, tumour necrosis factor- $\alpha$, or interleukin 6. Multiple lipid oxidation products in low density lipoproteins induce IL- $1 \beta$ release from human blood mononuclear cells (Thomas et al. 1994).
Glucocorticoids control IL-1 via inhibition of mitogen-induced transcription of the IL- $1 \beta$ gene and decreased stability of its mRNA in monocytes of several species (KNUDSEN et al. 1987, LEE et al. 1988, Amano et al. 1993, Huether et al. 1993). In contrast, the IL-1 receptor is induced by glucocorticoids in a protein and mRNA neosynthesisdependent fashion (Аканоsнi et al. 1988, GоттSCHALL et al. 1991), as is the type II receptor, a potential decoy target for IL-1 (RE et al. 1994).

The nitric oxide donor, S-nitroso- $N$-acetyl-D, Lpenicillamine induced dose-dependent inhibition of IL-1 production in LPS-stimulated rat alveolar macrophages in which endogenous nitric oxide production was blocked (Persoons et al. 1996).

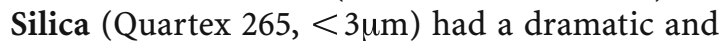
selective effect on mature IL-1 release from peritoneal macrophages obtained from BDF1 mice harvested without elicitation in a dose-dependent fashion (SARIH et al. 1993).

Levels of cytoplasmic oligonucleosomal DNA (cell death detection enzyme-linked immunosorbent assay) were significantly enhanced for chrysotile $(3-25 \mu \mathrm{g} / \mathrm{ml})$ and crocidolite $(25-75 \mu \mathrm{g} / \mathrm{ml})$ in a dose-dependent manner, a process that was inhibitable by $10 \mu \mathrm{M}$ Z-Val-Ala-Asp fluoromethyl ketone, an interleukin-converting enzyme inhibitor (Hamilton et al. 1996).

Crocidolite or potassium octatitanate whisker instilled intratracheally into male Wistar rats $(2 \mathrm{mg}$ in saline) peaked levels of IL- $1 \alpha$ mRNA in alveolar macrophages at 1 month thereafter (TsudA et al. 1997).

Rat alveolar macrophages lavaged 1 day after intratracheal instillation of welding fumes $(1 \mathrm{mg} / 100 \mathrm{~g}$ body weight) collected during flux-covered manual metal arc welding (using a stainless steel consumable electrode containing $\mathrm{Cr}, \mathrm{Ni}$, and $\mathrm{Mn}$ ), released significantly more $(P<0.05)$ IL-1 $\beta$ than did fumes from gas metal arc welding using a mild steel electrode (ANTONini et al. 1996).

A 30-min exposure to $\mathrm{SO}_{2}$ induced a significant $(P<0.05)$ decrease in lipopolysaccharide-stimulated IL-1 $\beta$ release from human alveolar macrophages obtained by bronchoalveolar lavage and cultured in vitro (KNORST et al. 1996).

When expressed by transfected tumour cells, IL1 decreased the tumorigenicity of oncogene cell lines (Douvdevani et al. 1992) and oncogene transformed $\mathrm{NIH}_{3} \mathrm{~T}_{3}$ fibroblasts (SAIto et al. 1994).

\subsubsection{2 Interleukin 4}

Interleukin-4 (IL-4) can prime murine macrophages (Phillips et al. (1990). Pre-treatment of murine bone-marrow derived macrophages with $10 \mathrm{U} / \mathrm{ml}$ 
murine IL- 4 for $48 \mathrm{~h}$ enhanced the respiratory burst following subsequent stimulation with $10^{-6} \mathrm{M}$ phorbol myristate acetate or $1 \mathrm{mg} / \mathrm{ml}$ zymosan.

IL-4 increased the motility of human blood monocyte-derived macrophages in vitro (DugAsT et al. 1997). The cells spread in thin cytoplasmic lamellas, regrouped in clusters, and within 1-3 weeks, differentiated into giant cells.

IL-4 in addition to TGF $\beta$ and TGF- $\alpha$ was shown to induce collagen synthesis by cultured fibroblasts (Elias et al. 1990, Sempowski et al. 1994). IL-4 was produced by macrophages during development of post-irradiation lung fibrosis in rats (BÜTTNER et al. 1997).

\subsubsection{3}

\section{Interleukin 6}

Interleukin-6 is a pluripotent cytokine with immunomodulatory effects (AKIRA et al. 1990). It is secreted mainly by cells of the monocyte or macrophage lineage and by endothelial cells (Hirano et al. 1990). Both cyclooxygenase and 5-lipoxygenase pathways are involved in platelet-activating factorinduced IL-6 production by alveolar macrophages (Thivierge and Rola-Pleszczynski 1994). Human alveolar macrophage response to endotoxin (LPS) includes the production of IL-6. LPS increased the relative abundance of NFxB and NF-IL6 (Hopkins et al. 1996). This increase in NFxB and NF-IL6 corresponded temporally with the rise in IL-6 mRNA. In RAW 264.7 macrophages upregulation of IL-6 production appeared to be protein kinase $\mathrm{C}$ independent, while its downregulation is mediated by a protein kinase Cdependent feedback mechanism (Tremblay et al. 1995).

In human venous blood IL-6 mean values were higher during the light period of the day without, however, any significant differences with respect to the nocturnal mean levels (Lissoni et al. 1998).

Hypoxia $\left(3 \% \mathrm{O}_{2}\right)$ in primary cultures of human pulmonary fibroblasts and pulmonary vascular smooth muscle cells induced transcription and translation of IL-6 (4- to 5-fold) in both cell types (TAMm et al. 1998). Hyperoxia-induced expression of IL- 6 was suppressed by $50 \%$ to $60 \%$ in the presence of platelet-activating factor antagonist WEB2170, or neutralising anti-platelet-derived growth factor antibodies.

IL-6 has been involved in the pathogenesis of inflammatoy and/or autoimmune diseases, including rheumatoid arthritis and systemic lupus erythematosus (Hirano et al. 1990). It could be implicated in the pathogenesis of systemic sclerosis, as IL-6 (NeEDleman et al. 1992) or anti-IL-6 auto antibo- dies (TAKemura et al. 1992) are more frequently detected in sera from systemic sclerosis patients than in sera from control patients. Crestani et al. (1994) demonstrated that during systemic sclerosis lung disease spontaneous and stimulated IL-6 secretion by blood monocytes is increased, compared with secretion by healthy control subjects. By contrast, IL- 6 secretion by alveolar macrophages recovered by bronchoalveolar lavage is normal despite of mild alveolitis.

$r$ IL- 6 by itself did not exhibit any chemotactic activity and it could not activate human mononuclear leucocytes for an oxidative burst response (KHARAZMI et al. 1989). Preincubation with $r$ IL-6 at concentrations of 5 and $50 \mathrm{ng} / \mathrm{ml}$ primed them for enhanced generation of reactive oxygen species as documented by the lucigenin-enhanced chemiluminescence following stimulation with the chemotactic peptide f-Met-Leu-Phe or phorbol myristate acetate.

Human alveolar epithelial cells exposed to residual oil fly ash release inflammatory cytokines including IL-6, IL-8, and tumour necrosis factor (CARTER et al. 1997). The IL-6 response was inhibited by the metal chelator deferoxamine and the free radical scavenger $N$-acetyl-L-cysteine, suggesting that the activation of NF $\mathrm{B}$ may be mediated through reactive oxygen intermediates generated by transition metals found in residual oil fly ash (QUAY et al. 1998).

$5 \mathrm{ppm} \mathrm{SO}_{2}$ exposure of human alveolar macrophages for 30 min yielded a small but not significant $(P>0.05)$ reduction from $611 \pm 76 \mathrm{pg}$ spontaneous IL-6 production $/ \mathrm{ml}$ to $585 \pm 85 \mathrm{pg} / \mathrm{ml}$ (KNORST et al. 1996).

Phorbol 12-myristate 13-acetate (PMA; $10 \mathrm{nM}$ ) strongly stimulated IL-6 production by human blood monocytes cultured in RPMI1640 supplemented with $2 \mathrm{mM}$ glutamine, $50 \mathrm{mg} / \mathrm{ml}$ gentamicin and $10 \%$ heat-inactivated foetal calf serum (Costanzo et al. 1999).

Enhanced secretion of IL- 6 by cultured sarcoid alveolar macrophages has been demonstrated, though previous studies show no difference in bronchoalveolar lavage fluid IL-6 levels between sarcoid patients and healthy individuals (JonEs et al. 1991, HomalKa and Müller-Quernheim 1993, StefFen et al. 1993, Girgis et al. 1995).

When expressed by transfected tumour cells, IL6 decreased the tumorigenicity of 3LL lung carcinoma cells by delaying their doubling time (PorgaDOR et al. 1992), but there was no influence on B16F10 melanoma cells (Dranoff et al. 1993) and TS/ A adenocarcinoma cells (Allione et al. 1994). 


\subsubsection{4 Interleukin 8}

Interleukin-8, part of a supergene family of novel chemotactic cytokines (CXC and CC chemokines), is secreted by macrophages/monocytes, neutrophils, fibroblasts, and endothelial and epithelial cells in response to a variety of stimuli. A potent chemoattractant and activator of neutrophils, IL-8 may also be chemotactic for T lymphocytes (LARSEN et al. 1989, BAgGioloni et al. 1993). IL-8 induces the respiratory burst in monocytes, and the structurally related chemokine monocyte chemotactic protein-1 (MCP-1) is a potent chemotactin for monocytes (BAGGiolini et al. 1993).

Oxidised LDL induces monocytic cell expression of IL-8 (Terkeltaub et al. 1994).

Hypoxia $\left(3 \% \mathrm{O}_{2}\right)$ in primary cultures of human pulmonary fibroblasts and pulmonary vascular smooth muscle cells induced transcription and translation of IL-8 (5- to 6-fold) in both cell types (TAMM et al. 1998).

Eosinophil infiltration in the bronchoalveolar lavage fluid in guinea pigs was induced by recombinant human IL-8 (LAGENTE et al. 1995). Administration of the phosphodiesterase IV isoenzyme inhibitor, rolipram $(5 \mathrm{mg} / \mathrm{kg})$ or betamethasone $(10 \mathrm{mg} / \mathrm{kg})$ significantly $(P<0.05)$ reduced the IL8 -induced eosinophil infiltration in bronchoalveolar lavage fluid. Betamethasone may act directly on eosinophils to inhibit their infiltration by IL-8.

In patients with fibrosing alveolitis, occurring alone or in association with rheumatological diseases such as systemic sclerosis, more monocytes secreted IL-8 than autologous macrophages and there was heterogeneity in the in vitro IL- 8 secretion by alveolar macrophages and peripheral blood monocytes (PANTELIDis et al. 1997). IL-8 secretion by alveolar macrophages was significantly higher in subjects with fibrosing alveolitis compared with subjects without fibrosing alveolitis, due to a higher percentage of IL-8-secreting alveolar macrophages in the fibrotic group both in the absence $(P<0.002)$ and presence of lipopolysaccharide $(P<0.04)$ and correlated with bronchoalveolar lavage neutrophil percentage. Using the MoAbs RFD1, RFD7 and RFD9, that distinguish subsets of alveolar macrophages, the authors have been able to identify associations between secretion of IL-8 and smaller cells and the cells identified by the MoAb RFD7. In situ hybridisation of the bronchoalveolar lavage cell population revealed that alveolar macrophages are the predominant source of IL-8 in the lung.

Haemolytic phospholipase C from Pseudomonas aeruginosa up to $1 \mathrm{unit} / 4 \times 10^{5}$ human monocytes induced a dose-dependent increase in IL-8 release and IL-8-specific mRNA expression (KöNIG et al. 1997).

The protein kinase $\mathrm{C}$ inhibitor, staurosporine $(100 \mathrm{nM})$ significantly inhibited the production of IL-8 by a human monocytic cell line, MonoMac6 stimulated by LPS, and so did the tyrosine kinase inhibitor, genistein $(25 \mu \mathrm{M})$ (Fujishima et al. 1997).

\subsubsection{5 Interleukin 10}

IL-10 is a potent antiinflammatory cytokine that shares with adenosine the ability to control TNF release after LPS challenge (DE WAAL MALEFYT et al. 1991, GÉrARD et al. 1993, MARChANT et al. 1994).

Macrophages from chronically infected lungs of cystic fibrosis patients appeared to be one source of IL-10, but Bonfield et al. (1995) found little or no intracellular IL-10 in bronchoalveolar lavage macrophages from healthy control subjects. MARTINEZ et al. (1997) quantified IL-10 and TNF- $\alpha$ mRNA levels in alveolar macrophages obtained by bronchoalveolar lavage from patients with idiopathic pulmonary fibrosis (IPF) and bronchiolitis obliterans with organising pneumonia (BOOP) and in normal healthy volunteers. While the levels of TNF- $\alpha$ mRNA in macrophages obtained from IPF and BOOP patients were not significantly different from normal healthy subjects, macrophages from patients with IPF and BOOP expressed increased levels of IL10 mRNA compared with healthy controls.

O'FARRELL et al. (1998) characterised IL-10 responses of the J774 mouse macrophage cell line, and of J774 cells expressing wild-type hIL-10R, mutant hIL-10R lacking two membrane-distal tyrosines involved in recruitment of Stat-3 (hIL-10R-Tyr ${ }^{\mathrm{FF}}$ ), a truncated Stat3 $(\Delta$ Stat3) which acts as a dominant negative, or an inducibly active Stat3-gyraseB chimera (Stat3-GyrB). A neutralising anti-mIL-10R monoclonal antibody was generated to block the function of endogenous mIL-10R. IL-10 inhibited proliferation of J774 cells and of normal bone marrow-derived macrophages, but not J774 cells expressing hIL-10RTyr ${ }^{\mathrm{FF}}$. Dimerization of Stat3-GyrB by coumermycin mimicked the effect of IL-10, and expression of $\Delta$ Stat 3 blocked the anti-proliferative activity of IL-10. For macrophage de-activation responses, hIL-10R-Tyr ${ }^{\mathrm{FF}}$ could not mediate inhibition of lipopolysaccharide-induced TNF $\alpha$, IL-1 $\beta$ or CD86 expression, while $\Delta$ Stat 3 did not interfere detectably with these IL-10 responses.

Pre-incubation with adenosine dose-dependently enhanced IL-10 release by TNF stimulated human monocytes $(+29,+58,+116 \%$ at 1,10 , and $100 \mu \mathrm{M}$, respectively). Adenosine also significantly enhanced 
IL-10 production after hydrogen peroxide and LPS stimulation and dose-dependently inhibited TNF secretion. The enhanced IL-10 production was not observed when cells were preincubated with adenosine $\mathrm{A}_{1}$ or $\mathrm{A}_{2}$ receptor agonists ( $R$-phenylisopropyladenosine, 5 '- $N$-ethylcarboxamide-adenosine, and 2-chloroadenosine) and was not affected by pre-treatment with theophylline, an antagonist of both $\mathrm{A}_{1}$ and $\mathrm{A}_{2}$ receptors, or with dipyridamole, an inhibitor of adenosine cellular uptake (LE MoINE et al. 1996).

In a mouse model of inflammatory lung reaction induced by intratracheal instillation of silica, the levels of IL-10 protein both in cells obtained after bronchoalveolar lavage and in lung tissue homogenates were significantly increased when compared with controls (HuAux et al. 1998). After in vitro lipopolysaccharide stimulation $(1 \mu \mathrm{g} / \mathrm{ml})$, bronchoalveolar lavage cells obtained from silica-treated animals produced significantly more IL-10 protein and mRNA than cells obtained from control animals.

When expressed by transfected tumour cells, IL10 decreased the tumorigenicity of Chinese hamster ovarian carcinoma cells (RICHTER et al. 1993), CL8-1 cells (SUzuKi et al. 1995), TS/A cells (GiovaRELLI et al. 1995) and B16-F1 melanoma cells (GÉrARD et al. 1996).

\subsubsection{6 Interleukin 12}

Interleukin 12 was originally discovered as a product of B cells in the spent medium of Epstein-Barr virus-transformed human lymphoblastoid cell lines (КоваYASHi et al. 1989). However, this cytokine is now known to be produced predominantly by macrophages and dendritic cells but minimally by $B$ cells, especially by resting B cells (D'ANDREA et al. 1992, TRINCHIERI 1994). Interleukin 12 production by macrophages/dendritic cells is induced by two different pathways of stimulation; one that occurs when the cells are exposed to various pathogens or their products (D'ANDREA et al. 1992, MA et al. 1995), and the other, when macrophages/dendritic cells as antigen-presenting cells interact with activated T cells (Macatonia et al. 1995, SHu et al. 1995, Maruo et al. 1996). TaKenaka et al. (1997) showed that $\mathrm{T}$ cell-dependent and -independent IL12 protein production is regulated by IL-10 and IL-4 corresponding to the levels of mRNA accumulation for the p40 and p35 IL-12 genes, whereas the presence of IL-6 during stimulation decreases IL-12 protein production without affecting steady-state mRNA levels. IsLer et al. (1999) studied the autocrine regulation of the heterodimeric, biologically active form of IL-12 (IL-12 p70), as well as the ex- pression of its subunits of $35 \mathrm{kDa}(\mathrm{p} 35)$ an $40 \mathrm{kDa}$ (p40). Alveolar macrophages cultured in medium alone expressed only p35 mRNA. Both p35 and p40 mRNA levels were induced by lipopolysaccharide and were further increased by interferon- $\gamma$. Lipopolysaccharide alone induced IL-12 p40 but not IL-12 p70 production in monocytes and in alveolar macrophages. However, IL-12 p70 was released when the autocrine production of IL-10 was neutralised by IL-10 blocking antibody, and IL- 12 p 40 production increased. In CD-1 mice, Brucella abortus 2308 was a potent inducer of IL-12p40 (FERnÁndEZLAGo et al. 1999).Secretion of IL-12p70 was also demonstrated in vivo, although at much lower levels. Production of IL-12 over the first 7 days after infection was accompanied by active multiplication of $B$. abortus in the spleens of infected mice. CD-1 cultured macrophages secreted only IL-12p40 in response to $B$. abortus infection and no production of IL-12p70 was observed. In contrast, CD-1 peritoneal macrophages secreted detectable amounts of IL-12p70 in response to purified lipopolysaccharide from B. abortus 2308.

In human macrophages, interferon- $\beta$, a naturally occurring protein produced by virus-infected cells - unlike interferon- $\gamma$ that can only prime the production of IL-12 - is able to produce this cytokine in response to antigenic stimuli (JIANG and DHIBJALBUT 1998).

In BALB/c mice treated with one dose of alcohol $(5 \mathrm{mg} / \mathrm{kg})$ or buffered saline and challenged $30 \mathrm{~min}$ later with lipopolysaccharide (LPS; $0.1 \mathrm{mg} / \mathrm{kg}$ ) or mycobacterial lipoarabinomannan (araLAM; $4 \mathrm{mg}$ / $\mathrm{kg}$ ) intratracheally under anaesthesia, and sacrificed $6 \mathrm{~h}$ later lung homogenates analysed for IL-12 (p75) showed a weak suppression of this cytokine by alcohol (MAson et al. 1997).

When expressed by transfected tumour cells, IL12 decreased the tumorigenicity of MCA-207 and MCA-102 fibrosarcoma cell lines (TAHARA et al. 1995), but had no influence on C26 colon carcinoma cells (MARTinotTi et al. 1995).

\subsubsection{7 \\ Interleukin 13}

Human interleukin 13 (IL-13) is a cytokine that has a profound effect on primary immune cells by inducing immunoglobulin production, proliferation of $B$ cells, and the differentiation of cells of the monocytic lineage. Alveolar macrophages obtained by bronchoalveolar lavage expressed more IL-13 mRNA in patients with fibrotic lung disease, in comparison with those from healthy volunteers (HANCOCK et al. 1998). IL-13 protein was detectable in the bronchoalveolar lavage fluid of 8 of 13 pa- 
tients with pulmonary fibrosis, but in none of the control subjects.

\subsubsection{8 \\ Interleukin 18}

In 6 day granulocyte-macrophage colony-stimulating factor-treated human macrophages, an "inactive" IL-18-recognizing monoclonal antibody detected the IL-18 proform $(24 \mathrm{kDa})$ and a $48-\mathrm{kDa}$ protein, which were gradually increased concomitant with maturation stage (KIKKAWA et al. 2001).

\subsubsection{9}

\section{Tumour Necrosis Factor- $\alpha$}

Tumour necrosis factor is a pleiotropic cytokine, initially isolated on the basis of its capacity to induce tumour necrosis (BEUTLER and CERAMI 1988). It exists in two forms, TNF- $\alpha$ and TNF- $\beta$, both having molecular masses around $17 \mathrm{kDa}$. TNF has a wide range of biological activities, including fibrogenic activity. In vitro, it enhances the proliferation of some fibroblast lines but inhibits others (SUgarman et al. 1985), and it inhibits collagen secretion (DAYER et al. 1985). In vivo, it is strongly fibrogenic (Piguet et al. 1990). In patients with adult respiratory distress syndrome, significant amounts of TNF were observed in the bronchopulmonary secretions, although no TNF was detected in samples of control patients (Millar et al. 1989). Using a human bronchial epithelial cell line (BEAS2B), Clara cell secretory protein mRNA levels increased in response to TNF- $\alpha(20 \mathrm{ng} / \mathrm{ml})$ stimulation after 8 to $36 \mathrm{~h}$ with the peak increase at $18 \mathrm{~h}$ (YAO et al. 1998). Immunoblotting of CCSP protein released into the culture media demonstrated that TNF- $\alpha$ induced the synthesis and secretion of CCSP protein released in a time dependent manner over 8 to $18 \mathrm{~h}$.

TNF has been shown to be present in the blood of patients with rheumatoid arthritis (COPE et al. 1992).

The gene of the TNF protein is located on the short arm of chromosome 6 , in close proximity to the major histocompatibility complex. It is closely related to another cytokine discovered by RUDDLE and WaKsman (1968) and Granger and WilliAMs (1968). The former group called the protein cytotoxic factor while the latter group named it lymphotoxin (LT). TNF and LT are $35 \%$ homologous (LI et al. 1987). They lie closely spaced within the class III region of the major histocompatibility complex, and they bind to the same receptors and share many activities, such as killing of L929 cells, induction of necrosis in Meth A sarcomas and acti- vation of polymorphonuclear leucocytes (GRAY et al. 1984; Shalaby et al. 1985). These observations prompted them to change the name LT to TNF- $\beta$, and that of TNF to TNF- $\alpha$.

ProTNF- $\alpha$ is initially produced as a membranebound form that is processes by TNF- $\alpha$ converting enzyme (TACE), a type I membrane-bound metalloproteinase (BLACK et al. 1997, Moss et al. 1997). TACE represents a potential target for the development of a therapeutic agent for inflammation and related diseases (PATEL et al. 1998). TACE is inhibited by TIMP-3 but not by TIMP-1, -2 and -4 (Amour et al. 1998).

TNF is a macrophage/monocyte-derived anticancer cytokine (CARSWELL et al. 1975, MATTHEWS 1978, NiITsu et al. 1985) with strong cytotoxicity to tumour cells in vitro (Helson et al. 1975, NiITsu et al. 1988, WATANABE et al. 1985, 1988). In the transformed murine macrophage cell line RAW 264.7, TNF- $\alpha$ up-regulation $4 \mathrm{~h}$ after lipopolysaccharide addition seems to involve protein kinase $\mathrm{C}$, as staurosporine strongly inhibited (over $70 \%$ ) the expression of TNF- $\alpha$ mRNA while calphostin had no significant influence (Tremblay et al. 1995).

Recombinant human TNF induced increased hydroxyl radical production in TNF-sensitive cells (YAMAUCHI et al. 1989). Liochev and Fridovich (1997) explained the $\mathrm{O}_{2}{ }^{--}$-dependent toxicity of TNF on the basis of a variant of the Fenton reaction:

$$
\mathrm{Fe}(\mathrm{II})+\mathrm{LOOH} \longrightarrow \mathrm{Fe}(\mathrm{III})+\mathrm{OH}^{-}+\mathrm{LO}^{\circ}
$$

where $\mathrm{L}$ denotes a polyunsaturated fatty acid such as arachidonate. The alkoxy radical $\left(\mathrm{LO}^{\circ}\right)$ so produced can initiate the oxidation of polyunsaturated lipids in membranes by a free radical chain reaction (TAPPEL 1972, BARCLAY and INGold 1981). The initiation and propagation reactions of lipid peroxidation can be blocked by antioxidants such as butylated hydroxy anisole (BREKKE et al. 1994), $N$-acetyl cyteine, pyrrolidine dithiocarbamate and nordihydroguaiaretic acid. Each of these antioxidants has been seen to diminish the cytotoxicity of TNF.

Using the stable free radical, 1,1-diphenyl-2picrylhydrazyl (DPPH) and the electron spin resonance technique, Matsubara et al. (1991) showed TNF- $\alpha$ to have scavenging properties in vitro.

TNF- $\alpha$ synthesis was induced in resting human blood monocyte cultures by actinomycin D (VoITENoK et al. 1989). Cycloheximide inhibited the production of TNF- $\alpha$. The data imply that TNF- $\alpha$ synthesis by human blood monocytes can be induced by posttranscriptional regulation of TNF- $\alpha$ mRNA presynthesized in vivo.

Serum TNF- $\alpha$ levels were increased, although nonsignificantly, in male adult older C57BL/6J mice 
that received either melatonin $(10 \mu \mathrm{g} / \mathrm{ml}$ drinking water, only during the night hours from 18.00 and 9.00) or $\mathbf{Z n}(22 \mu \mathrm{g} / \mathrm{ml}$ drinking water) supplementation for 3 months (CHEN et al. 1999).

$\mathrm{PbCl}_{2}$ increased TNF- $\alpha$ secretion from human peripheral blood mononuclear cells in a concentration- and time-dependent manner, suggesting that $\mathrm{PbCl}_{2}$ increased TNF- $\alpha$ expression by posttranscriptional mechanisms and enhanced the reactivity and uptake of TNF- $\alpha$ by increasing the surface expression of TNF-R p55 (Guo et al. 1996).

A 30-min exposure to $\mathrm{SO}_{2}$ induced a significant $(P<0.001)$ dose-dependent decrease in spontaneous and lipopolysaccharide-stimulated TNF- $\alpha$ release from human alveolar macrophages cultured in vitro (KNORST et al. 1996).

NO production by murine peritoneal macrophages co-cultured with $\mathrm{CD}^{+} \mathrm{T}$ cells prepared from BCG-infected mice was induced by TNF- $\alpha$ together with IFN- $\gamma$ (SAIto and NAKANo 1996).

Rat alveolar macrophages lavaged 1 day after intratracheal instillation of welding fumes $(1 \mathrm{mg} /$ $100 \mathrm{~g}$ body weight) collected during flux-covered manual metal arc welding (using a stainless steel consumable electrode containing $\mathrm{Cr}, \mathrm{Ni}$, and $\mathrm{Mn}$ ), released significantly more $(P<0.05)$ TNF- $\alpha$ than did fumes from gas metal arc welding using a mild steel electrode (ANTonini et al. 1996).

In NMRI mice bronchoalveolar lavage fluid gained 3 days after an intratracheal instillation of $2 \mathrm{mg}$ Dörentrup quartz DQ12 suspended in $100 \mu \mathrm{l}$ saline levels of TNF- $\alpha$ protein from fibrotic, inflammatory, and non-inflammatory models were significantly lower than in controls (HuAux et al. 1999). Although this downregulation of TNF- $\alpha$ was maintained for up to 120 days in the fibrotic model, TNF- $\alpha$ levels returned to control values after 15 and $30 \mathrm{~d}$ in the non-inflammatory and resolving inflammatory models, respectively. The pattern of TNF- $\alpha$ mRNA expression in the lung was opposite to that of the protein. In the resolving and inflammatory models, mRNA levels were upregulated as compared with those of controls. This effect was present for up to $30 \mathrm{~d}$ in the resolving alveolitis model, and at all time points examined in the fibrotic model. No effect on TNF- $\alpha$ mRNA levels was observed in the non-inflammatory model. The downregulation of TNF- $\alpha$ protein was concurrent with the accumulation of recruited polymorphonuclear neutrophils in alveoli, and coculture experiments showed that polymorphonuclear leucocytes explanted from the lungs of mice treated with silica particles were able to downregulate the expression of TNF- $\alpha$ protein by naive alveolar macrophages. In addition, polymorphonuclear leucocyte depletion prevented the downregulation of TNF- $\alpha$ induced by silica.
Significantly $(P<0.05$ vs. control $)$ increased amounts of TNF- $\alpha$ mRNA were found in Wistar rat alveolar macrophages exposed to crocidolite, chrysotile A, chrysotile $B$, MMVF 21 , CRF 1 , or SiC whiskers for $90 \mathrm{~min}$, and significantly $(P<0.05$ vs. control) increased activities of TNF- $\alpha$ were found in the medium of macrophages exposed to crocidolite, chrysotile A, chrysotile B or MMVF 21 for 4 h (LJUngman et al. 1994).

Long amosite stimulated more TNF release from HAN Wistar rat alveolar macrophages than short amosite $(P<0.05)$ (DonALDson 1992). Short chrysotile fibres augmented the production of TNF $(481 \pm 122 \mathrm{U} / \mathrm{ml})$ by male Wistar rat alveolar macrophages to a significantly greater extent than did the long fibres $(260 \pm 65 \mathrm{U} / \mathrm{ml} ; P<0.01)$ (MorIмото et al. 1993). While the short ceramic fibres appeared to stimulate TNF production less than did the long fibres $(112 \pm 21 \mathrm{U} / \mathrm{ml}$ vs. $161 \pm 76 \mathrm{U} / \mathrm{ml})$, the difference was not statistically significant.

Both wool and grain dust samples were capable of stimulating TNF release from rat alveolar macrophages isolated by bronchoalveolar lavage, in a dose-dependent manner (Brown and DonALDSON 1996). Leaches prepared from the dusts contained LPS and also caused TNF release but leachable LPS could not account for the TNF release and it was clear that non-LPS leachable activity was present in the grain dust and that wool dust particles themselves were capable of causing release of TNF.

The antibody for recombinant human TNF- $\alpha$ completely inhibited the formation of multinucleated giant cells from rat bone marrow-derived macrophages treated with acetyl lignin (SoRIMACHI et al. 1995). By an autocrine and/or paracrine mechanism TNF- $\alpha$ could interact immediately with its receptor.

TNF is inactivated rapidly in and cleared from the circulation. The clearance mechanism seems to consist of two phases. First, after the release of TNF in circulation, inactivation of the biologic activity of TNF is observed. Thereafter, clearance of the TNF protein takes place, mainly by the kidney and to a much lesser extent by the liver (BEutLer et al. 1985, Pessina et al. 1987).

When expressed by transfected tumour cells, TNF decreased tumorigenicity of Chinese hamster ovary carcinoma cells (OLIFF et al. 1987, Quin et al. 1995), J558L plasmacytoma cells (BLANKENSTEIN et al. 1991, НосH et al. 1993), 1591-RE skin tumour cells (Teng et al. 1991), MCA-205 fibrosarcoma cells (Asher et al. 1991), and TS/A murine mammary adenocarcinoma (AlLione et al. 1994), but had no influence on MCA-102 fibrosarcoma cells (KARP et al. 1993), and B16-F10 melanoma cells (Dranoff et al. 1993). TNA- $\alpha$ gene suppressed 
Friend leukaemia cells (Ferrantini et al. 1993, 1994), and TNF- $\gamma$ gene C1300 neuroblastoma cells (WATANABE et al. 1989), CMS-5 fibrosarcoma cells (GANSBACHER et al. 1990), SP1 and CT-26 colon carcinoma cells (Esumi et al. 1991).

\subsubsection{0}

\section{Interferons}

Interferons are a family of proteins secreted by vertebrate cells in response to various stimuli. They were identified by IsAAC and LINDEMAN (1957) as antiviral agents and they have long been known for their antitumoral and antiproliferative activities (PAucker et al. 1962, Sen and Lengyel 1992).

Initially classified according to their cellular origin, the interferons are now typed according to their sequences and their activities. Interferon type I regroups IFN- $\alpha$ (leukocyte IFN) and IFN- $\beta$ (fibroblast IFN); IFN type II: IFN- $\gamma$ (immune IFN).

After induction by 10 -carboxymethy-9-acridanone, murine bone marrow-derived macrophages produced two activities of interferon $\beta$ differing in the degree of glycosilation (BREHM et al. 1986).

Using the stable free radical, 1,1-diphenyl-2picrylhydrazyl (DPPH) and the electron spin resonance technique, Matsubara et al. (1991) showed both interferon $\alpha$ and interferon $\gamma$ to have scavenging properties in vitro.

IFN- $\gamma$ alone or combined with lipopolysaccharide induced iNOS expression and increased nitrite production in $\mathrm{iNOS}^{+/+}$macrophages, but not in iNOS $^{-/-}$macrophages (NIU et al. 2000). Formation of thiobarbituric acid reactive substances from lowdensity lipoprotein was suppressed in IFN- $\gamma$ - and IFN- $\gamma /$ lipopolysaccharide-treated $\mathrm{iNOS}^{+/+}$macrophages but was increased in IFN- $\gamma$-treated iNOS ${ }^{-1}$ macrophages. In the presence of $N^{G}$-monomethyl-Larginine the suppressive effect of IFN $-\gamma$ and IFN $-\gamma /$ lipopolysaccharide was abolished and the formation of thiobarbituric acid reactive substances was even increased to a level above that of untreated $\mathrm{iNOS}^{+/+}$macrophages. NOC 18, an NO donor, dosedependently inhibited macrophage-mediated lowdensity lipoprotein oxidation. IFN- $\gamma$ increased superoxide and thiol production in both types of macrophages.

Collagen synthesis in fibroblast cultures is suppressed by interferons (Rosenbloom et al. 1984, Rockey et al. 1992). Interferon- $\alpha$ is less potent than interferon- $\gamma$.

\subsubsection{1 Fibroblast Growth Factors (FGFs)}

Fibroblast growth factors (FGFs) comprises a family of peptides with a potent fibrogenic activity in vitro and in vivo (SPRUGEL et al. 1987) but whose biological role is poorly understood. They are present in a wide variety of cell types but it is not established whether their synthesis and secretion are regulated and/or if they are released only by damaged cells (D’Amore 1990).

\subsubsection{2 \\ Colony Stimulating Factor (CSF)}

The macrophage colony stimulating factor, CSF-1, is responsible for survival, proliferation, and differentiation of mononuclear phagocytes from bone marrow progenitor cells to mature macrophages (TUSHINSKI et al. 1982). It is a homodimeric glycoprotein growth factor, and its pleiotropic effects are mediated via a high affinity cell surface CSF-1 receptor (Guilbert and Stanley 1980, Byrne et al. 1981, Bartelmez and Stanley 1985) identical to the c-fms protooncogene product (SHERR et al. 1985). $1,25(\mathrm{OH})_{2}$ vitamin $\mathrm{D}_{3}$ prompted appearance of CSF-1 receptor in murine bone marrow precursors (Perkins and Teitelbaum 1991). Specific ${ }^{125} \mathrm{I}-$ CSF-1 binding by BAC 1,2F5 cells is enhanced ( $P$ $<0.001)$ after $24 \mathrm{~h}$ exposure to $10 \mathrm{nM} 1,25(\mathrm{OH})_{2}$ vitamin $\mathrm{D}_{3}$ (Meenakshi et al. 1993).

Macrophage activation with yeast glucan induced a pronounced elevation of colony stimulating activity in serum (SATOH et al. 1982, Chinara 1983). The increase of colony stimulating activity was dose dependent. While doses of $62.5 \mathrm{mg}$ krestin $(\beta-1,4 ; \beta-1,3 ; \beta-1,6$, protein complex; PSK)/kg and $125 \mathrm{mg} \mathrm{PSK} / \mathrm{kg}$ produced no increase in colony stimulating activity, a dose of $250-1000 \mathrm{mg} / \mathrm{kg}$ was associated with a rapid increase in colony stimulating activity followed by a rapid decline. Yeast glucan has been demonstrated to be very effective in enhancing CSF at doses where PSK is ineffective (PATCHEN and MCVittie 1983).

Overexpression of granulocyte-macrophage colony-stimulating factor induces pulmonary granulation tissue formation and fibrosis by induction of transforming growth factor- $\beta 1$ and myofibroblast accumulation (XING et al. 1997). 


\subsection{2 Complement Components}

Most of the serum complement proteins are produced primarily in the liver, but monocytes and macrophages constitute an important source of extrahepatic production of many complement components. Local production of complement at tissue sites of inflammation may be of considerable importance in host defences and immunopathological reactions.

Maturation of mononuclear phagocytes into tissue macrophages may be signalled or enhanced by changes in their capacity to synthesise and secrete biologically active complement proteins. Human breast milk macrophages secrete haemolytically active $\mathrm{C} 2$ and factor B immediately in vitro, while a lag of 3 to 6 days in complement production is noted in peripheral blood monocytes from the same woman (Cole et al. 1980). The rate of secretion of both $\mathrm{C} 2$ and $\mathrm{C} 4$ by macrophages from different tissues may be compared by simultaneous plaque assay and tissue culture (Cole et al. 1980). While alveolar macrophages from guinea pig secrete less $\mathrm{C} 2$ and $\mathrm{C} 4$ than peritoneal macrophages in culture, the rates of $\mathrm{C} 2$ and $\mathrm{C} 4$ secretion by complement producing alveolar macrophage are greater than the rates per complement producing peritoneal macrophage (Cole et al. 1980).

Posttranslational modification of precursor proteins appears to be an important regulatory step in the synthesis and secretion of complement proteins, as it is with other proteins (Oor et al. 1980). HALL and Colten (1978) showed in the guinea pig, that deficiency of $\mathrm{C} 3$ resulted from a translational defect in the synthesis of $\mathrm{C} 4$ protein. Under cell-free conditions, hepatic polysomes from C4-deficient guinea pigs synthesised only nascent $\mathrm{C} 4$ polypeptides that remain polysome-bound. Thus, the mRNA appears to be present but not completely translated.

The complement peptides C5a and C5a-desArg have been found to elicit the respiratory burst: $h u$ man C5a has a threshold of $10^{-9} \mathrm{M}$ for $\mathrm{O}_{2}{ }^{\circ-}$ generation; maximum is still not reached at $>10^{-7}$ ( $\mathrm{DA}^{-}$ HINDEN et al. 1983, McPhail and SNYderman 1983, GERARD et al. 1986). C5a-desArg from the same species is approximately 50 times less potent (WeBster et al. 1980). The activity of human C5a is greatly enhanced in the presence of a yet unidentified serum factor (protein?) (MALY et al. 1983).

\subsection{3}

\section{Platelet-Activating Factor}

The synthesis of the platelet-activating factor (PAF; 1-alkyl-2-acetyl-sn-glycero-3-phosphocholine) by the action of phospholipase $\mathrm{A}_{2}$ on phosphatidylcho- line, resulting in the generation of lysophosphatidylcholine. Alkyl species of phosphatidylcholine are a major source of PAF. They are converted to lysoplatelet-activating factor (lysoPAF) and then by PAF acetyltransferase to PAF (STURK et al. 1989, SNYDER 1990).

\subsection{4}

\section{Proteolytic Enzymes}

\subsubsection{1}

\section{Metalloproteinases}

Metalloproteinases are usually synthesised as proenzymes in balance with activating and inhibiting proteins. By blocking the synthesis of the major inhibitor TIMP (tissue inhibitor of metalloprotease by the antisense technique), the importance of the fine regulation of this balance was deduced. Upon removal of TIMP, the cells adopt a partially transformed phenotype, namely, anchorage-independent growth and soft agar colony formation (Кнокна et al. 1989). Another feature of metalloprotease activity has been revealed by overexpressing collagenase type I or stromelysin in rat 2 cells that had been transformed by the activated ras gene.

Human matrix metalloproteinases may be processed from their proenzyme forms to their active forms by two new and unique mechanisms (MAEDA et al. 1998): Firstly, by bacterial proteases such as Pseudomonas elastase and Vibrio cholerae protease, which cleave off the $N$-terminal autoinhibitory domain (so-called cysteine switch) from pro-matrix metalloproteinases. The second mechanism depends on free radical generation by activated polymorphonuclear leucocytes. In this case, peroxynitrite $\left(\mathrm{ONOO}^{-}\right)$or nitrogen dioxide radical $\left({ }^{\circ} \mathrm{NO}_{2}\right)$ are the key reagents.

$$
\begin{array}{lr}
\mathrm{O}_{2}^{--}+{ }^{-} \mathrm{NO} \longrightarrow \mathrm{ONOO}^{-}\left(\mathrm{pK}_{\mathrm{a}}=6.8\right) & {[82]} \\
\mathrm{ONOO}^{-}+\mathrm{H}^{+} \longrightarrow \mathrm{ONOOH}^{-} & {[83]} \\
\mathrm{ONOOH} \rightarrow \mathrm{HO}^{-}+{ }^{-} \mathrm{NO}_{2}(20-30 \% \text { yield }), \mathrm{NO}_{3}{ }^{-}+\mathrm{H}^{+} \text {(rest) }[84]
\end{array}
$$

Both $\mathrm{O}_{2}{ }^{--}$and ${ }^{\circ} \mathrm{NO}$ are generated by activated macrophages and polymorphonuclear leucocytes as a result of immunologic responses involving various proinflammatory cytokines. ${ }^{\circ} \mathrm{NO}_{2}$ or $\mathrm{ONOO}^{-}$seems to interact with a single cysteine residue in the propeptide autoinhibitory domain, or so called cysteine-switch of pro-matrix metalloproteinases, thus transforming pro-matrix metalloproteinases into their active conformation.

TIMP-3 inhibited TNF- $\alpha$ converting enzyme (AMour et al. 1998). 


\subsubsection{1}

\section{Elastase}

The detection and quantification of macrophagederived elastolytic enzymes are important considerations when studying model systems designed to evaluate the impact of macrophage secretory proteinases on lung and vascular elastin. The mouse peritoneal macrophage elastase is a neutral metalloproteinase (BANDA and WERB 1981) catalytically and immunochemically distinct from mouse pancreatic and mouse granulocyte elestases, both of which are serine proteinases. Macrophage elastase was inhibited by $\alpha_{2}$-macroglobulin, but not by $\alpha_{1}$-proteinase inhibitor. Mouse macrophage elastase catalysed the limited proteolysis of selected subclasses of mouse immunoglobulins, including monomeric $\mathrm{IgG}_{2 \mathrm{a}}$, $\mathrm{IgG}_{3}$, and some forms of $\mathrm{IgG}_{2 \mathrm{~b}}$ (BANDA et al. 1983). Mouse $\operatorname{IgG}_{1}$ was resistant to elastase degradation; however, human $\mathrm{IgG}_{1}$ was degraded. $\mathrm{IgG}_{3}$ in immune complexes was cleaved in a manner similar to that of monomeric $\mathrm{IgG}_{3}$. Degradation by macrophage elastase was limited to the heavy chain, resulting in products that did not compete for binding to the macrophage $\mathrm{Fc}$ receptor. Macrophage elastase usually produced a pepsin-like rather than a papain-like pattern of proteolysis, resulting in the release of $\mathrm{F}(\mathrm{ab})_{2}$ and $\mathrm{FC}^{\prime}$ subfragments. This degradation of IgG differed from the papain-like cleavage of IgG by granulocyte elastase. Macrophage elastase degraded papain-generated $\mathrm{Fc}$ fragments of $\mathrm{IgG}_{2 \mathrm{a}}$ into multiple fragments. Therefore, macrophage elastase at concentrations found in culture medium has a potential to regulate some aspects of cellular events associated with immunoglobulins.

The C-terminal domain of human macrophage elastase inhibited elastin degradation (GRONSKI jr. et al. 1996).

\subsubsection{2 \\ Collagenase}

Reactive oxygen species and serine proteases activated latent $70-75 \mathrm{kDa}$ human neutrophil collagenase (SAARI et al. 1990). The hydroxyl radical may cause the oxidative activation of collagenase by cleaving the bond between $\mathrm{Cys}^{73}$ and the forth coordination site of active site $\mathrm{Zn}$ atom and oxidise cysteine to cysteic acid that cannot be liganded by the $\mathrm{Zn}$ atom (SPRINGMAN et al. 1990). Hydrogen peroxide $\left(\mathrm{H}_{2} \mathrm{O}_{2}\right)$ increased the steady-state mRNA levels of collagenase/MMP-1 in human dermal fibroblasts (BRENNEISEN et al. 1997).

\subsubsection{3 \\ Stromelysin}

Stromelysin 1 (= matrix metalloproteinase 3 ) has been implicated as playing a pivotal role in jointdegrading diseases like arthritis (HASTY et al. 1990, HEMвRY et al. 1995). Its synthesis by chondrocytes and synoviocytes can be induced by inflammatory mediators like interleukin-1 (HASTy et al. 1990, MacNaul et al. 1990).

Stromelysin 2 (EC 3.4.24.22 = matrix metalloproteinase $10=$ transin 2 )

Stromelysin 3 (= matrix metalloproteinase 11) from rat skin has 491 amino acids, and shows 83, 95 and $58 \%$ homology with human, mouse and Xenopus ST3, respectively (OKADA et al. 1997). In contrast to other matrix metalloproteinases, ST3 is secreted into the extracellular space as a potentially active molecule (Pei and Weiss 1995, SantavicCa et al. 1996). By in situ hybridisation, Wolf et al. (1993) detected ST3 transcripts specifically in fibroblastic cells in primary breast carcinomas and so did OKada et al. (1997) in fibroblastic cells localised in the superficial dermis of healing rat skin wounds.

\subsubsection{4 \\ Gelatinase B (EC 3.4.24.35)}

This $92-\mathrm{kDa}$ gelatinase $(=$ matrix metalloproteinase 9 = type $\mathrm{V}$ collagenase $=92-\mathrm{kDa}$ type IV collagenase $=$ macrophage collagenase) is similar to collagenase A, but possesses a further domain. It is expressed in macrophages and eosinophils, and is stored in the tertiary granules of neutrophils as well. To assess the role of the $92 \mathrm{kDa}$ gelatinase in neutrophil, macrophage, and eosinophil function, Shipley et al. (1996) generated mice deficient in this enzyme by targeted mutagenesis. The murine $92 \mathrm{kDa}$ gelatinase gene was cloned from a 129Sv genomic P1 library. In the targeting construct, most of exon 2 is replaced with a cassette consisting of the neomycin phosphotransferase cDNA driven by the phosphoglycerate kinase promoter. The construct contains $5 \mathrm{~kb}$ of $5^{\prime}$ homolgy and $3 \mathrm{~kb}$ of $3^{\prime}$ homology. The targeting construct was introduced into 129Sv embryonic stem cells by electroporation, and eight independent correctly-targeted clones were obtained from 120 G418-resistant colonies. Chimeric mice were bred with C57BL/6J mice to generate mice heterozygous for the targeted allele.

FinLey et al. (1997) found that expression of gelatinase B mRNA was enhanced in macrophages from patients with chronic obstructive pulmonary disease. 
The functions of gelatinase $B$ as regulator and effector in leucocyte biology were reviewed by OPDENAKKER et al. (2001).

Matrix metalloproteinases- 2 and -9 facilitated tumour invasion via basement membrane degradation. Metastatic colorectal cancer cells (CRC line W620) induced matrix metalloproteinase release by human THP-1 monocytes (SWALlow et al. 1996).

\subsubsection{5 \\ 68 kDa Gelatinase}

\subsubsection{6}

\section{Angiotensin Convertase (EC 3.4.15.1)}

Angiotensin converting enzyme (EC 3.4.15.1) is a membrane-bound ectoenzyme of human blood monocytes augmented by dexamethasone in a biphasic dose-dependent manner with maximum effect after 6 days in culture at $10^{-8} \mathrm{M}$ concentration (Vuk-Pavlovic et al. 1989).

\subsubsection{2}

\section{Serine Proteinases}

\subsubsection{1}

\section{Urokinase-type Plasminogen Activator}

(EC 3.4.21.73)

Plasminogen activator, a neutral serine protease, is secreted by many different cell types and is thought to be important in cell migration, malignant cell transformation, tissue remodelling, and cytolysis of tumour cells. Alveolar macrophage plasminogen activator could be important in resolution of fibrin clots, promotion of inflammation, or defence against malignant cells in the lung. Secretion of plasminogen activator by rabbit alveolar macrophages derived from normal animals and rabbits pretreated with bacillus Calmette-Guérin (BCG) to activate these macrophages indicated by an increase in the phagocytic index was detected by SCHUYLER and FORMAN (1984) in the conditioned medium of day 2-3 alveolar macrophage cultures from 17 of 22 animals treated with BCG, opposed to 2 of 14 normal animals $\left(P<0.001, \chi^{2}\right.$ test $)$.

Endotoxin ( $5 \mathrm{mg}$ per kg body weight intraperitoneally) rapidly increased plasminogen activator release by rat alveolar macrophages (Етон et al. 1984). There was significant positive correlation between plasminogen activator release by alveolar macrophages and fibrinolytic activity in bronchoalveolar lavage fluid.

In murine $\mathrm{C} 57 \mathrm{BL} / 6$ peritoneal macrophages elicited with either proteose-peptone or fresh thioglycollate, enhancement of the secretion of plasmino- gen activator by lymphokine did not require bacterial lipopolysaccharide in addition to lyphokine (Jones et al. 1984).

Concanavalin A and phorbol myristate acetate greatly increased secretion of rabbit alveolar macrophage plasminogen activator, although the higher concentration of $10.0 \mu \mathrm{g}$ Con $\mathrm{A} / \mathrm{ml}$ had an adverse effect on viability (SCHUYLER and ForMAN 1984).

Urokinase produced by alveolar macrophages is operative not only at the alveolitis stage but also later in the fibrotic process, produced by silica particles, supporting the role of urokinase-type plasminogen activator in fibrogenesis (LARDOT et al. 1998).

Asbestos increased urokinase-type plasminogen activator receptor at the surface of rabbit and $h u$ man mesothelial cells, suggesting that altered expression of this receptor could be involved in asbestos-induced remodelling of the pleural mesothelium (Perkins et al. 1999).

Athersclerotic vessels studied by immunohistochemistry showed that urokinase plasminogen activator, plasminogen activator inhibitor type 2, and macrophages were mainly expressed within plaques while tissue plasminogen activator and plasminogen activator inhibitor type 1 were also expressed outside plaque lesions (FALKEnBERG et al. 1998).

\subsubsection{2}

\section{Cytolytic Proteinase}

\subsubsection{3 \\ Aspartyl Proteinases}

\subsubsection{1}

Cathepsin D (EC 3.4.23.5)

Cathepsin D is a lysosomal protease present in normal cells at a very low concentration. It is first produced in a precursor form, pro-cathepsin $D$ $(52 \mathrm{kDa})$, and the processed in the cell to an intermediate form of $48 \mathrm{kDa}$, then finally to the mature forms of $34 \mathrm{kDa}$ and $14 \mathrm{kDa}$. Phagocytosis of intravenously injected IgG-coated sheep red blood cells by rat liver Kupffer cells did not change the activity of cathepsin D (Brouwer et al. 1981).

\subsubsection{4 \\ Cysteine Proteinases (EC 3.4.22)}

Cysteine proteinases are most implicated in matrix degradation and thus in tumour growth and spontaneous metastasis. A comprehensive knowledge of their biochemistry, pharmacology and therapeutic influencabilty is useful as to tumour growth and metastasis prevention. 
L - trans - Epoxysuccinyl-leucylamido(guanidino) butane (E-64) and its analogues inhibited cysteine proteinases including cathepsins $\mathrm{B}, \mathrm{H}$, and L (BARRETT et al. 1982). Conway et al. (1996) tested some sulphamide derivatives by s.c. implantation of minipumps, which delivered compound throughout the period of primary tumour growth and spontaneous metastasis to the lung at steady state drug concentration orders of greater magnitude than the concentrations needed to either inhibit collagenase, gelatinase or stromelysin in vitro. Inhibitor treatment showed the growth of primary s.c. Mat Ly Lu rat prostate tumour, LOX human melanoma tumours by $40-60 \%$ but had no significant effect on the growth of primary M27 murine Lewis lung tumours.

\subsubsection{1}

\section{Cathepsin B (EC 3.4.22.1)}

Cathepsin B is a lysosomal cysteine endopeptidase with broad specificity for peptide bonds. It preferentially cleaves -Arg-Arg- bonds in small molecule substrates (thus differing from cathepsin $\mathrm{L}$ ).

Cathepsin B/L activity in microglia compared to both elicited or resident rat peritoneal macrophages was significantly $(P<0.001)$ higher (BANATI et al. 1993). The significanty higher $(P=0.029)$ fluorescence of thioglycolate-elicited versus resident rat peritoneal macrophages demonstrated that the activation of macrophages is accompanied by an increase of cathepsin B/L activity expression.

The extracellular release of cathepsin B was demonstrated in the amyloid deposits of Alzheimer brain (CATALDO and NiXON 1990), where microglial activation is a prominent neuropathological feature (McGeer et al. 1988, Styren et al. 1990).

Cigarette smoke (twice daily 10 puffs) stimulated cathepsin B activity in alveolar macrophages of Sprague-Dawley rats by $43 \%$ at both 4 - and 10 week exposure points (GAIrola et al. 1989).

\subsubsection{2}

Cathepsin L (EC 3.4.22.15)

Human alveolar macrophages synthesise and express an active form of cathepsin L (MAson et al. 1986, Chapman et al. 1987).

\subsection{5 \\ Other Enzymes}

\subsubsection{1}

Lipases

Human blood mononuclear leucocyte lysosomal acid lipase (triacylglycerol acylhydrolase, EC
3.1.1.3) in two individuals hydrolysed 9.5 and $9.0 \mathrm{nmol}$ substrate per min $\times \mathrm{mg}$ protein (COATES et al. 1979). The enzyme is under thyroid hormone regulation (CoATEs et al. 1982).

\subsubsection{2 \\ Glucosaminidase: Lysozyme}

Lysozyme (EC 3.2.1.17, mucopeptide $N$-acetylmuramoyl hydrolase or muramidase) catalyses hydrolysis of a $\beta(1 \rightarrow 4)$-glycosidic linkages of polysaccharide component of the peptidoglycan (mucopolymer of the bacterial cell wall), which is composed of alternating $N$-acetylmuramic acid and $N$-acetylglucosamine residues with alternating $\beta(1 \rightarrow 4)$ and $\beta(1 \rightarrow 6)$ linkages, cross-linked with peptide chains. The hydrolytic action of lysozyme gives rise to disaccharide units attached to the peptide chains called muropeptides.

High concentrations of lysozyme were found in monocytes (SchmalzL and Braunsteiner 1970, GoRDon et al. 1974) and alveolar macrophages (MYrvik et al. 1961, Cohn and Wiener 1963, Heise and Myrvik 1967, LeAKe and Myrvik 1968, McClelland and van Furth 1975). Lysozyme is considered to be one of the constitutive enzymes of the macrophages (Gordon et al. 1974, Gordon 1978). It is the major secretory product and forms about $25 \%$ of the extracellularly secreted protein of macrophages.

In 6 from 11 patients with sarcoidosis, initial 24$\mathrm{h}$ secretion of lysozyme by monocytes exceeded the normal range of values for cells from 11 age- and sex-matched control individuals (BODEL et al. 1979). Cells with initially augmented secretion rates continued to secrete increased amounts of lysozyme for 3 days. A correlation was noted between in vitro secretion of lysozyme by monocytes and serum levels of lysozyme in the same patient.

\subsubsection{3 \\ Lysosomal Acid Hydrolases}

\subsubsection{1}

Proteases

\subsubsection{2}

\section{Lysosomal Acid Lipase}

Lysosomal acid lipase (EC 3.1.1.13) is a lipolytic enzyme involved in the intracellular metabolism of cholesteryl esters and triacylglycerols derived from plasma lipoproterins (GolDSTEIN et al. 1975). The enzyme is synthesised in a variety of cells, including fibroblasts (SANDo and HeNkE 1982, SANDO and Rosenbaum 1985), mononuclear leucocytes 
(CoAtes et al. 1982), lymphocytes (CoAtes et al. 1975) and liver cells (AMEIs et al. 1994). After synthesis in the endoplasmic reticulum, the enzyme is targeted to the lysosomal compartment (SANDo and Henke 1982). Ameis et al. (1994) give a complete nucleotide sequence and deduced protein sequence for human LAL cDNA. There are some nucleotide differences of liver and fibroblast LAL cDNA.

\subsubsection{3 \\ (Deoxy)ribonuclease}

\subsubsection{4}

\section{Phosphatases}

Acid phosphatase (EC 3.1.3.2) is the classic lysosomal enzyme. The lead substitution method has localised acid phosphatase in the phagosomes of macrophages (CoHN and WIENER 1963) and can be used for electron microscopy.

\subsubsection{5}

\section{Glycosidases}

Glycosidase ( $N$-acetyl- $\beta$-D-glucosaminidase, $N$-acetyl- $\beta$-D-galactosaminidase, $\beta$-D-galactosidase, $\alpha$-Lgalactosidase, $\alpha$-D-mannosidase, $\alpha$-L-fucosidase and $\beta$-D-glucuronidase) activities were higher in the alveolar macrophages obtained by bronchoalveolar lavage from smokers than in those from nonsmokers (SCHARFMAN et al. 1980).

Swainsonine reversibly inhibited macrophage lysosomal $\alpha$-mannosidase (EC 3.2.1.24) in vitro (Greenaway et al. 1983).

\subsubsection{6}

\section{Sulphatases}

Arylsulphatase (EC 3.1.6.1) from a $96 \mathrm{~h}$ rabbit peritoneal exudate macrophage was present in segments of the rough endoplasmic reticulum, and perinuclear cisterna as well as within numerous small vesicles in the Golgi region, probably corresponding to secondary lysosomes (Nichols et al. 1971, DAVIES and BonNey 1980).

\subsubsection{4}

\section{Deaminase: Arginase}

Rat peritoneal macrophages elicited with $\lambda$ carrageenan had 10 times greater arginase activity than resident cells at harvest (AlbinA et al. 1988).

\subsection{6 Inhibitors of Enzymes}

Minactivin is a human monocyte product which specifically inactivates urokinase-type plasminogen activators (Golder and STEPHENs 1983). This inhibitory activity of monocyte culture supernatant was enhanced after culture with muramyl dipeptide. Inhibition was specific for $M_{r} 52,000$ and 36,000.

\subsection{7 \\ Proteins of Extracellular Matrix or Cell Adhesion}

\subsubsection{1}

\section{Fibronectin}

Alitalo et al. (1980) showed that cultured human blood monocyte-derived macrophages secreted fibronectin, but did not deposit a pericellular fibronectin matrix as do various connective tissue cell in culture.

Ozone exposure ( $0.1 \mathrm{ppm}$ for 10,20 , or $30 \mathrm{~min})$ to alveolar macrophages obtained by bronchoalveolar lavage from healthy, nonsmoking volunteers between the ages of 18 to 25 years, did not produce increased amounts of fibronectin (DEvLIN et al. 1994).

In idiopathic pulmonary fibrosis, alveolar macrophages have glucocorticoid receptors, but glucocorticoid therapy does not suppress alveolar macrophage release of fibronectin (LACRONIQUe et al. 1984).

Rom et al. (1984) studying alveolar macrophages obtained by bronchoalveolar lavage from 7 individuals exposed to asbestos, 4 coal miners and 2 patients with silicosis, suggested that similar to the pathogenesis of fibrosis in the interstitial lung disorders of unknown aetiology, activation of alveolar macrophages to release increased amounts of fibronectin and alveolar macrophage derived growth factor might play a role in the development of fibrosis in the pneumoconioses. In coalworkers' pneumoconiosis, fibronectin was detected by immunofluorescence microscopy on the surface of macrophages laden with dust particles (FrIEMANN et al. 1985). Fibronectin provides a mechanism for attaching the fibroblast to the connective tissue matrix and plays a role as a competence signal to move fibroblasts into the early portion of $G_{1}$ of the replication cycle (ERDOGDU and HASIRCI 1998).

$\mathrm{H}_{2} \mathrm{O}_{2}$ alone induced no changes of fibronectin purified from human plasma, even at an 800-fold molar excess (VISSERS and Winterbourn 1991). Radiolytic $\mathrm{HO}^{\bullet}$ caused a rapid loss of tryprophan fluorescence, an increase in bityrosine fluorescence, and extensive crosslinking. 


\subsubsection{2 Gelatin-Binding Protein/95 kDa Gelatinase}

VARTIo (1985) isolated a $95 \mathrm{kDa}$ protein from $h u$ man blood monocytes cultured in a 1:1 mixture of medium 199 and RPMI 1640 supplemented with $5 \%$ newborn calf serum by preparative polyacrylamide gel electrophoresis. The ability of this fraction to degrade gelatine was calcium-dependent and was inhibited by serum, sulfhydryl and metalchelating agents, but not with serine proteinase inhibitors. Gelatine was degraded optimally at $\mathrm{pH} 7-9$ and at $41^{\circ} \mathrm{C}$ and $37^{\circ} \mathrm{C}$ and less effectively at $22^{\circ} \mathrm{C}$. Native type I collagen was degraded at $41^{\circ} \mathrm{C}$ but not at $37^{\circ} \mathrm{C}$ or $22^{\circ} \mathrm{C}$. The results showed that cultured human macrophages secreted highly specific gelatine-degrading metal-proteinase activity associated with the $95 \mathrm{kDa}$ gelatine-binding protein.

\subsubsection{3}

\section{Thrombospondin}

\subsubsection{4}

\section{Chondroitin Sulphate Proteoglycans}

Resident mouse peritoneal macrophages synthesise and secrete equivalent amounts of chondroitin sulphate and heparan sulphate proteoglycans (KoLSET 1987).

\subsubsection{5 \\ Heparin Sulphate Proteoglycans}

Resident mouse peritoneal macrophages synthesise and secrete equivalent amounts of heparan sulphate and chondroitin sulphate proteoglycans (KOLSET 1987).

\subsection{8 \\ Bioactive Oligopeptides}

\subsubsection{1}

\section{Glutathione}

Glutathione (L- $\gamma$-glutamyl-L-cysteinyl-glycine, GSH) was released by rat pulmonary alveolar macrophages cultured in RPMI 1640 supplemented with $10 \%$ foetal calf serum exposed for $2 \mathrm{~h}$ to $0-100 \mu \mathrm{g}$ quartz particles (Min-U-Sil, $4.5 \pm 1.0 \mu \mathrm{m}$ ) or crocidolite asbestos (IUAC, fibre length $2.1 \pm 0.31 \mu \mathrm{m}$ ) in a concentration-dependent manner (Bовнme et al. 1992).

In plasma from 24 healthy individuals aged 25-35 years, Jones et al. (2000) found the concentration of GSH $(2.8 \pm 0.9 \mu \mathrm{M})$ much higher than that of GSSG $(0.14 \pm 0.04 \mu \mathrm{M})$. The redox potential of the

GSSG/2GSH pool $(-137 \pm 9 \mathrm{mV})$ was considerably more oxidised than values for tissues and cultured cells $(-185$ to $-258 \mathrm{mV})$. This indicates that a rapid oxidation of GSH occurs upon release into plasma. The difference in values between individuals was remarkably small, suggesting that the rates of reduction and oxidation in the plasma are closely balanced to maintain this redox potential.

Glutathione thiyl radicals (GS $)$ can be produced e.g. when radicals from oxidable drugs redox cycle (SCHREIBER et al. 1989), or in the repair of oxidative stress (O'BRIEN 1988) and radiation damage (QuiNTILIANI et al. 1977).

$$
\mathrm{GSH}+\mathrm{HO} \cdot \longrightarrow \mathrm{GS}^{*}+\mathrm{H}_{2} \mathrm{O}
$$

The fate of $\mathrm{GS}^{\bullet}$ depends critically on four key, rapidly established equilibria:

$$
\mathrm{GSH} \leftrightarrow \mathrm{GS}^{-}+\mathrm{H}^{+}
$$

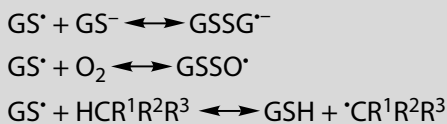

$\mathrm{GS}^{\cdot}+\mathrm{HCR}^{1} \mathrm{R}^{2} \mathrm{R}^{3} \longleftrightarrow \mathrm{GSH}+{ }^{\cdot} \mathrm{CR}^{1} \mathrm{R}^{2} \mathrm{R}^{3}$

and on a fast electron transfer reaction:

$$
\mathrm{GSSG}^{--}+\mathrm{O}_{2} \longrightarrow \mathrm{GSSG}+\mathrm{O}_{2}^{--}
$$

The kinetics of [164]-[167] have been investigated, mostly by pulse radiolysis, although some rate constants are known only for analogous reactions involving other thiols.

Glutathione esters have been shown to increase the levels of GSH in the liver and kidney of mice. Administration of $\gamma$-glutamylcysteinylglycine monomethyl or monoethyl esters at doses of $10 \mathrm{mmol} / \mathrm{kg}$ intraperitoneally raised GSH levels 4-fold in mice with GSH levels depleted by prior treatment with Lbuthionine sulphoxime (PURI and MeIster 1983). The improved uptake of the esters, and their subsequent intracellular hydrolysis to GSH may be explained by improved permeability through lysosomal membranes, and by specific hydrolysis by lysosomal esterases (GoldmAN 1973, Goldman and NAIDER 1974).

Glutathione has long been suspected to be the primary source of oxidative power for protein folding. It has now been shown to be just the opposite, namely a source of reductants (BADER et al. 1999). The ultimate origin of oxidants has become even more of a mystery.

Detoxification by glutathione is involved in the cellular resistance of cancer cells to anticancer drugs (MORROW and COWAN 1990). 
While the IGROV ovarian cancer cell line was very sensitive to cisplatin $\left(\mathrm{IC}_{50}=0.2 \mu \mathrm{g} / \mathrm{ml}\right)$, the $\mathrm{IC}_{50}$ of the drug-resistant subline IGROV $\mathrm{CDDP}_{\text {was }} 4.0 \mu \mathrm{g} /$ $\mathrm{ml}(P<0.01)$ (Schellens et al. 1995). Total GSH in the resistant cell line was not increased: $7.65 \pm 0.84$ in the parental cell line and $4.11 \pm 1.15 \mu \mathrm{g} \mathrm{GSH} / \mathrm{mg}$ protein in $\operatorname{IGROV}_{\mathrm{CDDP}}(P<001)$.

\subsection{9}

\section{Arachidonic Acid and Prostaglandins}

Elaboration of eicosanoids, oxygenated derivatives of arachidonic acid, is one means by which macrophages can regulate the functions of other effector cells, and thereby modulate inflammation and immune processes as well as tissue injury and repair (NATHAN 1987). Inasmuch as eicosanoids have been implicated in physiologic and pathologic processes in the lung (Henderson jr 1987), an understanding of the alterations in arachidonic acid metabolism that accompany macrophage differentiation in the lung is of substantial interest.

\subsubsection{1}

\section{Arachidonic Acid Release from Membrane Phospholipids}

Phospholipid breakdown is one of the earliest cellular events elicited by many extracellular signalling substances. Phospholipases $\mathrm{A}_{2}, \mathrm{C}$ and $\mathrm{D}$ are involved in these signal transduction pathways. Phospholipase $\mathrm{A}_{2}$ (EC 3.1.1.4) or phosphatidylcholine 2acylhydrolase is thought to be the key enzyme of the arachidonic acid cascade inducing the liberation of arachidonic acid from phospholipids and consequently the formation of eicosanoids, which are important biological mediators in many inflammatory processes. Phospholipase C (EC 3.1.4.3) hydrolysing inositol phospholipids produces diacylglycerol, which both serves as the major physiological regulator of protein kinase $\mathrm{C}$ and as substrate for diacylglycerol lipase (EC 3.1.1.34) leading to the liberation of arachidonic acid and thus to the formation of eicosanoids.

When rat pineal glands were incubated in culture, time-dependent release of arachidonic acid was significantly inhibited by a known $85-\mathrm{kDa}$ cytosolic phospholipase $\mathrm{A}_{2}$ inhibitor, methyl arachidonyl fluorophosphonate (LI et al. 2000). Coincubation with melatonin inhibited the arachidonic acid release in a concentration dependent manner, and this decrease was accompanied by a reduction of cytosolic phospholipase $\mathrm{A}_{2}$ protein and mRNA expression. Melatonin-receptor agonists, 2-iodo- $N$-butanoyl-5-methoxytryptamine and 5-methoxycarbonylamino- $\mathrm{N}$-acetyltryptamine, also decreased arachidonic acid release and cytosolic phospholipase $A_{2}$ protein and mRNA levels, while pre-incubation with the melatonin-receptor agonists luzindole and 2-phenylmelatonin abolished the melatonin effects.

Mineral oil-elicited guinea pig peritoneal macrophages released arachidonic acid in excess of that of unstimulated cells when stimulated with the ionophore, A23187, followed in decreasing potency by phorbol myristate acetate, a group of agents of similar activities (opsonized zymosan, phospholipase $\mathrm{C}, \mathrm{NaF}$, and fMet-Leu-Phe), and by concanavalin $\mathrm{A}$ and wheat germ agglutinin (BROMBERg and PICK 1983).

In rat alveolar macrophages incubated with $\leq 20$ $\mu \mathrm{M}$ eicosapentaenoic acid and further incubated with $1 \mathrm{mg} \mathrm{SiO}{ }_{2}$ for $90 \mathrm{~min}$, the production of $\mathrm{LTB}_{4}$ (eicosatrienoic acid) was inhibited dose-dependently. While production of $\mathrm{LTB}_{5}$, a metabolite of eicosapentaenoic acid, was increased at $\leq 10 \mu \mathrm{M}$ eicosapentaenoic acid and decreased at $>10 \mu \mathrm{M}$ (SAKU et al. 1996).

Rabbit alveolar macrophages exhibited an apparent discrepancy between the abundance of immunospecific cP450 4A protein and no 20-hydroxyeicosatetraenoic acid production (ZHU et al. 1998). "NO generated by alveolar macrophages may autoinhibit the P450 4A enzymes and production of 20HETE in these cells.

Human bronchoalveolar macrophages produce leukotriene $\mathrm{B}_{4}$ (Fels et al. 1982). A rapid conversion of $\mathrm{LTB}_{4}$ into dihydro- $\mathrm{LTB}_{4}$ (5,12-dihydroxyeicosatrienoic acid) is mediated by reduction of one of the conjugated double bonds, while no $\omega$-oxidised products were detected in pure macrophage suspensions (SСHÖNFELD et al. 1988).

LY293111 is a potent leukotriene $B_{4}$ receptor antagonist with exceptional oral activity (SoFIA et al. 1997). It inhibited binding of $\left[{ }^{3} \mathrm{H}\right] \mathrm{LTB}_{4}$ to guinea pig lung membrane receptors and to receptors on human neutrophils with a $\mathrm{K}_{\mathrm{i}}=7.1 \pm 1.1 \mathrm{nM}$ and an $\mathrm{IC}_{50}=17.6 \pm 4.8 \mathrm{nM}$, respectively. In vivo the agent blocked $\mathrm{LTB}_{4}$-induced airway obstruction when administered both intravenously $\left(\mathrm{ED}_{50}=0.014 \mathrm{mg} / \mathrm{kg}\right)$ and orally $\left(\mathrm{ED}_{50}=0.40 \mathrm{mg} / \mathrm{kg}\right)$. Histological evaluation of total granulocyte infiltration into lung tissue induced by $\mathrm{LTB}_{4}$ showed when given orally, LY29311 sodium salt inhibited cell migration with an $\mathrm{ED}_{50} \cong$ $3.0 \mathrm{mg} / \mathrm{kg}$. Analysis of the lung lavage fluid from these animals demonstrated that neutrophil migration into the lumen was also inhibited $\left(\mathrm{ED}_{50}=\right.$ $0.2 \mathrm{mg} / \mathrm{kg}$ ). 


\subsubsection{2 Inhibition of Arachidonic Acid Metabolism by Radical Scavengers}

Epidemiological and clinical evidence indicates a strong chemopreventive effect of prostaglandin synthesis by nonsteroidal antiinflammatory drugs on human colorectal cancer (MARKs et al. 1996). The initiation-promotion approach of mouse skin carcinogenesis provides a model for mechanistic evaluation and further improvement of such chemopreventive measures which are aiming at an interruption of tumour development at a premalignant stage.

Lipoxygenase (EC 1.13.11.12) catalyses the stereospecific dioxygenation of polyunsaturated fatty acids containing a 1,4-cis,cis-pentadiene system to a pentadienyl radical intermediate which reacts with molecular oxygen to yield cis,trans-conjugated diene hydroperoxides (WISEMAN et al. 1988).

The phospholipase $A_{2}$ inhibitor dibromoacetophenone, the anti-inflammatory steroid fluocinolone acetonide or the lipoxygenase inhibitor nordihydroguiaretic acid just prior to intraperitoneal injection of $100 \mathrm{ng}$ 12-O-tetradecanoylphorbol-13acetate into unmanipulated CD-1 female mice resulted in a dose-dependent decrease in the number of peritoneal exudate cells producing superoxide anion radical as assessed by the reduction of nitroblue tetrazolium, while the cycloxygenase inhibitor indomethacin had no effect on the number of formazan-positive peritoneal exudate cells caused by PMA treatment (CzERNIECKI and Witz 1989).

Bryostatin 1 could inhibit the effect of 12-Otetradecanoylphorbol-13-acetate both in stimulation of $\left[{ }^{3} \mathrm{H}\right]$ arachodonic acid release and the induction of prostaglandin $\mathrm{H}$ synthase in Madin Darby canine kidney cells cultivated in Dulbecco's modified Eagle's medium (PARKER et al. 1988).

$\boldsymbol{\gamma}$-Glutamyl transferase (EC 2.3.2.2), a glycoprotein enzyme widely distributed on cell surfaces, catalyses the conversion of leukotriene $\mathrm{C}_{4}$ to leukotriene $\mathrm{D}_{4}$. Since $\mathrm{LTD}_{4}$ is biologically much more potent than $\mathrm{LTC}_{4}$ (Lewis and Austen 1984, Piper 1984), the partial degradation of the glutathione moiety to the cysteinylglycine derivative $\mathrm{LTD}_{4}$ may be considered a biosynthetic reaction generating the ligand for the $\mathrm{LTD}_{4} / \mathrm{LTE}_{4}$ receptor.

Radical scavenging effects of leukotrienes

$\mathrm{LTD}_{4}>\mathrm{LTC}_{4}>\mathrm{LTB}_{4}$

\subsubsection{0 \\ Cyclic Nucleotides}

In RAW264 murine macrophages, TAKAHASHI et al. (2000) identified three cAMP inducible mRNAs, named cI-1, cI-2, and cI-3 (for cAMP inducible genes $1-3$. The cI-3 probe was identical to a previously known gene, gly96.

$\mathrm{PGE}_{2}$ within the range of $1.4 \times 10^{-9}$ to $1.2 \times 10^{-8} \mathrm{M}$ inhibited $\mathrm{PGI}_{2}\left(2.8 \times 10^{-6} \mathrm{M}\right)$ stimulation of the adenylate cyclase of starch elicited rat peritoneal macrophages at $\mathrm{PGE}_{2}$ concentrations that have no effect by themselves on the level of cyclic andenosine 3',5'-monophosphate (Adolfs and Bonta 1982). With higher concentrations of $\mathrm{PGE}_{2}$ the inhibition was either non-existent of masked by the effect of $\mathrm{PGE}_{2}$ per se on cyclic AMP levels. Cyclic AMP formation is stimulated in resident rat peritoneal cells by exogenous arachidonic acid (Elliott et al. 1983).

\subsubsection{1 \\ Cytotoxic Substances}

Normal mouse peritoneal macrophages cultivated for $<8 \mathrm{~h}$ exerted a potent cytolytic activity against extracellular ${ }^{51} \mathrm{Cr}$-labelled syngeneic erythrocytes, as demonstrated by isotope release to the medium (Melsom et al. 1975). This lytic reaction was due to the liberation of a labile macrophage cytolytic factor $<1 \mathrm{kDa}$ which required $10^{-4} \mathrm{M}$ cystine for its detection. Experiments with $10^{-4} \mathrm{M}$ 5,5'-dithiobis-(nitrobenzoic acid) demonstrated that the factor depended on free sulphydryl groups. $10 \mu \mathrm{g} \alpha$ Tocopherol/ml abolished both the sulphydryl and the ascorbic acid mediated lytic reaction supporting the theory of lipid peroxidation as the molecular mechanism of target cell lysis. While reduced glutathione and dihydrolipoic acid were effective, oxidised glutathone and lipoic acid were not.

Rat macrophages activated by endotoxin in vitro released a factor cytotoxic for sarcoma cells but not for normal cells (CURRIE and BASHAM 1975).

\section{5}

\section{Pharmacology}

\subsection{1}

Receptors

A receptor is an integral membrane protein which transduces biological information, conferred in signals. 


\subsubsection{1 \\ Cell Surface Receptors}

The macrophage cell surface as a crucial interface studded with receptors links antibody dependent molecular immune recognition triggering a broad spectrum of cellular responses including phagocytosis, cytolysis, release of lysosomal enzymes and mediators such as prostaglandin $\mathrm{E}_{2}$, and release of reactive oxygen metabolites.

Membrane receptors contain single, fourfold, sevenfold, and 24-fold membrane-spanning domains.

\subsubsection{1}

\section{Single Membrane-Spanning Receptors}

Characteristic for all partners of the single membrane-spanning receptors, which are stimulated by transformation growth factors, is the fact that endocytosis usually takes place after the ligand binds to the receptor.

\section{Growth Hormone Receptor}

The growth hormone receptor is especially interesting because a high degree of homology exists between the growth hormone receptor membrane protein and the growth hormone serum-binding protein (Hesch 1991). To determine if growth hormone would stimulate human alveolar macrophages, KeANE et al. (1996) incubated cells gained by bronchoalveolar lavage with $10 \mathrm{nM} \mathrm{HGH}$ and $100 \mathrm{nM}$ HGH, respectively, for $4 \mathrm{~h}$ and stimulated them with fMet-Leu-Phe and E. coli, respectively. There were no significant differences between control and HGH-incubated macrophages as to superoxide anion release.

\subsubsection{2 \\ Fourfold Membrane-Spanning Receptors}

The main function of the fourfold membranespanning receptors is at synapses, where they rapidly modulate informational intensity. GABA and glycine receptors contain mainly a positively charged area composed of positively charged amino acids located on the extracellular and cytosolic surfaces of the receptor, whereas the nicotinic acetylcholine receptor is thought to contain mainly negatively charged areas.

Phorbol esters were observed to reduce the rate of acetylcholine receptor synthesis in cultured chick myotubes (Miskin et al. 1978). These findings were confirmed by BurszTAJN et al. (1988) and by KLARSFELd et al. (1989), who, in addition, demonstrated that phorbol esters enhance receptor ex- pression upon chronic exposure, thought to lead to a depletion of protein kinase $\mathrm{C}$ from the treated cells.

\subsubsection{3}

\section{Sevenfold Membrane-Spanning Receptors}

\section{$\beta$-Adrenergic Receptor}

Computer analysis of $\left[{ }^{125} \mathrm{I}\right]$ iodocyanopindolol competition studies using the relatively selective $\beta 1$ adrenoceptor antagonist, ICI 89406, and the $\beta_{2-}$ selective antagonist, ICI 118551, on rabbit arterial blood mononuclear leucocyte plasmalemmal preparations favoured a two-site model indicating that both $\beta_{1^{-}}$and $\beta_{2}$-adrenoceptor subtypes were present in approximately equal numbers (TENNER jr. et al. 1989).

Exposure of THP-1 cells, a monocyte/macrophage cell line, to $10 \mu \mathrm{M}$ isoprenaline or $100 \mu \mathrm{M}$ salbutamol for $30 \mathrm{~min}$ before an adhesion assay to human bronchial epithelial cells did not result in an increase in adhesion (ROMBERGER et al. 2000).

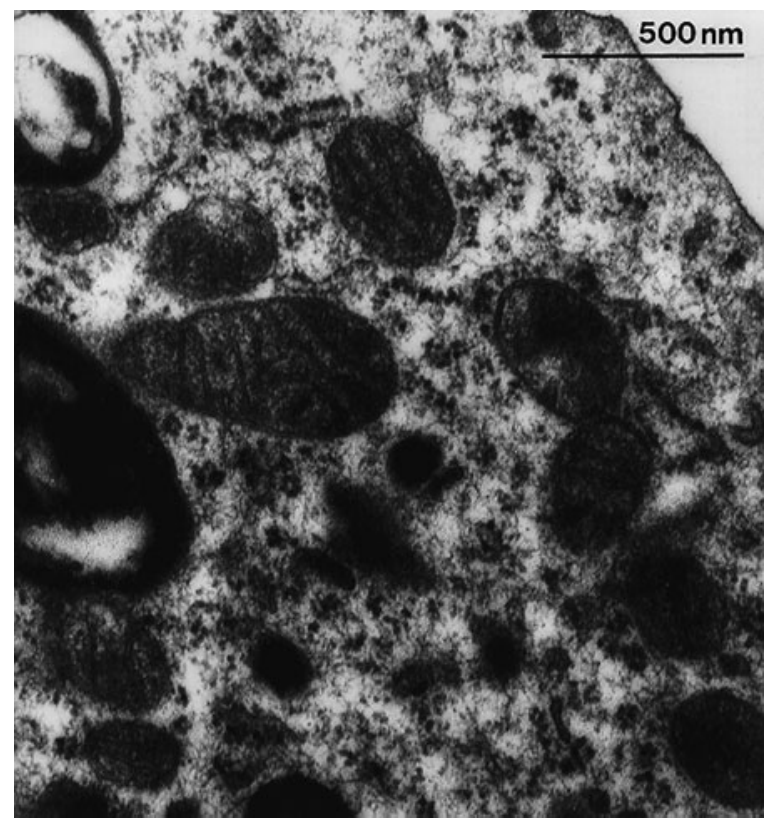

Fig. 122. Polysomes in an alveolar macrophage of a female rat (breeder: Winkelmann, Borchen-Kirchborchen) 30 min after inhaling $200 \mu \mathrm{g}$ micronized isoprenaline $\mathrm{HCl}+300 \mu \mathrm{g} \mathrm{mi}-$ cronized phenylephrine bitartrate/puff from a Medihaler ${ }^{\circledR} .12$ Puffs/min were dispersed into a $164.5 \mathrm{l}$ box where the animals stayed for $15 \mathrm{~min} .30 \mathrm{~min}$ later under methitural anaesthesia, the lung was fixed by intratracheal instillation of $2.5 \%$ glutaraldehyde in phosphate buffer ( $\mathrm{pH}$ 7.4) before opening the thorax. Postfixation with $1 \%$ osmium tetroxide in phosphate buffer ( $\mathrm{pH} 7.4$ ). Contrasted en bloc for $12 \mathrm{~h}$ with $0.5 \%$ uranyl acetate in $70 \%$ ethanol. Embedded in a $2: 8$ mixture of methyl and butyl methacrylate. Sectioned at $50 \mathrm{~nm}$. Lead citrate after REYNOLDS (1963). Film 345/84 
In human monocytes isoprenaline accumulates cAMP (GRIESE et al. 1990).

Formoterol induced a significant $(P<0.01)$ increase of basal $\mathrm{O}_{2}{ }^{--}$production from human alveolar macrophages recovered by bronchoalveolar lavage from 11 patients with chronic obstructive pulmonary disease (CAPELLI et al. 1989). The effect was independent from dose and comparable to control data obtained with zymosan.

\subsubsection{2 Thyrotropin Receptors}

Binding of $\left[{ }^{125} \mathrm{I}\right] \mathrm{TSH}$ to monocytes is reversible and saturable (СHABAUD and Lissitzky 1977). The cells exhibit high-affinity, low-capacity and low-affinity, high-capacity sites.

\subsubsection{1 \\ Cytosolic/Nuclear Receptors}

Cytosolic/nuclear receptors are primarily localised in the cytosol. They bind small lipophilic molecules, such as steroid or thyroid hormones, which pass the cell membrane by passive diffusion and, after activation, move towards the nucleus where the ligand-receptor complex exerts its specific function by directly or indirectly modifying DNA transcription.

\section{Vitamin D Receptor}

The active form of vitamin $\mathrm{D}_{3}, 1 \alpha, 25$-dihydroxyvitamin $\mathrm{D}_{3}$, is an important regulator of calcium metabolism and elicits most of its biological effects by binding to a high-affinity receptor in target tissues. In the formation of $1 \alpha, 25$-dihydroxyvitamin $D_{3}$, vitamin $D_{3}$ is hydroxylated in two sequential steps. An initial 25-hydroxylation in the liver is followed by an $1 \alpha$-hydroxylation in the kidney (DELuca and Schnoes 1983). The 25-hydroxyvitamin $\mathrm{D}_{3} 1 \alpha$-hydroxylase ( $1 \alpha$-hydroxylase) is the key enzyme in the determination of the level of $1 \alpha, 25$ dihydroxyvitamin $\mathrm{D}_{3}$ and plays a vital role in calcium homeostasis. It is present in the inner mitochondrial membrane of renal proximal tubular cells (Kawashima et al. 1981, Paulson and Deluca 1985). While under physiological conditions, the kidney is the only site of $1 \alpha, 25$-dihydroxyvitamin $\mathrm{D}_{3}$ (DeLuCa 1988), in sarcoidosis or lymphoma, $1 \alpha$-hydroxylase may be expressed at other sites (ARMBrecht et al. 1992).

The human vitamin D receptor is a member of the ligand-inducible nuclear transcription factors superfamily that regulate transcription through binding to hormone response elements in the target genes (Fuller 1991, Wahli and Martinez 1991, Gronemeyer 1992, Darwish and DeLuca 1993, Ross et al. 1994). These hormone response elements are basically divided into two groups: the first consists of palindromic sequences which include the glucocorticoid response elements subfamily (MAder et al. 1989, Umesono and Evans 1989). The second group which includes vitamin $D$ response elements consists of two direct repeats (Umesono et al. 1991, Carlberg 1995). In the latter group, the spacing between the two repeat elements plays an important role in determining hormone receptor specificity (UMEsono et al. 1991). The data of Jin et al. (1996) clearly demonstrated the existence of an activation domain in the human vitamin $\mathrm{D}$ receptor that is separable from the domain involved in dimerization. Factors that couple the human vitamin D receptor to the general transcription apparatus in yeast through the activation domain in the human vitamin D receptor, however, appear to be unrelated or dissimilar to those in COS- 1 cells.

The vitamin $\mathrm{D}$ receptor mediates the signal of $1 \alpha, 25$-dihydroxyvitamin $\mathrm{D}_{3}$ by binding to vitamin $\mathrm{D}$ responsive elements in DNA as a homodimer or as a heterodimer composed of one vitamin $\mathrm{D}$ receptor subunit and one retinoid $\mathrm{X}$ receptor subunit (NISHIKAWA et al. 1995). Exogenous $1,25(\mathrm{OH})_{2} \mathrm{D}_{3}$ stimulated $24,25(\mathrm{OH})_{2} \mathrm{D}_{3}$ synthesis in freshly isolated human monocytes, whereas matured macrophages showed no 24,25(OH) ${ }_{2} \mathrm{D}_{3}$ synthesis (KREUTz et al. 1992). Alveolar macrophages gained by bronchoalveolar lavage from 8 healthy volunteers and 6 patients with sarcoidosis II and incubated with various concentrations $\left(10^{-6}-10^{-12} \mathrm{~mol} / \mathrm{l}\right)$ of vitamin $\mathrm{D}_{3}$ and 24,25-dihydroxyvitamin $\mathrm{D}_{3}$ with and without priming with interferon- $\gamma$ expressed the intercellular adhesion molecule (ICAM-1) in a dosedependent manner and released TNF- $\alpha$ and reactive oxygen species in 6 of 8 healthy volunteers with highest values using a concentration of $10^{-6} \mathrm{~mol}$ of vitamin $\mathrm{D}_{3} / \mathrm{l}$, while in sarcoidosis these effects seemed to be less pronounced (BRAUN et al. 1996). $1,25(\mathrm{OH})_{2} \mathrm{D}_{3}$ induces human promyelocytic leukaemia cell line, HL-60, to differentiate into monocytes/macrophages (TANAKA et al. 1983, MANGelsDORF et al. 1984, INABA et al. 1986, 1987). While a high-salt extract of LG HL-60 cells displays specific $1,25(\mathrm{OH})_{2}\left[{ }^{3} \mathrm{H}\right] \mathrm{D}$ binding activity, ATCC HL-60 cells after sonication showed little or no specific binding explainable by the action of a serine protease in these cells (InAba and DeLuca 1989).

New analogues of vitamin $D$ have been developed in an attempt to separate the calcaemic actions from the effects on cell replication and differentiation. The most effective approach appeared to 
be modification of the side chain of the vitamin $\mathrm{D}$ molecule. EB1089 and KH1060 were more effective in the differentiation of U937 and HL-60 cells than the native hormone (JAMEs et al. 1997). KH1060 is characterised by altered stereochemistry at the carbon 20 position.

The conversion of vitamin D-binding protein to a very potent macrophage activating factor (YAMAмото and Номма 1991) involves stepwise deglycosilation of the glycosilated vitamin D-binding protein molecules (natural vitamin $\mathrm{D}$-binding protein is only $0 \%-5 \%$ glycosilated) by membranous $\beta$ galactosidase and sialidase of inflammation-primed $\mathrm{B}$ and $\mathrm{T}$ cells. While vitamin $\mathrm{D}$-binding proteinmacrophage activating factors obtained from natural vitamin D-binding protein significantly enhanced macrophage activity over the control, recombinant vitamin D-binding protein-macrophage activating factor had no effect (RAY 1996).

The absence of specific $1,25-(\mathrm{OH})_{2}\left[{ }^{3} \mathrm{H}\right] \mathrm{D}_{3}$ binding activity in HL-60 cell preparations is due to proteolysis of the $1,25-(\mathrm{OH})_{2}\left[{ }^{3} \mathrm{H}\right] \mathrm{D}_{3}$ receptor (INABA and DeLuCA 1989). An endogenous proteinase may act on the steroid-binding domain of the 1,25$(\mathrm{OH})_{2} \mathrm{D}_{3}$ receptor or in its immediate vicinity, because it destroyed the $1,25-(\mathrm{OH})_{2} \mathrm{D}_{3}$-binding ability of unoccupied receptor, but did not digest occupied receptor. The proteinase appeared to belong to the serine class of proteases, which is specifically inhibited with diisopropylfluorophosphate and not hydrolysed diisopropylfluorophosphate.

Contact with UMR106 rat osteoblast-like cells and the presence of 1 $\alpha 25$-dihydroxyvitamin $\mathrm{D}_{3}$ and human macrophage colony stimulating factor were absolute requirements for differentiation of human breast carcinoma tumour-associated macrophages into mature functional osteoclasts (QUINN et al. 1998).

\subsection{2}

\section{Serotonin}

In murine macrophages serotonin antagonists (250 $\mu \mathrm{M}$ methysergide, cyproheptadine or spiperone) inhibited the binding of serotonin and muramyl peptides and the respiratory burst induced by these stimuli (Silverman et al. 1985). These data suggest that muramyl peptides and serotonin act via the same receptor.

During the respiratory burst of human blood monocytes serotonin acts as a radical scavenger and is oxidised to a dimer, probably 5,5'-dihydroxy4,4'-bitryptamine (SCHUfF-Werner et al. 1995).

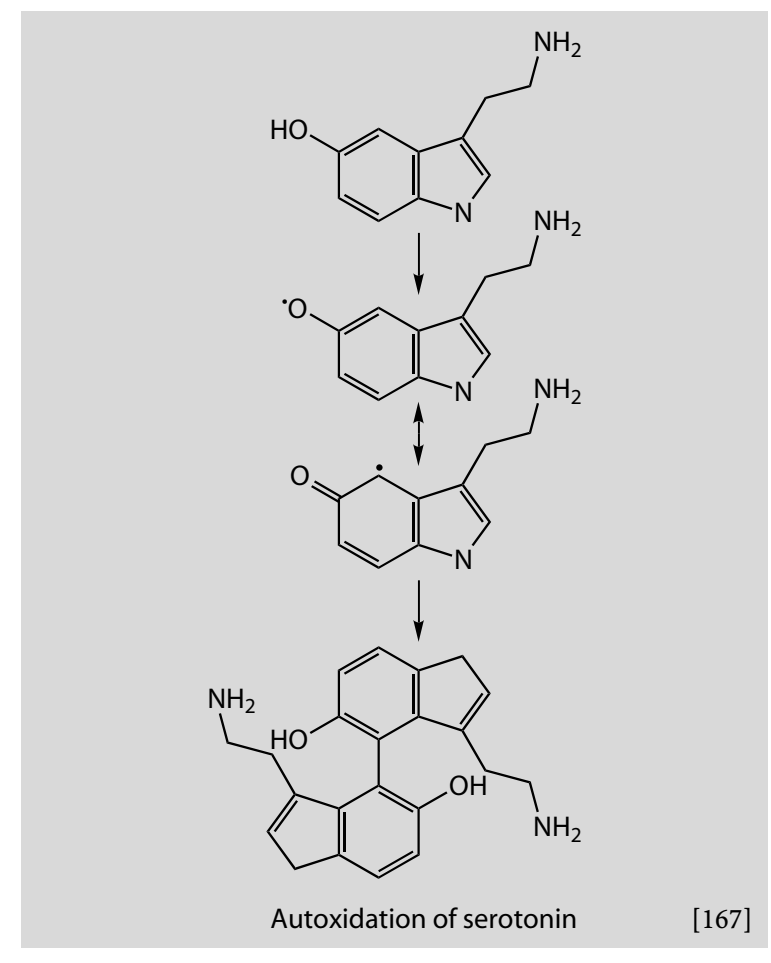

\subsection{3 \\ Histamine}

Bovine alveolar macrophages in response to histamine in a dose- $(P<0.001)$ and time- $(P<0.001)$ dependent manner released eosinophilic chemotactic activity (Nomura et al. 1996). The activity was predominantly ethyl acetate extractable. The release of eosinophilic chemotactic activity was inhibited by lipoxygenase inhibitors $(P<0.01)$. Leukotriene $\mathrm{B}_{4}$ receptor inhibitor inhibited the eosinophilic chemotactic activity $(P<0.01)$.

\subsection{4 Dexamethasone}

Glucocorticoid (dexamethasone or prednisolone)induced reduction of prostanoid synthesis in PMA (5 nM)-differentiated U937 cells is mainly due to a reduced cyclooxygenase activity (KoEHLER et al. 1990). Cyclooxygenase- 2 as a target of glucocorticoid regulation was confirmed by ex vivo studies (MASFERRER et al. 1994): cyclooxygenase-2 levels were undetected in macrophages from normal rats, but were elevated following adrenalectomy, and not detectable in adrenalectomized animals receiving dexamethasone replacement therapy. These data show that also under normal physiological conditions, cyclooxygenase- 2 levels are regulated by endogenous glucocorticoids. 
Treatment of RAW 264.7 cells with dexamethasone reduced the formation of nitrite, one of the stable end products of ${ }^{\circ} \mathrm{NO}$ production as measured in culture supernatants (WALKER et al. 1997). The $\mathrm{IC}_{50}$ is approximately $9 \mathrm{nM}$ of dexamethasone. The reduction of iNOS activity is caused by decreased iNOS protein levels as assessed by immunoblotting using a specific anti-iNOS-antibody. Dexamethasone treatment reduces the formation of iNOS mRNA steady state levels by about $50 \%$ of IFN- $\gamma$ induced iNOS mRNA levels. This is due to decreased iNOS gene transcription and iNOS mRNA stability.

Cortexolone (4-pregnene-17,21-diol-3,20-dione), a partial agonist of the glucocorticoid receptor, and RU 38486 [17 $\beta$-hydroxy-11 $\beta$-(4-dimethylaminophenol) $17 \alpha$-(prop-1-ynyl)estra-4,9-diene-3-one], a pure antagonist, were able to modulate and partially inhibit the suppressive effect of dexamethasone on the induction of $\mathrm{NO}_{2}^{-}$in specific pathogenfree male Wistar rat alveolar macrophages (JoRENS et al. 1992).

\subsection{5}

\section{Dehydroepiandrosterone}

Dehydroepiandrosterone added in vitro to alveolar macrophages lavaged from 11 non-smoking asbestos workers significantly reduced superoxide anion release (ROM and HARKIN 1991).

\subsection{6}

\section{Phosphodiesterase Inhibitors}

Theophylline, pentoxifylline, and amrinone [5amino-3,4'-bipyridin-6(1H)-on, a specific PDE 3 inhibitor] by therapeutic-range levels down-regulated the release of $\mathrm{H}_{2} \mathrm{O}_{2}$, nitrite and TNF- $\alpha$ in a rat alveolar macrophage cell line, NR8383, which responds vigorously and predictably to various soluble and particulate stimuli by producing these inflammatory mediators (HeLmKE et al. 1996). The release of IL-6, however, while inhibited by $\geq 20 \mu \mathrm{g} / \mathrm{ml}$ amirone, was enhanced by theophylline or pentoxifylline at concentrations of $\geq 5 \mathrm{mM}$. This effect was seen only after exposure to particulate zymosan and was absent with LPS, a soluble stimulus.

Pentoxifylline $(1 \mathrm{mM})$ stimulated the rate of $r a b$ bit alveolar macrophage spreading on glass coverslips more than twofold (WANG et al. 1996). It reduced superoxide generation induced by phorbolmyristate-acetate by $>50 \%$. Production of TNF- $\alpha$ induced by lipopolysaccharide was suppressed $>85 \%$.

\subsection{7 \\ $\mathrm{H}^{+}$-Transport Inhibitors}

A plasmalemmal V-type $\mathrm{H}^{+}$pump (V-ATPase) and a $\mathrm{Na}^{+} / \mathrm{H}^{+}$exchanger interact to control the cytosolic $\mathrm{pH}\left(\mathrm{pH}_{\mathrm{i}}\right)$ of alveolar macrophages. The NADPH oxidase system that generates superoxide anions in activated phagocytes is sensitive to $\mathrm{pH}$. In rabbit alveolar macrophages superoxide production induced by phorbol myristate acetate was reduced ( $\sim 50 \%)$ by $10 \mu \mathrm{M}$ bafilomycin $\mathrm{A}_{1}$, a specific inhibitor of ATPases, and marginally reduced $(\sim 15 \%)$ by $1 \mathrm{mM}$ amiloride, an inhibitor of $\mathrm{Na}^{+} / \mathrm{H}^{+}$exchange (Heming et al. 1996). Neither amiloride nor $\mathrm{Na}^{+}-$ free media altered macrophage $\mathrm{pH}_{\mathrm{i}}$. Molski et al. (1986) showed that most of the fMet-Leu-Phestimulated $\mathrm{Na}^{+}$influx is not coupled to $\mathrm{H}^{+}$efflux. $\mathrm{H}$ 7 [1-(5-isoquinolinesulfonyl)-2-methylpiperazine], a potent inhibitor of both protein kinase $\mathrm{C}$ and cyclic nucleotide-dependent protein kinases, blocks fMet-Leu-Phe-induced cell alkalinization without inhibiting superoxide anion formation, indicating that an increase of the intracellular $\mathrm{pH}$ is not obligatory required for activation of superoxide anion radical formation.

Bafilomycin (10 $\mu \mathrm{M}, \mathrm{pH}$ 7.4) caused shortening of microvilli, focal loss of surface ruffles, and marked cytoplasmic vacuolation in resident rabbit macrophages by $1 \mathrm{~h}$ incubation, with significant macrophage fragmentation and apoptosis at $48 \mathrm{~h}$ incubation (HAQUE et al. 1997). Amiloride $(100 \mu \mathrm{M}$, $\mathrm{pH}$ 7.4) caused elongation of microvilli and significant apoptosis by $1 \mathrm{~h}$ incubation.

In human alveolar macrophages gained by bronchoalveolar lavage from healthy subjects bafilomycin $\mathrm{A}_{1}$ inhibited phagolysosomal sealing and Fcmediated phagocytosis (COAKLEY et al. 1996).

\subsection{8 \\ Phenylbutazone}

Phenylbutazone $(100 \mu \mathrm{M})$ significantly $(P=0.01)$ inhibited $\mathrm{H}_{2} \mathrm{O}_{2}$ production by human monocytes/ macrophages stimulated by $1 \mathrm{mg}$ non-opsonized zymosan $/ 10^{6}$ cells or $0.1 \mu \mathrm{M} 12-O$-tetradecanoylphorbol-13-acetate (BAGGIOLINI et al. 1985).

A phenylbutazone radical has been identified spectrophotometrically using pulse radiolysis during which a solution of phenylbutazone was bombarded with hydroxyl radicals (Evans and Aruoma, unpublished work). It is therefore likely that ferryl systems oxidise the drug to free radical forms which cause oxidative damage to arachidonic acid and $\alpha_{1}$-antiproteinase. 


\subsection{9}

\section{Phenothiazines}

\subsubsection{1}

\section{Promethazine}

Promethazine or chlorpromazine but not thiazinamium chloride reduced the respiratory burst during phagocytosis of zymosan by rat alveolar macrophages gained by lung lavage in a dose-dependent manner (CHANG et al. 1983).

\subsubsection{2}

\section{Thiazinamium Chloride}

Thiazinamium chloride did not reduce the respiratory burst during phagocytosis of zymosan by rat alveolar macrophages gained by lung lavage (ChANg et al. 1983).

\subsubsection{3}

\section{Chlorpromazine}

The univalent oxidation of chlorpromazine in vitro to a free-radical intermediate has been clearly demonstrated in a variety of EPR experiments (BORG and Cotzias 1962, Piette and Forrest 1962). In each case oxidation was produced by either a metal ion $\left(\mathrm{Fe}^{3+}, \mathrm{Ce}^{4+}\right)$, concentrated acid, or controlled electrolysis. The univalent oxidation produced a coloured intermediate with an absorption maximum at $255 \mathrm{~nm}$ and $530 \mathrm{~nm}$. Cavanaugh (1957) reported chlorpromazine to be a substrate for both peroxidase (donor: $\mathrm{H}_{2} \mathrm{O}_{2}$ oxidoreductase, EC 1.11.1.7) and catalase $\left(\mathrm{H}_{2} \mathrm{O}_{2}: \mathrm{H}_{2} \mathrm{O}_{2}\right.$ oxidoreductase, $\mathrm{EC}$ 1.11.1.6) system. He reported the formation of a coloured product $(530 \mathrm{~nm})$ in the reaction of chlorpromazine with both peroxidase- $\mathrm{H}_{2} \mathrm{O}_{2}$ and catalase over a $\mathrm{pH}$ rase, $\mathrm{EC}$ 1.11.1.6) se, EC 1.11.1.6) nge of 3 to 6.3. GILLETTE and KАMM (1960) have shown that chlorpromazine is completely oxidised to both the sulphoxide and sulphone by the enzymatic action of liver microsomes.

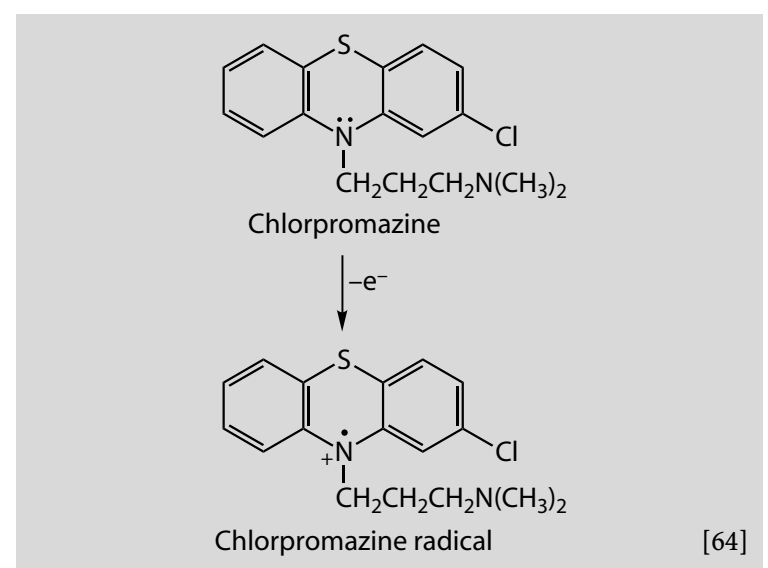

Piette et al. (1964) measured the dismutation reaction constant, $k_{\mathrm{d}}$, at $\mathrm{pH} 4.8$ to be $15.0 \mathrm{M}^{-1} \times \mathrm{s}^{-1}$. This extreme stability of the free radical allowed further oxidation of the free radical by horse radish peroxidase in the substrate-limited case; the rate constant for this reaction Piette et al. (1964) found to be $3.9 \times 10^{4} \mathrm{M}^{-1} \times \mathrm{s}^{-1}$, approximately 10 times slower than $k_{4}$, the first oxidation.

Chlorpromazine sulphoxide, a chlorpromazine metabolite formed in man and several other mammalian species, produced when irradiated with near-UV light large amounts of the highly reactive hydroxyl radical (BUETTNER et al. 1986).

\subsubsection{0}

\section{Thiosemicarbazones}

The primary lesion created in cells by the heterocyclic carboxaldehyde thiosemicarbazones is interference with the biosynthesis of DNA, and this action is primarily due to the potent inhibition of ribonucleoside diphosphate reductase activity. 5Hydroxypicolinaldehyde thiosemicarbazone (NSC107392) inhibits ribonucleoside diphosphate reductase by the chelation of iron. Krakoff et al. (1974) tried the drug by intravenous injection to $30 \mathrm{pa}$ tients with leukaemia or various solid tumours. Marked haemolysis, iron chelation, and urinary excretion of iron occurred.

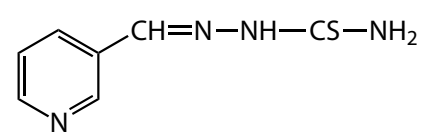

Pyridine-3-aldehyde thiosemicarbazone [168]<smiles>NS(N)=N/N=C/c1ccncc1</smiles>

Pyridine-4-aldehyde thiosemicarbazone [169]<smiles>NS(=O)N/N=C\C1C(=O)Nc2ccccc21</smiles>

2-Oxo-indole-3-carbaldehyde-thiosemicarbazone

[170]<smiles>Cc1ccc2c(c1)/C(=N/N[SiH][SiH2]N)C(=O)N2</smiles>

5-Methylisatin-3-thiosemicarbazone 
1-Methylisatin-3-thiosemicarbazones and $\mathrm{Cu}^{2+}$ ions form a stable complex with which any type of nucleic acid of at least $25 \mathrm{kDa}$ would firmly associate (Mikelens et al. 1976). Among the copper(II)bisthiosemicarbazone chelates, $\mathrm{Cu}(\mathrm{II})[2,3$-butanedionebis $\left(N^{4}\right.$-methylthiosemicarbazone $\left.)\right]$ is a most effective superoxide dismutase-like compound (WADA et al. 1994). Copper(II)bisthiosemicarbazones rapidly distributed to all tissues and readily crossed cell membranes as well as the blood-brain barrier with high distributions to the brain and the heart. The superoxide dismutase-like activities of $\mathrm{Cu}(\mathrm{II})\left[2,3\right.$ - butanedionebis $\left(N^{4}\right.$ - methylthiosemicarbazone)], as measured by several well-known methods, were compared to that of $\mathrm{Cu}(\mathrm{II})_{2} \mathrm{Zn}(\mathrm{II})_{2}$ superoxide dismutase. The $\mathrm{Cu}(\mathrm{II})$ [2,3-butanedionebis ( $\mathrm{N}^{4}$-methylthiosemicarbazone)] showed high $\mathrm{IC}_{50}$ values in these experiments. However these $\mathrm{IC}_{50}$ values were approximately two orders of magnitude higher than the corresponding $\mathrm{IC}_{50}$ values of $\mathrm{Cu}(\mathrm{II})_{2} \mathrm{Zn}(\mathrm{II})_{2}$ superoxide dismutase.

\subsubsection{1}

\section{Isoniazide}

Isoniazide, an antituberculous drug, was determined in alveolar macrophages by high-performance liquid chromatography (GUILlaUmont et al. 1982).

\subsubsection{2 \\ Histamine Liberator $\mathbf{4 8 / 8 0}$}

After an intravenous injection of Compound 48/80 ( $1 \mathrm{mg} / \mathrm{kg}$ body weight) the phagocytic function of the reticuloendothelial system (India ink clearance) of female albino rats remained unchanged (LÁzÁR et al. 1968). Carbon granules could not be seen in the lungs or kidneys.

\subsubsection{3 \\ Dinitrophenol}

Rats instilled intratracheally with 50 mg Dörentrup quartz and medicated with 2,4-dinitrophenol aerosol inhalations for 19 months initially showed some retardation in the development of pulmonary fibrosis, but after 9 and 12 months an increased proliferation of connective tissue occurred which was only insignificantly different from that in unmedicated quartz dustinstilled control rats (BECK and SCHLIPKÖTER 1960).

\subsubsection{4 \\ Chlorophyllin}

Chlorophyllin, the sodium-copper salt of chlorophyll is used in the treatment of several human ail- ments, particularly in geriatric patients (Young and BERGEI 1980). Under in vitro conditions chlorophyllin is known to be a highly efficient antimutagenic agent which can significantly reduce or eliminate the mutagenic activity of an impressive range of chemical mutagens/carcinogens like aflatoxin $\mathrm{B}_{1}, 2$-aminoanthracene, benzo[a]pyrene, $\mathrm{N}$ methyl- $N$-nitro- $N$-nitrosoguanidine, diemethylnitrosamine and nitrosamines related to tobacco, and complex mixtures such as cigarette smoke.

Treatment with chlorophyllin inhibited nitric oxide production in the lipopolysaccharidestimulated murine RAW 264.7 cells in a dose-related manner (СHо et al. 2000). Competitive RT-PCR analysis, using a DNA competitor as an internal standard, demonstrated that the treatment with 1 , 10 , and $50 \mu \mathrm{M}$ chlorophyllin decreased lipopolysaccharide-induced inducible nitric oxide synthase mRNA expression in a concentration-dependent manner. Chlorophyllin down-regulated the NF- $x \mathrm{~B}$ DNA binding on its cognate recognition site.

\subsubsection{5 Chloroquine}

Chloroquine is rapidly taken up by mouse peritoneal macrophages cultured on cover slips in Leighton tubes as observed by phase-contrast microscopy and recorded by motion pictures (FEDOR Ko et al. 1968). The drug is localised in small foci in the Golgi region.

Autophagic vacuoles containing various cytoplasmic components and acid phosphatase were produced in mouse peritoneal macrophages by addition of $30 \mu \mathrm{g}$ chloroquine $/ \mathrm{ml}$ culture medium (FEDorko et al. 1968). The early toxic vacuoles appeared in the perinuclear region within $15 \mathrm{~min}$; on electron microscopy, they showed irregular shape, amorphous moderately dense content, apparent double membranes, and in some instances curved thin tubular extensions with a central, dark linear element. When chloroquine was removed by changing the culture medium after $4 \mathrm{~h}$, the cells survived and $24 \mathrm{~h}$ later they exhibited no abnormality except for large cytoplasmic dense bodies packed with membrane lamellae. $24 \mathrm{~h}$ after exposure to chloroquine the macrophages had accumulated less hydrolases than control cells.

Inhibition of the serum treated zymosanstimulated superoxide anion generation from $h u-$ man blood mononuclear cells preincubated for $30 \mathrm{~min}$ or $60 \mathrm{~min}$ with $0.001 \mathrm{mM}-0.1 \mathrm{mM}$ chloroquine was time- and dose-dependent (Hurst et al. 1986). The drug could not markedly influence the oxygen burst induced by phorbol-myristate-acetate, A23187 or fluoride ion, excluding a significant ef- 
fect on protein kinase C, calmodulin-dependent kinase(s) or the membrane-bound superoxidegenerating $\mathrm{NADP}(\mathrm{H})$-oxidase.

Chloroquine $(0.1 \mathrm{mM})$ caused a decrease in mouse peritoneal macrophage antigen catabolism associated with inhibition of antigen presentation ( ${ }^{125} \mathrm{I}$-labeled Listeria monocytogenes) to $\mathrm{T}$ cells (Ziegler and UnANue 1982).

\subsubsection{6 \\ Azaspiranes}

The immunomodulatory azaspiranes are novel cationic amphiphilic drugs with beneficial effects in a number of animal models of autoimmune disease and transplantation. WAites et al. (1995) compared $N, N$-dimethyl-8,8-dipropyl-2-azaspiro[4,5]decane2-propanamine $\mathrm{HCl}$ (SK\&F 105685) and two analogues, SK\&F 106615 and SK\&F 103811 with chlorphentermine and chloroquine for their ability to induce phospholipid accumulation and suppressor cell activity. Oral administration of SK\&F 105685 and SK\&F 106615 caused phospholipid accumulation in bronchoalveolar lavaged rat macrophages but to a far lesser extent (three- to fivefold) than chlorphentermine. Neither the immunologically unreactive azaspirane SK\&F 103811 nor chloroquine affected phospholipid levels. Alveolar macrophages from rats treated with SK\&F 105685 or SK\&F 106615 expressed more potent suppressor cell activity than chlorphentermine. Neither SK\&F 103811 nor chloroquine induced suppressor cell activity.

\subsubsection{7 \\ Poly-2-vinylpyridine-1-oxide}

The uptake of poly-2-vinylpyridine-1-oxide into lysosomes of alveolar macrophages of rats (GRUNDMANN 1967) and guinea pigs (BECK and BoJE 1967) and hepatic Kupffer cells of mice (BAIRATI and Castano 1968) has been documented.

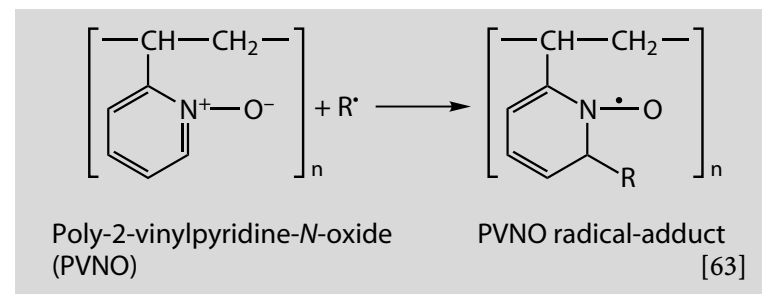

NAsH et al. (1966) synthesised another compound, polyvinylpyridinioacetic acid, which has even greater hydrogen bonding capacity than poly-2vinylpyridine-1-oxide and very efficiently protects macrophages from silica toxicity. This protection was confirmed by SAKABE and Koshi (1967), who also showed that it prevents the increase in acid phosphatase in macrophages following exposure to quartz particles $<2 \mu \mathrm{m}$ in diameter. However, polybetaine was slightly more active than poly-2vinylpyridine-1-oxide.

The protective effects of syndiotactic and isotactic poly-2-vinylpyridine-1-oxide remarkably differ in the spatial arrangement of the structural units (Holt et al. 1970). The isotactic polymer has probably a helical conformation. When the cultures

Fig. 123. Alveolar macrophage from a female rat (breeder: Winkelmann, Borchen-

Kirchborchen) which inhaled micronized poly-2-vinylpyridine-1-oxide for $15 \mathrm{~min}$ per day, 5 times per week from July 25, 1967 to January 15, 1968. Fixed on January 15, 1968 under methitural anaesthesia by intratracheal instillation of $2.5 \%$ glutaraldehyde in phosphate buffer ( $\mathrm{pH}$ 7.4) before opening the thorax. Postfixation with $1 \%$ osmium tetroxide in phosphate buffer ( $\mathrm{pH}$ 7.4). Contrasted en bloc for $12 \mathrm{~h}$ with $0.5 \%$ uranyl acetate in $70 \%$ ethanol. Embedded in a 2:8 mixture of methyl and butyl methacrylate. Sectioned at $50 \mathrm{~nm}$. Lead citrate after REYNOLDS (1963). Plate $47 / 07$

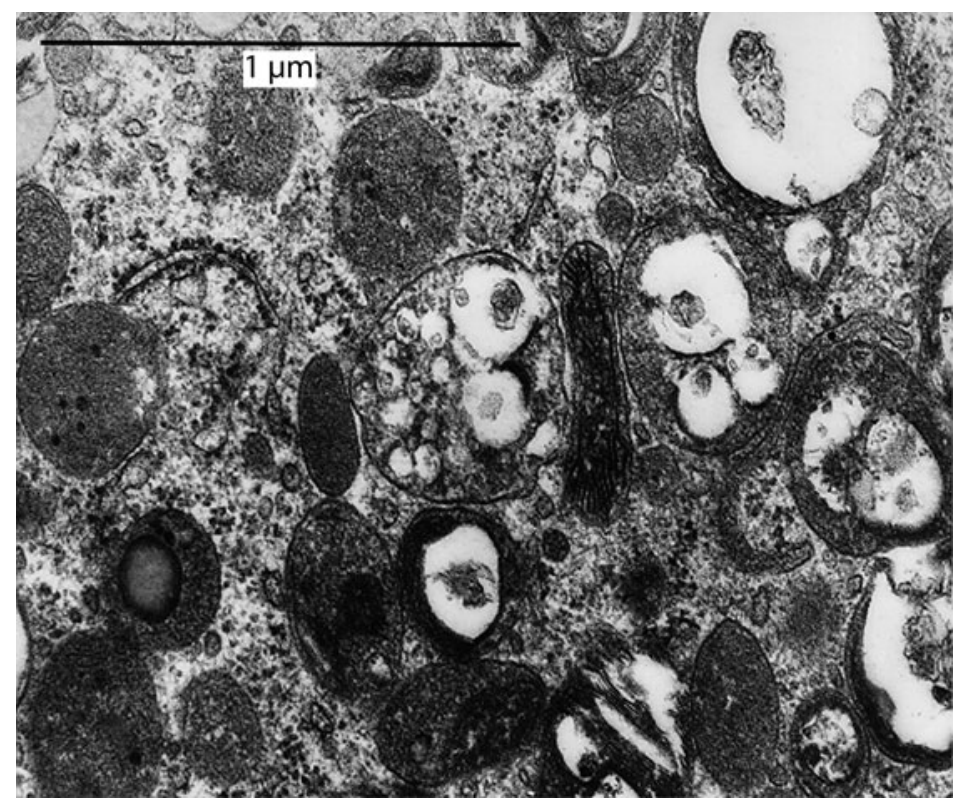




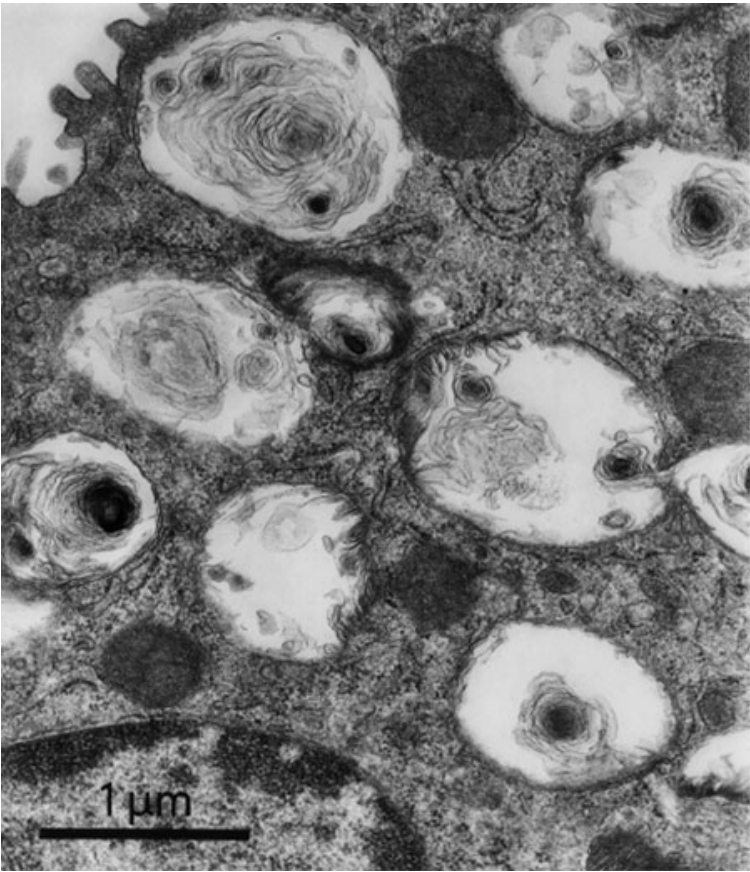

Fig. 124. Alveolar macrophage from a female rat (breeder: Winkelmann, Borchen-Kirchborchen) which inhaled micronized poly-2-vinylpyridine-1-oxide $15 \mathrm{~min}$ per day, 5 times per week from July 25, 1967 to January 15, 1968. Fixed on January 15, 1968 under methitural anaesthesia by intratracheal instillation of $2.5 \%$ glutaraldehyde in phosphate buffer (pH 7.4) before opening the thorax. Postfixation with $1 \%$ osmium tetroxide in phosphate buffer ( $\mathrm{pH} 7.4$ ). Contrasted en bloc for $12 \mathrm{~h}$ with $0.5 \%$ uranyl acetate in $70 \%$ ethanol. Embedded in a 2:8 mixture of methyl and butyl methacrylate. Sectioned at $50 \mathrm{~nm}$. Lead citrate after REY NOLDS (1963). Plate 40/08

of guinea pig peritoneal macrophages were pretreated it was as active against the pathogenic effects of Dörentrup quartz as the atactic polymer, but when the quartz was pre-treated it gave little protection. The syndiotactic polymer has a planar zigzag conformation. When the cell cultures were pre-treated, no protection was given to the cells against silica: when the quartz was pre-treated, the protection was comparable with that given by the atactic polymer previously used.

NATTA et al. (1966) found that polymers inhibiting of the lysis of macrophages that had engulfed silica particles belong to two chemical classes characterised by the presence in the monomeric unit either of the $\mathrm{N} \rightarrow \mathrm{O}$ function or the $\mathrm{N}$-methylene or $\mathrm{N}$-ethylene groups. Their activity decreased and vanished when molecular weight decreased.

Poly- $N$-(4-morpholino) ethyl acrylamide, poly$N$-ethyl- $N$ - $\beta$-(4-morpholino) ethyl acrylamide, and poly- $N$-isopropyl- $N-\beta$-(4-morpholino) ethyl acrylamide, though showing a good protection in vitro were completely inactive in protecting Swiss mice

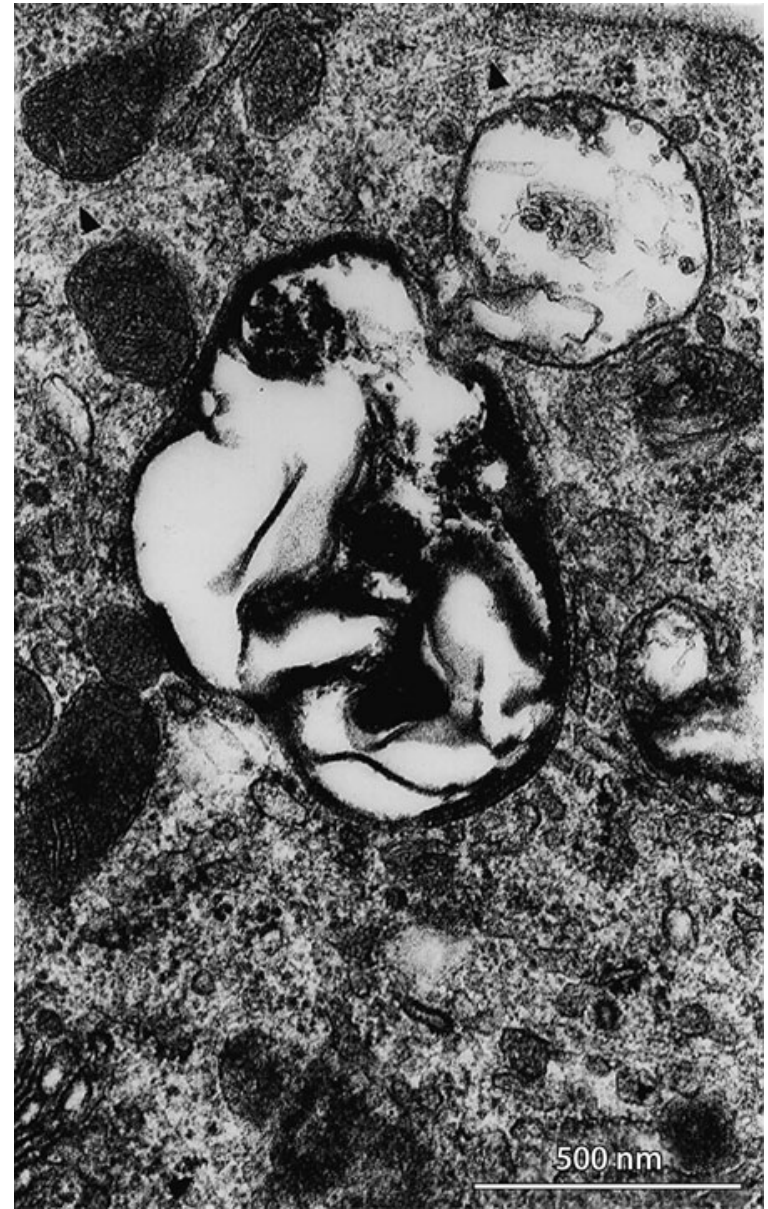

Fig. 125. Microtubules (arrowheads) and microfilaments (arrows) in an alveolar macrophage from a female rat (No.1; breeder: Winkelmann, Borchen-Kirchborchen) which inhaled micronized poly-2-vinylpyridine-1-oxide for $15 \mathrm{~min}$ per day, 5 times per week from July 25, 1967 to January 15, 1968. Fixed on January 15, 1968 under methitural anaesthesia by intratracheal injection of $2.5 \%$ glutaraldehyde in phosphate buffer ( $\mathrm{pH}$ 7.4) before opening the thorax. Postfixation with $1 \%$ osmium tetroxide in phosphate buffer ( $\mathrm{pH} 7.4$ ). Contrasted en bloc for $12 \mathrm{~h}$ with $0.5 \%$ uranyl acetate in $70 \%$ ethanol. Embedded in a 2:8 mixture of methyl and butyl methacrylate. Sectioned at $50 \mathrm{~nm}$. Lead citrate after REYNOLDS (1963). Plate 47/05

from hepatic silicosis after intravenous injection of $5 \mathrm{mg}$ tridymite dust (CHiappino et al. 1967).

NASH et al. (1966) synthesised another compound, polyvinylpyridinioacetic acid, which has even greater hydrogen bonding capacity than poly2 -vinylpyridine-1-oxide and very efficiently protects macrophages from silica toxicity. This protection was confirmed by SAKABE and Koshi (1967), who also showed that it prevents the increase in acid phosphatase in macrophages following exposure to silica. Holt et al. (1970) reported that some poly-2-vinylpyridinium salts prevented quartz toxicity in cultures. Thus the presence of hydrogen 
accepting groups (either $\mathrm{N}$-oxides of pyridinium groups) is frequently associated with protection. A difference between the protective effect of syndiotactic and isotactic polyvinylpyridine $\mathrm{N}$-oxide polymers differ only in the spatial arrangement of the structural units.

\subsubsection{8}

\section{Coumarins}

Coumarins used in postischaemic cardiomyopathy, have a reputation as anticoagulant, antimutagenic, tumoristatic (Von ANGerer et al. 1994, WeBER et al. 1998), antimetastatic, antiinflammatory (CHATURVEDI et al. 1974), immunostimulatory (BERKARDA et al. 1983), anticonvulsant and hypotensive agents. They are able to scavenge (quench) reactive oxygen species, stimulate respiration ionophoretically, inhibit 5- and 12-lipoxygenases and inhibit xanthine oxidase and phenylalanine hydroxylase.

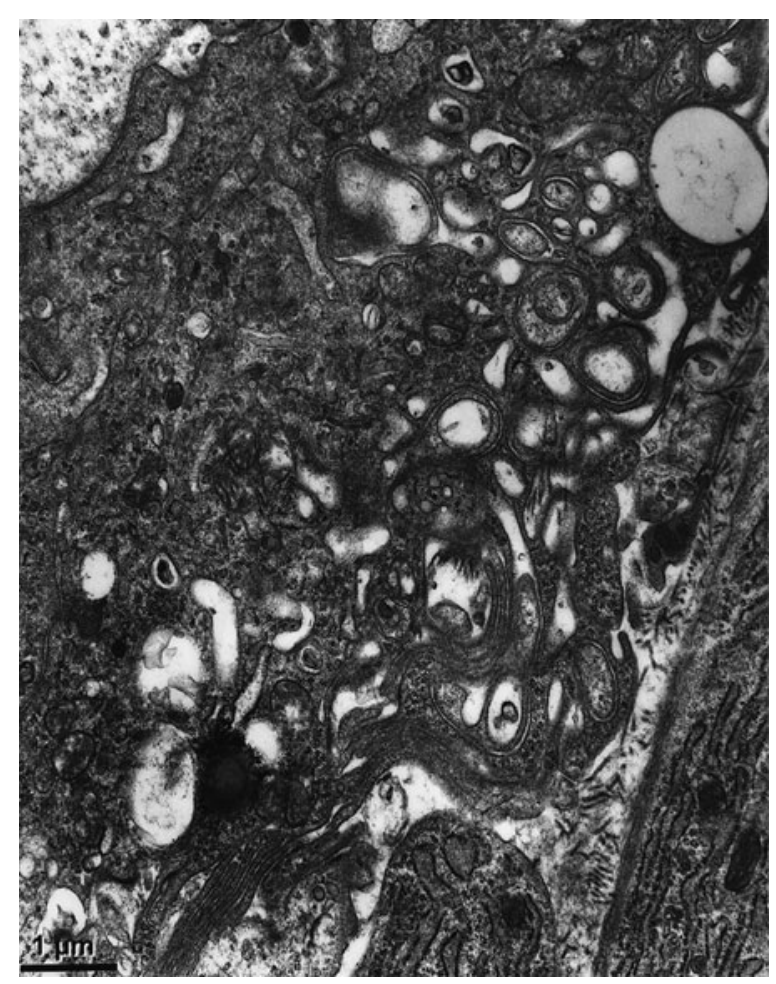

Fig. 126. Macrophage polykaryon with many microvilli, numerous cisternae of the endoplasmatic reticulum, polysomes, mitochondria, bundles of filaments and lipid droplets. Marbagelan ${ }^{\circledR}$-induced (7 days) resorption granuloma (block 171) of a male Sprague-Dawley rat (No.10) treated for 14 days with intragastric application of $15 \mathrm{mg}$ carbocromene per $\mathrm{kg}$ body weight $\times$ day. Perfused under pentobarbital anaesthesia $(30 \mathrm{mg} / \mathrm{kg})$ from the abdominal aorta with $2.5 \%$ glutaraldehyde in $0.1 \mathrm{M}$ sodium cacodylate buffer ( $\mathrm{pH} 7.4$ ). Postfixation with $1 \%$ osmium tetroxide in cacodylate buffer. Embedded in Epon 812 and sectioned at $50 \mathrm{~nm}$. Lead citrate and uranyl acetate. Plate 2846. Detail from Fig. 155

\subsubsection{1 \\ Carbocromene}

In Marbagelan ${ }^{\circledR}$-induced resorption granulomas, carbocromene enhanced the formation of polycaria, but prevented the translocation of nuclei to form typical Langhans cells (SCHILLER 1982).

\subsubsection{2 \\ Cloricromene}

Cloricromene or 8-monochloro-3 $\beta$-diethylaminoethyl-4-methyl-7-ethoxycarbonyl-methoxy-coumarine (APORTI et al. 1978) inhibited the induction of the inducible isoform of nitric oxide synthase in murine J774 macrophages (ZINGARELLI et al. 1993).

\subsubsection{9 \\ Benzhydryl Piperazines}

Analogues containing the benzhydryl piperazine structure are mainly $\mathrm{N}$-dealkylated (KING et al. 1965, Kuntzman et al. 1965, NARrod et al. 1965, KING and Howell (1966). A striking sex difference was observed for the in vitro metabolism of flunarizine in rat. In male rats, oxidative $N$-dealkylation at one of the piperazine nitrogens, resulting in bis(4fluorophenyl) methanol, was a major metabolic pathway, whereas aromatic hydroxylation at the phenyl of the cinnamyl moiety, resulting in hydroxyl-flunarizine, was a major metabolic pathway in female rats (LAVRIJSEN et al. 1992).

Epoxidation by the introduction of an oxygen atom into flunarizine produced 1-[bis(4-fluorophenyl)methyl] - 4 - [(3-phenyloxiran - 2 - yl)methyl] piperazine (metabolite 2) and epoxide hydration to a diol, 3-[4-[bis(4-fluorophenyl)methyl]-1-piperazinyl]-1-phenyl-1,2-propanediol (metabolite 10). LAVRIJSEN et al. (1992) found metabolites formed by epoxidation at the double bond (metabolite 2) and epoxide hydration (metabolite 10) in incubates with subcellular hepatocyte fractions of male and female rats. Metabolites formed by epoxidation and epoxide hydration were not detected in vivo (Meuldermans et al. 1983), probably because the resulting metabolites were metabolised in vivo, much more quickly than in vitro, into secondary metabolites. A diol metabolite, however, was described for the metabolism of 1-butyl-4-cinnamylpiperazine in guinea pigs (Morishita et al. 1978). With supernatant fractions a rapid disappearance of the epoxide intermediate from incubate was observed. This seems to indicate that, for the epoxide hydrolysis, besides microsomal epoxide hydrolase, cytosolic epoxide hydrolase might also be involved. 
Cinnarizine, 1-(diphenylmethyl)-4-(3-phenyl-2propenyl)-piperazine is a selective calcium entry blocker, and is extensively used in the treatment of cerebral and peripheral insufficiency (GoDFRAIND et al. 1982, SINGH 1986). Its oxidative metabolism to 1-(diphenylmethyl)piperazine (M-1), 1-(diphenylmethyl) -4-[3-(4'-hydroxyphenyl)-2-propenyl]-piperazine (M-2), benzophenone (M-3) and 1-[4'-hydroxyphenyl)-phenylmethyl] - 4-(3-phenyl-2-propenyl) piperazine (M-4) in rat liver microsomes required $\mathrm{NADPH}$, and was inhibited by carbon monoxide and SKF 525-A, typical inhibitors of $\mathrm{P}_{450}$ (KARIYA et al. 1992). Only M-2 formation was suppressed by sparteine or metoprolol, and was significantly lower in female Dark Agouti rats than in Wistar rats of both sexes.

Cinnarizine was found to inhibit spontaneous lipid peroxidation in rat liver homogenates, copperinduced lipid peroxidation in human plasma and copper-induced and hydrogen peroxide-induced lipid peroxidation in human erythrocytes (FeRNANDES et al. 1991).

Cyclicine, cyproheptadine and some other structurally related antihistaminics, when administered to rats, provoked a marked degranulation of the pancreatic beta cells together with a dramatic dilatation of the rough endoplasmic reticulum (HRUBAN et al. 1972, LONGNECKER et al. 1972, FISCHER et al. 1973, 1975). In fed, fasting and adrenalectomized rats cyproheptadine $(10-40 \mathrm{mg} / \mathrm{kg})$ caused a hyperglycaemia and a fall in plasma insulin (Poser et al. 1975).

\subsubsection{1 \\ $N$-Benzhydryl- $N^{\prime}$-p-hydroxybenzylpiperazine}

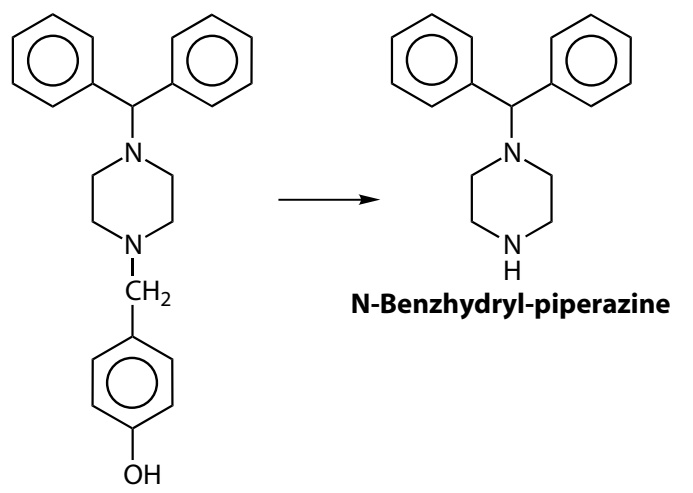

N-Benzhydryl-N'-p-hydroxybenzyl-piperazine

Biotransformation of $N$-Benzhydryl- $N$ '- $p$ -

hydroxybenzylpiperazine

[172]

\subsubsection{0 \\ Lazaroids}

The lazaroids, or 21-aminosteroids, are potent inhibitors of iron-dependent lipid peroxidation. U$83836 \mathrm{E}$ is a derivative of vitamin $\mathrm{E}$ which contains a trolox ring and the aminosteroid portion of the lazaroids. U-83836E protected a rat macrophage cell line (NR8383) against a dose-dependent lipid peroxidation $(13.8 \pm 2.4-423.7 \pm 76 \mu \mathrm{M}$ malondialdehyde $/ 10^{6}$ cells) induced by an exposure to $0.5-5 \mathrm{mM}$ $\mathrm{H}_{2} \mathrm{O}_{2}$ for $1 \mathrm{~h}$ (ALESSANDRINI et al. 1996). U-83836E pre-treatment reduced both lactate dehydrogenase activity $(46.58 \pm 13.5 \mathrm{U} / \mathrm{l}$ versus $124.91 \pm 13.5 \mathrm{U} / \mathrm{l})$ and proinflammatory cytokine mRNA induction (ALESSANDRINI et al. 1997).
Fig. 127. Alveolar macrophage (block BNh 3406) of a male Wistar rat (No.2569) $90 \mathrm{~min}$ after a single intraperitoneal injection of 5 mg $N$-benzhydryl- $N^{\prime}$ - $p$-hydroxybenzylpiperazine $\mathrm{HCl}$ (UCB F 241) per kg body weight. Under pentobarbital anaesthesia (30 $\mathrm{mg} / \mathrm{kg}$ ), the animal was perfused from the abdominal aorta with $2.5 \%$ glutaraldehyde in $0.1 \mathrm{M}$ sodium cacodylate buffer ( $\mathrm{pH}$ 7.4). Postfixation with $1 \%$ osmium tetroxide in sodium cacodylate buffer. Embedded in Epon and sectioned at $50 \mathrm{~nm}$. Lead citrate and uranyl acetate. Plate 2397

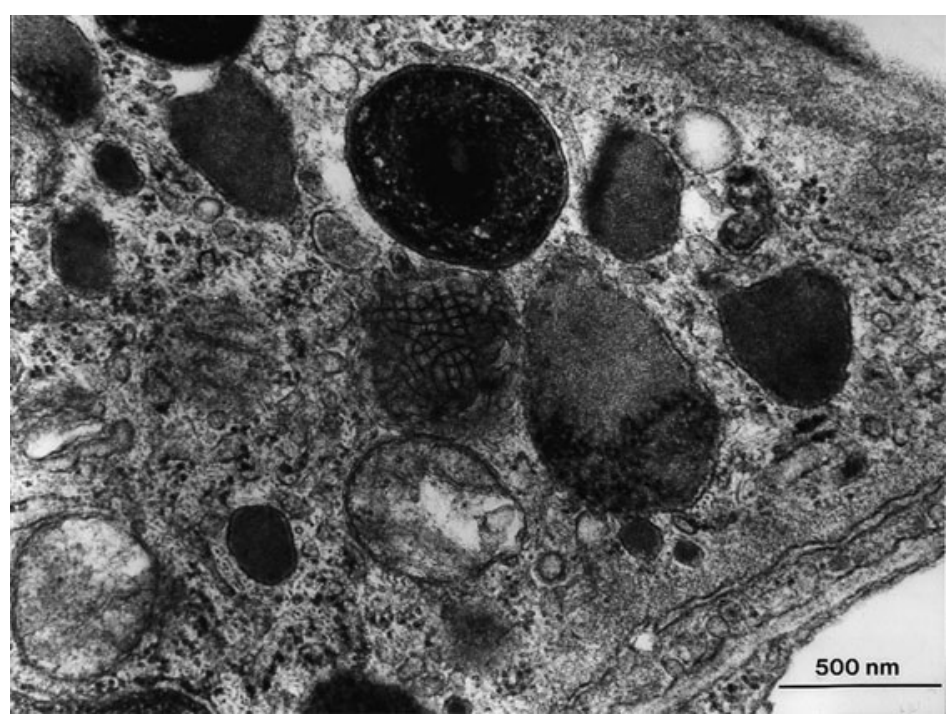




\subsubsection{1}

\section{Biphosphonates}

The effect of biphosponates on macrophages might explain the acute phase response occurring in some patients who received an amino-biposphonate intravenously for the first time showing a transient pyrexia of $1-2{ }^{\circ} \mathrm{C}$, sometimes more, accompanied by flu-like symptoms (ADAMI et al. 1987). The pyrexia has been shown to be accompanied by an increase in circulating IL-6 and TNF- $\alpha$ (SCHWEITZER et al. 1995, SAUTY et al. 1996).

\subsubsection{2}

\section{Ginkgo biloba Extract}

Oxidative burst triggered in murine J774-macrophages by zymosan was significantly $(P<0.05)$ attenuated when the cells were preincubated for $1 \mathrm{~h}$ with $\geq 50 \mu \mathrm{g}$ Ginkgo biloba extract/ml (RoNG et al. 1996).

\section{6 \\ Toxicology}

\subsection{1}

\section{Physical Hazards}

After a single thoracic $\mathrm{x}$-irradiation of $1,800 \mathrm{r}$, Gross and BALIs (1978) divided the murine alveolar macrophages lavaged 2, 4, and 8 weeks later, respectively, into two populations: the larger cell population consisted of cells that were either on the alveolar surface at the time of irradiation, or still in the interstitium but at a postmitotic radioresistant stage. As this population aged (4 weeks) the cell size increased. It was finally replaced by a small cell population which was absent 2 weeks after irradiation and appeared in the alveolar lumina in the following two weeks. Small cells reappeared in small numbers at 4 weeks. By 8 weeks they had increased in size and number, and had replaced the older large celled population.

\subsection{2}

\section{Chemical Agents}

\subsubsection{1}

\section{Inorganic Agents}

Intracellular dissolution of a large variety of inhaled inorganic particles not readily soluble in the pulmonary epithelial lining fluid constitutes an important long-term clearance mechanism of the lungs. Fluorescence microscope photometry and dual laser flow cytometry of intraphagolysosomal $\mathrm{pH}$ in canine and rat alveolar macrophages were compared using fluoresceinisothiocyanate labelled, amorphous silica particles (NyBERG et al. 1989, Heilmann et al. 1992). The higher intraphagolysosomal $\mathrm{pH}$ in rat alveolar macrophages correlated with the smaller intracellular particle dissolution rate in Wistar rat alveolar macrophages when compared with the data in Beagle dogs (KREYLING et al. 1992).

\subsubsection{1 \\ Aluminium}

Aluminium has the atomic number 13 and the valence electrons are those on the $3 s^{2} 3 p^{1}$ shell. Aluminium has a fixed oxidation number of +3 and therefor cannot participate in the redox reactions that lead to radical formation. However, aluminium salts accelerate peroxidation of membrane lipids stimulated by iron salts (GUTTERIDGE et al. 1985).

The soluble hexahydrates species of aluminium $\left[\mathrm{Al}\left(\mathrm{H}_{2} \mathrm{O}\right)_{6}\right]^{3+}$, as those of iron $\left[\mathrm{Fe}\left(\mathrm{H}_{2} \mathrm{O}\right)_{6}\right]^{3+}$, and zinc $\left[\mathrm{Zn}\left(\mathrm{H}_{2} \mathrm{O}\right)_{6}\right]^{2+}$, according to the law of mass action. is balanced with the pentahydrate, $\left[\mathrm{Al}(\mathrm{OH})\left(\mathrm{H}_{2} \mathrm{O}\right)_{5}\right]^{2+}$ $+\mathrm{H}^{+}$.

Aluminium is complexed by two carboxylates and the alkoxy group of citrate, leaving a free carboxylate (Gregor and Powell 1986). The availability of a free carboxylate may be of importance to the ability of $\mathrm{Al}$ citrate to cross membranes.

Aluminium can bind to phosphates and other oxygen donating ligands and form stable complexes. By doing so, Al may disrupt the enzyme activity in the mitochondria and thus effect the electron transport chain. This may lead to an increase in reactive oxygen species formation which in turn can lead to oxidative injury (HALliwell 1992). Mitochondrial respiratory activity in rat glioma (C-6) cells grown in RPMI medium with $10 \%$ foetal bovine serum was found to be significantly higher in aluminium $(500 \mu \mathrm{M})$-treated cells (CAMPBELL et al. 1999). At $500 \mu \mathrm{M}$, the oxidant effects of Al were maximal (Bondy and KiRSTEIN 1996).

Aluminium salts stimulated luminol-enhanced chemiluminescence production by human neutrophils (Stankovic and Mitrovic 1991). Kong et al. (1992) described an $\mathrm{Al}(\mathrm{III})$ complex with $\mathrm{O}_{2}{ }^{*-}$ which was a stronger oxidant than $\mathrm{O}_{2}{ }^{-2}$ itself and which may contribute to the adverse biological effects of $\mathrm{Al}(\mathrm{III})$. Aluminium can enhance hydroxyl radical production by iron, but it is not in itself capable of catalysing the generation of hydroxyl radical from the Fenton or Haber-Weiss reactions (GutTERIDGe et al. 1985). Aluminium facilitation of ironmediated lipid peroxidation is dependent on substrate, $\mathrm{pH}$ (greater at $\mathrm{pH} 5.5$ than 7.4), and alumi- 
nium and iron concentrations (XIE and YoKeL 1996). In male rhesus monkeys, $25 \mathrm{mg} \mathrm{Al}^{3+} / \mathrm{kg}$ body weight every alternate day for 52 weeks resulted in a significant $(P<0.001)$ enhancement in the levels of lipid peroxidation in hippocampus ( $82 \%)$, cerebral cortex (55\%), and corpus striatum (32\%) ( $\mathrm{SA}_{\mathrm{A}}$ RIN et al. 1997). Regional alterations in calcium homeostasis might enhance the production of free radicals as a cause of augmented lipid peroxidation.

Aluminium and iron may share important biological pathways (CANnAta et al. 1984, 1991, CANNATA and Diaz LóPEz 1991). Both are transported by transferrin (Trapp 1983, MARTIN 1986). Human serum transferrin released $\mathrm{Al}^{3+}$ when the $\mathrm{pH}$ varied from $\mathrm{pH} 7.4$ (FATEMI et al. 1991). The ubiquitous iron-storage protein, ferritin formed complexes with aluminium in the livers and brains of rats fed $\mathrm{AlCl}_{3}$ for 1 year (FLeming and Joshi 1987). Ferritin isolated from the brains of patients who died of Alzheimer's disease contained more aluminium and more iron than that from age-matched controls. In neuroblastoma cells, aluminium enhanced iron uptake and expression of neurofibrillary tangle protein (ABreo et al. 1999).

The iron-binding agent, 54 inducing a negative iron balance (BREITHAUPT et al. 1986) also chelated $\mathrm{Al}^{3+}$. It relieved the inhibitory action of aluminium on parathyroid cells and osteoblasts (RAPOPORT et al. 1987). However, Cournot-Witmer and PlaСнот (1990) showed that in the parathyroid glands of aluminium-intoxicated patients the presence of aluminium deposits neither induced cellular damage or chief cell necrosis nor interfered with the production of parathyroid hormone. In normal rats, Fe and $\mathrm{Al}$ after systemic administration chelated as ferroxamine and aluminoxamine, respectively (Allain et al. 1988).

1,2-Dimethyl-3-hydroxypyrid-4-one (deferiprone, L1) has been demonstrated to be a clinical effective iron and aluminium chelator, generally safe, and less expensive than desferrioxamine, being to date the most promising alternative to desferrioxamine for longer use in humans (AL-Refaie et al. 1995, Kontoghiorghes 1995, Olivieri et al. 1998). However, in some cases the long therapy with $\mathrm{L} 1$ has also been associated with complications such as neutropenia, agranulocytosis, hepatic fibrosis, arthralgias, and gastrointestinal disturbances (Domingo 1996, Olivieri et al. 1998, Töndury et al. 1998). In addition to the potential adverse effects, L1 has also a lower therapeutic ratio than desferrioxamine. Oral administration of L1 was the most effective treatment in enhancing urinary $\mathrm{Al}$ excretion in both young (21 days old) and old (18 months) Al-loaded rats (Gómez et al. 1999). Concurrent administration of desferrioxamine and L1 had no advantages over the use of either single agent, while 1-( $p$-methylbenzyl)-2-ethyl-3-hydroxypyrid-4-one was not effective in mobilising $\mathrm{Al}$ from Al-loaded rats.

Aluminium preferentially binds within the nuclear compartment (DEBoni et al. 1974, CRAPPER et al. 1978, WeN and WisNiewsKi 1985, WedRYCHOWSKI et al. 1986), particularly to heterochromatin (CRAPPER et al. 1980) and DNA phosphates and bases (KARLIK et al. 1980).

Preincubation of DNA and RNA from mouse and rat lung and liver with $\mathrm{AlCl}_{3}$ markedly inhibited the

Table 30. Effects of aluminium on enzyme activities

\begin{tabular}{|c|c|c|}
\hline Enzyme & $\mathrm{EC}$ & Authors \\
\hline $\mathrm{NAD}^{+}$-dependent isocitrate dehydrogenase & 1.1.1.41 & Yoshino et al. (1992) \\
\hline $\mathrm{NADP}^{+}$-dependent isocitrate dehydrogenase & 1.1 .1 .42 & Yoshino et al. (1992) \\
\hline Glucose-6-phosphate dehydrogenase & 1.1.1.49 & CHo and Joshi (1989) \\
\hline Cytochrome $c$ oxidase & 1.9.3.1 & ENGELBRECHT and JORDAN (1972) \\
\hline Dihydropteridine reductase & 1.14 .16 & Altmann et al. (1987) \\
\hline Ferroxidase & 1.16.3.1 & HUBER and FrIEDEN (1970) \\
\hline Choline acetyltransferase & 2.3.1.6 & $\begin{array}{l}\text { YATEs et al. (1980), Hofstetter et al. (1987), } \\
\text { BilKei-Gorzó (1994), CHERRoret et al. (1994) }\end{array}$ \\
\hline Hexokinase & 2.7 .1 & $\begin{array}{l}\text { HARRISON et al. (1972), WOMACK and CoLOWICK } \\
\text { (1979), TrAPP (1980), CHO and Joshi (1988) }\end{array}$ \\
\hline Acetylcholinesterase & 3.1.1.7 & MARQUis and LERRICK (1882), MARQUis (1983) \\
\hline Acid phosphatase (potato) & 3.1.3.2 & HELFERICH and SCHMitz (1953) \\
\hline $\mathrm{Ca}^{2+}$-calmodulin-dependent phosphodiesterase & 3.1 .4 & RICHARDT et al. (1985) \\
\hline Phospholipase C & 3.1 .4 .3 & SHI et al. (1993) \\
\hline Amylase & 3.2.1.1 & McGEACHin et al. (1962) \\
\hline$\alpha$-Chymotrypsin & 3.4 .21 .1 & Clauberg and Joshi (1993) \\
\hline Calpains I and II & 3.4.22.17 & ZHANG and JoHNSON (1992) \\
\hline ATPase & 3.6.1.3 & MissiAEn et al. (1989), HYPPÖNEN et al. (1993) \\
\hline $\mathrm{Na}^{+}-\mathrm{K}^{+}-\mathrm{ATPase}$ & 3.6.1.3 & LAI et al. (1980) \\
\hline $\mathrm{Mg}^{2+}$-ATPase & 3.6.1.3 & LAI et al. (1980), HYPPÖNEN et al.(1993) \\
\hline Glutamate decarboxylase & 4.1.1.15 & HOFSTETTER et al. (1987) \\
\hline$\delta$-Aminolaevulinic acid dehydratase & 4.2.1.2.24 & MEREDith et al. (1974), ZAMAN et al. (1993) \\
\hline
\end{tabular}


binding of 4-hydroxyaminoquinoline 1-oxide to either nucleic acid (Yamane and OHtawa 1978). Al exists in a variety of species in aqueous solution, depending upon the degree of hydroxylation (JÄGER and JÄGER 1941, JÄGER 1949). This complexity affects aluminium binding to DNA, which is $\mathrm{pH}$-depending and produces a shift from phosphate to base binding with decreasing $\mathrm{pH}$ (KARLIK et al. 1980).

\section{Human Pathology}

An open lung biopsy from a 44-year-old male employed for the previous 6 years as an aluminium rail grinder working in an extremely dusty environment without wearing a protective mask showed numerous areas where the alveolar spaces were filled with granular, hypocellular eosinophilic material, which was strongly and uniformly periodicacid-Schiff-positive material, diastase resistant, and metachromatic with toluidine blue (MiLler et al.

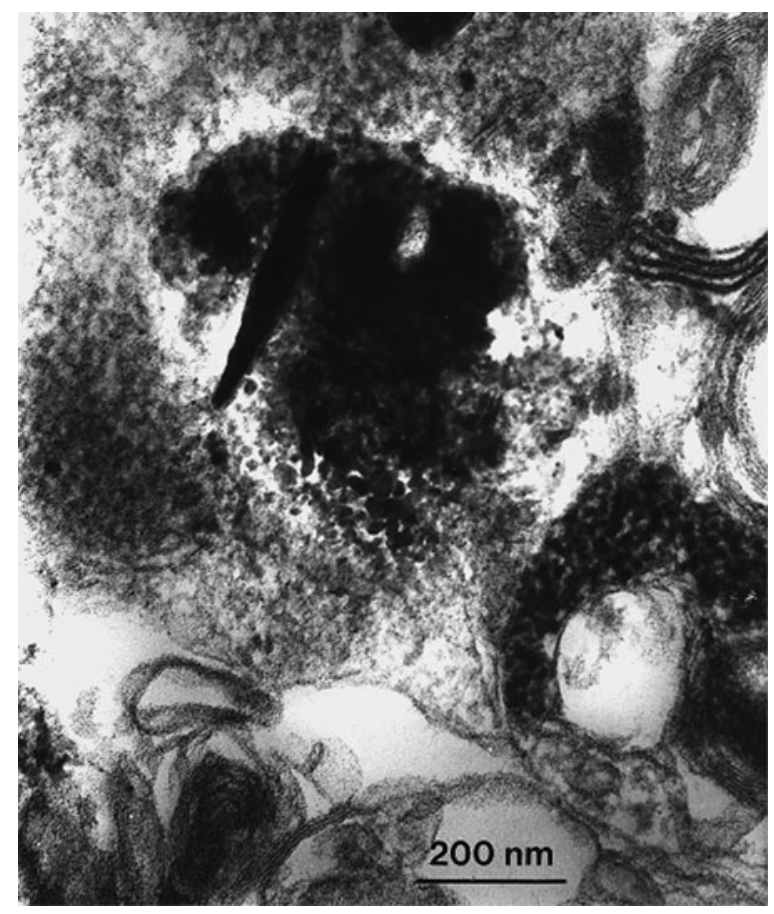

Fig. 128. Aluminium particle in an alveolar macrophage from a $296 \mathrm{~g}$ female white rat (No.3; breeder: Winkelmann Borchen-Kirchborchen) which inhaled $10 \mathrm{mg}$ powdered aluminium $/ \mathrm{m}^{3} 4 \mathrm{~h}$ per day, 5 days per week from August 16 to October 27, 1967 for a total of 51 days. Fixed on October 30, 1967 under methitural anaesthesia by intratracheal instillation of $2.5 \%$ glutaraldehyde in phosphate buffer $(\mathrm{pH} 7.4)$ before opening the thorax. Postfixation with $1 \%$ osmium tetroxide in phosphate buffer ( $\mathrm{pH} 7.4)$. Contrasted en bloc for 12 $\mathrm{h}$ with $0.5 \%$ uranyl acetate in $70 \%$ ethanol. Embedded in a 2:8 mixture of methyl and butyl methacrylate. Sectioned at $50 \mathrm{~nm}$. Lead citrate after REYNOLDS (1963). Plate 19/09
1984). The electron microscopic findings were typical for alveolar proteinosis. Only rare alveolar macrophages were found, and their cytoplasm was distended with granules similar to those free in alveolar spaces. The cytoplasmic borders of the macrophages were abnormally smooth and rounded and only occasional primary lysosomes were present.

\section{Experimental Pathology}

Inhalation experiments in rats demonstrated an eager phagocytosis of aluminium dust by alveolar macrophages and their elimination via the bronchi (SCHILler 1957). Although there is no disintegration of the alveolar macrophages produced by aluminium, characteristic phagolysosomal changes at the electron microscope level were described by SCHILler (1970, 1971). Rats inhaling aluminium dust up to $750 \mathrm{~h}$ accumulated the particulate matter in interstitial macrophages (SCHILLER 1959). After

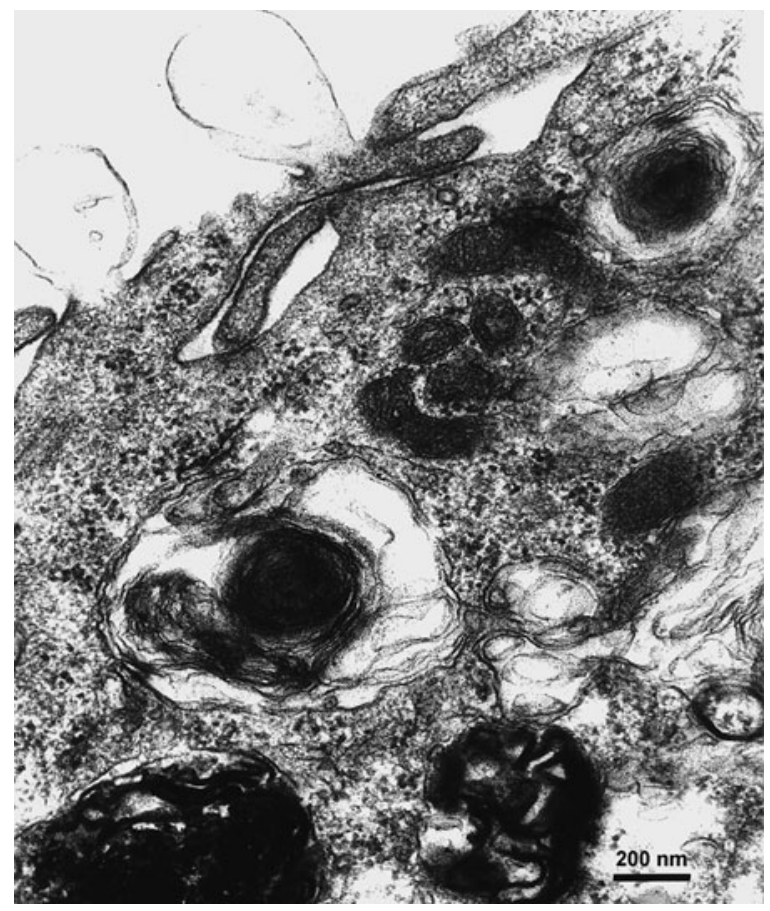

Fig. 129. Membrane blisters on an alveolar macrophage from a female rat (No.4; breeder: Winkelmann, BorchenKirchborchen) which inhaled $10 \mathrm{mg}$ powdered aluminium $/ \mathrm{m}^{3}$ $4 \mathrm{~h}$ per day, 5 days per week from August 16 to October 27, 1967 for a total of 51 days. Fixed on January 15, 1968 under methitural anaesthesia by intratracheal instillation of $2.5 \%$ glutaraldehyde in phosphate buffer ( $\mathrm{pH}$ 7.4) before opening the thorax. Postfixation with $1 \%$ osmium tetroxide in phosphate buffer ( $\mathrm{pH} 7.4)$. Contrasted en bloc for $12 \mathrm{~h}$ with $0.5 \%$ uranyl acetate in $70 \%$ ethanol. Embedded in a $2: 8$ mixture of methyl and butyl methacrylate. Sectioned at $50 \mathrm{~nm}$. Lead citrate after REYNOLDS (1963). Plate 45/05 


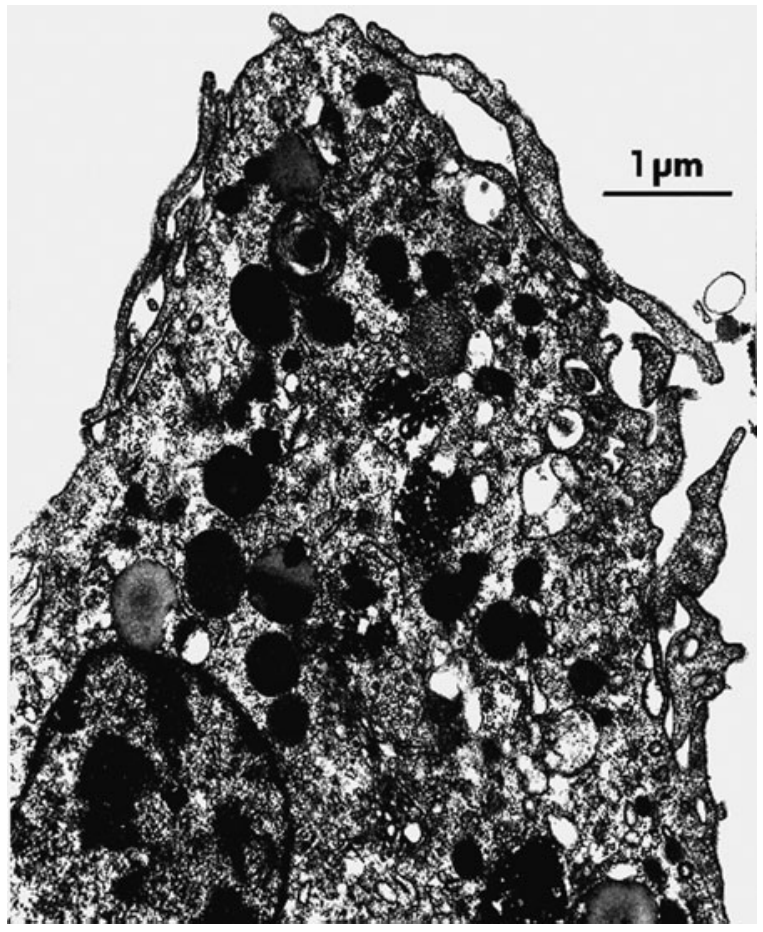

Fig. 130. Alveolar macrophage of a female rat (No.4; breeder Winkelmann, Borchen-Kirchborchen) which inhaled $10 \mathrm{mg}$ powdered aluminium $/ \mathrm{m}^{3} 4 \mathrm{~h}$ per day, 5 days per week from August 16 to October 27, 1967 for a total of 51 days. Fixed on January 15, 1968 under methitural anaesthesia by intratracheal instillation of $2.5 \%$ glutaraldehyde in phosphate buffer ( $\mathrm{pH}$ 7.4) before opening the thorax. Postfixation with $1 \%$ osmium tetroxide in phosphate buffer ( $\mathrm{pH} 7.4$ ). Contrasted en bloc for $12 \mathrm{~h}$ with $0.5 \%$ uranyl acetate in $70 \%$ ethanol. Embedded in a 2:8 mixture of methyl and butyl methacrylate. Sectioned at $50 \mathrm{~nm}$. Lead citrate after REYNOLDs (1963). Plate $38 / 10$

an inhalation of $\geq 28$ months KAHLAU (1955) found a diffuse thickening of the rabbit's alveolar walls characterised by deposits of a substance stained intensively red with acid fuchsin.

$10 \mathrm{mg}$ powdered aluminium $/ \mathrm{m}^{3}$ (for particle size see Table 8 ) inhaled by female rats over a period of 10 weeks and a half $(4 \mathrm{~h}$ per day for a total of 51 days) was phagocytosed by alveolar macrophages and became included within phagosomes (Fig. 128). Secondary lysosomes contain electron dense reaction products of $\mathrm{Al}^{3+}$ with cellular constituents. They are unchanged for several months, but their membranes will be disrupted finally. The number of free ribosomes is increased and so is the activity of the Golgi apparatus. Microtubules are well preserved, but there are also some macrotubules (Fig. 133). Microfilaments are preferably seen in the ruffled border. Some nuclei show elliptical vacuoles or fibrillary degeneration (Fig. 134).

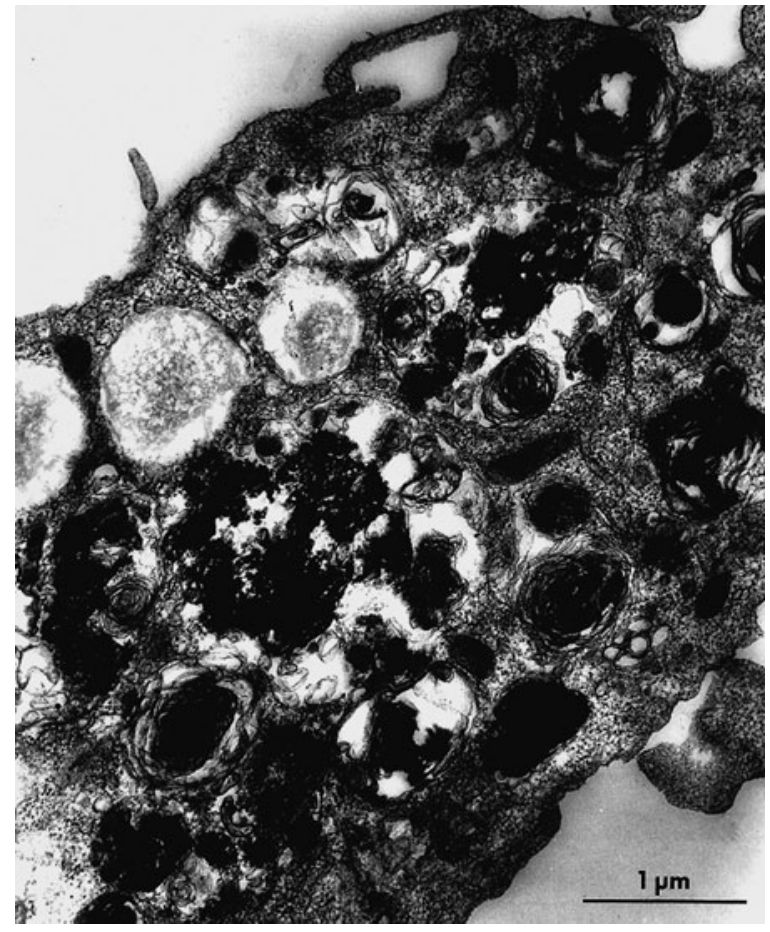

Fig. 131. Alveolar macrophage with myelin-figure inclusions and complex phagolysosomes from a $296 \mathrm{~g}$ female rat (No.3; breeder Winkelmann, Borchen-Kirchborchen) which inhaled $10 \mathrm{mg}$ powdered aluminium $/ \mathrm{m}^{3} 4 \mathrm{~h}$ per day, 5 days per week from August 16 to October 27, 1967 for a total of 51 days. Fixed on October 30, 1967 under methitural anaesthesia by intratracheal instillation of $2.5 \%$ glutaraldehyde in phosphate buffer ( $\mathrm{pH}$ 7.4) before opening the thorax. Postfixation with $1 \%$ osmium tetroxide in phosphate buffer ( $\mathrm{pH} 7.4$ ). Contrasted en bloc for $12 \mathrm{~h}$ with $0.5 \%$ uranyl acetate in $70 \%$ ethanol. Embedded in a 2:8 mixture of methyl and butyl methacrylate. Sectioned at $50 \mathrm{~nm}$. Lead citrate after REYNOLDS (1963). Plate 19/07

\subsubsection{2}

\section{Americium}

Americium has the atomic number 95 and the valence electrons are on the $5 f^{7}$ shell. Half life of ${ }^{241} \mathrm{Am}$ is 462 years.

At concentrations of $<0.125 \mu \mathrm{Ci}{ }^{241} \mathrm{AmO}_{2} / \mathrm{ml}$ $(4.63 \mathrm{kBq} / \mathrm{ml})$, Beagle dog alveolar macrophages obtained by bronchoalveolar lavage did not suffer significant changes of their viability and their ability to phagocytose opsoninized sheep erythrocytes (TAYA and Mewhinney 1987). Cell killing started at $0.5 \mu \mathrm{Ci}{ }^{241} \mathrm{Am} / \mathrm{ml}(18.5 \mathrm{kBq} / \mathrm{ml})$, whereas the reduction of phagocytic ability started at $0.125 \mu \mathrm{Ci}$ ${ }^{241} \mathrm{Am} / \mathrm{ml}$ at $20 \mathrm{~h}$ after exposure. After $72 \mathrm{~h}, 10 \%$ of the ${ }^{241} \mathrm{AmO}_{2}$ particles (physical diameter 0.1 to 1 $\mu \mathrm{m})$ were dissolved in vitro. 


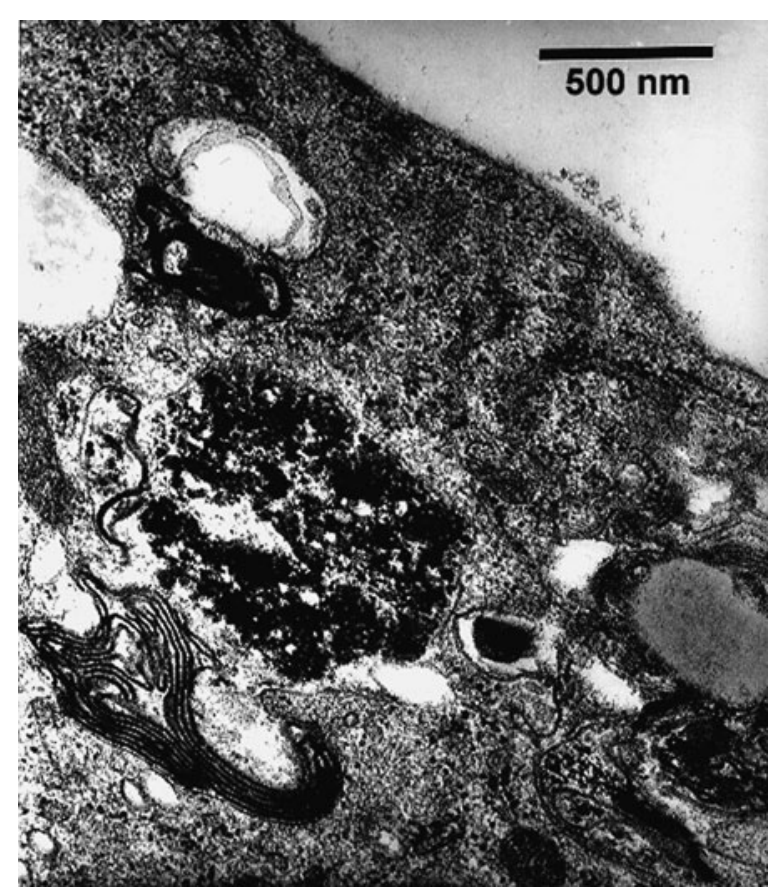

Fig. 132. Complex phagolysosomes and myelin figures in an alveolar macrophage of a female rat (No.4; breeder Winkelmann, Borchen-Kirchborchen) which inhaled $10 \mathrm{mg}$ powdered aluminium $/ \mathrm{m}^{3} 4 \mathrm{~h}$ per day, 5 days per week from August 16 to October 27, 1967 for a total of 51 days. Fixed on January 15, 1968 under methitural anaesthesia by intratracheal instillation of $2.5 \%$ glutaraldehyde in phosphate buffer ( $\mathrm{pH}$ 7.4) before opening the thorax. Postfixation with $1 \%$ osmium tetroxide in phosphate buffer ( $\mathrm{pH}$ 7.4). Contrasted en bloc for $12 \mathrm{~h}$ with $0.5 \%$ uranyl acetate in $70 \%$ ethanol. Embedded in a 2:8 mixture of methyl and butyl methacrylate. Sectioned at $50 \mathrm{~nm}$. Lead citrate after REy nolDs (1963). Plate $43 / 10$

\subsubsection{3}

\section{Antimony}

Antimony has the atomic number 51 and the valence electrons are on the $5 s^{2} 5 p^{4}$ shells. Its oxidation states are $-3,+3$ and +5 .

Inhalation of $1200 \mathrm{mg} \mathrm{Sb} \mathrm{O}_{3}$ dust $/ \mathrm{m}^{3}$ (average particle size $1.1 \mu \mathrm{m}$ ) in rats and $900 \mathrm{mg} \mathrm{Sb} \mathrm{O}_{3}$ dust/ $\mathrm{m}^{3}$ in hamsters, respectively, was associated with a significant increase in the mean macrophage count; whereas the inhalation of $\mathrm{Fe}_{2} \mathrm{O}_{3}$ or of $\mathrm{SiO}_{2}$ dusts did not result in a such significant increase in either species (Gross et al. 1969).

Zymosan-stimulated alveolar macrophages isolated from rabbits treated with Freund's adjuvant in vitro released less $\mathrm{O}_{2}{ }^{--}$when challenged with 200 $\mu \mathrm{g} / 10^{6}$ cells of a waste incinerator dust sonicated five times for 15 min (Gulyas et al. 1990). Decrease correlated best with logarithms of antimony, lead and arsenic contents and with logarithms of particle numbers and dust surfaces.

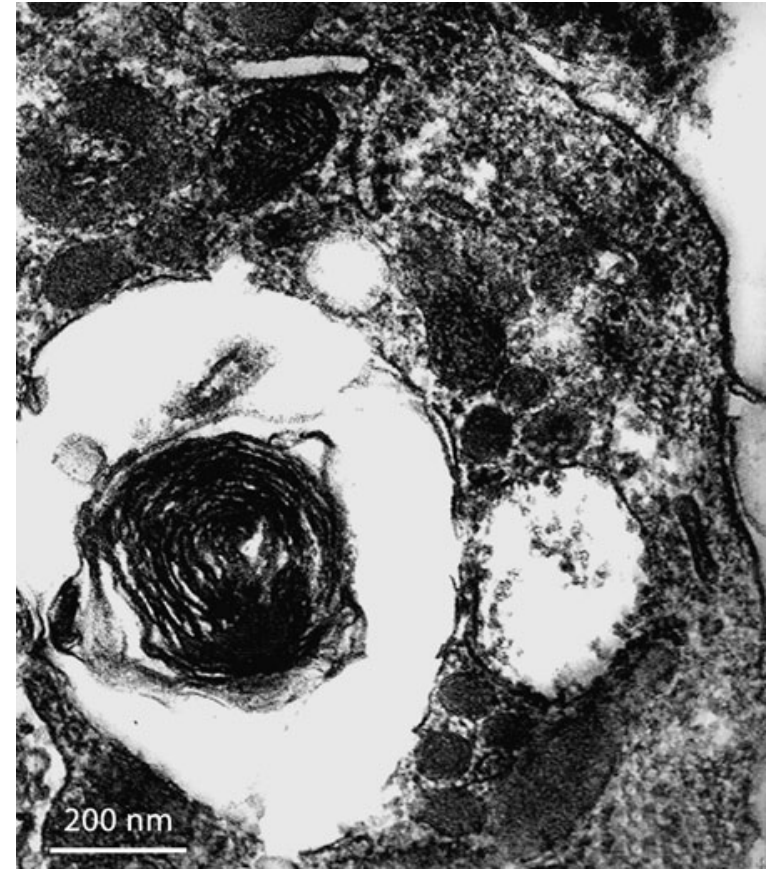

Fig. 133. Macrotubule in an alveolar macrophage from a $296 \mathrm{~g}$ female rat (No.3; breeder Winkelmann, BorchenKirchborchen) which inhaled $10 \mathrm{mg}$ powdered aluminium $/ \mathrm{m}^{3}$ $4 \mathrm{~h}$ per day, 5 days per week from August 16 to October 27, 1967 for a total of 51 days. Fixed on October 30, 1967 under methitural anaesthesia by intratracheal instillation of $2.5 \%$ glutaraldehyde in phosphate buffer ( $\mathrm{pH}$ 7.4) before opening the thorax. Postfixation with $1 \%$ osmium tetroxide in phosphate buffer ( $\mathrm{pH} 7.4)$. Contrasted en bloc for $12 \mathrm{~h}$ with $0.5 \%$ uranyl acetate in $70 \%$ ethanol. Embedded in a $2: 8$ mixture of methyl and butyl methacrylate. Sectioned at $50 \mathrm{~nm}$. Lead citrate after REYNOLDS (1963). Plate 18/07

\subsubsection{4 \\ Arsenic}

Arsenic has the atomic number 33 and the valence electrons are on the $4 p^{3}$ shell. Its oxidation states are $-3,+3$ and +5 .

Pulmonary alveolar macrophages lavaged from As $(V)$-exposed male Sprague-Dawley rats showed significant increases in $\mathrm{O}_{2}{ }^{--}$production and in basal release of TNF- $\alpha$, while As(III) did not induce significant alterations (LANTz et al. 1995). Neither arsenical inhibited prostaglandin $\mathrm{E}_{2}$ production.

\subsubsection{5 Beryllium}

Beryllium has the atomic number 4 and the valence electrons are those of the $2 s^{2}$ shell. +2 is its only oxidation state. Beryllium tends to form polynuclear complexes. 


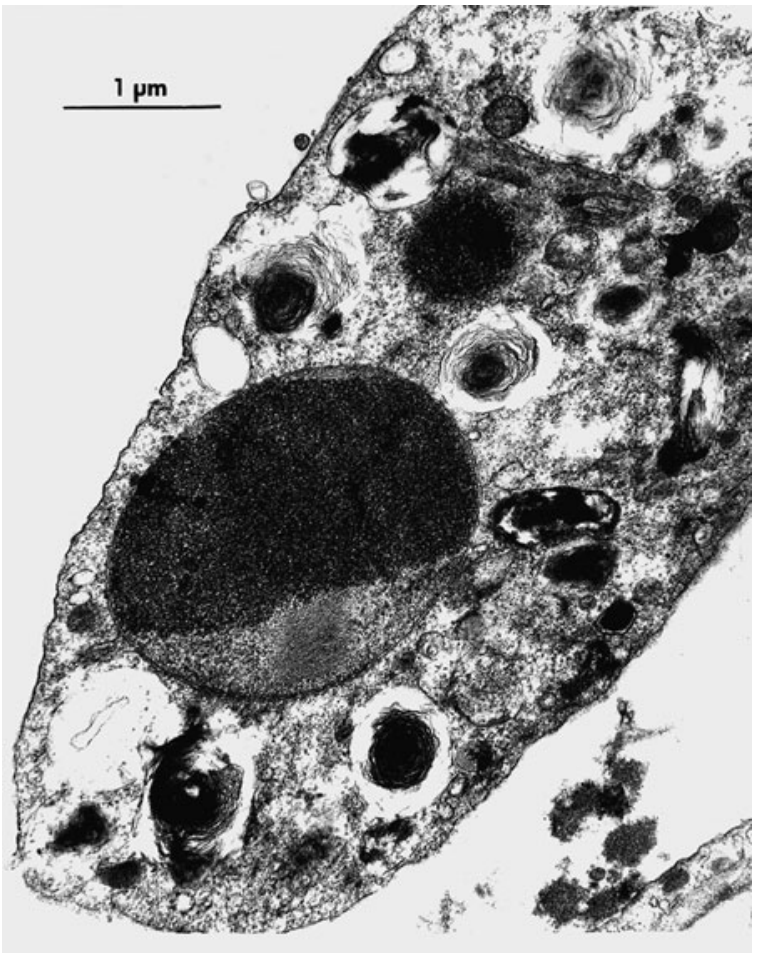

Fig. 134. Fibrillary nuclear degeneration in an alveolar macrophage of a female rat (No.4; breeder Winkelmann, Borchen-Kirchborchen) which inhaled $10 \mathrm{mg}$ powdered aluminium $/ \mathrm{m}^{3} 4 \mathrm{~h}$ per day, 5 days per week from August 16 to October 27, 1967 for a total of 51 days. Fixed on January 15, 1968 under methitural anaesthesia by intratracheal instillation of $2.5 \%$ glutaraldehyde in phosphate buffer ( $\mathrm{pH} 7.4$ ) before opening the thorax. Postfixation with $1 \%$ osmium tetroxide in phosphate buffer ( $\mathrm{pH} 7.4$ ). Contrasted en bloc for 12 $\mathrm{h}$ with $0.5 \%$ uranyl acetate in $70 \%$ ethanol. Embedded in a 2:8 mixture of methyl and butyl methacrylate. Sectioned at $50 \mathrm{~nm}$. Lead citrate after Reynolds (1963). Plate 45/09

Table 31. Effects of beryllium on enzyme activities

\begin{tabular}{|c|c|c|}
\hline Enzyme & EC & Authors \\
\hline Tryptophan pyrrolase & 1.13.11.11 & $\begin{array}{l}\text { Witschi and } \\
\text { MARCHAND (1971) }\end{array}$ \\
\hline $\begin{array}{l}\text { Glutamic-oxalacetic } \\
\text { aminotransferase }\end{array}$ & 2.6.1.1 & VACHER et al. (1975) \\
\hline $\begin{array}{l}\text { Glutamic-pyruvate } \\
\text { aminotransferase }\end{array}$ & 2.6.1.2 & VACHER et al. (1975) \\
\hline Alkaline phosphatase & 3.1.3.1 & $\begin{array}{l}\text { KLEMPERER et al. } \\
\text { (1949) }\end{array}$ \\
\hline$\beta$-Glucuronidase & 3.2.1.31 & VACHER et al. (1975) \\
\hline
\end{tabular}

Beryllium has been shown to induce an enhanced pathological and antibody response similar to Freund's adjuvant (SAlvagGio et al. 1965).

\section{Beryllium Metal}

In the guinea pig, intratracheal instillation of $75 \mathrm{mg}$ beryllium metal after 3 months induced focal lymphocytic and large mononuclear cell reaction immediately about visible deposits of the substance (VORWALD 1950).

\section{Beryllium Oxide}

In Macaca irus monkeys exposed to beryllium oxide ( 3.30 to $4.38 \mathrm{mg} \mathrm{Be} / \mathrm{m}^{3}$ ) three times, for 30 min each, at monthly intervals ConRadi et al. (1971) after 2 years found histiocytes in the pulmonary interstitial space containing heterogeneous dark granular material and lipid droplets within membrane-bounded space.

After intratracheal instillation of beryllium oxide to guinea-pigs, Policard (1950) found hypertrophied histiocytes, sometimes 30 to $40 \mu \mathrm{m}$ in diameter with voluminous nuclei $(20-25 \mu \mathrm{m})$, irregular wrinkled, abundant chromatin and several large nucleoli.

\section{Beryllium Hydroxide}

In the guinea pig, intratracheal instillation of 150 mg beryllium hydroxide after 4 months induced focal lymphocytic and large mononuclear cell reaction with proliferation of local tissue cells and mild fibrosis (VoRWALD 1950).

\section{Beryllium Sulphate}

Beryllium sulphate $\left(84.3 \mathrm{mg} \mathrm{BeSO}{ }_{4} \cdot 4 \mathrm{H}_{2} \mathrm{O} / \mathrm{m}^{3}\right)$ inhalation in rats induced rather marked pulmonary oedema accompanied by a moderate infiltration of neutrophils and monocytes (SPRAgUe et al. 1950).

H36.12j clonally derived, C57BL/6N-mouse hybrid precursor macrophages were found useful to evaluate the mechanisms by which Be stimulates macrophage cytokine production, and by which $\mathrm{T}$ cell derived IFN- $\gamma$ amplifies TNF- $\alpha$ production in granulomatous disease (SAWYER et al. 2000). The response was maximal at $100 \mu \mathrm{M} \mathrm{BeSO}_{4}$ and did not occur when $12 \mathrm{j}$ cells were stimulated with either aluminium sulphate of cobalt sulphate. Beryllium stimulated the production of $725 \pm 25 \mathrm{pg} / \mathrm{ml}$ TNF- $\alpha$ protein by $12 \mathrm{j}$ cells as measured by ELISA of culture supernatants after $24 \mathrm{~h}$. As measured by RT-PCR, Be-stimulated $12 \mathrm{j}$ cell TNF- $\alpha$ protein production was accompanied by an increased intracellular TNF- $\alpha$ mRNA at 3 and $24 \mathrm{~h}$. The addition of $10 \mathrm{U}$ or $100 \mathrm{U}$ of recombinant-Mu-IFN- $\gamma$ to Be-stimulated $12 \mathrm{j}$ cells further increased TNF- $\alpha$ production $1.5-4$ 
fold $(1.6 \pm 0.1 \mathrm{ng} / \mathrm{ml})$ respectively. Bacterial lipopolysaccharide (LPS, $1 \mu \mathrm{g} / \mathrm{ml}$ ) stimulated production of TNF- $\alpha$ in $12 \mathrm{j}$ culture supernatants after $6 \mathrm{~h}$ $(515 \pm 151 \mathrm{pg} / \mathrm{ml})$.

Recombinant-Mu-IFN- $\gamma$ (10 U) + beryllium (100 $\mu \mathrm{M} \mathrm{BeSO})_{4}$-stimulated $\mathrm{H} 36.12 \mathrm{j}$ macrophages produced $1195 \pm 225 \mathrm{pg}$ TNF- $\alpha / \mathrm{ml}$, while H36.12j cells stimulated with $\mathrm{rMu}-\mathrm{IFN}-\gamma$ by itself produced only $284 \pm 31$ pg TNF- $\alpha / \mathrm{ml}$ (Hamada et al. 2000).

\section{Beryllium Stearate}

12 Months after intratracheal instillation of $150 \mathrm{mg}$ beryllium stearate, the alveolar spaces of the guinea pig contained scattered dust laden macrophages in various stages of degeneration (VoRWALD 1950).

\section{Zinc Beryllium Manganese Silicate}

In the guinea pig, zinc beryllium manganese silicate inhalation $(8 \mathrm{~h} / \mathrm{d})$ for 40 months induced mild lymphocytic and large mononuclear cell reaction indicative of slight irritation about dust particles (VoRWALD 1950).

\subsubsection{6}

Cadmium

Cadmium has the atomic number 48 and the valence electrons are those of the $4 d^{10} 5 s^{2}$ shells. +2 is its only oxidation state. The co-ordination number in its complexes is 4 .

Table 32. Effects of cadmium on enzyme activities

\begin{tabular}{|c|c|c|}
\hline Enzyme & EC & Authors \\
\hline Lactate dehydrogenase & 1.1.1.27 & HART (1986) \\
\hline Catalase & 1.11.1.6 & ZIKIC et al. (1998) \\
\hline Glutathione peroxidase & 1.11.1.9 & ZIKIC et al. (1998) \\
\hline Lysine oxidase & 1.13 .12 .2 & $\begin{array}{l}\text { CHichester et al. } \\
\text { (1981) }\end{array}$ \\
\hline Proline hydroxylase & 1.14 .11 .2 & $\begin{array}{l}\text { CHICHESTER et al. } \\
\text { (1981) }\end{array}$ \\
\hline Superoxide dismutase & 1.15.1.1 & ZIKIC et al. (1998) \\
\hline Alkaline phosphatase & 3.1.3.1 & HART (1986) \\
\hline Acid phosphatase & 3.1.3.2 & HART (1986) \\
\hline $\mathrm{Na}^{+}-\mathrm{K}^{+}$-ATPase & 3.6.1.3 & LAI et al. (1980) \\
\hline $\mathrm{Mg}^{2+}$-ATPase & 3.6.1.3 & $\begin{array}{l}\text { LAI et al. (1980), } \\
\text { HyPPÖNEN et al. } \\
\text { (1993) }\end{array}$ \\
\hline
\end{tabular}

\section{Cadmium Oxide}

In specific-pathogen-free male Wistar rats, focal areas of epithelial hyperplasia, a mononuclear interstitial infiltrate, and increased numbers of alveolar macrophages were observed after a single 3-h exposure to an aerosol of insoluble CdO (BUCKLEY and BASSETt 1987).

In male Sprague-Dawley rats instilled intratracheally with $5 \mathrm{mg}$ CdO suspended in $0.5 \mathrm{ml}$ saline one week before, MurThy et al. (1982) noted two types of alveolar macrophages. The first showed a significant increase in size when compared to those seen in control animals. The cytoplasm of these cells was filled with numerous small vacuoles which contained bizarre-shaped electron-dense aggregations. Some vacuoles seemed to coalesce with others which resulted in larger vacuoles. The mitochondria were smaller in size and fewer in number when compared with controls. Some mitochondria were phagocytized in membrane-bound autophagosomes. A distinct nucleolus was lacking and chromatin material seemed to be fragmented and scattered. The second type of alveolar macrophages was smaller in size and their nucleus contained a prominent nucleolus. The cytoplasm contained fewer vacuoles, more profiles of rough endoplasmic reticulum, and mitochondria which were larger than those seen in the first type.

In alveolar macrophages from male SpragueDawley rats intratracheally intubuated with a suspension $(0.5 \mathrm{ml})$ of $0.1 \mathrm{mg} \mathrm{CdO}$ plus $0.2 \mathrm{mg} \mathrm{CuO}$ particles, Murthy and Holovack (1991) found large aggregates of multivesicular bodies.

\section{Cadmium Sulphide}

In conventionally kept Wistar rats into which $15 \mathrm{mg}$ cadmium sulphide (Merck, Darmstadt) had been injected intratracheally six times at weekly intervals, KISSLER (1983) observed pulmonary fibrosis of varying intensity from $2^{1 / 2}$ weeks after the last administration. Many alveolar septa were thickened by connective tissue. They did not show any inflammatory infiltrates. They were coated with continuous rows of isoprismatic type II pneumocytes with foamy cytoplasm. Numerous alveolar macrophages with foamy cytoplasm were seen from the beginning; the majority of them had phagocytosed CdS particles well recognised by their bright yellow colour. Abundant fine-granular but otherwise unstructured eosinophilic material was found in varying amounts between the phagocytes. Acid phosphatase reaction is intensified in intraalveolar macrophages only (KISSLER and SCHERBECK 1981, KISSLER 1983). 


\section{Cadmium Telluride}

Cadmium telluride (CdTe) particles (mean diameter $1.70 \mu \mathrm{m}$ ) suspended in sterile, phosphatebuffered saline, $\mathrm{pH}$ 7.2, and instilled intratracheally into specific pathogen-free female Sprague-Dawley rats at doses of $12 \mathrm{mg}, 24 \mathrm{mg}, 48 \mathrm{mg}$, or $96 \mathrm{mg} / \mathrm{kg}$ body weight after 3 days had induced an intaalveolar inflammatory exudate consisting of neutrophils, macrophages, fibrin, and protein (Morgan et al. 1995). A minimal amount of black cadmium telluride particles was present.

\section{$\mathrm{Cd}^{2+}$}

Goat alveolar macrophages gained by bronchoalveolar lavage of the isolated lung and exposed to $\geq 0.04 \mathrm{mM} \mathrm{CdCl}_{2}$ for $20 \mathrm{~h}$ showed strongly reduced viability $(P<0.01)$. The lactate dehydrogenase activity of the supernatant culture medium was elevated $(P<0.01)$. Phagocytic potential had decreased $(P<0.01)$. In cultures exposed to $0.20 \mathrm{mM}$ $\mathrm{CdCl}_{2}$ macrophages had withdrawn their pseudopodia, as seen by the scanning electron microscope (WASEEM et al. 1993).

In Long-Evans rats, cadmium ions depressed the oxidative burst of alveolar macrophages during phagocytosis of zymosan (CASTRANOvA et al. 1980): $10^{-3} \mathrm{M} \mathrm{Cd}^{2+}$ given in vitro for 15 min significantly inhibited oxygen consumption (by $76 \pm 1 \%$ ), glucose metabolism (by $83 \pm 7 \%$ ), and release of active oxygen species (by $73 \pm 1 \%$ ). This cadmiuminduced inhibition of oxidative processes of macrophages was postulated to diminish their antibacterial activity.

The oxidative metabolism of NMRI mouse peritoneal macrophages induced by phorbol myristate acetate was enhanced by $\mathrm{CdCl}_{2}$, the zymosaninduced oxidative metabolism was markedly re- duced as measured by means of the lucigeninenhanced chemiluminescence in a Berthold Biolumat 9500 up to 120 min (Hilbertz et al. 1986).

After a single incubation of mouse resident peritoneal macrophages for $18 \mathrm{~h}$ in medium containing $10 \mu \mathrm{M} \mathrm{Cd}^{2+}$, a five-fold increase in ${ }^{\circ} \mathrm{NO}$ production, with respect to incubation in medium alone, was observed (RAmirez and Gimenez 2000). After $18 \mathrm{~h}$ of culture, NO inductor agents induced significant expression of inducible nitric oxide synthase (iNOS) and ${ }^{\circ} \mathrm{NO}$ production in peritoneal macrophages. When cells were simultaneously incubated for $18 \mathrm{~h}$ in medium containing $10 \mu \mathrm{M} \mathrm{Cd}^{2+}$ and NO inductor agents, an increase in response to lipopolysaccharide $(1 \mu \mathrm{g} / \mathrm{ml})$, okadaic acid $(50 \mathrm{nM})$ and 12$O$-tetradecanoylphorbol-13-acetate (200 nM), and a decrease in the synergistic effect of lipopolysaccharide/okadaic acid, in relation to culture in medium without $\mathrm{Cd}^{2+}$ were observed.

\subsubsection{7 \\ Carbon}

While the surface of diamonds from volcanic chimneys is hydrophobic, that from secondary deposits is hydrophilic due to the formation of oxides (Boenm et al. 1964). Powdered diamonds with oxides on their surface show a higher wetting heat than those without. Treating diamonds oxidised on their surface with water forms carboxyl groups. Estimation of active hydrogen after ZEREWITINOFF or by deuterium exchange revealed that nearly all hydrogen atoms were carboxylic (SAPPOK and Boenm, quoted from Boenm 1966). Diamonds cleared of oxides at $10^{-5}$ to $10^{-6}$ torr in vacuo at $800^{\circ} \mathrm{C}$ do not show an essential resonance absorption (11 $\mu$ val free radicals/g diamond).

In the layers of the graphite lattice the atoms are combined with three neighbours by $\sigma$-bindings
Fig. 135. Alveolar macrophages having engulfed powdered diamond. 3-Day old culture of a 3-day old mouse. The preparation was fixed in Carnoy's fluid, stained with iron hematoxylum after Weigert, and mounted in toto in Rhenohistol ${ }^{\circledR}$. Eye-piece Leitz Periplan 4×; objective Leitz Pv Fl oil 70/1.15. Positive phase contrast (from SCHILlER 1954)

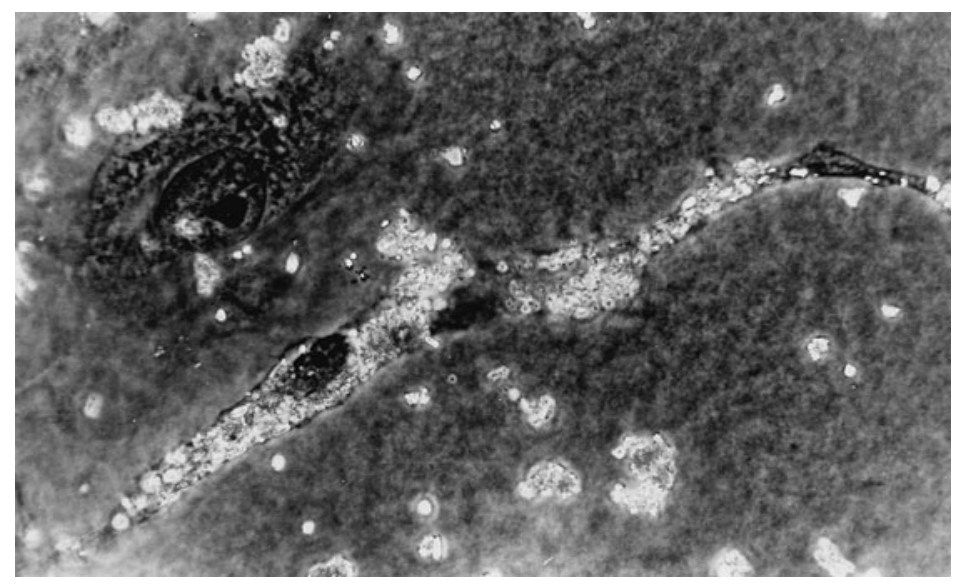


Fig. 136. Alveolar macrophages having engulfed powdered diamond. 4-Day old culture of a 3-day old mouse. The preparation was fixed in Carnoy's fluid, stained with iron hematoxylum after Weigert, and mounted in toto in Rhenohistol ${ }^{\circledR}$. Eye-piece Leitz Periplan $4 \times$; objective Leitz Pv Fl oil 70/1.15. Positive phase contrast (from ScHILler 1954)

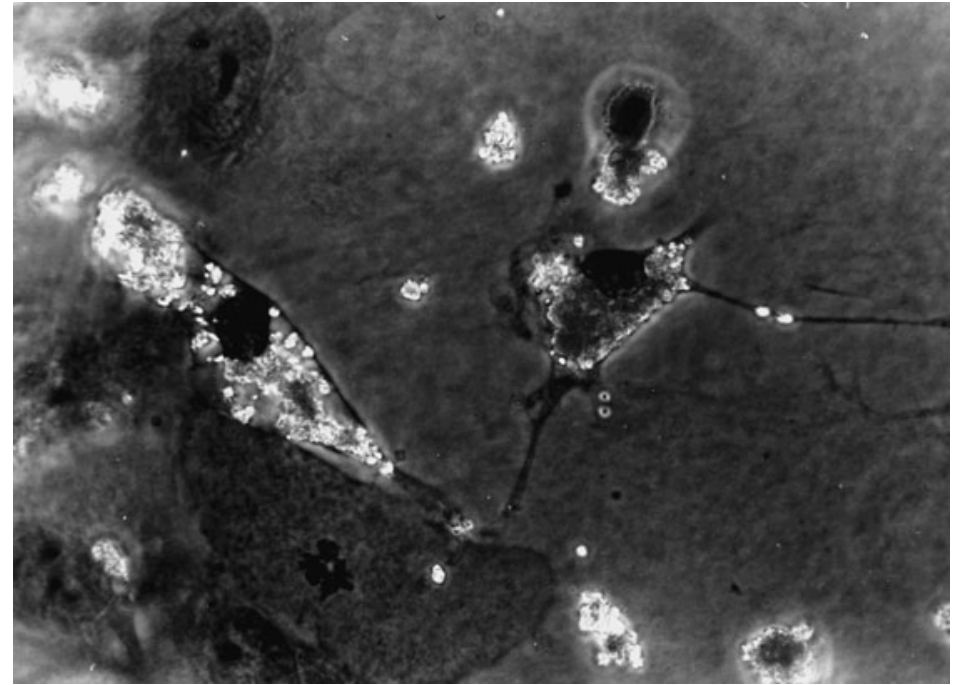

( $\mathrm{sp}^{2}$-hybridisation). The fourth valence electrons are bound as $\pi$-electrons which can easily change from one atom in the layer to another. The layers are held together by Van der Waals' forces (distance $0.335 \mathrm{~nm})$.

Twenty and $30 \mathrm{~s}$ following intravenous injection, BURKE and Simon (1970) localised carbon predominantly in tiny clumps in the sinuses of the marginal zone and the red pulp of the rabbit's spleen. At this stage some carbon was already being phagocytosed by macrophages arising from the Billroth cords.

In vitro, human alveolar macrophages collected by bronchoalveolar lavage and loaded with small masses $\left(0.03-3 \mu \mathrm{g} / 10^{6}\right.$ cells) of ultrafine particle aggregates induced a dose-related impairment of both the attachment and the ingestion processes of fluorescein-labelled silica particles $(3 \mu \mathrm{m})$ with a marked impairment down to a carbon particle dose around $0,2 \mu \mathrm{g} / 10^{6}$ cells (LundBorg et al. 2001). Incubation with interferon- $\gamma(12.5 \mathrm{U} / \mathrm{ml})$ also induced significant impairments in both the attachment and the ingestion processes. Loading with carbon further aggravated the effects of interferon- $\gamma$. In contrast to earlier studies in rat alveolar macrophages (LUNDBORG et al. 1999), interferon- $\gamma$ did not impair the oxidative metabolism (nitroblue tetrazolium reduction) at rest in these human alveolar macrophages; instead the oxidative metabolism was increased. This difference was due to a difference between rat and human alveolar macrophages and not between rat and human interferon- $\gamma$.

Unlike the characteristic progressive nature of silica-related lesions, all of respirable-sized carbon fibre-induced inflammatory effects were reversible within 10 days after exposure and any significant histopathologic effects were observed at any time post-exposure (WARHEIT et al. 1994).

\subsubsection{8}

\section{Cerium}

Cerium has the atomic number 58 and the valence electrons are those of the $4 f^{2} 6 s^{2}$ shells. The oxidation states of $\mathrm{Ce}$ are +3 and +4 .

Cerium forms complexes with riboflavin (SEKHON and CHOPRA 1974). Cerium chloride $(2 \mathrm{mg} / \mathrm{kg}$ rat intravenously) impaired the activities of cytochrome P-450 and NADPH-cytochrome $c$ reductase of rat liver, which could be prevented by $\alpha$-tocopherol acetate $(100 \mathrm{mg} / \mathrm{kg} \times$ day i.p.) pre-treatment (ARVELA 1974). $\mathrm{Ce}^{3+}$ promoted the binding of creatine kinase to Cibacron blue $F_{3}$ GA, the substrate analogue of the enzyme, even in the absence of $\mathrm{Mg}^{2+}$, its native cofactor (SHIVAKUMAR et al. 1989).

In a lung biopsy of a female aged 31 years, who had polished spectacle lenses with cerium oxide for three years, Sinico et al. (1982) found multiple macrophagic granulomas containing dust particles identified by analytical electron microscopy as cerium oxide.

Table 33. Effects of cerium on enzyme activities

\begin{tabular}{lll}
\hline Enzyme & EC & Author(s) \\
\hline $\begin{array}{l}\text { Glucose-6-phosphate } \\
\text { dehydrogenase }\end{array}$ & 1.1 .1 .49 & ArVELA (1974) \\
$\begin{array}{l}\text { NADPH-cytochrome } c \\
\text { reductase }\end{array}$ & 1.6 .99 .3 & ArVELA (1974) \\
$\begin{array}{l}\text { Creatine kinase } \\
\text { 2.7.3.3 }\end{array}$ & $\begin{array}{l}\text { SHIVAKUMAR et al. } \\
(1989) .\end{array}$ \\
\hline
\end{tabular}




\subsubsection{9}

\section{Chromium}

Chromium has the atomic number 24 and the valence electrons are those on the $3 d^{5} 4 s^{1}$ shells. The oxidation states of chromium include $+2,+3$ and +6 .

$$
\begin{aligned}
& {\left[\mathrm{CrO}_{4}\right]^{2-}+4 \mathrm{H}_{2} \mathrm{O}+3 \mathrm{e}^{-} \rightarrow \mathrm{Cr}(\mathrm{OH})_{3}(\mathrm{~s})+5 \mathrm{OH}^{-}} \\
& 2\left[\mathrm{CrO}_{4}\right]^{2-}+\mathrm{H}_{3} \mathrm{O}^{+} \rightarrow\left[\mathrm{Cr}_{2} \mathrm{O}_{7}\right]^{2-}+3 \mathrm{H}_{2} \mathrm{O} \\
& \mathrm{Cr}(\mathrm{VI})+3 \mathrm{~V}(\mathrm{VII}) \rightarrow \mathrm{Cr}(\mathrm{III})+3 \mathrm{~V}(\mathrm{~V}) \\
& \mathrm{Cr}(\mathrm{VI})+\mathrm{V}(\mathrm{IV}) \rightarrow \mathrm{Cr}(\mathrm{V})+\mathrm{V}(\mathrm{V}) \\
& \mathrm{Cr}(\mathrm{V})+\mathrm{NADH} \rightarrow \mathrm{Cr}(\mathrm{III})+\mathrm{NAD}
\end{aligned}
$$

$\mathrm{Cr}^{\mathrm{VI}}$ is formed from $\mathrm{Cr}^{\mathrm{III}}$ and from metallic $\mathrm{Cr}$ by oxidation, primarily by ozone. In gas shielded arc welding, ozone itself is formed by the interaction of oxygen and ultra-violet radiation emitted from the arc.

Hydrogen peroxide or superoxide form a paramagnetic complex in the reaction with chromium(VI) oxide in an alkaline water solution at room temperature (LAGERCRANTZ 1999). The complex $\left[\mathrm{Cr}(\mathrm{OH})_{5} \mathrm{O}_{2}\right]^{5-}$ with the g-value equal to 1.9734 is believed to contain hydroxyl groups derived from the alkaline solution and dioxygen derived from $\mathrm{H}_{2} \mathrm{O}_{2}$ or $\mathrm{O}_{2}{ }^{--}$.

$\mathrm{Cr}^{\mathrm{III}}$ supplementation prevented the increase in TNF- $\alpha$ levels and oxidative stress caused by high levels of glucose $(30 \mathrm{mM})$ in cultured U937 monocytic cells (JAIN and KanNan 2001). Similarly, chromium supplementation prevented elevated TNF- $\alpha$ secretion and lipid peroxidation levels in $\mathrm{H}_{2} \mathrm{O}_{2}$-treated U937 cells.

Alveolar macrophages from both guinea pig and man tolerated $\mathrm{Cr}$ (VI) salts up to $4.97 \mu \mathrm{g} / \mathrm{ml}$ (RATsCHEK et al. 1988). An increase in the number of macrophages in the bronchoalveolar lavage fluid was seen in rats inhaling $\mathrm{Na}_{2} \mathrm{Cr}_{2} \mathrm{O}_{7}$ for $22 \mathrm{~h}$ per day on 30 or 90 consecutive days (GLASER et al. 1990).

\section{Chromite}

Chromium ore (chromite, $\mathrm{FeO} \cdot \mathrm{Cr}_{2} \mathrm{O}_{3}$ ) has generally been absolved of playing in causal role in chromium-induced malignancies as chromite miners allegedly were free from lung cancers and because chromium ore was thought to be harmless on account of its insolubility in the body (KoELSCH 1938). A similar type of reasoning was advanced in placing the responsibility on zinc chromate as the cause of lung cancer among chrome pigment workers because it is more soluble than barium or lead chromate (Gross and Koelsch 1943). Mancuso and HuEPER (1951) added powdered chromium ore to citrated human blood for $16 \mathrm{~h}$ at room tempera- ture and found 0.000 and $0.001 \mathrm{mg} \mathrm{Cr} / \mathrm{ml}$ plasma, respectively, or 0.10 and $0.12 \mathrm{mg} \mathrm{Cr} / \mathrm{ml}$ washed cells, respectively. In genotoxic studies $\mathrm{Cr}$ (III) was absolutely inactive unless a direct interaction with purifies DNA was permitted by the test conditions (BIANCHI et al. 1983).

Macrophages outgrowing from mouse lung explants eagerly engulfed chromite dust particles (2 $\mu \mathrm{m})$ without suffering any damage detectable by phase contrast microscopy (WORTH and SCHILLER 1955). In the rabbit, inhaled $\mathrm{Cr}^{3+}$ reduced the capacity of alveolar macrophages to catabolize surfactant (Johansson et al. 1992).

\subsection{0 \\ Coal Dust}

Coal is characterised by a non-uniform molecular structure within which aromatic structural units can be distinguished. They are composed of one to five condensed or directly joined aromatic rings substituted by heterocyles and aliphatic (mainly methyl) groups. Nitrogen is involved in pyridine and pyrrole rings and a part of sulphur in thiophene rings. $\mathrm{C}$ - and $\mathrm{O}$-alkylation may occur by the reductive alkylation reactions of coal. LAzARov et al. (1984) presented a structural model which fits the values of the structural parameters obtained by ${ }^{1} \mathrm{H}$ and ${ }^{13} \mathrm{C}$ NMR spectroscpy of alkylated coal.

$\mathrm{H}_{2} \mathrm{O}_{2}$ was formed when $\mathrm{H}_{2} \mathrm{SO}_{4}$ was adsorbed on charcoal in the presence of freshly adsorbed $\mathrm{O}_{2}$, the yield increasing with the concentration of $\mathrm{H}_{2} \mathrm{SO}_{4}$ (BURSHTEIN and FrUMKIN 1941). The quantity of $\mathrm{H}_{2} \mathrm{O}_{2}$ reached a maximum in about $10 \mathrm{~min}$, and then decreased because of the catalytic decompensation on charcoal. The yield was very low when inert gases instead of $\mathrm{O}_{2}$ were bubbled through the solution. The formation of $\mathrm{H}_{2} \mathrm{O}_{2}$ stopped then the adsorption of the acid was complete. At a polarized cathode in the presence of freshly adsorbed $\mathrm{O}_{2}$, the yield of $\mathrm{H}_{2} \mathrm{O}_{2}$ as the concentration increased from $6.5 \times 10^{-5}$ to $130 \times 10^{-5} \mathrm{~A}$ per $0.1 \mathrm{~g}$ of charcoal with further increase of the concentration, the yield decreased. The yield was little dependent on the composition of the solution.

$$
\mathrm{C}_{\mathrm{x}} \mathrm{O}_{2}+2 \mathrm{H}^{+}+2 \mathrm{X}^{-} \rightarrow \mathrm{C}_{\mathrm{x}}^{2+}\left(\mathrm{X}^{-}\right)_{2}+\mathrm{H}_{2} \mathrm{O}_{2}
$$

Phagocytosis by mouse macrophages of dust particles from a coal miner's lung (Fig. 53) is not only a local reaction between the particle and the cell membrane, but a reaction in which both size and shape of the macrophage is changed (Figs.137, 138).

Coals have an appreciable concentration of free radicals in their native form, presumably formed in 


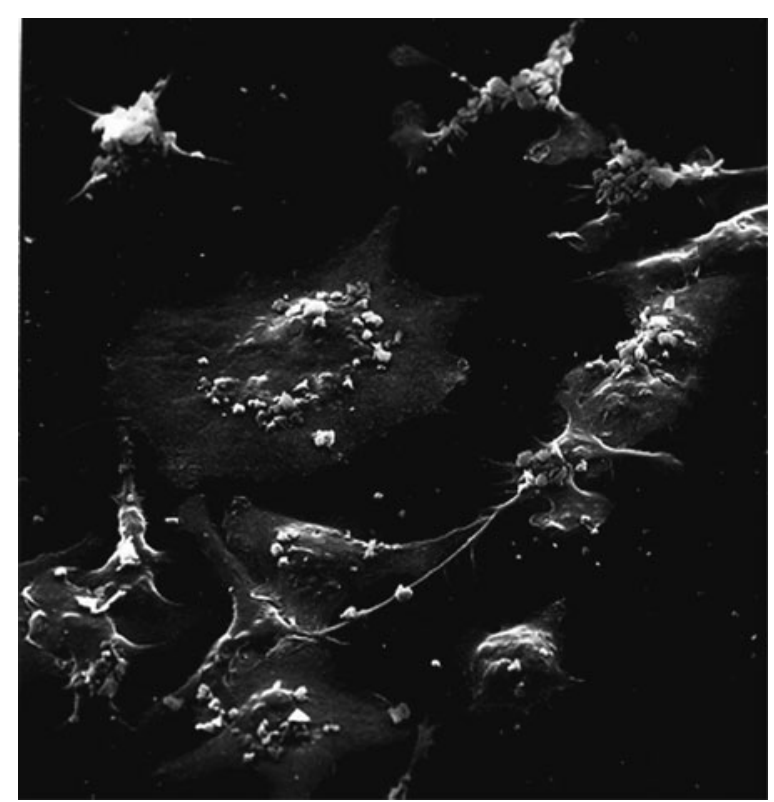

Fig. 137. Peritoneal macrophages from a SPF-NMRI mouse cultured in a Leighton tube after CHANG (1964) $4 \mathrm{~h}$ after addition of dust particles from a coal miner's lung (No.17 of EINBRODT 1965, aged 38 years, underground for 15 years), digested by formamide. Fixed with $1 \%$ glutaraldehyde in 0.07 $\mathrm{M}$ phosphate buffer ( $\mathrm{pH}$ 7.4). Ethanol, amyl acetate. Critical point drying. Gold coating. Cambridge Stereoscan Mark 2 A operated at $20 \mathrm{kV}$, negative 78-168/1.×600 (from SCHILLER 1982)

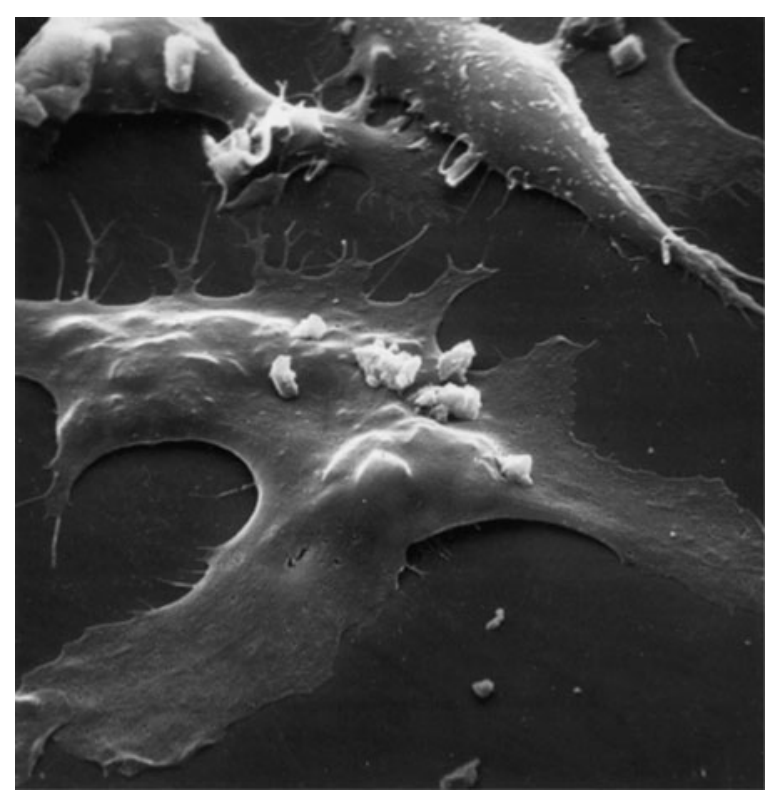

Fig. 138. Peritoneal macrophages from a SPF-NMRI mouse cultured in a Leighton tube after CHANG (1964) $4 \mathrm{~h}$ after addition of dust particles from a coal miner's lung (No.17 of EINBRODT 1965, aged 38 years, underground for 15 years), digested by formamide. Fixed with $1 \%$ glutaraldehyde in 0.07 M phosphate buffer ( $\mathrm{pH}$ 7.4). Ethanol, amyl acetate. Critical point drying. Gold coating. Cambridge Stereoscan Mark 2 A operated at $20 \mathrm{kV}$, negative 78-166/15. ×2,400 (from SCHILLER 1982) the "coalification" process. In addition, during crushing of coking coal paramagnetic centres are formed and their concentration increases with the milling time (LEBEDEV et al. 1978). These centres are free radicals produced by breaking chemical bonds with an unpaired electron on the primary and secondary carbon atoms.

The nature and concentration of free radicals present in a given coal are determined by rank, thermal history, and particle size (PETRAKIS and GRANDY 1978).

All the coal mine dust samples from Utah Blind Canyon and Western Virginia Pittsburgh seam examined by DALAL et al. (1995) generated varying levels of $\mathrm{HO}^{\circ}$ radicals from $\mathrm{H}_{2} \mathrm{O}_{2}$ in the presence of a $\mathrm{HO}^{\bullet}$ spin trap 5,5-dimethyl-1-pyrroline- $\mathrm{N}$-oxide. $\mathrm{HO}^{\circ}$ radical generation by the coal from $\mathrm{H}_{2} \mathrm{O}_{2}$ was effectively inhibited by deferoxamine and catalase, but only partly inhibited by superoxide dismutase. Metal chelators DETAPAC and EDTA enhanced the radical generation. The $\mathrm{HO}^{\circ}$-generating potential of all coal dusts showed a positive correlation with the surface iron content of coal mine dust.

Instillation of $500 \mathrm{mg}$ generic anthracite dust No. 867 (3.6\% silica; $7 \mu \mathrm{m}$ maximum diameter) and generic bituminous dust No. $1361(0.7 \%$ silica; 7 $\mu \mathrm{m}$ maximum diameter), respectively, suspended in $50 \mathrm{ml} \mathrm{PBS}$ into the right caudal lung lobe of $3 \mathrm{fe}-$ male pigtail macaque monkeys (Macaca nemestrina) each using a flexible fibreoptic bronchoscope increased the numbers of macrophages in the dust exposed lobes and significantly elevated the $N$ acetyl- $\beta$-D-glucosaminidase activity within the bronchoalveolar lavages taken at 2-weeks intervals for 12 weeks thereafter (MACK et al. 1995).

In the hamster, a single intratracheal instillation of $4 \mathrm{mg}$ bituminous coal dust increased both alveolar macrophages and neutrophils in bronchoalveolar lavage fluid (KLEINERMAN and IP 1990). The elastolytic activity of the culture fluid in which the macrophages were sustained was increased. The total concentration of the anti-proteases in the alveolar fluid, both $\alpha_{1}$ protease inhibitor and $\alpha_{2}$ macroglobulin were not significantly different from control values. Multiple coal dust instillations performed at 5-7 day intervals over 4-5 weeks showed an increase of neutrophils and macrophages in the broncho-alveolar lavage fluid obtained 3 and 90 days after the last instillation as compared with controls. The total elastolytic activity of the leucocytes was greater at day 3 than at day 90 . However, the $\alpha_{1}$ protease inhibitor and $\alpha_{2}$ macroglobulin concentrations in the alveolar fluid were also increased as compared to controls at both 3 and 90 days following the last dust instillation. The data indicate a simultaneous increase in the elastolytic burden and 
Table 34. Petrologic components (macerals) in coal and their groupings

\begin{tabular}{ll}
\hline Maceral groupings & Macerals or components \\
\hline Vitrinite & telinite \\
collinite \\
resinite \\
cerinite \\
sporinite (exinite) \\
cutinite \\
suberinite \\
alginite \\
massive micrinite \\
granular micrinite \\
sclerotinite \\
semifusinite \\
fusinite
\end{tabular}

in the protease inhibitor activity of the alveolar fluid. Emphysema was not present by histologic study suggesting that no significant imbalance between proteolytic and anti-proteolytic forces occurred as a result of the coal dust treatments.

Fenton's method of very fine grinding of the coals, followed by fractionation in liquids of differing specific gravity, resulted in exinite fractions rich in sporinite. BENT and BROWN (1961) noted that an absorption band at about $1,700 \mathrm{~cm}^{-1}$, attributed to a carbonyl group, and an increase in nonaromatic $\mathrm{C}-\mathrm{H}$ absorption, were the only significant differences between the spectra of lower rank sporinites and the dominant maceral of normal coals, vitrinite. The occurrence of margins and larger areas of higher reflectance than the main mass of resinite globules has frequently been observed on polished surfaces of coal examined under oil immersion in incident light (MURCHISON 1963). These more highly reflecting regions have been attributed by STACH (1962) to the effects of oxidation on the resin before it was finally incorporated in the peat in a reducing environment.

In dusts isolated by EINBRoDT from the fibrotic lungs of coal miners ScHulz et al. (1995) found a positive correlation of the amount of inertinite to the stage of fibrosis.

Fusinite, a coal component discernible from semifusinite and other opaque ingredients by its strong reflecting power in reflected light was used as a electron paramagnetic resonance (EPR) oxygen probe. It is highly inert in biological systems (VAHIDI et al. 1994, SANTINI et al. 1998). This stability is very important since fusinite particles are randomly internalised in the cytoplasm and may remain there for long periods of time (PETRAKIS and GRANDY 1978). From the physical point of view, fusinite has an exchange-narrowed EPR spectrum which can be broadened by interaction with oxygen principally through the adsorption of molecules of this gas to the fusinite surface. The feasibility of using this EPR probe in measuring the intracellular molecular oxygen concentration in cultured cells has been demonstrated by VAHIDI et al. (1994) and SANTINI et al. $(1995,1996)$.

The question of the biological importance of substances leached from coals of different rank (gasflame coal to anthracite) was first put forward by Thaer (1952, 1953, 1955). Sorenson et al. (1974) analysed the concentrations of $\mathrm{Cd}, \mathrm{Cu}, \mathrm{Fe}$, $\mathrm{Ni}, \mathrm{Pb}$ and $\mathrm{Zn}$ in bituminous coals from mines with differing incidences of coal workers' pneumoconiosis. Rosmanith et al. (1977) thought that $\mathrm{Cd}, \mathrm{Pb}$ and other trace elements might be engaged in the fibrogenicity of mineral coal. Kober et al. (1976) found that humic substances leached from Pennsylvania and Utah coals with high and low pathogenicities, respectively, differed in the quantities of material removed and in their metal-binding and galactose oxidase (EC 1.1.3.9) inhibiting activities.

Polycyclic aromatic hydrocarbons contained in coal dust were not eluted by pig lung homogenates or human gastric juices, nor were they capable of crossing the skin barrier in vitro (FoÀ et al. 1998).

In U937 cells and human macrophages aryl hydrocarbon receptor, with its partner cofactor aryl hydrocarbon receptor nuclear translocator, was expressed and CYP1A1 mRNA expression was induced in the presence of aryl hydrocarbon receptor ligand 3-methylcholanthrene (KomURA et al. 2001).

Coal workers' pneumoconiosis is associated with increased basal and PMA-stimulated $\mathrm{O}_{2}{ }^{-2}$ formation in their alveolar macrophages in comparison to control subjects (WALLAERT et al. 1990). In 96 coke oven workers NADIF et al. (1997) found erythrocyte catalase activity significantly higher and erythrocyte glutathione peroxidase activity significantly lower than in 105 randomly recruited non-exposed workers from a power plant.

Differences in the cytotoxicity (triphenyltetrazolium chloride method) of coal dusts to guinea-pig macrophages depend on coal rank expressed as the percentage of volatile matter (REISNER and RoBock 1977). The Horst strata, with a mineral content of nearly $50 \%$ (by weight), caused a $35 \%$ de-

Table 35. Classification of coal by rank

\begin{tabular}{lll}
\hline & Volatile matter [\%] & Strata \\
\hline Flame coal & $>40$ & Dorsten \\
Gasflame coal & $40-35$ & Horst \\
Gas coals & $35-28$ & Essen \\
Bituminous coals & $28-19$ & Bochum \\
Steam coals & $19-12$ & Witten \\
Semi-anthracites & $12-10$ & Sprockhövel \\
Anthracites & $<10$ & \\
\hline
\end{tabular}


pression of the biological activity (TTC-RA $=65 \%$ ). Similar depressions were caused by upper Essen strata dusts with $33 \%$ mineral; by lower Essen and Bochum strata dusts (mineral $\cong 20 \%$ ) and by Witten strata dusts (mineral $\cong 15 \%)$.

As measured by electron spin resonance spectroscopy, a freshly ground anthracite coal ( $95 \%$ carbon) produced greater concentration of free radicals than a bituminous coal ( $72 \%$ carbon), and the radical reactivity was also greater for the anthracite (DALAL et al. 1989). The reactivity of the newly produced radicals in the anthracite dust correlated with the dust's toxicity. Furthermore, similar coal-based free radicals were detected in the lung tissue of autopsied coal miners, suggestive of persistent reactivity by the embedded coal dust leading to the progressive disease process.

In the rat, long-term clearance of low rank coal dust (Lea Hall, Staffordshire) tended to be greater than that of high rank (Ffeldau, South Wales), although a peculiar macrophage reaction to low rank dust may have affected its removal from the lung (Heppleston et al. 1971).

The particulate matter in the lungs of rats exposed to aerosols $\left(6.6 \pm 1.9 \mathrm{mg} / \mathrm{m}^{3}\right.$ and $14.9 \pm 6.2$ $\mathrm{mg} / \mathrm{m}^{3}$ ) of high-potential coal workers' pneumoconiosis bituminous coal dust from a mine in Appalachia for up to 20 months was contained almost entirely within the cytoplasm of alveolar macrophages so burdened with the material that cellular detail was obscured (Busch et al. 1981).

Interferon induction by influenza virus in monkey kidney $\left(\mathrm{LLC}-\mathrm{MK}_{2}\right.$ ) cell monolayers pre-treated with coal dust was inhibited in relation to coal rank (HAHON 1983). Maximal inhibition of viral interferon induction was noted with high rank coal and the degression of this activity was related to coal's position in the carboniferous series: anthracite, bituminous, subbituminous, lignite, and peat. Adsorption of poly(4-vinylpyridine- $N$-oxide) to bituminous coal dust from the Pittsburgh seam, Cambria County, Pa., not only occurred at a more rapid rate than to cell monolayers, but also less polymer was required to pretreat coal dust than cell monolayers to achieve comparable amelioration of interferon production (HAHON 1976).

The exact consequences of the cellular mechanisms that occur in the lungs of subjects chronically exposed to coal dust are still much of a puzzle (SCHINS and Borm 1999).

\subsection{1}

Cobalt

Cobalt has the atomic number 27 and the valence electrons are those of the $3 d^{7} 4 s^{2}$ shells. The oxidation states of cobalt are +2 and +3 .
Most complex compounds of cobalt are of oxidation state +3 and six-co-ordinate, or of oxidation state +2 and four- or six-co-ordinate. Five-coordinate complexes are the anions $\left[\mathrm{Co}(\mathrm{CN})_{5}\right]^{3-}$ and $\left[\mathrm{Co}(\mathrm{CN})_{5}\right]^{2-}$, and the oxygen-carrying Schiff's base compounds described by EARNSHAw et al. (1963). The readiness of cobalt to form five-co-ordinate compounds can be related to the tendency of the metal to achieve (cobalt (I), $3 d^{8}$ ) or approach (cobalt (II), $3 d^{7}$ ) the effective atomic number of the next inert gas (Nуноцм 1961). The correlation between the co-ordination number, stereochemistry, spectra, and magnetism of bivalent cobalt complexes has been widely investigated. The crystalfield stabilisation energy of the $d_{\gamma}^{4} d_{\varepsilon}^{3-}$ configuration is favourable for a tetrahedral arrangement and in many compounds of the type $\mathrm{MX}_{2}(\mathrm{Hal})_{2}$ where $\mathrm{X}$ can be, for example, pyridine (GILl and NyHolm 1961). In general, the ligands of high electronegativity, such as water, favour a co-ordination number of six, whereas those which are more easily polarised, such as the halide ions, give rise to fourco-ordinate tetrahedral complexes.

As models for natural oxygen carriers, many cobalt(II) complexes coordinated with nitrogen-bases have been shown to reversibly interact with molecular oxygen to form $\mathrm{CoO}_{2}$ or $\mathrm{Co}_{2} \mathrm{O}_{2}$ complexes depending upon the nature of the ligands.

HEARON (1947) showed that a co-ordination complex formed between histidine and cobaltous ion possesses the ability to combine reversibly with molecular oxygen. In contrast with this, histidine complexes with other transition metals tested $\left(\mathrm{Cu}^{2+}\right.$, $\mathrm{Cu}^{+}, \mathrm{Ni}^{2+}, \mathrm{Fe}^{2+}, \mathrm{Mn}^{2+}$ ) failed to exhibit the property of combining reversibly with oxygen gas. The $\mathrm{Co}^{2+}-$ histidine complex does not readily, if ever, become oxidised to $\mathrm{Co}^{3+}$ from the presence of $\mathrm{O}_{2}$ (HEAron 1948). Cobalt complexes of various histidine derivatives: histamine, 1-methyl histidine, 3-benzyl histidine, dibenzyl histidine, and the peptides anserine, carnosine, L-alanyl-L-histidine, and $\alpha$-L-aspartylD-histidine combined with $\mathrm{Co}^{2+}$ and the resulting complex was capable of carrying oxygen reversibly, although in certain cases (most notably carnosine) the irreversible reaction sets in exceedingly rapidly even at room temperature (HEARoN et al. 1949).

$\mathrm{Co}($ Salen) catalyses the oxygenation of 3-substituted indoles and flavonols via a proton transfer process, representing a model for tryptophan 2,3dioxygenase (EC 1.13.11.11) or indoleamine 2,3dioxygenase (heme enzyme) and quercetinase (EC 1.13.11.24; $\mathrm{Cu}$ enzyme). The 3-substituted indoles give formylkynurenine-type products (NishinaGA 1975, Nishinaga et al. 1981).

Phosphate buffer solutions of carnosine and anserine produced active oxygen species as measured 
by bleaching of $N, N$-dimethyl-4-nitrosoaniline (HARTMAN and Hartman 1992). The formation of active oxygen species appeared to depend predominantly on the presence of metal ions contaminating the phosphate buffer since prior Chelex treatment of buffer substantially reduced bleaching activity. Activity was restored by addition of micromolecular levels of $\mathrm{Cu}(\mathrm{I}), \mathrm{Cu}(\mathrm{II})$ and to a lesser extent, by $\mathrm{Co}$ (II) but not by addition of ferrous or ferric ions. Catalase eliminated most activity but superoxide dismutase had little effect.

$$
\begin{aligned}
& \text { One-electron transfer catalysed by cobalt } \\
& \begin{array}{l}
\mathrm{O}_{2}+\mathrm{Co}^{2+} \leftrightarrow \mathrm{Co}^{3+}-\mathrm{O}_{2}^{--} \rightarrow \mathrm{O}_{2}^{--}+\mathrm{Co}^{3+} \\
\mathrm{O}_{2}^{--}+\mathrm{Co}^{2+}+2 \mathrm{H}^{+} \longrightarrow \mathrm{H}_{2} \mathrm{O}_{2}+\mathrm{Co}^{3+} \\
\mathrm{H}_{2} \mathrm{O}_{2}+\mathrm{Co}^{2+} \longrightarrow \mathrm{HO}^{\circ}+\mathrm{HO}^{-}+\mathrm{Co}^{3+}
\end{array}
\end{aligned}
$$

Cobalt(II) reacts with $\mathrm{H}_{2} \mathrm{O}_{2}$, to produce $\mathrm{HO}^{\circ},{ }^{1} \mathrm{O}_{2}$, and metal-oxygen complexes, which cause sitespecific DNA damage (ҮАмамото et al. 1989, KAWANISHI et al. 1994).

Though essential in forming cobalamin (vitamin $B_{12}$ ), cobalt is an industrial poison taken up from the catalyst in the Fischer-Tropsch synthesis (FISCHER and PiCHLER 1936) of benzene and in the sintered carbide metal industry (SCHILlER 1958, 1961, BeCH et al. 1962, KüHNe 1962, 1965, CoATES and Watson 1971, Hartung et al. 1982, HillerDal and Hartung 1983, Figueroa et al. 1992, Lison 1996). When both cobalt and tungsten carbide particles are associated, electrons provided by cobalt metal are easily transferred to the surface of the carbide particles where reduction of oxygen can occur at a rate greatly increased (Lison et al. 1995). Further, cobalt compounds are used as dryers in paints (e.g. cobalt linoleates), and as pigments in glass and pottering industries.

$\mathrm{Co}^{2}$ is effective in stimulating cyclic GMP dependent protein kinase from a variety of sources, with optimal concentration of $0.5 \mathrm{mM}$, equivalent to a $\mathrm{K}_{\mathrm{m}}$ of about $0.1 \mathrm{mM}$ (Kuo and SHOJ 1982). The maximal activity stimulated by $\mathrm{Co}^{2+}$ is about $40 \%-80 \%$ of that by $\mathrm{Mg}^{2+}$ for the mammalian cGMP dependent protein kinase (Kuo et al. 1976, SHoji et al. $1977 \mathrm{a}, \mathrm{b}) . \mathrm{Co}^{2+}$ stimulates mammalian cAMP dependent protein kinase to a greater extent than does $\mathrm{Mg}^{2+}$ (Kuo et al. 1970). $5 \mathrm{mM} \mathrm{Co}^{2+}$ almost completely inhibits the activity of bovine lung cGMP stimulated protein kinase stimulated by 10 $\mathrm{mM} \mathrm{Mg}^{2+}$ (GiLl et al. 1977).

Alveolar macrophages of rabbits exposed for 4 months (5 days/week, $6 \mathrm{~h} /$ day) to $0.6 \pm 0.5 \mathrm{mg} / \mathrm{m}^{3}$ $\mathrm{Co}^{2+}$ as $\mathrm{CoCl}_{2}$, had significantly more laminated, surfactant-like inclusion, compared with the controls (Johansson et al. 1992).
The carbonyls of cobalt, like other metal carbonyls [see $\mathrm{Ni}(\mathrm{CO})_{4}$ ], are the most toxic cobalt compounds. $\mathrm{Co}_{2}(\mathrm{CO})_{8}, \mathrm{Co}_{4}(\mathrm{CO})_{12}$ and $\mathrm{Co}_{6}(\mathrm{CO})_{16}$ are solid substances which are prepared from cobalt and carbon monoxide using elevated temperature and pressure.

Inhalation of cobalt hydrocarbonyl, $\mathrm{HOCo}(\mathrm{CO})_{4}$, for 30 min established a $\mathrm{LD}_{50}$ of $165 \mathrm{mg} \mathrm{Co} / \mathrm{m}^{3}$ (PALMEs et al. 1959).

\section{Cobalt-Nickel Alloy}

The toxicity of cobalt and cobalt-nickel alloys in experimental hard metal pneumoconiosis is well documented. Metallic cobalt powder instilled intratracheally in the lungs of rats had an acute irritant action and lead to severe alterations in capillaries (HARDing 1950, SCHILler 1958, 1961). The solubility of cobalt in plasma is some 500 times greater than in saline, but a fairly large $(10 \mathrm{ml})$ intraperitoneal dose of plasma saturated with cobalt was without evident effects on the rat (HARDING 1950).

In rabbits exposed to soluble cobalt $0.4-2 \mathrm{mg} / \mathrm{m}^{3}$ $\mathrm{CoCl}_{2}$ for 1-4 months, the number of alveolar macrophages was increased and some of them, close to the type II cell nodules, were enlarged and filled with laminated, surfactant like inclusions (JoHANsson et al. 1983, 1986). In lavage fluids the number of macrophages and the percentage of these cells with smooth surface and intracellular surfactantlike inclusions were more increased in group $\mathrm{Co}^{2+}+$ $\mathrm{Cr}^{3+}$ than in group $\mathrm{Co}^{2+}$ as were oxidative metabolic and phagocytic activities of the macrophages (JoHANSSON et al. 1992).

In hard-metal workers, giant cells have been found in the lung lavage (DAVIDSON et al. 1983, TABATOWSKI et al. 1988).

\subsection{2 \\ Copper}

Copper has the atomic number 29 and the valence electrons are those on the $3 d^{10} 4 s^{1}$ shells. Its oxidation states are +1 and +2 . The difference of $1 \mathrm{e}^{-}$in the $3 d$ shells of cuprous and cupric ions allow a participation in radical reactions. Copper does not really fit the definition of a transition element since its $3 d-$ orbitals are full, but it readily forms the $\mathrm{Cu}^{2+}$ ion by loss of two electrons, one from the $4 s$ - and one from the $3 d$-orbital. This leaves an unpaired electron.

$$
\begin{aligned}
& \text { One-electron transfer catalysed by copper } \\
& \qquad \begin{array}{l}
\mathrm{O}_{2}+\mathrm{Cu}^{+} \rightarrow \mathrm{O}_{2}^{-}+\mathrm{Cu}^{2+} \\
\mathrm{O}_{2}^{-}+\mathrm{Cu}^{+}+2 \mathrm{H}^{+} \rightarrow \mathrm{H}_{2} \mathrm{O}_{2}+\mathrm{Cu}^{2+} \\
\mathrm{H}_{2} \mathrm{O}_{2}+\mathrm{Cu}^{+} \rightarrow \mathrm{HO}^{-}+\mathrm{HO}^{-}+\mathrm{Co}^{2+}
\end{array}
\end{aligned}
$$


Fig. 139. Alveolar macrophage with a tubular invagination from a $220 \mathrm{~g}$ female white rat (No. 1 breeder: Winkelmann, BorchenKirchborchen) which inhaled $10 \mathrm{mg}$ of a powdered cobalt-nickel alloy $/ \mathrm{m}^{3}$, used in the production of hard metal by Deutsche Edelstahlwerke in Krefeld, $4 \mathrm{~h}$ per day, 5 times per week from October 2-28, 1967 for a total of 19 days. Fixed on October 30, 1967 under methitural anaesthesia by intratracheal instillation of $2.5 \%$ glutaraldehyde in phosphate buffer ( $\mathrm{pH}$ 7.4) before opening the thorax. Postfixation with $1 \%$ osmium tetroxide in phosphate buffer ( $\mathrm{pH}$ 7.4). Contrasted en bloc for $12 \mathrm{~h}$ with $0.5 \%$ uranyl acetate in $70 \%$ ethanol. Embedded in a 2:8 mixture of methyl and butyl methacrylate. Sectioned at $50 \mathrm{~nm}$. Lead citrate after REYNOLDS (1963). Plate $16 / 01$

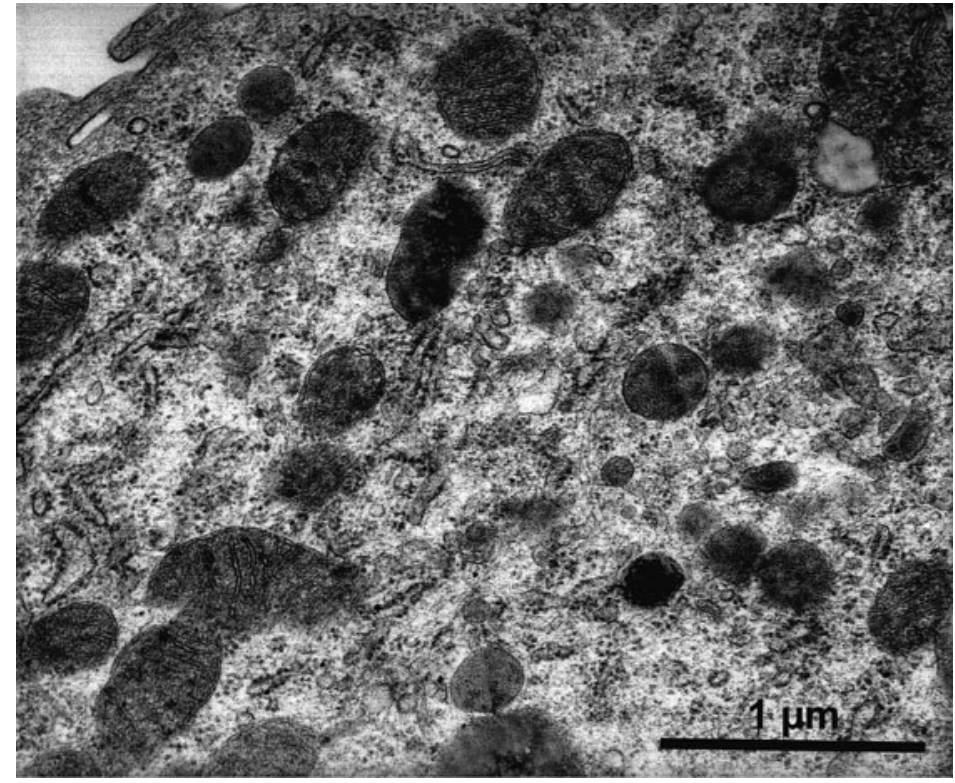

As with iron, copper serves to convert superoxide $\left(\mathrm{O}_{2}{ }^{\circ-}\right)$ and hydrogen peroxide, formed during the metabolism of oxygen (particularly in the presence of redox-cycling xenobiotics; RUMYANTSEva and WEINER 1988, MASON 1990) to the highly reactive

Fig. 140. Phagophore (arrow) in an alveolar macrophage from a $225 \mathrm{~g}$ female white rat (breeder: Winkelmann, Borchen-Kirchborchen) which inhaled $10 \mathrm{mg}$ of a powdered cobalt-nickel alloy $/ \mathrm{m}^{3}$, used in the production of hard metal by Deutsche Edelstahlwerke in Krefeld, 4 h per day, 5 times per week from October 2-28, 1967 for a total of 19 days. Fixed on October 30, 1967 under methitural anaesthesia by intratracheal instillation of $2.5 \%$ glutaraldehyde in phosphate buffer ( $\mathrm{pH} 7.4)$ before opening the thorax. Postfixation with $1 \%$ osmium tetroxide in phosphate buffer $(\mathrm{pH}$ 7.4). Contrasted en bloc for $12 \mathrm{~h}$ with $0.5 \%$ uranyl acetate in $70 \%$ ethanol. Embedded in a 2:8 mixture of methyl and butyl methacrylate. Sectioned at $50 \mathrm{~nm}$. Lead citrate after REYNOLDS (1963). Plate 35/11 hydroxyl radical (equations [181]-[183]), which is considered to be the oxidising species responsible for the induction of biomolecular damage.

Copper-dependent hydroxyl radical formation has been demonstrated in many model systems,

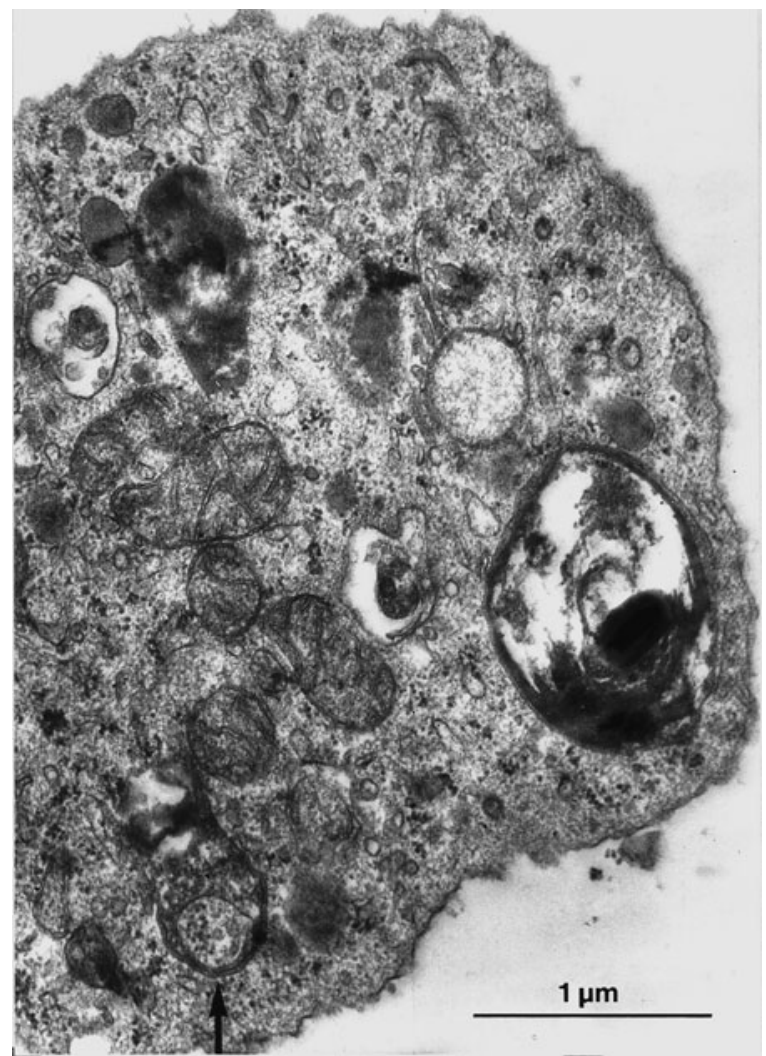


generally via the application of standard methods of $\mathrm{HO}^{\circ}$ determination. VAN STEVENINCK et al. (1985) have used salicylate hydroxylation in systems containing $\mathrm{H}_{2} \mathrm{O}_{2}, \mathrm{Cu}^{\mathrm{II}}$ and reducing agents (equation [184]). However, the most direct method of hydroxyl radical detection is electron spin resonance (ESR) spectroscopy in conjunction with a spin trapping reagent (BUETTNER and MAson 1990, MAsON et al. 1994). The $\mathrm{HO}^{\circ}$ radical was detected as its adduct to the spin trap 5,5-dimethyl-1-pyrroline $\mathrm{N}$-oxide (DMPO) (equation [8]).

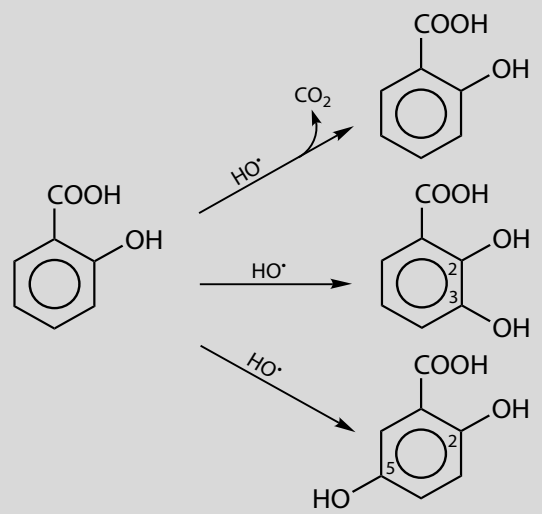

Salicylate hydroxylation by $\mathrm{HO}^{\circ}$

[184]

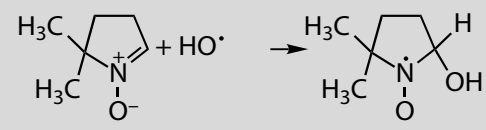

5,5-Dimethyl-1-pyrroline-1-oxide-HO` adduct

The possible involvement of $\mathrm{Cu}(\mathrm{III}), \mathrm{Fe}(\mathrm{IV})$, or $\mathrm{Mn}(\mathrm{III})$ in the mechanism of the toxicity of $\mathrm{O}_{2}{ }^{-}$is not surprising as the redox potential of the couple $\mathrm{O}_{2}{ }^{-} / \mathrm{H}_{2} \mathrm{O}_{2}$ at $\mathrm{pH} 7.4$ is $0.94 \mathrm{~V}$ (Koppenol and ButLER 1985), which implies a strong oxidising capability for $\mathrm{O}_{2}{ }^{\circ-}$ (GoldsTEIN and CzAPSKI 1990). However, there is no direct evidence for the formation of a $\mathrm{Cu}^{\mathrm{III}}$ species obtainable from electron spin resonance spin trapping studies (BURKITT et al. 1995).

Tetrakis- $\mu$-3,5-diisopropylsalicylatodiaquodicopper(II) is known to disproportionate $\mathrm{O}_{2}{ }^{-{ }^{-}}$at the same rate as superoxide dismutase (SORENSEN 1989).

Copper bound to 1,10-phenanthroline was found to be a good catalyst of hydroxyl radical formation: the $\mathrm{Cu}^{\mathrm{II}}(1,10 \text {-phenanthroline) })_{2}$ complex can be reduced by $\mathrm{H}_{2} \mathrm{O}_{2}$ and the $\mathrm{Cu}^{\mathrm{I}}$ (1,10-phenanthroline $)_{2}$ generated reacts with the peroxide to form $\mathrm{HO}^{\circ}$.<smiles>c1cnc2c(c1)ccc1cccnc12</smiles>

1,10-Phenanthroline

The rate constant for the Fenton reaction is higher for copper than for iron (Halliwell and GutTeRIDGE 1989).

2,9-Dimethyl-1,10-phenanthroline $(10 \mu \mathrm{M})$ significantly increased the inhibitory effects of both $\mathrm{CuSO}_{4}$ and $\mathrm{Cu}(2,9-\text { dimethyl-1,10-phenanthroline })_{2}$ $\mathrm{NO}_{3}$ on NADH oxidase activity (SMit et al. 1982).

A transition complex with triplet molecular oxygen may also be formed:

$$
\mathrm{Cu}^{+}+{ }^{3} \mathrm{Sg}^{-} \mathrm{O}_{2} \rightarrow\left(\mathrm{Cu}^{2+}-\mathrm{O}_{2}^{-}\right)
$$

Copper and its co-ordination compounds are known to produce severe cytotoxicity (FreEDMAN et al. 1986, BYrnes et al. 1990).

Tetrahedral co-ordination dominates with two and three co-ordination much less common. The co-ordination number rarely exceeds four although five co-ordination is known (GAGNÉ 1976).

In solutions of $\mathrm{CuCl}_{2}$ and adenine copper can be bound to adenine. Two $\mathrm{Cu}$ (adenine) ${ }_{2}$ complexes $\left.\left[\mathrm{Cu}\left(\mathrm{C}_{5} \mathrm{H}_{4} \mathrm{~N}_{5}\right)_{2}\right]^{2+} / \mathrm{Cu}\left(\mathrm{C}_{5} \mathrm{H}_{4} \mathrm{~N}_{2}\right)_{2}\right)$ are in equilibrium with free adenine (BRUston et al. 1999). Copperadenine complexes present a catalytic activity (e.g., $\mathrm{H}_{2} \mathrm{O}_{2}$ disproportionation into $\mathrm{O}_{2}$ and water) but depending on complex concentration $\mathrm{H}_{2} \mathrm{O}_{2}$ also strongly oxidises the adenine within the complexes. Raman spectroscopy quantifies copper-adenine complex formation and $\mathrm{H}_{2} \mathrm{O}_{2}$ consumption.; polarography quantifies $\mathrm{O}_{2}$ production. As for $\mathrm{C}_{40}$ catalase, optimal catalytic capacities depend on physiological conditions, such as $\mathrm{pH}$ and temperature. The comparative analysis of kinetic parameters shows that the affinity for $\mathrm{H}_{2} \mathrm{O}_{2}$ of $\mathrm{Cu}$ (adenine) $)_{2}$ is 37-fold lower than that of $\mathrm{C}_{40}$ catalase and that the molar activity for $\mathrm{O}_{2}$ production is 200 -fold weaker for $\mathrm{Cu}$ (adenine) $)_{2}$ than for the enzyme. In the $10^{-6}$ to $10^{-3} \mathrm{M}$ range, the strong decrease of activity with raising complex concentration is explained by aggregation or stacking, which protects $\mathrm{Cu}$ (adenine) ${ }_{2}$ entities from $\mathrm{H}_{2} \mathrm{O}_{2}$ oxidation, but also decreases $\mathrm{O}_{2}$ production.

Glutathione and $\mathrm{Cu}^{2+}$ form several complexes (PETIT et al. 1975). Reduction by glutathione triggered a $N, N^{\prime}$-bis(2-pyridylmethylene)-1,4-butanediamine $\left(N, N^{\prime}, N^{\prime \prime}, N^{\prime \prime}\right)-\mathrm{Cu}(\mathrm{II})$ diperchlorate-supported redox cycle with oxygen yielding $\mathrm{H}_{2} \mathrm{O}_{2}$ (STEINKÜHLER et al. 1991). Whereas reduction by ascorbate was reversible.

Under physiological conditions of $\mathrm{pH}$, peptide (class A) chelates are only formed by those bidentate amide ligands with $\mathrm{X}$ being either an imidazole $\left(\mathrm{sp}^{2}\right)$ nitrogen or a terminal $\left(\mathrm{sp}^{3}\right)$ amino nitrogen. Mercaptide sulphur must also be considered to belong to this group of strong copper(II) binding sites. 
The apolipoprotein B of low-density lipoprotein has two sulphydryl groups exposed to its surface and they could play a role in the reductive activation of the transition metal ions, e.g. $\mathrm{Cu}^{2+}$. Once bound, $\mathrm{Cu}^{2+}$ must be reductively activated by a net transfer of one electron:

$$
\mathrm{Cu}^{2+}+\mathrm{e}^{-} \rightarrow \mathrm{Cu}^{+}
$$

The formation of hydroxyl radicals $\left(\mathrm{HO}^{\circ}\right)$ by the reaction of the $\mathrm{Cu}$ (II) complex of ethylenediamine tetraacetic acid (EDTA) with $\mathrm{H}_{2} \mathrm{O}_{2}$ in the presence of biological reductants, such as L-ascorbic acid and Lcysteine, has been demonstrated by ESR spectroscopy using water-soluble spin-traps (OzAWA et al. 1992).<smiles>O=C([O-])CN(CCN(CC(=O)[O-])CC(=O)[O-])CC(=O)[O-]</smiles>

Ethylenediamine tetraacetic acid (EDTA)
[187]

Thiourea (1-10 $\mathrm{mM}$ ) provided marked and dosedependent protection against protein oxidation in three copper-containing systems: $\mathrm{Cu}(\mathrm{II}) /$ ascorbate, $\mathrm{Cu}(\mathrm{II}) / \mathrm{H}_{2} \mathrm{O}_{2}$, and $\mathrm{Cu}(\mathrm{II}) / \mathrm{H}_{2} \mathrm{O}_{2}$ /ascorbate [Cu(II), 0.1 $\mathrm{mM}$; ascorbate, $1 \mathrm{mM} ; \mathrm{H}_{2} \mathrm{O}_{2} ; 1 \mathrm{mM}$ ) (ZHU et al. 2002). In contrast, only minor protection was observed with dimethyl sulphoxide and mannitol, even at concentrations as high as $100 \mathrm{mM}$. Strong protection was also observed with dimethylthiourea, but not with urea or dimethylurea. Thiourea also significantly inhibited copper-catalysed oxidation of ascorbate, and competed effectively with histidine and 1,10-phenanthroline for binding of cuprous, but not cupric, copper, as demonstrated by both UV-visible and low temperature electron spin resonance measurements.

Both phospholipid-containing delipidated $h u-$ man low-density lipoprotein ghosts and trilinoleinreconstituted low-density lipoprotein were devoid of antioxidants and were extremely susceptible to 2,2'-azobis-(2-amidino propane) hydrochlorideinduced oxidation but, paradoxically, were rather resistant to copper-mediated oxidation (VISIOLI et al. 2000). The dynamic reduction of $\mathrm{Cu}(\mathrm{II})$ to $\mathrm{Cu}(\mathrm{I})$ was quantitatively decreased in low-density lipoprotein ghosts and in trilinolein-reconstituted lowdensity lipoprotein, also lacking the initial rapid reduction and the subsequent inhibition phases, due to the absence of endogenous antioxidants. Conversely, the rate of copper reduction was linear and likely due to lipid peroxides, either already present of formed during copper-induced oxidation.

Ternary copper(II) complexes constituted by a 1,10-phenanthroline and an amino acid as ligands accelerated both the decomposition of peroxynitrite and its nitration of 4-hydroxyphenylacetic acid at $\mathrm{pH}>7$ (FerRer-SuEta et al. 1997). The enhancing effect of $\mathrm{Cu}^{2+}$ on the decomposition of $\mathrm{N}$-ethyl- $\mathrm{N}$ nitrosourea (Preussmann et al. 1975) can be inhibited by complex formation with ethylenediamine tetraacetic acid (ZELLer and IVANCovic 1972).

The formation of oxidising species from bolus $\mathrm{H}_{2} \mathrm{O}_{2}$ and cupric ions was found to be profoundly dependent on the choice of buffer. In phosphate and cacodylate buffers, as well as unbuffered water, SANDSTRÖM et al. (1994) found barely detectable amounts of methane sulphinic acid. However, in Hepes buffer they could detect a strong formation of methane sulfinic acid. The addition of 2-[(2bis[carboxymethyl]amino-5-methylphenoxy)methyl]-6-methoxy-8-bis[carboxymethyl]-

aminoquinolin (quin2) inhibited $97 \%$ of the methane sulfinic acid formation. With the deoxyribose assay, a significant 2-thiobarbituric acid reaction was found in all media tested, the strongest in cacodylate buffer and water, and weaker in Hepes and phosphate. In this assay quin 2 inhibited the formation of oxidising species by $65 \%$ in phosphate buffer, $85 \%$ in water and more than $95 \%$ in both Hepes and cacodylate buffers.

$\mathrm{Cu}^{2+}(2-10 \mu \mathrm{M})$ catalysed the conversion of xanthine dehydrogenase (EC 1.1.1.204) to xanthine oxidase (EC 1.1.3.22) which was prevented by oleic acid, arachidonic acid, eicosapentaenoic acid and docosahexaenoic acid (50-200 $\mu \mathrm{M})$ as shown in the perfused rabbit liver (Fujita et al. 1995).

The contribution of Esterbauer and RAMOS (1996) on the chemistry and pathophysiology of oxidation of low-density lipoprotein focuses mainly on in vitro oxidation of $\mathrm{LDL}$ by $\mathrm{Cu}^{2+}$.

LDL was able to reduce $\mathrm{Cu}^{2+}$ to $\mathrm{Cu}^{+}$in a time and concentration-dependent manner (PROUdFOoT et al. 1997a). Blocking of free $-\mathrm{SH}$ groups on LDL apoprotein B by preincubation with dithionitrobenzoic acid had no significant effect on the rate and extent of $\mathrm{Cu}^{2+}$ reduction. Consumption of tocopherol in LDL undergoing oxidation with $\mathrm{Cu}$ was very rapid (rate $=6 \times 10^{-10} \mathrm{M} \mathrm{s}^{-1}$ ). When $\mathrm{Cu}^{+}$formed during incubation with LDL was complexed with neocuproine, there was significant inhibition of LDL oxidation, as indicated by lipid peroxide formation and mobility on agarose gel electrophoresis. Tocopherol consumption was even more rapid in the presence of neocuproine, consistent with a shift in $\mathrm{Cu}^{2+} / \mathrm{Cu}^{+}$equilibrium and faster reduction of $\mathrm{Cu}^{2+}$ by $\alpha$-tocopherol.

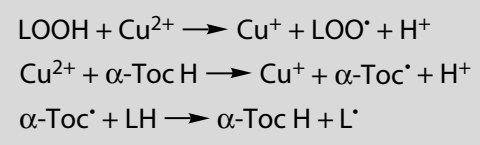


When oxidising isolated LDL, there was a decrease in lag time with increasing concentration of $\mathrm{Cu}^{2+}$ until a minimum "lag time" was reached at a $\mathrm{Cu}: \mathrm{LDL}$ ratio of about 50:1 (Proudfoot et al. 1997b). In serum, an initial decrease in "lag time" occurred with increasing $\mathrm{Cu}$ concentrations up to $12.5 \mu \mathrm{M}$. However, with higher $\mathrm{Cu}$ concentrations "lag time" to oxidation increased, contrary to expectation, until a maximum was reached at about $50 \mu \mathrm{M} \mathrm{Cu}$. This dose response observed for $\mathrm{Cu}$ oxidation of diluted serum was highly reproducible in a number of individual subjects. When serum was gel-filtered to remove low molecular weight compounds, the resulting filtrate behaved the same as isolated LDL. Uric acid was found to be an important component of the low molecular weight fraction for the paradoxical effect of $\mathrm{Cu}$ concentration on serum oxidation. The same paradoxical effect was found when isolated LDL was incubated with uric acid in the presence of human serum albumin and $\mathrm{Cu}$. The incubation of human serum albumin with reducing agents such as uric acid or bilirubin in the presence of high $\mathrm{Cu}$ concentration, produced a "peroxidase-like" activity, capable of breaking down hydrogen peroxide as well as lipid hydroperoxides.

The oxidation of low-density lipoprotein by mouse resident peritoneal macrophages or that initiated by $\mathrm{Cu}^{2+}$ ions was inhibited by SB209995, a metabolite of carvedilol in human, with $\mathrm{IC}_{50} \mathrm{~s}$ of 59 $\mathrm{nmol} / \mathrm{l}$ and $1.7 \mu \mathrm{mol} / \mathrm{l}$, respectively (FEUERSTEIN and YuE 1994). Under the same conditions, the $\mathrm{IC}_{50} \mathrm{~S}$ of carvedilol were 3.8 and $17.1 \mu \mathrm{mol} / \mathrm{l}$, respectively.

There are at least two pathways by which copper is transported across the cell membrane (DIDoNATO and SARKAR 1997). Ceruloplasmin, the most abundant copper-protein in plasma, can contribute copper to cells (HSIE and Frieden 1975, CAMPBELL et al. 1981, Dameron and Harris 1987, MAS and SAKAR 1992, SAENKo et al. 1994). Studies of ceruloplasmin-mediated copper transport in cells have shown that copper derived from ceruloplasmin enters the cell but the protein does not (PERCIVAL and HARRIS 1990). Stimulation of copper uptake by ascorbate and the inhibition of the process by cuprous chelators suggest that copper takes place in the form of $\mathrm{Cu}(\mathrm{I})$ rather than $\mathrm{Cu}(\mathrm{II})$ (Percival and HARRIS 1989, HARRIS 1991).

Ceruloplasmin is a plasma glyoprotein containing about seven $\mathrm{Cu}$ atoms per molecule and $\approx 95 \%$ of the total plasma Cu (Rydén 1984, Fox et al. 1995). It is a $132-\mathrm{kDa}$ monomer comprised almost entirely of three major domains that have $35-40 \%$ homology to each other (OrTEL et al. 1984) and to three homologous domains in factors Va and VIIIa
(CHuRch et al. 1984). The prooxidant site was localised to a region containing $\mathrm{His}_{426}$ because ceruloplasmin $_{\text {His426A }}$ almost completely lacked prooxidant activity whereas the other mutants expressed normal activity (MuKHOPADHYAY et al. 1997).

Copper is distributed in all cellular organelles including the nucleus, mitochondria, lysosomes, endoplasmic reticulum and cytosol (LINDER 1991). LINDQUIST (1968) presented biochemical and electron microscopic evidence that $\mathrm{CuCl}_{2}$ injected intraperitoneally into rats was sequestered by liver lysosomes and initiated lipid peroxidation of lysosomal membranes with resulting lysosomal rupture and release of hydrolytic enzymes. Glutathione has been implicated as the main chelator of copper in the cytoplasm. In copper-loaded hepatoma cells, over $60 \%$ of cytosolic copper exists as a $\mathrm{Cu}(\mathrm{I})-\mathrm{GSH}$ complex (FrEedMAN et al. 1989). ${ }^{1} \mathrm{H}$ NMR of copper(II) complexes of adenosine (2') (3') (5')-monophosphates have been compared with the spectra of adenosine $2^{\prime}, 3^{\prime}-$ and $3^{\prime}, 5^{\prime}$-cyclic phosphates. A comparison of the chemical shifts and broadening effects with varying copper(II) concentrations suggested that greater base stacking and no phosphate-base deshielding occurred in the cyclic nucleotides relative to the nucleoside monophosphates (BERGER and EICHHORN 1971). Activation of guanylate cyclase in a postmitochondrial supernatant fraction of rat lung homogenates was found to be dependent on temperature, $\mathrm{Cu}^{2+}$, and the presence of oxygen (White et al. 1976). The activation was inhibited by thiols, bovine serum albumin, $\mathrm{KCN}$, and sodium diethyldithiocarbamate, a $\mathrm{Cu}^{2+}$ chelator.

Viral interferon induction was inhibited in rhesus monkey kidney (LLC-MK ${ }_{2}$ ) cells treated with 0.1 mg copper at $49.4 \%$ (HAHON et al. 1980). Interferon yields were maximally inhibited by the smallest particles $(1 \mu \mathrm{m})$. Particles of increased size $(10 \mu \mathrm{m})$ were progressively ineffectual in depressing interferon production.

Alveolar macrophages from male SpragueDawley rats instilled intratracheally with $5 \mathrm{mg} \mathrm{CuO}$ suspended in $0.5 \mathrm{ml}$ saline one week before showed significant increase in size and contained numerous vacuoles (MURTHY et al. 1982). Their polymorphic nuclei with margination of the chromatin and well developed nucleoli were eccentrically placed. Lipid droplets in the cytoplasm were numerous, and often appeared to fuse to form large vacuoles. Other cytoplasmic constituents included numerous bizarre-shaped membranous bodies, myelin figures, and crystalloid-like inclusions. The mitochondria appeared smaller when compared with those of the control animals. The plasmalemma displayed considerable undulations and numerous cytoplasmic processes. 


\section{Copper as a DNA-Cleaving Agent}

By the change of valences, copper salts can act as a Fenton reagent in the presence of hyrogen peroxide (von SonNTAG 1987). $\mathrm{Cu}^{2+}$ and $\mathrm{H}_{2} \mathrm{O}_{2}$ induced strong cleavage of both calf thymus DNA and mammalian chromatin at quite low concentrations $\left(10^{-6}\right.$ and $10^{-5} \mathrm{M}$ ) (SAgRipanti and Kraemer 1989, YAMAMOTO and KAWANISHI 1989) in a reaction that appears to involve $\mathrm{Cu}^{2+}$. In contrast to studies with iron ions, complexation of copper with either nitrilotriacetate or with $N, N, N^{\prime}, N^{\prime}$-ethylenediaminetetraacetic acid inhibits the damage to DNA bases. $\mathrm{Cu}^{2+}$ can bind to the DNA (ҮАмамото and KAWANISHI 1989), but whether the mechanism goes via the hydroxyl radical (SAGRIPANTI and KRAEMER 1989) or some sort of metal bound complex (ҮАмамото and KaWANISHI 1989) is uncertain. Studies directly on $\mathrm{Cu}^{+} / \mathrm{H}_{2} \mathrm{O}_{2}$ suggested that free hydroxyl radicals are not formed but may remain bound to the copper (Johnson et al. 1985). Drouin et al. (1996) induced base modifications by $\mathrm{Cu}(\mathrm{II}) /$ ascorbate $/ \mathrm{H}_{2} \mathrm{O}_{2}$ distinguishing base damage from frank strand break. Modified base production predicted by computer simulation at an initial $\mathrm{Cu}$ (II) concentration of 50-70 $\mu \mathrm{M}$ was experimentally validated. The copper ion binding sites on DNA were saturated at $50 \mu \mathrm{M}$ bound copper ion, or when $\approx 40 \%$ of the DNA phosphates were occupied. The $\mathrm{k}_{\text {app }}$ indicated that DNA base damage occurred slowly in relation to the rate of DNA-Cu(I) oxidation (Stoewe and Prutz 1987).

$$
\begin{aligned}
& \mathrm{DNA}-\mathrm{Cu}^{2+}+\mathrm{Asc}^{2-} \rightarrow \mathrm{DNA}-\mathrm{Cu}^{+}+\mathrm{Asc}^{--} \\
& \mathrm{DNA}-\mathrm{Cu}^{+}+\mathrm{H}_{2} \mathrm{O}_{2} \rightarrow \mathrm{DNA}-\mathrm{Cu}^{2+}+\mathrm{OH}^{-}+\mathrm{HO}^{\circ}
\end{aligned}
$$

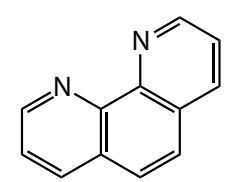

[137]
1,10-Phenanthroline

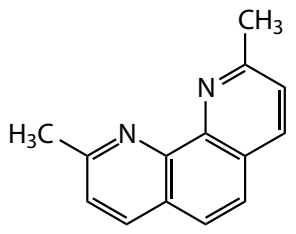

2,9-Dimethyl-1,10-phenanthroline (neocuproine)
Although 1,10-phenanthroline and 2,9-dimethyl1,10-phenanthroline (neocuproine) will each chelate both copper and iron, the effect on the redox chemistry of the two metal ions are distinct: $\mathrm{Cu}(\mathrm{I})$ chelated to 1,10-phenanthroline reacts readily with $\mathrm{H}_{2} \mathrm{O}_{2}$ to form HO (Goldstein and Czapski 1986), whereas reaction of the $\mathrm{Fe}(\mathrm{II})$ complex is inhibited (Mello-Filho and Meneghini 1991). Neocuproine inhibits the reaction of $\mathrm{Cu}(\mathrm{I})$ with $\mathrm{H}_{2} \mathrm{O}_{2}$ (CZAPSKI and GolDSTEIN 1986) but appears to have little effect of Fe(II) (Mello-Filho and Meneghini 1991).

Copper-thiosemicarbazide complexes interact with both the bases and the phosphate groups of native calf thymus DNA (Pillai et al. 1977).

\section{Malignancies in Copper Miners and Smelters}

In the Mansfield copper-bearing shales (Kupferschiefer) miners' pneumoconiosis WäTJEN (1933) did not find any malignancy in 54 necropsies. In 1936, under additional 65 cases WäTJEN detected three primary bronchial carcinomas: two miners had severe pneumoconioses after 41 and 43 years of work underground, respectively, the third miner, who had worked underground for 43 years had a slight degree of pneumoconiosis.

Epidermoid or squamous cell carcinoma of the lung has been found to be predominant among copper smelter workers (New MAN et al. 1976, AxELson et al. 1978). Of 71 consecutive occupational lung cancers among male Japanese copper smelter workers Kreyberg group I (squamous, large cell, and small cell carcinomas) was prevalent, which could not be explained by age, sex, ethnicity, air pollution, or smoking per se (ToKudome et al. 1988).

Copper fume is likely to provide the principal potential health hazard in the arc-air gouging process (SANDERSON 1968).

\section{Copper Gallium Diselenide and Copper Indium Diselenide}

Copper gallium diselenide $\left(\mathrm{CuGaSe}_{2}\right)$ and copper indium diselenide $\left(\mathrm{CuInSe}_{2}\right)$ particles (mean diameters $2.47 \mu \mathrm{m}$ and $2.55 \mu \mathrm{m}$, respectively) suspended

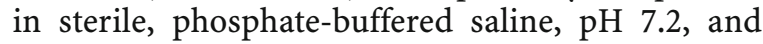
instilled intratracheally into specific pathogen-free female Sprague-Dawley rats at doses at doses of $12 \mathrm{mg}, 24 \mathrm{mg}, 48 \mathrm{mg}$, or $96 \mathrm{mg} / \mathrm{kg}$ body weight after 3 days were found within the cytoplasma of macrophages (Morgan et al. 1995).

\subsection{3 \\ Gallium}

Gallium has the atomic number 31 and the valence electrons are those on the $4 p^{1}$ shell. Its only oxidation state is +3 .

In two patients inhaling an aerosol containing ${ }^{67} \mathrm{Ga}$ citrate $24 \mathrm{~h}$ before resection surgery KenNedY et al. (1985) found the nuclide only in alveolar macrophages but not in epithelial cells and interstitial mononuclear phagocytes.

Pulmonary lesion of rats exposed for 4 weeks ( $2 \mathrm{~h} / \mathrm{d}, 5 \mathrm{~d} /$ week) to $27 \mathrm{mg} \mathrm{Ga} \mathrm{O}_{3} / \mathrm{m}^{3}$ (particle size 
$220 \mathrm{~nm}) 1$ day thereafter consisted primarily of accumulations of proteinaceous debris in the alveoli (alveolar proteinosis) and a slight increase in the number of alveolar macrophages and neutrophils in alveolar air spaces within or adjacent to alveolar ducts. In the rats sacrificed at 6 months after exposure, several large foci of alveolar macrophages were scattered throughout the parenchyma, but most often centred within centriacinal regions or subpleural locations. In these areas, the macrophages were large and markedly vacuolated (WoLFF et al. 1989).

After intratracheal instillation into rats, $\mathrm{Ga}_{2} \mathrm{O}_{3}$ particles were being actively phagocytized while nodules of macrophages and $\mathrm{Ga}_{2} \mathrm{O}_{3}$ were found adhered to the alveolar walls (Wевв et al. 1986).

\subsection{4}

\section{Germanium}

Germanium has the atomic number 32 and the valence electrons are those on the $4 p^{2}$ shell. Its only oxidation state is +4 .

$\mathrm{GeO}_{2}$ occurs in three modifications: one is isostructural to $\alpha$-quartz, the other to cristobalite (Вӧнм 1968), and the third isostructural to rutile. The rutile form was prepared at $5 \times 10^{9} \mathrm{~Pa}$ and $1000^{\circ} \mathrm{C}$ from the hexagonal modification in a belttype apparatus (SCLAR et al. 1964). KlOSTERKöTTER (1956) in 3 of 55 animals found fibrohyalin nodules after intraperitoneal application of hexagonal $\mathrm{GeO}_{2}$. Phagocytes containing dust particles showed a honeycomb-like eosinophilic protoplasm.

\subsection{5}

Indium

Indium has the atomic number 49 and the valence electrons are those of the $5 s^{2} 5 p^{1}$ shells. The usual oxidation states of indium are +1 and +3 .

Indium trichloride $\left(\mathrm{InCl}_{3}\right)$ inhaled by female Fischer 344 rats initiated an inflammatory response (BLAzKA et al. 1994). Seven days following inhalation of $20 \mathrm{mg} \mathrm{InCl} / \mathrm{m}^{3}$ for $1 \mathrm{~h}$ the total cell number, fibronectin, and TNF- $\alpha$ levels in the bronchoalveolar lavage fluid were 8,40 , and 5 times higher than the control, respectively. While the increase was due primarily to an influx of polymorphonuclear leucocytes, a portion could be attributed to alveolar macrophages, which increased from 10.4 to $24.0 \times 10^{6}$.

Indium phosphide (InP) is a II-V compound of semiconductor materials widely employed for optoelectronic devices. KABE et al. (1996) showed that InP particles were hardly soluble in the synthetic lung fluid in vitro and that the $\mathrm{LDL}_{0}$ of InP was $>5,000 \mathrm{mg} / \mathrm{kg}$, which was considered relative low toxicity. Uemura et al. (1997) showed phagocytosis of InP by macrophages in bronchoalveolar lavage fluid of male Fischer 344 rats. After intratracheal instillation of $0,1.2,6.0$ and $62.0 \mu \mathrm{g} \mathrm{InP} / \mathrm{kg} \mathrm{rat}$, respectively, OdA (1997) found a dose-related mild elevation of superoxide dismutase activity and lactate dehydrogenase activity in bronchoalveolar fluid on day 1 without increase of inflammatory cells and total protein in BAL, which suggested the response of neutrophils and alveolar macrophages to instilled InP, and/or the manifestation of a very early stage of inflammation.

Autoradiography of human alveolar macrophages after phagocytosis of ${ }^{111}$ In-oxine showed a highly significant $(P<0.001)$ concentration of grains over the nucleus (DAVIs et al. 1980). All cytoplasmic structures except the lysosomes showed less than predicted labelling. If nuclear labelling was not considered, and only cytoplasmic labelling compared, there was a significant increase in labelling of lysosomes $(P<0.0001)$. The cell bound activity at $1 \mathrm{~h}$ was $93 \%$ and at $24 \mathrm{~h}$ it was $83 \%$. Radiolabelled macrophages were similar to unlabeled cells by dye exclusion, adherence and phagocytosis both at 1 and $24 \mathrm{~h}$ after labelling.

\subsection{6 Iron}

Iron has the atomic number 26 and the valence electrons are those of the $3 d^{6} 4 s^{2}$ shells. The usual oxidation states of iron are +2 and +3 , less frequently +4 and +6 .

As all the metals in the first row of the $d$-block in the Periodic Table with the sole exception of zinc, iron contains impaired electrons and can thus qualify as a radical (HALliWELl and GutTERIDGe 1989, p. 15), and ferrous ions can participate in electron transfer reactions with molecular oxygen.

In his review on potential use of iron chelators against oxidative damage, GALEY (1997) recalled that although triplet dioxygen cannot directly react with biomolecules in the ground state, iron, as well as other transition metals, can relieve the spin restriction of oxygen and dramatically enhances the rates of oxidation (MiLLER et al. 1990).

Heme, an essential iron chelate, serves in respiration, oxygen transport, detoxification, and signal transduction processes. The potential toxicity of heme and hemoproteins points to a critical role for heme degradation in cellular metabolism. The heme oxygenases provide this function and participate in cellular defence.

The autoxidation of ferrohemoglobin to methemoglobin generates $\mathrm{O}_{2}{ }^{--}$and its dismutation product $\mathrm{H}_{2} \mathrm{O}_{2}$ that could react with reduced iron to form 
HO` (Kalyanaraman et al. 1983). However, it remains unclear if such a process would be catalysed in vivo by the heme iron itself, or by iron released from heme (CADENAS 1989). Indeed, whether any form of free heme or hemoprotein can act as a true Fenton catalyst to generate $\mathrm{HO}^{\bullet}$ remains controversial (RYter and TyrRell 2000).

Various biological iron chelates were assayed for efficiency of Fenton catalysis (percentage conversion of $\mathrm{O}_{2}{ }^{--}$to $\mathrm{HO}^{\circ}$ ) and ranked:

citrate $>>$ pyrophosphate $>$ lactate $>$ adenosine triphosphate $>$ hematin, transferrin, hemin, haemoglobin.

Polynuclear ferric citrate complex (metal-to-ligand molar ration 1:1)-preincubated mural peritoneal macrophages gave a positive Prussian blue reaction, while mononuclear iron complex (metal-to-ligand molar ration 1:10)-preincubated cells failed to do so (Gebran et al. 1993).<smiles>O=N[P+](N=O)(OP(=O)([O-])O)OP(=O)([O-])O</smiles><smiles>O=N[P+]1(N=O)OP(=O)([O-])OP(=O)([O-])O1</smiles>

Fe(II)-NO-phosphate and pyrophosphate complexes

[194]

$\mathrm{Fe}^{2+}$ is complexed by dihydrolipoic acid (PAGANI et al. 1989).

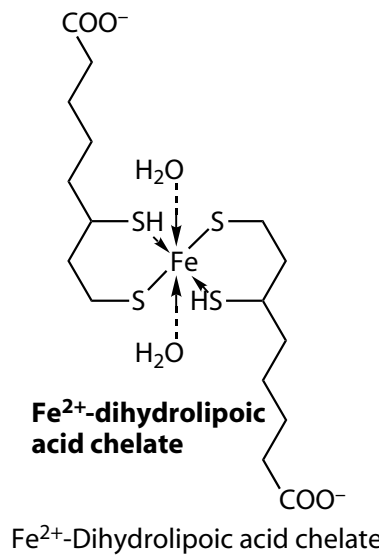

Complexation of ferric iron with D-tagatose, a cellpermeable zero-energy producing ketohexose, protected against oxidative cell injury (VALERI et al. 1997). Lethal liver cell injury induced in vitro by $300 \mu \mathrm{M}$ nitrofurantoin was completely prevented by high concentrations $(20 \mathrm{mM})$ of $\mathrm{D}$-tagatose, whereas equimolar concentrations of glucose, mannitol, or xylose were ineffective (PATERNA et al. 1998).
Surface-complexed iron $\left[\mathrm{Fe}^{3+}\right]$ observed after intrapleural injection of $30 \mathrm{mg}$ of either amosite, crocidolite, and chrysotile both genuine and saturated with $\mathrm{Fe}^{3+}$ in Sprague-Dawley rats corresponded to oxidant generation, measured as barbituric acid reactive products of deoxyribose, and more covalently closed, circular DNA strand scission induced by these asbestos fibres (GHio et al. 1994).

Iron chelates as $\mathrm{Fe}(\mathrm{III})$ nitrilotriacetate were genotoxic in HeLa cells and V79 cells (HARTwIG et al. 1992, 1993). Ferric nitrilotriacetate promoted $N$ diethylnitrosamine-induced renal tumorigenesis in the rat (ATHAR and IQBAL 1998). In renal cell carcinoma induced by ferric nitrilotriacetate, glutathione $S$-transferase Yp isozyme was over expressed and Ya isozyme concomitantly down-regulated (TANAKA et al. 1998).

$\mathrm{N}$-(2-hydroxybenzyl)-L-serine is a tridentate iron chelator (KitAZAWA and IWASAKI 1999) that does not induce hypoxia inducible factor-1 (CREIGHTONGutteridge and Tyrrell 2002).

Lactobionic acid, a major constituent of a solution used to preserve organs prior to transplantation, can chelate ferric iron (IsAACson et al. 1989). Relative to iron(III) chelated to ethylenediamine tetraacetic acid (EDTA), the lactobionic acid-iron complex is less able to participate in the Fenton reaction as measured by formaldehyde generation from dimethylsulphoxide and bleaching of $p-N, N-$ dimetylnitrosoaniline.

$N, N^{\prime}$-bis (2-hydroxybenzyl) ethylenediamine$N, N^{\prime}$-diacetic acid (HBED) facilitated $\mathrm{Fe}(\mathrm{II})$ oxidation but blocked $\mathrm{O}_{2}{ }^{--}$-induced reduction of $\mathrm{Fe}(\mathrm{III})$ and consequently pre-empted production of $\mathrm{HO}^{\bullet}$ or hypervalent iron through the Haber-Weiss reaction cycle (SAMUni et al. 2001). The efficacy of HBED as a 1-electron donor was demonstrated by reduction of the 2,2'-azino-bis(3-ethylbenzothiazoline-6-sulphonate)-derived nitrogen-centred radical cation $\left(\mathrm{ABTS}^{\bullet+}\right)$, accompanied with a short-lived phenoxy radical.

Ferrocene, di-2,4-cyclopentadien-1-yliron, $\mathrm{Fe}\left(\mathrm{C}_{5} \mathrm{H}_{5}\right)_{2}$, which is used in the manufacture of integrated circuits (Fox and RADER 1988) and acetylferrocene react with cyanomethyl and ethoxycarbonylmethyl carbon-centred radicals leading to homo- and heteroannular disubstituted products (BACIOCCHI et al. 1993). Histochemists used 1chloromercuriferrocene as an electron-opaque stain for aldehydes and thiol groups (Allen and Perrin 1974, SWift 1975).

The findings from several animal model studies of the pathology of iron indicate that oxidative damage to the membranes of cell organelles may be a crucial event in toxicity. There also exists a large body of indirect evidence from in vitro studies 
involving isolated organelles (BURKITT and GILBERT 1989, Rice-Evans et al. 1989, BACON and Britton 1990, Sevanian et al. 1990), cells (Poli et al. 1987), and tissue homogenates (ARTHUR et al. 1988) to suggest that oxidative damage due to hydroxyl radical formation is responsible for the toxic effects of iron. Aust et al. (1993) have employed a secondary radical-trapping technique in which the hydroxyl radical reacts with dimethyl sulfoxide to form the methyl radical ${ }^{\circ} \mathrm{CH}_{3}$, which is then detected as its adduct of the spin trap PBN in the bile of animals given an intragastric dose of ferrous sulphate.

$$
\begin{aligned}
& \text { One-electron transfer catalysed by iron } \\
& \mathrm{O}_{2}+\mathrm{Fe}^{2+} \leftrightarrow \mathrm{Fe}^{3+}-\mathrm{O}_{2}^{--} \rightarrow \mathrm{O}_{2}^{--}+\mathrm{Fe}^{3+} \\
& \mathrm{O}_{2}^{--}+\mathrm{Fe}^{2+}+2 \mathrm{H}^{+} \rightarrow \mathrm{H}_{2} \mathrm{O}_{2}+\mathrm{Fe}^{3+} \\
& \mathrm{H}_{2} \mathrm{O}_{2}+\mathrm{Fe}^{2+} \longrightarrow \mathrm{HO}^{-}+\mathrm{HO}^{-}+\mathrm{Fe}^{3+}
\end{aligned}
$$

The intermediate formed in equation [196] is the perferryl ion, a resonance hybrid of $\mathrm{Fe}^{3+}-\mathrm{O}_{2}{ }^{\cdot-}$ and $\mathrm{Fe}^{2+}-\mathrm{O}_{2}$. Perferryl has been widely implicated as an initiating species (Pederson and Aust 1975, SvinGEN et al. 1978, Tien et al. 1981, Sugioka et al. 1983) since the concept was first introduced by Hochstein and Ernster (1963) and Hochstein et al. 1964). However, iron in the ferryl ion $\left(\mathrm{FeO}^{2+}\right)$ is considerably more reactive than in the perferryl ion, and the ferryl ion has been considered as alternative to the $\mathrm{HO}^{-}$in Fenton chemistry (KopPENOL 1985).

Ferric ions readily precipitate out of neutral aerobic solution to form insoluble ferric hydroxides, and it was recognised that complexing with ligands such as adenosine diphosphate (HochsteIn and ERNSTER 1963, HochSTEIN et al. 1964) or ethylenediaminetetraacetate (EDTA) overcame this problem. Complexing with EDTA helps to keep iron in a reactive form in solution and may favourably alter the redox potential of the iron (Aust et al. 1985; Grootveld and Halliwell 1986). Depending on its molecular ratio to iron, EDTA can either stimulate of inhibit lipid peroxidation (GUTTERIDGE et al. 1979). EDTA promotes the autoxidation of ferrous ions $\left(\mathrm{Fe}^{2+}\right)$ and formation of HO${ }^{\bullet}$ (CoHEN and SINET 1980), but EDTA can inhibit lipid peroxide decomposition (GUTTERIDGe 1984).

LAMB and Elder (1931) found solutions of $\mathrm{FeSO}_{4}$ containing sodium pyrophosphates to oxidise 1000 times faster than $\mathrm{FeSO}_{4}$ in 0.1 to $3.0 \mathrm{M}$ $\mathrm{H}_{2} \mathrm{SO}_{4}$. While the rate expression for $\mathrm{Fe}^{2+}$ autoxidation in the presence of sulphate shows as secondorder dependence of $\mathrm{Fe}^{2}$ concentration, that for phosphate is first order (CHER and DAvidson 1954). The rate of $\mathrm{Fe}^{2+}$ autoxidation is also depend- ent upon the nature of its chelators. Anions with low affinity for $\mathrm{Fe}^{2+}$ do not appear to affect the rate of autoxidation (LAMBETH et al. 1982). However, chelation of $\mathrm{Fe}^{2+}$ by compounds with oxygen donor atoms tends to enhance $\mathrm{Fe}^{2+}$ autoxidation, perhaps because of their affinity for $\mathrm{Fe}^{3+}$ (CHER and DAVIDSON 1954, HARris and AISEN 1973).

Table 36. Spin state of oxidised iron $\left(\mathrm{Fe}^{3+}\right)$

\begin{tabular}{rrrlll}
\hline 1 & 1 & 1 & 1 & 1 & $\mathrm{~S}=5 / 2$ (high-spin state) \\
11 & 11 & 1 & & & $\mathrm{~S}=1 / 2$ (low-spin state) \\
11 & 1 & 1 & 1 & & $\mathrm{~S}=3 / 2$ (intermediate state) \\
\hline
\end{tabular}

$$
\left[\mathrm{Fe}\left(\mathrm{H}_{2} \mathrm{O}\right)_{6}\right]^{3+}+\mathrm{e}^{-} \rightarrow\left[\mathrm{Fe}\left(\mathrm{H}_{2} \mathrm{O}\right)_{6}\right]^{2+}
$$

Octahedral $d^{5}$ complexes have been shown to exist in two different magnetic and thermodynamic stable forms only: the low- or the high-spin state. The temperature dependence of the spin equilibrium shows a very low energy barrier between the two spin states.

White and Coon (1980) have postulated a highly resonance-stabilised perferryl species:

$\mathrm{S}^{-} \mathrm{Fe}(5+) \mathrm{O}^{2-} \mathrm{S}^{-}(4+) \mathrm{O}^{-} \leftrightarrow \mathrm{S}^{-} \mathrm{Fe}(3+) \mathrm{O} \leftrightarrow \mathrm{S}^{\circ} \mathrm{Fe}(3+) \mathrm{O}^{-}[200]$

Under physiological conditions, iron is only slightly soluble, tending to form a precipitate with anions such as $\mathrm{OH}^{-}$and inorganic phosphate. However, a variety of chelating agents greatly increase the solubility of iron. For example, the addition of EDTA to an $\mathrm{O}_{2}{ }^{--}$generating system in the presence of $\mathrm{Fe}^{3+}$ markedly potentiated cytotoxicity (Rosen et al. 1981), HO formation (MCCORD and DAY 1978, RoSEN et al. 1981), and lipid peroxidation (GuTTERIDGE et al. 1979). The powerful iron chelators DTPA and Desferal (desferrioxamine B methanesulfonate) totally inactivate $\mathrm{Fe}^{3+}$. Desferrioxamine is a hexadentate ligand, i.e. it occupies all six coordination sites of an iron ion. The desferrioxamine molecule 'wraps around' the iron ion, encasing it in an envelope of organic material.

Hydroxypyridones are bidentate ligands, i.e. they can occupy only two coordination sites. Hence three molecules of hydroxypyridones are needed to complex one Fe(III) completely. Upon dilution, $3: 1$ hydroxypyridone:iron ion complexes might dissociate into $2: 1$ complexes, leaving available coordination sites on the iron that could allow catalysis on free radical reactions.

Quinolinic acid, $\alpha$-picolinic acid, fusaric acid, and 2,6-pyridinedicarboxylic acid enhanced the Fenton reaction in phosphate buffer, respectively (IwAHAshi et al. 1999). The ultraviolet-visible ab- 
sorption spectrum of the mixture of $\alpha$-picolinic acid with ferrous ion showed a characteristic band with a $\lambda_{\max }$ at $443 \mathrm{~nm}$, suggesting that $\alpha$-picolinic acid chelate of $\mathrm{Fe}^{2+}$ ion forms in the solution. Similar characteristic absorbance band was also observed for the mixture of $\mathrm{Fe}^{2+}$ ion with quinolinic acid (or fusaric acid, or 2,6-pyridinedicarboxylic acid). The chelation seems to be related to the enhancement by quinolinic acid, $\alpha$-picolinic acid, fusaric acid, and 2,6-pyridinedicarboxylic acid of the Fenton reaction.

The inhibitory effect of diethyldithiocarbamate on Fenton reagent-generated hydroxyl radicals may be due either to its capacity to scavenge hydroxyl radicals or to chelate and then oxidise ferrous ions (Liv et al. 1996).

Peroxidation of lysosomal membranes can be induced by ascorbate/Fe(III)complex (MAK and WEGLICKI 1985) or by the autoxidizing, superoxide producing, compound dihydroxyfumarate in combination with a Fe(III)complex (MAK et al. 1983). These processes probably involve the initiation of lipid peroxidation by either iron-centred radicals or by hydroxyl radicals. Zdolsek and Svensson (1993) found that the damaging effects of hydrogen peroxide in the presence of $\mathrm{Fe}$ (III)-complexes depended on the additional presence of a reducing agent, such as cysteine. The reducing agents would reduce $\mathrm{Fe}(\mathrm{III})$ to $\mathrm{Fe}(\mathrm{II})$ making it prone to catalyse Fenton reactions generating hydroxyl radicals in the presence of hydrogen peroxide.

The mechanism of $\mathrm{Fe}^{2+}$-initiated lipid peroxidation in a liposomal system was studied by TANG et al. (2000). They found that a second addition of ferrous ions within the latent period lengthened the time lag before lipid peroxidation started. The apparent time lag depended on the total dose of $\mathrm{Fe}^{2+}$ whenever the second dose of $\mathrm{Fe}^{2+}$ was added, which indicated that $\mathrm{Fe}^{2+}$ has a dual function: to initiate lipid peroxidation on one hand and suppress the species responsible for the initiation of the peroxidation on the other. When the pre-existing peroxides $(\mathrm{LOOH})$ were removed by incorporating triphenylphosphine into liposomes, $\mathrm{Fe}^{2+}$ could no longer initiate lipid peroxidation and the acceleration of the $\mathrm{Fe}^{2+}$ oxidation by the liposomes disappeared. However, when extra $\mathrm{LOOH}$ were introduced into liposomes, both enhancement of the lipid peroxidation and shortening of the latent period were observed. When the scavenger of lipid peroxyl radicals $\left(\mathrm{LOO}^{\circ}\right), N, N^{\prime}$-diphenyl-p-phenylene-diamine, was incorporated into liposomes, neither initiation of the lipid peroxidation nor acceleration of the $\mathrm{Fe}^{2+}$ oxidation could be detected.

Lipid peroxidation, measured by FonTECAVE et al. (1990) by malonaldehyde formation is induced in rat liver microsomes by the insoluble ironcontaining minerals, pyrite, magnetite, nemalite (a fibrous brucite where about $8 \%$ of $\mathrm{Mg}^{2+}$ is substituted by $\mathrm{Fe}^{2+}$ ) and minette de Lorraine.

$\mathrm{Fe}^{3+}$ reacts with the silanol groups at the surface of amorphous silica forming

$$
\mathrm{Fe}^{3+}+\mathrm{m}(\mathrm{SiOH}) \longleftrightarrow \mathrm{Fe}(\mathrm{OSi}) \mathrm{m}^{3 \mathrm{~m}+}+\mathrm{mH}^{+}
$$

$\mathrm{Fe}^{3+}$ reacts with prostaglandin $\mathrm{G}_{2}$ as follows:

$$
\mathrm{Fe}^{3+}+\mathrm{PGG}_{2} \rightarrow[\mathrm{FeO}]^{3+}+\mathrm{PGH}_{2}
$$

$$
[\mathrm{FeO}]^{3+}+\mathrm{PGG}_{2} \rightarrow \mathrm{Fe}^{3+}+\mathrm{PGH}_{2}+{ }^{1} \mathbf{O}_{2}
$$

Rötig et al. (2001) examined the expression levels of various genes involved in iron uptake, reduction, and storage, in $\mathrm{Fe}-\mathrm{S}$ protein biogenesis, in mitochondrial electron transport chain, plus the two superoxide dismutase genes, in human adult tissues by Northern blot analysis. Most of these genes were ubiquitously expressed, but their transcription showed strongly different levels in the various tissues investigated denoting different mechanisms for iron utilisation in various organs.

Normally the entry of iron(III) into cells and its intracellular distribution is carefully controlled. However, if iron(III) is complexed by nitrilotriacetate (NTA), it circumvents the controlled transferrin path, and may even penetrate into the cell nucleus where it catalyses detrimental oxidative reactions with DNA. The Fe(III) NTA-complex has been shown to cause cancer in rats (ЕвINA et al. 1986) and DNA breaks and increased frequency of sister chromatid exchanges in Chinese hamster V79 cells (Hartwig et al. 1993).

Sequestration of iron by macrophages diminishes the potential of the metal to catalyse oxidant generation (Olakanmi et al. 1993). The storage of iron within intracellular ferritin confers an antioxidant function to this protein and, in certain cells, provides cytoprotection in vitro against oxidants (CozzI et al. 1990, BALLA et al. 1995). Ferritin expression is regulated by a posttranscriptional mechanism. A specific sequence at the 5'-untranslated end of ferritin mRNA called the iron responsive element (IRE) binds a cubane iron-sulphur cluster, the iron regulatory protein (IRP), when the IRP exists in the apoprotein form (LEIBOLD and Guo 1992, BEINERT and KenNedy 1993). Iron included in atmospheric particles could react with IRP, which alters its conformation. This decreases the affinity of the protein to mRNA, and it is displaced, allowing translation of ferritin to proceed. 
The binding activity of IRP-1 was increased and the ferritin synthesis was suppressed when the macrophages were cultures under hyperoxia, and the reverse occurred under hypoxia (KuriYamaMatsumura et al. 2001). Iron diminished the IRP1-binding activity and the enhanced synthesis of ferritin. However, this effect was arrested under hyperoxia. Consistently, hypoxia-induced loss of binding activity of IRP-1 and the enhanced synthesis of ferritin was blocked in the presence of an iron chelator deferoxamine. These alteration in the binding activity of IRP-1 in response to oxygen were not reproduced in the cell-free extract.

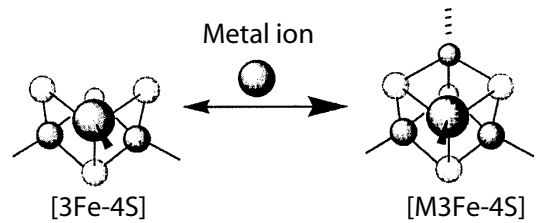

Addition of a metal ion to a [3Fe-4S] cuboidal cluster to yield a [4Fe-4S] cubane

The loading of iron into ferritin needs to be initiated with $\mathrm{Fe}(\mathrm{II})$, with oxygen as an oxidant, to deposit iron in the core of ferritin as ferric oxyhydroxides.

The iron stored in ferritin can be released by $\mathrm{O}_{2}{ }^{--}$ (Thomas et al. 1985) and other reducing radicals, e.g. dipyridyls, nitroaromatics, and semiquinones (Thомas and Aust 1986). Reducing agents like flavins (SIRIVECH et al. 1974) and ascorbate (BOYER and McCleary 1987) also release iron slowly from ferritin. Polyhydroxypyrimidines: dialuric acid, isouramil, divicine, and acid-hydrolysed vicine in aerated phosphate-buffered saline, $\mathrm{pH} 7.3$, at $37^{\circ} \mathrm{C}$, all caused concentration-dependent release of iron from ferritin, which was measured as the progressive formation of $\mathrm{Fe}^{2+}$-ferrozine complex (MoNTERO and Winterbourn 1989). When superoxide was anaerobically generated by dissolving $\mathrm{KO}_{2}$, no metal complex with bathophenantroline built up from horse spleen ferritin (CASSANELli and MouLIS 2001). Similar to superoxide, initial reports (Reif and Simmons 1990) of iron release by nitric oxide have been questioned (LAULHÈRE and FonTECAVE 1996).

The addition of NifS to horse spleen ferritin does not sustain iron release at a high rate after an initial burst (CAssanelli and Moulis 2001). This increase in soluble iron can be attributed to endogenous compounds able to expel iron from ferritin. Addition of the NifS substrate, L-cysteine, increases the rate.

Organically bound iron is one of the most potent initiators of phagocytic activity in macrophages (RHodes et al. 1969, BAKer and Morgan 1970, RHODEs 1970, WADA et al. 1970).
Intramacrophagic iron was $0.33 \pm 0.21 \mu \mathrm{g} \times 10^{-6}$ cells in healthy non-smoking subjects without occupational exposure (CoRHAY et al. 1992). Intramacrophagic iron was increased in smokers, ironsteelworkers, and in patients with chronic obstructive pulmonary disease or lung cancer even in the absence of pulmonary haemorrhage. About $80 \%$ of the whole bronchoalveolar lavage fluid iron content was in the cells.

To study the fate of internalised $\mathrm{Fe}$, McGowan and PARRISH (1985) and McGowan et al. (1986) incubated human alveolar macrophages with ${ }^{59} \mathrm{Fe}$ saturated transferrin at $37^{\circ} \mathrm{C}$, lysed the cells in a French pressure cell at $8000 \mathrm{psi}$ and $4^{\circ}$, and fractionated the lysate by differential centrifugation (at $900 \times \mathrm{g}$ for $5 \mathrm{~min}$ and then at $11,500 \times \mathrm{g}$ for $20 \mathrm{~min}$ ). ${ }^{59} \mathrm{Fe}$ internalised initially bound to transferrin was distributed to a cytoplasmic, largely ferritinassociated, pool more slowly in smokers than in non-smokers, during a 24-hour incubation in vitro. Significantly less newly internalised iron was returned to the culture medium by alveolar macrophages from smokers, which by $24 \mathrm{~h}$ had released $11.0 \pm 3.7 \%$ of the initially internalised ${ }^{59} \mathrm{Fe}$ compared with $36 \pm 2,3 \%$ for non-smokers $(P<0.01)$. According to McGowan and Henley (1988) the majority of the iron contained in alveolar macrophages is insoluble and is most likely incorporated into haemosiderin. However, approximately $25 \%$ of the intracellular iron is associated with ferritin and could potentially be released to catalyse the formation of hydroxyl radicals through the Haber-Weiss reaction.

The iron burden in alveolar macrophages of smokers considerably exceeds that of non-smokers. Of $440-1,150 \mu \mathrm{g}$ iron contained in $1 \mathrm{~g}$ cigarette $0.1 \%$ gets into the mainstream smoke (MussaloRAUHAMmaA et al. 1986). Furthermore, bronchitis increases the iron content of alveolar macrophages from 3.2times to 5.4times of that of a healthy nonsmoker.

A study on monocytic THP-1 cells induced to differentiate with phorbol myristate acetate showed that further stimulation with interferon- $\gamma$ strongly increased $\mathrm{H}$-ferritin expression and also induced a greater release of TNF- $\alpha$ (SCACCABAROZZI et al. 2000). This effect is possibly mediated by a stabilization of $\mathrm{H}$-ferritin transcript caused by phorbol myristate acetate and the relevant mRNA region was localised within a pyrimidine rich region in the 3' untranslated region (AI et al. 1999). Most of the stimuli related to inflammation and directed to ferritin synthesis seem to upregulate $\mathrm{H}$-ferritin preferentially over the L-ferritin, thus determining an increase of the catalytic sites and a reduction of cell iron availability. These events may be accompanied 
by indirect effects via the induction of nitric oxide that modify the activity of iron regulatory proteins and regulate equally both subunits.

When stimulated with either phorbol myristate acetate $(10 \mathrm{ng} / \mathrm{ml})$ of opsonized zymosan $(3 \mathrm{mg} / \mathrm{ml})$ neither human monocytes nor monocyte-derived macrophages exhibited spin trap evidence of hydroxyl radical formation unless provided with an exogenous $\mathrm{Fe}^{3+}$ catalyst (BRITIGAN et al. 1988). Using a spin adduct resistant to superoxide-mediated destruction, BRITIGAN et al. (1990) confirmed the lack for an endogenous capacity of significant $\mathrm{HO}^{\circ}$ production during the respiratory burst of human phagocytes.

In HL-60 cells treated with $\mathrm{H}_{2} \mathrm{O}_{2}$, BYRNEs (1996) measured diffusion distances, i.e. the distances between the sites of generation of presumed hydroxyl radicals by low molecular weight forms of $\mathrm{Fe}$ and the site of their reaction with substrate. In HL-60 cells made deficient of Fe by treatment with bathophenanthroline disulphonic acid and ascorbic acid, and subsequently allowed to incubate in fresh media during short periods, an additional diffusion distance of $1.9 \mathrm{~nm}$ was measured. This was assumed to derive from $\mathrm{Fe}$ acquired from extracellular sources and was associated with ATP, as depletion of cellular ATP led to decreased single-strand break generation (LI and BYRNES 1999). Li et al. (1999) measured two diffusion distances for $\mathrm{Fe}$ complexes each of ethylene diamminetetraacetic acid (FeEDTA) and nitrilotriacetic acid for generation of malondialdehyde-type products from deoxyribose and of single-strand breaks in the plasmid pBR322. The closer diffusion distances for pBR322 single-strand break generation $(5-6 \mathrm{~nm})$ were considerably greater than the diffusion distances for malondialdehyde generation in the deoxyribose assay $(2-3 \mathrm{~nm})$. This is consistent with charge-charge interactions playing an important role in defining diffusion distance. The diffusion distances for FeNTA, FeEDTA, and other Fe species generating single-strand breaks in isolated Ehrlich ascites tumour cell nuclei ranged from 2.1 to $14 \mathrm{~nm}$.

Nitric oxide $\left({ }^{\circ} \mathrm{NO}\right)$ in a concentration-dependent (0.4-1.8 $\mu \mathrm{M})$ manner inhibited lipid peroxidation induced in HL-60 cells by an oxidative stress of 20 $\mu \mathrm{M} \mathrm{Fe}^{2+}$ (KeLley et al. 1999).

Lipid peroxyl radical can be scavenged by nitric oxide $\mathrm{LOO}^{*}+{ }^{+} \mathrm{NO} \rightarrow$ LOONO chain determination [204]

The phagocytic capacity of ferric citrate-treated peritoneal macrophages of Balb/c mice was inhibited in a dose-related manner, $50 \mu \mathrm{M}$ being the lowest concentration achieving a significant inhibition of phagocytosis in vitro (GEBRAN et al. 1993). How- ever, such inhibition was only observed in the presence of ferric citrate with a metal-to-ligand molar ratio of $1: 1$, but not with ferric citrate with a metalto-ligand molar ratio of $1: 10$ in which the hydrolyzation and polymerization of iron in physiological solutions is prevented. Lipid peroxidation at concentrations of $125 \mu \mathrm{M}$ ferric citrate showed an absorbance of thiobarbituric acid reactive substances of $0.403 \pm 0.012$ and $0.154 \pm 0.005$, respectively, while the untreated control macrophages gave figures of $0.029 \pm 0.003$, read at $532 \mathrm{~nm}$.

\section{Iron Oxides}

The three iron oxides correspond to the idealised compositions $\mathrm{FeO}, \mathrm{Fe}_{2} \mathrm{O}_{3}$ and $\mathrm{Fe}_{3} \mathrm{O}_{4}$. However, often they were not composed stoichiometrically.

Three distinct submicronic aerosols of iron oxide can be reproducibly generated by combustion of iron pentacarbonyl vapours under varying conditions (Valberg and Brain 1979). The "feathers" haematite has a mass median aerodynamic diameter of $0.17 \mu \mathrm{m}$ and a large surface area because it is an agglomerate of units $0.005 \mu \mathrm{m}$ in diameter. The "birdshot" haematite has a median aerodynamic diameter of $0.31 \mu \mathrm{m}$, but has a smaller surface area because the subunits are $0.03 \mu \mathrm{m}$ in diameter. The $\gamma$-oxide has a median aerodynamic diameter of 0.73 $\mu \mathrm{m}$ and has crystalline subunits of $0.2 \mu \mathrm{m}$ in diameter; it is a magnetic form of haematite, $\gamma-\mathrm{Fe}_{2} \mathrm{O}_{3}$. The magnetic properties of $\gamma-\mathrm{Fe}_{2} \mathrm{O}_{3}$ permit enhancement of deposition and non-invasive detection.

Taconite, a low-grade siliceous formation in Minnesota, from which high grade iron ore is derived, consists of fine-grained silica with variable ratios of hæmatite and magnetite totalling less than 30 per cent iron.

Table 37. Iron Ores

\begin{tabular}{|c|c|c|c|}
\hline Mineral & Formula & $\mathrm{Fe}(\%)$ & Deposit \\
\hline Magnetite & $\mathrm{Fe}_{3} \mathrm{O}_{4}$ & 72 & $\begin{array}{l}\text { Norway, Sweden } \\
\text { (Kiruna) }\end{array}$ \\
\hline Hæmatite & $\alpha-\mathrm{Fe}_{2} \mathrm{O}_{3}$ & 70 & $\begin{array}{l}\text { Sieg area, Cumber- } \\
\text { land, Spain, } \\
\text { Morocco }\end{array}$ \\
\hline Goethite & $\alpha-\mathrm{Fe}^{3+} \mathrm{O}(\mathrm{OH})$ & 63 & Central Europa \\
\hline Lepidocrocite & $\gamma-\mathrm{FeO}(\mathrm{OH})$ & 63 & Sieg area, Hesse \\
\hline Linonite & $\mathrm{FeO}(\mathrm{OH})$ & 63 & $\begin{array}{l}\text { Salzgitter, Wester- } \\
\text { wald }\end{array}$ \\
\hline Siderite & $\mathrm{FeCO}_{3}$ & 48 & $\begin{array}{l}\text { Sieg area, Styria, } \\
\text { Bergamo }\end{array}$ \\
\hline Chamosite & & $36-42$ & $\begin{array}{l}\text { Lorraine, Luxem- } \\
\text { bourg }\end{array}$ \\
\hline Thuringite & & $31-35$ & $\begin{array}{l}\text { Lorraine, Luxem- } \\
\text { bourg }\end{array}$ \\
\hline
\end{tabular}




\section{Hæmatite}

Hæmatite $\left(\alpha-\mathrm{Fe}_{2} \mathrm{O}_{3}\right)$ is hexagonal. It is most widely encountered as the henna-red pigment of many diverse rocks. Some sedimentary rocks and their metamorphosed products contain such high percentages of hæmatite that they have been recovered as iron ore.

Following intrapulmonary instillation of 2.6- $\mu \mathrm{m}$ diameter $\mathrm{Fe}_{2} \mathrm{O}_{3}$ particles in human subjects, particles were cleared from the lavagable alveolar macrophage compartment in a biphasic pattern, with a rapid-phase clearance half-time of $0.5 \mathrm{~d}$ and longterm clearance half-time of $110 \mathrm{~d}$ (LAY et al. 1998). The intracellular distribution of particles within lavaged alveolar macrophages was similar in bronchial and alveolar bronchoalveolar lavage fractions. Alveolar macrophages with high intracellular particle burdens disappeared from the lavagable phagocytic alveolar macrophage population disproportionately more rapidly (shorter clearance half-time) than did alveolar macrophages with lower particle burdens.

The zymosan-stimulated hydrogen peroxide and superoxide anion release of alveolar macrophages isolated from rabbits treated with Freund's adjuvant were not significantly decreased by $\leq 50 \mu \mathrm{g} \mathrm{Fe}_{2} \mathrm{O}_{3} /$ $10^{6}$ cells prior to zymosan stimulation (GuLYAs et al. 1990).

\section{Magnetite}

Magnetite is a cubic mineral and member of the spinel structure type, space group $\mathrm{Fd} 3 \mathrm{~m}$, with composition $\left[\mathrm{Fe}^{3+}\right]^{\mathrm{IV}}\left[\mathrm{Fe}^{2+} \mathrm{Fe}^{3+}\right]^{\mathrm{VI}} \mathrm{O}_{4}$. The most spectacu- lar ore body occurs at Kiruna in northern Sweden, but magnetite can also be prepared by heating hæmatite $\left(\alpha-\mathrm{Fe}_{2} \mathrm{O}_{3}\right)$ in a reducing atmosphere. Oxidation of magnetite leads to maghemite $\left(\gamma-\mathrm{Fe}_{2} \mathrm{O}_{3}\right)$, which gradually inverts to hæmatite.

Magnetite particles of an aerodynamic diameter of $2.8 \mu \mathrm{m}$ allowed to deposit in the alveolar region of the human lung by the non-invasive method of magnetopneumography characterised two important properties of the alveolar macrophage: the cellenergy and the viscosity (STAHLHOFEN and MöLLER 1992). Cell energy was much larger than thermal energy at $37^{\circ} \mathrm{C}: \mathrm{kT}=4.3 \times 10^{-21} \mathrm{~J}$. The Newtonviscous properties of the cytoskeleton could be determined as $\eta=87.1 \pm 34.6 \mathrm{~Pa} \times \mathrm{s}$.

Alveolar macrophages of rats dusted with magnetite from Grängesberg (Central Sweden) for 8 weeks contained large autophagous vacuoles (SCHILler 1971, 1989; Fig. 141). X-ray microanalysis of the telolysosomes contained in the alveolar macrophages of rats instilled intrabronchially with iron dextran showed plenty of iron and phosphorus (FASSKE and MorgenRoth 1985) suggesting an involvement of surfactant. Even 3 months after the last inhalation of $\mathrm{Fe}_{3} \mathrm{O}_{4}$ alveolar macrophages manifested eager phagocytosis of surfactant (Fig. 143).

J774 murine macrophage cell line was used to verify the dynamic processes of spherical magnetite particles of different sizes within the cells in vitro (Stahlhofen and Möller 1994). Relaxation, that is the decay of a remanent magnetic field after primary magnetisation, was particle-size-dependent. The results might indicate that relaxation is not a volume effect, but depends more on the surface area of the phagolysosomes.
Fig. 141. Alveolar macrophage from a $286 \mathrm{~g}$ female white rat (No. 3; breeder: Winkelmann, Borchen-Kirchborchen) which inhaled $10 \mathrm{mg}$ powdered Grängesberg magnetite $/ \mathrm{m}^{3}$ $4 \mathrm{~h}$ per day, 5 days per week from August 24 to October 19, 1967 for a total of 40 days. Fixed on October 30, 1967 under methitural anaesthesia by intratracheal instillation of $2.5 \%$ glutaraldehyde in phosphate buffer $(\mathrm{pH}$ 7.4) before opening the thorax. Postfixation with $1 \%$ osmium tetroxide in phosphate buffer. Contrasted en bloc for $12 \mathrm{~h}$ with $0,5 \%$ uranyl acetate in $70 \%$ ethanol. Embedded in a $2: 8$ mixture of methyl and butyl methacrylate. Sectioned at $50 \mathrm{~nm}$. Lead citrate after REYNOLDS (1963). Plate 34/12

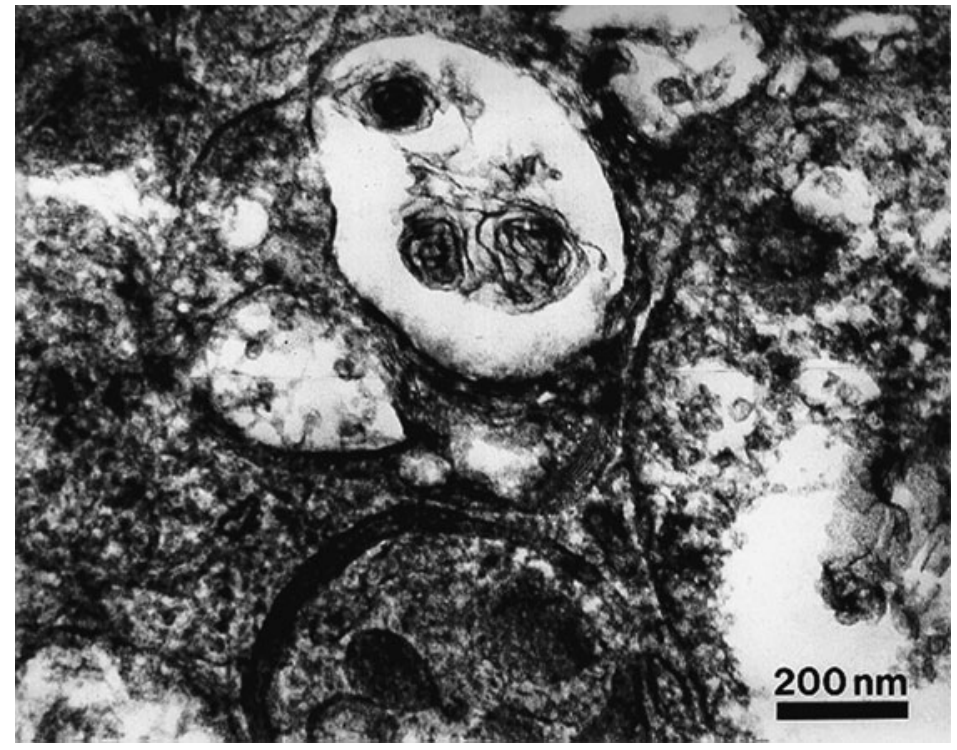




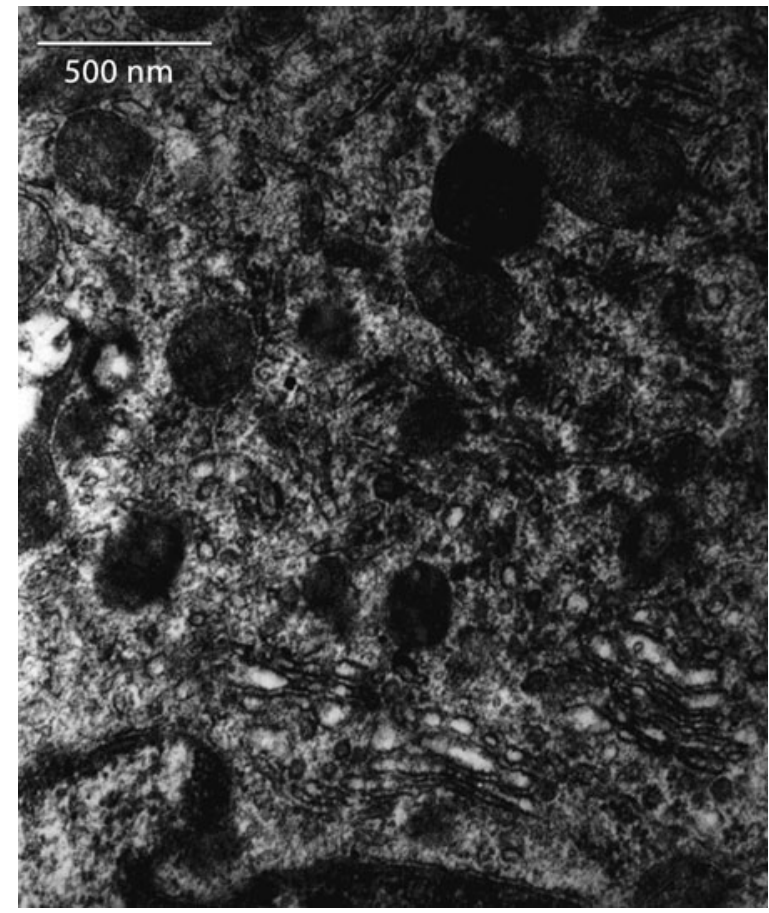

Fig. 142. Rough endoplasmic reticulum and dilated Golgi cisternae in an alveolar macrophage from a $286 \mathrm{~g}$ female white rat (No.3, breeder: Winkelmann, Borchen-Kirchborchen) which inhaled $10 \mathrm{mg}$ powdered Grängesberg magnetite $/ \mathrm{m}^{3} 4$ hours per day, 5 days per week from August 24 to October 19, 1967 for a total of 40 days. Fixed on October 30, 1967 under methitural anaesthesia by intratracheal instillation of $2.5 \%$ glutaraldehyde in phosphate buffer ( $\mathrm{pH}$ 7.4) before opening the thorax. Postfixation with $1 \%$ osmium tetroxide in phosphate buffer. Contrasted en bloc for $12 \mathrm{~h}$ with $0.5 \%$ uranyl acetate in $70 \%$ ethanol. Embedded in a 2:8 mixture of methyl and butyl methacrylate. Sectioned at $50 \mathrm{~nm}$. Lead citrate after REYNOLDS (1963). Plate 46/06

\section{Carbonyl Iron}

Pentacarbonyliron, $\mathrm{Fe}(\mathrm{CO})_{5}$, is able to form stable $\pi$-complexes with hydrocarbons. RHEILEN et al. (1930) reported the first example of such a complex, tricarbonyl $\left[\eta^{4}\right.$-1,3-butadiene]iron.

Carbonyl iron inhaled by male Crl:CD BR rats for $6 \mathrm{~h}$ at $100 \mathrm{mg} / \mathrm{m}^{3}$ did not produce cellular or biochemical indices for pulmonary inflammation, either in normal or complement-depleted animals (WARHEIT et al. 1991).

Carbonyl iron instilled intratracheally in SPF male Fischer 344 rats did not significantly elevate iNOS mRNA in alveolar macrophages lavaged $24 \mathrm{~h}$ after treatment (BLACKFORD et al. 1997).

\section{Ferric Ions}

2-[(2-Bis[carboxymethyl]amino-5-methylphenoxy)methyl] - 6-methoxy-8-bis[carboxymethyl] - amino-

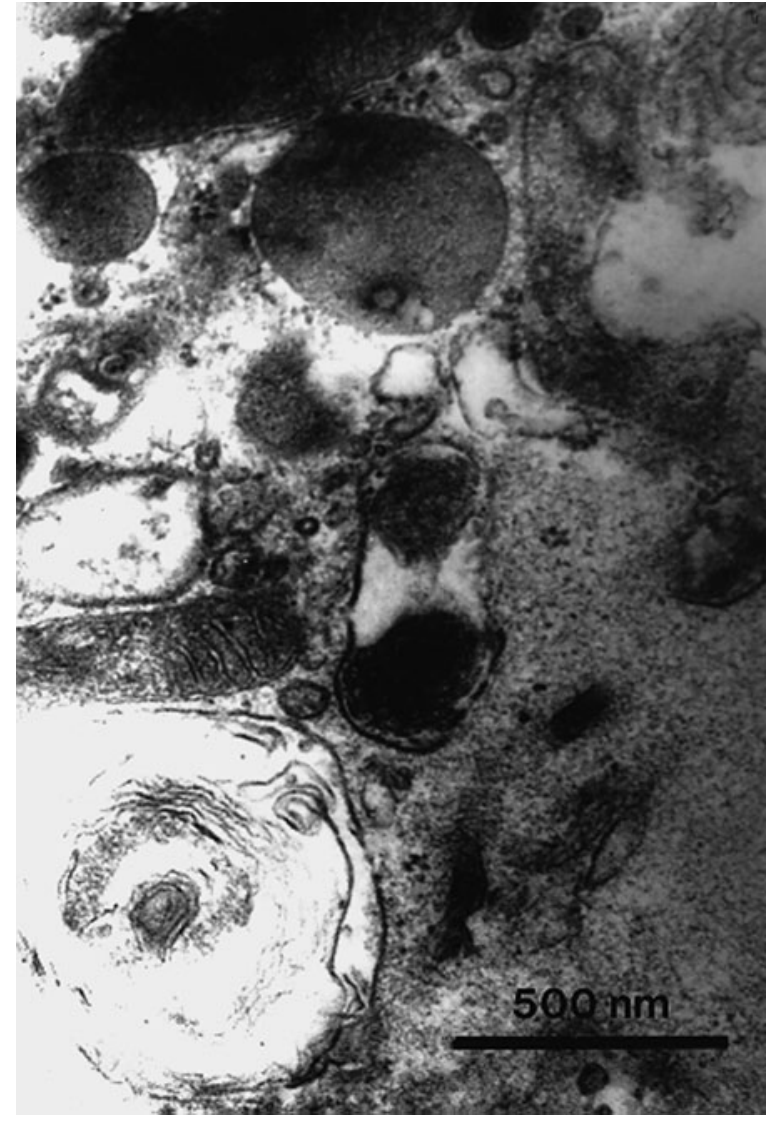

Fig. 143. Tubular myelin engulfed by an alveolar macrophage from a female white rat (breeder: Winkelmann, BorchenKirchborchen) which inhaled $10 \mathrm{mg}$ powdered Grängesberg magnetite $/ \mathrm{m}^{3} 4$ hours per day during a 5-day work week. Inhalation took place from August 24 to October 19, 1967 for a total of 40 days. Fixed on January 15, 1968 under methitural anaesthesia by intratracheal instillation of $2.5 \%$ glutaraldehyde in phosphate buffer ( $\mathrm{pH}$ 7.4) before opening the thorax. Postfixation with $1 \%$ osmium tetroxide in phosphate buffer. Contrasted en bloc for $12 \mathrm{~h}$ with $0,5 \%$ uranyl acetate in $70 \%$ ethanol. Embedded in a 2:8 mixture of methyl and butyl methacrylate. Sectioned at $50 \mathrm{~nm}$. Lead citrate after REYNOLDS (1963). Film 33188

quinolin (quin2) is a powerful transition metal ion chelator. It can efficiently catalyse the formation of oxidising species from the generally unreactive combination of $\mathrm{Fe}^{3}$ and $\mathrm{H}_{2} \mathrm{O}_{2}$ (SANDSTRÖM et al. 1994). Using the $\mathrm{Fe}^{2+}$ indicator ferrozine, Sandström et al. (1997) found evidence for direct reduction of $\mathrm{Fe}^{3+}$-quin2 by $\mathrm{H}_{2} \mathrm{O}_{2}$. Superoxide anion radical appeared to be less efficient than $\mathrm{H}_{2} \mathrm{O}_{2}$ as reductant of $\mathrm{Fe}^{3+}$-quin2 as addition of superoxide dismutase in the ferrozine experiments only decreased the amount of $\mathrm{Fe}^{2+}$ available for Fenton reaction by $10-20 \%$.

Nitric oxide synthase activity was decreased by about $50 \%$ in homogenates obtained from J744A.1 macrophages treated with interferon $\gamma$ plus lipopo- 
lysaccharide in the presence of $50 \mu \mathrm{M} \mathrm{Fe}^{3+}$ as compared with extracts of cells treated with IFN- $\gamma$ plus LPS alone (WeIss et al. 1994).

\section{Gaucher Cells}

LEE et al. (1977) in a child aged $2^{1 / 2}$ years observed a remarkable number of Gaucher cells in alveolar space and in terminal bronchioles heavily laden with iron.

\subsection{7 \\ Lead}

Lead has the atomic number 82 and the valence electrons are those of the $6 s^{2} 6 p^{2}$ shells. The oxidation states of lead include +2 and +4 .

Lead, an immunomodulator and potential human carcinogen, is a major airborne pollutant in industrial environment which possesses a serious threat to human health.

In 66 secondary lead smelter workers leadexposed for $8.09 \pm 4.03$ years there was a positive correlation between the presence of $\mathrm{Pb}$ in blood and significant increases in malondialdehyde levels and superoxide dismutase activity (YE et al. 1999). A positive correlation was found between malondialdehyde and DNA damage.

Inhalation for $3 \mathrm{~h} / \mathrm{d}$ for $4 \mathrm{~d}$ of $30 \mu \mathrm{g} / \mathrm{m}^{3} \mathrm{PbO}$ by male New Zealand white rabbits enhanced $\mathrm{H}_{2} \mathrm{O}_{2}$ and $\mathrm{O}_{2}{ }^{\circ}$ - production by their pulmonary macrophages (Zelikoff et al. 1993). Phagocytic uptake of latex particles was reduced with increasing postexposure time reaching a maximum inhibition at $72 \mathrm{~h}$. Effects of TNF- $\alpha$ release/activity appeared earliest and were persistent up to $72 \mathrm{~h}$. Immediately and $24 \mathrm{~h}$ after exposure, lipopolysaccharidestimulated activity of TNF- $\alpha$ was depressed by $62 \%$ and $50 \%$, respectively. After $72 \mathrm{~h}$, TNF- $\alpha$ release was significantly enhanced compared to control levels. Guo et al. (1996) reported that $\mathrm{PbCl}_{2}$ increased TNF- $\alpha$ secretion from human peripheral blood mononuclear cells in a concentration- and time-dependent manner. They suggested that $\mathrm{PbCl}_{2}$ increased TNF- $\alpha$ expression by posttranscriptional mechanisms and enhanced the reactivity and uptake of TNF- $\alpha$ by increasing the surface expression of TNF-R p55.

The oxidative metabolism of NMRI mouse peritoneal macrophages induced by phorbol myristate acetate was enhanced by $\mathrm{PbCl}_{2}$, the zymosaninduced oxidative metabolism was weakly reduced as measured by means of the lucigenin-enhanced chemiluminescence in a Berthold Biolumat 9500 up to $120 \mathrm{~min}$. After $20 \mathrm{~h}$ of exposure, $\mathrm{PbCl}_{2}$ concentration-dependently $\left(10^{-4}-10^{-8} \mathrm{M}\right)$ suppressed the oxidative metabolism induced by $100 \mu \mathrm{g}$ zymosan/ $\mathrm{ml}$ and $0.5 \mu \mathrm{g}$ PMA $/ \mathrm{ml}$ as well as phagocytosis of latex particles (HILberTz et al. 1986).

Quinlan et al. (1988) observed that lead (0.01-0.4 mM) stimulated $\mathrm{Fe}^{2+}$-induced lipid peroxidation, determined by quantification of thiobarbituric acid-reactive species.

5-Aminolaevulinic acid accumulates in all tissues in lead poisoning and could be a source of oxygen radical formation and cellular damage (MoNTEIRO et al. 1989). The intermediateness of reactive oxygen species in these processes was evidenced in the inhibitory effects of superoxide dismutase, catalase, and mannitol (MonTEIRo et al. 1986). Melatonin, mannitol and trolox, all of which are free radical scavengers, inhibited the formation of 8-hydroxydeoxyguanosine in a concentration-dependent manner (QI et al. 2001). The concentration of each to reduce DNA damage by $50 \%$, i.e., the $\mathrm{IC}_{50}$, was $0.52,0.84$ and $0.90 \mathrm{mM}$, respectively.

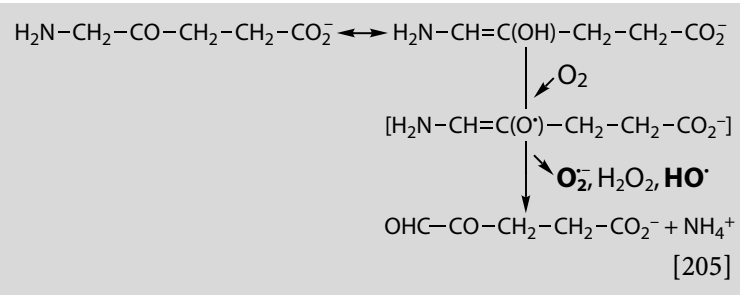

Iron is released from horse spleen ferritin by both 5-aminolaevulinic acid-generated $\mathrm{O}_{2}{ }^{--}$and enoyl radical, which amplifies the chain of 5-aminolaevulinic acid oxidation (RocHA et al. 2000). Iron chelators such as ethylenediaminetetraacetic acid (EDTA), ATP, but not citrate, and phosphate accelerate this process and 5-aminolaevulinic acidpromoted iron release from horse spleen ferritin is faster in horse spleen isoferritins containing larger amounts of phosphate in the core.

The intensity of spontaneous chemiluminescence from liver, soleus muscle, and parietal brain region of 5-aminolaevulinic acid-treated rats was markedly increased after two doses $40 \mathrm{mg} / \mathrm{kg}$ body weight injected intraperitoneally on alternate days (DEMASI et al. 1997). The plasma trapping capacity evaluated by the luminol/2-aminopropane system, gave a parallel response: maximum values after two doses and decreased values after prolonged treatment. After eight doses, the 5-aminolaevulinic acid concentration was found to be 3-fold above the normal value in plasma, $48 \%$ higher in liver and $38 \%$ higher in total brain. These data indicated that the plasma antioxidant system responded to 5aminolaevulinic acid treatment and was correlated with tissue chemiluminescence. 
Free radicals and $\alpha$-ketoglutaraldehyde, a source of Schiff adducts with proteins (ACHARYA and MANNING 1980), might be formed in vivo when 5aminolaevulinic acid accumulates in lead poisoning and might constitute a causative factor of tissue damage (Hermes-Lima et al. 1991).

CARNEIRo and REITER (1998) have reported that the incubation of rat cerebral, hippocampal and cerebellar homogenates with $\delta$-aminolaevulinic acid increases the formation of lipid peroxidation products presumably as a result of the induction of free radicals by $\delta$-aminolaevulinic acid. Melatonin coincubation, in both a concentration-dependent and time-dependent manner, prevented the rises in the damaged lipid products malondialdehyde and 4hydroxyalkenals. In vivo as well, acute melatonin administration reduced damaged lipid products in the brain of rats treated with $\delta$-aminolaevulinic acid. PRINC et al. (1997) in similar studies like CARNEIRO and REITER (1998) felt that the ability of melatonin to protect against $\delta$-aminolaevulinic acid toxicity relates to the free radical scavenging activity of the indolamine. Furthermore, PRINC et al. (1998) showed that melatonin administered in vivo not only reduced lipid peroxidation in the brain of rats treated with $\delta$-aminolaevulinic acid, but also increased enzyme activities of the heme pathway.

The dithiol meso-2,3-dimercaptosuccinic acid was the first orally administered metal chelating agent to receive U.S. Food and Drug Administration (FDA) approval for the treatment of childhood plumbism. It reduced oxidative stress in lead exposed C57BL/6 mice in vivo (ERCAL et al. 1996).

Lead sulphide, 6 times $15 \mathrm{mg}$ given intratracheally to rats, was phagocytosed by alveolar macrophages inducing foamy cytoplasm within two months (KISSLER 1983). Alveolar lipoproteinosis occurred 6 months after intratracheal instillation of $50 \mathrm{mg} \mathrm{PbS}$ (Rosmanith et al. 1989).

Lead citrate inhibited oxidative metabolism of mouse peritoneal macrophages exposed to macrophage-activating factor (BUCHMÜLLER-ROUILLER et al. 1989).

Particulate $\mathrm{PbO}, \mathrm{Pb}_{3} \mathrm{O}_{4}$, and $\mathrm{PbO}$-coated coal fly ash of respirable size were ingested by New Zealand white rabbits' lavaged bronchoalveolar macrophages cultured in vitro (DE VRIEs et al. 1983). Swelling of the mitochondria, nuclear membrane, and endoplasmic reticulum was common, as well as were characteristic reprecipitation complexes of lead, phosphorus, and calcium within the nuclear heterochromatin and cytoplasm of the cell. It was suggested that solubilization of lead from the ingested particles in phagosomes of macrophages results in the liberation of intracellular lead with the resultant formation of reprecipitation complexes.
Triethyllead $\left.\left[\mathrm{C}_{2} \mathrm{H}_{5}\right) \mathrm{PbCl}\right]$ in high $(\geq 50 \mu \mathrm{M})$, but not in low concentrations $(\leq 1 \mu \mathrm{M})$ inhibited the incorporation of exogenous $\left[{ }^{14} \mathrm{C}\right]$ arachidonic acid into HL-60 cells (KRUG et al. 1994). Calcium ionophore A 23187-induced stimulation of acetyltransferase, the key enzyme of PAF synthesis, was inhibited only by $40 \mu \mathrm{M}$ of triethyllead, while $50 \mu \mathrm{M}$ blocked the enzyme below basal activity.

Lead caused cell rounding in epithelioid cells by altering the fluidity of the membrane bilayer, making it more vulnerable to osmotic shock (FILERMAN and BerLINER (1980).

\subsection{8 \\ Manganese}

Manganese has the atomic number 25 and the valence electrons are those of the $3 d^{5} 4 s^{2}$ shells. The oxidation states of manganese include $+1,+2,+3$, $+4,+5,+6$, or +7 .

Unlike $\mathrm{Fe}$ and $\mathrm{Cu}$, inorganic complexes of $\mathrm{Mn}$ are known to exist in high concentrations in certain cells as the chlorplast stroma (Kono et al. 1976) and in many lactic acid bacteria (ARCHIBALD and FRIDovich 1981, Archibald 1986). Cheton et al. (1988) used three different free radical generating systems and four $\mathrm{HO}^{\circ}$ detecting methods to investigate the activity of biologically relevant inorganic Mn complexes. When using $\mathrm{HO}^{\bullet}$ generator systems (hypoxanthine-xanthine oxidase system; formula [45]) in which $\mathrm{O}_{2}$ derived $\mathrm{O}_{2}{ }^{--}$and $\mathrm{H}_{2} \mathrm{O}_{2}$ are apparently precursors of $\mathrm{HO}^{\circ}$, simple $\mathrm{Mn}$ complexes were effective in reducing oxidative effects generally believed to be $\mathrm{HO}^{\circ}$ dependent, while in systems not requiring $\mathrm{O}_{2}{ }^{--}$and $\mathrm{H}_{2} \mathrm{O}_{2}$ as precursors for $\mathrm{HO}^{\circ}$ generation $\left({ }^{137} \mathrm{Cs} \gamma\right.$-irradiation), Mn complexes were ineffective.

Magnetic resonance techniques have been applied to study the stability of the complexes formed between $\mathrm{Mn}$ (II) ions and NADP in aqueous solutions at a pH of 7.5 and $20^{\circ}$ (GreEN and КотоWyCZ 1979).

A purely chemical system for $\mathrm{NAD}(\mathrm{P}) \mathrm{H}$ oxidation to biologically active $\mathrm{NAD}(\mathrm{P})^{+}$has been developed and characterised by PAOLEtTi et al. (1990). Suitable amounts of EDTA, manganous ions and mercaptoethanol, combined at physiological $\mathrm{pH}$, induce nucleotide oxidation through a chain length also involving molecular oxygen, which eventually undergoes quantitative reduction to hydrogen peroxide. $\mathrm{Mn}^{2+}$ is specifically required for activity, while both EDTA and mercaptoethanol can be replaced by analogues. Two steps appear of special importance in nucleotide oxidation: (a) the supposed transient formation of $\operatorname{NAD}(\mathrm{P})^{\bullet}$ from the reaction between $\mathrm{NAD}(\mathrm{P}) \mathrm{H}$ and thiyl radicals; (b) 
the oxidation of the reduced complex by superoxide to keep thiol oxidation cycling.

The manganese in resting MnSOD is trivalent, and the catalytic cycle of this enzyme involves reduction and then reoxidation of the metal centre during successive encounters with $\mathrm{O}_{2}{ }^{-}$. In contrast, the stable form of manganese in aqueous solutions is $\mathrm{Mn}(\mathrm{II})$, while $\mathrm{Mn}(\mathrm{III})$, a strong oxidant, tends to equilibrate with $\mathrm{Mn}$ (II) and $\mathrm{Mn}$ (IV).

Replacement of haeme in prostaglandin $\mathrm{H}$ synthase-1 by mangano protoporphyrin IX gives a holoenzyme that retains most cyclooxygenase activity but only $\sim 4 \%$ of the peroxidase activity (ODENWALler et al. 1992, Strieder et al. 1992, KulmaCZ et al. 1994). Lassmann et al. (1991) and KulmaCz et al. (1994) demonstrated that a 35-38 G radical was produced in mangano prostaglandin $\mathrm{H}$ synthase-1 during aerobic reaction with either fatty acid peroxide, 15-hydroperoxyeicosatetraenoic acid or arachidonic acid. The mangano prostaglandin $\mathrm{H}$ synthase-1 radical structure was sensitive to the tyrosine modifying agent, tetranitromethane, and the kinetics of its formation appeared to correlate with production of prostaglandin $\mathrm{G}_{2} /$ prostaglandin $\mathrm{H}_{2}$ (KulmaCz et al. 1994). The EPR data of TsaI et al. (1998) for the arachidonic acid-derived radical formed by prostaglandin $\mathrm{H}$ synthase- 2 and mangano prostaglandin $\mathrm{H}$ synthase-1 could be accounted for by a planar pentadienyl radical with two strongly interacting $\beta$-protons at $\mathrm{C} 10$ of arachidonic acid.

$\mathrm{Mn}^{\mathrm{II}}$ forms an adduct with $\mathrm{O}_{2}{ }^{\circ-}$, much as vanadate does (BIELSKI and CHAN 1978):

$$
\mathrm{Mn}^{\prime \prime}+\mathrm{O}_{2}^{--} \longleftrightarrow \mathrm{Mn}^{\prime} \mathrm{OO}
$$

$\mathrm{Mn}^{\mathrm{I}} \mathrm{OO}^{\bullet}$ can either act as an oxidant directly or, in the presence of chelating agents capable of stabilising $\mathrm{Mn}^{\mathrm{III}}$, can yield $\mathrm{Mn}^{\mathrm{III}}$ which, in turn, acts as an oxidant:

$\mathrm{Mn}^{\prime} \mathrm{OO}^{\circ}+\mathrm{NADPH} \leftrightarrow \mathrm{Mn}^{\mathrm{O} O O H}+\mathrm{NADP} \cdot$

$$
\mathrm{Mn} \mathrm{OOH}+\mathrm{H}^{+} \leftrightarrow \mathrm{Mn}^{\mathrm{Il}}+\mathrm{H}_{2} \mathrm{O}_{2}
$$

Ligand-Mn'OO* $+2 \mathrm{H}^{+} \leftrightarrow$ Ligand- $\mathrm{Mn}^{\text {III }}+\mathrm{H}_{2} \mathrm{O}_{2}$

Ligand-Mn"II $+\mathrm{NADPH} \leftrightarrow$ Ligand-Mn" $+\mathrm{NADP}+\mathrm{H}^{+}$

$\mathrm{H}_{2} \mathrm{O}_{2}$ is a powerful agent reducing $\mathrm{Mn}^{\mathrm{III}}$. ARCHIBALD and FRIDOVICH (1982) quote the following reactions:

$$
\begin{aligned}
& \mathrm{Mn}^{\prime \prime \prime}+\mathrm{H}_{2} \mathrm{O}_{2} \rightarrow \mathrm{MnO}_{2}{ }^{+}+{ }^{2} \mathrm{H}^{+} \\
& \mathrm{MnO}_{2}{ }^{+}+\mathrm{Mn}^{\mathrm{III}} \rightarrow(\mathrm{Mn}-\mathrm{O}-\mathrm{O}-\mathrm{Mn})^{+4} \\
& (\mathrm{Mn}-\mathrm{O}-\mathrm{O}-\mathrm{Mn})^{+4} \rightarrow 2 \mathrm{Mn}(\mathrm{II})+\mathrm{O}_{2}
\end{aligned}
$$

The hydroxyl radical, $\mathrm{HO}^{*}$, reacts with $\mathrm{Mn}^{2+}$ :

$$
\mathrm{Mn}^{2+}+\mathrm{HO}^{*} \rightarrow \mathrm{Mn}(\mathrm{OH})^{2+}
$$

A purely chemical system for $\mathrm{NAD}(\mathrm{P}) \mathrm{H}$ oxidation to biologically active $\mathrm{NAD}(\mathrm{P})^{+}$has been developed by PAOLETti et al. (1990). $\mathrm{Mn}^{2}$ is specifically required for activity, while both EDTA and thioethanol can be replaced by analogues. Optimal molar ratios of chelator/metal ion $(2: 1)$ yield an active coordination compound which catalyses thiol autoxidation to thiyl radical. The latter is further oxidised to disulphide by molecular oxygen whose one-electron reduction generates superoxide radical.

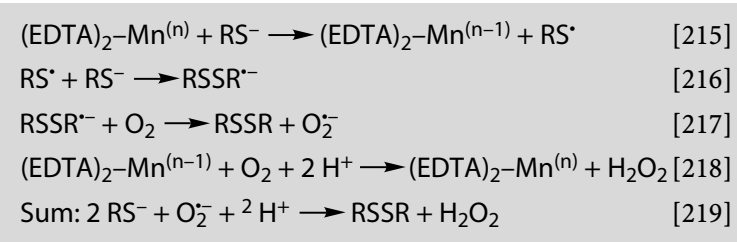

Reaction [215] represents the initial event in thiyl radical formation as proposed for the so-called spontaneous autoxidation of thiols catalysed by metal ion complexes (Misra 1974, SAEZ et al. 1982, HARMAN et al. 1984). The propagation (reactions [216] and [217]) leads to thiol oxidation and contemporary superoxide radical formation. The latter might be reduced to hydrogen peroxide by reacting with $(\mathrm{EDTA})_{2}-\mathrm{Mn}^{(\mathrm{n}-1)}$ to yield back the oxidised complex and close the catalytic cycle.

The importance of manganese and its compounds for industrial medicine and the uncertainty of the MPC values valuable now were emphasised by Pflaumenbaum et al. (1990).

Manganese is extracted from pyrolusite $\left(\mathrm{MnO}_{2}\right)$, braunite $\left(\mathrm{Mn}_{2} \mathrm{O}_{3}\right)$, hausmannite $\left(\mathrm{Mn}_{3} \mathrm{O}_{4}\right)$, and rhodochromite $\left(\mathrm{MnCO}_{3}\right.$, manganese spar).

CAMner et al. (1985) found an increase in the size of alveolar macrophages lavaged from rabbits exposed to an aerosol of $3.9 \pm 1.0 \mathrm{mg} \mathrm{Mn} / \mathrm{m}^{3}$ for 4-6 weeks ( 5 days/week, $6 \mathrm{~h} /$ day).

The toxicity of insoluble manganese dioxide dusts depends on the particle surface area as demonstrated in vitro by lactate dehydrogenase release from NMRI mouse peritoneal macrophages elicited by casein hydrolysate (LISON et al. 1997). In vivo, the lung inflammatory response was assessed by analysis of bronchoalveolar lavage after intratracheal instillation in mice (LDH activity and protein concentration in the cell-free fraction, recruitment of polymorphonuclear leucocytes). Freshly ground particles with a specific surface area of $5 \mathrm{~m}^{2} / \mathrm{g}$ in 
vitro exhibited an enhanced cytotoxic activity, which was almost equivalent to that of $62 \mathrm{~m}^{2} / \mathrm{g}$ particles, indicating that undefined reactive sites produced at the particle surface by mechanical cleavage may also contribute to the toxicity of insoluble particles.

Respiratory burst activity in lung macrophages increased at $61 \%$ and $68 \%$ after exposure to $10 \mu \mathrm{M}$ and $50 \mu \mathrm{M}$ manganese, respectively (GRABOwsKI et al. 1996). No increase in the degree of tyrosine phosphorylation in lysate from manganese exposed lung macrophages was evident. Despite the lack of increased tyrosine phosphorylation, the respiratory burst by manganese was reduced approximately $50 \%$ by the NADPH oxidase inhibitor, 2-deoxy-Dglucose.

\subsection{9}

\section{Mercury}

Mercury has the atomic number 80 and the valence electrons are those of the $6 s^{1}$ shell. The oxidation states of mercury include +1 and +2 .

Nitrosothiols are very readily decomposed by the addition of mercuric ion (well-known for its coordinating ability to sulphur). This reaction results in $\mathrm{NO}^{+}$expulsion leading to nitrous acid in acid solution and nitrite anion formation at $\mathrm{pH}>$ ca 3 (equation [220]).

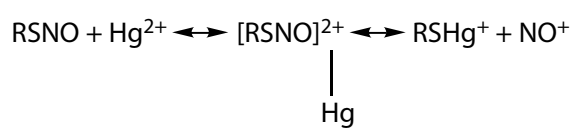

[220]

Even when carried out anaerobically there is no -NO formation and this reactions shows very little of the structural dependence so pronounced in the copper reactions (SWIFT and Williams).

Primary exposure to mercury occurs through environmental contamination as the result of mining, smelting, and industrial discharge, and include ingestion via inhalation and the food chain (GOYER 1991). The decrease in free sulfhydryl groups may lead to the formation of an oxidative stress, resulting in tissue-damaging effects.

Alveolar macrophages gained by lung lavage of freshly slaughtered, healthy calves, incubated with $\mathrm{Hg}^{2+}$ in the range of $0.25,0.5,1.0,1.5,2.0$, and 5.0 $\mu \mathrm{mol} / \mathrm{l}$ showed almost no rosetting with opsonized erythrocytes in the range of 1 to $5 \mu \mathrm{mol} / \mathrm{l}$ after $1 \mathrm{~h}$ incubation (Buss et al. 1991).

\subsection{0}

\section{Montmorillonite}

The K10 montmorillonite clay catalysed the clean backbone rearrangement of cholest-5-ene into cholest-13(17)-enes; the transformation shown is quantitative and can be run on the gram scale (Sieskind and Albrecht 1985).

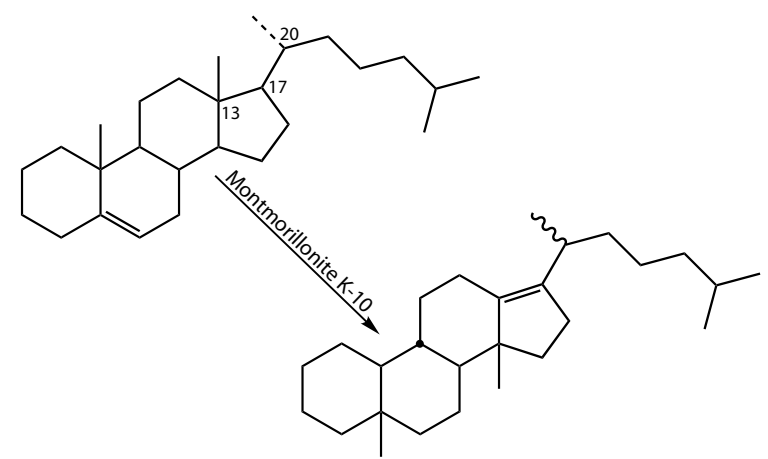

Rearrangement of cholest-5-ene into cholest-13(17)-enes [221]

The term "fuller's earth" is applied to a number of clay minerals capable to remove impurities from animal, plant and mineral oils. The soft, flocky, mica like minerals are open-pit mined and purified by calcination and washing.

Pneumoconioses after the inhalation of particulate fuller's earth from the Nutfield district were reported by Middleton (1940) and CAMPBELl and Gloyne (1942), also from Surrey by TonNing (1949), and from Olmstead, Illinois, USA by MCNAlly and Trostler (1941). After an exposure of 38 years, CAmpbell and Gloyne (1942) found the alveoli in the more severely affected areas of the lung involved in a reticular fibrosis surrounding the dust particles; macrophages, mononuclear cells, fibroblasts and leucocytes were present, but they were not very numerous and no foreign body-giant cells were observed. In Tonning's case, the exposure was some 50 years ago, and the dust was contained in large macrophages held in a mesh of fine reticulin fibres.

In rats, illite and muscovite, but not kaolin produced lesions of alveolar proteinosis (MARTIN et al. 1977). Reisner et al. (1982) and Rosmanith et al. (1982) emphasised that the relation between the kaolinite content of the lymph nodes and that of the lungs six months after an intratracheal injection of different West German coal mine dusts showed an exponential regression function by the method of least squares.

In vitro illite and bentonite decreased intracellular lactate dehydrogenase activity of glycogen elicited peritoneal macrophages (ADAMIS and TIMÁr 1976, 1978). Groups which adsorbed paraquat cations may have a role in the cytotoxic effect of bentonite (ADAMIS and Timár 1976).

The complex multilayered structure of mica with its surface exposed negatively charged honeycomb arrangements of $\mathrm{Si}(\mathrm{Al}) \mathrm{O}_{4}$ tetrahedra has been used 
as a substrate for monolayer formation of amphiphilic organic molecules, such as alkylphosphonic acids (WoodWARD and Schwartz 1996) and organosilanes (SCHWARTz et al. 1992, OKusa et al. 1994, Hu et al. 1996, XIAO et al. 1996). As an alternative to the attachment of a two-dimensional siloxane network onto the mica surface, efforts have been made to alter the surface chemistry by exchanging the surface cations (mostly potassium) at the basal $\{001\}$ cleavage plane with other inorganic and organic ions (Shelden et al. 1993, HèHner et al. 1996). Surfactant adsorption of long-chain alkylammonium salts, such as hexadecyl-trimethylammonium bromide (ERIKsSON et al. 1996, SHARMA et al. $1996)$ and $N$-dodecyl-pyridinium chloride (SHEL-

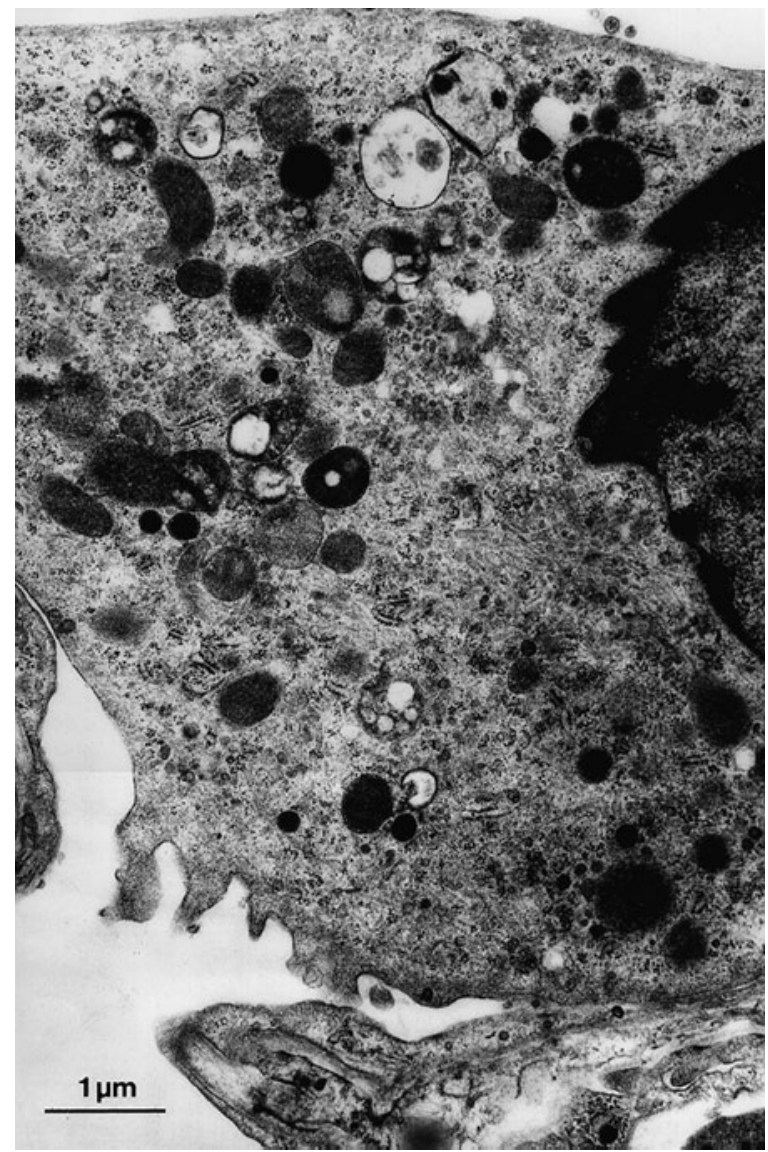

Fig. 144. Alveolar macrophage (block 4480) of a $201 \mathrm{~g}$ Sprague-Dawley rat (Charles River, France) instilled intratracheally with $50 \mathrm{mg}$ montmorillonite API H 26 suspended in $0.5 \mathrm{ml}$ saline. The animal was treated daily for 4 consecutive days with intraperitoneal injection of $15 \mathrm{mg}$ carbochromene per $\mathrm{kg}$ body weight. Under pentobarbital anaesthesia $(30 \mathrm{mg} /$ $\mathrm{kg}$ ), the lung was fixed by intratracheal instillation of $2.5 \%$ glutaraldehyde in $0.1 \mathrm{M}$ sodium cacodylate buffer ( $\mathrm{pH} 7.4$ ). Postfixation with $1 \%$ osmium tetroxide in sodium cacodylate buffer. Embedded in Epon 812 and sectioned at $50 \mathrm{~nm}$. Lead citrate and uranyl acetate. Plate 4132
DEN et al. 1993) are known to hydrophobize negatively charged minerals. Exchange with bivalent cations has been used to mediate binding of DNA for SPM studies (SHELDEN et al. 1994). Muscovite surfaces reversibly, site-specifically immobilised polyarginine-tagged fusion proteins (Nock et al. 1997).

Alveolar macrophages were stimulated by montmorillonite not only after inhalation or intratracheal instillation (Fig. 144) of the dust, but also by intraperitoneal application (Figs. 145, 146).

A circulus vitiosus (Von ZgLinicki and BRUNK 1993) comes into play because several damaged mitochondria have to be degraded in lysosomes. If lipofuscin accumulation in lysosomes decreases their degradative efficiency, damaged mitochondria producing high amounts of hydrogen peroxide will stay undegraded for longer times both outside and within lysomes.

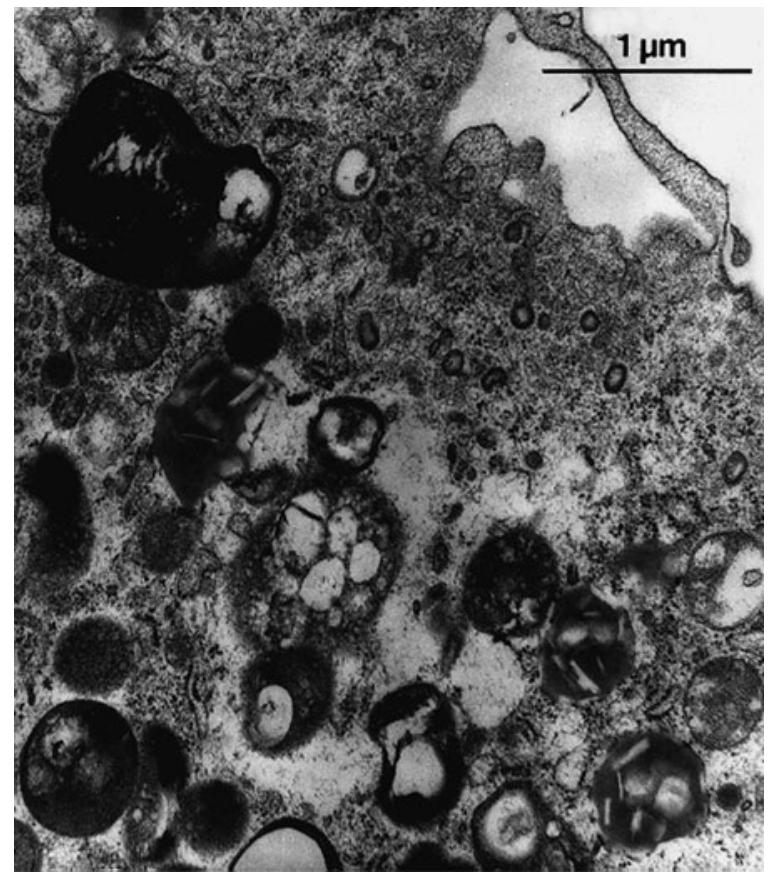

Fig. 145. Alveolar macrophage (block 4473) from a $215 \mathrm{~g}$ Sprague-Dawley rat (Charles River, France) injected intraperitoneally with $50 \mathrm{mg}$ montmorillonite API H 26 suspended in $2 \mathrm{ml}$ saline. Four days later, on July 25, 1978 under pentobarbital anaesthesia $(30 \mathrm{mg} / \mathrm{kg})$, the animal was perfused from the abdominal aorta with $2.5 \%$ glutaraldehyde in $0.1 \mathrm{M}$ sodium cacodylate buffer ( $\mathrm{pH} 7.4$ ). Postfixation with $1 \%$ osmium tetroxide in sodium cacodylate buffer. Embedded in Epon 812 and sectioned at $50 \mathrm{~nm}$. Lead citrate and uranyl acetate. Film 288/79 


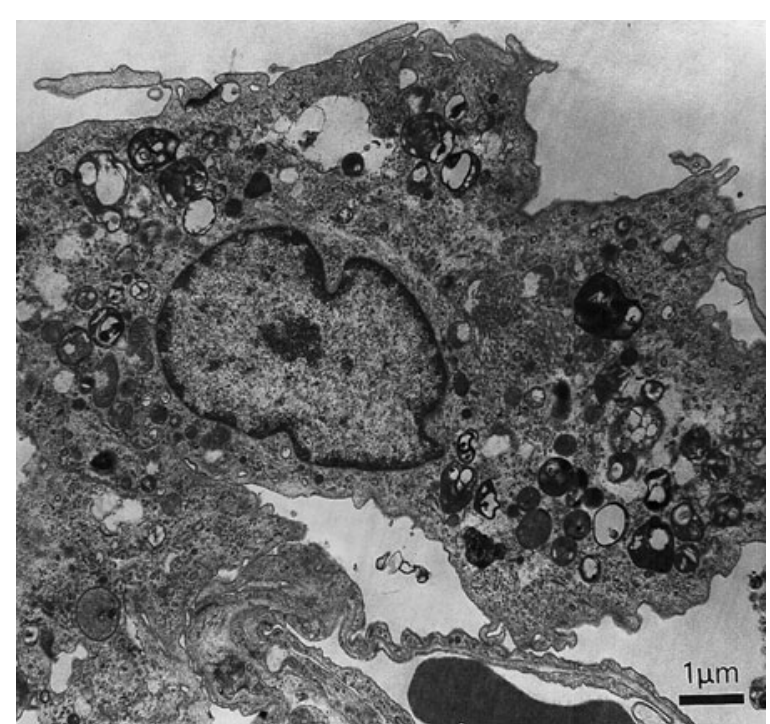

Fig. 146. Alveolar macrophage (block 4473) of a $215 \mathrm{~g}$ Sprague-Dawley rat (Charles River, France) injected intraperitoneally with $50 \mathrm{mg}$ montmorillonite suspended in $2 \mathrm{ml} \mathrm{sa}-$ line. 4 days later under pentobarbital anaesthesia $(30 \mathrm{mg} / \mathrm{kg})$, the animal was perfused from the abdominal aorta with $2.5 \%$ glutaraldehyde in $0.1 \mathrm{M}$ sodium cacodylate buffer ( $\mathrm{pH}$ 7.4). Postfixation with $1 \%$ osmium tetroxide in sodium cacodylate buffer. Embedded in Epon 812 and sectioned at $50 \mathrm{~nm}$. Lead citrate and uranyl acetate. Film 286/79

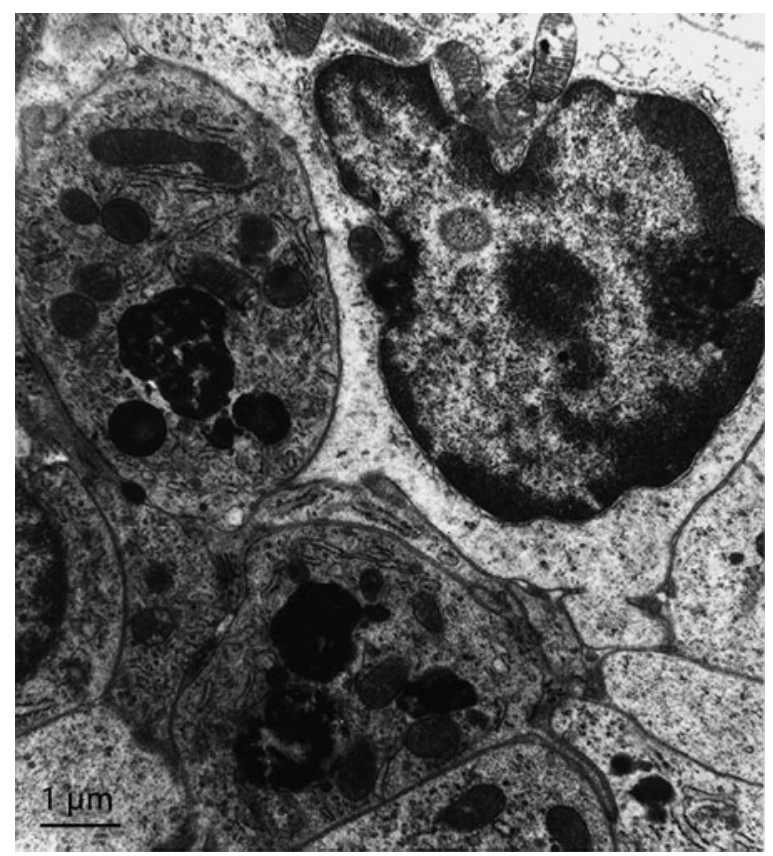

Fig. 147. Montmorillonite API H 26-laden macrophages in a thoracic lymph node (block 4471) of a $224 \mathrm{~g}$ Sprague-Dawley rat (Charles River, France) injected intraperitoneally with 50 mg montmorillonite API $\mathrm{H} 26$ suspended in $2 \mathrm{ml}$ saline. 4 days later under pentobarbital anaesthesia $(30 \mathrm{mg} / \mathrm{kg})$, the animal was perfused from the abdominal aorta with $2.5 \%$ glutaraldehyde in $0.1 \mathrm{ml}$ sodium cacodylate buffer ( $\mathrm{pH} 7.4)$. Postfixation with $1 \%$ osmium tetroxide in sodium cacodylate buffer. Embedded in Epon 812 and sectioned at $50 \mathrm{~nm}$. Lead citrate and uranyl acetate. Film 295/79
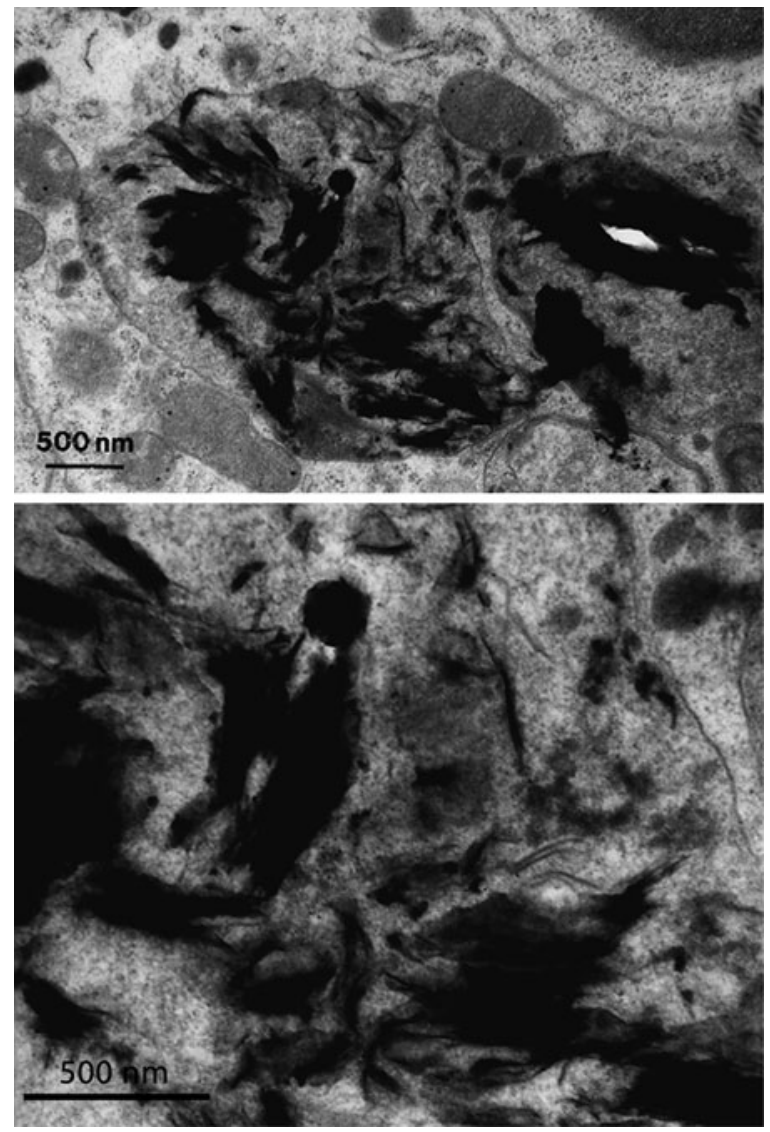

Fig. 148. Details of the montmorillonite API H 26 particles in a dust-laden macrophage in a thoracic lymph node (block 4471) of a 224 g Sprague-Dawley rat (Charles River, France) injected intraperitoneally with $50 \mathrm{mg}$ montmorillonite suspended in $2 \mathrm{ml}$ saline. 4 days later under pentobarbital anaesthesia $(30 \mathrm{mg} / \mathrm{kg})$, the animal was perfused from the abdominal aorta with $2.5 \%$ glutaraldehyde in $0.1 \mathrm{ml}$ sodium cacodylate buffer ( $\mathrm{pH}$ 7.4). Postfixation with $1 \%$ osmium tetroxide in sodium cacodylate buffer. Embedded in Epon 812 and sectioned at $50 \mathrm{~nm}$. Lead citrate and uranyl acetate. Films 33121 and 33122 


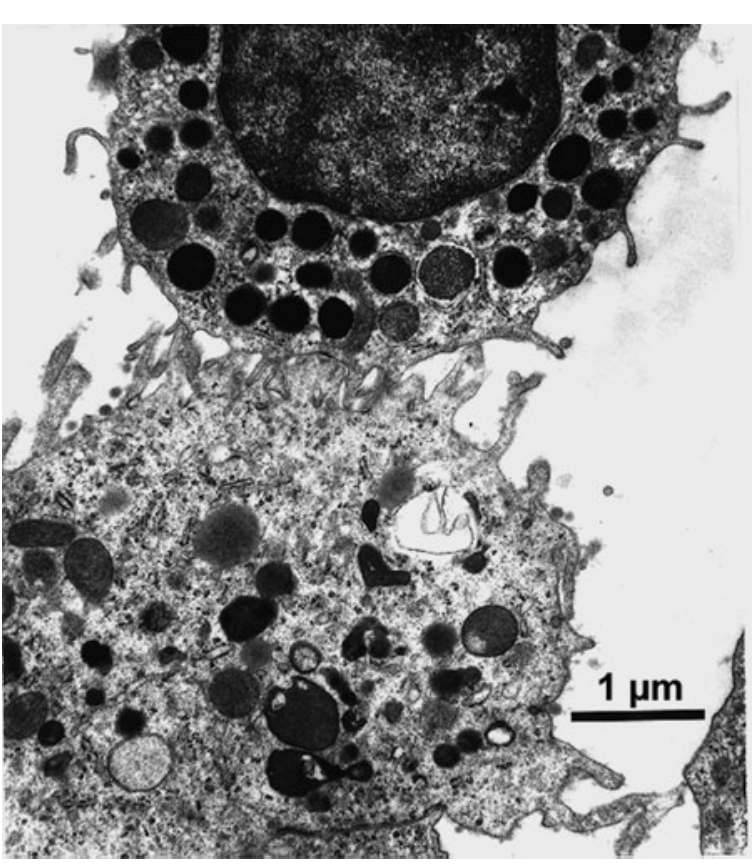

Fig. 149. Intimate contact of the plasmalemmata of a mononuclear phagocyte (bottom) and a mast cell (top) in a mesenteric lymph node (block 4477) of a $215 \mathrm{~g}$ Sprague-Dawley rat (Charles River, France) injected intraperitoneally with $50 \mathrm{mg}$ montmorillonite API H 26 suspended in $2 \mathrm{ml}$ saline. 4 days later under pentobarbital anaesthesia $(30 \mathrm{mg} / \mathrm{kg})$, the animal was perfused from the abdominal aorta with $2.5 \%$ glutaraldehyde in $0.1 \mathrm{ml}$ sodium cacodylate buffer ( $\mathrm{pH} 7.4$ ). Postfixation with $1 \%$ osmium tetroxide in sodium cacodylate buffer. Embedded in Epon 812 and sectioned at $50 \mathrm{~nm}$. Lead citrate and uranyl acetate. Film 271/79

Fig. 151. Segregation of a damaged mitochondrium, cisternae of the rough endoplasmic reticulum, and free ribosomes by a phagophore within a macrophage from a mesenteric lymph node (block 4470) of a $224 \mathrm{~g}$ Sprague-Dawley rat (Charles River, France) injected intraperitoneally with $50 \mathrm{mg}$ montmorillonite suspended in $2 \mathrm{ml}$ saline. 4 days later under pentobarbital anaesthesia $(30 \mathrm{mg} / \mathrm{kg})$, the animal was perfused from the abdominal aorta with $2.5 \%$ glutaraldehyde in $0.1 \mathrm{M}$ sodium cacodylate buffer ( $\mathrm{pH} 7.4$ ). Postfixation with $1 \%$ osmium tetroxide in sodium cacodylate buffer. Embedded in Epon 812 and sectioned at $50 \mathrm{~nm}$. Lead citrate and uranyl acetate. Film 299/79

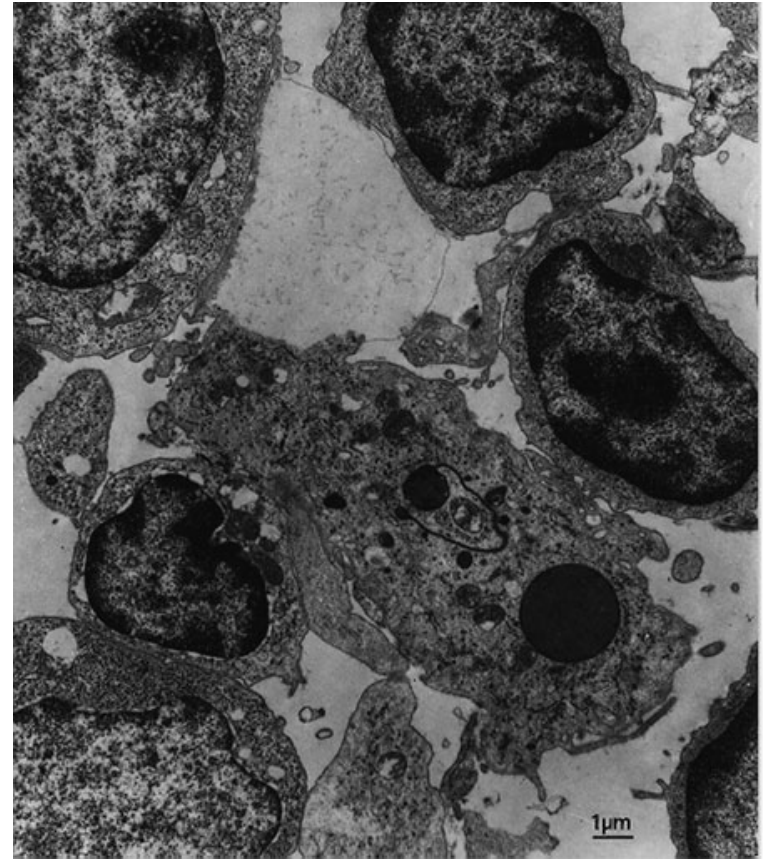

Fig. 150. Segregation of a damaged mitochondrium, cisternae of the rough endoplasmic reticulum, and free ribosomes by a limiting membrane within a macrophage from a mesenteric lymph node (block 4470) of a $224 \mathrm{~g}$ Sprague-Dawley rat (Charles River, France) injected intraperitoneally with $50 \mathrm{mg}$ montmorillonite suspended in $2 \mathrm{ml}$ saline. 4 days later under pentobarbital anaesthesia $(30 \mathrm{mg} / \mathrm{kg})$, the animal was perfused from the abdominal aorta with $2.5 \%$ glutaraldehyde in $0.1 \mathrm{M}$ sodium cacodylate buffer ( $\mathrm{pH}$ 7.4). Postfixation with $1 \%$ osmium tetroxide in sodium cacodylate buffer. Embedded in Epon 812 and sectioned at $50 \mathrm{~nm}$. Lead citrate and uranyl acetate. Film 298/79

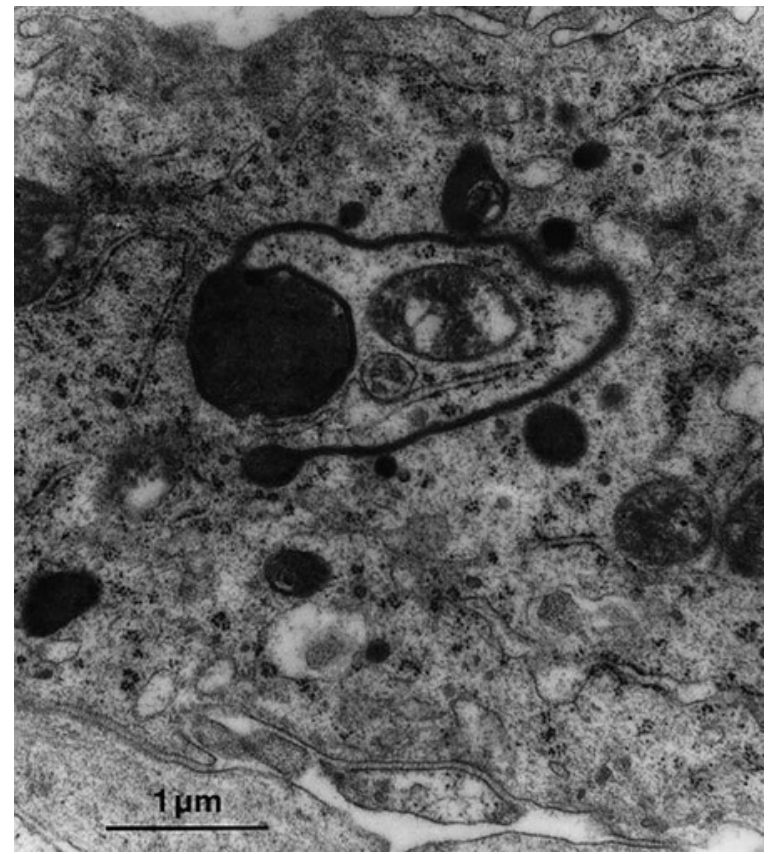




\subsection{1 Nickel}

Nickel has the atomic number 28 and the valence electrons are those of the $3 d^{8} 4 s^{2}$ shells. The oxidation states of nickel include $-1,0,+1,+2,+3$, or +4 , but the most prevalent oxidation state is +2 .

The chemical and biological properties of nickel are highly dependent on its surrounding ligands. Both the coordination geometry and oxidation-reduction potential are significantly altered when simple salts forming $\mathrm{Ni}-\left(\mathrm{H}_{2} \mathrm{O}\right)_{6}{ }^{2+}$ in aqueous solution are chelated by strong donor ligands. Under physiological conditions, simple nickel salts exhibit little intrinsic oxidation or reduction chemistry particularly in contrast to iron, copper and cobalt (KASPRZAK 1991, HAUSINGER 1993). However, nickel binds to the backbone of peptides and proteins, it becomes quite easily oxidised by $\mathrm{O}_{2}, \mathrm{O}_{2}{ }^{-}$, and $\mathrm{H}_{2} \mathrm{O}_{2}$ (Bossu et al. 1977, 1978). GGH$\mathrm{Ni}(\mathrm{II})$, glutathione-Ni(II), and other oligopeptide complexes of $\mathrm{Ni}$ (II) have been shown to promote lipid and protein oxidation in the presence of peroxides to a much greater extent than either free $\mathrm{Ni}^{2+}$ or ligand alone (SHI et al. 1992). Similarly, GGGG-Ni(II) induced polymerisation of histones (KASPRZAK and BARE 1989), and histidine-Ni facilitated oxidation of deoxyguanosine (DATTA et al. 1992).

Mononuclear thiolate complexes containing nickel sites in different oxidation state as $\left[\mathrm{Ni}\left(\mathrm{SC}_{6} \mathrm{H}_{4} \mathrm{O}\right)_{2}\right]^{2-}$ and $\left[\mathrm{Ni}\left(\mathrm{SC}_{6} \mathrm{H}_{4} \mathrm{O}\right)_{2}\right]^{-}$were described by Köckerling and Henkel (1993). With respect to the chemical as well as electrochemical properties of the nickel sites of various dehydrogenases, $\left[\mathrm{Ni}\left(\mathrm{SC}_{6} \mathrm{H}_{4} \mathrm{O}\right)_{2}\right]^{-}$is a relevant model complex of coordination number four.

$\mathrm{Ni}^{2+}$ forms a lipophilic complex with pyridinethione by bonding through the oxygenated sulphur atoms. Lipophilic complex formation may promote metal absorption and tissue penetration. Oral administration of sodium pyridinethione together with ${ }^{63} \mathrm{Ni}^{2+}$ to rats, guinea-pigs and ferrets was found to induce highly increased tissue levels of the metal in comparison with animals given the $\mathrm{Ni}^{2+}$ alone (Borg-NecZaK and TJäLve 1994).

Nickel exists in many forms as an inhalable pollutant which include metallic nickel, nickel subsulphide, nickel chloride, nickel oxide, and nickel carbonyl. Of these compounds, $\mathrm{Ni}_{3} \mathrm{~S}_{2}$ is recognised as the most carcinogenic (SUNDERMAN 1983). The majority of nickel-associated lesions described in man have been caused by inhalation exposure (WEHNER et al. 1975). Immune mechanisms appear to be particularly vulnerable to the effects of nickel compounds.

In an aqueous medium reactive oxygen species were generated by powdered $\mathrm{Ni}_{2} \mathrm{O}_{3}$ when $\mathrm{Ni}(\mathrm{II})$ were converted to Ni(III) ions (SAWATARI 1988).

\section{One-electron transfer catalysed by nickel}

$\mathrm{O}_{2}+\mathrm{Ni}^{2+} \longleftrightarrow \mathrm{Ni}^{3+}-\mathrm{O}_{2}^{-} \longrightarrow \mathrm{O}_{2}^{-}+\mathrm{Ni}^{3+}$

$\mathrm{O}_{2}^{--}+\mathrm{Ni}^{2+}+2 \mathrm{H}^{+} \rightarrow \mathrm{H}_{2} \mathrm{O}_{2}+\mathrm{Ni}^{3+}$

$\mathrm{H}_{2} \mathrm{O}_{2}+\mathrm{Ni}^{2+} \rightarrow \mathrm{HO}^{\circ}+\mathrm{HO}^{-}+\mathrm{Ni}^{3+}$

Solubilities in distilled water and saline were in the order of nickel fumes $\left(97 \% \mathrm{NiO}, 3 \% \mathrm{Ni}_{2} \mathrm{O}_{3}\right.$; particle size 5-10 nm) $>\mathrm{Ni}_{2} \mathrm{O}_{3}$ powder (particle size $2.0 \pm 1.69 \mu \mathrm{m})>>\mathrm{NiO}$ powder (particle size $2.2 \pm 1.68 \mu \mathrm{m}$ ) (ToyA et al. 1997).

Phagocytic indices of particulate nickel compounds by rat peritoneal macrophages in vitro were ranked by KuEHN et al. (1982) as follows: $\mathrm{NiO}>$ $\mathrm{Ni}_{4} \mathrm{FeS}_{4}>\mathrm{NiTiO}_{3}>\mathrm{NiSe}>\alpha \mathrm{Ni}_{3} \mathrm{~S}_{2}>\mathrm{Ni}>\mathrm{Ni}_{5} \mathrm{As}_{2}$ $>\mathrm{NiS}_{2}>\mathrm{NiFe}$ alloy $>\mathrm{NiSb}>\mathrm{Ni}_{11} \mathrm{As}_{8}>\mathrm{Ni}_{3} \mathrm{Se}_{2}>$ $\beta \mathrm{NiS}>\mathrm{NiTe}>\mathrm{NiAs}>\mathrm{NiAsS}>$ amorphous NiS. Rank-correlation $(P<0.03)$ was observed between the phagocytic indices of the nickel compounds and their dissolution half-times in rat serum. Nickel subsulphide, $\alpha \mathrm{Ni}_{3} \mathrm{~S}_{2}$ was a notable exception to the general concordance between phagocytic indices and dissolution half-times: $\alpha \mathrm{Ni}_{3} \mathrm{~S}_{2}$ was avidly phagocytized by macrophages, yet it had one of the shortest dissolution half-times. Preliminary results of the carcinogenesis tests of 14 of the nickel compounds did not indicate significant rankcorrelation between the phagocytic indices of the nickel compounds and the sarcoma indices at 1 year after intramuscular administration of the compounds to rats.

\section{Metallic Nickel}

In manual metal arc and shielding gas welding fumes nickel is dissolved in the iron oxide fume particles and in small amounts it exists as separate nickel oxide particles.

Alveolar macrophages from rabbits exposed to $0.5 \mathrm{mg} \mathrm{Ni}$ dust $/ \mathrm{m}^{3} 6 \mathrm{~h}$ per day during a 5 -day work week after 4 weeks showed highly undulating membranes, numerous slender microvilli and long protrusions from the cell surface, excentrically placed polymorphic nuclei with chromatin marginations, and well-developed nucleoli (CAMNER et al. 1978). There were also membrane-bound bodies containing laminated structures of different sizes, similar to the laminated structures of alveolar type II cells. Lysosomal structures were absent in nickelexposed cells found to contain large amounts of laminated structures, which was in contrast to the control macrophages. X-ray microanalysis revealed no intracellular $\mathrm{Ni}$ in the in vivo experiment, while in vitro macrophages internalized large amounts of Ni particles. 
Instillation of $0.1 \mathrm{~g}$ Ni dust suspended in $1 \mathrm{ml}$ isotonic saline into the rabbit's trachea induced macrophages with a surface rich in long protrusions and microvilli (WIERNIK et al. 1981). The cytoplasm contained many lamellar bodies and lipid droplets. The nuclei of the cells were polymorphic and had well developed nucleoli. The Golgi areas were prominent and so was the granular endoplasmic reticulum. The nitroblue tetrazolium reduction in macrophages from nickel-exposed rabbits showed, without any incubation, values of the same magnitude as from the corresponding cells lavaged from the lungs of unexposed rabbits after incubation with the surfactant from nickel-treated animals.

\section{Nickel Subsulphide}

In vitro, phagocytosis of particulate $\mathrm{Ni}_{3} \mathrm{~S}_{2}$ resulted in a rapid dissolution of the compound once it had entered the macrophage with subsequent release of $\mathrm{Ni}^{2+}$ ion (CostA et al. 1981). Scanning electron micrographs of bovine macrophages exposed to $\mathrm{Ni}_{3} \mathrm{~S}_{2}$ showed loss of surface features, membrane disruption, and bleb formation (FISHER and PlaCKE 1987).

'NO generation from RAW 264.7 Abelson virustransformed murine macrophages stimulated with lipopolysaccharide $(0.5$ or $1 \mu \mathrm{g} / \mathrm{ml})$ was enhanced by $\mathrm{Ni}_{3} \mathrm{~S}_{2}$ in a dose-dependent manner (KAWANISHI et al. 2001). The addition of $100 \mu \mathrm{g} \mathrm{Ni}_{3} \mathrm{~S}_{2} / \mathrm{ml}$ caused strong cell toxicity. Nickel compounds did not induce ${ }^{\circ} \mathrm{NO}$ production in the absence of lipopolysaccharide. When RAW 264.7 cells were stimulated with $100 \mathrm{U} / \mathrm{ml}$ interferon- $\gamma, \mathrm{Ni}_{3} \mathrm{~S}_{2}$ enhanced the $\mathrm{NO}_{2}{ }^{-}$accumulation. A nonselective NOS inhibitor, $N^{\mathrm{G}}$-monomethyl-L-arginine, inhibited enhancement of ${ }^{\circ} \mathrm{NO}$ production, suggesting that the enhanced accumulation of $\mathrm{NO}_{2}^{-}$was due to induction of NOS.

Repeated inhalation of 0.6 or $2.5 \mathrm{mg} \beta-\mathrm{Ni}_{3} \mathrm{~S}_{2} / \mathrm{m}^{3}$, $6 \mathrm{~h} /$ day for up to 22 days by male and female F344/ $\mathrm{N}$ rats aged 7 weeks at the start of the exposure resulted in an exposure-concentration-dependent increase of lactate dehydrogenase activity in the bronchoalveolar lavage fluid (BENSON et al. 1995). $\beta$ Glucuronidase activity increased more pronounced in rats exposed to $2.5 \mathrm{mg}$ than to $0.6 \mathrm{mg} \mathrm{Ni} \mathrm{Ni}_{2} /$ $\mathrm{m}^{3}$.The viability of the alveolar macrophages was significantly reduced after 2 days of exposure, but more slowly thereafter.

Male Cynomolgus monkeys instilled intratracheally with $800 \mathrm{nM} \mathrm{Ni}_{3} \mathrm{~S}_{2} / \mathrm{g}$ lung showed mild focal accumulation of macrophages and lymphocytes within the pulmonary interstitium and alveoli, perivenular lymphoid infiltration and follicles with a central core of large monocytes, macrophages, and lymphoblasts surrounded by a mantle of small, darkly basophil lymphocytes (HALEY et al. 1987).

\section{Nickel Chloride}

Acute and chronic inhalation exposure of rabbits and mice to $\mathrm{NiCl}_{2}$ consistently resulted in deficient macrophage function as indicated by decreased phagocytic and bactericidal capacity of pulmonary alveolar macrophages (ADKINs et al. 1979, WIERNIK et al. 1983). Viability of macrophages in these studies was unchanged, reflecting a subtile functional abnormality that has been reproduced in vitro (Graham et al. 1975).

Washed alveolar macrophages from rats exposed ( $8 \mathrm{~h}$ per day, 5 days per week, for 18 days) to $\mathrm{NiCl}_{2}$ $\left(109 \mu \mathrm{g} / \mathrm{m}^{3}\right)$ aerosols were found to contain reduced quantities of the various hydrolytic enzymes (except for acetylesterase) when compared with those from control rats (MURTHY et al. 1983). On the other hand, a significant increase in enzymatic activity was noted in lung washout fluid from exposed animals.

\section{Nickel Sulphate}

The numbers of macrophages and polymorphonuclear leucocytes were increased in bronchoalveolar lavage fluid following the intratracheal instillation of $0.4 \mathrm{ml} \mathrm{NiSO}_{4}$ solution $(50 \mu \mathrm{g} \mathrm{Ni} /$ rat $)$ or inhalation (36.5 mg Ni/m $\mathrm{m}^{3}$ for $2 \mathrm{~h}$ ) of nickel sulphate (Hirano et al. 1994).

Mice deficient in the cytoplasmic domain of Ron (Ron tk-/-) when exposed to aerosolised $\mathrm{NiSO}_{4}$ $\left(115 \pm 7 \mu \mathrm{g} / \mathrm{m}^{3}\right)$ succumbed to nickel-induced acute lung injury earlier, expressed larger, early increases in interleukin-6, monocyte chemoattractant protein-1, and macrophage inflammatory protein2, displayed greater serum nitrite levels, and exhibited earlier onset of pulmonary pathology and augmented pulmonary tyrosine nitrosylation (McDowELL et al. 2002).

\section{Nickel Oxides}

In $\mathrm{F} 344 / \mathrm{N}$ rats and $\mathrm{B} 6 \mathrm{C} 3 \mathrm{~F}_{1}$ mice, repeated inhalation of $\mathrm{NiO}$ at levels resulting in alveolar macrophage hyperplasia and alveolitis impaired clearance of subsequently inhaled $7.5-16.2 \mathrm{mg}{ }^{63} \mathrm{NiO} / \mathrm{m}^{3}$ of a mass median aerodynamic diameter of 1.1-2.2 $\mu \mathrm{m}$ (BENSON et al. 1995).

In the rat, single $(3.8 \mathrm{mg} / \mathrm{kg})$ or repeated $(14.3$ $\mathrm{mg} / \mathrm{kg}$ ) intratracheal instillations of nickel fumes 
induced a marked mobilisation of macrophages into the alveoli, the formation of foam cells, and their destruction (Toya et al. 1997).

Alveolar macrophages of rats injected intratracheally with $\mathrm{NiO}$ contained invaginated bizarreshaped nuclei, which lacked prominent nucleoli, numerous primary and secondary lysosomes, pigment aggregations, and several membranous whirls (Migally et al. 1982). In vitro, $>500 \mu \mathrm{M} \mathrm{Ni}_{2} \mathrm{O}_{3}$ inhibited $\mathrm{H}_{2} \mathrm{O}_{2}$ and $\mathrm{O}_{2}{ }^{--}$release from alveolar macrophages of rabbits injected intravenously with complete Freund's adjuvant by $50 \%$ (LABEDZKA et al. 1989). $100 \mu \mathrm{M} \mathrm{Ni}_{2} \mathrm{O}_{3}$ increased extracellular lactate dehydrogenase activity to $140.0 \pm 68.8 \%$ of the controls.

Washed alveolar macrophages from rats exposed ( $8 \mathrm{~h}$ per day, 5 days per week, for 18 days) to $\mathrm{NiO}$ $\left(120 \mu \mathrm{g} / \mathrm{m}^{3}\right)$ aerosols were found to contain reduced quantities of the various hydrolytic enzymes (except for acetylesterase) when compared with those from control rats (MURTHY et al. 1983). On the other hand, a significant increase in enzymatic activity was noted in lung washout fluid from exposed animals.

In the lungs of rats instilled with $0.5 \mathrm{ml}$ of a suspension of $1 \mathrm{mg} \mathrm{NiO}+0.1 \mathrm{mg} \mathrm{CdO}$ one week before, the alveolar macrophages appeared smaller than those of control, with numerous lamellar bodies of different sizes. The lamellar bodies consisted of membranous whorls, disintegrating structures, as well as accumulation of surfactant-like material (MurThy and Holovack 1991). In addition, the cytoplasm was filled with multivesicular bodies, degenerating mitochondria, as well as primary and secondary lysosomes.

\section{Nickel Carbonyl}

Owing to its volatility (boiling point at $43^{\circ} \mathrm{C}$ ), lack of strong odour, and propensity for inadvertent formation, nickel carbonyl, $\mathrm{Ni}(\mathrm{CO})_{4}$, is generally considered the most hazardous compound encountered in the workplace. 38 per cent of the administered ${ }^{63} \mathrm{Ni}(\mathrm{CO})_{4}$ was detected in the expired air for 6 $\mathrm{h}$ after intravenous injection into male SpragueDawley rats, demonstration that the lung is a major route for excretion of $\mathrm{Ni}(\mathrm{CO})_{4}$ (SUNDERMAN and Selin 1968). Barnes and Denz (1951) and GhIRINGHelli and AgAmennone (1957) demonstrated that nickel was rapidly mobilized from lung, liver, kidney, brain and blood of rats following inhalation of nickel carbonyl.

Chromatographic fractionations performed on the ultracentrifugal supernatants of homogenates of lung and liver of rats exposed to $\mathrm{Ni}(\mathrm{CO})_{4}$ demon- strated nickel firmly bound to macromolecular constituents (SUNDERMAN 1964). A Ni ${ }^{2+}$-binding protein, $p N i X a$, was identified in Xenopus oocytes and embryos ( $45 \mathrm{kDa}$, isoelectric point $\sim 8.5)$ with a strong homology to human $\alpha_{1}$-antitrypsin, $\alpha_{1-}$ antichymotrypsin, and other serine protease inhibitors (Sunderman 1993).

\subsection{2 \\ Nitrogen 0xides}

Nitrogen has the atomic number 7 and the molecular orbitals of $\mathrm{N}_{2}$ are $K K \sigma^{2} 2 s \sigma^{\star 2} 2 s\left(\pi 2 p_{\mathrm{y}} \pi 2 p_{\mathrm{z}}\right)^{4} \sigma^{2} 2 p_{\mathrm{x}}$. The oxidation states of nitrogen include $-3,-2,-1$, $+1,+2,+3,+4$, and +5 .

Nitrogen dioxide and nitric oxide have been associated with industrial pulmonary disease for a long time (e.g. Haldane 1902, Low RY and SCHUMAN 1956). In coal mines these gases are present both in diesel exhaust and shotfiring fume. Particular concern has been expressed about possible adverse effects on the health of coal miners from high levels of oxides of nitrogen present after shotfiring (Nicholas and Wall 1971) and Kennedy (1970, $1972,1974)$ has suggested that exposure to oxides of nitrogen in shotfiring fume may cause emphysema in coal miners. Robertson et al. (1982) found both nitric oxide and nitrogen dioxide readily absorbed on coal, DQ12 quartz and kaolinite dusts.

Nitroxides have been used to study the membranes of macrophages and also to investigate some of the oxidative intermediates that are produced by macrophages as part of their mechanism of cell killing. As in other cell lines, the reduction of lipophilic nitroxides, which spontaneously localise in membranes, is not preceded by active internalisation and cytoplasmic reduction and does not occur by penetration of external ascorbic acid into the lipid bilayer. The reduction is enzymatic and can be described by first-order kinetics; it can be reversed by potassium ferricyanide, decreased by disulphides, and increased by $\mathrm{NO}_{2}$ (Rowlands et al. 1978).

\section{Nitrogen Dioxide}

Similar to ozone, $\mathrm{NO}_{2}$ is relatively insoluble and penetrates deep into the respiratory tract.

Concanavalin A agglutinated alveolar macrophages of female Sprague-Dawley rats that had inhaled $12.1 \mathrm{ppm}$ nitrogen dioxide for $2 \mathrm{~h}$ (GoldSTEIN et al. 1977). Agglutinability was almost completely inhibited by $\alpha$-methylmannose. Exposure of male Long-Evans rats to $40 \mathrm{ppm} \mathrm{NO}_{2}$ for $5 \mathrm{~h}$ resulted in a significant increase in NADPH cytochrome $c$ reductase activity (46\%) in macrophages, 
but no change in any biosynthetic enzymes (Wright et al. 1982).

Adsorption of nitrogen dioxide onto kaolinites produced a small but significant decrease in toxicity to $\mathrm{P}_{388 \mathrm{D}_{1}}$ cells (RoBERTSON et al. 1982).

\section{Nitrogen Oxide and ${ }^{\circ} \mathrm{O}$-forming Drugs}

A simple molecular orbital diagram for ${ }^{\circ} \mathrm{NO}$ shows that the unpaired electron resides in an antibondal orbital. The fact that ${ }^{\circ} \mathrm{NO}$ contains one electron in an antibonding orbital explains the bond order of 2.5 (i.e., the total bonding is described as three net bonds gained from the filled $\sigma_{z}, \pi_{x}$, and $\pi_{y}$ molecular orbitals minus half a bond from the partially filled $\pi^{*}$ antibonding orbital). Since the highest occupied molecular orbital is antibonding in nature, it may be expected that this electron is loosely held and should be easily lost to generate ${ }^{+} \mathrm{NO}$. This is indeed the case, as the ionisation potential of $\mathrm{NO}$ is only $9.25 \mathrm{eV}$ (compared to, e.g. $15.56 \mathrm{eV}$ for $\mathrm{N}_{2}$ or $14.1 \mathrm{eV}$ for $\mathrm{CO}$ ).

NO fluxes could be detected at distances from RAW 264.7 macrophages of 100-500 $\mu$ m (PorTERFIELD et al. 2001). The initial flux and the distance from the cells at which NO could be detected were directly related to the number of cells in the immediate vicinity of the probe releasing NO. Thus, whereas NO fluxes of $\sim 1 \mathrm{pmol} \times \mathrm{cm}^{-2} \times \mathrm{s}^{-1}$ were measured from individual macrophages, aggregates composed of groups of cells varying in number from 18 to 48 cells produces NO fluxes between $\sim 4$ and $10 \mathrm{pmol} \times \mathrm{cm}^{-2} \times \mathrm{s}^{-1}$. NO fluxes required the presence of L-arginine. Signals were significantly reduced by the addition of haemoglobin and by $N$ nitro-L-arginine methyl ester. NO fluxes were greatest when the sensor was placed immediately adjacent to cell membranes and declined as the distance from the cell increased. The NO signal was markedly reduced in the presence of the protein albumin but not by either oxidised or reduced glutathione. A reduction in the NO signal was also noted after the edition of lipid micelles to the culture medium.

One of the most biologically significant aspects of ${ }^{\circ} \mathrm{NO}$ chemistry is its ability to bind to and/or react with metals and metal containing proteins. The activation of the iron heme-containing enzyme guanylate cyclase occurs through a ligation of ${ }^{\circ} \mathrm{NO}$ to the iron heme (see, e.g. IgNarro 1989, 1992, and the references therein). Pulmonary soluble guanylate cyclase is a hemoprotein containing $1 \mathrm{~mol}$ of ferroprotoporphyrin-IX per mol of heterodimer (GERZER et al. 1981). Enzyme stimulation is mediated by binding of ${ }^{\circ} \mathrm{NO}$ to the heme (IGNARRO 1991). Stone and Marletta (1994) showed that soluble guanylate cyclase contains a pentacoordin- ated high-spin ferrous heme with histidine as the proximal ligand.

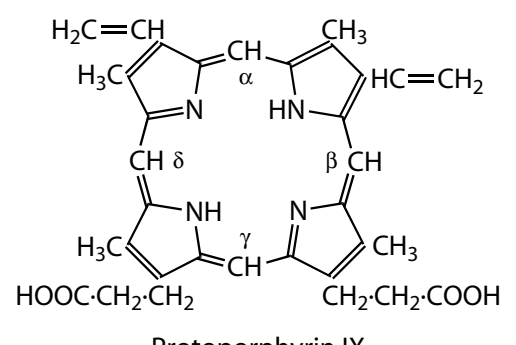

Protoporphyrin IX [222], the iron-free precursor of heme, stimulates soluble guanylate cyclase (EC 4.6.1.2) independently of ${ }^{\circ} \mathrm{NO}$ (IgNARRo et al. 1982). As protoporphyrin IX does not contain $\mathrm{Fe}$, its structure resembles that of NO-heme complex in which the iron is moved out of the plane of the porphyrin ring. In both cases, i.e. in the protoporphyrin IX- and the ${ }^{\circ} \mathrm{NO}-$ stimulated enzyme, the axial histidine is unbound.

Phantom solutions of synthetic nitrosyl-iron complexes altered the signal intensity of protonelectron-double-resonance imaging (MüLsCH et al. 1999). The dinitrosyl-iron complex with serum albumin induced a significantly larger signal alteration than the mononitrosyl-iron complex with dithiocarbamate. Exposure of rat liver to sodium nitroprusside by ex vivo and in situ perfusion induced a composite X-band electron spin resonance spectrum of the isolated liver characteristic of a mononitrosyl-iron complex and dinitrosyl-iron complex. On storage of the tissue, the mononitrosyl-iron complex signal disappeared and the dinitrosyl-iron complex signal intensity increased. Correspondingly, in cross-sectional proton-electron-doubleresonance images taken at room temperature, the sodium nitroprusside-exposed livers initially exhibited a weak signal that strongly increased with time.

Upon activation with monocyte chemoattractant protein-1, murine peritoneal macrophages showed a dose- and time-dependent production of ${ }^{~} \mathrm{NO}$ together with increased tumoricidal activity against P815 mastocytoma cells (BIswas et al. 2001). NMonomethyl-L-arginine inhibited the monocyte chemoattractant protein-1-induced ${ }^{\circ} \mathrm{NO}$ secretion and generation of macrophage-mediated tumoricidal activity against $\mathrm{P} 815$ (NO-sensitive, TNF-resistant) cells but not the L929 (TNF-sensitive, NO-resistant) cells.

'NO produced by the cells upon cytokine induction, or added as a gas to the recombinant protein, converts the iron-regulatory protein (also called iron-regulatory factor [IRF] or iron-responsive element-binding protein [IRE-BP]) to the same condition as low iron, causing a binding of the protein to mRNA (Drapier et al. 1993, Weiss et al. 1993). 
Macrophages isolated 0-48 $\mathrm{h}$ after an exposure of male Balb/c mice to $80-100 \mathrm{ppm}{ }^{\circ} \mathrm{NO}$ for $5 \mathrm{~h}$ produced low levels of ${ }^{\circ} \mathrm{NO}$, but stimulation with lipopolysaccharide (LPS) and interferon- $\gamma$ resulted in a time-dependent induction of ${ }^{\circ} \mathrm{NO}$ (LASKIN et al. 1997). Alveolar macrophages from $\mathrm{NO}$-exposed mice produced significantly more $\mathrm{NO}$ than cells from control animals which was maximum in cells isolated 24-48 $\mathrm{h}$ after exposure. This was correlated with increased inducible nitric oxide synthase (iNOS) expression by the cells. Alveolar macrophages from "NO-exposed mice were also found to produce significantly more superoxide anion than cells from control mice. This was evident in vitro in isolated cells and in situ in histological slides. ${ }^{\circ} \mathrm{NO}$ reacts rapidly with superoxide anion to form peroxynitrite, a potent oxidising agent. Following inhalation of ${ }^{\circ} \mathrm{NO}$, alveolar macrophages were also found to produce significant quantities of peroxynitrite.

Nitroxide radicals without charged groups were reduced significantly in the murine lung, while radicals with charged groups were only slightly reduced (TAKeshita et al. 1999). Permeation rates across lung plasma membrane were not limiting of the stage of reduction of noncharged nitroxides.

\section{L-Arginine}

Arginine can be synthesised from citrulline in the body, the kidneys being the major site (DнаNAкот I et al. 1990). L-Citrulline produced by nitric oxide synthase can be recycled to L-arginine in endothelial cells (Hecker et al. 1990). Sessa et al. (1990) reported that the synthesis of arginine from citrulline in endothelial cells was markedly inhibited by Lglutamine or L-arginine, pointing to a possible role for amino acids in controlling the availability of arginine and the production of ${ }^{\circ} \mathrm{NO}$. The synthesis of $\left[{ }^{14} \mathrm{C}\right]$-arginine from $0.1 \mathrm{mM}\left[{ }^{14} \mathrm{C}\right]$-citrulline by rat peritoneal macrophages in vitro was about 300 $\mathrm{pmol} / \mathrm{h}$ per $10^{6}$ cells (Wu and BRosNan 1992). Both arginine synthesis from citrulline and nitrate production as an indicator of ${ }^{\circ} \mathrm{NO}$ generation were increased about 3-fold in the cells from lipopolysaccharide-treated animals. The rate of arginine synthesis from citrulline was inhibited by about $20 \%$ by $0.5 \mathrm{mM}$ L-glutamine in both control and lipopolysaccharide-treated rat cells, but was inhibited by $0.5 \mathrm{mM}$ L-arginine only in control cells.

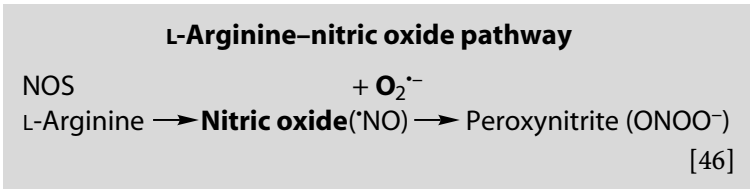

When cultured rat alveolar macrophages were stimulated with LPS, substantial amounts of nitric oxide were produced (Persoons et al. 1996). Inhibition of the nitric oxide production by the Larginine analogue, $N^{\mathrm{G}}$-monomethyl-L-arginine resulted in an increase of IL- $1 \beta$ and IL- 6 , whereas TNF- $\alpha$ concentrations remained unchanged. Conversely, the nitric oxide donor, S-nitroso- $\mathrm{N}$-acetylD, L-penicillamine induced dose dependent inhibition of IL-1 production in LPS-stimulated alveolar macrophages in which endogenous nitric oxide production was blocked.

For macrophage cell death, activation of endogenous ${ }^{\circ} \mathrm{NO}$ generation or exogenously applied ${ }^{\circ} \mathrm{NO}$ causes apoptosis. Morphological criteria as well as biochemical analysis revealed characteristic apoptotic features (Messmer and Brüne, unpublished, quoted from BRÜNE et al. 1995). A chemically heterogeneous group of NO-releasing compounds like sodium nitroprusside, SIN-1, S-nitroso- $\mathrm{N}$-acetylD, L-penicillamine, spermine-NO, and the diethylamine-nitric oxide complex produced a time- and concentration-dependent effect.

While increasing concentrations of free calcium $\left(E_{50} 300\right.$ and $30 \mathrm{nM}$, respectively) increased ${ }^{\circ} \mathrm{NO}$ synthesis in porcine endothelial and cerebellar cytosol, it was unaffected in murine bone marrow macrophage cytosol (MüLsCH et al. 1991).

Endogenous nitric oxide production may be linked to 1,25-dihydroxyvitamin $\mathrm{D}_{3}$ synthesis in chick myeolomonocytic HD-11 cells in vitro, indicating that macrophage NO-generating capacity could be functionally linked to endogenous synthesis of the active vitamin D metabolite (ADAms et al. 1994).

There has been considerable controversy about whether human macrophages make nitric oxide. Treatment in culture with the same sorts of regimens that stimulate nitric oxide production in rodent cells generally failed to elicit significant amounts of nitric oxide production (Padgett and Pruett 1992), though Munoz-Fernandez et al. (1992) described activation for the killing of intracellular Trypanosoma cruzi by TNF- $\alpha$ and TNF- $\gamma$ through a nitric oxide dependent mechanism, and SHERman et al. (1991) cytokine- and Pneumocystis carinii-induced Larginine oxidation by human alveolar macrophages.

\section{Sydnonimines}

The sydnonimines are one of the most interesting classes of organic nitro compounds as they are highly active in treating coronary artery disease and are nearly devoid of tolerance (i.e. in contrast with organic nitrates long-term therapy does not become ineffective). 
Sydnonimines are unstable in aqueous solutions. The sydnonimine ring opens, by a base-catalysed mechanism to give SIN-1A. SIN-1A reduces oxygen, in a one-electron transfer reaction, to give superoxide anion radical $\left(\mathrm{O}_{2}{ }^{--}\right)$and $\mathrm{SIN}-1^{\bullet+}$, a cation radical. SIN- $1^{\circ+}$ decomposes to $\mathrm{NO}$ and biologically inactive metabolites like SIN-1C (FeELISCH et al. 1989). By high-pressure liquid chromatography, NoACK and Feelisch (1989) found that $500 \mu \mathrm{M}$ SIN-1 were converted to SIN-1A with a half-time of $126 \mathrm{~min}$, while SIN-1C was formed three to four times more quickly with a half-time of $38 \mathrm{~min}$. The thiomorpholinyl analogue of SIN-1, compound C 78-0698, showed that the A form was more rapidly formed than with SIN-1, but was considerably more stable.

$30 \mathrm{~min}$ after an oral application of $6 \mathrm{mg}{ }^{14} \mathrm{C}-\mathrm{N}$ ethoxycarbonyl-3-morpholino-sydnonimine (molsidomine) per $\mathrm{kg}$ rat, pulmonary radioactivity $(2.98 \pm 0.892 \mu \mathrm{g} / \mathrm{g}$ wet weight $)$ was increased in relation to blood $(1.74 \pm 0.714 \mu \mathrm{g} / \mathrm{g}$ wet weight) (TANAYAMA et al. 1970).

In totally hepatectomized rats, the $\mathrm{N}$-ethoxycarbonyl-3-morpholinosydnonimine level did not decline during the first hour after intravenous injection of the drug (Tanayama et al. 1974). Furthermore, the amounts of the metabolites in the blood did not increase with time.

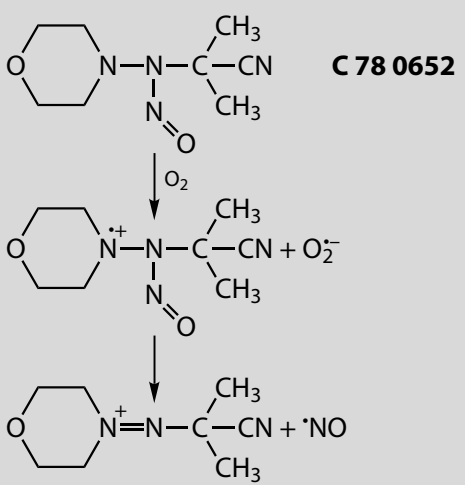

Formation of both ' $\mathrm{NO}$ and $\mathrm{O}_{2}{ }^{--}$from sydnonimine metabolites, e.g. open ring compount C 78-0652

[136]

The very different chemical reactivity towards $\alpha$ keto- $\gamma$-methiolbutyric acid by the SIN- 1 and ironascorbate-generated oxidants indicates that hydroxyl radical is not a major oxidant produced by the SIN-1 system (REgoli and Winston 1999).

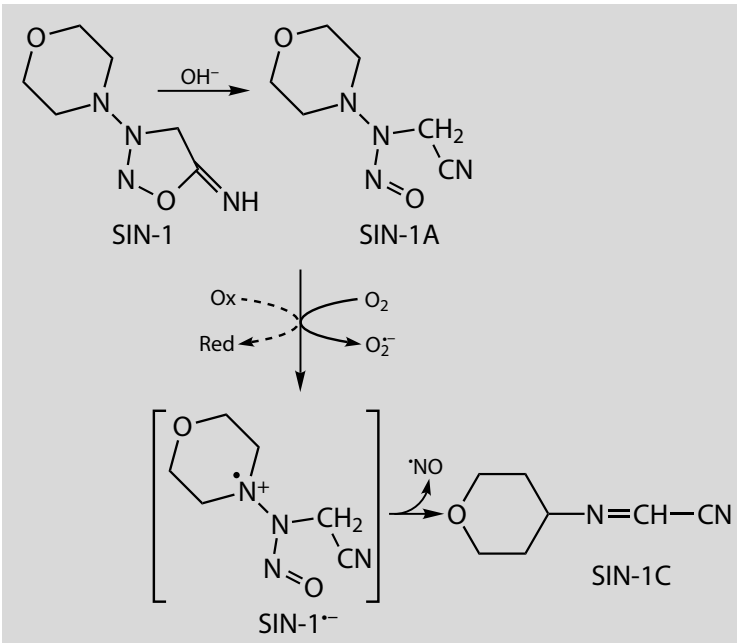

Mechanism of "NO production and formation of an imino nitroxide during the reaction of $\mathrm{SIN}^{-1}$ and nitronyl nitroxide (SINGH et al. 1999)

[223]

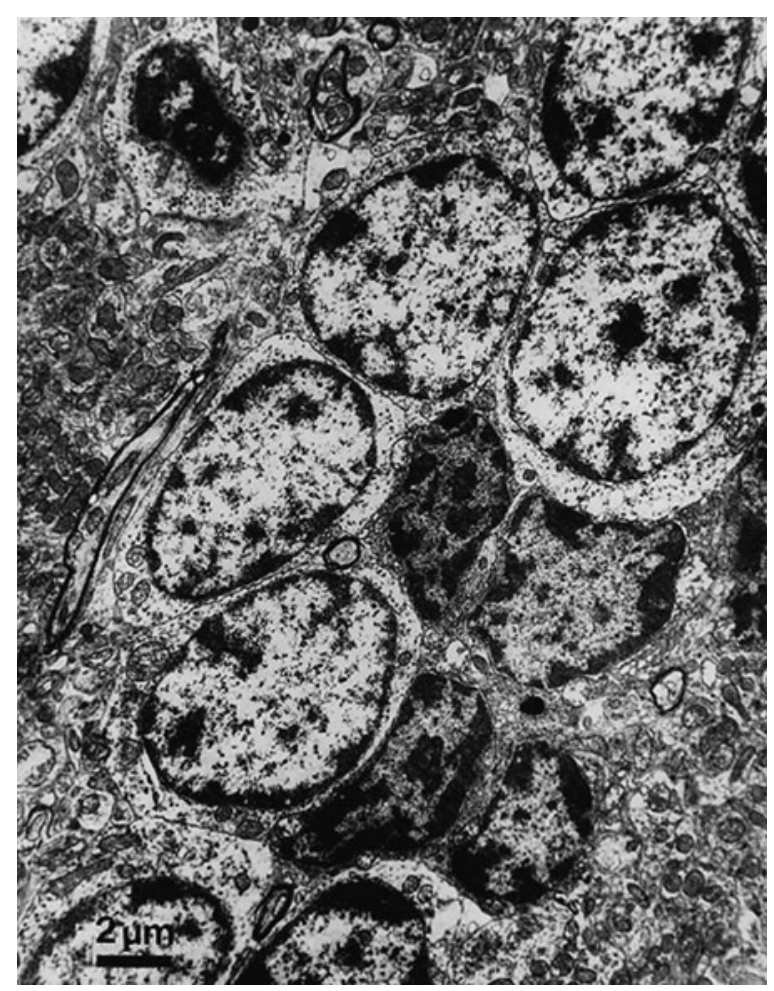

Fig. 152. Microglial cell infiltration in the granule layer of the cerebellum (block 4766) of a $221 \mathrm{~g}$ female rat (No.668) medicated with 5,000 ppm molsidomine added to the food (powdered Altromin ${ }^{\circledR}$ R) from July 17/18, 1978 to November 30, 1978. After discontinuation of the medication for 7 weeks, under pentobarbital anaesthesia $(30 \mathrm{mg} / \mathrm{kg})$, the animal was perfused from the abdominal aorta with $2.5 \%$ glutaraldehyde in $0.1 \mathrm{M}$ sodium cacodylate buffer ( $\mathrm{pH}$ 7.4). Postfixation with $1 \%$ osmium tetroxide in sodium cacodylate buffer. Embedded in Epon 812 and sectioned at $50 \mathrm{~nm}$. Lead citrate and uranyl acetate. Film 182/79 
Nitronyl nitroxide has been widely used for detecting and antagonising the effect of "NO (AKAIKE et al. 1993, Hogg et al. 1995, Konorev et al. 1995). Nitronyl nitroxide is converted to an imino nitroxide during its reaction with ${ }^{\circ} \mathrm{NO}$ with a stoichiometry of approximately 0.5:1 (HogG et al. 1995). Pfeiffer et al. (1997) demonstrated that nitronyl nitroxide enhanced the formation of ${ }^{\circ} \mathrm{NO}$ from SIN1 , as determined by an increase in cyclic GMP levels. Sing et al. (1999) reported that one-electron oxidising agents, in addition to oxygen, can oxidise SIN-1A, resulting in the release of ${ }^{\circ} \mathrm{NO}$ without the concomitant formation of $\mathrm{O}_{2}{ }^{-}$. Easily reducible nitroxides, such as the nitronyl and imino nitroxides, are able to oxidise SIN-1. Biological oxidising agents such as ferricytochrome $c$ also stimulate ${ }^{\circ} \mathrm{NO}$ production from SIN-1. Decomposition of SIN-1 by human plasma or by the homogenate of rat liver, kidney and heart tissues resulted in the formation of ${ }^{\circ} \mathrm{NO}$.

\section{Nitric oxide Synthase Induction as Influenced by Sydnonimines}

A single dose of the molsidomine metabolite SIN-1 given in the postinduction phase did not inhibit NO release from mouse macrophages (line RAW 264.7) induced by LPS/IFN- $\gamma$, but ${ }^{\circ} \mathrm{NO}$ formation was reduced by about $60 \%$ when $500 \mu \mathrm{M}$ SIN-1 were applied for $4 \mathrm{~h}$ in the induction phase (OsTRowsKi et al. 1992). Superoxide dismutase antagonised this effect. The pharmacologically inactive molsidomine metabolite SIN-1C and sydnonimine C 3754, a metabolite of pirsidomine, did not influence the induction of NO synthase activity in this cell line, while LPS/IFN-dependent induction was lost by 40 and $60 \%$, respectively, when LPS/IFN or mouse macrophages were preincubated with SIN-1 for $1 \mathrm{~h}$. $\left[{ }^{3} \mathrm{H}\right] \mathrm{L}$-citrulline formation mediated by $c y-$ tosolic mouse macrophage NO synthase was reduced by SIN-1, which could not be compensated by superoxide dismutase. Pirsidomine metabolite $\mathrm{C}$ 3786 was unable to change nitric oxide synthase activity. In bovine alveolar macrophages SIN-1 (100 $\mu \mathrm{M})$ increased iNOS mRNA after isolation procedure comparable with untreated cells (HöcKELE et al. 1997).

\section{Oxatriazole-type NO-donors}

The novel oxatriazole-type ${ }^{\circ}$ NO-donors (GEA compounds) are mesoionic 3-aryl substituted oxatriazole-5-imine derivatives releasing ${ }^{\circ} \mathrm{NO}$. They have vasodilator, antiplatelet and fibrinolytic activities (Corell et al. 1994, Karup et al. 1994, KanKaAN-
RANTA et al. 1996). They are also effective in reducing blood pressure (NURMinen and VapaAtalo 1996) and inhibiting the growth of tumour and haematopoietic cells (Vilpo et al. 1994, 1997).

After $60 \mathrm{~min}$ incubation of rat vascular smooth muscle cells the order of the compounds in their capability to release ${ }^{\circ} \mathrm{NO}$ was GEA $3162=$ SIN $1>S$-nitroso- $N$-acetylpenicillamine $>$ GEA 5624 (LÄHTEENMÄKI et al. 1998).

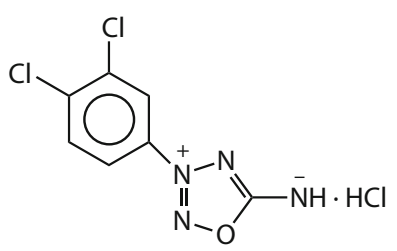

(1,2,3,4-oxatriazolium-5-amino-3-(3,4-dichlorophenyl)

[224]

Co-culture of human neutrophils with the NO donors GEA 3162 (1,2,3,4-oxatriazolium,5-amino3-(3,4-dichlorophenyl)-chloride) $(10-100 \mu \mathrm{M})$ and 3-morpholino-sydnonimine (SIN-1) (0.3-3 mM) caused a dramatic and concentration-dependent induction of apoptosis (WARD et al. 2000).

\section{4-Ethyl-2[(Z)-hydroxyiminol $]-5-n i t r o-3(E)-$ hexeneamide}

4-Ethyl-2[(Z)-hydroxyiminol]-5-nitro-3(E)-hexeneamide (FK409) is a semi-artificial fermentation product of Streptomyces griseosporeus No.16917 that spontaneously generates $\mathrm{NO}$ and possesses potent vaso-relaxant and anti-platelet activities (Funuyama et al. 1995). It is relative unstable, with a half-life of approximately $45 \mathrm{~min}$ at $37^{\circ} \mathrm{C}$ in phosphate buffer. FK 409 has been reported to attenuate experimental liver (OHMORI et al. 1998), cardiac (IsONo et al. 1993), pulmonary (TAKeYoshi et al. 2000) and renal (Matsumura et al. 1998) ischaemia/reperfusion injury. FK409 improved survival and reduced microcirculatory disturbances after intestinal ischaemia/reperfusion injury (KALIA et al. 2001).

\section{Inhibitors of ${ }^{\bullet} \mathrm{NO}$ Synthesis}

Taurine chloramine inhibited the synthesis of nitric oxide and the release of tumour necrosis factor in activated RAW 264.7 cells (PARK et al. 1993). Inhibition of ${ }^{\circ} \mathrm{NO}$ production was dependent on taurine chloramine concentration and was accounted for by reduced expression of inducible nitric oxide synthase mRNA, regardless of activator combinations (PARK et al. 1997). 
Cloricromene (2, 20 or $200 \mu \mathrm{M})$ inhibited the expression but not the activity of the inducible form of nitric oxide synthase in lipopolysaccharide (100 $\mathrm{ng} / \mathrm{ml}$ )-stimulated murine $\mathrm{J774}$ macrophages in a concentration-dependent manner (ZINGARELLI et al. 1993). Maximal inhibition $(84.0 \pm 8.0 \%)$ was observed when cloricromene $(200 \mu \mathrm{M})$ was added to the cells $6 \mathrm{~h}$ before lipopolysaccharide, whereas it was ineffective when given $6 \mathrm{~h}$ after endotoxin.

$\Delta^{9}$-Tetrahydrocannabinol inhibited lipopolysaccharide- and IFN- $\gamma$-induced nitric oxide production by thioglycollate-elicited peritoneal macrophages of female BALB/c mice (Coffey et al. 1996).

\section{Nitrous Oxide}

Nitrous oxide $\left(\mathrm{N}_{2} \mathrm{O}\right)$ is a colourless gas (boiling point $-88.5^{\circ} \mathrm{C}$ ) with a slightly sweet taste and odour at high concentration.

$$
\mathrm{N}_{2} \mathrm{O}+\mathrm{e}_{\mathrm{aq}}^{-} \rightarrow \mathrm{N}_{2}+\mathrm{HO}^{\circ}+\mathrm{OH}^{-}
$$

It has been known for many years that the inhalation of "nitrous fumes' may cause acute pulmonary oedema and death in man. In the United Kingdom, nitrous fume poisoning is listed under occupational diseases prescribed under the National Insurance Act, 1965 (P.D.17). KenNedy (1972) summarised some of the many situations where nitrous fumes may occur. In another paper (KENNEDY 1972, 1974) he listed animal studies on the toxicology of nitrous fumes. In rabbits, KleINERMAN and Wright (1961) found macrophage infiltration 4 days after an exposure of $25 \mathrm{ppm}$ for $2 \mathrm{~h}$.

Interactions of nitrous oxide with bovine heart dioxygen utilisation (SowA et al. 1987) and cytochrome $c$ oxidase (EINARSDótTIR and CAUGHeY 1988) were reported.

\subsection{3}

Ozone

Ozone is formed in both the upper (stratosphere) and lower (troposphere) sections of the earth atmosphere. Stratospheric $\mathrm{O}_{3}$ is generated by intensive ultraviolet light. The high energy of this radiation splits molecular $\mathrm{O}_{2}$ into oxygen atom radicals, $\mathrm{O}^{*}$, which then react with oxygen molecules:

$$
\begin{aligned}
& \mathrm{O}_{2}+\mathrm{hv} \longrightarrow 2 \mathrm{O}^{\circ} \\
& \mathrm{O}_{2}+\mathrm{O}^{\circ} \longrightarrow \mathrm{O}_{3}
\end{aligned}
$$

Cyclic generation of ozone in the troposphere occurs as follows:

$$
\begin{aligned}
& \mathrm{O}_{3}+\mathrm{H}_{2} \mathrm{O} \rightarrow \cdot \mathrm{NO}+\mathrm{O}^{\cdot} \\
& \mathrm{O}_{2}+\mathrm{O}^{\cdot} \rightarrow \mathrm{O}_{3} \\
& \mathrm{O}_{3}+{ }^{\mathrm{NO}} \rightarrow \mathrm{NO}_{2}+\mathrm{O}_{2}
\end{aligned}
$$

Ozone is a major component of photochemical smog. High levels of this pollutant, sufficient to affect human health, are found in many urban areas world-wide (LIPMANN 1991).

$$
\begin{aligned}
& \mathrm{O}_{3}+\mathrm{H}_{2} \mathrm{O} \rightarrow 2 \mathrm{O}_{2}^{--}+2 \mathrm{H}^{+} \\
& \mathrm{O}_{3}^{-} \leftrightarrow \mathrm{O}_{2}+\mathrm{O}^{--} \\
& \mathrm{O}_{3}^{-}+\mathrm{H}^{+} \longrightarrow \mathrm{O}_{2}+\mathrm{HO}^{\circ} \\
& \mathrm{O}_{3}^{-}+2 \mathrm{H}^{+}+\mathrm{e}^{-} \rightarrow \mathrm{O}_{2}+\mathrm{H}_{2} \mathrm{O} \\
& \mathrm{O}_{3}^{--}+\mathrm{O}_{3}^{--}+2 \mathrm{H}^{+} \rightarrow \mathrm{O}_{2}+\mathrm{H}_{2} \mathrm{O}_{2}
\end{aligned}
$$

In Escherichia coli K-12, glyceraldehyde-3-phosphate dehydrogenase (EC 1.2.1.12) showed the greatest susceptibility to ozone (Komanapalli et al. 1997). When the active site sulphydryl (cysteine) is reversibly blocked by tetrathionate during ozone treatment, enzyme activity is completely retained despite the oxidation of tryptophan, methionine, and histidine residues (KNIGHT and MudD 1984).

\section{Accumulation of Macrophages In Vivo}

After an exposure of male Wistar rats to $0.5 \mathrm{ppm}$ of $\mathrm{O}_{3}, 6 \mathrm{~h}$ a day 6 days a week for 2 months, alveolar macrophages accumulated in the centroacinar alveoli (Hiroshima et al. 1989). These macrophages were false positive for periodic acid-Schiff staining and partially positive for Berlin blue staining. The intraluminar accumulation of alveolar macrophages lasted until 12 months after exposure.

In C57BL/6 mice, steady-state mRNA levels for monocyte chemoattractant protein-1 (MCP-1) in the lung increased at $0.5 \mathrm{ppm}$ ozone and were maximal at $2.0 \mathrm{ppm}$ ozone (ZHAO et al. 1998). After exposure to $2 \mathrm{ppm}$ ozone, macrophage inflammatory protein -2 (MIP-2) mRNA levels peaked at $4 \mathrm{~h}$ postexposure, whereas MCP-1 mRNA levels peaked at $24 \mathrm{~h}$ postexposure. Neutrophils and monocytes recovered in bronchoalveolar lavage fluid peaked at 24 and $72 \mathrm{~h}$, respectively.

\section{Influence on the Number and Viability of BAL Macrophages}

After a 15 min inhalation of $0.5 \mathrm{ppm}, 1.0 \mathrm{ppm}$, and $2.0 \mathrm{ppm}$ ozone, respectively, the number of cells in bronchoalveolar lavage of female Wistar rats was dose-dependent reduced (STALDER et al. 1987). In vitro, peritoneal macrophages elicited by glycogen 
showed a reduced phagocytosis of yeast cells (as shown by bioluminescence) and at $\geq 2 \mathrm{ppm}$ a permeability for nigrosine.

In male Wistar rats immediately following an exposure to $1.77 \pm 0.03 \mathrm{ppm} \mathrm{O}_{3}$ for 2 and $4 \mathrm{~h}$, respectively, the number of lavaged macrophages significantly $(P<0.05)$ decreased from $1.44 \pm 0.14 \times 10^{6}$ cells in the controls to $0.82 \pm 0.09$ and $0.94 \pm 0.14 \times 10^{6}$ cells per lung (BASSETt et al. 1988).

2 days after the initiation of exposure of male Fischer 344 rats to 0.25 and $0.50 \mathrm{ppm}$ ozone for $20 \mathrm{~h} /$ $\mathrm{d}$, alveolar macrophages showed a transient wave of proliferation, as demonstrated by labelling with [methyl- ${ }^{3} \mathrm{H}$ ]-thymidine (Wright et al. 1987).

\section{Phagocytosis In Vitro}

Rat alveolar macrophages exposed to ozone for $1 \mathrm{~h}$ in concentrations of $0.74,1.35,2.48$ or $4.48 \mathrm{ppm}$ showed a concentration-related inhibition of phagocytosis of latex particles (Wenzel and Morgan 1983). The $\mathrm{O}_{3}$ inhibition was not influenced by the presence of either bovine serum in the medium, or by alternately removing and replacing the cultured medium (rotated exposure) or both.

$30 \mathrm{~min}$ after an intratracheal injection of approximately $10^{8}$ organisms of Lancefield C Streptococci, bronchoalveolar lavage from albino rabbits of either sex exposed to ozone concentrations from 0.67 to $9.50 \mathrm{ppm}$ showed a significant $(P<0.001)$ decrease in the numbers of both phagocytic cells and bacteria ingested (Coffin et al. 1968).

Cells lavaged from male Wistar rats exposed to $0.5 \mathrm{ppm}$ ozone for 1 week phagocytised $0.9 \mu \mathrm{m}$ polystyrene beads at the same levels as that from the control animals, both to the percentage of cells (index I) and the number of particles per cell (index II) (Creutzenberg et al. 1995). After an exposure for 2 months, however, the number of phagocytized particles per macrophage increased from $4.8 \pm 2.7$ to $11.1 \pm 4.5$.

\section{Lysosomal Enzymes In Vivo}

In rats infected with aerosols of Staphylococcus aureus and then exposed for $5 \mathrm{~h}$ to $2.5 \mathrm{ppm}$ of ozone, bacterial ingestion and clearance by alveolar macrophages were impaired due to the absence of lysosomal acid phosphatase and $\beta$-glucuronidase activities in those cells subjected to the dual insults (Goldstein et al. 1978).

\section{Formation of Superoxide Anion Radicals}

The formation of superoxide anion radicals by $0.2 \times 10^{6}$ lavaged cells was not changed after a 7 -day exposure of rats to $0.5 \mathrm{ppm}$ ozone compared to the control group, but was increased after a two months ozone exposure (CREUtzenberg et al. 1995).

In murine macrophages, 3-(4,5-dimethylthiazol2-yl)-diphenyltetrazolium bromide metabolism (monitored by optical density) $24 \mathrm{~h}$ after treatment with ozone showed a slight but significant increase $(P=0.01)$ compared to controls for the $20 \mu \mathrm{g} / \mathrm{ml}$ dose and a decrease $(P=0.02)$ for $100 \mu \mathrm{g} / \mathrm{ml}$ dose (CARDIle et al. 1995).

\section{4-Hydroxynonenal Protein Adducts After Ozone Exposure}

To determine whether 4-hydroxynonenal could account for the acute effects of ozone on human alveolar macrophages, Hamilton jr. et al. (1998) exposed healthy, non-smoking volunteers to $0.4 \mathrm{ppm}$ ozone or air for $1 \mathrm{~h}$ with exercise (each subject served as his/her own control). Six hours after ozone exposure, cells obtained by airway lavage were examined for apoptotic cell injury, presence of 4-hydroxynonenal adducts, and expression of stress proteins. Significant apoptosis was evident in airway lung cells after ozone exposure. Western analysis demonstrated an increase in a $32-\mathrm{kDa} 4$-hydroxynonenal protein adduct and a number of stress proteins, viz., $72-\mathrm{kDa}$ heat shock protein and ferritin, in alveolar macrophages after ozone exposure. All these effects could be replicated by in vitro exposure of alveolar macrophages to 4-hydroxynonenal.

\section{Synthesis of Inflammatory Cytokines}

Exposure of guinea pig alveolar macrophages to 0.4 ppm $\mathrm{O}_{3}$ for 60 min increased the IL- 6 activity by $252 \pm 60 \%$ and TNF activity by $202 \pm 35 \%$ (ARSALANE et al. 1995). The increase of monokine production by human alveolar macrophages was $443 \pm 208 \%$ for TNF $\alpha \quad 484 \pm 171 \%$ for IL- $1 \beta$, $383 \pm 147 \%$ for IL- 6 , and $226 \pm 45 \%$ for IL- 8 after a $60 \mathrm{~min}$ exposure to $0.4 \mathrm{ppm} \mathrm{O}_{3}$. Lowest $\mathrm{O}_{3}$ concentrations ( 0.1 and $0.2 \mathrm{ppm}$ ) only increased TNF $\alpha$ secretion. Shorter (30 min) or longer (120 min) exposure duration to $0.4 \mathrm{ppm}_{3}$ were also associated with a significant increase in monokine production, suggesting that the effect of ozone on cytokine production did not depend on the exposure duration. The mRNA expression of TNF $\alpha$, IL-1 $\beta$, IL- 6 , and IL8 was increased in human alveolar macrophages, 
whereas similar amounts of mRNA were detected in each sample.

A statistically significant 29-fold increase in bronchoalveolar lavage fluid IL-6 levels was observed in rats exposed to $0.5 \mathrm{ppm} \mathrm{O}_{3}$ during nighttime hours then compared with daytime hours even though similar kinetics of inflammation were induced by each exposure (McKinney et al. 1998). Animals receiving an initial night-time exposure showed a lesser degree of inflammation following a subsequent $\mathrm{O}_{3}$ exposure when compared with animals which received an initial daytime exposure.

\section{Ozone and Serum Ceruloplasmin}

Decreased serum ceruloplasmin concentration and the cumulated worktime along the week showed a linear relationship in aluminium welders exposed to ozone, which is the major pollutant in arc welding (PIERRE et al. 1988). Ceruloplasmin may be a protective molecule against direct oxidant injury to the human lung in cigarette smoke and air pollution (GALDSTON et al. 1984).

\subsection{4}

Platinum

Platinum has the atomic number 78 and the valence electrons are those of the $5 d^{9} 6 s^{1}$ shells. The oxidation states of platinum are +2 and +4 .

Platinum(IV) complexes of halogenopentammine type are reduced irreversibly to platinum(II) complexes in one step (HALl and Plow Man 1955) The polarographic stability of the pentammineplatinum(IV) complexes, $\left[\mathrm{Pt}\left(\mathrm{NH}_{3}\right)_{3} \mathrm{X}\right]$, increases in the following order of ligand $\mathrm{X}$ :

$$
\mathrm{Br}^{-}<\mathrm{Cl}^{-}<\mathrm{NH}_{3}<\mathrm{OH}^{-}
$$

\section{Cisplatin}

The subcutaneous injection of cis-diamminedichloroplatinum(II) into ABD2F1 mice $(8 \mathrm{mg} / \mathrm{kg})$ resulted in alterations of size and surface structure of peritoneal macrophages and in a reduction of the mean number of their concanavalin A binding sites (Peschke et al. 1992).

Murine peritoneal macrophages treated with cisplatin showed an enhanced production of nitric oxide and increased tumoricidal activity against P815 mastocytoma cells (SodHI and Kumar 1994). The $\mathrm{NO}$ secretion and generation of macrophagemediated tumoricidal activity were significantly inhibited by $\mathrm{L}-\mathrm{N}$-monomethyl arginine, a specific inhibitor of L-arginine pathway. Induction of ${ }^{\circ} \mathrm{NO}$ production in macrophages on activation with cisplatin or lipopolysaccharide is inhibited by EGTA, nifedipine, TMB-8 and $\mathrm{W}-7$, suggesting the probable involvement of $\mathrm{Ca}^{2+}$ and calmodulin in the induction of ${ }^{\circ} \mathrm{NO}$ release. Protein kinase $\mathrm{C}$ and tyrosine kinase inhibitors significantly inhibited the cisplatin/lipopolysaccharide induced ${ }^{\circ} \mathrm{NO}$ production, suggesting that phosphorylation via these kinases may up-regulate the ${ }^{\circ} \mathrm{NO}$ synthase activity in macrophages.

Cisplatin-treated macrophages from pathogen free $\mathrm{BALB} / \mathrm{c}$ mice synthesised and secreted oncostatin M (SINGH and SodHI 1998). The protein kinase $\mathrm{C}$ and protein tyrosine kinase inhibitors significantly inhibited oncostatin $M$ production of cisplatin-treated macrophages. The oncostatin $M$ production of cisplatin-treated macrophages was also inhibited in the presence of $\mathrm{Ca}^{2+}$ chelators, $\mathrm{Ca}^{2+}$ channel blockers and calmodulin/calmodulindependent kinase inhibitors.

Cisplatin treatment of pathogen free BALB/c mouse macrophages increased the expression and activation of lyn, a protein tyrosine kinase of src family, within 5 min (SINGH and Sodhi 1998). Cisplatin-induced expression and activation of lyn involved serine/threonine phosphatases $1 / 2 \mathrm{~A}$, protein tyrosine phosphatases, protein tyrosine kinase and protein kinase C. It was also observed that $\mathrm{Ca}^{2+} /$ calmodulin and calmodulin-dependent kinases are involved in the regulation of cisplatininduced lyn expression and activation in macrophages.

\subsection{5 \\ Plutonium Oxide $\left({ }^{239} \mathrm{PuO}_{2}\right)$}

Plutonium particles were located and identified within alveolar macrophages by autoradiography. The paths of the $\alpha$ particles emitted from the plutonium within the cell formed a "star" of tracks of reduced silver halide grains (MORROW and CASARETT 1961, SANDERS 1970).

Migration of alveolar macrophages lavaged from rabbits dusted with $\mathrm{PuO}_{2}$ was inhibited (NolibE 1972).

\subsection{6 \\ Selenium}

Selenium has the atomic number 34 and the valence electrons are those of the $4 s^{2} 4 p^{4}$ shells. Se is classified in group VIa of the periodic table below sulphur and its chemical and physical properties are very similar to those of the latter. Like sulphur, selenium occurs in the oxidation states $-2,0,+2,+4$ and +6 and is present in compounds analogues to 
those of sulphur such as the inorganic selenides, selenites and selenates and the various organic compounds in which the element is present mainly in the -2 oxidation state such as dimethylselenide, trimethyl selenonium, selenocysteine, selenomethionine and Se-methylselenocysteine.

The two elements differ, as Kyriakopoulos and BeHNe (2002) stated in a review on seleniumcontaining proteins, with regard to the reduction of their oxyanions. In biological systems the selenium compounds tend to be more easily reduced than sulphur, which is thermodynamically stable in the +6 state. Most important for the significance of selenium in biological systems, however, is the lower $\mathrm{pK}_{\mathrm{a}}$ value of the selenohydryl group of selenocysteine $\left(\mathrm{pK}_{\mathrm{a}} 5.24\right)$ as compared with that of the sulphydryl group of cysteine $\left(\mathrm{pK}_{\mathrm{a}}\right.$ 8.25) (HUBER and CRIDDLE 1967). Accordingly, the selenols of the selenocysteine-containing proteins are anionic at the physiological $\mathrm{pH}$, while the thiols in the cysteine-containing proteins are mainly protonated under these conditions. Replacement of selenocysteine in the active centre of a selenoenzyme with cysteine therefore results in a drastic decrease in its catalytic activity as was shown, for instance, in the case of formate dehydrogenase $\mathrm{H}$ from Escherichia coli (AXLEY et al. 1991), type I iodothyronine deiodinase (BERRY et al. 1991) and thioredoxin reductase (GASDASKA et al. 1999, LeE et al. 2000).

Selenium is an essential trace element that constitutes the active centre of glutathione peroxidase (Forstrom et al. 1978, LANDESTEIN et al. 1979, Wingler and BRIgELIUS-FlohÉ 1999).

Selenium compounds are more active electron donors compared to analogous sulphur compounds and play a wide role in biochemical systems. They can decompose peroxides (CALDWELL and TAPPEL 1964, 1965) and can act as antioxidants (Dillard et al. 1978) and free radical scavengers (URSINI and Bindoli 1987). Masumoto and Siess (1996) and Masumoto et al. (1996) have shown that peroxynitrite rapidly oxidises ebselen and its main metabolite to the corresponding selenoxides. The results of PAdmaja et al. (1997) suggest that $\mathrm{CO}_{2}$ partially protects methionine selenoxide from peroxynitritemediated oxidation and that $\mathrm{O}=\mathrm{N}-\mathrm{OO}-\mathrm{CO}_{2}^{-}$or its derivatives do not mediate the oxidation of $\mathrm{D}, \mathrm{L}-$ selenomethionine or methionine selenoxide.

$\mathrm{SeO}_{3}{ }^{2-}$ and $\mathrm{SeO}_{4}{ }^{2-}$ are weakly mutagenic in the Ames test (TAKano and Sakurai 1979).

In vitro, guinea-pig peritoneal macrophages showed nontoxic responses to variable concentrations of $\mathrm{Na}_{2} \mathrm{SeO}_{3} \times 5 \mathrm{H}_{2} \mathrm{O}$ : cell viability was unaffected at $10^{-6} \mathrm{M}$, migration at $10^{-6}$ and $10^{-5} \mathrm{M}$ selenite, while for $10^{-4} \mathrm{M}$ selenite no excessive lipid peroxidation occurred (GABOR et al. 1985).
In vivo, rats' lung and lymph node weights were not influenced by selenium supplementation in animals given quartz (GABOR et al. 1986). However, a decrease of lung lipids, phospholipids, and hydroxyproline was observed in rats with experimental silicosis.

\subsection{7}

\section{Silicon}

Silicon has the atomic number 14 and the valence electrons are those of the $3 s^{2} 3 p^{2}$ shells. There are 3 stable isotopes naturally occurring, ${ }^{28} \mathrm{Si},{ }^{29} \mathrm{Si}$, and ${ }^{30} \mathrm{Si}$. Their relative frequencies are $92.18 \%, 4.71 \%$ and $3.12 \%$, respectively. ${ }^{31} \mathrm{Si}$ and ${ }^{32} \mathrm{Si}$ are artificial radionuclides with half-lives of $2.62 \mathrm{~h}$ and 101 years, respectively. The annual limit of intake of ${ }^{32} \mathrm{SiO}_{2}$ by inhalation is $2 \times 10^{5} \mathrm{~Bq} / \mathrm{m}^{3}$ (BURKART 1987). The oxidation state of $\mathrm{Si}$ is +4 .

Silicon and oxygen are the two most important elements in the crust and the form is a fundamental $\mathrm{SiO}_{4}$ tetrahedral unitconsisting of a central silicon ion with oxygen ions attached three-dimensionally at the four "corners" of a tetrahedron. All pathogenic forms of "silica", i.e. silicon dioxide $\left(\mathrm{SiO}_{2}\right)$, are composed of these tetrahedra joined by oxygen atoms so that each crystal consists of a giant molecule with an average stoichiometric formula of $\mathrm{SiO}_{2}$. Being uncombined they are referred to as "free silica". The tetrahedra are linked in various ways by $\equiv \mathrm{Si}-\mathrm{O}-\mathrm{Si} \equiv$ chains, and the manner in which metallic cations are included in this linkage decides their form and characteristics.

Electron spin resonance studies of a series of airannealed samples of glassy $\mathrm{SiO}_{2}$ having various degrees of enrichment (or depletion) in the ${ }^{29} \mathrm{Si}$ isotope have confirmed that a $\gamma$-ray-induced doublet of $420-\mathrm{G}$ splitting is the ${ }^{29} \mathrm{Si}$ hyperfine structure of the well-known E' centre (GRISCOM 1979). This finding validated the widely accepted model of the $E$ ' centre as an unpaired electron spin in a dangling $s p^{3}$ hybrid orbital of a silicon bonded to three oxygens in the glass structure and eliminates an alternative model proposed by SHENDRIK and YUDIN (1978).

An experimental study by Wu et al. (1998) on the competitive adsorption resulted in the magnitude order of metal ions $\left(\mathrm{Ag}^{+}, \mathrm{Ni}^{2+}, \mathrm{Zn}^{2+}, \mathrm{Cu}^{2+}, \mathrm{Cd}^{2+}, \mathrm{Pb}^{2+}\right.$, $\mathrm{Cr}^{3+}$ ) adsorbed onto oxide and silicate minerals in near-neutral solution with low ionic strength in $\mathrm{mol} / \mathrm{nm}^{2}$ as follows:

$\mathrm{CaCO}_{3}>$ quartz $>$ hydromuscovite $>$ kaolinite $>$ Ca-montmorillonite $>$ goethite $>$ gibbsite.

$\mathrm{Cu}^{2+}$ and $\mathrm{Cr}^{3+}$ were adsorbed onto quartz at a higher rate than on calcite. 
The distinction between free and combined silica is important: combined silica is $\mathrm{SiO}_{2}$ in combination with various cations as silicates.

Free silica is the most widespread substance in nature with a fibrogenic potential for living tissues. Free silica (silicon dioxide) occurs in three forms: polymorphic crystalline, cryptocristalline (i.e., minute crystals), and amorphous (i.e., noncrystalline).

The tetrahedral - and thus pathogenic - crystalline phases of silica are as follows:

1. Quartz which is stable up to $867^{\circ} \mathrm{C}$ but is capable of metastable existence at higher temperatures.

2. Tridymite which is stable from $867^{\circ} \mathrm{C}$ to $1470^{\circ} \mathrm{C}$ and is capable of metastable both above $1470^{\circ} \mathrm{C}$ and below $867^{\circ} \mathrm{C}$.

3. Cristobalite which is stable from $1470^{\circ} \mathrm{C}$ up to its melting point of $1723^{\circ} \mathrm{C}$ but is capable of unstable existence at any temperature below $1470^{\circ} \mathrm{C}$. Opaline silica is a variety of cristobalite (Sosman 1965). Pure quartz when heated to temperatures between temperatures between $867^{\circ} \mathrm{C}$ and $1470^{\circ} \mathrm{C}$ is nearly always converted to cristobalite and not to tridymite unless a catalyst is present.

4. Coesite was first synthesised at a pressure of about $3.4 \mathrm{GPa}$, in the temperature range of 500 $800^{\circ} \mathrm{C}$ in the laboratory by CoEs (1953) and later discovered and identified from shocked Coconino sandstone of the Meteor Crater in Arizona (Сhao et al. 1960, Bohn and StöвER 1965), and from the Ries Crater in Nördlingen, Bavaria. It has a specific gravity of $2.915 \pm 0.015$ and a hardness of about 8 . It is biaxial positive with $2 \mathrm{~V}$ about $64^{\circ}$. Its indices of refraction are $\alpha 1.5940$, $\beta 1.5955$, and $\gamma 1.5970 \pm 0.0005$. It is nearly insoluble in $5 \% \mathrm{HF}$ at room temperature.

Octahedral stishovite is $46 \%$ denser than coesite. It has a specific gravity of 4.35 for the synthestic material. In stishovite each oxygen is co-ordinated to three ${ }^{\mathrm{VI}} \mathrm{Si}$, as opposed to two ${ }^{\mathrm{IV}} \mathrm{Si}$ in lower-pressure silica minerals. The ${ }^{\mathrm{IV}} \mathrm{Si} \rightarrow{ }^{\mathrm{VI}} \mathrm{Si}$ transitions increase the Si-O separation as well as the packing efficiency. The ${ }^{\mathrm{IV}} \mathrm{Si} \rightarrow{ }^{\mathrm{VI}} \mathrm{Si}$ transformations occur over a limited range of pressure.

Stishovite is biologically inert (STRECKER 1965, BRIEGER and Gross 1967).

Different investigators have suggested a number of possible post-stishovite phases of $\mathrm{SiO}_{2}$. Such phases have been obtained from theoretical simulations or observed experimentally in related systems; these are $\mathrm{CaCl}_{2}, \alpha-\mathrm{PbO}_{2}$, and modified $\alpha$ $\mathrm{PbO}_{2}$ (space group I2/a), $\alpha-\mathrm{PbCl}_{2}$, fluorite and modified fluorite (space group $\mathrm{Pa} 3$ ) structures (TsuCHIDA and YAGI 1989, Tse et al. 1992, LACKs and
Gordon 1993, Kingma et al. 1995, 1996). A new high-pressure phase of silica (space group Pnc2) was synthesised by heating silica gel or quartz to $2000 \pm 50 \mathrm{~K}$ at $\approx 80 \mathrm{GPa}$ (DUBrovinsKy et al. 1997). The phase transitions: stishovite $\rightarrow \mathrm{CaCl}_{2}$-like phase $\rightarrow$ Pnc2 structure $\rightarrow P a 3$ were found at 45 $\mathrm{GPa}, 80 \mathrm{GPa}$, and $220 \mathrm{GPa}$, respectively.

Moganite, a very rare microcrystalline silica mineral, consists of systemic twinning of one R- and one L-quartz $\{1011\}$ lattice slice per unit cell.

"Colloidal silica", also defined acid (LüHNING 1954), is a poisonous substance (GYE and PURDY 1922). It reacts with rat macrophages without affecting cell integrity (CANTRELL and Ellis 1983).

The toxicity of silica has been attributed to hydrogen donation by polymeric silicic acid, forming hydrogen-bonded polymeric complexes notably with phospholipids of cell membranes (Allison and DAVIEs 1974). Breaking a hydrogen bond can result in a significant structural change in biomolecules. Specifically, hydrogen bonding between silica and phosphate groups of membrane phospholipids could be responsible for silica toxicity (НовzA et al. 1981).

The ability to destroy the cell membrane depends upon the particle size: the denaturing effect was found to increase with the increase in size of colloidal silica particles. The proposed mechanism was that large particles would stretch the protein molecule of the membrane by adsorption forces, whereas small particles $(2-3 \mathrm{~nm})$ would be too small to separate the protein coils (HARLEY and Margolis 1961).

Using human erythrocytes as a model of interaction, Diociaiuti et al. (1999) due to the high surface/volume of aerosil particles obtained considerable membrane damage with small weight concentrations. Silica induced a considerable reduction of the intramembranous density in the endoplasmic fracture face, while the protoplasmic fracture face was practically unchanged.

The adsorption of water on the silica surfaces provided a useful tool for surface analysis (STÖBER 1955,1956 , Bolis et al. 1983). Water is in fact a constituent of a silica surface exposed to the atmosphere, for when equilibrated with water vapour the surface is mostly hydroxylated (surface silanols) and molecular water is bound to the silanols. Water can be released by heating under vacuum, and the process is reversible or irreversible depending on the outgassing temperature. The silanol groups interact with the protein or phospholipid constituents of the membrane and thus damage it (NASH et al. 1966, Summerton et al. 1977).

After intratracheal injection of a fresh suspension of $20 \mathrm{mg}$ of condensed silica in saline, the 
spherical particles of $<30 \mathrm{~nm}$ diameter formed agglomerates. After $4 \mathrm{~h}$ numerous polynuclear phagocytes have emigrated from the alveolar capillaries, followed by macrophages, and adhered to the masses of particles (Policard et al. 1955). After 7 days, Policard and Collet (1958) distinguished histiocytes capable to phagocytize and non-phagocytic reticulo-histiocytes with plenty of ergastoplasm. Aerosil ${ }^{\circledR}$ particles $(20-50 \mathrm{~nm})$ given intraperitoneally to rats after 8 days were found conglutinated by a biologic substance and thus detoxified (KLosTERKöTtER and THEMANN 1958).

Min-U-Sil 5 quartz, together with Sikron F-600 the most likely candidate for an international $\alpha$ quartz calibration standard (VERMA and SHAw 2001), shifted the phenotypic ratio of human alveolar macrophages obtained by bronchoalveolar lavage of normal, non-smoking adult volunteers of either gender to a more inflammatory condition (HoLIAN et al. 1997). The macrophage phenotypes were characterised by flow cytometry targeting the RFD1 and RFD7 epitopes. Results demonstrated that crystalline silica, as well as chrysotile and crocidolite, but not titanium dioxide or wollastonite, increased the $\mathrm{RFD} 1^{+}$phenotype (inducer or immune activator macrophages) and decreased the RFD $1^{+} \mathrm{RFD}^{+}$phenotype (suppressor macrophages).

Alveolar macrophages obtained by bronchoalveolar lavage from 25 silicotic patients under the electron microscope showed silica particles, activated nuclei, autophagic vacuoles and destroyed cell organelles (REISz et al. 1988). Lysosomal acid phosphatase activity was elevated as compared with healthy volunteers.

Instillation of $500 \mathrm{mg}$ generic quartz dust $(7 \mu \mathrm{m}$ maximum diameter) suspended in $50 \mathrm{ml}$ PBS into the right caudal lung lobe of 3 female pigtail macaque monkeys (Macaca nemestrina) using a flexible fibreoptic bronchoscope increased the numbers of macrophages in the dust exposed lobes and significantly elevated the $N$-acetyl- $\beta$-D-glucosaminidase activity within the bronchoalveolar lavages taken at 2-weeks intervals for 12 weeks thereafter (MACK et al. 1995).

In Macaca fascicularis, DQ 12 inhalation caused a shift from large, active (with respect to phagocytosis) alveolar macrophages to small, monocyte-like, less active alveolar macrophages (HILDEMANN et al. 1992).

Silica directly injured rat alveolar macrophages as evidenced by a cytotoxic index of $32.9 \pm 2.5$, whereas the addition of surfactant protein A $(5 \mu \mathrm{g} /$ $\mathrm{ml})$ significantly $(P<0.001)$ reduced the cytotoxic index to $16.6 \pm 1.2$ (SРech et al. 2000). The effect was reversed when surfactant protein A was incubated with either polyclonal rabbit anti-rat surfactant protein A antibody or D-mannose.
Table 38. Macrophages $\left(\times 10^{6}\right)$ in bronchoalveolar lavage fluid. 1 day after intratracheal instillation in hamsters (BECK et al. 1987)

\begin{tabular}{lcl}
\hline$\alpha$-Quartz & $\mathrm{CDM}=1.3 \mu \mathrm{m}$ & $3.1 \pm 0.3$ \\
Iron oxide & $1.3 \mu \mathrm{m}$ & $6.3 \pm 0.3$ \\
Talc & $0.8 \mu \mathrm{m}$ & $5.5 \pm 0.3$ \\
Granite & $0.55 \mu \mathrm{m}$ & $6.7 \pm 1.0$ \\
Saline & - & $6.6 \pm 0.5$ \\
\hline
\end{tabular}

$\mathrm{CDM}=$ Count Median Diameter

The role of functionalities on the surface of $\alpha$ quartz may play specific roles in the initiating of the cascade of events resulting in the fibrotic response (Fubini et al. 1990). Sianols and surface radicals that both decreased upon thermal heating, leaving isolated sianols, siloxanes and distorted bridges, and siloxane groups appear to be linked to the phagocytosis and transport of particles, whereas the surface radicals and distorted bridges might be more closely associated with the fibrogenic response (Hemenway et al. 1994).

After stimulation with lipopolysaccharide lavaged Sprague-Dawley rat alveolar macrophages ( $10^{6}$ cells/cuvette) in a dose-dependent manner generated oxygen radicals when 0.05 to $5 \mathrm{mg}$ silica were added (Lim et al. 1997). At higher concentrations silica-induced chemiluminescence abruptly decreased. Cytosolic $\left[\mathrm{Ca}^{2+}\right]_{\mathrm{i}}$ was suppressed only in part, perhaps due to a release of calcium from intracellular calcium stores. Staurosporine, a protein kinase $\mathrm{C}$ inhibitor to bind to the catalytic domain of protein kinase C (TAMAOKI et al. 1986), inhibited silica-induced chemiluminescence by $66 \%$ at 0.2 $\mu \mathrm{M}$ and at $99 \%$ at $2 \mu \mathrm{M}$. Sphingosine, an agent that competes with diacylglycerol/phorbol ester for binding protein kinase C (HANNUN et al. 1991) inhibited silica-induced chemiluminescence by $53 \%$ at $2.5 \mu \mathrm{M}$ and by $99 \%$ at $50 \mu \mathrm{M}$. Of the phospholipase C inhibitors, neomycin inhibited silicainduced chemiluminescence by $29 \%$ at $0.2 \mathrm{mM}$ and at $72 \%$ at $2 \mathrm{mM}$, and $\mathrm{U}-73122$ by $25 \%$ at $0.1 \mu \mathrm{M}$ and by $71 \%$ at $1 \mu \mathrm{M}$. Genistein which is known to inhibit protein tyrosine kinase activity by binding to ATP binding sites in a competitive manner (Akiyama and Ogawara 1991), inhibited silicainduced chemiluminescence by $83 \%$ at $20 \mu \mathrm{M}$, and erbstatin by $99 \%$ at $5 \mu \mathrm{M}$.

To re-evaluate macrophages as accessory cells by treating various cell preparations with either silica (O'Rourke et al. 1978, THIELE and Lipsky 1982) or L-leucine methyl ester (THIELE et al. 1983), which have been reported to be cytotoxic to macrophages, Bowers et al. (1988) showed that L-leucine methyl ester failed to kill rat macrophages or dendritic cells, whereas silica was specifically toxic for rat macrophages. 
There is an increase in L-arginine uptake and metabolism by rat bronchoalveolar lavage fluid inflammatory cells following in vivo exposure to silica, suggesting an increase in L-arginine utilisation by both arginase and nitric oxide synthase (SCHAPIRA et al. 1996). From 1 day after intratracheal instillation of silica iNOS showed positive reaction, peaked at 3 days and then decreased (SeToguchi et al. 1996). NOS activity, as measured by conversion of $\left[{ }^{14} \mathrm{C}\right]$ arginine to $\left[{ }^{14} \mathrm{C}\right]$ citrulline, took the same time profile. In situ hybridisation showed that iNOS mRNA was detected in the inflammatory cells phagocytizing silica. iNOS was expressed on macrophages, neutrophils and bronchial epithelial cells by immunohistochemical double staining. The administration of $N^{\omega}$-nitro-L-arginine methyl ester resulted in significant reducing the number of inflammatory cells such as monocyte-derived exudate macrophages and neutrophils in the granulomatous lesions.

The amount of macrophage-derived nitric oxide release varies remarkably between animal species, i.e. rat and hamster (DöRGER et al. 1997).

HL-60 cell derived macrophages were recommended as an easily manageable model system for toxicity studies of particulate bronchopulmonary noxious agents (Zoller et al. 1996, Zoller and Zeller 2000). During the differentiation of HL-60 cells, both an optimal concentration of calcitriol $\left(10^{-8} \mathrm{M}\right.$ to $\left.10^{-7} \mathrm{M}\right)$ and of ethanol is necessary. Differentiation of HL-60 cells in the presence of $0.1 \%(\mathrm{v} /$ $v)$ ethanol significantly reduced their ability to produce $\mathrm{O}_{2}{ }^{--}$; an ethanol concentration of $2 \%(\mathrm{v} / \mathrm{v})$, on the other hand, was toxic. HL-60-G cells exposed to a high concentration of calcitriol $(400 \mathrm{nM})$ slowly developed the ability to reduce nitroblue tetrazolium and did not proliferate (STUDZINSKI et al. 1997). HL-60 cells differentiated with phorbol myristate acetate were resistant to cytotoxic effects of quartz (BRÜCKNER-NIEDER et al. 1992). HL-60-M cells produced tumour necrosis factor after stimulation with lipopolysaccharide but not with quartz. SCHEDLE et al. (1995) induced apoptosis by 33-1000 $\mu \mathrm{M}$ metal cations/l. Crocidolite asbestos suppressed the differentiation of HL-60 cells induced by DMSO (Ueki et al. 1992).

Some bisbenzimides, Hoechst 33342, but not Hoechst 33258, induced apoptosis in the HL-60 cell in a time- and dose-dependent manner (ZHANG et al. 1999). Endogenous nuclear topisomerase 1 activity in HL-60 cells was inhibited by Hoechst 33342, but not Hoechst 33258.

U-937 cells, a myelomonocytic cell line derived from a patient with histiocytic lymphoma, when differentiated by phorbol myristate acetate and exposed to DQ12 quartz (specific area $3 \mathrm{~m}^{2} / \mathrm{g}$ ), $\mathrm{MnO}_{2}$ (specific area $59 \mathrm{~m}^{2} / \mathrm{g}$ ) and $\mathrm{TiO}_{2}$ (specific area 7.5 $\mathrm{m}^{2} / \mathrm{g}$ ), particles, respectively, showed specific patterns of changes in the carbohydrate moiety of glycoproteins (Trabelsi et al. 1997). Given the biological implications of carbohydrates, these changes may be critical in modulating macrophage functions. Melatonin reduced $\mathrm{H}_{2} \mathrm{O}_{2}$-induced DNA damage in U-937 cells (Romero et al. 1999).

P388D $_{1}$ macrophage-like cells were used in France to test cytotoxicity (viability and lactate dehydrogenase and acid phosphatase levels) at a serum content of $4 \%$ (DAVIs et al. 1982) Because uncontrolled variation occurring in animal cells ( $r a b$ bit alveolar macrophages obtained by pulmonary lavage 3-4 weeks after stimulation by i.v. injection of Freund's adjuvant, thioglycolate evoked rat peritoneal macrophages) DANIEL and Le Bouffant

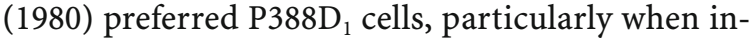
vestigating dusts with low toxicities. The extent of killing of $\mathrm{P}_{388 \mathrm{D}_{1}}$ macrophages is dependent on both the dose of silica and the concentration of $\mathrm{Ca}^{2+}$ ions in the medium (KANE et al. 1980). In the presence of extracellular calcium ions, after $3 \mathrm{~h}$ of exposure to $350 \mu \mathrm{g}$ of silica, $69 \%$ of the cells had lost viability, and ATP content was reduced to $21 \%$ of the control level (KANE et al. 1985). P388D 1 cells injured by silica showed a similar dose and time relationship between loss of viability and increased chlorotetracycline fluorescence (GooDGLICK et al. 1986): after $6 \mathrm{~h}$ of silica exposure $58.1 \pm 4.7 \%$ of the cells were killed and $45.4 \pm 5.4 \%$ showed increased chlorotetracycline fluorescence. To test whether disruption of mitochondrial $\mathrm{Ca}^{2+}$ sequestration was sufficient to cause cell death, two types of metabolic inhibitors were used. First, the mitochondrial membrane potential was disrupted by $p$-trifluoromethoxy-phenylhydrazone or carbonyl cyanide $m$ chlorophenylhydrazone which caused leakage of rhodamine 123 and release of mitochondrial $\mathrm{Ca}^{2+}$ as detected by fura2. After $6 \mathrm{~h}$ of $p$-trifluoro-methoxyphenylhydrazone or carbonyl cyanide $m$-chlorophenylhydrazone exposure, $98.3 \pm 5.6 \%$ and $96.7 \pm 7.7 \%$ of the cells were viable and $2.5 \pm 1.0 \%$ showed increased chlorotetracycline fluorescence after 6 h. Lиото et al. (1998) investigated the dissolution of short and long rockwool and glasswool fibres by $\mathrm{P}_{388} \mathrm{D}_{1}$ cells by assessing the dissolution of $\mathrm{Si}, \mathrm{Fe}$, and $\mathrm{Al}$ from the fibres.

The contact of dust particles with cell membranes is the first event during the process of phagocytosis, whatever the way of particle uptake. Membrane fluidity of alveolar macrophages gained from guinea pigs by bronchoalveolar lavage was changed more by quartz than by $\mathrm{TiO}_{2}$ as shown by 1,6 diphenyl-1,3,5-hexatriene fluorescence polarization probe (CAO 1990). Compared with quartz controls, 
fluidity was decreased when quartz plus aluminium citrate were added to the cell cultures simultaneously, although aluminium citrate did not influence membrane fluidity by itself.

Experiments with autoxidation of linolate, haemolysis of red blood cells, lipid peroxidation in macrophages, and in vivo effects of antioxidants on collagen produced by quartz injected subcutaneously, have shown that peroxidation of membrane lipids is not the primary mechanism whereby quartz attacks membranes (KilRoe-SMith 1974).

If silica or chrysotile particles are added to macrophages in serum-free medium, early cytotoxic effects are seen within minutes of exposure (ALLISON and Davies 1974). These effects are analogous to the haemolysis produced by silica and chrysotile and are thought to be due to the interaction with the plasmalemma. NAsH et al. (1966) have pointed out that silica particles unlike inert dusts, have on their surface in aqueous media silicic acid with multiple, rigidly placed hydroxyl groups which can act as hydrogen donors in hydrogen-bonding reactions with membrane phospholipids. Release by silica particles from liposomes composed of phospholipid and cholesterol has been reported.

Phagocytosis of DQ 12 quartz (MUNDER and Modolell 1987) and Brazilian crystallised quartz (BREHM 1996, BreHm et al. 1996) by bone marrow macrophages and bovine alveolar macrophages, respectively, induced different periods and intensities of luminescence demonstrating the formation and degradation of oxygen radicals. RAW 264.7 mouse macrophages treated with $\alpha$-quartz for $48 \mathrm{~h}$ indicated many regulatory mechanisms of gene expression were simultaneously triggered (SEGADE et al. 1995). The silica-induced genes SIG-12, -14 , and -20 corresponded to the genes for ribosomal proteins L13a, L32, and L26, respectively. SIG-61 is the mouse homologue of p21 RhoC. SIG-91 is identical to the $67-\mathrm{kDa}$ high-affinity laminin receptor. Four genes (SIG-41,SIG-81, SIG-92, and SIG-111) were not identified and are novel. In rat alveolar macrophages standard crystalline silica obtained from the Institute of Occupational Medicine, Chinese Academy of Preventive Medicine (Beijing) with a particle size of $<95 \%$ less than $5 \mu \mathrm{m}$ in diameter enhanced $\mathrm{O}_{2}{ }^{\circ-}$ and $\mathrm{H}_{2} \mathrm{O}_{2}$ formation (ZHANG et al. 2000). There were clear dose- and time-dependent relationships in silica-induced cytotoxicity and genotoxicity.

By phase-contrast microcinematography, ScHILLER $(1953,1954)$ showed a swift decline in the phagocytosis rate of quartz particles by muine alveolar macrophages as compared with that of powdered diamonds of the same size. Quartz dust poisoned the macrophages very quickly. Vacuolisation oc- curred only in cells that had taken up some quartz particles, while dust-free cells in the neighbourhood entered mitosis, and so did cells that had taken up powdered diamond (SCHILLER 1961). Interspecies comparisons gave no principal variation of the response between different animal species (SEIDEL et al. 1990). BRÜCKNER-NIEDER et al. (1992) developed a human cell model using HL-60 cells, a promyelocytic line. These cells can be differentiated by phorbol myristate acetate into non-proliferating macrophage-like cells (HL-60-M). Sikron F600 quartz particles were engulfed. At the ultrastructural level, the quartz was seen inside phagolysosomes, but the morphology of the cell remained normal.

Treatment of human alveolar macrophages with crystalline silica resulted in significant apoptosis within $6 \mathrm{~h}$. Macrophage scavenger receptors are trimeric integral membrane proteins which exhibit unique ligand-binding characteristics. The scavenger receptor may play an important role in the uptake of charged particulates (e.g. negatively charged silica) by alveolar macrophages. Silica treatment of human alveolar macrophages resulted in increased interleukin-converting enzyme (detection of p20 fragments) and cpp32 $\beta$ (enhanced degradation of cpp32) activity (Iyer and Holian 1997). Pretreatment of cells with the interleukin-converting enzyme and cpp32 $\beta$ inhibitors, Z-Val-Ala-Aspfluoromethyl ketone $(10 \mu \mathrm{M})$ and DEVD, respectively, significantly inhibited apoptosis. In specific pathogen-free Wistar rats, $2.5 \mathrm{mg}, 7.5 \mathrm{mg}$, and 22.5 mg Min-U-Sil 5 silica suspended in $0.5 \mathrm{ml}$ saline, respectively, 10 days after intratracheal instillation induced apoptosis in a dose-related quantity of bronchoalveolar lavage cells (LEIGH et al. 1997). Engulfment of apoptotic cells by macrophages was also noted. 56 days after instillation, morphologically apoptotic cells, the majority of which were considered to be differentiated macrophages, could be identified in granulomas.

The RAW 264.7 macrophage cell line was more sensitive, and the IC-21 cell line more tolerant to silica particle $(<1 \mu \mathrm{m})$ exposure $(0.2$ or $1 \mathrm{mg} / \mathrm{ml}$ for $6 \mathrm{~h})$ as evidenced by significantly higher apoptotic responses in RAW $264.7(P<0.05)$ (GozAL et al. 2002). RAW 264.7 macrophages exhibited enhanced TNF- $\alpha$ production and NF- $x$ B activation in response to silica, whereas IC-21 macrophages did not produce TNF- $\alpha$ in response to silica and did not induce NF- $x \mathrm{~B}$ nuclear binding. Inhibition of NF- $x \mathrm{~B}$ in RAW 264.7 cells with $50 \mu \mathrm{M}$ BAY 11-7082, an inhibitor of I $x \mathrm{~B}$ phosphorylation, significantly increased apoptosis while inhibiting TNF- $\alpha$ release.

In rat alveolar macrophages, SHEN et al. (2001) showed a temporal pattern of apoptotic events 
starting with the formation of reactive oxygen species and followed by caspase- 9 and caspase- 3 activation, poly(ADP-ribose)polymerase clevage and DNA fragmentation. Silica-induced apoptosis was significantly attenuated by a caspase-3 inhibitor, $\mathrm{N}$ acetyl-Asp-Glu-Val-Asp aldehyde, and ebselen (formula [65]).

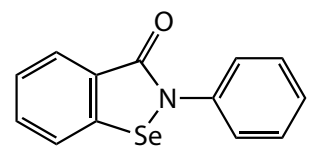

Ebselen, 2-phenyl-1,2-benzisoselenazol-3(2H)-one

KIM et al. (2001) showed that pan-caspase inhibitor benzyloxycarbonyl-Val-Ala-Asp-fluoromethyketone or $t$-butyloxycarbonyl-Asp-fluoromethylketone caused the death of lipopolysaccharide-activated RAW 264.7 cells with apoptotic features.

Exposure of $1 \times 10^{6}$ human alveolar macrophages to $100 \mu \mathrm{g}$ silica/ml for $4 \mathrm{~h}$ increased ferritin protein concentrations by approximately $50 \%$ of the baseline value in both supernatants and lysates (GHIO et al. 1997). Inclusion of $1.0 \mathrm{mM}$ deferoxamine, an iron chelator, in the reaction mixtures inhibited increases after silica. There were no increases in ferritin after incubation with acid-washed particles or silica with complexed zinc cation. There were no significant differences in levels of ferritin cDNA between any of the exposures suggesting a posttranscriptional control of ferritin expression.

Cortisone ( $5 \mathrm{mg}$ given to rats 5 days a week for 3 weeks and then 3 days a week for the rest of the experiment) produced relative immobilisation of the dust cells, so that they were unable to migrate, and therefore unable to concentrate the quartz $(50 \mathrm{mg}$ instilled intratracheally) into foci (HARRISON et al. 1952, KING and HARRISON 1960).

Lazaroids (21-aminosteroid U-75412E) at a concentration of $5 \mu \mathrm{M}$ protected rat alveolar macrophages against crystalline silica (Min-U-Sil $<5 \mu \mathrm{m}$ )induced cytotoxicity (HuANG et al. 1998). Maximal inhibition of lactate dehydrogenase (EC 1.1.1.27) release from macrophages was achieved at a lazaroid concentration of $20 \mu \mathrm{M} . \mathrm{H}_{2} \mathrm{O}_{2}$ secretion from alveolar macrophages was also inhibited at $5 \mu \mathrm{M}$ lazaroid concentration. $N$-Acetyl- $\beta$-D-glucosaminidase (EC 3.2.1.17) showed minimal inhibition (17\%) at all concentration of lazaroid used. Superoxide dismutase (EC 1.15.1.1) and glutathione peroxidase (EC 1.11.1.9) showed concentration-dependent decreases at all the concentration of lazaroids.

In vitro, disodium cromoglycate, the disodium salt of 1,3-bis 2-carboxycromon-5-yloxy-hydroxypropan, an electron scavenger (CARMICHAEL et al. 1988), was effective in protecting Chinchilla male rabbit alveolar macrophages from silica-induced necrosis only when the quartz dust was pre-treated with the drug dissolved in saline for $60 \mathrm{~min}$ at $37^{\circ} \mathrm{C}$, while pre-treatment of the macrophages with cromoglycate for $30 \mathrm{~min}$ before phagocytosis of the Dörentrup quartz particles had no or a negligible protective effect on the cells (VLCKoví et al. 1976).

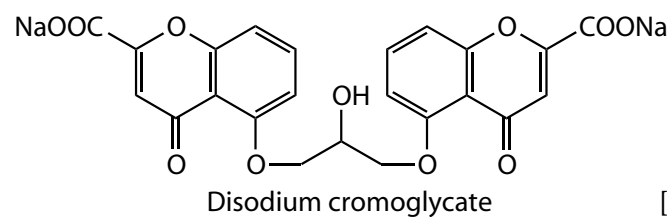

Macrophage inflammatory protein (MIP) $1 \alpha$ mRNA was upregulated in human peripheral blood monocytes after a 4-6 h exposure to $100 \mu \mathrm{g}$ Min-U-Sil/ml (ERKAN et al. 1997).

Treatment of RAW 264.7 murine macrophage cultures with dimethyl sulphoxide, extracellular glutathione, or $\mathrm{N}$-acetyl-L-cyteine decreased cristobalite (particle size range 0.08-1.5 $\mu \mathrm{m}$; particle dose $35 \mu \mathrm{g} / \mathrm{cm}^{2}$ or 54-78 particles/cell)-induced tumour necrosis factor- $\alpha$ mRNA levels by $40 \%, 20 \%$, and $42 \%$, respectively (BARRETT et al. 1999). Both MIP- $1 \alpha$ and MIP- $1 \beta$ mRNA levels were reduced at a magnitude similar to the reduction in TNF- $\alpha$ mRNA levels, whereas monocyte chemotactic protein (MCP)-1 mRNA levels were reduced at a magnitude similar to the reduction in MIP-2 mRNA levels following antioxidant treatment.

Pure cristobalite was considerably less cleared from the lungs of male Fischer 344 rats exposed for $6 \mathrm{~h}$ per day to $13 \pm 2.4 \mathrm{mg} / \mathrm{m}^{3}, 40 \pm 12.4 \mathrm{mg} / \mathrm{m}^{3}$, and $81 \pm 15.3 \mathrm{mg} / \mathrm{m}^{3}$, respectively, on twice 4 days with an interval of 2 days compared with two quartz materials (Hemenway et al. 1990). There was little or no clearance after the initial 30 days post exposure. Cristobalite showed an early and sustained response with an elevated macrophage, neutrophil, and lymphocyte count through 180 days post exposure.

Human alveolar macrophages produced more reactive oxygen species in response to particles than rat alveolar macrophages (RAHMAN et al. 1997).

Granite dust containing quartz crystal (10 to $30 \%$ silicon dioxide) and other aluminium, magnesium, and calcium silicates was present within the majority of alveolar macrophages lavaged from Vermont granite workers with 4 to $36 \mathrm{yr}$ of employment in the industry compared with those from control subjects, as determined by polarising light microscopy and confirmed by scanning electron microscopy with X-ray energy spectroscopy (CHRistman et al. 1985). There were no differences in the phagocytosis of zymosan particles (labelled 
with ${ }^{99 \mathrm{~m}} \mathrm{Tc}$ ) or the viability of macrophages (defined by trypan blue dye exclusion) from granite workers compared with those from nonexposed volunteers.

Kaolin is a nonfibrous hydrated aluminium silicate $\left(\mathrm{Al}_{2} \mathrm{O}_{3} \cdot \mathrm{SiO}_{2} \cdot 2 \mathrm{H}_{2} \mathrm{O}\right)$ that can cause pneumoconiosis in workers processing the clay commercially (Thomas 1952, LynCH and McIver 1954, Hale et al. 1956, Sheer 1964, Kennedy et al. 1983, SepulVEDA et al. 1983, ALteKruse et al. 1984, LAPENAS et al. 1984, Morgan et al. 1988). Its basic unit of structure consists of tetrahedral and octahedral sheets in which the anions at the exposed surface of the octahedral sheet are hydroxyls. The catalytic activity of kaolin for generating hydroxyl radicals from hydrogen peroxide was studied in a chemical system that measured $\mathrm{HO}^{\circ}$ as evolution of methane from dimethylsulfoxide. In the presence of $1 \mathrm{mM}$ ascorbate as a reducing agent, and $10 \mathrm{mM} \mathrm{H}_{2} \mathrm{O}_{2}$, hydrous and calcined kaolin generated $\mathrm{CH}_{4}$ concentrations of $1634 \pm 328 \mathrm{ppm}$ and $1395 \pm 29 \mathrm{ppm}$, respectively (BASER et al. 1990). Surface modification with dipalmitoyl lecithin, the lipid of pulmonary surfactant, blocked generation of $\mathrm{HO}^{\bullet}$ in hydrous kaolin $\left(38 \pm 38 \mathrm{ppm} \mathrm{CH}_{4}\right)$ but not in calcined kaolin $(875 \pm 262 \mathrm{ppm})$. Generation was inhibited by the $\mathrm{HO}^{\circ}$ scavenger dimethylthiourea $(15 \mathrm{mM})$ or by preincubating kaolin for $48 \mathrm{~h}$ with the iron chelator deferoxamine $(0.5 \mathrm{mg} \mathrm{kaolin} / \mathrm{ml}$ in aqueous solution of $18 \mathrm{mM}$ deferoxamine).

$$
\begin{aligned}
& \text { Decomposition of kaolin on heating } \\
& 3\left(\mathrm{Al}_{2} \mathrm{O}_{3} \cdot 2 \mathrm{SiO}_{2}\right) \rightarrow 3 \mathrm{Al}_{2} \mathrm{O}_{3} \cdot 2 \mathrm{SiO}_{2}+4 \mathrm{SiO}_{2} \\
& \text { kaolin } \\
& \text { mullite free silica }
\end{aligned}
$$

In human pulmonary tissue obtained at thoracotomy or autopsy from 5 kaolin workers from Georgia particulate-filled macrophages distended the interstitium, both in septa and in peribronchialperivascular areas (LAPENAs et al. 1984). The particles were predominantly intracellular in macules. Only in one patient were large numbers of particleladen macrophages found in the alveolar spaces, presumably because of ongoing kaolinite exposure.

Rats instilled intratracheally with untreated Cornish kaolin showed foci of alveoli filled with large macrophages which contained some dust (KING et al. 1948). Ignited Cornish kaolin in 14 to 73 days produced a simple phagocytic reaction without reticulinosis. In a rat which surviving for 140 days there was a phagocytic reaction in the perivascular connective tissue, associated with a strictly local reticulinosis.

Riebeckite, a non-asbestiform polymorph of crocidolite in vitro was non-toxic to alveolar macrophages at doses on a mass basis where crocidolite exhibited substantial activity (Mossman and Sesкo 1990). CAstranova et al. (1994) starting from surface areas found that riebeckite exhibited cytotoxicity to rat alveolar macrophages in culture at doses equal to or less than those for crocidolite.

Asbestos dust was readily phagocytosed by macrophages (DAVIs 1963, 1967). Each cell could have several dozen dust containing phagosomes at any one time. Although it was obvious from these experiments that asbestos was not rapidly toxic in the same way as silica, SMIth and DAVIs (1971) investigated the occurrence of acid phosphatase in guinea-pig granulomas produced by the intrapleural injection of chrysotile asbestos dust by histochemical staining combined with electron microscopy. In dust-containing macrophages and giant cells, often fewer than 50 per cent of primary lysosomes and as few as 10 per cent of dust-containing phagosomes showed evidence of acid phosphatase. There was no increase in enzyme activity between 2 and 4 weeks.

Phagocytosis of asbestos particles up to $0.5 \mu \mathrm{m}$ in length occurs by alveolar macrophages (MoRGENROTH 1973) attracted to the alveolar duct bifurcations where inhaled asbestos fibres are deposited (WARHeit et al. 1984). Inhaled asbestos activates a complement-dependent chemoattractant for macrophages (WARHEIT et al. 1985). Phagocytosis is followed by the appearance of hæmosiderin in the cytoplasm, then by intracellular transport of iron micelles from hæmosiderin granules into the phagosomes with the asbestos fibres. Iron micelles progressively concentrate in the vicinity if the fibres. The central fibre, with its coating of hæmosiderin, and the investing membranes of the phago-

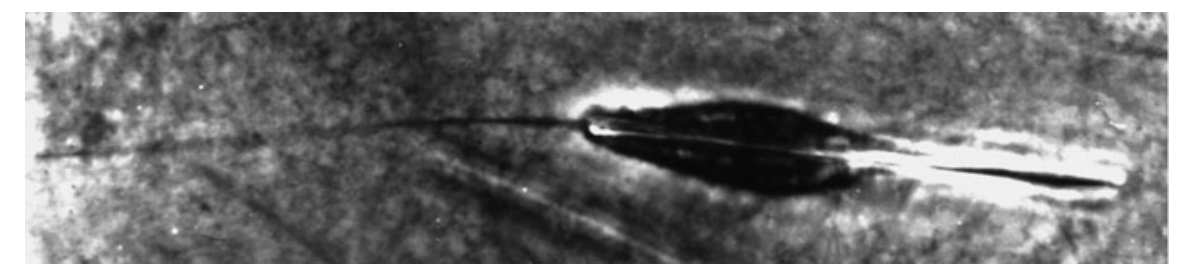

Fig. 153. Frustrated phagocytosis of powdered crocidolite from Griqualand. Organ culture of the lung of a 3-day old mouse. The preparation was fixed in Carnoy's fluid, stained with haematoxylin after Böhmer, and mounted in toto in Rhenohistol ${ }^{\circledR}$. Eyepiece Leitz Periplan $4 \times$; objective Leitz Pv Fl oil 70/1.15. Positive phase contrast (from ScHILler 1954) 
some are regarded as essential elements of the ferruginous body.

Scanning electron micrographs of alveolar macrophages incubated with fibres show the cell membrane placing itself around the fibre like a sleeve (RoBOCK and KLOSTERKÖTTER 1977). In mouse Lfibroblasts, the incomplete incorporation increased permeability of the plasmalemma in the area of invagination (BECK et al. 1971). The early cytotoxic effects of different types of asbestos are correlated with their magnesium content (HARINGTON et al. 1974) and it seems likely that the positively charged magnesium groups on the surface of the fibres interact with negatively charged membrane sialoglycoproteins. Clustering of the membrane proteins follows, and this is associated with increased passive ion flux through the membranes in excess of active cation transport capacity. As a result of the osmotic pressure exerted by entrapped protein, water accumulates and the cell swells until it bursts. Protection against lysis by asbestos but not by silica can be achieved if a nonpenetrating solute, such as sucrose, is present in the extracellular medium, counterbalancing the osmotic pressure of intracellular protein (ALLISON 1974).

With either successful or frustrated (Fig. 153) phagocytosis, a direct contact between lysosomal content (acidic $\mathrm{pH}$, strongly oxidising medium) and fibre will occur, which may yield different redox reactions. This on one hand modifies the surface of the mineral itself, and on the other hand provokes a prolonged generation of reactive oxygen species, the release of which into the surrounding medium can be demonstrated by lucigenin-enhanced chemiluminescence (ZeLler et al. 1993, BREHM 1996).

30-min exposure of $10^{6}$ human alveolar macrophages to $100 \mu \mathrm{g}$ chrysotile B caused a significant (up to 2.5 -fold) increase in reactive oxygen intermediates release compared with control experiments (Oettinger et al. 1999). $100 \mu \mathrm{g}$ Chrysotile B induced a significant maximum 4.0 -fold up-regulation of NK- $x \mathrm{~B}$ gene expression.

From rabbit alveolar macrophages, UICC chrysotile $\mathrm{A}$ and leached $(0.1 \mathrm{~N}$ oxalic acid) amphiboles selectively released $\beta$-galactosidase (JAURAND et al. 1980).

Macrophages obtained from male BD-IX rats dusted with UICC crocidolite were able to stimulate both dusted and control lymphocytes to proliferate, demonstrating that crocidolite inhalation resulted in alveolar macrophages with different properties from those of control macrophages (MiLler et al. 1980).

Alveolar macrophages are activated and release growth factors that stimulate mesenchymal cell proliferation and enhanced formation of extracellular matrix. Both insulin-like growth factor-I (IGF-I), and transforming growth factor $\beta$ (TGF- $\beta$ ) regulate cellular growth and promote matrix accumulation and are hypothesised to play important roles in asbestosis. LEE et al. (1997) performed immunohistochemistry using polyclonal antibodies to specific synthetic peptides of the three mammalian isoforms of TGF- $\beta$ (TGF- $\beta 1,-\beta 2,-\beta 3$ ) and to TGF-I on lungs of sheep treated intratracheally with UICC Canadian chrysotile asbestos fibres. All three TGF- $\beta$ isoforms were found in alveolar macrophages. While asbestos had minimal acute effects on cytokine production by the human alveolar macrophage, intense chronic exposure to asbestos lead to the enhanced basal release of significant amounts of several cytokines that have activity for the fibroblast, even in the absence of overt fibrosis (PERKINs et al. 1993).

Quantification of IGF-I also manifested elevated IGF-I levels in silicotic rat bronchoalveolar lavage fluids, which tended to increase with silica exposure in vivo, but no alteration in IGF-I level could be found in sera (CHEN et al. 1994).

Chrysotile, but not amphiboles (crocidolite, anthophyllite, or amosite), stimulated a rapid $(<1$ $\mathrm{min})$ and dose-dependent $(2.5-50 \mu \mathrm{g} / \mathrm{ml})$ production of superoxide anion at noncytotoxic dose $(2.5-25 \mu \mathrm{g} / \mathrm{ml})$ by guinea pig alveolar macrophages as monitored by measuring the rate of ferricytochrome $c$ reduction at $550 \mathrm{~nm}$ (RoNeY and Holian (1989). The stimulation of superoxide anion production by chrysotile could be blocked by putative protein kinase $\mathrm{C}$ inhibitors (staurosporine, sphingosine, and fluphenazine). Chrysotile also stimulated phosphatidylinositol turnover as measured using ${ }^{32} \mathrm{P}_{\mathrm{i}}$ incorporation into phospholipids, $\left[{ }^{3} \mathrm{H}\right]$ diacylglycerol levels, and intracellular calcium mobilisation as measured using fura- 2 and ${ }^{45} \mathrm{Ca}$. Pertussis toxin partially blocked chrysotile stimulated superoxide anion production.

Asbestos initiates the formation of oxygen radicals through at least two mechanisms: activation of respiratory burst in phagocytic cells and ironcatalysed reactions (KAMP et al. 1992). The dramatic enhancement of release of superoxide anions found by Donaldson et al. (1995) and Hill et al. (1995) when long fibre amosite was opsonized with IgG, confirmed the greatly increased biological activity after opsonization (SCHEUle and HoliaN 1989, Nyberg and Klockars 1990, Perkins et al. 1991, Donaldson et al. 1992). All asbestos fibres were able to induce chemiluminescence but chrysotile induced maximal chemiluminescence at higher concentrations than amosite and crocidolite (LIM et al. 1997). Protein kinase C inhibitors (sphingosine and staurosporine) suppressed the ability of asbestos to induce oxygen radical generation. Phospholipase C inhibitors (U73122 and neomycin) and protein tyrosine kinase inhibitors (erbstatin and genis- 
tein) decreased oxygen radical generation of asbestos stimulated rat alveolar macrophages. Oxygen radical formation was not suppressed by an adenylate cyclase activator (forskolin), a protein kinase A inhibitor (H-8), and a protein serine-threonin phosphatase inhibitor (okadaic acid). Phospholipase $C$ and protein tyrosine kinase inhibitors suppressed the increment of phosphoinositide turnover by amosite.

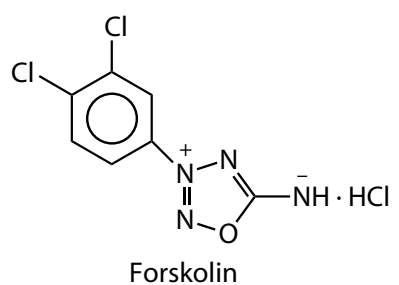

Alveolar macrophages from Fischer 344 rats inhaling crocidolite or chrysotile asbestos showed significant elevation $(P<0.05)$ in nitrite/nitrate levels which were ameliorated by $N^{G}$-monomethyl-Larginine (QUINLAN et al. 1998). Temporal patterns of ${ }^{\circ} \mathrm{NO}$ generation from alveolar macrophages correlated with neutrophil influx in bronchoalveolar lavage samples after asbestos inhalation. To determine the molecular mechanisms and specificity of iNOS promoter activation by asbestos, RAW 264.7 cells and alveolar macrophages isolated from control rats were exposed to crocidolite in vitro. These cells showed increases in steady-state levels of iNOS mRNA in response to asbestos and more dramatic increases in both iNOS mRNA and immunoreactive protein after addition of lipopolysaccharide (LPS). After transfection of an iNOS promoter/luciferase

Table 39. Toxicity of silicates containing iron

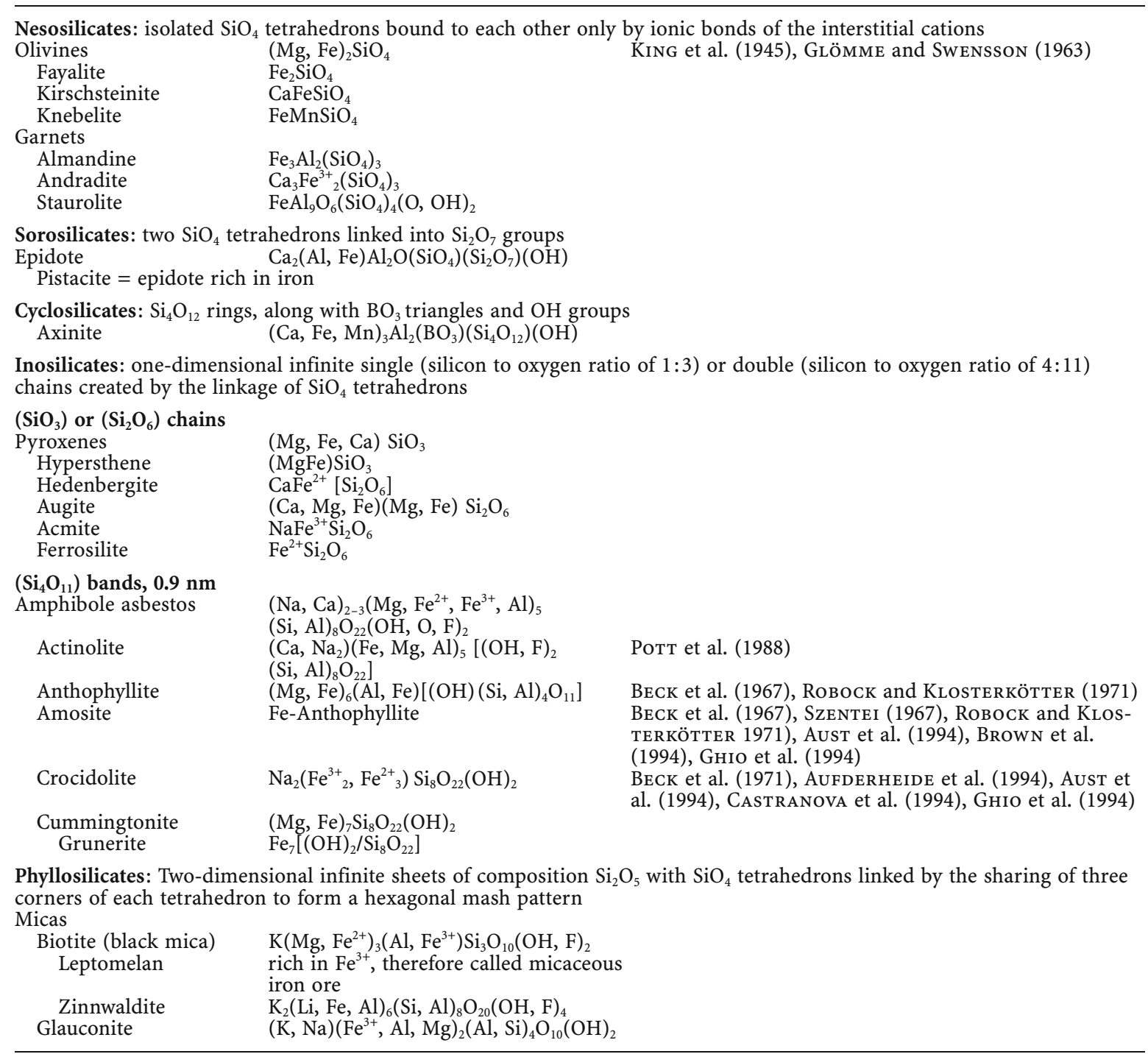


reporter construct, RAW 264.7 cells exposed to LPS, crocidolite asbestos and its nonfibrous analogue, riebeckite, revealed increases in luciferase activity whereas cristobalite had no effects.

Silicate complexes with $\mathrm{Fe}^{3+}$ were observed by Hazel et al. (1949). Weber and Stumm (1965) investigating the equilibria,

$\mathrm{Fe}^{3+}+\mathrm{Si}(\mathrm{OH})_{4} \leftrightarrow \mathrm{FeSiO}(\mathrm{OH})_{3}{ }^{2+}+\mathrm{H}^{+}$

found a formation constant, $\beta_{111}=0.57$. For the reaction

$\mathrm{Fe}^{3+}+\mathrm{SiO}(\mathrm{OH})_{3}{ }^{-} \leftrightarrow \mathrm{FeSiO}(\mathrm{OH})_{3}{ }^{2+}$

they deduced, by using a value of 9.5 for the $\mathrm{pk}_{1}$ value of silicic acid, a formation constant of $\beta_{1}=$ $1.8 \times 10^{9}\left(\log \beta_{1}=9.26\right)$.

Comparing the preference of $\mathrm{SiO}(\mathrm{OH})_{3}{ }^{-}$to form complexes with different cations one obtains the series: $\mathrm{Ca}^{2+}<\mathrm{Mg}^{2+}<<\mathrm{Fe}^{3+}<\mathrm{H}^{+}$.

$\mathrm{Fe}^{3+}$ forms considerable stronger complexes which may, in natural water, be present in significant amounts.

Surface-complexed iron $\left[\mathrm{Fe}^{3+}\right]$ observed after intrapleural injection of $30 \mathrm{mg}$ of either amosite, crocidolite, and chrysotile both genuine and saturated with $\mathrm{Fe}^{3+}$ in Sprague-Dawley rats corresponded to oxidant generation, measured as barbituric acid reactive products of deoxyribose, and more covalently closed, circular DNA strand scission induced by these asbestos fibres (GHIO et al. 1994).

Metal complexes of flavonoids were adsorbed by chrysotile fibres considerably better than uncomplexed compounds and probably for this reason flavonoid metal complexes have better protective properties against asbestos-induced haemolysis (Kostyuk et al. 2001).

The endocytosis of the fibre is associated with oxidant generation and therefor should increase with the concentration of complexed iron and surface area (Hobson et al. 1990, GHio et al. 1992). Such endocytosis positions the silicate surface with the complexed transition metal in the proximity of DNA, which also can co-ordinate this metal (ANDRonIKASHVILI et al. 1974, BARRY et al. 1983). Subsequent oxidant generation could then result in strand breaks, genotoxicity, and cancer (LiBBus et al. 1989).

Table 40. Surface area, percentage $\mathrm{SiO}_{2}$, and surface silanol density of fibrous silicates (from GHIo et al. 1994)

\begin{tabular}{lrll}
\hline Silicate & $\begin{array}{l}\text { Surface area } \\
\left(\mathrm{m}^{2} / \mathrm{g}\right)\end{array}$ & $\begin{array}{l}\text { Percentage } \\
\mathrm{SiO}_{2}\end{array}$ & $\begin{array}{l}\text { Surface silanol } \\
\text { density } \\
\text { (groups/nm }\end{array}$ \\
\hline Amosite & $2.3 \pm 0.4$ & $49.2 \pm 0.5$ & $7.5 \pm 1.8$ \\
Crocidolite & $8.7 \pm 1.0$ & $48.5 \pm 0.3$ & $4.7 \pm 0.6$ \\
Chrysotile & $28.8 \pm 1.4$ & $39.8 \pm 0.9$ & $1.8 \pm 0.1$ \\
\hline
\end{tabular}

Nitric oxide may interact with the surface of mineral fibres. Cigarette smoke which increases the risk of asbestos-induced lung cancer contains up to $600 \mu \mathrm{g}{ }^{\circ} \mathrm{NO}$ per cigarette (IARC 1985) raised the fibre-bound "NO from 34 to $85 \mu \mathrm{g} \mathrm{NO} / \mathrm{g}$ fibre (LeANDERson et al. 1997). "NO was found in different amounts on chrysotile B, crocidolite, amosite and silicon carbide whiskers. There was a strong correlation between the amount of ${ }^{\circ} \mathrm{NO}$ and the specific surface area of these fibres $(r=0.98) .{ }^{\circ} \mathrm{NO}$ could not be demonstrate on rockwool fibres, manmade vitreous fibres MMV21 and MMV22 or silicon nitride whiskers.

The haemolytic and early cytotoxic effects of different types of asbestos fibres are correlated with their magnesium content (HARING TON et al. 1974). It seem likely that the positively charged magnesium groups on the surface of the fibres interact with the negatively charged membrane sialoglycoproteins. Clustering of the membrane proteins follows, and this is associated with increased passive ion flux through the membranes in excess of the active cation transport capacity. As a result of the osmotic pressure exerted by entrapped protein, water accumulates and the cells swells until it bursts. Protection against lysis by asbestos but not by silica can be achieved if a non-penetrating solute, such as sucrose, is present in the extracellular medium, counterbalancing the osmotic pressure of intracellular protein (ALLISON 1974). When asbestos fibres are incubated with cells in the presence of fresh serum, haemolysis can occur by activation of the complement system (HARINGTON et al. 1974).

The surface of chrysotile asbestos modified with a chemically bound coating of an alkyl hydrocarbon altered phagocytic activity, cytotoxicity and mutagenicity in human alveolar macrophages obtained by fibre optic bronchial lavage (MACE jr et al. 1981). Scanning electron microscopy of cell particle interactions and phagocytosis showed no impairment of the processes by the coated fibres. Cytotoxicity studies by trypan blue exclusion revealed a significant reduction in alveolar macrophage mortality over 24 and $48 \mathrm{~h}$ incubation periods, compared to uncoated fibres.

Erionite particles (sizes from colloidal to about 10-15 $\mu \mathrm{m}$ in length) upon phagocytosis by the rat lung alveolar macrophage cell line, NR8383 increased generation of reactive oxygen metabolites as shown by fluorescence imaging with 5-(and 6) carboxy-2',7'-dichlorodihydrofluorescein diacetate (LoNG et al. 1997). Sharply delineated fluorescence characteristically evolved within fibre-exposed cells during the period up to $90 \mathrm{~min}$ following the cellfibre contact. For the $20-40 \mathrm{~min}$ period, the erionite-exposed cells had a mean cellular fluores- 
cence more than three times that of controls. During the 40-60 $\mathrm{min}$ period, the erionite-exposed cells had a mean cellular fluorescence still more than twice that of controls. Only during the 60-80 min period did the mean erionite cellular fluorescence decline to a level relatively indistinguishable compared to controls.

Wollastonite, an aciculate or fibrous silicate mineral $\left(\mathrm{CaSiO}_{3}\right)$, in a dose of $25 \mu \mathrm{g} / \mathrm{ml}$ in vitro reduced the viability of rat alveolar macrophages from $77.0 \pm 11.0 \%$ to $66.4 \pm 8.9 \%(P<0.05)$ and increased lactate dehydrogenase release from $37.2 \pm 10.2 \%$ to $46.5 \% \pm 7.3 \%(P<0.05)$ within $24 \mathrm{~h}$ (PASANEN et al. 1984). Wollastonite $(<10 \mu \mathrm{m})$ was moderately cytotoxic in both haemolysis and alveolar macrophage enzyme release (lactate dehydrogenase, $\beta$-glucuronidase, $\beta$ - $N$-acetylglucosaminidase) studies whereas shorter fibres $(<5 \mu \mathrm{m})$ were only mildly cytotoxic (VALlyathan et al. 1984).

Wollastonite (up to $400 \mu \mathrm{g} / \mathrm{ml}$ ) produced no significant DNA fragmentation in a 24 -h culture of $h u$ man alveolar macrophages (HАMiLton et al. 1996).

Glass fibres with diameters $\leq 3 \mu \mathrm{m}$ and lengths $\leq 10 \mu \mathrm{m}$ are cleared quite efficiently from the rat lung, presumably by macrophage-mediated processes (Morgan et al. 1982). For MMVF34, the inhalation test at different duration showed a similar persistence patter, while the intratracheal test longer elimination half-times especially for long fibres (KAMstrup et al. 1998). The MMVF34 fibre is considerably less biopersistent than the traditional MMVF21 fibre when comparing the calculated elimination half-times after short-term inhalation. Throughout the 18 month period, only slight macrophage reaction was seen in the lungs of rats exposed to MMVF34 (Wagner grade 2) although in some animals occasional microgranulomas were seen (Wagner grade 3 ).

Pulmonary lavage washings from glass-fibredusted inbred male BD-IX rats consisted of 5-10\% lymphocytes and a heterogeneous macrophage population, varying in size from $10-40 \mu \mathrm{m}$ (MilleR 1980). Many cells contained a great number of glass fibres, and multinucleate giant cells comprised between $10-20 \%$ of the population. Many macrophages also contained protruding fibres. Such cells still adhered to the glass substrate after $22 \mathrm{~h}$ of incubation and retained their characteristic surface ruffles.

Dust- and fibre-induced release of lactate dehydrogenase from rat alveolar macrophages at a dose of $10 \mathrm{mg} / \mathrm{ml}$ by man-made vitreous fibres was less than by quartz, that by RCF 1 the least of all the fibres, even smaller than that induced by titanium dioxide (Luото et al. 1997). MMVF 10 glasswool fibre caused less LDH release than MMVF 21 rockwool fibre, the MMVF 22 slagwool fibre or the ceramic fibre RCF $3(P<0.005)$. Refractory ceramic fibres (RF2) and rock wool (RW1) increased the release of lactate dehydrogenase with increasing fibre concentration (KIM et al. 2001). From these parameters, RF2 was shown to exhibit greater cytotoxicity than did RW1.

For each of 5 lengths (generated by a dielectrophoretic classifier) of Manville Code 100 fibres to rat macrophages cultured on 96-well plates for $18 \mathrm{~h}$, toxicity depended on concentration ranging from 0-500 $\mu \mathrm{g}$ fibres/ml (BLAKe et al. 1997, 1998). Lucigenin-enhanced chemiluminescence declined and lactate dehydrogenase release increased with increasing fibre concentration. On a $\mu \mathrm{g} / \mathrm{ml}$ basis, an intermediate-length fibre (mean length $=17 \mu \mathrm{m}$ ) showed the greatest toxicity, while both shorter and longer fibres caused less damage.

A significantly higher percentage of rat alveolar macrophages than peritoneal macrophages endured frustrated phagocytosis of MMVF10 and MMVF21 fibres (DöRger et al. 2001). In line with these findings, alveolar macrophages generated higher levels of oxygen radicals than peritoneal macrophages upon exposure to MMVF21 fibres. In contrast, MMVF10 fibres failed to induce the generation of reactive oxygen species by both alveolar and peritoneal macrophages.

$\mathrm{P}_{388 \mathrm{D}_{1}}$ macrophages grown in RPMI 1640 medium flowthrough $(2-3 \mathrm{ml} / \mathrm{h})$ culture dissolved manmade vitreous fibres leading to subsequent morphological changes of fibres as shown by scanning electron microscopy, while the dissolution of $\mathrm{Si}, \mathrm{Fe}$, and $\mathrm{Al}$ was measured on days $4,8,16$, and 28 using an atomic absorption spectrometer (Lиото et al. 1998).

HL-60-M cells challenged by glass wool code A or stone wool code $\mathrm{G}$ fibres preincubated in unbuffered saline for about 4 weeks, produced less intense luminol-enhanced chemiluminescence, as compared with freshly suspended fibres (Zoller and Zeller 2000). MMVF 21 and HT-N fibres as well as crocidolite and erionite did not show any decrease in chemiluminescence intensity after incubation in aqueous solutions.

Intracellular adenosine triphosphate content of rat alveolar macrophages obtained by bronchoalveolar lavage was decreased by refractory ceramic fibres (RF2) and rock wool (RW1) in a concentration-dependent manner (KIM et al. 2001). This fibres suppressed succinate-triggered oxygen consumption. Polyinsosinic acid, a ligand of the scavenger receptor, inhibited the man-made vitreous fibre-induced decrease in ATP concentration.

Silicon nitride $\left(\mathrm{Si}_{3} \mathrm{~N}_{4}\right)$, a fibrous material, increased the release of lactate dehydrogenase, but 
not of acid phosphatase, from lavaged bovine pulmonary macrophages in vitro (FISHER et al. 1989). Scanning electron micrographs indicated that the fibrous $\mathrm{Si}_{3} \mathrm{~N}_{4}$ is cytotoxic and poorly tolerated by macrophages.

Complexes with catechol and other $o$-diphenols (Rosenheim et al. 1931, Weiss et al. 1956, 1959, BAUMANN 1963) form as follows:

$3 \mathrm{C}_{6} \mathrm{H}_{4}(\mathrm{OH})_{2}+\mathrm{Si}(\mathrm{OH})_{4} \leftrightarrow\left[\mathrm{Si}\left(\mathrm{C}_{6} \mathrm{H}_{4} \mathrm{O}_{2}\right)_{3}\right]^{2-}+4 \mathrm{H}_{2} \mathrm{O}+2 \mathrm{H}^{+}[241]$

In these complexes silicon is octahedrally surrounded by six oxygens (three dihydroxybenzenes, didentately bound to $\mathrm{Si}$ ). According to WeIss et al. (1961), besides these mononuclear silicon complexes, also binuclear complexes (dimers) seem to be formed.

The relationship between silicate minerals and the interferon system was studied by HAHON and Воотн (1987) in monkey kidney cell monolayers. Minerals within the classes nesosilicate, sorosilicate, cyclosilicate, and inosilicate exhibited either little or marked ( $50 \%$ or greater) inhibition of interferon induction. Within the inosilicate class, however, minerals of the pyroxenoid group (wollastonite, pectolite, and rhodonite) all significantly showed a two- to threefold increase in interferon production. Silicate materials in the phyllosilicate and tectosilicate classes all showed inhibitory activity for the induction process. When silicate minerals were coated with poly(4-vinylpyridine- $\mathrm{N}$-oxide), the inhibitory activity of silicates on viral interferon induction was counteracted.

In the lung-associates lymph nodes of rats instilled intratracheally with DQ12 quartz (surface area $9.4 \mathrm{~m}^{2} / \mathrm{g}$ ) or Aerosil ${ }^{\circledR} 150$ (surface area $150 \pm 15 \mathrm{~m}^{2} /$ g), poly-2-vinylpyridine- $N$-oxide treatment significantly reduced the incidence and severity of inflammation as evidenced by the presence of well cir- cumscribed aggregates of intact particle-laden macrophages without signs of degeneration and accompanying granulocyte infiltrations and fibrosis (ERNST et al. 2002).

\section{Granuloma Formation After Intravenous Injections of Quartz}

In an extended study of experimental tissue reactions following intravenous injections of silica and other dusts Simson and Strachan (1940) stated that the distribution of the microscopically visible dust in the organs and tissues of the rabbit in the early stage is the same irrespective of whether the dust later exhibits toxic or non-toxic properties. The chief situations in which it can be found are the organs provided with sinusoids and an active reticulo-endothelial tissue in relation to the blood vascular system, e.g. liver, spleen, bone-marrow and lymph-nodes.

\section{Liver}

GARDNER et al. (1944) produced massive fibrosis in the liver by 20 intravenous injections of powdered quartz (1-3 $\mathrm{mm}$; total dose $500 \mathrm{mg})$ suspended in saline into the ear veins of rabbits. When the quartz powder was suspended in 0.33 per cent gelatinous colloidal alumina this silicosis failed to develop, only a marked foreign body reaction being produced in the liver despite the accumulation there of a large amount of quartz mixed with aluminium.

\section{Spleen}

Simson and Strachan (1940) noted great variation in the size of the rabbit spleen, quartz dust
Fig. 154. Silicotic granuloma in the liver $(20 \mathrm{~g})$ of a $410 \mathrm{~g}$ male white rat (No.36) 12 weeks after an intravenous application of $25 \mathrm{mg}$ Brazilian crystallised quartz (specific surface $2 \mathrm{~m}^{2} / \mathrm{g}$ ). Fixed by immersion in Bouin's fluid. Paraplast. Haematoxylin and eosin. Objective Leitz Pl 40/0.65. LeitzOrthomat ${ }^{\circledR}(\times 3.2)$. Agfachrome 50 L professional

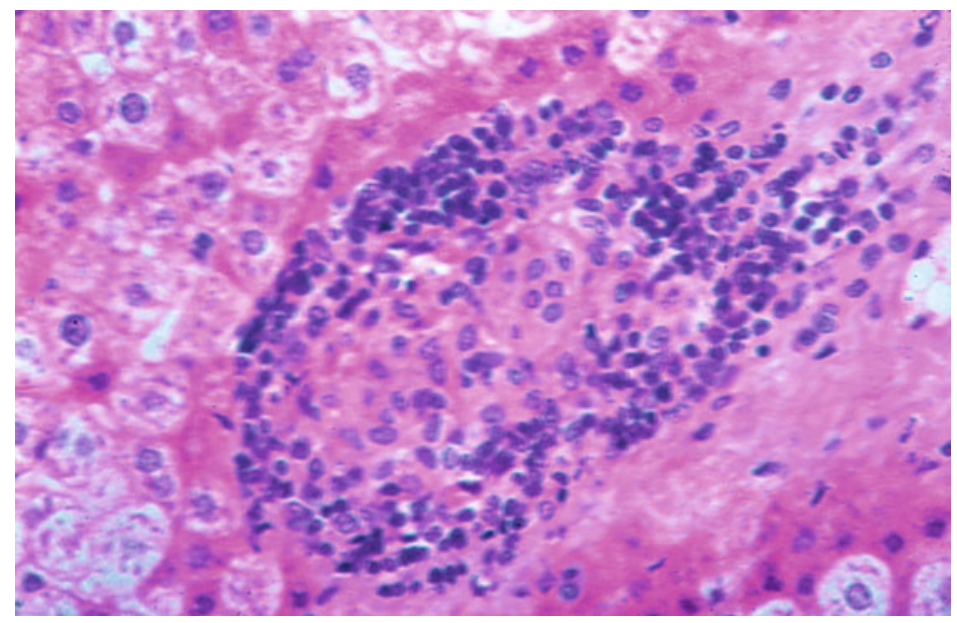


Fig. 155. Macrophages in the marginal zone of the splenic white pulp of a male white rat (No.30) 8 weeks after an intravenous application of $25 \mathrm{mg}$ Brazilian crystallised quartz (specific surface $2 \mathrm{~m}^{2} / \mathrm{g}$ ). Fixed by immersion in Bouin's fluid. Paraplast. Azan modification using rubine fast red and aniline blue (Specht 1973). Objective Leitz Pl 40/0.65. Leitz-Orthomat ${ }^{\circledR}(\times 3.2)$. Elliptic polarised light. Agfachrome $50 \mathrm{~L}$ professional

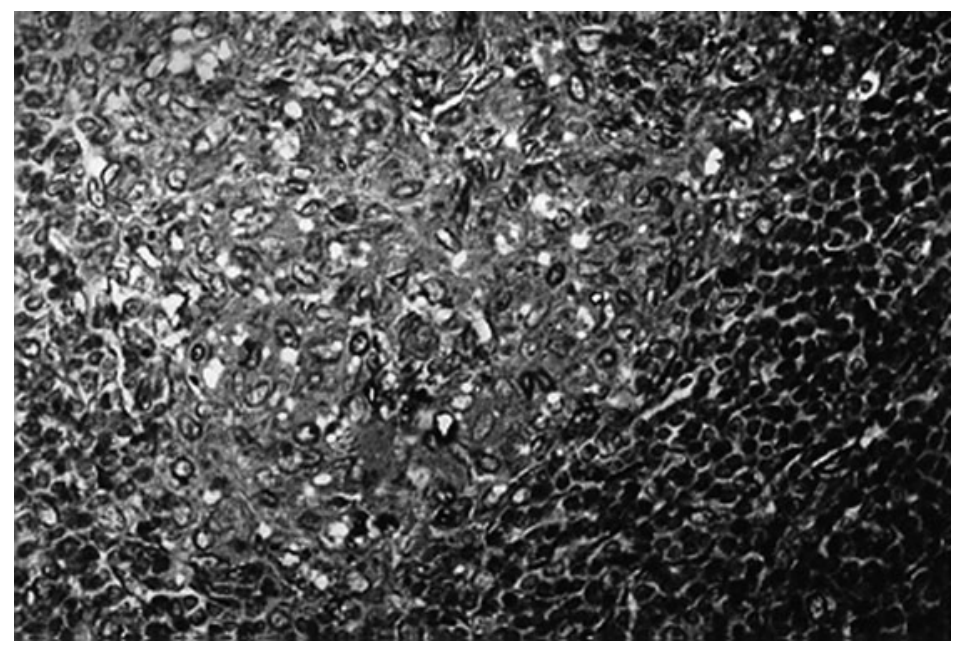

being responsible for the greatest enlargement. The spleen in texture, colour and consistence was indistinguishable from that of Banti's splenomegaly.

Colloidal, molybdate-inactive silicic acid after prolonged intravenous injection up to 11 months, accumulated in the rabbit spleen (up to $4.580 \mathrm{mg} / \mathrm{g}$ ) inducing splenomegaly (АMMON and МонN 1958). In the rat, colloidal silicic acid was accumulated in histiocytes (МоHN 1963). Lymphocytes did not contain any $\mathrm{SiO}_{2}$, and there were no lymphocytes in mature silicotic granulomas. Necrosis occurred at an increased rate of $\mathrm{SiO}_{2}$ administration.

Polyvinylpyridine- $N$-oxide retarded splenic fibrosis of mice given $3 \mathrm{mg}$ quartz intravenously as judged from the hydroxyproline content of the organ (Dolgner and PotT 1967).

\subsection{8}

\section{Sulphur}

Sulphur has the atomic number 16 and the valence electrons are those of the $3 s^{2} 3 p^{4}$ shells. The oxidation states of sulphur are $-2,+4$ and +6 . These many valence states make sulphur an ideal redox regulator.

\section{Sulphur Dioxide}

In the nasal passages and lung, $\mathrm{SO}_{2}$ is hydrated rapidly according to the equation

$$
\mathrm{SO}_{2}+\mathrm{H}_{2} \mathrm{O} \leftrightarrow \mathrm{HSO}_{3}{ }^{-}+\mathrm{H}^{+}
$$

with the equilibrium constant $1.7 \times 10^{2} \mathrm{M} / \mathrm{l}$ (PeterING 1977). Bisulfite, which predominates at physiological $\mathrm{pH}$, is a weak acid which dissociates according to the reaction

$$
\mathrm{HSO}_{3}{ }^{-}+\mathrm{H}_{2} \mathrm{O} \leftrightarrow \mathrm{SO}_{3}{ }^{-2}+\mathrm{H}_{3} \mathrm{O}^{+}
$$

with an equilibrium constant $1.02 \times 10^{7} \mathrm{M} / 1$.

Prostaglandin synthase (EC 1.14.99.1), which is immunoreactive in human and other mammalian alveolar macrophages (Yu et al. 1988),

Human alveolar macrophages gained by bronchoalveolar lavage were placed on a polycarbonate membrane through which they were supplied with RPMI 1640 for nutrient. Exposed for $10 \mathrm{~min}, 20 \mathrm{~min}$ and $30 \mathrm{~min}$ to $2.5 \mathrm{ppm}, 7.5 \mathrm{ppm}$ and $12.5 \mathrm{ppm} \mathrm{SO}_{2}$, respectively, they showed a significant increase in luminol-enhanced chemiluminescence due to the production of reactive oxygen species (KIENAST et al. 1993). The $12.5 \mathrm{ppm}$ concentration of $\mathrm{SO}_{2}$ killed $62 \pm 9 \%$ of the alveolar macrophages and thus reduced chemiluminescence by $63 \%$ compared with the $2.5 \mathrm{ppm}$ exposure. A 30-min exposure to $\mathrm{SO}_{2}$ induced a significant decrease in spontaneous and lipopolysaccharide-stimulated tumour necrosis factor- $\alpha(P$ $<0.001$ ) and lipopolysaccharide-stimulated interleukin- $1 \beta$ release $(P<0.059)$, while the release of interleukin- 6 and tansforming growth factor- $\beta$ was not significantly affected (KNORST et al. 1996).

\subsection{9 \\ Tantalum}

Tantalum has the atomic number 73 and the valence electrons are those of the $4 f^{4} 5 d^{3} 6 s^{2}$ shells. The oxidation state of tantalum is +5 .

Tantalum oxide $\left(150 \mathrm{mg} \mathrm{Ta} \mathrm{O}_{5} / \mathrm{m}^{3}\right)$ dust inhaled for 10 days $(10 \mathrm{~h} / \mathrm{d})$ was phagocytized by rat alveolar macrophages. The cells contained agglomerations of particles of different size, all $<1 \mu \mathrm{m}$ within phagolysosomes, unchanged mitochondria and numerous free and membrane-attached ribosomes (NemetscheK-Gansler et al. 1975). 
In vitro, at two hours more than $90 \%$ of alveolar macrophages obtained from New Zealand white rabbits by pulmonary lavage had ingested tantalum oxide particles ( $1 \mu \mathrm{m}$ mean particle diameter); at 12,24 and $30 \mathrm{~h}$ lactate dehydrogenase release was significantly higher than in control and latex (0.81 $\mu \mathrm{m}$ mean particle diameter) preparations (MATTHAY et al. 1978). Loss of cell viability and cell sloughing was observed first at $6 \mathrm{~h}$, and by $24 \mathrm{~h}$ when LDH levels were high $(29.16 \pm 5.58 \mu \mathrm{g})$ few cells remained adherent and viable. At $12 \mathrm{~h}$ lysozyme release in tantalum oxide preparations $(26.00 \pm 3.12 \mu \mathrm{g})$ was equal to that in quartz $(0.28$ $\mu \mathrm{m}$ mean particle diameter) preparations $(25.19 \pm 2.20 \mu \mathrm{g})$, and both tantalum oxide and silica media lysozyme levels were significantly $(P$ $<0.01)$ higher than those in control $(10.53 \pm 1.37$ $\mu \mathrm{g})$ and latex preparations $(9.75 \pm 0.32 \mu \mathrm{g})$.

\subsection{0 \\ Thallium}

Thallium has the atomic number 81 and the valence electrons are those of the $6 s^{2} 6 p^{1}$ shells. The oxidation states of thallium are +1 and $+3 . \mathrm{Tl}^{+}$ tends to form stable complexes with soft ligand donors, such as sulphur-containing compounds. The well-known mechanism of thallium toxicity is related to the interference with the vital potassiumdependent processes, substitution of potassium in the $\left(\mathrm{Na}^{+} / \mathrm{K}^{+}\right)$-ATPase, as well as a high affinity for sulfhydryl groups from proteins and other biomolecules (Aoyama et al. 1988). Lipid peroxidation particularly in the kidney - was increased with a marked decrease in non-protein sulfhydryls as an indicator of glutathion levels, as well as a depletion of glutathione peroxidase (EC 1.11.1.9) activity, suggesting that the tissue damage induced by thallium can be also associated to peroxidative processes.

The effect of thallium on lipid peroxidation was first tested by HASAN and ALI (1981) and compared to nickel and cobalt administration.

In patients intoxicated with thallium CAVANAGH et al. (1974) described oedema, moderate congestion, patchy early bronchopneumonia and inflammation.

\subsection{1 \\ Titanium}

Titanium has the atomic number 22 and the valence electrons are those of the $3 d^{2} 4 s^{2}$ shells. The oxidation states of titanium include $+2,+3$ and +4 . The tetravalent form is the is the most commonly encountered.
The violet cation $\mathrm{Ti}^{3+}$ was introduced by Dixon and Norman (1962) to produce $\mathrm{HO}^{\circ}$ from $\mathrm{H}_{2} \mathrm{O}_{2}$ in the laboratory:

$$
\mathrm{Ti}^{3+}+\mathrm{H}_{2} \mathrm{O}_{2} \rightarrow \mathrm{Ti}(\mathrm{IV})+\mathrm{HO}^{\circ}+\mathrm{OH}^{-}
$$

The generation of singlet oxygen $\left({ }^{1} \mathrm{O}_{2}\right)$ by irradiated $\mathrm{TiO}_{2}$ (anatase) in ethanol was measured by ESR spectroscopy using 2,2,6,6-tetramethyl-4-piperidone as a ${ }^{1} \mathrm{O}_{2}$-sensitive trapping agent (KonAKA et al. 1999).

\section{Titanium Dioxides}

There are three modification of $\mathrm{TiO}_{2}$ : rutile, anatase, and brookite.

Alveolar lumina completely filled with phagocytes some of which have been destroyed were found in a 38 years old patient, who had worked in a titanium dioxide factory for about nine years, all this time as a repair worker in the dusty departments (Elo et al. 1972). Specimens from this and another patient showed alveolar phagocytes with large lysosomes containing electron-dense $\mathrm{TiO}_{2}$ particles.

Macrophages which contained carbon-like, but birefringent pigment were found in the sputum as well as in extracellular areas of biopsy samples from former workers of $\mathrm{TiO}_{2}$ pigment factory, who had been put on pension because of chronic bronchitis and partial pulmonary dysfunction (Mё̈̈TTA and Arstila 1975). Dispersive X-ray analyses from the extremely electron-dense, round or oval-shaped particles inside the lysosomes gave distinct peaks for titanium $\mathrm{K}_{\alpha}$ and $\mathrm{K}_{\beta}$ in every case. The electrondense, more or less rectangular particles inside the lysosomes gave typical peaks for silicon $\mathrm{K}_{\alpha}$ and weak signal for aluminium $\mathrm{K}_{\alpha}$ and potassium $\mathrm{K}_{\alpha}$, as one of the coating materials in the industrial process was aluminium silicate.

Autopsy findings in a 55-year-old man known to have been occupationally heavily exposed to titanium dioxide dust showed extensive pulmonary deposition of white pigment identified by energy dispersive X-ray analysis and electron X-ray diffraction as rutile (RoDE et al. 1981). The pigment was mainly distributed in the perivascular tissue, but smaller amounts were found in the alveolar walls and also in alveolar macrophages.

The biological activity of ultrafine $\mathrm{TiO}_{2}$ maybe a consequence of its size and Donaldson and GiLMOUR (1995) hypothesised that there could be free radical activity associated with the surface of ultrafine $\mathrm{TiO}_{2}$, which caused strand breakage electrondense detectable as $100 \%$ depletion of supercoiled DNA whilst normal sized $\mathrm{TiO}_{2}$ had no effect; the injury could be ameliorated by including mannitol, implicating hydroxyl radical. 
Fig. 156. Alveolar macrophage (block 4459) of a $194 \mathrm{~g}$ male Sprague-Dawley rat (Charles River, France), injected intraperitoneally with $50 \mathrm{mg}$ anatase (titanium dioxide P 25, Degussa, Frankfort on the Main) suspended in $2 \mathrm{ml}$ saline. 4 days later, on July 25,1978 under pentobarbital anaesthesia $(30 \mathrm{mg} / \mathrm{kg})$, the animal was perfused from the abdominal aorta with $2.5 \%$ glutaraldehyde in $0.1 \mathrm{M}$ sodium cacodylate buffer ( $\mathrm{pH}$ 7.4). Postfixation with $1 \%$ osmium tetroxide in sodium cacodylate buffer. Embedded in Epon 812 and sectioned at $50 \mathrm{~nm}$. Lead citrate and uranyl acetate. Film 362/79

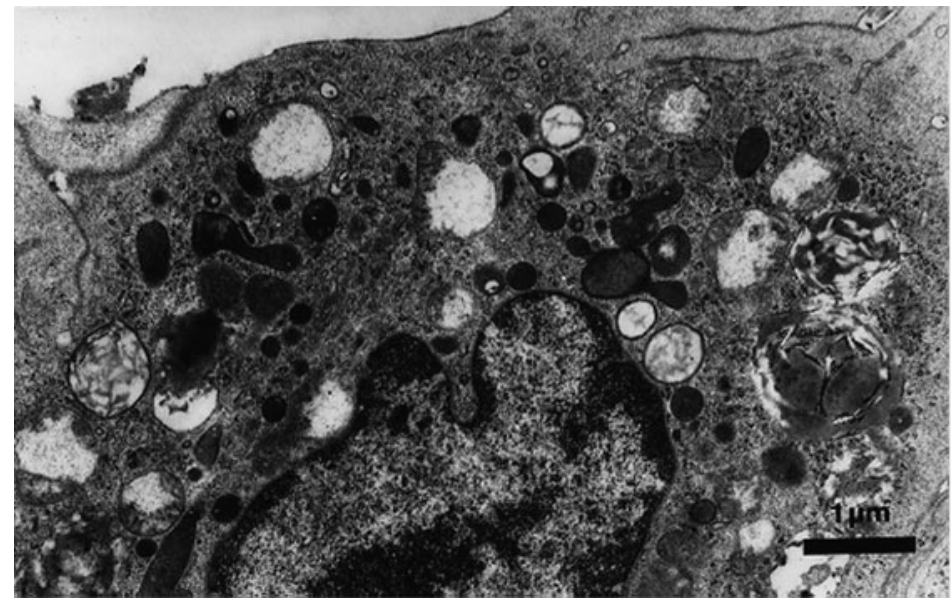

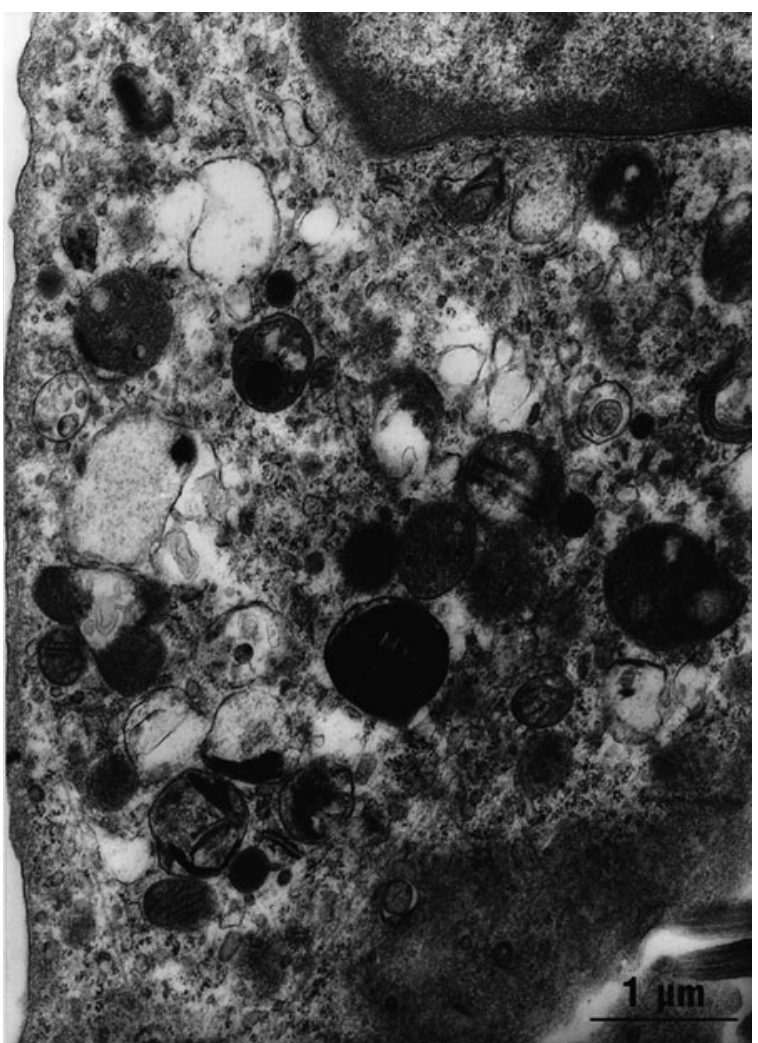

Fig. 157. Macrophage on the bronchiolar epithelium (block 4482) from a $200 \mathrm{~g}$ Sprague-Dawley rat (Charles River, France) instilled intratracheally with $50 \mathrm{mg}$ anatase (titanium dioxide P 25, Degussa, Frankfort on the Main) suspended in $0.5 \mathrm{ml}$ saline. Animal treated for 4 consecutive days with intraperitoneal injection of $15 \mathrm{mg}$ carbocromen in $10 \%$ methylcellulose per $\mathrm{kg}$ body weight $\times$ day. On July 25, 1978 under pentobarbital anaesthesia $(30 \mathrm{mg} / \mathrm{kg})$, the lung was fixed by intratracheal instillation of $2.5 \%$ glutaraldehyde in $0.1 \mathrm{M}$ sodium cacodylate buffer ( $\mathrm{pH}$ 7.4) before opening the thorax. Postfixation with $1 \%$ osmium tetroxide in sodium cacodylate buffer. Embedded in Epon 812 and sectioned at $50 \mathrm{~nm}$. Lead citrate and uranyl acetate. Plate 4138

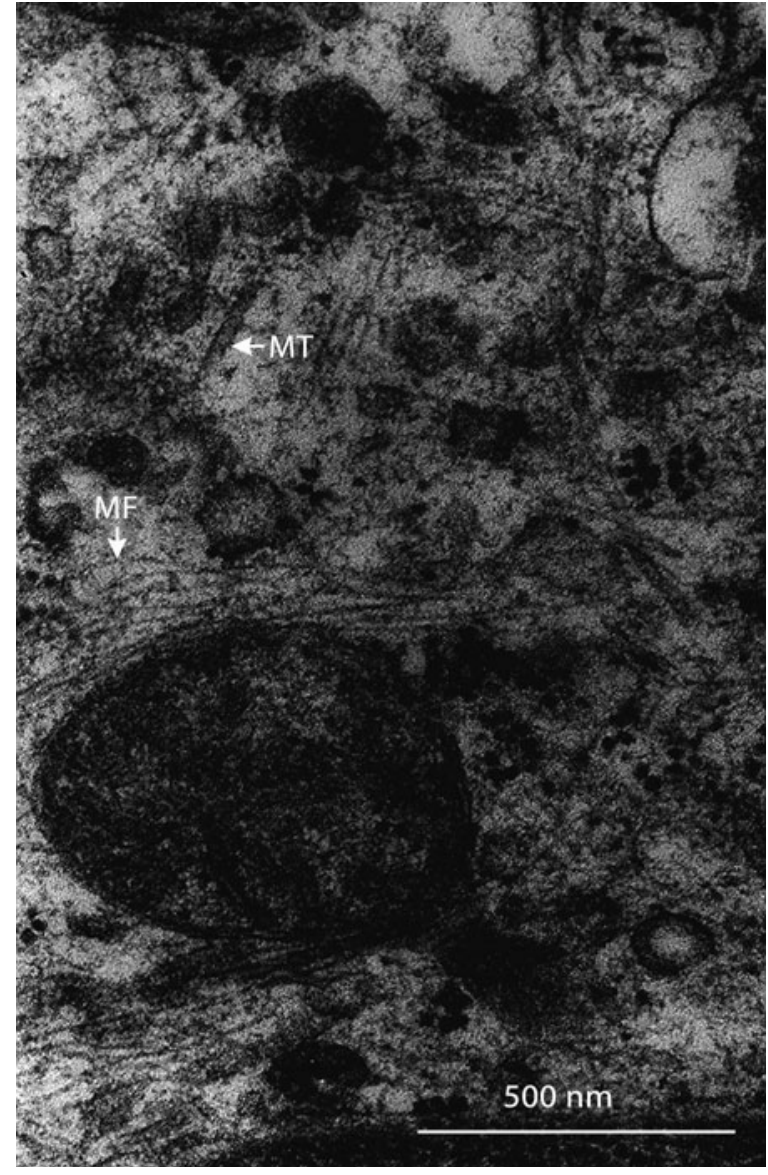

Fig. 158. Microfilaments (MF) and microtubules (MT) in an alveolar macrophage (block 4481) from a 199 g SpragueDawley rat (Charles River, France) instilled intratracheally with $50 \mathrm{mg}$ anatase (titanium dioxide P 25, Degussa, Frankfort on the Main) suspended in $0.5 \mathrm{ml}$ saline. Animal treated for 4 consecutive days with intraperitoneal injection of $15 \mathrm{mg}$ carbocromen in $10 \%$ methylcellulose per $\mathrm{kg}$ body weight $\mathrm{X}$ day. On July 25, 1978 under pentobarbital anaesthesia $(30 \mathrm{mg} /$ $\mathrm{kg}$ ), the lung was fixed by intratracheal instillation of $2.5 \%$ glutaraldehyde in $0.1 \mathrm{M}$ sodium cacodylate buffer ( $\mathrm{pH} 7.4$ ) before opening the thorax. Postfixation with $1 \%$ osmium tetroxide in sodium cacodylate buffer. Embedded in Epon 812 and sectioned at $50 \mathrm{~nm}$. Lead citrate and uranyl acetate. Plate 4134 
Guinea pigs' alveolar macrophages after 14 days' exposure to rutile $\left(23 \pm 7.3 \mathrm{mg} / \mathrm{m}^{3}\right.$ for $\left.20 \mathrm{~h} / \mathrm{d}\right)$ contained $\mathrm{TiO}_{2}$ particles in phagolysosomes (BASKERVILLE et al. 1988). The macrophage blockade by $\mathrm{TiO}_{2}$ did not alter the animals' susceptibility to Legionnaires' disease nor increase mortality.

Human alveolar macrophages produced more reactive oxygen species in response to ultrafine titanium dioxide particles than rat alveolar macrophages (RAHMAN et al. 1997). In case of human alveolar macrophages, the peak level of chemiluminescence was reached in less than $1 \mathrm{~min}$ after the addition of ultrafine titanium dioxide particles.

Instillation of $500 \mathrm{mg} \mathrm{\textrm {TiO } _ { 2 }}$ dust ( $7 \mu \mathrm{m}$ maximum diameter) suspended in $50 \mathrm{ml}$ PBS into the right caudal lung lobe of 3 female pigtail macaque monkeys (Macaca nemestrina) using a flexible fibreoptic bronchoscope increased the numbers of macrophages in the dust exposed lobes and significantly elevated the $N$-acetyl- $\beta$-D-glucosaminidase (EC 3.2.1.17) activity within the bronchoalveolar lavages taken at 2-weeks intervals for 12 weeks thereafter (MACK et al. 1995).

\section{Titanium Tetrachloride}

Rats exposed to titanium tetrachloride $\left(\mathrm{TiCl}_{4}\right)$ hydrolysis products by inhalation exposure at aerosol concentrations of $0.1,1.0$ and $10 \mathrm{mg} / \mathrm{m}^{3}$ for $6 \mathrm{~h} /$ day, 5 days/week, for 2 years showed alveoli filled with two types of alveolar macrophages: dust-laden macrophages were filled with densely aggregated particles and foamy alveolar macrophages that only contained a small amount of dust particles (foamy dust cells). Many foamy dust cells showed degenerative changes and were disintegrated in the alveolar air spaces releasing dust particles and granular or fibrinous cellular debris. Cholesterol crystal clefts were formed within the proteinaceous material or foamy alveolar macrophages. Subsequently, cholesterol granulomas were developed with accumulation of proteinaceous material, foamy dust cells, and cellular debris from disintegrated foamy dust cells. At the electron microscopic level, the cytoplasm was packed with numerous myelin inclusions, scanty lysosomes, and phagosomes containing a few dust particles (LeE et al. 1986).

\section{Titanium Phosphate}

Titanium phosphate, a manmade fibre of $0.2 \mu \mathrm{m}$ to $0.3 \mu \mathrm{m}$ in diameter and from $10 \mu \mathrm{m}$ to $20 \mu \mathrm{m}$ in length, has squared-off ends and forms tight bundles.

Intratracheal injection of suspension in physiologic saline into rats' lungs induced accumulation of mononucleated cells including the fibres and forming syncytia and giant cells and a slight, doserelated fibrogenic response (Gross et al. 1977). After 17 months macrophages had undergone lipidic degeneration.

\section{Potassium Titanate}

Four weeks after intratracheal instillation of $2 \mathrm{mg}$ of respirable potassium titanate whiskers into male Wistar rats RNA extracted from LPS stimulated alveolar macrophages expression of IL- $1 \alpha$ was increased (TsudA et al. 1996, 1997). The expression of IL- $1 \alpha$ mRNA was by fibres was greatest in potassium octatitanate whisker $>$ UICC crocidolite $>$ UICC chrysotile $>$ refractory ceramic fibreinstilled rat alveolar macrophages. The levels of IL$1 \alpha$ and TNF- $\alpha$ mRNA in alveolar macrophages (expressed in their ratio to $\beta$-actin) peaked at 1 month and 3 days after exposure to octatitanate, respectively.

\subsection{2 \\ Tungsten}

Tungsten has the atomic number 74 and the valence electrons are those of the $\left(4 f^{4}\right) 5 d^{4} 6 s^{2}$ shells. The oxidation states of tungsten are $+2,+3,+4,+5$ and +6 .

Calcium tungstate $(250 \mu \mathrm{g})$ instilled intratracheally in CD-1 mice was ingested by alveolar macrophages which showed a high density of surface microvilli (PEÃo et al. 1993).

\subsection{3 \\ Uranium}

Uranium has the atomic number 92 and the valence electrons are those of the $5 f^{3} 6 d 7 s^{2}$ shells. The oxidation states of uranium are $+3,+4,+5$ and +6 .

$\alpha$-Tracks emanating from ${ }^{234} \mathrm{UO}_{2}$ particles within macrophages of rats exposed for $100 \mathrm{~min}$ to $45 \pm 1.3$ $\mathrm{mg} / \mathrm{m}^{3}$ and $87.0 \pm 2.6 \mathrm{mg} / \mathrm{m}^{3}$ uranium dioxide, respectively, were shown in autoradiographs of the airways at times between 2 and 35 days postinhalation (Gore and THORNe 1977).

\subsection{4 \\ Vanadium}

Vanadium has the atomic number 23 and the valence electrons are those of the $3 d^{3} 4 s^{2}$ shells. The oxidation states of vanadium are $+2,+3,+4$ and +5 .

Vanadium, like molybdenum, has two very different catalytic activities associated with it and two very different biological functions. Both elements 
have a rich oxygen chemistry associated with the oxyanion and oxycation species. Vanadium compounds of a low oxidation state are reductants, while vanadium $(\mathrm{V})$ compounds are oxidants. In aqueous solutions $\mathrm{V}^{2+} \mathrm{aq}, \mathrm{V}^{3+} \mathrm{aq}, \mathrm{VO}^{2+}$, and $\mathrm{VO}_{3}^{-}$ ions exist, but also numerous complexes and polynuclear complexes.

The second class of chemistry of these two metals belongs to sulphur-rich reducing conditions, where vanadium like molybdenum, can form sulphur-containing anionic centres, e.g. $\mathrm{VS}_{4}{ }^{3-}\left(\mathrm{MoS}_{4}{ }^{2-}\right)$; sulphur-containing cationic centres, e.g. $\mathrm{VS}^{2+}(\mathrm{com}-$ pare $\mathrm{MoOS}^{2+}$ ); and iron-sulphur clusters $\mathrm{Fe}_{6} \mathrm{MS}_{8}$, associated in biology with nitrogen fixation.

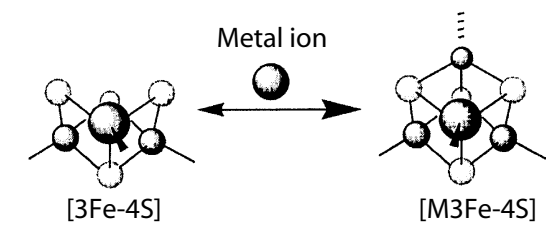

Addition of a metal ion to a [3Fe-4S] cuboidal cluster to yield a [4Fe-4S] cubane

In toadstools biology has used another curious device, an apparently simple ligand - N-hydroxyimino-di- $\alpha$-propionate - which forms an extraordinary stable 2:1 complex with V(IV), and is therefore able to extract the metal from soils in which it is scarce relative to its potential competitors, e.g. $\mathrm{Fe}^{3+}$ and $\mathrm{Cu}^{2+}$ (Fraústo da Silva 1989).

Vanadium compounds of a low oxidation state are reductants, while vanadium $(\mathrm{V})$ compounds are oxidants. In aqueous solutions $\mathrm{V}^{2+} \mathrm{aq}, \mathrm{V}^{3+} \mathrm{aq}, \mathrm{VO}^{2+}$, and $\mathrm{VO}_{3}{ }^{-}$ions exist, but also numerous complexes and polynuclear complexes.

\section{Vanadium Pentoxide $\left(\mathrm{V}_{2} \mathrm{O}_{5}\right)$ and Vanadates}

$$
\begin{aligned}
& \mathrm{V}^{\mathrm{V}}+\mathrm{O}_{2}^{--} \leftrightarrow \mathrm{V}^{\mathrm{IV}}-\mathrm{OO}^{\circ} \\
& \mathrm{V}^{\mathrm{IV}}-\mathrm{OO}^{\cdot}+\mathrm{NADPH} \leftrightarrow \mathrm{V}^{\mathrm{IV}}-\mathrm{OOH}+\mathrm{NADP} \\
& \mathrm{NADP}^{\cdot}+\mathrm{O}_{2} \leftrightarrow \mathrm{NADP}^{+}+\mathrm{O}_{2}^{--} \\
& \mathrm{V}^{\mathrm{IV}}-\mathrm{OOH}+\mathrm{H}^{+} \leftrightarrow \mathrm{V}^{\mathrm{V}}+\mathrm{H}_{2} \mathrm{O}_{2}
\end{aligned}
$$

This process has been shown to account for the vanadate stimulation of NADPH oxidation by biological membranes (Liochev and Fridovich 1986). A number of sugars and sugar phosphates can reduce $\mathrm{V}^{\mathrm{V}}$ to $\mathrm{V}^{\mathrm{IV}}$, and $\mathrm{V}^{\mathrm{IV}}$ can reduce dioxygen to yield $\mathrm{O}_{2}{ }^{-}$. It follows that $\mathrm{V}^{\mathrm{V}}$ plus sugar will, under aurobic conditions, generate $\mathrm{O}_{2}{ }^{\circ-}$, which in the presence of $\mathrm{V}^{\mathrm{V}}$ will then initiate the oxidation of NADPH (LIOcheV and Fridovich 1987).

Rabbit alveolar macrophages incubated for $20 \mathrm{~h}$ in supplemented Medium 199 with particulate forms of $\mathbf{V}_{2} \mathbf{O}_{5}(13 \mu \mathrm{g} \mathrm{V} / \mathrm{ml}), \mathbf{V}_{2} \mathbf{O}_{3}(21 \mu \mathrm{g} / \mathrm{ml})$ or $\mathbf{V O}_{2}$ $(33 \mu \mathrm{g} \mathrm{V} / \mathrm{ml})$ showed a reduction of their viability by $50 \%$, while only $9 \mu \mathrm{g} \mathrm{V} / \mathrm{ml}$ were required when $\mathrm{V}_{2} \mathrm{O}_{5}$ was dissolved in media prior to exposure of cells (WATERs et al. 1974).

$100 \mu \mathrm{M}$ Desferal was able to suppress the stimulatory effect of $\leq 200 \mu \mathrm{M} \mathrm{V}_{2} \mathrm{O}_{5}$ on the oxidation of NADH by $\mathrm{O}_{2}{ }^{\circ-}$ (DARR and Fridovich 1985). This chelating agent could form 1:1 complexes with metavanadate $\left(\mathrm{V}_{4} \mathrm{O}_{12}{ }^{-4}\right)$ or orthovanadate $\left(\mathrm{VO}_{4}^{-3}\right)$. The latter was the catalytically active species.

Supernatants from rat alveolar macrophages treated with $\mathrm{V}_{2} \mathrm{O}_{5}$ in vitro released upregulatory activity for platelet-derived growth factor receptor- $\alpha$ on cultured lung myofibroblasts, and this activity was blocked by the interleukin-1-receptor antagonist (BonNer et al. 1998).

Ammonium metavanadate exposure affected mouse WEHI-3 macrophage interferon- $\gamma$-binding and -inducible responses (CoHen et al. 1996). It interfered with both the uptake and ultimate intralysosomal killing of Listeria monocytogenes by mouse peritoneal macrophages (CoHEN et al. 1989).

Respiratory burst in lung macrophages significantly increased within $5 \mathrm{~min}$ of exposure to metavanadate $(10-100 \mu \mathrm{M})$, with a maximum increase of $173 \%$ above baseline after $15 \mathrm{~min}$, measured using a DCF-DCFHDA fluorescence assay (GRABowski et al. 1996). Metavanadate $(10-100 \mu \mathrm{M})$ inhibition of phosphotyrosine phosphatase activity in membrane and cytoplasmic fractions of lung macrophages was dose-dependent with a range of $28-89 \%$. Suppression of the metavanadate-induced burst by $37-47 \%$ was accomplished using $10 \mu \mathrm{M}$ of Tyrphostin B50, implicating epidermal growth factor receptor kinase activity in the respiratory burst.

The inducible production of interleukin- 6 and interferon- $\gamma$ by alveolar macrophages lavaged from Fischer 344 rats exposed to ammonium metavanadate $\left(2 \mathrm{mg} \mathrm{V} / \mathrm{m}^{3}\right)$ for $8 \mathrm{~h} / \mathrm{d}$ for $4 \mathrm{~d}$, followed, $24 \mathrm{~h}$ later by intratracheal instillation of $0.15 \mathrm{mg}$ polyinosinic:polycytidylic acid in $100 \mu \mathrm{l}$ saline, within 24 $\mathrm{h}$ increased 11-fold and 20-fold, respectively, vs. $\approx 30$-fold and 7-fold, respectively, in air control rats (Conen et al. 1997). At $24 \mathrm{~h}$ following poly I:C instillation, there were no significant host exposure dependent differences in either spontaneous of opsonized zymosan-stimulated $\mathrm{O}_{2}{ }^{-0}$ formation. At 48 $\mathrm{h}$ a time post poly I:C-dependent effect upon opsonized zymosan-stimulated pulmonary alveolar macrophage $\mathrm{O}_{2}{ }^{--}$production was evident; this effect was greater in macrophages from rats exposed to $\mathrm{V}$ prior to poly I:C instillation than in similarly instilled air control animals.

In HL-60 cells, vanadate stimulated $\mathrm{O}_{2}$ consumption for the synthesis of superoxide, which trig- 
gered the formation of peroxovanadyl $\left[\mathrm{V}^{(4+)}-\mathrm{OO}\right]$ and vanadyl hydroperoxide $\left[\mathrm{V}^{(4+)}-\mathrm{OOH}\right)$, one or both of these species, rather than vanadate itself being responsible for the respiratory burst (TRUDEL et al. 1991).

Rat alveolar macrophages exposed in vitro to 100 $\mu \mathrm{M}$ vanadyl chloride $/ 1 \mu \mathrm{Ci}{ }^{48} \mathrm{~V}$ incorporated $8.3 \%$ of the metal after $30 \mathrm{~min}$ (GRABOwSKI et al. 1999). Exposure of alveolar macrophages to increasing concentrations of sodium metavanadate resulted in a dose-dependent increase in production of reactive oxygen intermediates as measured by dichlorofluorescin oxidation. The lowest dose yielding a measurable response was $50 \mu \mathrm{M}$, whereas $1000 \mu \mathrm{M}$ increased respiratory burst activity by $173 \%$. NADPH oxidase inhibitors deoxy-D-glucose $(100 \mathrm{mM})$ and diphenylene iodonium $(25 \mu \mathrm{M})$ reduced the metavanadate-induced respiratory burst by 62 and $71 \%$, respectively, implicating $\mathrm{NADPH}$ oxidase as the primary cellular source of reactive oxygen intermediates. Enhanced cerium chloride oxidation in response to metavanadate localised to the plasma membrane consistent with increased NADPH oxidase activity. Pre-treatment of alveolar macrophages with the epidermal growth factor receptor inhibitor, tyrphostin B50 $(10 \mu \mathrm{M})$, reduced the metavanadate-induced respiratory burst, but did not influence overall tyrosine phosphorylation. Metavanadate and $\mathrm{H}_{2} \mathrm{O}_{2}$ exposure greatly increased overall tyrosine phosphorylation, yielding a similar but distinguishable pattern of phosphorylation in these cells.

Acid phosphatase activity in $10^{6} \mathrm{~B}_{6} \mathrm{C}_{3} \mathrm{~F}_{1}$ mouse peritoneal macrophages was depressed by 22.8 and $44.7 \%$ when ammonium metavanadate $(2.5 \mathrm{mg}$ and $10 \mathrm{mg} \mathrm{V} / \mathrm{kg}$ ) was given i.p.every $3 \mathrm{~d}$ for 6 weeks, while the activities of $\beta$-glucuronidase, $\mathrm{N}$-acetyl- $\beta$ $D$-glucosaminidase, and lysozyme were not significantly influenced (VADDI and WeI 1991).

\subsection{5}

\section{Yttrium}

Yttrium has the atomic number 39 and the valence electrons are those of the $4 d^{1} 5 s^{2}$ shells. Its only oxidation state is +3 .

\section{Yttrium Chloride}

MARUbashi et al. (1998) investigated pulmonary clearance of yttrium and acute lung injury following intratracheal instillation of $\mathrm{YCl}_{3}$ in saline- or $\mathrm{YCl}_{3}$-pretrated male Wistar rats (30 days before the second challenge). About $67 \%$ of the initial dose of $\mathrm{Y}$ remained in the lung even 31 days after intratra- cheal treatment. The pre-treatment with $\mathrm{YCl}_{3}$ significantly reduced $\mathrm{YCl}_{3}$-induced increases in biochemical inflammatory indicators in bronchoalveolar lavage fluid, such as lactate dehydrogenase, $\beta$ glucuronidase, and alkaline phosphatase activities and protein concentration, while the pre-treatment increased the number of polymorphonuclear leucocyte in bronchoalveolar lavage fluid.

\section{Yttrium-Barium-Copper Oxide $\left(\mathrm{YBa}_{2} \mathrm{Cu}_{3} \mathrm{O}_{7}\right)$ Superconducting Material}

Bovine alveolar macrophages incubated for $20 \mathrm{~h}$ with $100 \mu \mathrm{g} \mathrm{YBa}_{2} \mathrm{Cu}_{3} \mathrm{O}_{7} / \mathrm{ml}$ PPMI 1640 exhibited a sponge-like structure. After $60 \mathrm{~min}$ the dust was detected in vacuoles increasing with time. Viability was reduced in a dose-dependent manner (WILCZEK et al. 1990).

\subsection{6}

Zinc

Zinc has the atomic number 30 and the valence electrons are those of the $3 d^{10} 4 s^{2}$ shells. Its only oxidation state is +2 . Zinc appears in the active site of a variety of enzymes and, unlike other well-known constituents of metalloenzymes like iron and copper, does not function as a redox site but rather acts as a Lewis acid that can accept an electron pair (Prasad 1977).

Zinc forms a lipophilic complex with pyridinethione by bonding through the oxygenated sulphur atoms. Lipophilic complex formation may promote metal absorption and tissue penetration. Zinc reacts with several peptide hormones. Adrenocorticotropic hormone (ACTH) binds zinc and copper(II) ions in acid solution (CARR et al. 1952). Zinc glucagon is somewhat less soluble than glucagon, and exhibits prolonged biological effects (WEINGES 1959, Sokal 1960, Bromer and Chance 1969, TARDing et al. 1969, Assan and Delaunay 1972). If protamine is added to the zinc glucagon suspension, a greater prolongation of action is observed, even though the protamine is not bound to the zinc glucagon (TARDING et al. 1969).

Working with purified plasma membranes of alveolar macrophages from rabbits, CHVAPIL et al. (1976) found a definite but small amount of zinc present in the membrane, and most of this metal being linked to a certain fraction.

The viability of thioglycolate-elicited peritoneal macrophages isolated from male $\mathrm{CD} 1$ mice pretreated for 4 days with $0.25 \mathrm{mg} \mathrm{ZnCl}_{2}$ per day in $\mathrm{He}$ pes buffer i.p. and exposed to $1 \mu \mathrm{m}$ particles of silica $\left(0.5-0.7 \mathrm{mg}\right.$ crystalline $\left.\mathrm{SiO}_{2} / \mathrm{ml}\right)$ in vitro was 
decreased from $55.9 \pm 2.8 \%$ to $37.6 \pm 13.7 \%$ (KARL et al. 1973). $0.05 \mathrm{mg} \mathrm{ZnCl}$, however, increased the viability of silica-treated macrophages to $79.1 \pm 2.8 \%$.

One week after an intratracheal instillation of 0.5 $\mathrm{ml}$ suspension of $10 \mathrm{mg} \mathrm{ZnO} / \mathrm{ml}$ saline the size of rat pulmonary alveolar macrophages was considerably reduced (MigALLY et al. 1982). The cells contained a prominent nucleolus within an invaginated nucleus. Several types of lysosomes were considered to be primary, secondary, and residual bodies. Short profiles of rough endoplasmic reticulum, small dense mitochondria and numerous profiles of Golgi membranes were also noted.

\subsection{7}

Zirconium

Zirconium has the atomic number 40 and the valence electrons are those of the $4 d^{2} 5 s^{2}$ shells. The oxidation states of zirconium are +3 and +4 .

In the hamster, multiple short-term intratracheal instillations of zirconium lactate produced lesions beginning with exudative pneumonia followed by pneumonitis (interstitial pneumonia) and foreign body granulomas. Electron microscope microprobe analysis demonstrated the metallic component of the instilled compound in membrane-bound cytoplasmic inclusions of macrophages (LEININGER et al. 1977).

In the rat, zircon $\left(\mathrm{ZrSiO}_{4}\right)$ dust (180 mesh) injected intratracheally was found 7 to 9 months later in swollen alveolar histiocytes with pyknotic nuclei and a faintly staining hydropic cytoplasm in which were a few tiny granules of pigment (HARDING 1948).

In the guinea-pig immunised with $0.2 \mathrm{mg}$ sodium zirconium lactate in Freund's complete adjuvant 14 days before, weekly intradermal injections of $25 \mu \mathrm{g}$ of the metal salt in $0.1 \mathrm{ml}$ saline produced nodular granulomas (Turk et al. 1978). Lesions taken at the peak of granuloma development 7 days after skin testing contained epithelioid cells which could be recognised particularly by their content of rough endoplasmic reticulum, fimbriated cell membrane and typical nuclear appearance. Multinucleate giant cells were also seen. They were characterised by their large number of lysosome-like dense bodies and phagocytic structures. A constant feature of the guinea-pig lesions was the presence of large numbers of apparently active fibroblasts.

\subsubsection{2 Organic Agents}

9.6.2.2.1

Acrolein

Acrolein is a pollutant found in both indoor and outdoor environments. It is manufactured as an intermediate in chemical synthesis, used as an aquatic biocide, and is also formed as a combustion product of organic matter including tobacco.

Human alveolar macrophages developed a dose dependent $(1-50 \mu \mathrm{M})$ induction of stress response, inhibition of cytokine release, apoptosis, and necrosis (Li et al. 1997).

\subsubsection{2}

\section{Amiodarone}

Amiodarone (INN) is a iodinated benzofuran derivative, the chemical structure of which resembles that of thyroxin (HEger et al. 1981). It was introduced into Belgium, France, Germany (Cordarex $\left.{ }^{\circledR}\right)$, the Netherlands (Sotalol ${ }^{\circledR}$ ), and South Africa for use as a coronary vasodilator, but proved to be antiarrhythmic (Rosenbaum et al. 1974, 1976). An attempt to classify amiodarone according to its toxic ability to interfere with the integrated function of electron transport enzymes (RIBEIRo et al. 1997) confirmed the effect of the antiarrhythmic drug on complex I and allowed the placement of amiodarone in class $\mathrm{A}$ of the classification established by KNOBELOCH et al. (1990) i.e. together with rotenone, amytal, detergents and solvents. The drug itself and its main metabolite, desethylamiodarone accumulate in lung (DANIELs et al. 1989, REASOR et al. 1989), liver (Pirovino et al. 1986) and eye (BAHEL et al. 1970) cells and within the ubiquitous monocyte/macrophage system to inhibit the lysosomal phospholipases. Pichler et al. (1988) noted that most patients receiving a cumulative dose of $>100 \mathrm{~g}$ amiodarone had side effects and that only one patient with an anti-amiodarone titre of $>10$ density units had no side effect. 
<smiles>CCCCc1oc2ccccc2c1C(=O)c1cc(I)c(OCCN(CC)CC)c(I)c1</smiles>

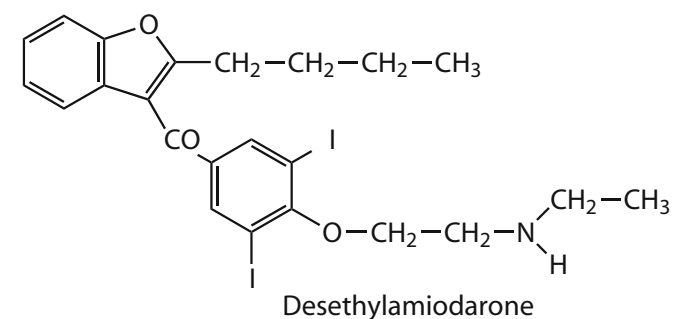<smiles>CCCCc1oc2ccccc2c1C(=O)c1cc(I)c(OCCNN)c(I)c1</smiles><smiles>CCCCc1oc2ccccc2c1C(=O)c1ccc(OCCNCC)c(I)c1</smiles>

Monodeiodinated-desethylamiodarone Amiodarone and its derivatives

[249-252]

The most serious side effect is the development of pulmonary toxicity (AKoun et al. 1987, MARTIN and Rosenow 1988), characterised by phospholipidosis (REAsor et al. 1989) and the presence of interstitial and/or alveolar infiltrates (MARTIN and Rosenow 1988), which may progress to pulmonary fibrosis.

In a 55-year-old male patient Colgan et al. (1984) described alveolar spaces containing large macrophages with abundant pale foamy cytoplasm. Numerous heterogenous, dense, lamellar inclusions within their cytoplasm, which showed a decreased number of normal cytoplasmic organelles.

Transbronchial and open lung biopsies in a 29year-old man treated with amiodarone $(800 \mathrm{mg} /$ day) for 10 months showed filling of the alveoli by foamy macrophages (CostA-Jussà et al. 1984).

In isolated single-pass perfused rat lungs amiodarone $\left(30 \mu \mathrm{M}+1 \mu \mathrm{Ci}{ }^{14} \mathrm{C}\right.$-amiodarone) uptake at 20 min averaged $1312 \pm 225$ nmoles/g, correspond- ing to a tissue to medium ration of $44 \pm 7.5$ (CAMUs et al. 1989). Efflux of amiodarone was incomplete, only $34 \pm 10 \%$ of the amount present at $20 \mathrm{~min}$ had effluxed from the lung at $40 \mathrm{~min}$. Chlorphentermine significantly accelerated the efflux of amiodarone. When amiodarone was raised in the inflowing perfusate, accumulation in lung increased proportionally, except at $120 \mu \mathrm{M}$ where the tissue to medium ration decreased. This concentration also induced pulmonary oedema.

Alveolar macrophages from Fischer-344 rats treated with amiodarone showed a dose $(50 \mathrm{mg} /$ $\mathrm{kg}-200 \mathrm{mg} / \mathrm{kg}$ ) and time (1 day to 9 weeks)dependent increase of all individual classes of phospholipids (REAsor et al. 1988). Using an oesophageal cannula male mice dosed with $200 \mathrm{mg}$ amiodarone $/ \mathrm{kg} \times$ day suspended in 5 per cent methylcellulose up to three months and young adult SpragueDawley rats given $400 \mathrm{mg}$ amiodarone $/ \mathrm{kg} \times$ day for up to one month showed lamellated bodies in their pulmonary alveolar macrophages after glutaraldehyde/paraformaldehyde perfusion (CosTA-Jussà et al. 1984).

The adhesion molecules, CD18 and CD54 (ICAM-1) were significantly more expressed by bronchoalveolar cells lavaged from rats treated for 6 weeks with $175 \mathrm{mg}$ amiodarone/ $\mathrm{kg} \times$ day by gavage (CAstro Silva et al. 1997). After 12 weeks of treatment, however, the expression of these molecules significantly decreased. Throughout the experiment the cellularity of the BAL was elevated in experimental and control groups with great predominance of alveolar macrophages; the number of neutrophils was higher in experimental groups as well as the percentage of macrophage spreading. Release of $\mathrm{H}_{2} \mathrm{O}_{2}$, both spontaneously or stimulated by PMA, was variable being equal or higher than the amount released by control alveolar macrophages.

After intratracheal administration of amiodarone $(1.83 \mu \mathrm{mol} /$ day of days 0 and 2$)$ or an equivalent volume $(0.4 \mathrm{ml})$ of distilled water to male $\mathrm{Fi}$ scher 344 rats, Northern and immunoblot analyses demonstrated that lung transforming growth factor (TGF)- $\beta 1$ (mRNA and protein) expression was increased 1.5- to 1.8-fold relative to control during the early inflammation period and 1 day, 1 week, and 2 weeks post amiodarone treatment (CHUNG et al. 2001). Lung c-jun protein was increased 3.3-fold relative to control.

Tumour necrosis factor- $\alpha$ release by lavaged alveolar macrophages of female Fischer 344 rats significantly $(P<0.05)$ increased 3 and 6 weeks after an intratracheal instillation of $6.25 \mathrm{mg}$ amiodarone/ $\mathrm{kg}$ dissolved in $\sim 200 \mu \mathrm{l}$ of sterile deionized water (Reinhart and Gairola 1997). 5 mg Desethylamiodarone $/ \mathrm{kg}$ had no effect. 
Pulmonary phospholipidosis induced by $150 \mathrm{mg}$ amiodarone $/ \mathrm{kg}$ rat $\times$ day attenuated the acute toxicity of $2.5 \mathrm{mg}$ and $10 \mathrm{mg} \alpha$-quartz (median area equivalent diameter $3.5 \mu \mathrm{m}$ ) per $100 \mathrm{~g}$ body weight (ANTONINI et al. 1994).

In vitro, amiodarone decreased the surface density of rabbit alveolar macrophage mitochondria and lysosomes while increasing the surface density of inclusion bodies, increased the incorporation of choline into dipalmitoylphosphatidylcholine, modified the distribution of lysosomal enzymes, and did not affect the uptake and processing of diphtheria toxin $(10 \mathrm{nM})$ but inhibited the degradation of surfactant protein A (BARITUssio et al. 2001).

\subsubsection{3}

\section{Benzo[a]pyrene}

Benzo[a]pyrene is biotransformed by guinea pig alveolar macrophages to give 3-OH-benzo[a]pyrene, 7,8-dihydro-7,8-dihydroxybenzo[a]pyrene, 9,10-dihydro-9,10-dihydroxybenzo[a]pyrene, several chinones and three other unidentified substances (DeHnen 1975). Benson et al. (1987) incubated resting canine alveolar macrophages in vitro with ${ }^{14} \mathrm{C}$ benzo[a]pyrene and determined the effect of addition of SKF-525A and metyrapone (cytochrome $\mathrm{P}_{450}$ inhibitors), indomethacin (cyclooxygenase inhibitor), and nordihydroguiaretic acid (lipoxygenase inhibitor) on the extent of benzo[a]pyrene metabolism and the pattern of metabolites formed. Indomethacin and nordihydroguiaretic acid inhibited benzo[a]pyrene metabolism in resting alveolar macrophages by $35 \%$ and $30 \%$, respectively, while SKF-525A and metyrapone had no effect. Activation of alveolar macrophages by zymosan resulted in an approximately $60 \%$ reduction in benzo[a]pyrene metabolism.

Due to the presence of polycyclic aromatic hydrocarbons in asphalt, most studies of the health hazards for asphalt exposure have been concentrated on carcinogenicity, while very few studies have been devoted to characterisation of the effects of asphalt fumes on non-cancerous responses in the lung. In vitro exposure to paving asphalt fume condensate $(<200 \mu \mathrm{g} / \mathrm{ml})$ to rat alveolar macrophage functions did not induce cytotoxicity, oxidant generation, or IL-1 production by alveolar macrophages, but it did a small but significant increase in TNF- $\alpha$ release from alveolar macrophages (MA et al. 2000). In vitro exposure of alveolar macrophages resulted in a significant decline of chemiluminescence in response to zymosan or 12-O-tetradecanoylphorbol13-acetate stimulation. In vivo studies in rats instilled intratracheally with 0.1 or $0.5 \mathrm{mg}$ paving asphalt fume condensate showed that this toxicant did not induce significant neutrophil infiltration or alter lactate dehydrogenase or protein content in acellular lavage samples. Macrophages obtained from these rats did not show significant differences in oxidant production or cytokine secretion at rest or in response to lipopolysaccharide in comparison with control macrophages.

\subsubsection{4 \\ Bleomycin}

Bleomycin has been shown to exert its cytotoxic effects through the intracellular generation of oxygen radicals by forming complexes with iron and entering redox cycles (SAUSville et al. 1978, Sugiura and KikUChi 1978, Mahmutoglu et al. 1987).

The number of alveolar macrophages recovered by bronchoalveolar lavage from bleomycin-injected rat lungs was more than double control values at 10 days and remained above normal at 6 weeks (Young and AdAmson 1993). Both hyaluronan content in bronchoalveolar lavage and staining for hyaluronan in macrophages accumulating in injured areas of the rat lung were maximal at $4 \mathrm{~d}$ (SAVANI et al. 2000). Increased hyaluronan in bronchoalveolar lavage correlated with increased locomotion of isolated alveolar macrophages. Hyaluronan-binding peptide was able to specifically block macrophage motility in vitro. Importantly, systemic administration of hyaluronan-binding peptide to rats before injury not only decreased alveolar macrophage motility and accumulation in the lung, but also reduced lung collagen $\alpha$ (I) mRNA and hydroxyproline contents.

As compared to control rats, production of $\mathrm{O}_{2}{ }^{--}$ by alveolar macrophages from bleomycin-medicated $(20 \mathrm{U} / \mathrm{kg}$ body weight $\times$ day for 5 days) animals was increased upon stimulation with either phorbol myristate acetate $(21.04 \pm 21.78$ vs. $11.45 \pm 2.26 \mathrm{nM} /$ $10^{6}$ cells $\times 20 \mathrm{~min} ; P<0.05$ ) or opsonized zymosan $\left(9.35 \pm 0.87\right.$ vs. $7.03 \pm 0.66 \mathrm{nM} / 10^{6}$ cells $\times 20 \mathrm{~min} ; P$ $<0.05$ ) (SLosman et al. 1990).

Intratracheal instillation of bleomycin $(0.1 \mathrm{U} /$ mouse) stimulated an early influx of neutrophils followed by an increase in lymphocytes and macrophages in the bleomycin + control diet group (Gurujeyalakshmi et al. 2000). Taurine (1\% in water) and niacin (2.5\% in diet) treatment significantly reduced the numbers of neutrophils, lymphocytes and macrophages in the bleomycin + taurine/niacin group and caused significant reductions in bleomycin-induced increases in the lung hydroxyproline content at 14 and 21 days in the bleomycin + taurine/niacin group. The mice in the salineinstilled + control diet and the saline-instilled + taurine/niacin control groups had lower levels of 
'NO in bronchoalveolar lavage fluid, whereas mice in the bleomycin-instilled + control diet group as compared to the saline-instilled + control diet group had elevated levels of $\mathrm{NO}$ from day 3 through day 21 . The increases in "NO levels in bronchoalveolar lavage fluid from bleomycin + control diet group were associated with elevated levels of iNOS gene expression and protein in the lungs. Taurine and niacin suppressed the bleomycin-induced increases in iNOS massage and iNOS protein.

Alveolar macrophages from rats or hamsters with bleomycin-induced fibrosis secrete cytokines (Jordana et al. 1988, Suwabe et al. 1988), fibroblast growth (Kovacs and Kelley 1985, Denholm and Phan 1989, PhAN 1989) and inhibiting (CLARK et al. 1983) factors, and leucocyte chemotactic factors (Kaelin et al. 1983, Denholm et al. 1989), all of which are thought to play important roles in mediating the fibrotic reaction of the lungs to this drug. Alveolar macrophages from normal rats (JoRDANA et al. 1988) or hamsters (SUWABE et al. 1988) stimulated with bleomycin in vitro induced similar secretory activities.

Activin A immunoreactive and bioactive protein belonging to the transforming growth factor $\beta$ (TGF- $\beta$ ) supergene family was demonstrated in lavaged murine alveolar macrophages 14 days after bleomycin $(10 \mathrm{mg} / \mathrm{kg} \times$ day for 10 days $)$ treatment (Matsuse et al. 1995).

\subsubsection{5 \\ Carrageenan}

Carrageenan was first described as a mononuclear phagocyte toxin by ALLISON et al. (1966), who ascribed its toxic effect to destabilisation of the membranes of the secondary lysosomes, resulting in release of hydrolytic enzymes into the cytoplasm of treated cells. The $\lambda$-form of carrageenan appears in several reports to be the most potent macrophage toxin, compared to other forms of this material (BECKer and RudBACH 1979). However, carrageenan has a broad spectrum of effects apart from its suppression of mononuclear phagocytes (LEBLANC and Russell 1981), as it can have either a depressive of adjuvant effect on antibody production to either cellular or soluble antigens (AsCHHEIM and RAFFel 1972, Turner and Higginbotham 1977, BECKER and RudBach 1979). In light of these facts, LEBLANC and RusSell (1981) consider carrageenan as one of the least specific macrophage inhibitors. Reнм (1983) injected type II and type V lcarrageenan intraperitoneally into BALB/c and outbred ICR mice: viable recoverable macrophages progressively declined, reaching a nadir of $15-45 \%$ of control values. After administration of the chem- ically purer type $\mathrm{V}$, more rapid macrophage depletion was achieved (nadir at days 2-5). Thus ReHM (1983) recommended l-carrageenan as an investigational tool for manipulating alveolar macrophages.

Carrageenan-activated macrophages showed tumoricidal activity against a tissue culture line of BN472 cells (malignant mammary adenocarcinoma) (Bijma et al. 1988).

\subsubsection{6 Cellulose Dust}

One month after a single intratracheal instillation of $15 \mathrm{mg}$ cellulose powder MN 300 as used for thin layer chromatography (Nagel, Düren), male CFY rats showed irregularly shaped, periodic acid-Schiff positive foreign bodies inside the macrophages (TÁtrai and UngVÁRY 1992). Three months after the istillation several multinucleated foreign body giant cells were seen in the granulation tissue filling the lumina of brochioli and alveoli.

\subsubsection{7 \\ Chlorphentermine}

$p$-Chlorphentermine, $p$-chloro- $\alpha, \alpha$-dimethylphenethylamine $\mathrm{HCl}$ applied intraperitoneally to male Sprague-Dawley rats at doses of $50 \mathrm{mg} / \mathrm{kg}$ for one to 30 days or at doses of $20 \mathrm{mg} / \mathrm{kg}$ for 60 and 72 days, respectively, induced large intraalveolar foam cells (LÜLLMANN-RAUCH et al. 1972). Their tightly packed concentric lamellar bodies (periodicity 4-5 $\mathrm{nm})$ proved to be phospholipids. Foam cells of chlorphentermine-treated $(80 \mathrm{mg} / \mathrm{kg} \times$ day $)$ female Siv 50 rats contained mainly phospholipids, i.e. lecithin and only small amounts of neutral lipids, i.e. cholesterol (KARABELNIK and ZBINDEN 1975). Chlorphentermine $(60 \mathrm{mg} / \mathrm{kg} \times$ day) inhibited the incorporation of $1-{ }^{14} \mathrm{C}$-palmitic acid into lung phospholipids in a time-dependent manner (KARABELNIK and ZBINDEN 1976). The levels of thiobarbituric acid-reactive material, an index of lipid peroxidation, was increased in the lung tissue above saline vehicle controls in all vitamin E dietary rat groups $(0,60,300 \mathrm{ppm})$ with the vitamin Edeficient group being the highest (GAIrola et al. 1983).

\subsubsection{8 \\ Cork Dust}

A man aged 54 years, who was exposed to cork dust for about 14 years, showed intraalveolar giant cells loaded with coal and cork particles. Some had asteroid bodies in their cytoplasm (Remmele and 
EINBrodt 1962). The formamide method (Thomas and Stegemann 1954) discovered some pseudoasbestos bodies.

Guinea pigs and rabbits inhaling cork dust in different factory workshops from 56 to 1,278 and from 276 to 1,100 days, respectively, showed suberophages and multinucleated giant cells containing larger particles (Lopo DE CARvalho CANCElla 1959). Pneumoconiotic focal lesions were observed after an exposure for more than a year. In some of these nodules fibroblasts had proliferated scarcely accompanied by the formation of pre-collagenous fibres.

\subsubsection{9}

\section{Diesel Exhaust}

Free radical generation from diesel particulate was characterized by Ross et al. (1982) using electron paramagnetic resonance spectroscopy. The free radical signals were sensitive to oxygen, nitric oxide ( ${ }^{\mathrm{NO}}$ ), nitrogen dioxide, and ultraviolet radiation. Vogl and Elstner (1989) showed that diesel soot particulate could catalyse the release of ethylene from $\alpha$-keto- $\gamma$-methylthiobutyrate, which suggests the release of strong oxidants. They showed that the photodynamic catalysis reaction could be inhibited by radical scavengers ( $\alpha$-tocopherol, $100 \%$; catalase, $83 \%$, azide, $40 \%$; 1,4-diazabicyclo[2,2,2] octane, $83 \%$ ).

Clinical Investigations: Diesel exhaust (mass median diameter $<10 \mu \mathrm{m}$ ) at $300 \mu \mathrm{g} / \mathrm{m}^{3}$ associated with concentrations of $\mathrm{NO}_{2}$ of $1.6 \mathrm{ppm}$, $\mathrm{NO}$ of 4.5 ppm for $1 \mathrm{~h}$, CO of $7.5 \mathrm{ppm}$, total hydrocarbons of $4.3 \mathrm{ppm}$, formaldehyde of $0.26 \mathrm{mg} / \mathrm{m}^{3}$, and $4.3 \times 10^{6}$ suspended particles/ml in healthy human volunteers exposed for $1 \mathrm{~h}$ induced a $240 \%$ median relative increase in interleukin-8 mRNA gene transcripts in the cells contained in bronchial lavage performed $6 \mathrm{~h}$ later (SALVI et al. 2000). There were no changes in the mRNA transcript levels for ILL$1 \beta$, ILL-4, TNF- $\alpha$, interferon- $\gamma$, and granulocytemacrophage colony-stimulating factor after exposure to diesel exhaust compared with air.

Animal Experiments: Diesel exhaust particles were inhaled by Fischer-344 rats for 6 month and 1 year, respectively, at concentrations of $250 \mu \mathrm{g} / \mathrm{m}^{3}, 750 \mu \mathrm{g} /$ $\mathrm{m}^{3}, 1500 \mu \mathrm{g} / \mathrm{m}^{3}$ and $6000 \mu \mathrm{g} / \mathrm{m}^{3}$. Bronchoalveolar lavage showed nearly all the macrophages containing particle-filled phagosomes, their amount varying with the concentration of the exposure, with some macrophages appearing several times the size of the control macrophages (SтRом 1984). The number of alveolar macrophages increased primar- ily with the inhaled concentration of particles, but was not different from controls in the group exposed to $250 \mu \mathrm{g} / \mathrm{m}^{3}$. The macrophages did not increase significantly with the duration of the exposure.

Female ddY mice after intratracheal instillation of $0.05 \mathrm{mg}$ diesel exhaust particles suspended in 50 $\mathrm{mM}$ phosphate-buffered saline ( $\mathrm{pH}$ 7.4) containing $0.05 \%$ Tween 80 and sonicated for 3 min showed $\mathrm{HO}^{\circ}$ generation in their lungs as demonstrated in vivo by non-invasive L-band ESR spectroscopy and a membrane-impermeable nitroxyl probe (HAN et al. 2001). HO generation was confirmed with the enhancement of in vivo ESR signal decay rate of the probe. The decay rate at mid-thorax was significantly enhanced in diesel exhaust particle-treated mice compared to that in vehicle treated animals. The enhancement was completely suppressed by the administration of either $\mathrm{HO}^{*}$ scavengers, catalase, or desferrioxamine, while the administration of superoxide dismutase further increased the rate. The administration of Fenton's reagents into the lung also enhanced the decay of the probe at midthorax of mice.

Male ICR mice instilled intratracheally with suspensions containing 1 or $2 \mathrm{mg}$ of diesel exhaust particles in $1 \mathrm{ml}$ of $50 \mathrm{mM}$ phosphate-buffered saline (pH 7.4) and $0.05 \%$ Tween 80 prepared by sonication once a week for 10 weeks showed increased levels of inducible NO synthase in the macrophages (Lim et al. 1998).

Rat lung inflammation $24 \mathrm{~h}$ after intratracheal instillation of diesel exhaust particles (Standard Reference Material 2975) initially exposed to 0.1 ppm $\left[{ }^{18} \mathrm{O}_{3} \mathrm{O}_{3}\right.$ for $48 \mathrm{~h}$ was more potent in increasing neutrophilia, lavage total protein, and lactate dehydrogenase (EC 1.1.1.1.27) activity compared to unexposed diesel exhaust particles (MADDEN et al. 2000). Exposure of diesel exhaust particles to $1 \mathrm{ppm}$ $\mathrm{O}_{3}$ led to a decreased bioactivity of the particles.

\subsection{0 \\ Diphenyl-methane 4,4'-diisocyanate}

The early acute pulmonary response of Wistar rats exposed nose-only to respirable polymeric diphenyl-methane 4,4'-diisocyanate aerosol for $6 \mathrm{~h}$ were bronchoalveolar lavage cells markedly loaded with phosphatidylcholine (PAuluhn 2000). Bronchoalveolar lavage cells from rats repeatedly exposed to $12.9 \mathrm{mg}$ polymeric diphenyl-methane 4,4 'diisocyanate $\mathrm{m}^{-3}$ air $(6 \mathrm{~h} /$ day, 5 days/week for 14 days; exposure was from days $0-17$ followed by a post-exposure period to day 35) increased in number and phospholipid content significantly in a time-dependent manner and returned to almost 
normal levels within 10 post-exposure days (PAULUHN 2002).

\subsection{1}

Ethanol

The detrimental influence of alcoholic beverages on host susceptibility to infection is, historically, a well known clinical observation (Rush 1784, Koch 1884). The incidence of tuberculosis and its severity is profoundly higher for alcoholics (FEINGOLD 1976). The proportion of persons isolating M.tuberculosis among the new cases of tuberculosis complicated by chronic alcoholism was 1.3 times higher than that among patients with tuberculosis not complicated by alcoholism (Rudor and CHUBAKov 1984). Drew et al. (1984) found both unstimulated and pokeweed mitogen stimulated immunoglobulin synthesis by peripheral blood mononuclear cells in vitro and plasma immunoglobulin concentrations abnormal in alcoholics. Glucan significantly modified the susceptibility of chronic ethanol-treated mice to Staphyloccocus aureus infection by possibly enhancing macrophage-mediated host defence mechanisms (Di Luzio and Williams 1980). The ANIMALS MAINTAINED ON A CHRONIC ETHANOL DIET WHICH WERE PRE-TREATED WITH GLUCAN EXHIBITED THE WELL-DOCUMENTED GLUCANINDUCED GRANULOMA BUT NO HEPATIC INJURY AS DID THE CONTROL mice. ${ }^{131} \mathrm{I}$ labeled microaggregated albumin clearance is significantly depressed in rats given ethanol (Ali and Nolan 1967, Nolan et al. 1980), but two similar studies in humans conflicted on whether the delayed clearance of the colloid is due to decreased perfusion (CooksLey et al. 1973) or a direct depression of the R.E.S. function (Liu 1975).

The role of short-chain aliphatic alcohols in the regulation of the respirarory burst is controversial, and several mechanisms may be involved in their effects (Seifert and Schultz 1991). Alcohol metabolism has at least four pathways, the most important one being the oxidation of ethanol by alcohol dehydrogenase. After chronic consumption of ethanol or in the presence of high concentration of ethanol, minor pathways such as the microsomal ethanol oxidising system

$\mathrm{H}_{3} \mathrm{C}-\mathrm{CH}_{2} \mathrm{OH}+\mathrm{NAD}^{+} \rightarrow \mathrm{NADH}+\mathrm{H}^{+}+\mathrm{H}_{3} \mathrm{C}-\mathrm{CHO}$

become more important. At least two ethanol microsomal oxidation pathways have been characterised by electron spin resonance (KRIKUN et al. 1984, Albano et al. 1988). The $\alpha$-hydroxyethyl radical formed by the one-electron oxidation of ethanol has been detected by spin trapping with $\alpha$-(4pyridyl 1-oxide)- $N$-tert-butylnitrone (POBN) in

microsomal incubations (Gonthier et al. 1991). Radical production resulted in part from Fentontype chemistry due to microsomal hydrogen peroxide and trace iron present in the microsomal preparations (McCAY et al. 1992). However, with a Desferal-treated system, radical adducts from ethanol and other alcohols could still be detected and were attributed to enzymatic activity by cytochrome P450 IIE1 (Albano et al. $(1988,1991)$.

The expression of the ethanol-metabolising cytochrome P-450 (CYP2E1) in human monocytederived macrophages cultured for 3 and 6 days in RPMI 1640, $15 \%$ human serum, and $80 \mu \mathrm{g} / \mathrm{ml}$ gentamicin was studied at the mRNA and protein levels. Hutson and Wickramasinghe (1999) showed that CYP2E1 is expressed at a level similar to that demonstrated in other extrahepatic tissues.

Ethanol may scavenge the highly reactive hydroxyl radical to form a much less reactive hydroxyethyl radical:

$$
\mathrm{H}_{3} \mathrm{CCH}_{2} \mathrm{OH}+\mathrm{HO}^{\cdot} \rightarrow \mathrm{H}_{3} \mathrm{CC} \cdot \mathrm{HOH}+\mathrm{H}_{2} \mathrm{O}
$$

This reaction is made use of in spin-trapping experiments with 5,5-dimethylpyrroline- $N$-oxide.

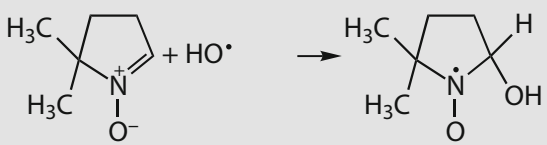

5,5-Dimethyl-1-pyrroline-1-oxide-HO^adduct
[8]

Catalase (EC 1.11.1.6) was progressively inactivated by exposure to 1,1'-dihydroxyazoethane-generated hydroxyethyl radical in a time and hydroxyethyl radical concentration-dependent manner (PUNTARULO et al. 1999). Ascorbic acid and phenyl-t-butyl nitrone gave full protection to catalase against hydroxyethyl radical-dependent inactivation. The antioxidants 2-tert-butyl-4-methylphenol, pyrogallate, and $\alpha$-tocopherol protected catalase against inactivation by 84,88 , and $39 \%$, respectively. Other antioxidant enzymes were also sensitive to exposure to hydroxyethyl radical. Glutathione reductase (EC 1.6.4.2), glutathione peroxidase (EC 1.11.1.9), and superoxide dismutase (EC 1.15.1.1) were inactivated by 46,36 , and $39 \%$, respectively, by hydroxyethyl radical.

In the rat, TAKahashi et al. (1992), French et al. (1993), Morimoto et al. 1994, 1995) and Albani et al. (1996) correlated the level of CYP2E1 with liver pathology, in vitro lipid peroxidation, and the formation of $\alpha$-hydroxyethyl radical adduct.

Mitochondrial DNA isolated from the livers of rats fed ethanol for $42-76$ days showed a $21 \%$ increase in the level of 8-hydroxydeoxyguanosine 
compared with the controls $(4.8 \pm 0.9$ versus $3.98 \pm 0.58-\mathrm{OH}-\mathrm{dG} / 10^{5} \mathrm{dG}$ ) (CAHILL et al. 1997). This difference increased to $43 \%$ in animals that had been fed ethanol for 105-164 days (control, 6.9 \pm 1.0 ; ethanol, $9.9 \pm 1.18-\mathrm{OH}-\mathrm{dG} / 10^{5} \mathrm{dG}$ ).

Ethanol inhibited ${ }^{\circ} \mathrm{NO}$ production by thioglycollate elicited mouse macrophages as measured by nitrite production (GoLdIN et al. 1993). Some significant differences between strains suggested that genetic factors may be important in alcoholic liver disease.

\subsection{2}

\section{Formaldehyde}

The major pathways of metabolism of inhaled formaldehyde are oxidation to formate and incorporation into biological macromolecules via tetrahydrofolate-dependent one-carbon biosynthetic pathways (HuENNEKens and Osborne 1959, KoIvusAlo et al. 1982). The most important pathway for oxidation appears to be that catalysed by formaldehyde dehydrogenase (EC 1.2.1.1), an enzyme that requires both glutathione and $\mathrm{NAD}^{+}$as cofactors. Uotila and KoIvusalo (1974) showed that the true substrate is the hemithioacetal adduct of formaldehyde and glutathione and the product formed is the thiol ester of formic acid, $S$ formylglutathione.

$\mathrm{H}_{2} \mathrm{CO}+\mathrm{GSH}+\mathrm{NAD}(\mathrm{P})^{+} \longleftrightarrow \mathrm{GSCHO}+\mathrm{NAD}(\mathrm{P}) \mathrm{H}+\mathrm{H}^{+}$

[255]

Rat alveolar macrophages obtained by bronchoalveolar lavage showed a concentration- and timedependent depression of zymosan induced and lucigenin enhanced chemiluminescence when exposed to formaldehyde (Schroers and Tilkes 1990).

In the male $200 \mathrm{~g}$ Sprague-Dawley rat, $20 \mathrm{ppm}$ formaldehyde exposure for $4 \mathrm{~h}$ at rest delayed short-term clearance $(0-50 \mathrm{~h})$ of monodisperse polystyrene latex microspheres (about $1.7 \mu \mathrm{m}$ in diameter) labelled with ${ }^{51} \mathrm{Cr}$ and inhaled for $20 \mathrm{~min}$ just prior to pollutant exposure. Exposure to 10 ppm formaldehyde did not produce significant effects; however, this same concentration during treadmill exercise $(8 \mathrm{~m} / \mathrm{min})$ caused a delay in short-term clearance (PHALEN et al. 1994).

\subsection{3}

\section{Glutaraldehyde}

Rat alveolar macrophages obtained by bronchoalveolar lavage showed a concentration- and timedependent depression of zymosan induced and lucigenin enhanced chemiluminescence when ex- posed to glutaraldehyde (Schroers and Tilkes 1990).

\subsection{4 \\ Glyoxal}

The $\alpha$-oxoaldehydes glyoxal, methylglyoxal and 3deoxyglucosone are physiological metabolites. Glyoxal is formed by the slow, spontaneous oxidative degradation of glucose (WELLS-KNECHT et al. 1995), the degradation of glycated proteins (NAMIKI and HAYASHi 1983), and lipid peroxidation (Mlakar and Spiteller 1996).

Rat alveolar macrophages obtained by bronchoalveolar lavage showed a concentration- and time-dependent depression of zymosan induced and lucigenin enhanced chemiluminescence when exposed to glyoxal (Schroers and Tilkes 1990).

Incubation of murine $\mathrm{P}_{388} \mathrm{D}_{1}$ macrophages with $30 \mathrm{mM} \mathrm{H}_{2} \mathrm{O}_{2}$ increased the cellular concentration of glyoxal after $3 \mathrm{~h}(P<0.05)$ and decreased the concentration of glyoxal in the medium $(P<0.01)$ with respect to control values (АвоRDo et al. 1999).

\subsection{5 \\ 4-Hydroxynonenal}

4-Hydroxynonenal is one of the major aldehyde products of lipid peroxidation and has been reported to be the most toxic aldehyde formed (Benedetti et al. 1979, 1980, Benedetti and Comporti 1987). Li et al. (1996) found a formation of protein adducts in alveolar macrophages from $\mathrm{C} 3 \mathrm{H} / \mathrm{HeJ}$ and C57BL/6J mice in a dose-dependent manner. Alveolar macrophages from both strains had extensive apoptosis at $100 \mu \mathrm{M}$ 4-hydroxyonenal.

\subsection{6 \\ Lindane}

$1 \alpha, 2 \alpha, 3 \beta, 4 \alpha, 5 \alpha, 6 \beta$-Hexachlorocyclohexane, lindane at a concentration of $125 \mu \mathrm{M}$ in vitro stimulated $\mathrm{O}_{2}{ }^{--}$- production by guinea pig alveolar macrophages (Holian et al. 1984). $1.5 \times 10^{6}$ Human neutrophil granulocytes exposed to $400 \mu \mathrm{M}$ lindan formed $>11 \mathrm{nM} \mathrm{O}_{2}{ }^{-}-1 \mathrm{~min}$ (ENGLISH et al. 1986),

\subsection{7 \\ Local Anaesthetics}

Lidocaine solution (12 mM), as proposed by STEWART (1981) to remove adherent murine monocytes, activated superoxide dismutase (Gulyakwa et al. 1987). Lidocaine and related local anaesthetics disrupt ion transport across cell membranes through changes in membrane fluidity. Lidocaine 
(2.5 mM, 1-h pre-treatment) caused a small but significant decrease in baseline cytosolic $\mathrm{pH}\left(\mathrm{pH}_{\mathrm{i}}\right)$ of $0.14 \pm 0.02$ units (BIDANI et al. 1996). Ammoniaprepulse studies with bafilomycin $A_{1}$ (a specific inhibitor of V-type $\mathrm{H}^{+}$pumps, i.e., V-ATPase) and amiloride (an inhibitor of the $\mathrm{Na}^{+} / \mathrm{H}^{+}$exchanger, i.e., NHE) showed that $2.5 \mathrm{mM}$ lidocaine suppressed plasmalemmal V-APTase activity by $\sim 50 \%$ and NHE activity by $\sim 90 \%$. The median effective concentration $\left(\mathrm{EC}_{50}\right)$ of lidocaine (1-h pre-treatment) against $\mathrm{pH}_{\mathrm{i}}$ recovery from ammonia-prepulse acidloads was $0.97 \pm 0.04 \mathrm{mM}$. Lidocaine $(2.5 \mathrm{mM})$ diminished the generation of superoxide anions induced using PMA by $>60 \%$ and reduced the release of TNF- $\alpha$ induced using lipopolysaccharide by $>50 \%$.

\subsection{8}

\section{Melamine Resin Semifinished Products}

Powdered Trimethylolmelamine (Madurit ${ }^{\circledR}$ )

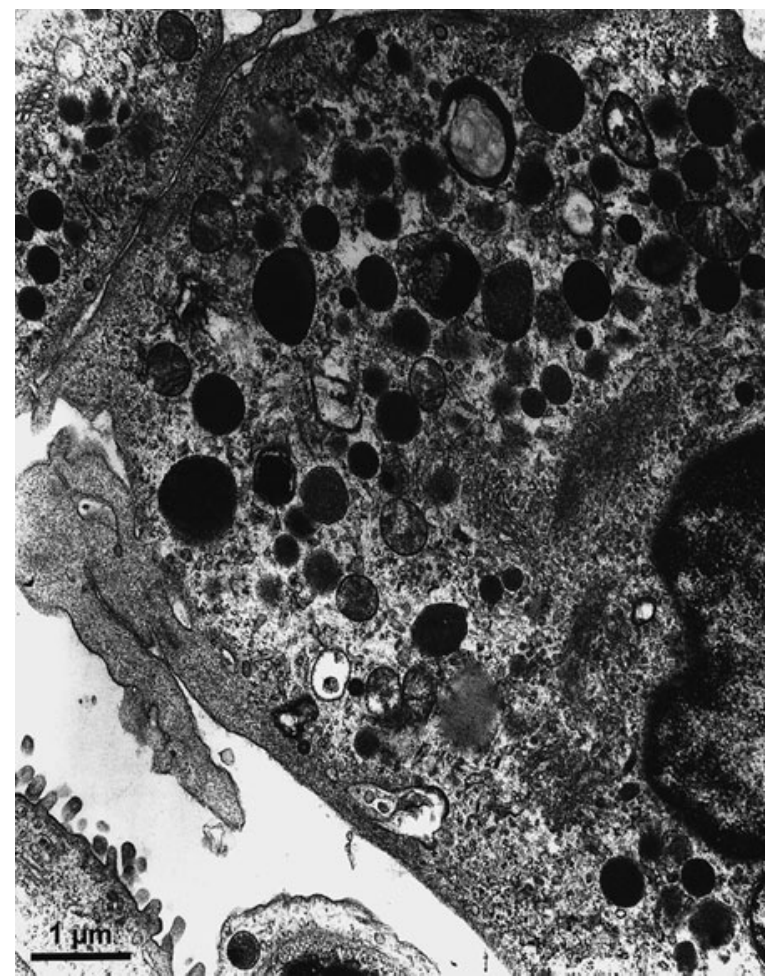

Fig. 159. Alveolar macrophage (block 490) from a male rat which inhaled $73.6 \mathrm{mg}$ trimethylol melamine (Madurit ${ }^{\circledR}$ ) dust/l for a total of $125 \mathrm{~h} 15 \mathrm{~min}$ on 5 days/week for 16 days. Fixed under pentobarbital anaesthesia $(30 \mathrm{mg} / \mathrm{kg})$ by intratracheal instillaTion of $2.5 \%$ glutaraldehyde in $0.1 \mathrm{M}$ sodium cacodylate buffer. Postfixation with $1 \%$ osmium tetroxide in sodium cacodylate buffer. Embedded in Epon 812 and sectioned at $50 \mathrm{~nm}$. Lead citrate and uranyl acetate. Plate 4068

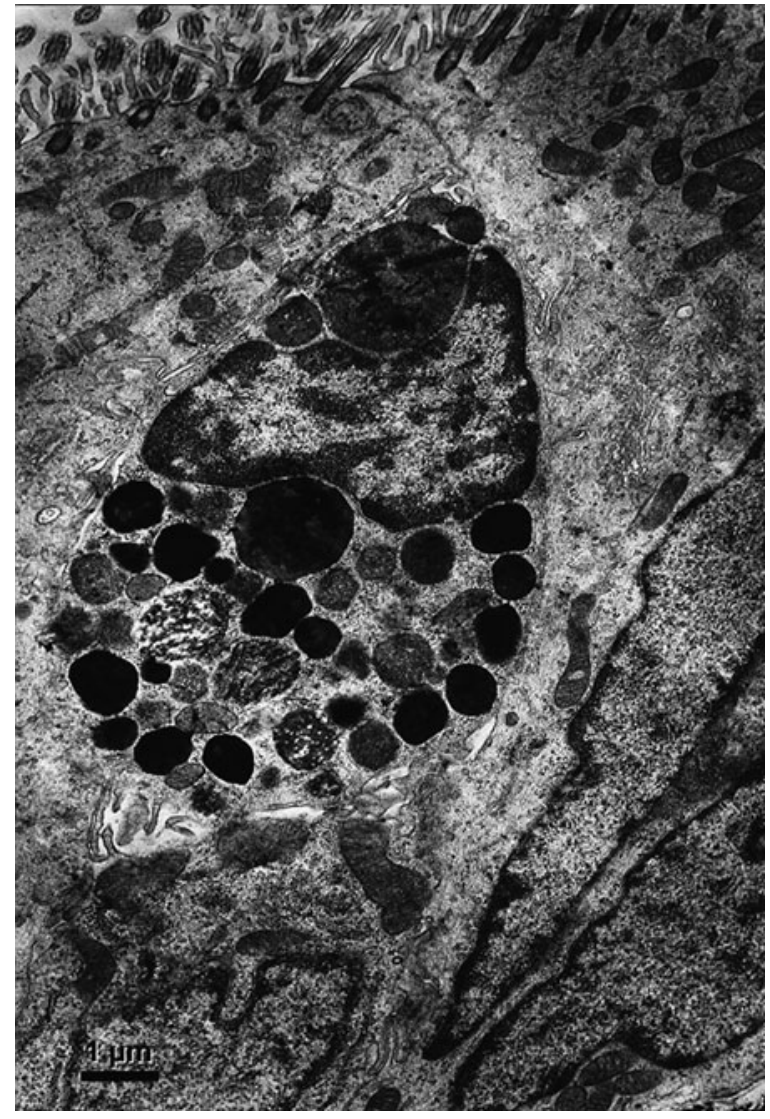

Fig. 160. Mononuclear phagocyte in the bronchial epithelium (block 490) of a male rat which inhaled $73.6 \mathrm{mg}$ trimethylol melamine (Madurit ${ }^{\circledR}$ ) dust/l for a total of $125 \mathrm{~h} 15 \mathrm{~min}$ on 5 days/week for 16 days. Fixed under pentobarbital anaesthesia $(30 \mathrm{mg} / \mathrm{kg})$ by intratracheal instillation of $2.5 \%$ glutaraldehyde in $0.1 \mathrm{M}$ sodium cacodylate buffer. Postfixation with $1 \%$ osmium tetroxide in sodium cacodylate buffer. Embedded in Epon 812 and sectioned at $50 \mathrm{~nm}$. Lead citrate and uranyl acetate. Plate 4108

\subsection{9 Methyl Cellulose}

The anæmia caused by administration of methyl cellulose is due to excessive destruction of normal erythrocytes by the cells of the reticulo-endothelial system, especially those in the spleen, which have undergone hypertrophy and hyperplasia in response to the injected inert colloidal material (PALMER et al. 1953, Giblett et al. 1956). 


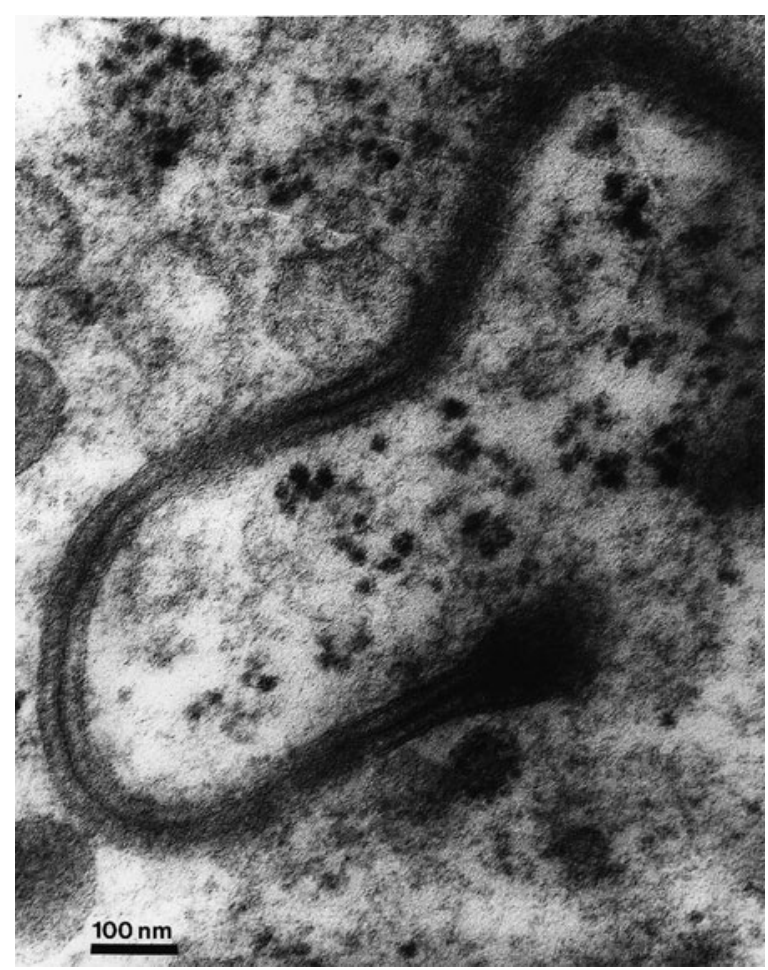

Fig. 161. Phagophore in a lymph node macrophage (block 4435) from a $225 \mathrm{~g}$ Sprague-Dawley rat (Charles River, France) four days after intraperitoneal injection of $10 \mathrm{mg}$ methylcellulose $/ \mathrm{kg}$ body weight. Under pentobarbital anaesthesia $(30 \mathrm{mg} / \mathrm{kg})$, the animal was perfused from the abdominal aorta with $2.5 \%$ glutaraldehyde in $0.1 \mathrm{M}$ sodium cacodylate buffer ( $\mathrm{pH}$ 7.4). Postfixation with $1 \%$ osmium tetroxide in sodium cacodylate buffer. Embedded in Epon 812 and sectioned at $50 \mathrm{~nm}$. Stained with lead citrate and uranyl acetate. Plate 4144

\subsection{0}

\section{Paprika Dust}

One month after a single intratracheal instillation of $15 \mathrm{mg}$ paprika dust suspended in $1 \mathrm{ml}$ saline, male CFY rats showed some phagocytosed, irregularly shaped periodic acid-Schiff positive foreign bodies in their alveolar macrophages (TÁTRAI and UNGVÁRY 1992). 3 Months after instillation intraalveolar foci of foreign body type giant cells had formed.

\subsection{1 \\ Pyridinium Compounds}

The pyridinyl radical may be oxidised or reduced in one-electron reactions. Each oxidation state is capable of complexation, with the pyridinium ion acting as acceptor, the 1,4-dihydropyridine ring acting as a donor, and the pyridinyl radical functioning as both a donor and an acceptor towards itself.

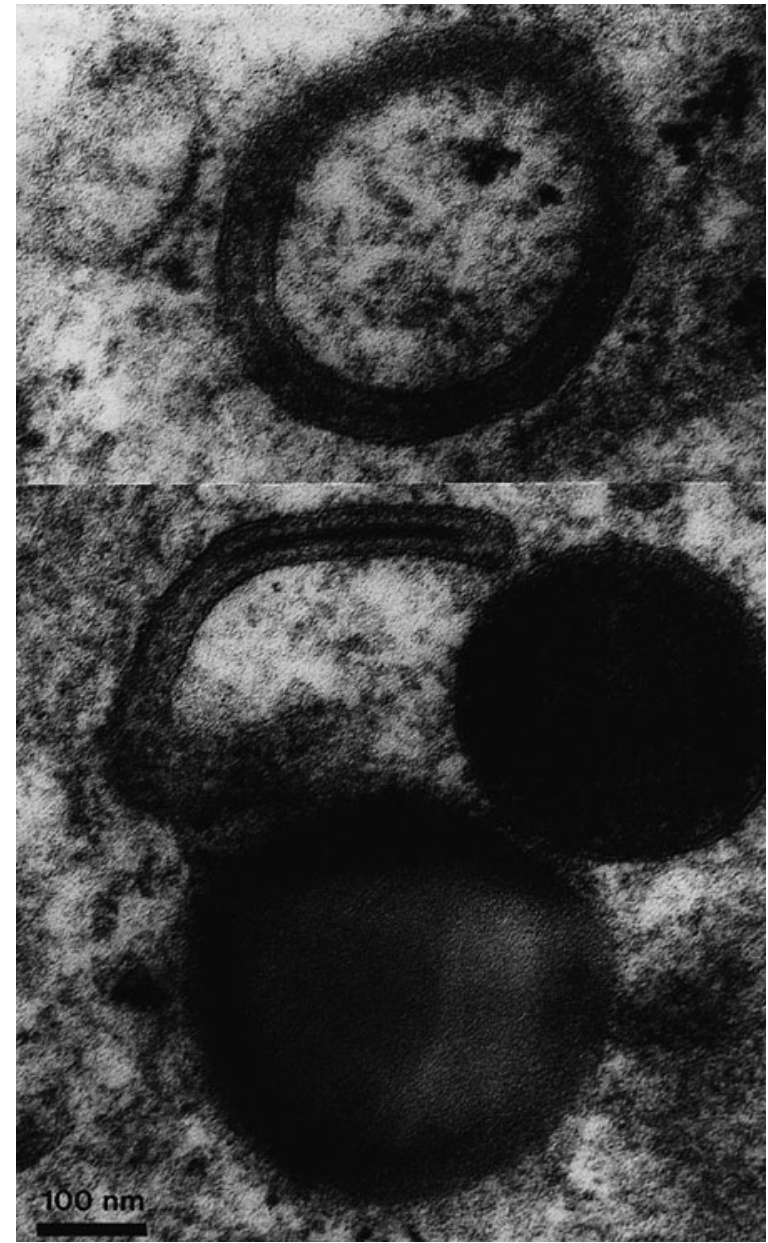

Fig. 162. Phagophores in a lymph node macrophage. Omentum (block 4435) of a 225 g male Sprague-Dawley rat (Charles River, France). On the 4th day after an intraperitoneal injection of $10 \mathrm{mg}$ methylcellulose/kg body weight, under pentobarbital anaesthesia $(30 \mathrm{mg} / \mathrm{kg})$, the animal was perfused from the abdominal aorta with $2.5 \%$ glutaraldehyde in $0.1 \mathrm{M}$ sodium cacodylate buffer ( $\mathrm{pH}$ 7.4). Postfixation with $1 \%$ osmium tetroxide in sodium cacodylate buffer. Embedded in Epon 812 and sectioned at $50 \mathrm{~nm}$. Lead citrate and uranyl acetate. Plates 4145 and 4146

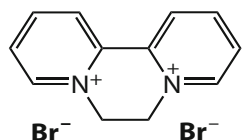

Diquat<smiles>C[n+]1ccc(-c2cc[n+](C)cc2)cc1</smiles>

Paraquat

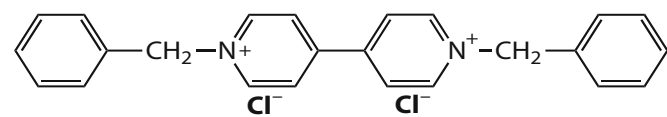

Benzylviologen

Pyridinium compounds [256] 
Pyridinium compounds, e.g. diquat, paraquat and benzylviologen, by enzymatic one-electron transfer become radicals. These radicals react with $\mathrm{O}_{2}$ forming $\mathrm{O}_{2}{ }^{-}$and the starting materials. By redox cycling $\mathrm{O}_{2}{ }^{-}$is produced again and again (Bus et al. 1974). Hydroxyl radicals generated via $\mathrm{H}_{2} \mathrm{O}_{2}$ induce lipid peroxidation and denaturation of proteins and nucleic acids, which are segregated and degraded by lysosomal enzymes. $4 \mathrm{~h}$ after an inhalation of hexadecylpyridinium chloride, phagophores were detected in alveolar macrophages (Fig. 162). The Fenton catalyst, $\mathrm{Fe}^{2+}$ is segregated from cytoplasmic areas containing ferritin. The autophagous vacuoles included other organelles, membrane structures and fat droplets.

\section{Hexadecylpyridinium Chloride}

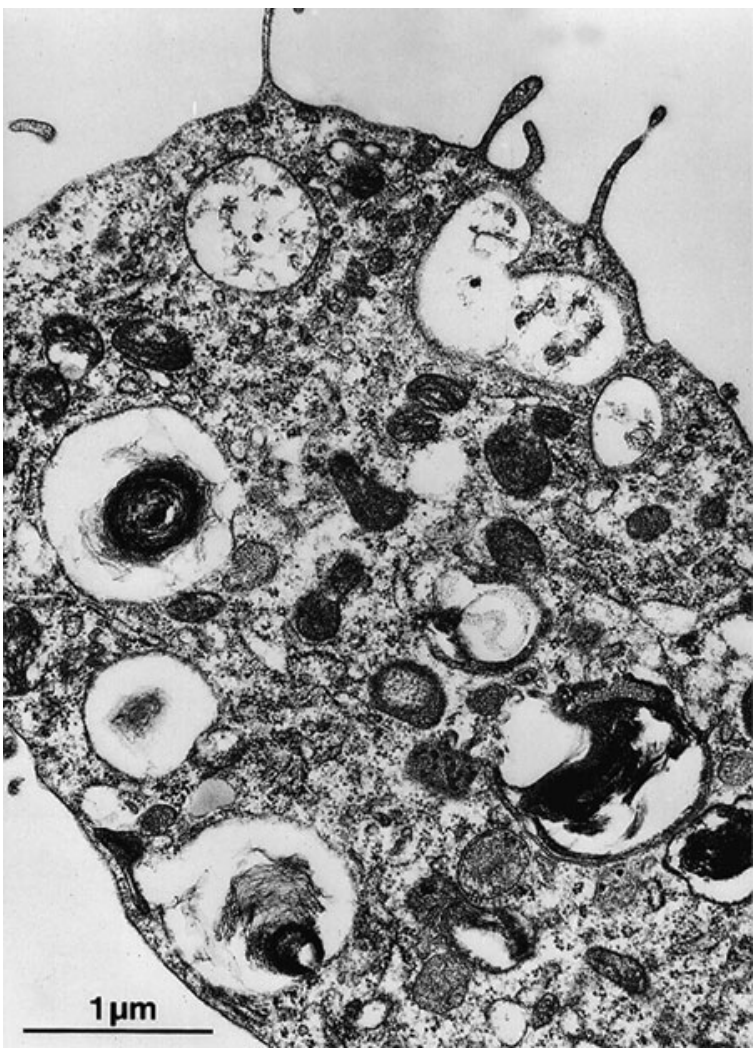

Fig. 163. Phagosomes in a stimulated alveolar macrophages from a female rat (breeder: Winkelmann, BorchenKirchborchen) 4 hours after an inhalation of a mixture of 28 $\mu \mathrm{g}$ hexadecylpyridinium chloride/puff + sorbitan trioleate (surfactants for metered aerosols) dispersed from a Medihaler ${ }^{\circledR} ; .12 \mathrm{puffs} / \mathrm{min}$ were dispersed into a $164.5 \mathrm{l}$ box where the animals stayed for 15 min. Fixed under methitural anaesthesia by intratracheal instillation of $2.5 \%$ glutaraldehyde in phosphate buffer ( $\mathrm{pH}$ 7.4) before opening the thorax. Postfixation with $1 \%$ osmium tetroxide in phosphate buffer $(\mathrm{pH}$ 7.4). Contrasted en bloc for $12 \mathrm{~h}$ with $0.5 \%$ uranyl acetate in $70 \%$ ethanol. Embedded in a 2:8 mixture of methyl and butyl methacrylate. Sectioned at $50 \mathrm{~nm}$. Lead citrate after Reynolds (1963). Plate 48/05

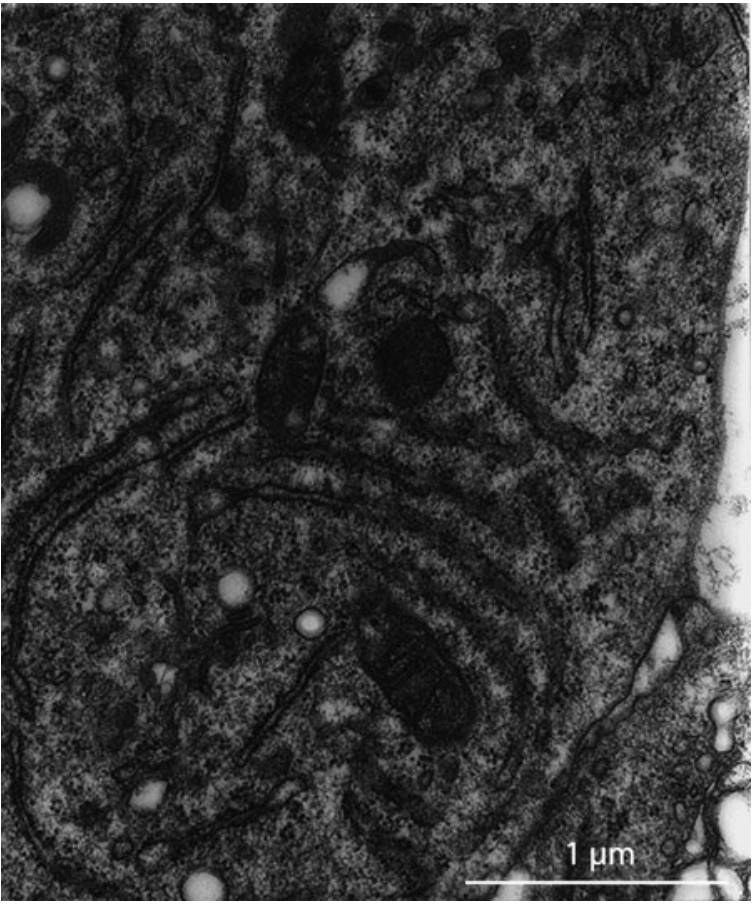

Fig. 164. Rough endoplasmic reticulum in a stimulated alveolar macrophages from a female rat (breeder: Winkelmann, Borchen-Kirchborchen) 4 hours after an inhalation of a mixture of $28 \mu \mathrm{g}$ hexadecylpyridinium chloride/puff + sorbitan trioleate (surfactants for metered aerosols) dispersed from a Medihaler ${ }^{\circledR} .12$ puffs/min were dispersed into a 164.51 box where the animals stayed for 15 min. Fixed under methitural anaesthesia by intratracheal instillation of $2.5 \%$ glutaraldehyde in phosphate buffer ( $\mathrm{pH}$ 7.4) before opening the thorax. Postfixation with $1 \%$ osmium tetroxide in phosphate buffer (pH 7.4). Contrasted en bloc for $12 \mathrm{~h}$ with $0.5 \%$ uranyl acetate in $70 \%$ ethanol. Embedded in a 2:8 mixture of methyl and butyl methacrylate. Sectioned at $50 \mathrm{~nm}$. Lead citrate after Reynolds (1963). Plate 49/08 


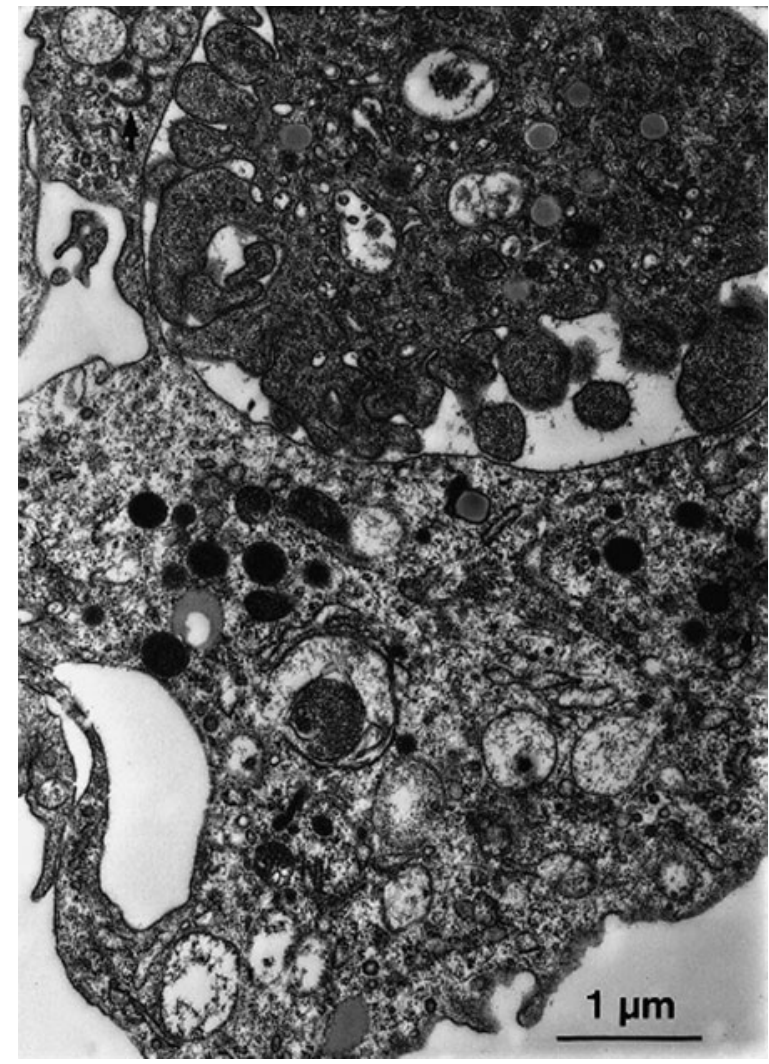

Fig. 165. Phagophore (arrow) in an alveolar macrophage from a female rat (breeder: Winkelmann, Borchen-Kirchborchen) 4 hours after an inhalation of a mixture of $28 \mu \mathrm{g}$ hexadecylpyridinium chloride/puff + sorbitan trioleate (surfactants for metered aerosols) dispersed from a Medihaler ${ }^{\circledR}$. $12 \mathrm{puffs} / \mathrm{min}$ were dispersed into a $164.5 \mathrm{l}$ box where the animals stayed for 15 min. Fixed under methitural anaesthesia by intratracheal instillation of $2.5 \%$ glutaraldehyde in phosphate buffer ( $\mathrm{pH}$ 7.4) before opening the thorax. Postfixation with $1 \%$ osmium tetroxide in phosphate buffer ( $\mathrm{pH} 7.4$ ). Contrasted en bloc for $12 \mathrm{~h}$ with $0.5 \%$ uranyl acetate in $70 \%$ ethanol. Embedded in a 2:8 mixture of methyl and butyl methacrylate. Sectioned at $50 \mathrm{~nm}$. Lead citrate after REYNOLDS (1963). Plate $49 / 11$

\section{Paraquat}

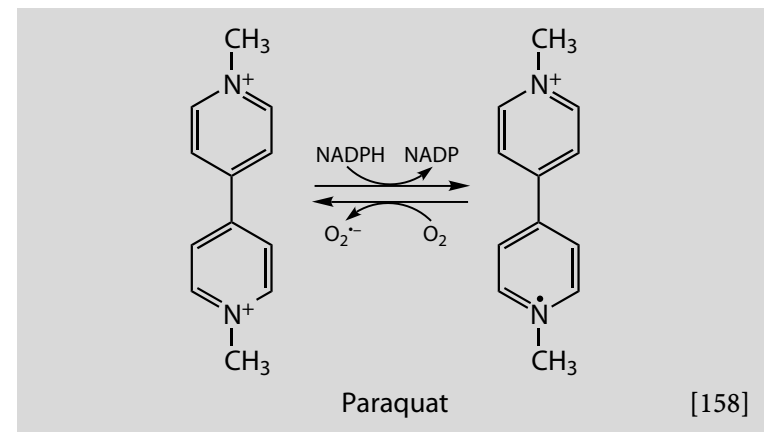

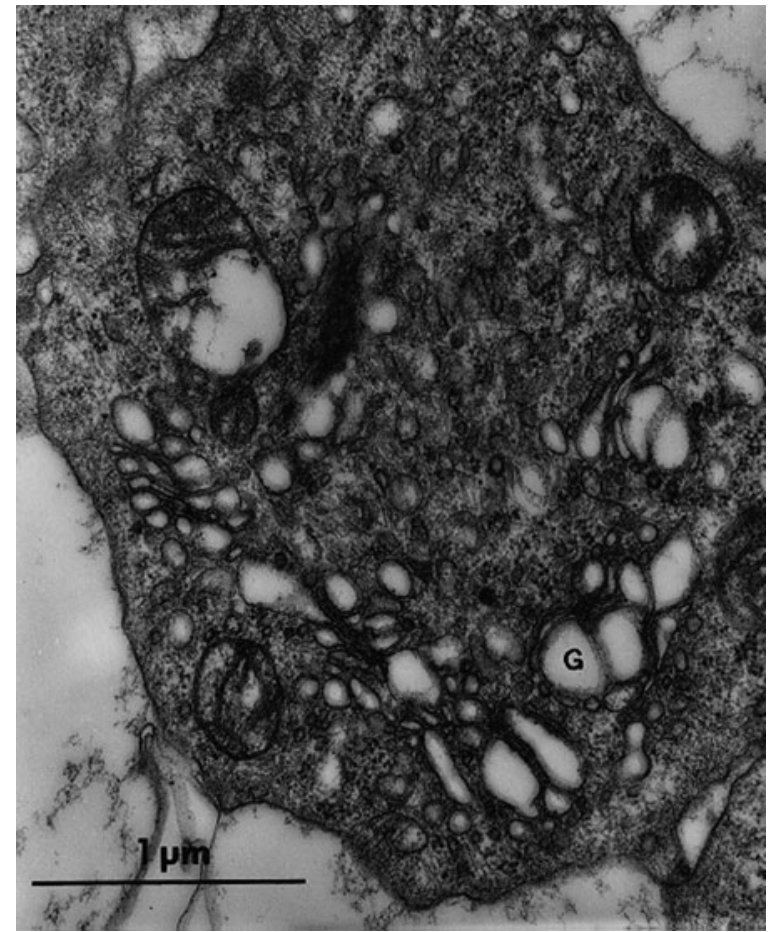

Fig. 166. Dilated Golgi cisternae $(G)$ in a stimulated alveolar macrophage from a female rat (breeder: Winkelmann, Borchen-Kirchborchen) 4 hours after an inhalation of a mixture of $28 \mu \mathrm{g}$ hexadecylpyridinium chloride/puff + sorbitan trioleate (surfactants for metered aerosols) dispersed from a Medihaler ${ }^{\circledR} .12$ puffs/min were dispersed into a $164.5 \mathrm{l}$ box where the animals stayed for $15 \mathrm{~min}$. Fixed under methitural anaesthesia by intratracheal instillation of $2.5 \%$ glutaraldehyde in phosphate buffer ( $\mathrm{pH}$ 7.4) before opening the thorax. Postfixation with $1 \%$ osmium tetroxide in phosphate buffer ( $\mathrm{pH}$ 7.4). Contrasted en bloc for $12 \mathrm{~h}$ with $0.5 \%$ uranyl acetate in $70 \%$ ethanol. Embedded in a $2: 8$ mixture of methyl and butyl methacrylate. Sectioned at $50 \mathrm{~nm}$. Lead citrate after REYNOLDS (1963). Plate 49/07

Paraquat (1,1'-dimethyl-4,4'-bipyrdilium dichloride) is a widely used herbicide causing severe injury to the lungs and other organs mediated by reactive oxygen intermediates, NADP cytochrome $c$ reductase in microsomal drug metabolising enzyme systems forming radicals (GAGE 1968, Bus et al. 1974, 1976). SHIMADA et al. (1998), however, demonstrated a mitochondrial NADH-quinone oxidoreductase of the outer mitochondrial membrane responsible for paraquat cytotoxicity in rat livers.

Paraquat significantly reduced concavalin Astimulated $\mathrm{O}_{2}^{--}$production when incubated at 1 $\mathrm{mM}$ with rat alveolar macrophages in the absence of glucose (Forman et al. 1980). The effect of paraquat was reversed by glucose, but fructose, lactate, and pyruvate could not reverse paraquat inhibition. 
Paraquat enhanced oxidation of NADPH (but not $\mathrm{NADH}$ ) by cell supernatant and increased pentose phosphate shunt activity in resting macrophages, but did not affect mitochondrial respiration or ATP content of alveolar macrophages.

Paraquat (respective $\mathrm{LD}_{10}$ dose given intraperitoneally) induced different biochemical responses including different protective responses in Wistar rats and Swiss mice (ALI et al. 2000). As a protective response, NADPH-specific quinone reductase is induced in rats, while catalase is induced in mice. It is implied that an early induction of catalase in mice as opposed to rats may account for the resistance of Swiss mice to paraquat toxicity. Xanthine oxidase, which was induced in rats, remains unaffected in mice indicating that the enzyme contributed to paraquat toxicity only in Wistar rats.

The "lipid peroxide stimulation' mechanism of paraquat acute toxicity has been questioned by SHU et al. (1979), who have shown that it is possible by pre-treatment with $N, N^{\prime}$-diphenyl- $p$-phenylenediamine, an antioxidant, to prevent the paraquat stimulation of lipid peroxidation without protecting the animals against its lethal effects.

\subsection{2}

\section{Tannin from Cotton Bract}

Condensed tannin, a polymer of monoflavonoid subunits, is one of the major water-soluble components present in the airborne dust generated from the processing of a wide variety of woody plants (Heming Way 1989). In vitro studies have shown that condensed tannin can profoundly change the functional capacity of alveolar macrophages (Rohrbach et al. 1989, 1992, Kreofsky et al. 1990, 1992, Vuk PaVlovic and Rohrbach 1990).

Arachidonic acid release from rabbit alveolar macrophages gained by bronchoalveolar lavage was increased by tannin in a dose-dependent manner (RALston and Rohrbach 1994).

Using the luciferin luciferase reaction, PrÉvost et al. (1996) showed that tannin markedly depleted ATP content of rabbit alveolar macrophages in vitro. In inositol-labeled cells, tannin increased inositol-phosphate release in a dose-dependent manner. In lyso-PAF-labeled cells, tannin induced synthesis of phosphatidic acid and diglycerides. In the presence of ethanol, the level of tannin-induced phosphatidic acid was slightly reduced, and phosphatidylethanol was synthesised. No phosphatidylethanol was found in alveolar macrophages stimulated by zymosan in the presence of ethanol. GF 109203X, a specific inhibotor of protein kinase C decreased only tannin-induced phosphatidylethanol synthesis.

\subsection{3}

\section{Tetrandrine}

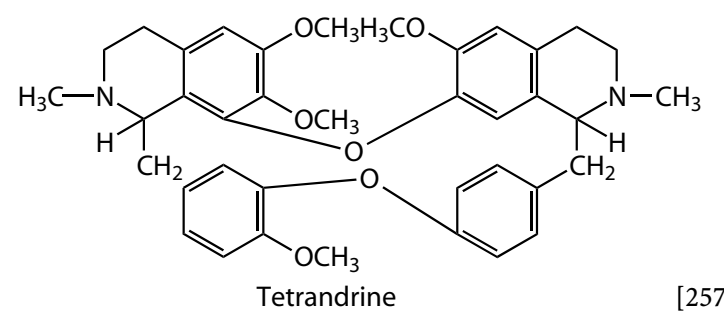

Tetrandrine, an antiinflammatory immunosuppressive bisbenzylisoquinoline alkaloid isolated from the Chinese herb Stephania tetranda, at $10^{-7} \mathrm{M}$ to $10^{-4} \mathrm{M}$ caused dose- and time-dependent loss of cell viability of guinea pig alveolar macrophages, mouse peritoneal macrophages, and mouse macrophage-like J774 cells (PANG and Hoult 1997). Loss of macrophage viability after tetrandrine treatment was accompanied by the generation of large amounts of prostaglandin $\mathrm{E}_{2}$, to levels $285-877 \%$ of control. Coincubation with indomethacin abolished $\mathrm{PGE}_{2}$ generation, but did not prevent cell death. Tetrandrine did not cause generation of nitric oxide. Verapamil also reduced the viability of mouse peritoneal macrophages and J774 cells, but did not cause $\mathrm{PGE}_{2}$ overproduction, except at $10^{-4} \mathrm{M}$ in mouse peritoneal macrophages. In macrophages cultured with lipopolysaccharide and interferon- $\gamma$ to induce the generation of large amounts of both $\mathrm{PGE}_{2}$ and nitric oxide, tetrandrine reduced mediator release and their forming enzymes (cyclooxygenase- 2 and inducible nitric oxide synthase), secondary to cytotoxicity.

Tetrandrine interacted with slowly inactivating calcium channels by completely blocking $d$-cis$\left[{ }^{3} \mathrm{H}\right]$ diltiazem binding, partially inhibiting $( \pm)-\left[{ }^{3} \mathrm{H}\right] \mathrm{D}-$ 600 binding, and markedly stimulating $\left[{ }^{3} \mathrm{H}\right]$ nitrendipine binding in porcine cardiac sarcolemmal membrane vesicles (KING et al. 1988). Tetrandrine reversibly blocked inward $\mathrm{Ca}^{2+}$ currents through L-type $\mathrm{Ca}^{2+}$ channels in $\mathrm{GH}_{3}$ anterior pituitary cells grown in a humidified atmosphere containing $5 \% \mathrm{CO}_{2}$.

Tetrandrine was a potent inhibitor in vitro of zymosan-stimulated oxygen consumption, superoxide anion release, and hydrogen peroxide secretion by rat alveolar macrophages (CASTRANOVA et al. 1991). Tetrandrine (33 $\mu \mathrm{g} / \mathrm{kg} \times$ day) was also effective in vivo in preventing activation of alveolar macrophages after inhalation $\left(117.5 \pm 12.5 \mathrm{mg} / \mathrm{m}^{3}\right.$ of $1.54 \pm 0.01 \mu \mathrm{m}$ mass median diameter for $6 \mathrm{~h}$ per day) or intratracheal instillation ( $40 \mathrm{mg}$ in $0.3 \mathrm{ml}$ of saline, specific surface $3.97 \mathrm{~m}^{2} / \mathrm{g}$ ) silica. Tetrandrine also inhibited stimulant-induced chemiluminescence by polymorphonuclear leucocytes. 
Lipid peroxidation of liposomes induced by $\mathrm{Fe}^{2+}$ was inhibited by tetrandrine at $88 \%$, while cepharanthine, another biscoclaurine head-to-head diether, inhibited lipid peroxidation at $97 \%$ (SHIRAISHI et al. 1980).

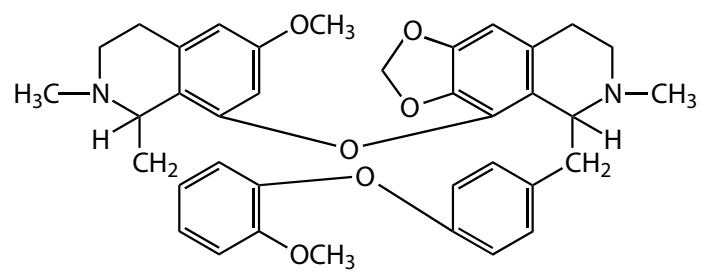

Cepharanthine

In alveolar rat macrophages (NR8383), tetrandrine inhibited the activation of $\mathrm{NF}-\varkappa \mathrm{B}$ and NF- $\varkappa \mathrm{B}-$ dependent reporter gene expression by lipopolysaccharide, 12-O-tetradecanoylphorbol-13-acetate and silica in a dose dependent manner (CHEN et al. 1997).

\subsection{4}

\section{Tobacco Smoke}

By using the electron spin resonance (ESR) spin trapping technique with the $N$-methyl-D-glucamine dithiocarbamate-Fe(II) complex, the generation of "NO was observed in NO spin trapping solution bubbled with the filtered mainstream of cigarette smoke (Токімото and SHinagawa 2002). The ESR signal, with a three-line spectrum characteristic of a 'NO radical, which was not observed immediately after bubbling of smoke, started rapidly increasing with time up to around $25 \mathrm{~min}$ after the addition of $\mathrm{Fe}^{2+}$, and the slowly approached a peak value dependent on the burned cigarette mass and on the smoking speed. The production of "NO was, however, much affected by air oxidation and enhanced by the addition of ascorbic acid. A certain concentration of $\mathrm{NaNO}_{2}$ solution, in which $\mathrm{NO}_{2}^{-}$is assumed as the main origin of the ${ }^{\circ} \mathrm{NO}$ mimicked closely the time course of "NO generation resulting from the smoke of one cigarette. The cigarette smoke was passed through alkaline pyrogallol solution as a deoxidiser; however, it exhibited an unchanged intensity of ${ }^{\circ} \mathrm{NO}$ signal throughout the measurement.

Cigarette smoke serves as a relevant model of alveolar macrophage toxicology, since it represents one of the most common and universal insults to lung defences, and its effects on human alveolar macrophages have been extensively studied. In response of chronic cigarette exposure the macrophage population increases in number and individual cell size (DAvis et al. 1980), and multiple changes occur in microbicidal function, substrate transport (Low and BulmaN (1977), protein biosynthesis (Low 1974), and immunologic responsiveness, as reviewed by MARTIN and WARR (1977). The absolute increase in CD11/CD18-positive alveolar macrophages found by SCHABERG et al. (1995) was mainly due to the increased absolute number of positive alveolar macrophages with high density from fractions $3(>1.40$ to $\leq 1.050 \mathrm{~g} / \mathrm{ml})$ and 4 $(>1.050$ to $\leq 1.070 \mathrm{~g} / \mathrm{ml})$ compared with nonsmokers $(P<0.05$ to $<0.01)$. These high-density alveolar macrophages of smokers are mainly responsible for the spontaneous $\mathrm{O}_{2}{ }^{\circ-}$ production of these cells.

Zwilling (1981) proposed the following sequence of events underlying the formation of emphysematous lesions:

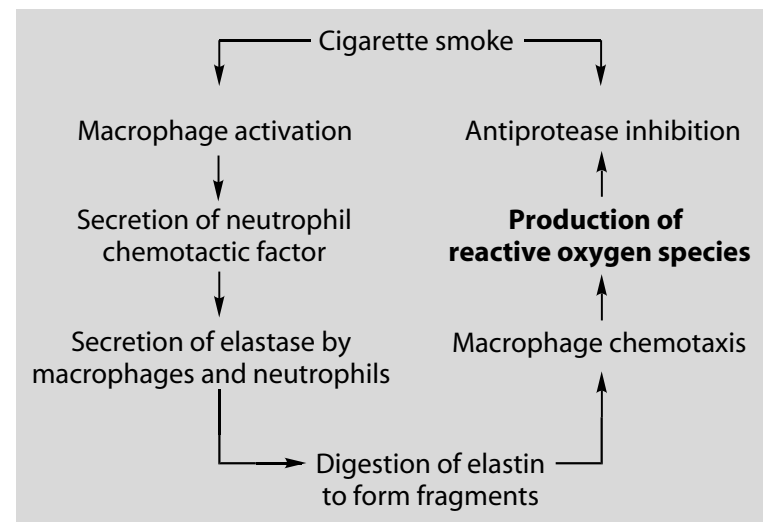

Lavaged pulmonary alveolar macrophages obtained from chronic cigarette smokers contain various quantities of brownish-yellow pigment (WARR and MARTIN 1978). In the presence of serum, alveolar macrophages from smokers cultured on glass in TCM 199 were significantly larger in area than those from non-smokers.

Reiter (1983) identified sudanophilic chromolipids in paraffin sections of cigarette smokers' alveolar macrophages, even 11 months after burial. Electron micrographs of human transbronchial lung biopsies showed typical heterogeneous osmiophilic intralysosomal smokers' inclusions (SÉBAsTIEN et al. 1994). Only the five heavy smokers out of 15 patients exhibited similar inclusions on their interstitial macrophages.

Glycosidase ( $N$-acetyl- $\beta$-D-glucosaminidase, $N$ acetyl- $\beta$-D-galactosaminidase, $\beta$-D-galactosidase, $\alpha$-L-galactosidase, $\alpha$-D-mannosidase, $\alpha$-L-fucosidase and $\beta$-D-glucuronidase) activities were higher in the alveolar macrophages obtained by bronchoalveolar lavage from smokers than in those from non-smokers (SCHARFMAN et al. 1980).

In the alveolar macrophages of rats exposed to tobacco smoke for 4 hours per day for two weeks, 
5 days each week, and for 6 weeks, 4 days each week, respectively, JEFFERY and REID (1981) found macrophages containing "tar bodies' showing that smoke did reach this distal site.

Bronchoalveolar lavage fluid recovered from upper lobes of smokers contained higher concentrations of iron $(P<0.01)$ and ferritin $(P<0.006)$ and lower concentrations of transferrin $(P<0.003)$ compared with the lower lobes (Nelson et al. 1996). In contrast, bronchoalveolar lavage fluid recovered from upper and lower lobes of nonsmokers contained much lower concentrations of iron and ferritin, and concentrations were similar in both sites.

Production of oxygen radical species was significantly $(P<0.01)$ elevated in alveolar macrophages from current smokers compared with those from non-smokers in the presence $\left(29.8 \pm 15,5 \mu \mathrm{M} / 10^{6}\right.$ cells versus $13.7 \pm 8,2 \mu \mathrm{M} / 10^{6}$ cells) and absence $\left(3.8 \pm 1.6 \mu \mathrm{M} / 10^{6}\right.$ cells versus $2.2 \pm 1.2 \mu \mathrm{M} / 10^{6}$ cells) of $1 \mathrm{ng}$ phorbol myristate acetate/ml (Kondo et al. 1994). Decreases in antioxidant activities were observed in cells from smokers versus those from non-smokers for $\mathrm{Cu}, \mathrm{Zn}$-superoxide dismutase $(114 \pm 41$ versus $210 \pm 73$ units/mg protein, respectively, $P<0.01)$, glutathione S-transferase $(0.217 \pm 0.091$ versus $0.368 \pm 0.017$ units $/ \mathrm{mg}$ protein, $P<0.01)$, and glutathione peroxidase $(0.736 \pm 0.779$ versus $1.590 \pm 0.879$ units/mg protein, $P<0.05)$. Immunologic estimation showed a decrease in the levels of $\mathrm{Cu}, \mathrm{Zn}$-superoxide dismutase in cells from smokers $(104.3 \pm 46.6$ versus $184.1 \pm 64.4 \mathrm{ng}$ enzyme/mg protein, respectively, $P<0.01$ ). Northern blot analysis of $\mathrm{Cu}, \mathrm{Zn}$-superoxide dismutase mRNA showed no apparent difference between the two groups, suggesting not the inactivation of this enzyme but a reduction in the translational step or increased proteolysis.

J774A.1 macrophage cells incubated with $250 \mu \mathrm{g} /$ $\mathrm{ml}$ of an aqueous smokeless tobacco extract released lactate dehydrogenase into the media as an indicator of cellular membrane damage. The amount released was both concentration- and time dependent (BAGCHI et al. 1995). Superoxide dismutase, catalase, mannitol and allopurinol by themselves had no significant effect, while a combination of the four free radical scavengers resulted in a $59 \%$ decrease in the release of lactate dehydrogenase.

Arachidonic acid metabolism: In vitro, a significant decrease in prostaglandin $\mathrm{E}_{2}$ and thromboxane $\mathrm{B}_{2}$ synthesis but not in prostaglandin $\mathrm{F}_{2 \alpha}$ synthesis by the smoker pulmonary alveolar macrophages compared with non-smoker PAM was observed by LAVIOLETTE et al. (1981).

\subsection{5 \\ Trifluoperazine}

Trifluoperazine, 10-[3-(4-methyl-1-piperazinyl)propyl]-2-trifluoromethylthiophenazine, a drug that binds to $\mathrm{Ca}^{2+}$-calmodulin and inhibits its interaction with other proteins, reversibly inhibited growth and phagocytosis of polyvinyltoluene latex beads $(2.97 \mu \mathrm{m}$ diameter $)$ in a macrophagelike cell line, J774.16 (Horwitz et al. 1981).

\subsection{6 \\ Wood Smoke Particles}

RAW 264.7 mouse macrophages exposed to wood smoke from western bark thermolysis showed significant DNA damage, which was intensified by the addition of $\mathrm{H}_{2} \mathrm{O}_{2}$ (LEONARD et al. 2000). Both sodium formate, an $\mathrm{HO}^{\bullet}$ radical scavenger, and deferoxamine, a metal chelator, inhibited DNA damage caused by wood smoke plus $\mathrm{H}_{2} \mathrm{O}_{2}$ and completely blocked DNA damage from wood smoke in the absence of $\mathrm{H}_{2} \mathrm{O}_{2}$. Elemental analysis performed on the filter samples presented $\mathrm{Fe}$ as the only transition metal present in measurable amounts.

\subsection{7 \\ Wool and Grain Dusts}

Wool and grain (wheat and barley) dusts stimulated TNF secretion by rat alveolar macrophages in vitro in a dose-dependent manner as measured in supernatants with the mouse fibroblast cell line L929 bioassay (BRown and Donaldson 1996).

\section{7 Transformation of Macrophages to Epithelioid Cells}

Macrophages turn into epithelioid cells when they become immobilised at the site of inflammation without being called upon to undertake phagocytosis or when phagocytosis or pinocytosis leads to complete elimination of the particle within a few days. On the other hand, uptake of indigestible, non-excretable material prevents macrophages from becoming epithelioid cells (PAPADIMITRIOU and SPECTOR 1971).

\subsection{1 \\ Epithelioid Cells in Resorption Granulomas}

The mature epithelioid cell has a large nucleus fairly regular in outline with finely marginated nuclear chromatin (Fig. 167). The presence of one or more large, spherical, reticulated nucleoli is typical. 
In type A epithelioid cells both smooth- and roughsurfaced endoplasmic reticulum was predominant (Fig. 165). The cytoplasm of type B epithelioid cells

Fig. 167. Type A epithelioid cells with much rough surfaced endoplamic reticulum and type B epithelioid cells showing vesicles of different sizes from a Marbagelan ${ }^{\circledR}$-induced ( 7 days) resorption granuloma (block 171) of a male SpragueDawley rat (No.10). Animal treated for 14 days with intragastric application of $15 \mathrm{mg}$ carbocromene per $\mathrm{kg}$ body weight $\times$ day. Perfused under pentobarbital anaesthesia (30 $\mathrm{mg} / \mathrm{kg}$ ) from the abdominal aorta with $2.5 \%$ glutaraldehyde in $0.1 \mathrm{M}$ sodium cacodylate buffer ( $\mathrm{pH}$ 7.4). Postfixation with $1 \%$ osmium tetroxide in cacodylate buffer. Embedded in Epon 812 and sectioned at $50 \mathrm{~nm}$. Lead citrate and uranyl acetate. Plate 2850 contained a well developed Golgi apparatus and a variety of vesicles (Figs. 167, 168).
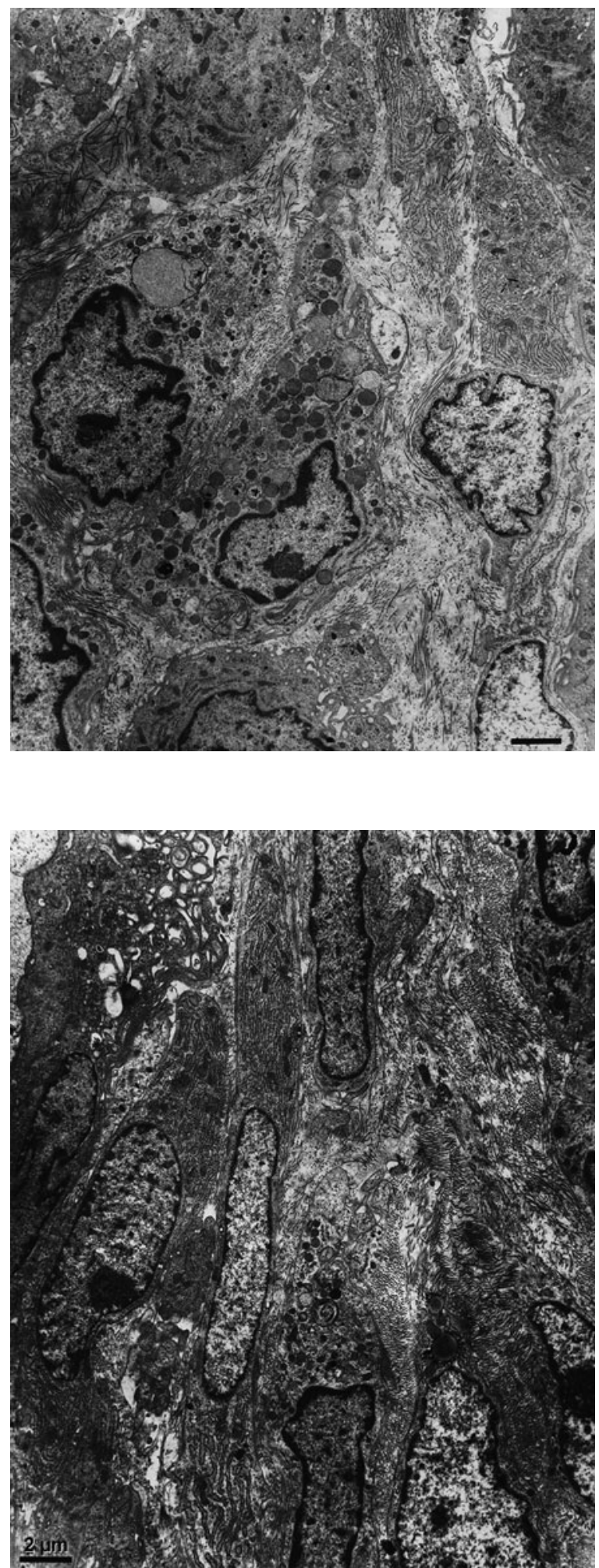

Fig. 168. Type A epithelioid cells with much rough surfaced endoplamic reticulum and type B epithelioid cells showing vesicles of different sizes from a Marbagelan ${ }^{\circledR}$-induced (7 days) resorption granuloma (block 171) of a male SpragueDawley rat (No.10). Animal treated for 14 days with intragastric application of $15 \mathrm{mg}$ carbocromene per $\mathrm{kg}$ body weight $\times$ day. Perfused under pentobarbital anaesthesia $(30$ $\mathrm{mg} / \mathrm{kg}$ ) from the abdominal aorta with $2.5 \%$ glutaraldehyde in $0.1 \mathrm{M}$ sodium cacodylate buffer ( $\mathrm{pH}$ 7.4). Postfixation with $1 \%$ osmium tetroxide in cacodylate buffer. Embedded in Epon 812 and sectioned at $50 \mathrm{~nm}$. Lead citrate and uranyl acetate. Plate 2845 


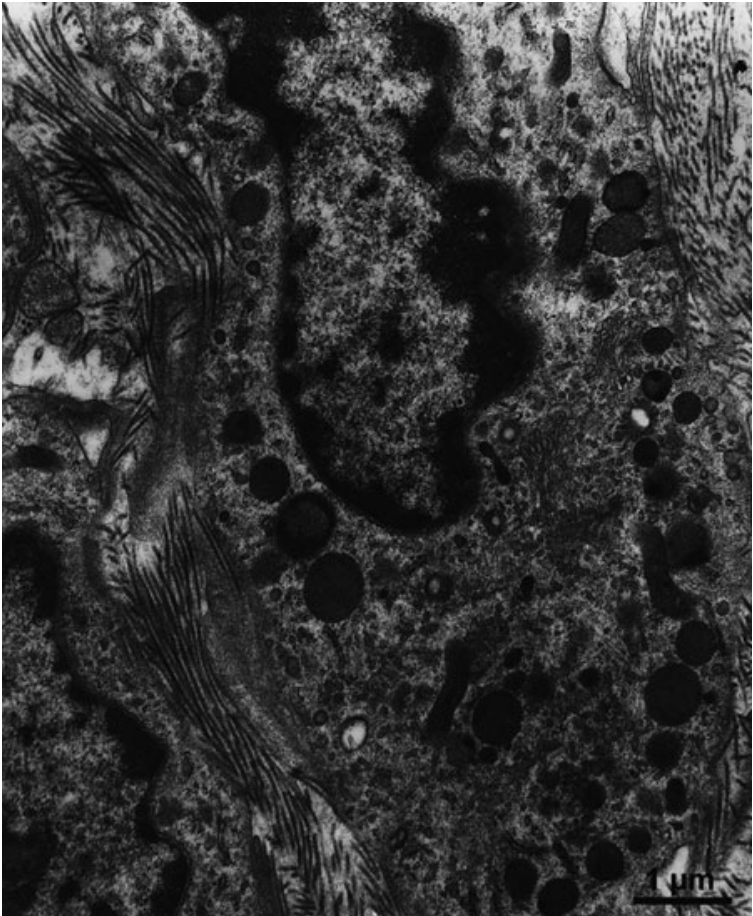

Fig. 169. Type B epithelioid cell showing vesicles of different sizes, few mitochondria and indistinct plasmalemma from a Marbagelan ${ }^{\circledR}$-induced (7 days) resorption granuloma (block 171) of a male Sprague-Dawley rat (No.10). Animal medicated for 14 days with intragastric application of $15 \mathrm{mg}$ carbocromene per kg body weight $\times$ day. Perfused under pentobarbital anaesthesia $(30 \mathrm{mg} / \mathrm{kg})$ from the abdominal aorta with $2.5 \%$ glutaraldehyde in $0.1 \mathrm{M}$ sodium cacodylate buffer (pH 7.4). Postfixation with $1 \%$ osmium tetroxide in cacodylate buffer. Embedded in Epon 812 and sectioned at $50 \mathrm{~nm}$. Lead citrate and uranyl acetate. Plate 2849

\section{8 \\ Stimulation, Priming and Activation of Macrophages}

The word stimulus can be used as a noun, as in inflammatory stimulus, but in this context not in the adjectival form, as in stimulated macrophages (VAN FURTH 1989). The term stimulated macrophages is imprecise, because stimulation means that a stimulus has been applied that may result in elicitation and/or activation of cells.

The term "immunomodulators" has been employed to define agents or approaches that will modify the relationship between tumour and host by modifying a host's biologic response to tumour cells, with an enhanced therapeutic benefit. The cascade of the cellular immune system is initiated by the uptake and processing of antigen by the macrophage.

Several agents which increase macrophage and lymphocyte tumour cell cytotoxicity appear to do so by interferon production. Poly I:C and maleic

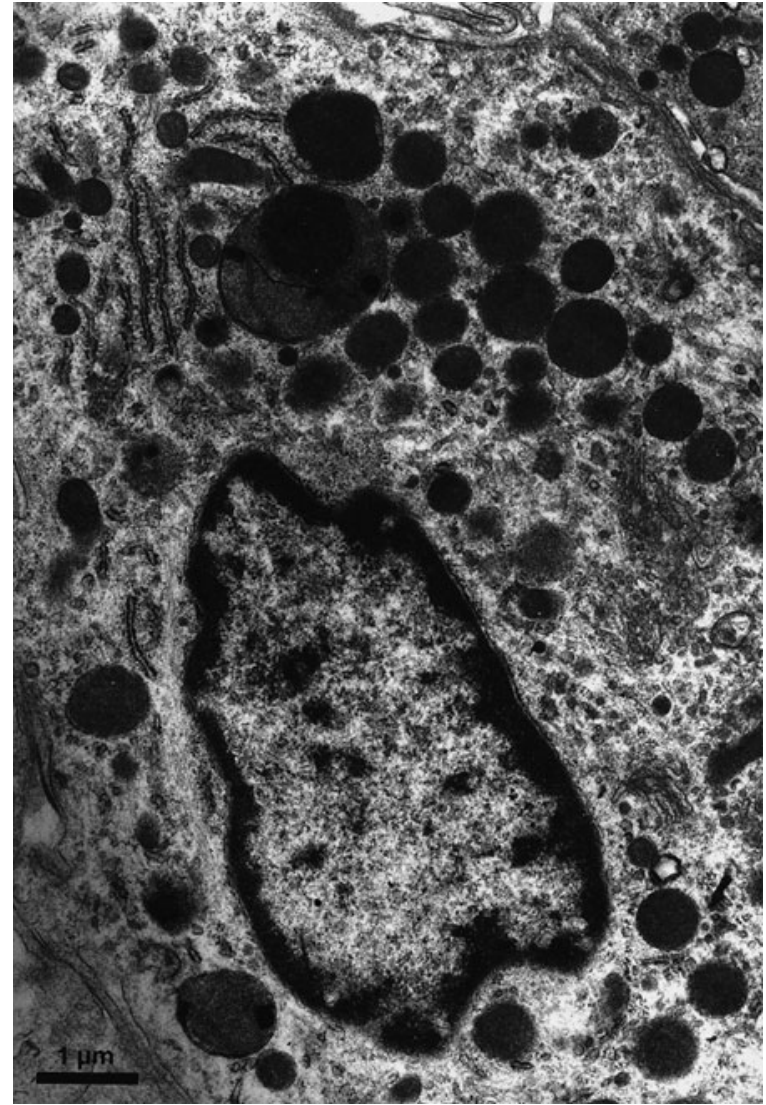

Fig. 170. Prominent arrangement of intermediate filaments around the cell nucleus and large autophagosome in a type B epithelioid cell from a Marbagelan ${ }^{\circledR}$-induced (7 days) resorption granuloma (block 171) of a male Sprague-Dawley rat (No.10). Animal medicated for 14 days with intragastric application of $15 \mathrm{mg}$ carbocromene per $\mathrm{kg}$ body weight $\times$ day. Perfused under pentobarbital anaesthesia $(30 \mathrm{mg} / \mathrm{kg})$ from the abdominal aorta with $2.5 \%$ glutaraldehyde in $0.1 \mathrm{M}$ sodium cacodylate buffer ( $\mathrm{pH} 7.4$ ). Postfixation with $1 \%$ osmium tetroxide in cacodylate buffer. Embedded in Epon 812 and sectioned at $50 \mathrm{~nm}$. Lead citrate and uranyl acetate. Plate 2848

anhydride divenyl ether copolymer incubated in vitro with resting macrophages and tumour cells caused macrophage killing of the tumour cells. Adding a specific antibody neutralising interferon will abandon tumoricidy.

\subsection{1 \\ Non-immunological Stimulation}

Di Luzio (1976) established the criteria for an agent which would function as a macrophage stimulant as follows:

1. Non-viable entity

2. Defined chemical structure

3. Non-toxic at employed dose

4. Non-antigenic 
5. Activate all phases of macrophage mediated events
a. Chemotaxis
b. Phagocytosis
c. Intracellular killing
d. Extracellular killing
e. Expression of humoral and cell mediated im- munity
f. Secretory aspects

6. Induced macrophage response, i.e., hyperfunction-hyperplasia and hypertrophy of RES - must be reversible in nature

7. Readily available and relatively inexpensive.

\subsubsection{1}

\section{Immunostimulators}

The term "immunomodulators" has been applied to define agents or approaches that will modify the relationship between tumour and host by modifying a host biologic response to tumour cells, with an enhanced therapeutic benefit. Several terms have appeared in the literature describing the action of these agents: immuno-augmentators, immunostimulators, immunoenhancers, immunoregulators, immunomodifiers, and immunorestorators. As most agents produce a direct or indirect effect on a cellular component of the immune system, the term "biologic response modifiers" includes the many agents and approaches that have a mechanism of action which involves the individual's biologic responses.

The macrophage appears to play a major role in initiating the cellular immune system with the production and secretion of soluble factors (monokines) which regulate various cellular components. These monokines, along with lymphokines (lymphocyte secreted cell regulators) and specific chemicals comprise the bulk of the list of biologic response modifiers.

\section{Hormones}

As reviewed by Yамамото (1985) and Веато (1989) steroid hormones influence gene expression at essentially all known levels of regulation.

In certain cases, steroid hormones were converted into corresponding radical intermediates in either enzymatic or non-enzymatic systems (KoDADMA et al. 1997). Although detection of the free radicals was limited to oestrogens, evidence suggests that glucocorticoids as well as androgens may also share the physiological formation of free radicals.

In general, the concentrations of hormones needed to inhibit peroxidation in vitro seem much larger than physiological levels (picomolar range). The phenoxyl radicals produced during antioxidant activity are reactive and capable of damaging proteins and DNA in vitro (LIEHR 1996).

\section{Glucocorticoids}

Phagocytosis of Staphylococcus aureus by macrophages elicited in rabbits by intrapleural application of Liebig's meat extract plus carboxymethylcellulose was $\leq 50 \%$ enhanced when the animals had been injected subcutaneously with $0.2 \mathrm{mg}$ cortisone acetate per kg body weight $\times$ day (CRABBÉ 1956).

On glucocorticoid stimulation, macrophages elaborate anti-phospholipase proteins such as macrocortin (Flower and BLACKWELL 1979) and lipomodulin (Hirata et al. 1980). Glucocorticoids reduced the mRNA levels of the rate-limiting enzyme in cholesterol synthesis, 3-hydroxy-3-methylglutaryl CoA reductase, in a mouse macrophage-like cell

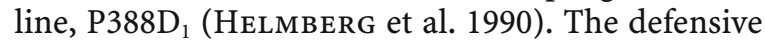
role of neutrophils and mononuclear phagocytes are impaired. Fauve and Pierce-Chase (1967), however, have suggested that some synthetic steroids can retain the beneficial anti-inflammatory activities without drastically compromising the defensive role of the macrophage.

While glucocorticoids dampen most immune responses, they stimulate polyclonal immunoglobulin production, e.g., by human peripheral blood lymphocytes (Goodwin and ATLURU 1986), increase the synthesis of IgE by IL-4-stimulated human lymphocytes (Wu et al. 1991) and induce IgA in serum (but not at mucosal surfaces) and secretory components in serum, saliva, and bile of rats (WIRA et al. 1990, Wira and Rossoll 1991).

\section{Oestrogens}

Female sex hormones may contribute to the gender-related differences in the immune response (Robert and Spitzer 1997). Nitric oxide production by rat alveolar macrophages cultured in the presence or in the absence of lipopolysaccharide was higher from female than from male rats, but without statistical significance. However, ovariectomy induced significant inhibition in spontaneous production of ${ }^{\circ} \mathrm{NO}$ by alveolar macrophages. In orichidectomized rats, the "NO response by alveolar macrophages to lipopolysaccharide stimulation relative to spontaneous ${ }^{\circ O}$ production was significantly downregulated.

Oestrogen is a potent initiator of multiplication, mobilisation and phagocytic activities of macrophages and the infiltration of the uterus is presum- 
ably a result of the oestrogen surge during oestrus (EUfinger 1932, Nicol and Vernon-Roberts 1965, Vernon-Roberts 1969).

Radicophilic moieties (Kitagawa et al. 1992, Cynshi et al. 1995) in oestrogens are important for efficient antioxidant and radical scavenging activities. Römer et al. (1998) designed and synthesised a novel series of $17 \beta$-œstradiol and its homologue $\Delta^{9(11)}$-dyhydro-17 $\beta$-œstradiol linked with aromatic moieties at the $\mathrm{C}-17$ position, with an aim to improve scavenging of lipid peroxyl and/or superoxide anion radicals ant to enhance $\mathrm{Fe}$ (II) chelation as well as stimulation of $\mathrm{Fe}(\mathrm{III})$ reduction. While the classical oestrogen, $17 \beta$-œstradiol as well as selected phenolic compounds only moderately inhibited iron-dependent lipid peroxidation and stimulated total antioxidant activity, $\Delta^{9(11)}$-dyhydro$17 \beta$-œstradiol and its $17 \alpha$-substituted analogues directly altered the iron redox chemistry and diminished superoxide anion formation in the xanthine xanthine oxidase system.

$17 \beta$-Oestradiol is a good inhibitor of lipid peroxidation in microsomal and pre-formed liposomal systems (WISEMAN 1995). The order of effectiveness in the microsomal system was 4hydroxytamoxifen $>17 \beta$-œstradiol $>$ tamoxifen $>$ ICI 164384 and in the liposomal system 4hydroxytamoxifen $>17 \beta$-œstradiol > ICI 164384 $>$ tamoxifen. However, tamoxifen also functions as a genotoxic carcinogen (HARD et al. 1993), a therapeutic regimen including melatonin plus tamoxifen may allow for a lower dose of the latter agent thereby reducing its collateral toxicity (REITER 1998). Metabolism of tamoxifen by rat liver microsomes gave equal amounts of the $R$ and $S$ enantiomers (Osborne et al. 2001). They have the same chemical properties but, on treatment of rat hepatocytes in culture, $R-(+)$ - $\alpha$-hydroxytamoxifen gave at least eight times as many DNA adducts as the $S-(-)$ isomer.

YEN et al. (2001) utilized the fluorescent dye 2',7'dichlorofluorescin diacetate to measure the generation of reactive oxygen species in human umbilical vein endothelial cells. $17 \beta$-Oestradiol $(54 \mu \mathrm{M})$ pretreatment for $18 \mathrm{~h}$ or direct co-incubation significantly suppressed both tert-butylhydroperoxideand oxidised LDL-induced stimulation of the generation of reactive oxygen species.

$17 \beta$-Oestradiol afforded protection against oxidative nerve cell apoptosis. $17 \beta$-Oestradiol is indeed a potent antioxidant comparable to other phenolic compound such as $\alpha$-tocopherol (BEHL 1999, Moosmann and BeHL 1999).

Increasing evidence indicates that the effector molecule crucial for œstrogen signalling is the short-lived messenger ${ }^{\circ} \mathrm{NO}$. The observation that estrogens not only increases expression of NO synthesizing enzymes (NOS; WEINER et al. 1994), but also triggers direct activation of eNOS in the first minutes after the addition to cells (CHEN et al. 1999, Chambliss et al. 2000, Haynes et al. 2000, Hisaмото et al. 2001), opens the possibility that part of the ${ }^{\circ} \mathrm{NO}$ effects on œestrogen biological actions are also mediated through regulation of the signal transduction cascades initiated by the hormone. Exposure of the MCF-7 human breast cancer cell line to $17 \beta$-œstradiol in the presence of ${ }^{\circ} \mathrm{NO}$ gave rise to activation of signalling events additional to those triggered by $17 \beta$-œstradiol alone, namely tyrosine phosphorylation of specific proteins, including the insulin receptor substrate-1, with recruitment to this adapter of the phosphatidylinositol 3'kinase and persistent activation of Akt (protein kinase B; FAlCONE et al. 2002). Active Akt, in turn, prevented $17 \beta$-œstradiol from activating $\mathrm{p} 42 / 44 \mathrm{ex}-$ tracellular signal-regulated kinases (ERK 1/2; Kelly and LeVin 2001).

Older references: Nicol and Helmy (1951), Abou-Zirky and Nicol (1952), Heller et al. (1957), NiCol and SNELl (1957), Bilbey and Nicol (1958), Nicol et al. (1964)

\section{Diethylstilbestrol}

It is widely believed that metabolic activation by peroxidases plays a role in the biological effects of diethylstilbestrol, a synthetic oestrogen which is also a tumour-inducing agent (Metzler 1987). When diethylstilbestrol is exposed to horseradish peroxidase $/ \mathrm{H}_{2} \mathrm{O}_{2}$ in the presence of the cationic surfactant hexadecyltrimethylammonium bromide, the rapid dioxygen consumption is accompanied by a burst of light emission (KNUDSon et al. 1992). Lipoxygenase (EC 1.13.11.12) catalyses the oxidation of diethylstilbestrol to its corresponding quinone to yield free radical species intermediates (diethylstilbestrol semiquinone and diethylstilbestrol quinone), which are associated with the adverse effects of this synthetic oestrogen (NúÑEZ-DELICADo et al. 1997). Due to the low degree of water solubility of diethylstilbestrol, the enzyme works in a range of diethylstilbestrol concentrations below $K_{m}$.

The E-diethylstilbestrol-4',4'-semiquinone oxygen-centred radical is converted to a carbon centred radical which reacts with $\mathrm{O}_{2}$ forming a dioxetane. Unless the dioxetane is rapidly protonated, it will almost instantly cleave into excited carbonyl products because of the intramolecular chemically initiated electron exchange luminescence. In the latter, electron transfer from the phenoxide moiety to the peroxide ring promotes the fluorescence observed from the singlet-excited 4-hydroxy propio- 
phenone product. If any triplet species are formed, they derive from the protonated dioxetane. The latter is expected to go fast intersystem crossing to the lowest triplet state, which is of the $\pi, \pi^{*}$ type.
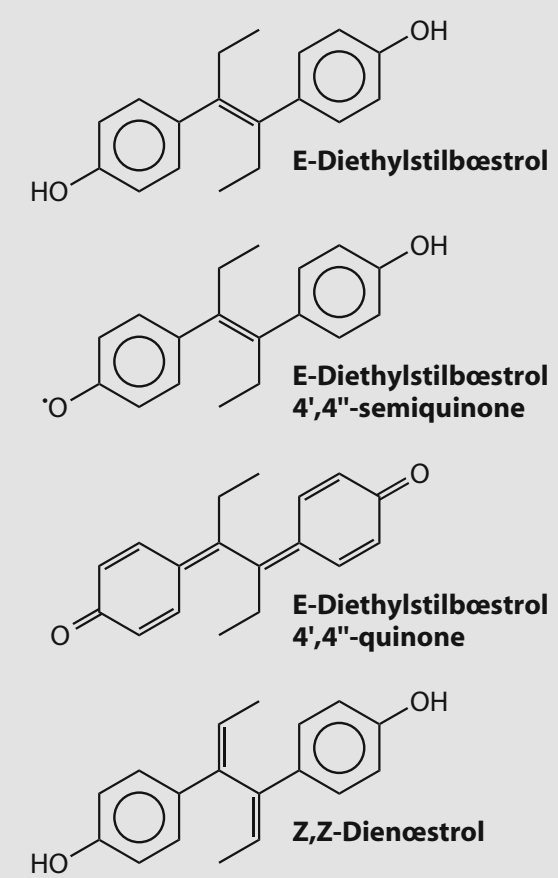

Diethylstilbœstrol biotransformation

When diethylstilbœstrol was administered to female and male CD-1 mice as four subcutaneous injections for 1 week at $0,5,15$, and $30 \mu \mathrm{g} / \mathrm{kg}$ body weight doses, female thymus glands were significantly larger than their male counterparts (CALEMINE et al. 2002). Short-term administration of diethylstilbœstrol to female of male mice neither induced thymic atrophy nor altered the relative percentages of thymic subsets. Nevertheless, diethylstilbœstrol treatment of female of male mice induced a dose-related apoptosis of $\mathrm{CD}^{+} 8^{+}, \mathrm{CD}^{+} 8^{-}$and $\mathrm{CD} 4^{-} 8^{+}$subsets as analysed by 7 -amino-actinomycin. Immature $\mathrm{CD} 4^{-} 8^{-}$subsets of thymocytes from females was also affected by high dose diethylstilbœstrol. The pattern of mitogen-induced proliferation of splenic lymphocytes varied with the dose of hormone and the gender. In females, splenic lymphocytes from low dose diethylstilbœstrol (5 $\mu \mathrm{g} / \mathrm{kg}$ body weight)-treated mice exhibited an increased proliferative response to Con-A, lipopolysaccharide or PMA/ionomycin compared with controls.

Experimental dose dependent palatoschisis due to diethylstilbœstrol treatment of pregnant Swiss mice has been reported by GABRIEL-RoBEz et al. (1972).

Further references: Nicol and Helmy (1951), Nicol et al. (1958, 1960, 1961, 1964), NicOL and Ware (1960), Trejo et al. (1972), Steven and SNook (1975), Boorman et al. (1980), DeAn et al. $(1980,1986)$

trans-Resveratrol (3,5,4'-trihydroxy-trans-stilbene) is a naturally occurring stilbene found in grapes and certain medicinal plants, where it is believed to provide protection from fungal infections and other stresses (SCHWEKENDIEK et al. 1992, SoLEAs et al. 1997). It has been reported to have a diverse range of pharmacological properties, including antiinflammatory, antiplatelet (PACE-AsCIAC et al. 1995), and antioxidant activities (BELGUENDOUZ et al. 1997, Chanvitayapongs et al. 1997).

\section{Progesterone}

In the male guinea pig, progesterone ( $3 \mathrm{mg}$ per day for 3 weeks) had little or no effect on the phagocytic activity of reticulo-endothelial system (NICOL and SNELL 1957).

Progesterone at $10^{-4} \mathrm{M}$ inhibited basal and in vitro lipopolysaccharide-stimulated nitric oxide generation by alveolar macrophages of both saline and lipopolysaccharide-treated male rats (RoBERT and SPITZER 1997).

\section{Lipids}

Human monocytes cultured with a mixture of serum lipids and lipoproteins (cardiolipids) changed morphologically after overnight culture (CHU et al. 1999). Most of them detached from the bottom of the plate and assumed a rounded morphology. IL$1 \beta$, IL-6, platelet-derived growth factor, and transforming growth factor $\beta 1$ all exhibited a significant reduction $(P<0.05)$ in lipid-treated cultures compared to control cultures.

\section{Glyceryl trioleate}

Peritoneal macrophages of male mice $8 \mathrm{~h}$ to $14 \mathrm{~d}$ after intraperitoneal injection of $10 \mathrm{mg}$ glyceryl trioleate emulsified in Hanks' solution had longer processes, more acid phosphatase and more lysosomes than controls (CARR 1967).

Further references: Cooper and STUART (1961, 1962), Cooper and West (1962), Stuart and Cooper (1962), Stuart and Davidson (1964), Carr (1966), Mouton et al. (1975)

\section{Polyunsaturated Fatty Acid (PUFAs)}

Six all cis polyunsaturated fatty acids (PUFAs) in HL-60 cells induced apoptosis in correlation with 
the number of double bonds (HAw KINs et al. 1998). Cell death was preceded by a progressively increasing lipid peroxidation. PUFA-induced apoptosis was oxidative, being blocked by both vitamin E acetate $(5$ or $50 \mu \mathrm{M})$ and sodium selenite $(10$ or 100 $\mathrm{nM})$, the latter in a critically time-dependent manner, as the cytotoxic effect of cis-parinaric acid $\left(\Delta^{9,11,13,15}\right.$, cis, trans, trans, cis; $\left.5 \mu \mathrm{M}\right)$ on Mia-Pa-Ca2 cells was prevented only when the selenite was added simultaneously or within $2 \mathrm{~h}$. In combination, addition of PUFA and $\gamma$-irradiation induced a significantly greater cell kill than either agent alone.

\section{Lipoarabanomannan}

The mycobacterial cell wall glycolipid lipoarabanomannan (LAM) is capable of regulating several macrophage functions. AraLAM, lacking terminal mannosyl units and derived from nonpathogenic organisms, is a potent inducer of proinflammatory cytokines and chemokines. Terminally-mannosylated LAM (ManLAM)from virulent M.hominis, only weakly induces these proteins. BERNARDO et al. (1996) studies LAM-induced cell migration, intracellular $\mathrm{Ca}^{2+}$-fluxes and surface receptor expression and function in fresh human monocytes and in 48-h monocyte-derived macrophages. Using modified Boyden chambers, they found that AraLAM but not ManLAM (0.01-10 $\mu \mathrm{g} / \mathrm{ml})$ was chemotactic for both monocytes and macrophages. Chemotaxis was blocked by anti-CD14 mAb or by the lipopolysaccharide antagonist Rhodobacter sphaeroides lipid A. ManLAM ' but not AraLAM, induced a transient rise in cytosolic $\mathrm{Ca}^{2+}$ in a subpopulation of macrophages, but not in monocytes.

\section{Lipopolysaccharides}

Lipopolysaccharide initiates a variety of events which Adams and Hamilton (1987, 1989), HaMILTON and ADAMs (1987) and ADAMs et al. (1988) collectively have termed cascades II and III. Included in cascade II are enhanced levels of massage for the protooncogenes c-fos and c-myc (which can be attributed to enhances transcription of these genes), and enhanced mRNA for the competence gene JE (which can be attributed to accumulation or stabilisation of massage). Many of the events in cascade II can be mimicked by platelet activating factor (PAF), a potent bioactive ether lipid for which a receptor has been demonstrated on macrophages (PrPIC et al. 1988). Cascade III is typified by synthesis of a distinct set of new polypeptides as well as transcription of the competence gene KC.
Human alveolar macrophages stimulated with lipopolysaccharide $(1 \mu \mathrm{g} / \mathrm{ml})$ activated extracellular regulated kinase/mitogen-activated protein kinase, in addition to JUN amino-terminal kinase (JNK) and p38, and showed translocation of nuclear factor xB (Mondal et al. 2000). In contrast to alveolar macrophages, monocyte adhesion or exposure to residual oil fly ash particles $\left(10 \mu \mathrm{g} / 2 \times 10^{5}\right.$ cells $)$ in suspension rapidly activated p38, JNK, and extracellular regulated kinase/mitogen-activated protein kinase, and activated nuclear factor $\chi \mathrm{B}$ binding as well as interleukin-8 mRNA expression.

Lipopolysaccharide inhibited the expression of the scavenger receptor Cla-1 in human monocytes and macrophages (Buechler et al. 1999). Downregulation of Cla-1 mRNA by LPS is likely due to a modification and destabilisation of the mRNA.

Lipopolysaccharides caused a time- and dosedependent superoxide release in nonadherent purified human blood monocytes (LANDMANN et al. 1995). The effect appeared after $5 \mathrm{~min}$, peaked at 30 min, and disappeared after $2 \mathrm{~h}$. It was maximal with $10 \mathrm{ng} / \mathrm{ml}$ lipid A $(+148 \pm 22 \%, P<0.001), 1 \mathrm{ng} / \mathrm{ml}$ LPS Escherichia coli Re $(+226 \pm 68 \%, P<0.001)$ and $100 \mathrm{ng} / \mathrm{ml}$ LPS Salmonella abortus equi sm $(+272 \pm 52 \%, P<0.001)$, respectively. Nitric oxide synthase inhibitors, $0.1 \mathrm{mM} \mathrm{N}$-monomethyl arginine or $20 \mathrm{mM}$ spermine- $\mathrm{HCl}$ lowered the enhanced levels of superoxide released by LPS treated rat peritoneal macrophages (JoE and LoKESH 1997). The superoxide dismutase activity in LPS treated macrophages was $51 \%$ lower than that observed in resident cells. NO synthase inhibitors prevented the loss of SOD activity in LPS treated cells. Exogenously added SOD during sensitisation of cells with LPS also inactivated the enzyme. This inactivation of SOD is inhibited by NO synthase inhibitors. While human alveolar macrophages incubated for $18 \mathrm{~h}$ in media supplemented with $50 \mu \mathrm{g}, 100 \mu \mathrm{g}$ or $250 \mu \mathrm{g} \mathrm{Fe}_{2} \mathrm{O}_{3}$ or $\mathrm{TiO}_{2}$ dust $/ \mathrm{ml}$ did not increase release of LDH, TNF$\alpha$, or IL- $1 \beta$, supplementation by $0.1 \mu \mathrm{g}$ lipopolysaccharide $/ \mathrm{ml}$ induced alveolar macrophage release of both cytokines with decreased release of IL-1 $\beta$ by alveolar macrophages from smokers compared with non-smokers (BING and Wesselius 1996). Both murine peritoneal exudate cells and a murine macrophage cell line, RAW 264.7 produced surface and secreted TNF- $\alpha$ in response to lipopolysaccharide in a dose-dependent manner (CHAUdHRi 1997). However, much lower concentrations of LPS (100 ng/ml) were needed for optimal expression of surface TNF$\alpha$ than for secreted TNF- $\alpha(1 \mu \mathrm{g} / \mathrm{ml})$. Furthermore, concentrations of actinomycin $\mathrm{D}$ that inhibit the synthesis of new mRNA and the production of secreted TNF- $\alpha$ did not block the expression of surface TNF- $\alpha$ on LPS-stimulated cells. 
Lipopolysaccharide priming amplifies lung macrophage tumour necrosis factor production in response to air particles (IMRICH et al. 1999).

Activation of nuclear factor- $x \mathbf{B}$ is necessary for the induction of both TNF- $\alpha$ and Mn superoxide dismutase mRNAs by LPS (White et al. 2000). Neither hypoxia $\left(1 \% \mathrm{O}_{2}, 5 \% \mathrm{CO}_{2}\right.$, and $\left.94 \% \mathrm{~N}_{2}\right)$ nor diphenylene iodonium $(0.5$ to $5 \mu \mathrm{M})$ had any effect on LPS activation of nuclear factor- $x \mathrm{~B}$ in human monocytes (White and Tsan 2001). Treatment of human alveolar macrophages with LPS, polymericand secretory-IgA, but not 12-O-tetradecanoylphorbol-13-acetate, induced NF- $x \mathrm{~B}$ activation through $\mathrm{I} x \mathrm{~B} \alpha$ phosphorylation and subsequent proteolysis (OUADRHIRI et al. 2002).

While low levels of IL-6 were detected in medium from uninduced human blood monocytes cultured in RPMI1640 supplemented with $2 \mathrm{mM}$ glutamine, $50 \mathrm{mg} / \mathrm{ml}$ gentamicin and $10 \%$ heat-inactivated foetal calf serum, incubation of these cells with LPS and IFN- $\gamma$ led to a very high IL-6 protein secretion (Costanzo et al. 1999).

The intracellular oxidation of fluorescent probes dichlorofluorescin, dihydrorhodamine, and hydroethidine by hamster alveolar macrophages in response to lipopolysaccharide did not shoe any significant increase in intracellular reactive oxygen species (IMRICH et al. 1999).

In gene transfection studies performed with cytomegalovirus-luciferase as a reporter plasmid and cationic liposome as a transfecting agent, the presence of endotoxin in plasmid DNA preparations severely limited transgene expression in macrophages but had little or no effect in other cell types tested (Doкka et al. 2000). This decreased transfection was dependent on ROS-mediated cellular toxicity induced by endotoxin. Neutralising the endotoxin by the addition of polymyxin B effectively increased transfection efficiency and reduced toxicity. Electron spin resonance studies confirmed the formation of reactive oxygen species in endotoxin-treated cells and their inhibition by free radical scavengers The ROS scavenger $\mathrm{N}$-tert-butyl$\alpha$-phenylnitrone, the $\mathrm{H}_{2} \mathrm{O}_{2}$ scavenger catalase (EC 1.11.1.6), and the $\mathrm{HO}^{\bullet}$ scavenger sodium formate effectively inhibited endotoxin-induced effects, whereas the $\mathrm{O}_{2}{ }^{--}$scavenger superoxide dismutase (EC 1.15.1.1) had lesser effects.

The initial events of the cellular response to LPS have now been characterised; endotoxin responses are initiated by the binding of complexes of LPS and LPS-binding protein to CD14, which is a phosphatidylinositol-linked surface receptor present mainly in myelomonocytic cells (SCHUMAN et al. 1990, Wright et al. 1990, Ziegler-Heitbrock et al. 1994). Subsequently, activation of these cells oc- curs, leading to the release of biologically active products. This system is very sensitive, and triggered by picogram levels of LPS corresponding to concentrations that induce endotoxin shock. The levels of rat CD14 mRNA expression in resident peritoneal macrophages, alveolar macrophages, and peripheral blood monocytes are constitutively high, whereas that in Kupffer cells is low (TAKaI et al. 1997). IL-1 $\beta$ and iNOS mRNA expressions in blood monocytes, and both peritoneal and alveolar macrophages after stimulation with LPS in vivo and in vitro are high, whereas those in Kupffer cells are low. Scavenger receptors may regulate nitric oxide production from mouse peritoneal macrophages stimulated by LPS (MATsuno et al. 1997).

Sensitivity to LPS and monophosphoryl lipid A can be enhanced by concurrently loading mice with D-galactosamine (Elliot et al. 1991). Significant diurnal variation in susceptibility to lethal toxicity was observed in D-galactosamine loaded in mice upon LPS or monophosphoryl lipid A immunostimulant challenge. In mice treated with either monophosphoryl lipid A or monophosphoryl lipid A plus D-galactosamine, at the time of greatest toxic sensitivity, serum TNF levels were significantly higher than was seen in mice treated at the time of low sensitivity. Peritoneal exudates cells harvested from mice treated with either D-galactosamine or monophosphoryl lipid A displayed enhanced in vitro superoxide $\left(\mathrm{O}_{2}{ }^{--}\right)$production. Simultaneous treatment with $\mathrm{D}$-galactosamine and monophosphoryl lipid A led to a synergistic enhancement of $\mathrm{O}_{2}{ }^{--}$production above that induced by either xenobiotic alone. Pre-treatment with superoxide dismutase mimic $\mathrm{Cu}(\mathrm{II})$ (diisopropylsalicylate) ${ }_{2}$ significantly protected mice from the lethal toxicity of $\mathrm{D}$ galactosamine-monophosphoryl lipid A challenge.

Twenty hours after LPS and IFN- $\gamma$ stimulation, alveolar macrophages recovered by bronchoalveolar lavage from 3-day-old specific pathogen-free, virusfree rats produced $>2$ times more $\mathrm{NO}_{2}^{-}$and $\mathrm{NO}_{3}{ }^{-}$ than did macrophages more mature animals (SHERMAN et al. 1996). Basal and stimulated $\mathrm{O}_{2}{ }^{-{ }^{-}}$was similar among 3-day-old, 10-day-old and adult alveolar macrophages. EU and STAMLER (1997) investigated the intracellular fate of ${ }^{\circ} \mathrm{NO}$ and its relationship to the redox state of the RAW 264.7 cell line immunostimulated by LPS and interferon- $\gamma$. Following induction of iNOS, the rates of $\mathrm{NO}_{2}{ }^{-} / \mathrm{NO}_{3}{ }^{-}$accumulation in the media remained steady of $24 \mathrm{~h}$. The redox state within the cell, as measured by the intracellular concentrations of reduced glutathione and the ratios of GSH/GSSG, remained stable for $12 \mathrm{~h}$ following immunostimulation and declined thereafter, i.e. the cell became progressively oxidised. Three to $4 \%$ of the intracellular protein thiols were 
S-nitrosylated during the steady state production of $\mathrm{NO}_{2}{ }^{-} / \mathrm{NO}_{3}{ }^{-}$. The depletion of $\mathrm{GSH}$ by buthionine sulfoxime led to further increases in protein $\mathrm{S}$ nitrosothiols, but did not alter the rate of $\mathrm{NO}_{2}{ }^{-} / \mathrm{NO}_{3}{ }^{-}$ production.

The combination of aerosolised LPS and IFN- $\gamma$ to induce enhanced pulmonary alveolar macrophage activation in C57BL/6 mice was significantly better in killing of B16-F10 melanoma cells than either treatment modality alone (EISENBERG et al. 1991). Activated pulmonary alveolar macrophages selectively killed tumour cells, but did not kill the 3T3 fibroblast cell line. Peritoneal macrophages from mice treated with LPS + IFN- $\gamma$ by inhalation were enhanced (indicating a systemic effect), but not to the same extent as pulmonary alveolar macrophages.

The incubation of resident peritoneal macrophages with bacterial lipopolysaccharide induced high arginase (EC 3.5.3.1) activity as judged by the consumption of ${ }^{14} \mathrm{C}(\mathrm{U})$-L-arginine and the release of labelled ornithine into the cell supernatant (KrIEgBAUM and Dröge 1985).

NO production in LPS-stimulated RAW 264.7 cells was inhibited by flavonoids such as apigenin, wogonin, luteolin, tectorigenin, and quercetin as measured by nitrite formation at $10-100 \mu \mathrm{M}$ (KIM et al. 1999). Apigenin, chrysin, and kaempferol strongly enhanced the inhibition of inducible cyclooxygenase and inducible nitric oxide synthase promoter activities in lipopolysaccharide-stimulated RAW 264.7 macrophages, which contain the peroxisome proliferator-activated receptor $\gamma$ expression plasmids (LiAng et al. 2001). A synthetic carbazole compound, 9-(2-chlorobenzyl)-9H-carbazole-3-carbaldehyde, was found to have an inhibitory effect $\left(\mathrm{IC}_{50}=1.3 \pm 0.4 \mu \mathrm{M}\right)$ on lipopolysaccharide-stimulated ${ }^{\circ} \mathrm{NO}$ generation in RAW 264.7 cells (TsAo et al. 2002). This carbazole decreased the transcription of iNOS mRNA through a signal pathway that did not involve NF- $x \mathrm{~B}$ activation.

Murine peritoneal exudate cells pre-exposed to bacterial lipopolysaccharide showed augmented -NO production by LPS restimulation, in contrast to LPS tolerance with reduced production of TNF- $\alpha$ and IL-6 (Tominaga et al. 1999). Significant amounts of IFN- $\gamma$ were detected in the peritoneal exudate cell cultures on LPS stimulation, and antiIFN- $\gamma$ antibody suppressed the LPS-induced ${ }^{\circ} \mathrm{NO}$, but not TNF- $\alpha$ and IL-6, production. Addition of anti-IFN- $\gamma$ antibody to the cultures in the LPS preexposure step strongly suppressed the augmented -NO production on LPS restimulation. Anti-IL-12 antibody, which suppressed the LPS-induced IFN- $\gamma$ production, also suppressed the augmented $\mathrm{NO}$ production, as did anti-IFN- $\gamma$ antibody. Mouse peritoneal macrophages fed with synthetic $\beta$-hematin, structurally identical to native hemozoin, no longer produced tumour necrosis factor- $\alpha$ and ${ }^{\circ} \mathrm{NO}$ in response to LPS (TARAMELLI et al. 2000). $\beta$-Hematinmediated inhibition of macrophage function cannot be ascribed to iron release from $\beta$-hematin because neither prevention by iron chelators nor downregulation of iron-regulatory protein activity was detected. Inhibition appeared to be related to pigment-induced oxidative stress because (a) thiol compounds partially restored macrophage functions, (b) heme oxygenase and catalase mRNA levels were up-regulated, and (c) free radicals production increased in $\beta$-hematin-treated cells.

In a mixed cell culture system of astrocytes and microglial cells from the neocortex of new-born Wistar rats, nitrite levels, used as an indicator of nitric oxide production, were elevated after the addition of LPS and cytokines (PosSEL et al. 2000). Immunohistochemistry and the NADPH diaphorase technique demonstrated selective localisation of the iNOS protein in microglial cells, whereas no iNOS protein or NADPH diaphorase activity was detected in astrocytes.

The NO donor DETA NONOate $(200 \mu \mathrm{M})$ inhibited mouse bone marrow-derived macrophage proliferation by approx. $80 \%$ (VADIVELoo et al. 2001). However, despite ${ }^{\circ} \mathrm{NO}$ being an antimitogen, LPS was as potent at inhibiting proliferation in marrow-derived macrophages from NOSII-/- mice as from wild-type mice. Consistent with these findings, LPS-induced cell cycle arrest in normal bone marrow-derived macrophages was not reversed by the addition of NOSII inhibitor $S$-methylisothiourea. Moreover, in both normal and NOSII-/- bone marrow-derived macrophages, LPS inhibited the expression of cyclin D1, a protein that is essential for proliferation of many cell types. Despite inhibiting proliferation DETA NONOate had no effect on cyclin D1 expression.

Blocking of NO synthase with aminoguanidine (4 mg/mouse intraperitoneally $1 \mathrm{~h}$ before an intravenous application of $300 \mu \mathrm{g}$ LPS) decreased the levels of NO derivatives in the serum and bronchoalveolar lavage fluid by $45-50 \%$ (HEREMANs et al. 2000). TNF production in the serum was also reduced, but there was no effect on the development of lung oedema unless the dose of aminoguanidine was increased to 8 or $10 \mathrm{mg}$. Inhibition of NOS synthase by 2-amino-5,6-dihydro-6-methyl- $4 \mathrm{H}$-1,3tiazine abrogated LPS-induced increase of Larginine uptake in rat alveolar macrophages (HAMMERMANN et al. 2000).

Cytokine-induced neutrophil chemoattractant production in lipopolysaccharide-stimulated rat alveolar macrophages is regulated by the polyamine, putrescine (KamoI et al. 1997). 
Arachidonic acid metabolism of THP-1 monocytic cells grown in RPMI supplemented with $10 \%$ foetal bovine serum, $100 \mathrm{U}$ penicillin, $0.1 \mathrm{mg} / \mathrm{ml}$ streptomycin, and $2 \mathrm{mM} \mathrm{L}$-glutamine was activated by purified lipopolysaccharide R595 from S.minnesota (PFAU et al. 1998).

\section{Peptides}

\section{Glycyl-leucyl-phenylalanine}

Gattegno et al. (1988)

\section{Heptanoyl- $\gamma$-D-Glu-(L)meso- $\alpha, \in-A_{2}$ pm-(L)-AlaOH}

Heptanoyl- $\gamma$-D-Glu-(L)meso- $\alpha, \in-\mathrm{A}_{2}$ pm-(L)-AlaOH (FK-565) activated peritoneal macrophages harvested from C57BL/6 mice to become cytotoxic to syngeneic B16 melanoma cells (INAMURA et al. 1985).

\section{Lauroyltripeptide (RP 56 142)}

Lauroyltripeptide (RP 56 142) $\left(N^{2}\right.$-[N-(N-lauroyl-Lalanyl)- $\gamma$-D-glutamyl]-L, L-2,6-diaminopimelamic acid) induced macrophage activation and enhanced cytotoxicity of natural killer cells in spleen, blood, and liver (Fizames et al. 1989).

\section{Tuftsin (Threonyl-lysyl-prolyl-arginine)}

The tetrapeptide tuftsin represents the active moiety of leukokinin, the leukophilic fraction of immunoglobulin G. It is derived from leukokinin in two steps by enzymatic cleavage, one by tuftsin endocarboxypeptidase occurring in the spleen, the other by leukokinase on the surface of phagocytic cells. Neutrophils and monocytes possess highaffinity binding sites for tuftsin which cross-react with substance $P$, suggesting that tuftsin receptors may be considered a subtype of substance $P$ receptors (STABINSKY et al. 1978, BAR-SHAVit et al. 1980, Fridkin and GotTlieb 1981, WATson 1984). Tuftsin has been shown to activate superoxide radical $\left(\mathrm{O}_{2}{ }^{--}\right)$formation in murine macrophages with a biphasic concentration-response function (TRITSCH and Niswander 1982). The time course of thromboxane $\mathrm{B}_{2}$ production by albumin-elicited guinea pig peritoneal macrophages differed from that of $\mathrm{O}_{2}^{-{ }^{--}}$and $\mathrm{H}_{2} \mathrm{O}_{2}$ release by Corynebacterum parvuminduced cells in that considerable amounts were measurable at $2 \mathrm{~h}$ and plateau values were reached at $12 \mathrm{~h}$ (HARTUNG and Toyka 1984).

Macrophage monolayers from mice injected intraperitoneally with thioglycollate broth pulsed in vitro with keyhole limpet hemocyanin-tuftsin conjugates exerted a stronger immunogenic response effect than keyhole limpet hemocyanin alone (DAGAN et al. 1987). Bovine serum albumin, which by itself was not immunogenic, when applied to macrophages as tuftsin conjugate evoked a high lymphoproliferative immune response. In vivo, bovine serum albumin conjugated to tuftsin, when injected in aqueous solutions, augmented significantly antibody production, whereas administration of bovine serum albumin alone or bovine serum albumin admixed with tuftsin had no immunogenic effect. Studies conducted to elucidate the mechanisms underlying the activation of the immunogenic function of macrophages by the peptide revealed that treatment of cells with antigen and tuftsin increases secretion of interleukin-1 and expression of cell surface Ia encoded antigens.

Antigen-tuftsin covalent conjugates, injected in aqueous solution intramuscularly or intravenously into mice, significantly augmented antibody production (DAgAN et al. 1988).

Tuftsin deficiency in humans has been observed by Constantopoulos et al. (1972) and NaJjar and Constantopoulos (1973).

Further references: NAJJAR and Nishioka (1970), NajJar and Constantopoulos (1972), NajJar $(1975,1985)$, Spirer et al. (1977), Tzehoval (1978), Tzehoval et al. (1978), Stabinsky et al. (1980), Bruley-Rosset et al. (1981), MARtinez and Winternitz (1981), NajJar et al. (1981), NiSHIOKA et al. (1981), BAR-SHAVIT and GoldMAN (1982), Goldman and Bar-Shavit (1983), Tritsch and Niswander (1983), Chirigos and Talmadge (1985), Fridkin and NajJar (1989), SoROKIN et al. (1989), KACHEL et al. (1996)

\section{Dalargin (D-Ala-Gly-Phe-Leu-Arg)}

Dalargin $\left(10^{-3}\right.$ to $\left.10^{-9} \mathrm{M}\right)$ stimulated the luminoldependent chemiluminescence of mouse whole blood during phagocytosis of polystyrene latex beads $(0.8 \mu \mathrm{m}$ in diameter) recorded over $10 \mathrm{~s}$ (RoGovine and Mushtakova 1995).

\section{Valyl-glutamyl-prolyl-isoleucyl-prolyl-tyrosine}

Gattegno et al. (1988)

\section{Synthetic Hexapeptide $C 3 a_{72-77}$}

The synthetic hexapeptide $\mathrm{C} 3 \mathrm{a}_{72-77}$ induced generation of thromboxane $\mathrm{B}_{2}$ from guinea pig peritoneal macrophages (HARTUNG et al. 1984). This effect is specific as it can be blocked by an appropriate anti- 
body. It is not dependent on T cells. Phorbol myristate acetate given as a second stimulus excited release of large amounts of thromboxane $B_{2}$.

\section{Substance P}

The tachykinin substance $\mathrm{P}$ is an undecapeptide

$$
\begin{gathered}
\text { H-Arg-Pro-Lys-Pro-Gln-Gln-Phe-Phe-Gly- } \\
\text { Leu-Met- } \mathrm{NH}_{2}
\end{gathered}
$$

structurally related to the formyl peptides in its Cterminal portion. Substance $\mathrm{P}$ binds to specific plasma membrane receptors which can be divided into subtypes (WATSON 1984, 1987, REgoli et al. 1987). High-affinity binding sides have been identified on guinea pig macrophages (MARAsco et al. 1981). Substance $P$ has been reported to activate the respiratory burst in guinea pig macrophages (HARTUNG and ToYKA 1983). Activation of NADPH oxidase (EC 1.6.99.6) by substance $\mathrm{P}$ is accompanied by phospholipase $\mathrm{C}$ activation and $\mathrm{Ca}^{2+}$ mobilisation. Cytochalasin $\mathrm{B}$ enhances substance $\mathrm{P}$-induced phosphoinositide turnover and $\mathrm{Ca}^{2+}$ mobilisation, but somewhat surprisingly, cytochalasin B inhibits the respiratory burst (SERRA et al. 1988). Unlike the respiratory burst induced by fMet-Leu-Phe, that induced by substance $\mathrm{P}$ is only partially pertussis toxin-sensitive (SERRA et al. 1988).

Neurotensin (H-pyroGlu-Leu-Tyr-Glu-Asn-LysPro-Arg-Arg-Pro-Tyr-Ile-Leu-OH)

BAR-SHAVIT et al. (1982) found the specific binding of $\left[{ }^{3} \mathrm{H}\right]$ neurotensin to thioglycollate-elicited mouse peritoneal macrophages cultivated in vitro to be concentration dependent. Neurotensin was competitively displaced by tuftsin, substance P (SP) and SP $81-84$.

Gramicidin (HCO-Val-Gly-Ala-D-Leu-Ala-D-ValVal-D-Val-Trp-D-Leu-Trp-D-Leu-Trp-D-Leu-Trp$\mathrm{NHCH}_{2} \mathrm{CH}_{2} \mathrm{OH}$ )

Gramicidins are linear pentadecapeptide ethanolamide antibiotics with a formyl group at the $\mathrm{N}$ terminus (BAMBERg et al. 1976). Gramicidin is similarly potent but less effective than fMet-Leu-Phe. A competitive antagonist at formyl peptide receptors prevents activation by gramicidin, suggesting that this peptide is partial agonist at formyl peptide receptors (ЈАСОВ 1988).

\section{Gliadin}

Gliadin is an ethanol soluble fraction of gluten. Synthesized gliadin dodecapeptide sequence
FQQPQQQYPSSQ elicited the highest TNF- $\alpha$, IL-10, and RANTES secretion and increased IFN- $\gamma$ primed NO production by female $\mathrm{Balb} / \mathrm{c} / \mathrm{Ph}$ mouse macrophages (TučKovÁ et al. 2002).

\section{Cyclomunine}

Cyclomunine is a hexacyclodepsipeptide extracted from the fungus Fusarium equiseti. In vitro incubation of resident peritoneal macrophages from rats with $10^{-1}$ to $10^{-6} \mu \mathrm{g}$ cyclomunine $/ \mathrm{ml}$ for $3-24 \mathrm{~h}$ significantly increased the intracellular levels of proteins and lysosomal $\beta$-D-glucuronidase, as well as the neutral protease release and glucosamine incorporation (JosEPH et al. 1981). Superoxide anion generation during zymosan phagocytosis was also increased by preincubation of these cells with cyclomunine.

\section{Cyclosporin}

Cyclosporin is a cyclic hendecapeptide extracted from the fungus Trichoderma polysporum. There is some evidence that this immunosuppressant acts by interfering with the process by which antigens raise intracellular $\mathrm{Ca}^{2+}$ levels (Button and Palacios 1982). Interleukin 2 producing $T$ cells do not express receptors for interleukin 1 in the presence of cyclosporin A. Thus, this subset of $\mathrm{T}$ cells is rendered unable to produce and/or secrete interleukin 2 , which is a requirement for $\mathrm{T}$ cell proliferation and growth. When $\mathrm{T}$ cells are rendered unresponsive to interleukin 2, progression of $\mathrm{T}$ cell help for development of $\mathrm{T}$ cytotoxic cells, which are instrumental in rejecting foreign tissue is effectively stopped. It does not appear to inhibit $\mathrm{T}$ suppressor cells; rather through its selective toxicity for $\mathrm{T}$ helper cells, it permits a greater expression of the suppressive reactivity. In addition, the lymphokine, macrophage activating factor thought to be synonymous with interferon $\gamma$, may not be released in the presence of cyclosporin A.

Pulmonary eosinophilia in allergic mice (challenged with nebulized ovalbumin) was inhibited by cyclosporin A when given orally at $24 \mathrm{~h}$ and $1 \mathrm{~h}$ before challenge (UMLAND et al. 1999). There was a complete, dose-dependent inhibition of eosinophils in the bronchoalveolar lavage fluid of challenged animals, with only a partial reduction in interleukin-5 mRNA levels in the lung. For each concentration of cyclosporin A tested there was a greater reduction in eosinophils than of IL-5.

Cyclosporin A, an inhibitor of cyclophilin (TAKAHASHI et al. 1989), does not inhibit but slows the folding of the monomeric protein transferrrin in 
the endoplasmic reticulum (Lodish and Kong 1991), since it is active in concentrations which only block a minor percentage of the cell's peptidylprolyl isomerases. Its complex with peptidyl-prolyl isomerase interferes with signal transduction pathways of eukaryotic cells, and the inhibitor mediates pharmacological and toxic effects primarily by interfering with regulatory functions in the cell (Kunz and Hall 1993). The same cyclophilin can act in a late step of protein folding by acting as a prolyl isomerase, but also in an early step, by preventing the aggregation of a denatured substrate protein. Both activities are inhibited in the presence of cyclosporin A (FRESGARD et al. 1992). Cyclosporin A slows collagen triple-helix formation in vivo (STEINMANN et al. 1991). This was accompanied by a remarkable post-translational overmodification and increased degradation.

Reactive oxygen species generated during its metabolism and inducing microsomal lipid peroxidation were shown to be a possible explanation for the toxicity of this immunosuppressive drug (SERINo et al. 1993).

All seven subsets of epithelial cells were markedly changed in the rat thymus after oral application of $30 \mathrm{mg}$ cyclosporin A per kg body weight for 21 consecutive days (Milicevic and Milicevic 1997). The abundance of cytokeratin bundles was registered in type 1-,subcapsular/paraseptal/perivascular' - epithelial cells.

Diabetes mellitus spontaneously occurring in $40-60 \%$ of colony of BioBreeding/Worcester rats was significantly reduced in its frequency and delayed in its onset by pre-treatment of susceptible animals for 10-day intervals prior to 70 days of age with cyclosporin A (LiKE et al. 1984).

Cyclosporin A exhibits very little myelotoxicity and does not interfere with phagocytosis by macrophages. Cardiotoxicity of the drug was seen in swine (Topalidis et al. 1989). The ready reversibility of both the nephrotoxicity (SIBLEy et al. 1983, Minatsch et al. 1988, 1989) and hepatotoxicity (ANDRÉs et al. 2000) of cyclosporin A has aided its acceptance as a drug of choice.

\section{Poly-L-lysine}

Poly-L-lysine treatment of lipopolysaccharidecoated particles greatly increased the attachment rate of thioglycolate-elicited peritoneal macrophages of 7-week-old female mice (strain ddY) closely correlated with the difference in surface charge density of the particles caused by such treatments, increasing with the decrease in negative charge density (MATsui et al. 1983).
Poly- $L$-lysine appeared as a potent dissolver of preformed $\beta$-amyloid fibrils in vitro (NGUYEN et al. 2002). Its efficiency is instantaneous. Poly-L-lysine can be used as a universal dissolver of all types of oligomeric $\beta$-sheet conformation, precursor of the fibrils.

\section{Poly-L-glutamic Acid}

AxLine et al. (1973)

\section{Glycopeptides}

Muramyl dipeptide ( $N$-acetyl-muramyl-L-alanyl-Disoglutamine), which has been synthesized along with hundreds of analogues, has been shown to be effective at both enhancing and suppressing the immune response.

Macrophages were stimulated by muramyl dipeptide to induce polymorphonuclear leucocyte accumulation in the peritoneal cavities of guinea pigs (Nagao et al. 1990).

Muramyl dipeptide-induced immunosuppression appears to be mediated through the generation of suppressor T cells (LECLERC et al. 1984).

Mouse alveolar macrophages were rendered tumoricidal after the intravenous administration of liposomes containing muramyl tripeptide phosphatidyl-ethanolamine, a lipophilic derivative of muramyl dipeptide (FIDLER et al. 1989).

\section{Nucleotides}

\section{Isoprinosine}

Isoprenosine, a complex of $p$-acetamidobenzoic acid, $N, N$-dimethylamino-2-propanol and inosine at a molar ratio of $3: 1$, is a white crystalline powder, slightly bitter in taste, soluble in water at room temperature to an extent of $25 \%(250 \mathrm{mg} / \mathrm{ml})$ and stable in neutral solution. The dosage commonly administered to man is $500 \mathrm{mg}$ tablets (HADDEN and Giner-Sorolla 1981). Of particular importance is the metabolic lability of the inosine moiety of isoprinosine, the half life of which is $3 \mathrm{~min}$ and $50 \mathrm{~min}$, respectively, following intravenous and oral administrations in the rhesus monkey. More than $90 \%$ of the labelled inosine is excreted as allantoin and uric acid.

Further references: HADDEN (1978), HADDEN et al. (1979), HAdDEN and Giner-Sorolla (1981) 
NPT 15392 [erythro-9(2-hydroxy, 3-nonyl) hypoxynthine]

NPT 15392 augmented lymphokine-induced guinea pig macrophage proliferation and phagocytosisinduced chemiluminescence (HADDEN and GINERSOROLla 1981).

\section{5-Iodo-2'-deoxyuridine}

ANACLERIO et al. (1976)

\section{Synthetic Oligonucleotides}

Pre-treatment of murine bone marrow macrophages, J774 and RAW264.7 macrophages with synthetic 30-mer phosphorothionate oligonucleotides inhibited NO production induced by $\mathrm{CpG}$ oligonucleotides, E. coli DNA, or lipopolysaccharide (ZHU et al. 2002).

\section{Synthetic Polynucleotide Complexes}

Synthetic polynucleotide complexes have been shown to be effective immune response modulators in animals and man (BRAUN et al. 1971, JoHNSON 1979). The polynucleotides are formed following the action of an enzyme, polynucleotide phosphorylase on the synthetic mononucleotide diphosphates. Complexing takes place following the mixing of polymers composed of opposite base pairs. Two have been utilised, polyinosinic acid complexed with polycytidylic acid (poly I poly C) and polyadenylic acid complexed with polyuridylic acid (poly A poly $U$ ). The single strands mononucleotides are ineffective.

\section{Polyinosine-Polycytidylic Acid}

As polyinosine-polycytidylic acid failed to enhance killing of Escherichia coli by mouse peritoneal macrophages in vitro, Thalinger and MANDELL (1972) suggested that it acted not directly on mononuclear phagocytes.

Polyriboinosinic-polyribocytidylic acid augmented resistance of thioglycolate-elicited inflammatory macrophages to infection with herpes simplex virus type 1 (Pyo et al. 1991). Polyriboinosinic-polyribocytidylic acid antiviral activity was completely abrogated by antibodies to interferon- $\beta$ whereas antibodies to other interferons or to other cytokines had no effect.

LEE et al. (1990) used a murine mammary tumour model to test the efficacy of a combination of heparin and the interferon inducer, polyriboinosinic-polyribocytidylic acid on spontaneous metastasis from a subcutaneous primary tumour and on experimental metastasis following intravenous injection of tumour cells. This treatment had no effect on the growth of primary tumours, but lung metastases arising from these tumours were reduced.

Further references: Evans and Alexander (1976), Hamburg et al. (1978), Pugh-Humphreys and Thomson (1979), Martinez et al. (1980), TARAMELli and VARESIo (1981), REIDARSON et al. (1982), Chirigos and Talmadge (1985), Chirigos et al. (1985), Souvannavong and Adam (1990), CoHen et al. (1997)

\section{Polyadenyl-Polyuridylic Acid}

The double stranded polynucleotide, poly A:U, when administered intraperitoneally at the same time as intravenous infection with Brucella abortus, suppressed the growth of that organism in the spleen and liver of mice (MADraso and CHEERS 1978). The antiviral function of spleen cells induced by poly A:U was evident in the supernatant fluid when cultured for $48 \mathrm{~h}$ at $37^{\circ} \mathrm{C}$ (LeE et al. 1992). Murine cytomegalovirus-induced plaques were reduced to 52 and $5 \%$ of controls in the plaque assays performed at $37^{\circ}$ and $40^{\circ}$, respectively.

Further references: Evans and Alexander (1976), Pugh-Humphreys and Thomson (1979), Chirigos and Talmadge (1985)

\section{Compounds Containing a Sulphur Moiety}

Both levamisole and isoprinosine have received extensive in vivo immunopharmacologic testing in animals and man. Many of the immune functions that are affected by thymic hormones are shown also to be affected by levamisole, and isoprinosine in vitro and have been confirmed in vivo following administration to experimental animals or human subjects (SPECTER and HAdDEN 1985). Both have additional actions on macrophages which have not been tested extensively for the thymus hormones. Regarding their therapeutic usefulness, both have limitations as they are relatively mild in their actions. Levamisole treatment often takes weeks to months to achieve effects, and agranulocytosis as a side effect is significant.

Some of the other sulphur-containing compounds are less toxic and may be more potent. 
Levamisole ( $L-2,3,5,6$-Tetrahydro-6-phenylimidazo- $[2,1-b]$ thiazole- $\mathrm{HCl})$

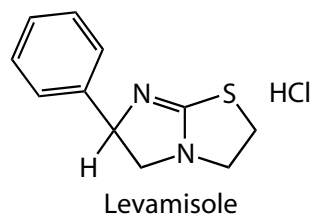

[260]

Levamisole is rapidly absorbed by both oral and parenteral routes, with extensive metabolism and rapid excretion of both unchanged drug and metabolites in urine and faeces of rats. Two major metabolites and six minor ones have been identified in experimental animals. In man, most of the urinary activity consists of hydrophilic compounds: $p$-hydroxy-levamisole $\{(+)-2,3,5,6$-tetrahydro-6-(4-hydroxyphenyl)imidazo $[2,1-b]$ thiazole $\}$ and its glucuronide conjugate have been identified (ADAMs 1978). A possible mechanism for the formation of (-)-2-oxo-3-(mercaptoethyl) -5phenylimidazolidine, one of the major metabolites of levamisole, has been proposed by VAN BELLE and JANSSEN (1979). $\alpha$-Ketoaldehydes, particularly glyoxal and methylglyoxal, are specific catalysts for the hydrolysis of levamisole to (-)-2-oxo-3-(mercaptoethyl)-5-phenylimidazolidine. The proposed mechanism involves a charge-transfer complex followed by a concerted mechanism in which water is carried from the hydrated aldehyde to levamisole with concomitant ring opening.

Further references: RÈnOuX and Rènoux (1971), Schuermans (1975), Van Ginckel and Hoebeke (1975), Huskisson et al. (1976), Renoux et al. (1976), Schmidt and Douglas (1976), DaughaDAY et al. (1977), Symoens and Rosenthal (1977), Hadden (1978), Kelly (1978), Nathanson et al. (1978), Plewig and Luderschmidt (1978), HadDEN et al. (1979), Boura et al. (1984), JoHNSON and Regal (1985), J.H. Schiller et al. (1991), Kimball et al. (1992); against: ZIPRIN et al. (1977)

\section{Diethyldithiocarbamate}

Sodium diethyldithiocarbamate inhibits prostaglandin synthetase at $\sim 10 \mu \mathrm{g} / \mathrm{ml}$. The site of action is not at the fatty acid substrate site. It is a copper chelating agent and probably prevents the interaction of oxygen with the enzyme.

\section{Thiabendazole [2-(4-thiazolyl)-benzimidazole]}

Thiabendazole primarily used as an anthelmintic in male ICR mice depleted of glutathione by treatment with DL-buthionine sulphoximine was much more nephrotoxic than in females (Mizutani et al. 1992).

\section{Thioglycollate}

Karl et al. (1973), Rabinovitch and De Stefano (1973), Gordon et al. 1974), UnKeless et al. (1974), BiAnco et al. (1975), Edelson et al. (1975), Werb and Gordon (1975), Wellek et al. (1977), Dy and Astoin (1978), Dy et al. (1978, 1979), Schnyder and Baggiolini (1978), Swenson and Kozel (1978), TANSEy et al. (1978), vAN DER Zeijst et al. (1978), Werb et al. (1978), Ано et al. (1979), ERICKSON and Hu (1979), Jones and SCOTTBurden (1979), Vetvicka et al. (1979), DAhLgren et al. (1980), Hagmann and Fishman (1980), Johnson and Balish (1980), MiYasaka et al. (1980), Phaire-Washington et al. (1980), SaintGuillain et al. 1980), Weidemann et al. (1980), Beller and Unanue (1981), Fornusek et al. (1981), Imai and Tanaka (1981), Khoo et al. (1981), Vray et al. (1981), WAldrep and Reese (1981), Roubin et al. (1982), Thyberg et al. (1982), BAggiolini et al. (1983), BeElen and Walker (1983), Berton and Gordon (1983), DAHA and Van Es (1983), Gottlieb et al. (1983), Werb and Chin (1983), Hume and Gordon (1984), De BAetSelier et al. (1985), Mellman and UkKonen (1985), Metzger et al. (1986), Veerhuis et al. (1986), Morse and Moore (1988), Newburg et al. (1988), Аве et al. (1990), Goldin et al. (1993), Tritsch and Evans (1993), Zdolsek et al. (1993), Costa Rosa et al. (1994), Brisseau et al. (1995), Watanabe et al. (1998), Han et al. (2001)

\section{DL-2-Oxo-3-(2-mercaptoethyl)-5- phenylimidazolidone}

Van Ginckel and De Brabander (1979)

\section{Carbohydrates}

\section{Glycogen}

Heidelberger et al. (1954), Comolli and Perin (1963), Vaughan (1965), Borowski et al. (1972), Kurisu et al. (1978), Stalder and Schräder (1980), Cianciola and Snyderman (1981), ManTOVANi (1981), Glass et al. (1983), YamazaKi et al. (1983), Glass et al. (1984), BradLey et al. (1989)

\section{Zymosan}

$\alpha$-Mannans and $\beta$-glucans are the major constituents of zymosan, which is prepared from yeast cell walls (Pillemer and Ecker 1941, Phaff 1963, BACON et al. 1969). 
The mannosyl-fucosyl receptor has broad specificity and is able to bind various glycoconjugates in the order L-fucose $=\mathrm{D}$-mannose $>\mathrm{N}$-acetyl glucosamine $\approx$ D-glucose $>$ D-xylose $>>$ D-galactose $=\mathrm{L}$ arabinose $=\mathrm{D}$-fucose (STAHL et al. 1980, SHEPHERD et al. 1981). The ligand binding site of type 3 complement receptors is involved in macrophagezymosan binding and uptake with Fab fragments of anti-C3 antibodies and monoclonal antireceptor antibodies M01 and OKM10 (EzEKowITZ et al. 1985). Unopsonized zymosan is a poor trigger of respiratory burst activity in 7-d adherent human blood monocyte-derived macrophages, but induced cell aggregation and secretion of large amounts of superoxide anion, when these cells were co-cultivated with neutrophils in serum-free medium and challenged with zymosan. Mouse serum-treated zymosan activated the respiratory burst in primed murine macrophages (BERTON and GoRDON 1983).

Measuring the production of oxygen free radicals in response to zymosan, KREIPE et al. (1988) found a 20-30 fold increase in multinucleated giant cells when compared with unfused cultured human blood monocyte-derived macrophages. This augmentation, however, could not be noted when chemiluminescence was related to the nuclei counted in the syncytia. Opsonization of zymosan is not necessary for stimulation of the murine bronchoalveolar macrophage oxidative burst (SUGAR and Field 1988). In rat cerebral microglia cell cultures, opsonized zymosan produced superoxide radical anions (Colton and Gilbert 1987).

By injecting zymosan A (50 mg) into an air pouch on male CD (Sprague-Dawley) and Fisher 344 rats, SAMs et al. (2000) found significant increases $(P<0.01)$ in 8 -hydroxy-2'-deoxyguanosine after 1 day in the DNA from cells lining the air pouch from zymosan-treated versus control rats. By 28 days, 8-hydroxy-2'-deoxyguanosine levels had returned to background in Sprague-Dawley rats, but remained elevated in F-344 rats.

Pre-treatment of murine bone-marrow derived macrophages with $10 \mathrm{U} / \mathrm{ml}$ murine IL- 4 for $48 \mathrm{~h}$ enhanced the respiratory burst following subsequent stimulation with $10^{-6} \mathrm{M}$ phorbol myristate acetate or $1 \mathrm{mg} / \mathrm{ml}$ zymosan (Phillips et al. (1990).

Zymosan-bound histamine activated $\mathrm{O}_{2}{ }^{--}$formation in guinea pig alveolar macrophages via $\mathrm{H} 1$ receptors (Diaz et al. 1979).

After intravenous injection of quartz dust (particle size 1-2 $\mu \mathrm{m}$ ), a serum factor appeared in 8 out of 10 rabbits which agglutinated zymosan to a dilution of $1: 256$ (PERnis et al. 1959).

Further references: Riggi and Di Luzio (1961), DiCARlo et al. (1963), Wisse (1974), Ringrose et al.
(1975), Dean et al. (1979), Bitter-SuermanN (1980), Castranova et al. (1980, 1981, 1994), Czop et al. (1981), Roubin and Benveniste (1981), RouZER et al. (1981, 1982), BERRY (1982), Fels et al. (1982), Schade and Rietschel (1982), BaggioLINI et al. (1983), BAXTER et al. (1983), BERTON and Gordon (1983), Bodmer and DeAN (1983), Chang et al. (1983), Schopf and Lemmel (1983), D’ONofrio and Lohmann-Matthes (1984), Ezekowitz et al. (1984, 1985), Peters-Golden et al. (1984), Paterson et al. (1985), De Maroussem et al. (1986), Gairola and Tai (1986), Kadish et al. (1986), Colton and Gilbert (1987), PetersGolden and TheBert (1987), ANDre et al. (1988), Kreipe et al. (1988), Kuroiwa et al. (1988), Sugar and Field (1988), SChroers and Tilkes (1990), JoRens et al. (1991), Polzer et al. (1992, 1993), HuWiler and Pfeilschifter (1993), Costa Rosa et al. (1994), TAPPer and Sundler (1995), Helmke et al. (1996), Sherman et al. (1996), Sporn et al. (1996), Cohen et al. (1997), Ushijima et al. (1997), Blake et al. (1998), Fernández et al. (1999), NAGAISHi et al. (1999), AкiвA et al. (2002)

\section{Glucan ( $\beta$-1,3-poly-glucopyranose)}

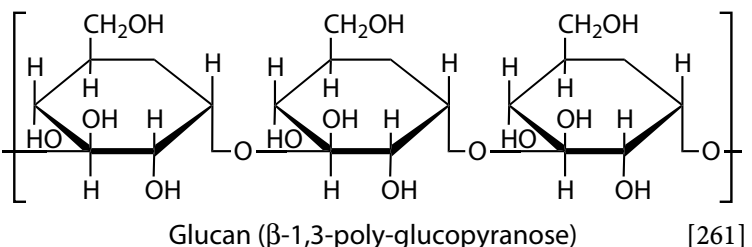

Macrophage activation with yeast glucan induced a pronounced elevation of colony stimulating activity in serum (SATOH et al. 1982, Chinara 1983). The increase of colony stimulating activity was dose dependent. While doses of $62.5 \mathrm{mg}$ krestin $(\beta-1,4 ; \beta-1,3 ; \beta$ 1,6 , protein complex; PSK) $/ \mathrm{kg}$ and $125 \mathrm{mg}$ PSK $/ \mathrm{kg}$ produced no increase in colony stimulating activity, a dose of $250-1000 \mathrm{mg} / \mathrm{kg}$ was associated with a rapid increase in colony stimulating activity followed by a rapid decline. Yeast glucan has been demonstrated to be very effective in enhancing CSF at doses where PSK is ineffective (Patchen and McVittie 1983).

Pulmonary granuloma formation induced by infusion of $5 \mathrm{mg}$ particulate glucan/rat was markedly reduced when neutrophils were depleted by rabbit antiserum directed against rat peripheral blood neutrophils or by catalase $(15,000 \mathrm{U} /$ rat $)$ at the time of injection and $24 \mathrm{~h}$ after glucan infusion (KILGORE et al. 1997). Neutrophils and reactive oxygen intermediates $\left(\mathrm{H}_{2} \mathrm{O}_{2}\right)$ are required for the local induction of monocyte attractant protein-1 to be secreted by endothelial cells. 
Further references: Di Luzio and BierMAN (1964), MANSEll et al. (1975), Browder et al. (1976, 1977, 1978), Di Luzio (1976, 1977, 1983, 1985), Di Luzio et al. (1976, 1978, 1984), Burgaleta and Golde (1977), Burgaleta et al. (1978), Cook et al. (1978, 1980, 1982), Di LuZio and Williams (1978, 1979, 1980), Кокоsнis et al. (1978), Niskanen et al. (1978), Stiteler et al. (1978), Williams et al. (1978), Deimann and Fahimi (1980), Way et al. (1980), Bärlin et al. (1981), SELjelid et al. (1981), Agostini et al. (1982), Benach et al. (1982), CHIhara (1983), Patchen and MacVittie (1983), JaNiAK et al. (1984), Kimura et al. (1984), PATchen et al. (1984), LEW et al. (1986), SHERwood et al. (1986), Ralston and Rohrbach (1994), Kilgore et al. (1997)

\section{Lentinan}

Lentinan is a $\beta$-1,3-glucan having two $\beta-1,6-$ glucopyranoside branchings for every five $\beta-1,3-$ glucopyranoside linear linkage (SASAKI et al. 1976). According to TAlmadge and Chirigos (1985) there is no influence on the macrophage-mediated cytotoxicity in vitro and in vivo.

\section{Alginate}

Alginates are polysaccharides wih gel-forming properties composed of 1,4-linked $\beta$-D-mannuronic acid, $\alpha$-L-guluronic acid and alternating $\beta$-Dmannuronic acid- $\alpha$-L-guluronic acid blocks. Alginates stimulated human monocytes to produce high levels of tumour necrosis factor- $\alpha$, interleukin6 and interleukin-1 (OtTERLEI et al. 1991).

\section{Scholler Lignin}

The substance has an unusually high adsorbing power; cell activation could possibly by achieved through physico-chemical influences on the cell and/or intracellular membranes (FLEMMING 1974).

Further references: GRAACK and FlEMming (1966), Flemming (1967), Flemming and GraACK (1967)

\section{Chitin Derivatives}

Chitin-based tissues are absent in the human body, but $\mathrm{N}$-acetylglucosamine, the repeating unit of chitin, and chitobiose are present in glycosaminoglycans and in glycoproteins (Muzzarelli et al. 1986). About $1 \%$ of the secretory proteins of macrophages is chitinase (Воот et al. 1995). Еsсотт and ADAMs (1995) showed experimentally that on differentiation of the monocytes into macrophages in vitro, chitinase is released into the growth medium. Chitinase activity was found to be associated with the monocyte cell line, THP-1 following culture with colloidal chitin (EscotT et al. 1996).

The induction of cytotoxic macrophages was enhanced by an increase of negative charge at O- 6 and decreased by further modification at $\mathrm{O}-3$ of the glucopyranose- $\mathrm{N}$-acetyl residue of $\mathrm{O}$-(carboxymethyl)chitins (Nishimura et al. 1986). O-(Carboxymethyl)chitins had a minor effect on mitogenic activity that was independent of the site of modification; partially $N$-deacetylated chitins had little activity. Although there was remarkable enhancement of accessibility to lysozyme upon modification at $\mathrm{O}-6$ of the glucopyranose- $\mathrm{N}$-acetyl residue, the accessibility was decreased by further substitution of O-3.

The secretion of enzymes, interleukins, tumour necrosis factor, nitric oxide, peroxide and other compounds by activated macrophages provides macroscopic evidence of the biochemical significance of chitosans (Muzzarelli 1996). Various chitosans were found to induce production of tumour necrosis factor- $\alpha$ in human monocytes (OTTERLEI et al. 1994). Lipopolysaccharides and watersoluble chitosans recognise a binding site on monocytes which involves CD14, a receptor of lipopolysaccharides also present in macrophages. GoRBACH et al. (1994) found immunostimulating and antitumour activities in chitooligosaccharide derivatives containing $N$-linked side chains which mimic the lipopolysaccharides.

Intravenous administration of phagocytable chitin particles $(1-10 \mu \mathrm{m})$ in C57BL/6 mice and SCID mice primed alveolar macrophages within 3 days up to a 50-fold increase in their oxidative burst when elicited in vitro with phorbol myristate acetate (Shibata et al. 1997). C57BL/6 mice pre-treated with monoclonal antibodies against mouse $\gamma$ interferon showed a markedly decreased level of alveolar macrophage priming following injection of chitin particles.

The consequences of chitosan application to living tissues could possibly go far beyond the enhanced production of nitric oxide by macrophages; additional NO could be produced chemically, but some could be consumed as nitrite by the chitosan amino groups. Thus, extra amounts of NO could be beneficial and help resorb chitosan. The presence of chitosan could presumably have some as yet undocumented consequence on the iron-enzymes (MuzZARELLI 1997). 


\section{Polymers}

Pyran (Divinylether-maleic Anhydride) Copolymer

Merigan and Regelson (1967), Regelson (1967), Braun et al. (1970), Munson et al. (1970), Kapila et al. (1971), Hirsch et al. (1972), Kaplan et al. (1974), Regelson et al. (1974), BAIRD and KAPLAN (1975), Harmel and Zbar (1975), Herling (1975), Elzay and Regelson (1976), Mohr et al. (1976), Schultz et al. (1976, 1977), Levine et al. (1977), Majeski and Stinnett (1977), Puccetti et al. (1979), Stinnett et al. (1979), Kaplan et al. (1980), Miller et al. (1980), Bärlin et al. (1981), DeAn et al. (1981), Loveless and Munson (1981), Männel (1982), AdAMs et al. (1983), Werb and Chin (1983), JaniaK et al. (1984), Kataoka and Оh-Hashi (1985), KuUs et al. (1985)

\section{Cyclohexyl-1,3-dioxepin Maleic Anhydride Copolymer}

KuUs et al. (1985)

\section{4-Methyl-2-pentenoyl Maleic Anhydride Copolymer}

KuUs et al. (1985)

Poly-2-vinylpyridine- $\mathrm{N}$-oxide

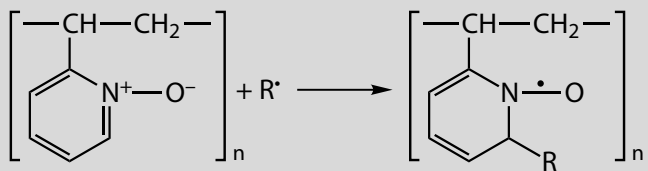

Poly-2-vinylpyridin $\mathrm{N}$-oxid forming radical adducts [63]

Flemming and Nothdurft (1968), Nothdurft and Flemming (1970), Flemming (1974)

\section{Polyacrylic Acid}

Synthetic polyanions derived from polyacrylic acid (REgelson et al. 1960, 1974, SAKAmoto et al. 1982) having molecular mass $<10^{4}$ are potent stimuli to macrophage phagocytic function and exhibit antitumour activity. Polymers with higher molecular mass inhibit macrophage function, and only those with molecular mass $>15,000$ have antiviral and immunological stimulating capacities (REGELSON 1979). Kief et al. (1974) $24 \mathrm{~h}$ after a single subcutaneous injection of $10 \mathrm{mg}$ polyacrylic acid $(\sim 15$ $\mathrm{kDa}$ ) per animal saw many vacuoles in the Kupffer cells of mice in connection with a histochemically demonstrable activation of acid phosphatase.

In mouse liver microsomes, cytochrome $\mathrm{P}-450_{47}$ increased after monomeric methylmetacrylate at a dose of $45 \mathrm{mg} / \mathrm{kg} \times$ day administered intraperitoneally for 4 days but was totally repressed at a dose of $600 \mathrm{mg} / \mathrm{kg} \times$ day, while the total amount of cytochrome P-450 was unaltered (NiLSEN et al. 1978).

Fig. 171. Two non-immunologically stimulated peritoneal macrophages from a SPFNMRI mouse $2 \mathrm{~h}$ after a single intraperitoneal injection of $150 \mathrm{mg}$ isotactic polyacrylic acid (Vi 2329 molecular mass 8-12 kDa, MücK et al. 1977) as sodium salt per kg body weight. The produced ascites were centrifuged for $10 \mathrm{~min}$ at 1000 r.p.m. and incubated in TCM $199+20 \%$ foetal calf serum on a plastic cover slip for $2 \mathrm{~h}$, rinsed with phosphate buffered saline after Dulbecco and fixed in $1 \%$ glutaraldehyde in $0.07 \mathrm{M}$ phosphate buffer (pH 7.4). Ethanol, amyl acetate. Critical point drying. Gold coating. Cambridge Stereoscan 150 operated at $19 \mathrm{kV}$. APhR. 856/80, negative 07168 . - The cells are rounded showing surface ruffles and attach themselves to the underlying substrate by means of thin veils of cytoplasm spreading beneath the dome-shaped nuclear pole.

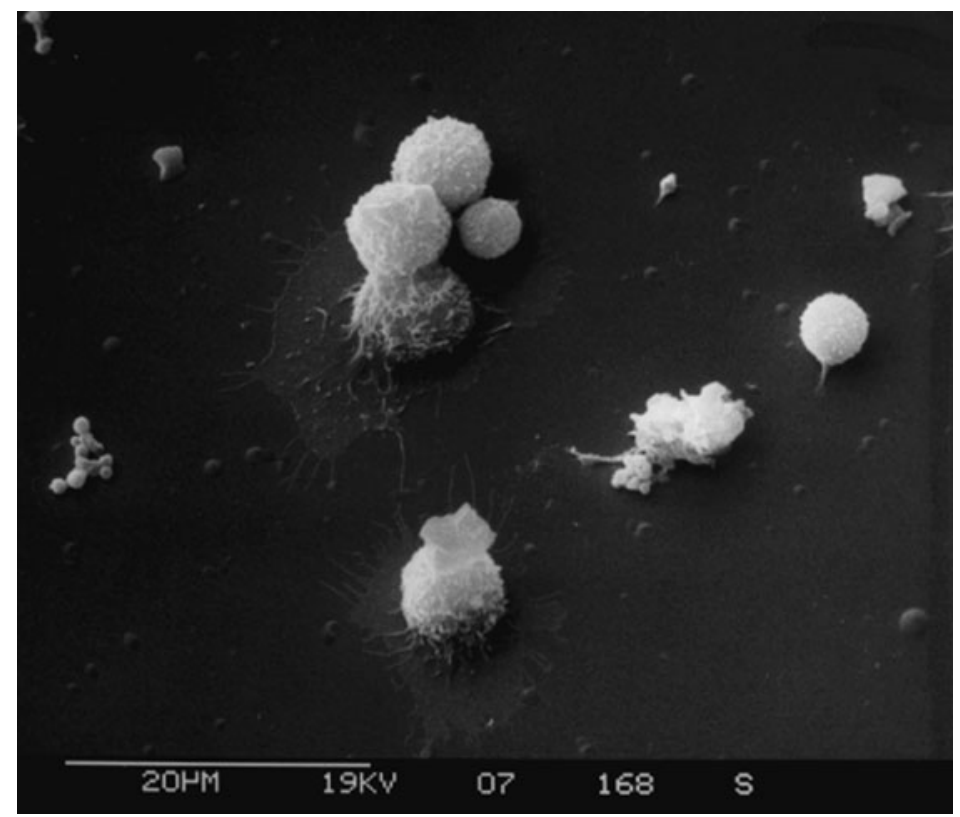


Fig. 172. Spreading of a non-immunologically stimulated peritoneal macrophages from a SPF-NMRI mouse $8 \mathrm{~h}$ after a single intraperitoneal injection of $150 \mathrm{mg}$ isotactic polyacrylic acid (Vi 2329 molecular mass $8-12 \mathrm{kDa}$, MüCK et al. 1977) as sodium salt per kg body weight. The produced ascites were centrifuged for $10 \mathrm{~min}$ at 1000 r.p.m. and incubated in TCM $199+20 \%$ foetal calf serum on a plastic cover slip for $2 \mathrm{~h}$, rinsed with phosphate buffered saline after Dulbecco and fixed in $1 \%$ glutaraldehyde in $0.07 \mathrm{M}$ phosphate buffer ( $\mathrm{pH}$ 7.4). Ethanol, amyl acetate. Critical point drying. Gold coating. Cambridge Stereoscan 150 operated at 19 kV. APh-R. 859/80, negative 07 198. (from SCHILLER 1982)

Fig. 173. Spreading of some nonimmunologically stimulated peritoneal macrophages from a SPF-NMRI mouse $24 \mathrm{~h}$ after a single intraperitoneal injection of $150 \mathrm{mg}$ isotactic polyacrylic acid (Vi 2329; molecular mass 8-12 kDa; cf. MücK et al. 1977) as sodium salt per kg body weight. The produced ascites were centrifuged for $10 \mathrm{~min}$ at 1000 r.p.m. and incubated in TCM $199+20 \%$ foetal calf serum on a plastic cover slip for $2 \mathrm{~h}$, rinsed with phosphate buffered saline after Dulbecco and fixed in $1 \%$ glutaraldehyde in $0.07 \mathrm{M}$ phosphate buffer $(\mathrm{pH}$ 7.4). Ethanol, amyl acetate. Critical point drying. Gold coating. Cambridge Stereoscan 150 operated at $19 \mathrm{kV}$. APh-R. 861/80, negative 07 201

Fig. 174. Spreading of a non-immunologically stimulated peritoneal macrophages from a SPF-NMRI mouse $48 \mathrm{~h}$ after a single intraperitoneal injection of $150 \mathrm{mg}$ isotactic polyacrylic acid (Vi 2329; molecular mass 8-12 $\mathrm{kDa}$; cf. MücK et al. 1977) as sodium salt per kg body weight. The produced ascites were centrifuged for $10 \mathrm{~min}$ at 1000 r.p.m. and incubated in TCM $199+20 \%$ foetal calf serum on a plastic cover slip for $2 \mathrm{~h}$, rinsed with phosphate buffered saline after Dulbecco and fixed in $1 \%$ glutaraldehyde in $0.07 \mathrm{M}$ phosphate buffer $(\mathrm{pH}$ 7.4). Ethanol, amyl acetate. Critical point drying. Gold coating. Cambridge Stereoscan 150 operated at $19 \mathrm{kV}$. APh-R. 101/80, negative 03 795. (from SCHILLER 1982)
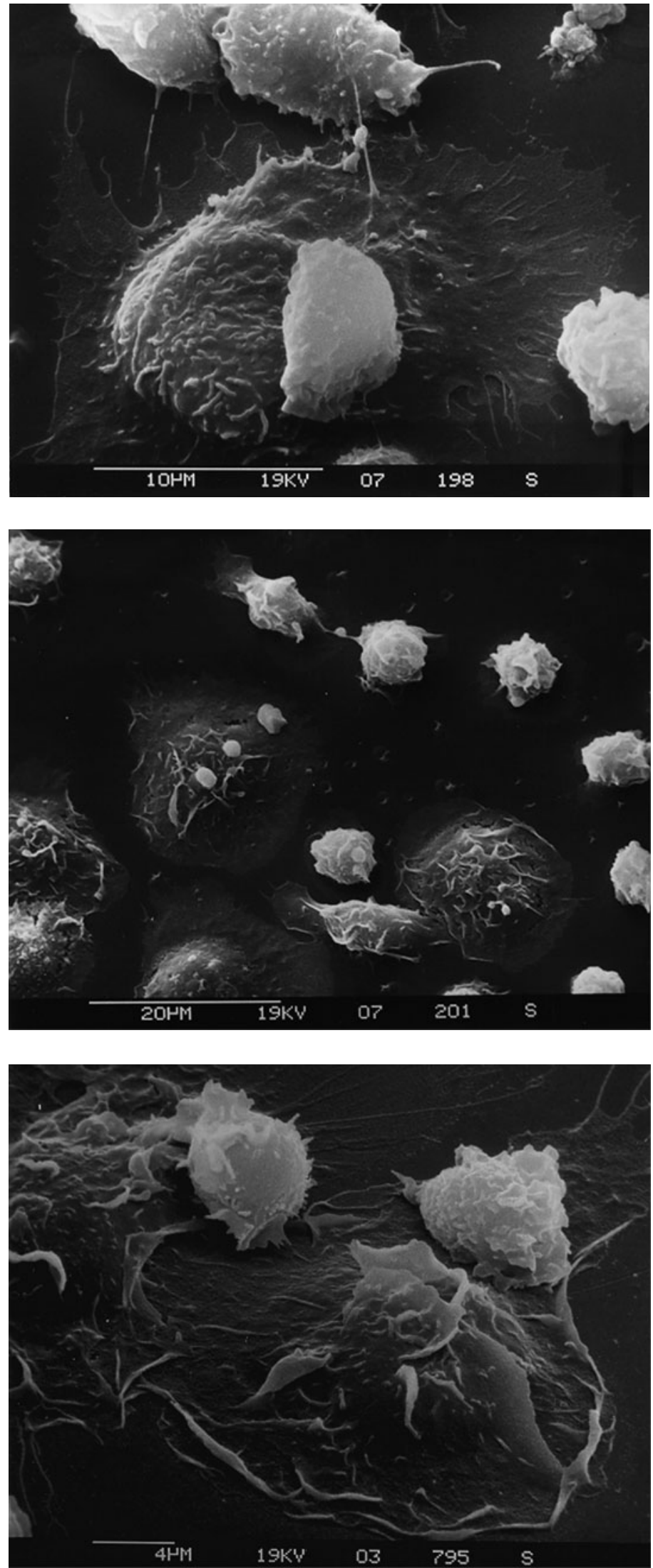
Fig. 175. Non-immunologically stimulated peritoneal macrophages from a SPF-NMRI mouse $48 \mathrm{~h}$ after a single intraperitoneal injection of $150 \mathrm{mg}$ isotactic polyacrylic acid (Vi 2329; molecular mass 8-12 kDa; cf. MücK et al. 1977) as sodium salt per kg body weight. The produced ascites were centrifuged for $10 \mathrm{~min}$ at 1000 r.p.m. and incubated in TCM $199+20 \%$ foetal calf serum on a plastic cover slip for $2 \mathrm{~h}$, rinsed with phosphate buffered saline after Dulbecco and fixed in $1 \%$ glutaraldehyde in $0.07 \mathrm{M}$ phosphate buffer (pH 7.4). Ethanol, amyl acetate. Critical point drying. Gold coating. Cambridge Stereoscan 150 operated at $19 \mathrm{kV}$. APhR. 101/80, negative 03 773. (from SCHILLER 1982)

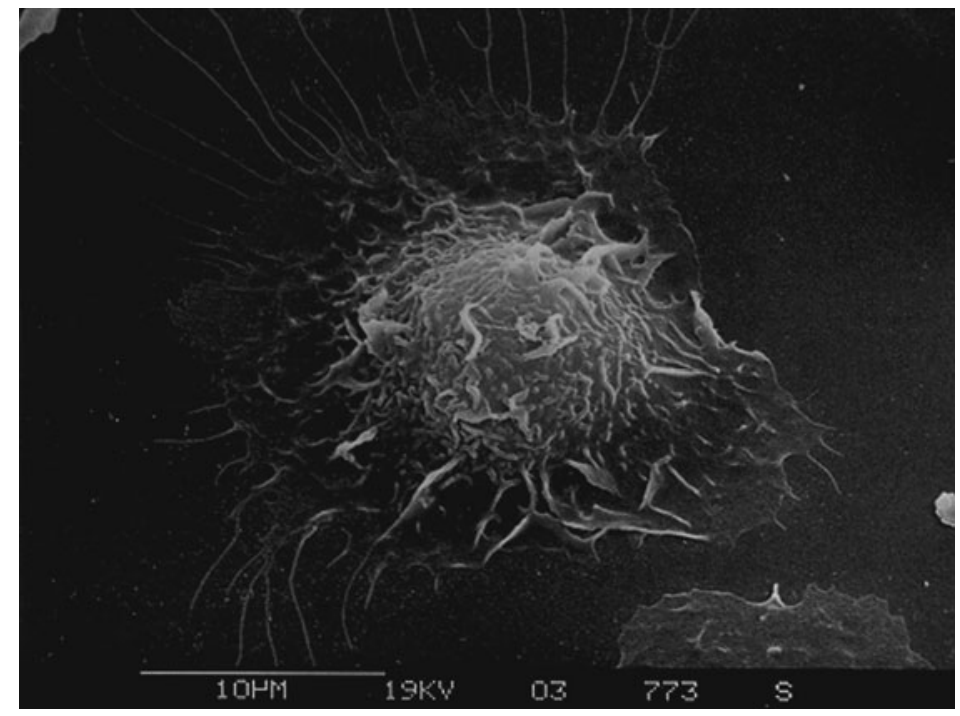

Fig. 176. Spreading of two non-immunologically stimulated peritoneal macrophages from a SPF-NMRI mouse $48 \mathrm{~h}$ after a single intraperitoneal injection of $150 \mathrm{mg}$ isotactic polyacrylic acid (Vi 2329; molecular mass 8-12 kDa; cf. MücK et al. 1977) as sodium salt per kg body weight. The produced ascites were centrifuged for $10 \mathrm{~min}$ at 1000 r.p.m. and incubated in TCM $199+20 \%$ foetal calf serum on a plastic cover slip for $2 \mathrm{~h}$, rinsed with phosphate buffered saline after Dulbecco and fixed in $1 \%$ glutaraldehyde in $0.07 \mathrm{M}$ phosphate buffer (pH 7.4). Ethanol, amyl acetate. Critical point drying. Gold coating. Cambridge Stereoscan 150 operated at $19 \mathrm{kV}$. APh-R. 856/80, negative 07186

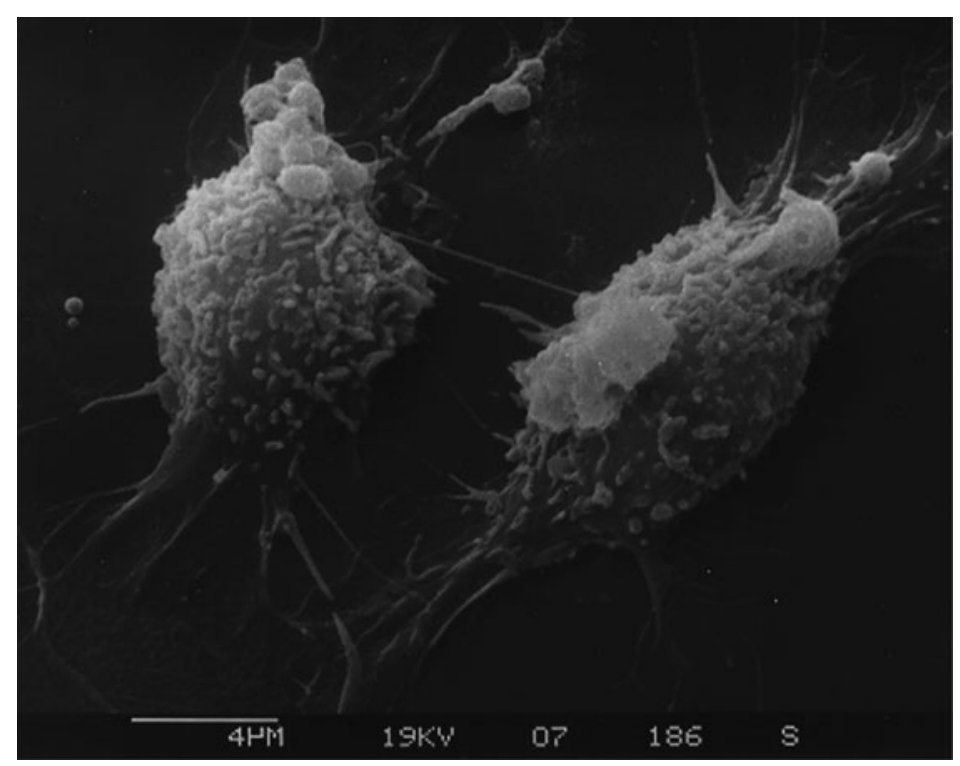

Further references: De ClercQ and DE Somers (1968), Remington and Merigan (1969), VANDePUTte et al. (1970), VAN DiJCK (1970), RiCHMOND (1971), de ClercQ (1973), Eyckmans et al. (1973), SCHILLER (1980, 1982)

\section{Methylmethacrylate}

Friedmanmor et al. (1977)

\section{Drugs Activated to Free Radicals}

\section{Adriamycin}

Stoychkov et al. (1979), HASkill (1981), Martin et al. (1982)

\section{Mitomycin C}

Ogura et al. (1980), SHindo et al. (1985)

\subsection{2}

\section{Immunologically Specific Activation}

The term "macrophage activation" was introduced by MaCKAnEss $(1969,1970)$ on account of morphological changes of mononuclear phagocytes after immunisation of the host against Listeria monocytogenes. Activated macrophages adhere to the glass wall of the culture vessel and spread quickly (NATHAN et al. 1971) showing increased phagocytosis.

ALLISON (1978) preferred the term "activation" for the marked metabolic changes that occur in 
macrophages over several hours or days, by analogy with those induced in lymphocytes by antigens or mitogens. "Stimulation" can be used for short-term responses to stimuli such as phagocytosis.

Theoretically, activation in one or an other form is necessary to be cured from infective diseases, in which the antigen survives or multiplies in normal macrophages.

FIDLER and RAZ (1981) reserved the term "macrophage activation" for the process whereby noncytotoxic macrophages acquire tumour cytotoxic or tumoricidal properties.

The target specificity of activated macrophages has been controversial:

1. Specifically immune macrophages whose target specificity has been associated with the presence of cytophilic antibody that enhances macrophage-tumour interaction (Pels and DEN OTTER 1974).

2. Armed macrophages that demonstrate specific tumour cell killing after becoming activated by specific macrophage-activating factor (MAF) release by T lymphocytes (Evans and Alexander 1971, 1972; Grant et al. 1972; LOHMANNMAtThes et al. 1973, 1974; KRIPKE et al. 1977).

3. Macrophages that are activated by non-specific agents, including MAF, are rendered tumoricidal.

AdAms and Marino (1984) defined "activation" as a competence to produce a given output (i.e. to complete a given function). They pose several critical questions:

1. What functions of macrophages can be activated?

2. What capacities of macrophages are necessary for completion of each function?

3. What signal(s) regulates each capacity?

4. How specific is the relationship between each signal and each capacity? (i.e., How many capacities are induced by each signal? How many signals induce each capacity?)

5. What is the interplay between the various signals? (i.e., Does receipt of one signal alter the responsiveness or the response(s) of the macrophages to a second signal?) Does induction of one preclude development of another?

6. What governs the path of activation followed? Is the path simply a matter of the first signal received or are more complicated regulatory circuits involved?

ad 1) Examples of functions might represent kill tumour cells or microbes, phagocytosis of a given type of particle, processing and presentation of antigen, or chemotaxis. ad 2) Capacities represent either the expression of distinct gene products or covalent modifications thereof, while functions represent the combined interaction of several capacities.

ad 3) Macrophage activation is known to be regulated by a large number of signals (for review see AdAms and Hamilton 1987, Hamilton and ADAMs 1987). Inductive signals include IFN $\gamma$, interferons $\alpha / \beta$, ganulocyte-macrophage colony-stimulating factor (GM-CSF 1), B cell stimulating factor (BSF 1 ), vitamin $\mathrm{D}_{3}$, retinoic acid, bacterial lipopolysaccharide (LPS), maleylated or acetylated proteins, tumour necrosis factor (TNF), heat-killed gram-positive microorganisms, such as L.monocytogenes, and liposome encapsulated muramyl dipeptide. N-Acetylmuramyl-Lalanyl-D-isoglutamine, a synthetic substance of a minimal structure required for the adjuvant activity of bacterial cell walls was found to activate macrophages in mice, whereas its diastereomer $\quad \mathrm{N}$-acetylmuramyl-L-alanyl-Lisoglutamine, which is inactive as adjuvant, did not activate them (TANAKA et al. 1977). Muramyl dipeptide bound to glycosylated serum albumin or bound to gluconoylated and glycosilated poly-L-lysine were more efficient than free muramyl dipeptide in rendering mouse peritoneal macrophages and rat alveolar macrophages cytostatic against various tumour cells (Petit et al. 1990).

ad 4) Many of these activating signals act through defined surface receptors (ADAMS and HAmilton 1987, Hamilton and Adams 1987).

\subsubsection{1 \\ Activation of the Mononuclear Phagocyte System by Complete Freund's Adjuvant}

Experimental animals given a course of injections of homologous antigen and Freund's complete adjuvant develop delayed hypersensitivity to the antigen. These immunised animals may also develop granulomatous lesions at the injection sites (in muscles if the primary injection was made intramuscular, and internally if the injection was made either intavenous or intraperitoneal).

Freund's adjuvant $\left(1.4-140 \mu 1 / 25 \times 10^{6}\right.$ peritoneal exudate cells of mice) induced a significant activation of phospholipase A (EC 3.1.1.4) (MUNDER et al. 1973). The relative concentrations of lysolecithin (1-acyl-sn-glycero-3-phosphorylcholine), neutral lipids and cephalin changed. Cephalin (1,2-acylglycero-3-phosphorylethanolamine) was attacked by phospholipase $\mathrm{A}$ and degraded at the same rate as lecithin (1,2-acyl-glycero-3-phosphorylcholine). 


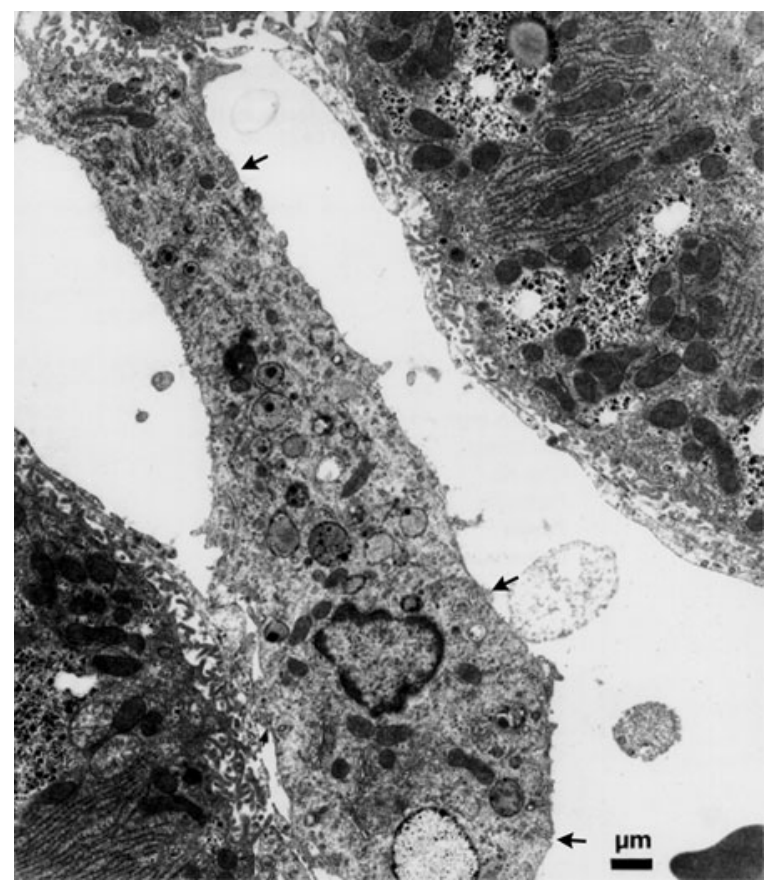

Fig. 177. Kupffer cell (block 559) from an unmedicated female rat. Under pentobarbital anaesthesia $(30 \mathrm{mg} / \mathrm{kg})$, the animal was perfused from the abdominal aorta with $2.5 \%$ glutaraldehyde in $0.1 \mathrm{M}$ sodium cacodylate buffer ( $\mathrm{pH} 7.4$ ). Postfixation with $1 \%$ osmium tetroxide in sodium cacodylate buffer. Embedded in Epon 812 and sectioned at $50 \mathrm{~nm}$. Lead citrate and uranyl acetate. Film 376/79. - Several tubular invaginations are marked by arrows

In the presence of $1.4 \mu \mathrm{l}$ Freund's complete adjuvant ${ }^{14} \mathrm{C}$-oleic acid $(10 \mathrm{nM})$ was transesterified from phospholipid to triglyceride.

While bentonite and bentonite mixed with wax fractions were unable to induce enhancement of carbon clearance on antigen recall in the chicken, neutral delipidated $M$. avium cell walls made good potentiators of antigen recall in carbon clearance (Aiyedun 1972).

Adjuvant fibrosis due to complete and incomplete Freund's adjuvant was used for a model of experimental pulmonary fibrosis by KISSLER (1983), while Antweiler et al. (1963), Schiller (1980) and KissLer (1983) studied the influence of adjuvant in pneumoconiosis.

Intravenous injection of complete Freund's adjuvant induced infiltrative and follicular lesion in the rat lung, resembling human noncaseating granulomatoses of specific type in their localisation and cellular patter comparable in particular to sar- coidal and hypersensitivity granulomatous lesions (BASSET et al. 1974). The rat appeared particularly suitable because its pulmonary lesions, in spite of an excellent tolerance, regress more slowly than those in the $\operatorname{dog}$ (STRAuss et al. 1970) and rabbit (Rupp et al. 1960, Moore and Schoenberg 1963, Moore 1973).

MAsin et al. (1979) have found that in guinea pigs immunised i.v. with $0.15 \mathrm{ml}$ of complete Freund's adjuvant and challenged five days later with $1 \mathrm{mg}$ of BCG i.v. $(2 \mathrm{mg} / \mathrm{ml})$ most of the lung parenchyma was replaced by multiple granulomas consisting of epithelioid cells and a few giant cells. Mitogenic and macrophage migration inhibition (MIF) activity were found in aqueous extracts of the lungs of these animals.

In the rabbit, subpleural pulmonary lesions were the preferential reactions after an intravenous injection of Freund's adjuvant (AMEMori and ALTSCHUL 1963). Initial granulamas resemble normal solitary follicles. Later they grow and exceed the lymph follicles by size. Epithelioid cells appear. Advanced granulomas gain a considerable size and undergo central necrosis.

Saccharated iron oxide premedication modified the response of the reticulo-endothelial cells in the liver and spleen of the rabbit. There was a decrease in the proliferative response (MOORE and SCHOENBERG 1963).

Histamine release from human basophils and mast cells is mediated by nondialyzable factor(s) (molecular mass $>2 \mathrm{kDa}$ ) secreted by lung macrophages when cocultured for $24 \mathrm{~h}$ (SCHULMAN et al. (1985).

$\mathrm{N}$-Acetylmuramyl-L-alanyl-D-isoglutamine (muramyl dipeptide) first isolated from mycobacteria (Elouz et al. 1974, Kotani 1976) and later synthesized (LEDERER 1980), was found to be the minimal structural unit that can replace mycobacterial organisms in Freund's complete adjuvant for activation of macrophages (CHEDID et al. 1979). However, although synthetic muramyl dipeptide affects several macrophage functions in vitro (CHEDID et al. 1979), it did not have these effects in vivo. This might be, as PARENT et al. (1979) showed, due to the clearance of soluble muramyl dipeptide from the body within 60 min. Multilamellar vesicle liposomes containing muramyl dipeptide were more effective than free muramyl dipeptide in potentiating the tumoridical activity of human alveolar macrophages during culture for $4 \mathrm{~h}$ (Sone et al. 1984). 


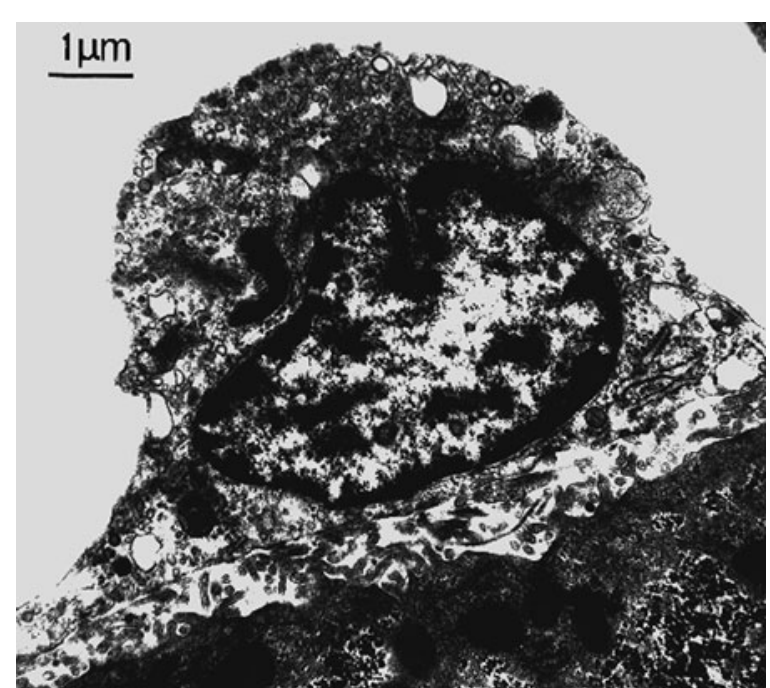

Fig. 178. Kupffer cells (block 4790) from a male SpragueDawley rat (Charles River, France) 2 weeks after an activation of the mononuclear phagocyte system by intravenous injection of $0.05 \mathrm{ml}$ complete Freund's adjuvant on two consecutive days. Under pentobarbital anaesthesia $(30 \mathrm{mg} / \mathrm{kg})$, the animal was perfused from the abdominal aorta with $2.5 \%$ glutaraldehyde in $0.1 \mathrm{M}$ sodium cacodylate buffer ( $\mathrm{pH} 7.4$ ). Postfixation with $1 \%$ osmium tetroxide in sodium cacodylate buffer. Embedded in Epon 812 and sectioned at $50 \mathrm{~nm}$. Lead citrate and uranyl acetate. Film 983/79 and Film 979/79

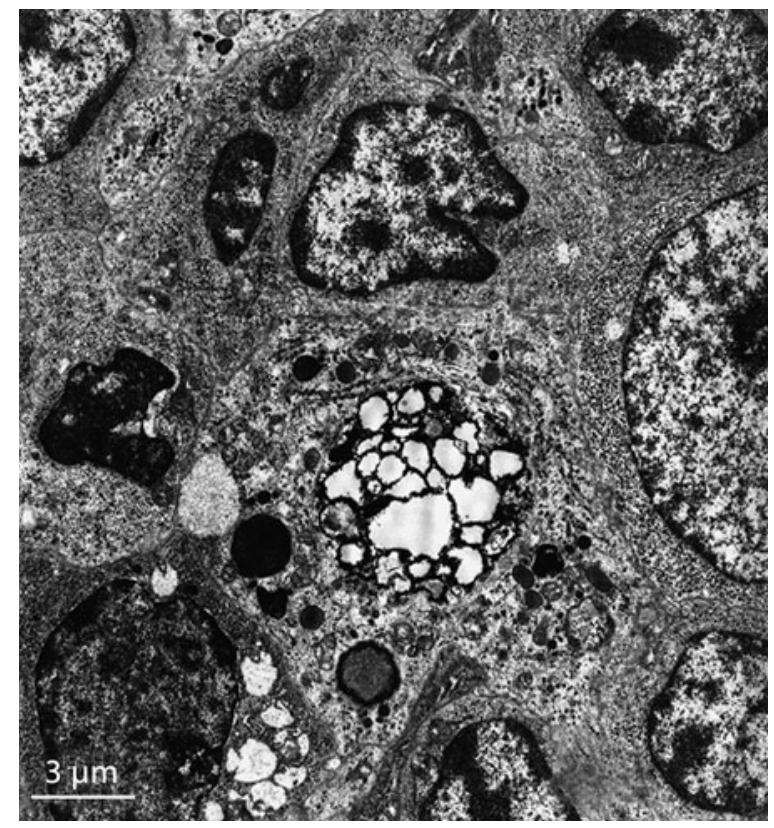

Fig. 180. Lymph node (block 4800) from a male SpragueDawley rat (Charles River, France) 2 weeks after an activation of the mononuclear phagocyte system by intravenous injection of $0.05 \mathrm{ml}$ complete Freund's adjuvant on two consecutive days. Under pentobarbital anaesthesia $(30 \mathrm{mg} / \mathrm{kg})$, the animal was perfused from the abdominal aorta with $2.5 \%$ glutaraldehyde in $0.1 \mathrm{M}$ sodium cacodylate buffer ( $\mathrm{pH}$ 7.4). Postfixation with $1 \%$ osmium tetroxide in sodium cacodylate buffer. Embedded in Epon 812 and sectioned at $50 \mathrm{~nm}$. Lead citrate and uranyl acetate. Film 1006/79

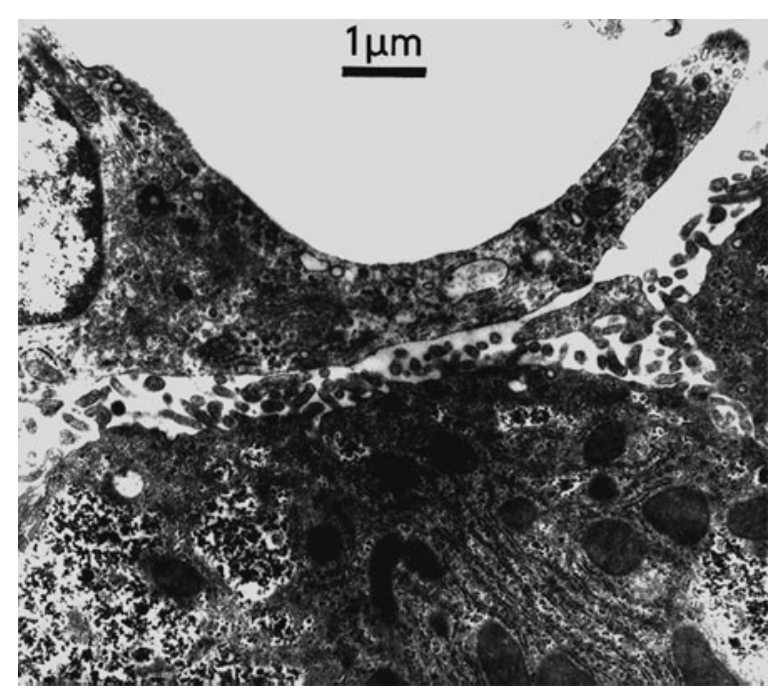

Fig. 179. Lymph node (block 4800) from a male SpragueDawley rat (Charles River, France) 2 weeks after an activation of the mononuclear phagocyte system by intravenous injection of $0.05 \mathrm{ml}$ complete Freund's adjuvant on two consecutive days. Under pentobarbital anaesthesia $(30 \mathrm{mg} / \mathrm{kg})$, the animal was perfused from the abdominal aorta with $2.5 \%$ glutaraldehyde in $0.1 \mathrm{M}$ sodium cacodylate buffer ( $\mathrm{pH}$ 7.4). Postfixation with $1 \%$ osmium tetroxide in sodium cacodylate buffer. Embedded in Epon 812 and sectioned at $50 \mathrm{~nm}$. Lead citrate and uranyl acetate. Film 1003/79

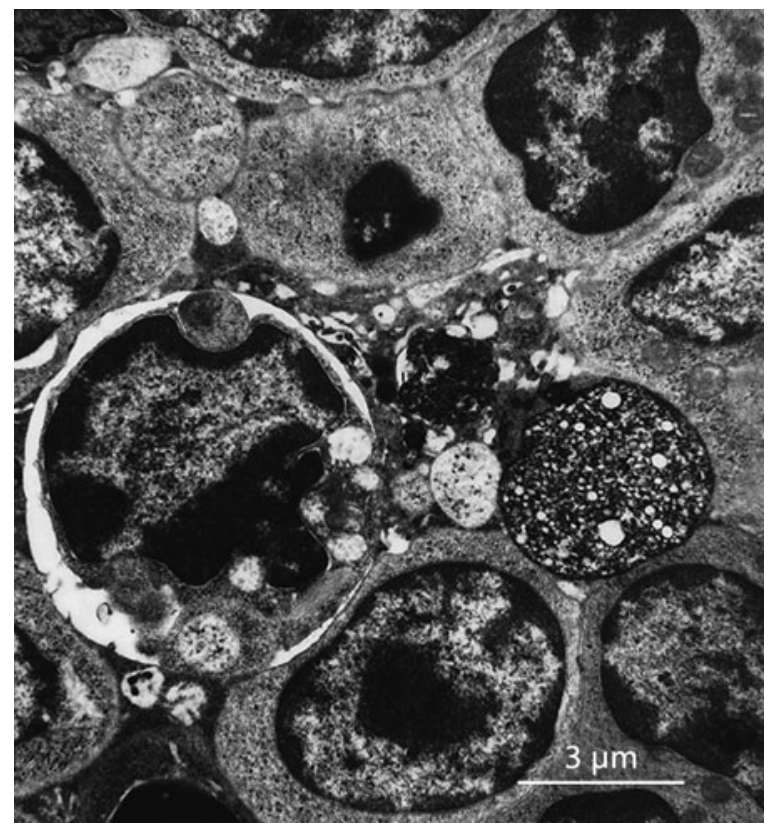

Fig. 181. Splenic white pulp (block 4791) from a male Sprague-Dawley rat (Charles River, France) 2 weeks after an activation of the mononuclear phagocyte system by intravenous injection of $0.05 \mathrm{ml}$ complete Freund's adjuvant on two consecutive days. Under pentobarbital anaesthesia $(30 \mathrm{mg} /$ $\mathrm{kg}$ ), the animal was perfused from the abdominal aorta with $2.5 \%$ glutaraldehyde in $0.1 \mathrm{M}$ sodium cacodylate buffer $(\mathrm{pH}$ 7.4). Postfixation with $1 \%$ osmium tetroxide in sodium cacodylate buffer. Embedded in Epon 812 and sectioned at $50 \mathrm{~nm}$. Lead citrate and uranyl acetate. Film 991/79 


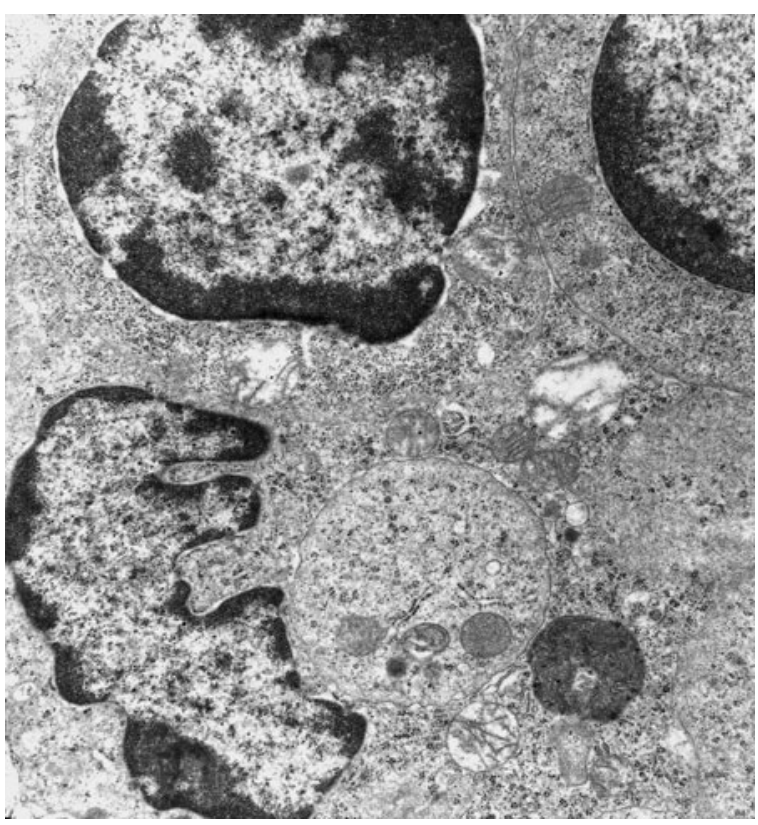

Fig. 182. Lymph node (block 4812) from a $258 \mathrm{~g}$ male Sprague-Dawley rat (Charles River, France) 4 weeks after an activation of the mononuclear phagocyte system by intravenous injection of $0.05 \mathrm{ml}$ complete Freund's adjuvant on two consecutive days. Under pentobarbital anaesthesia $(30 \mathrm{mg} /$ $\mathrm{kg}$ ), the animal was perfused from the abdominal aorta with $2.5 \%$ glutaraldehyde in $0.1 \mathrm{M}$ sodium cacodylate buffer $(\mathrm{pH}$ 7.4). Postfixation with $1 \%$ osmium tetroxide in sodium cacodylate buffer. Embedded in Epon 812 and sectioned at $50 \mathrm{~nm}$. Lead citrate and uranyl acetate. Film 1010/79

Fig. 184. Dilated Golgi cisternae (G) in a Kupffer cell (block 4838) from a male Sprague-Dawley rat (Charles River, France) 16 weeks after an activation of the mononuclear phagocyte system by intravenous injection of $0.05 \mathrm{ml} \mathrm{com-}$ plete Freund's adjuvant on two consecutive days. Under pentobarbital anaesthesia $(30 \mathrm{mg} / \mathrm{kg})$, the animal was perfused from the abdominal aorta with $2.5 \%$ glutaraldehyde in $0.1 \mathrm{M}$ sodium cacodylate buffer ( $\mathrm{pH}$ 7.4). Postfixation with $1 \%$ osmium tetroxide in sodium cacodylate buffer. Embedded in Epon 812 and sectioned at $50 \mathrm{~nm}$. Lead citrate and uranyl acetate. Film 33059/88

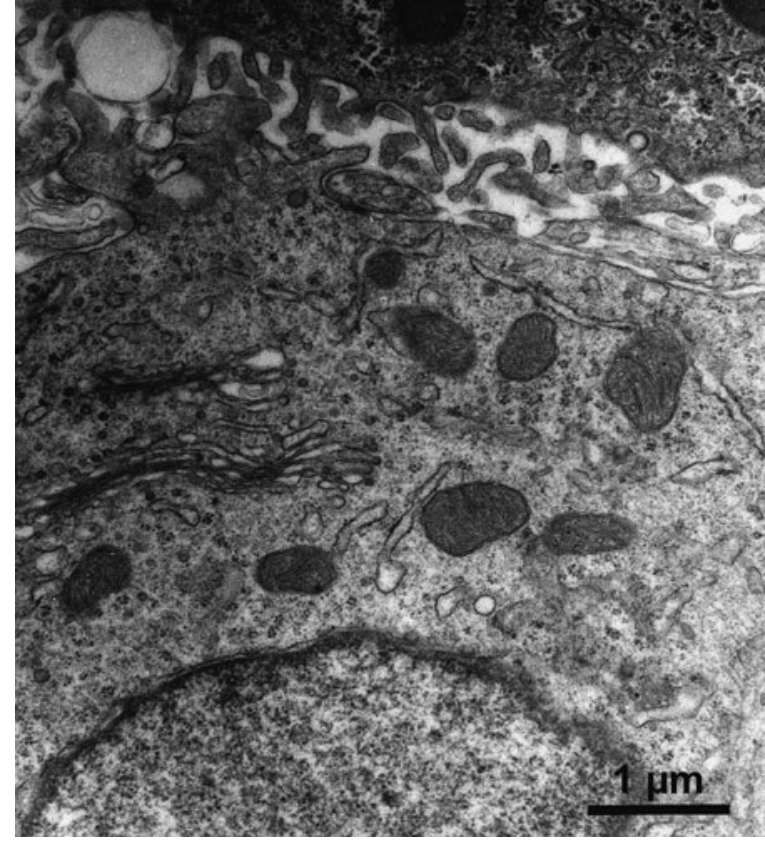

Fig. 183. Prominent Golgi area in a Kupffer cell (block 4838) from a male Sprague-Dawley rat (Charles River, France) 16 weeks after an activation of the mononuclear phagocyte system by intravenous injction of $0.05 \mathrm{ml}$ complete Freund's adjuvant on two consecutive days. Under pentobarbital anaesthesia $(30 \mathrm{mg} / \mathrm{kg})$, the animal was perfused from the abdominal aorta with $2.5 \%$ glutaraldehyde in $0.1 \mathrm{M}$ sodium cacodylate buffer ( $\mathrm{pH}$ 7.4). Postfixation with $1 \%$ osmium tetroxide in sodium cacodylate buffer. Embedded in Epon 812 and sectioned at $50 \mathrm{~nm}$. Lead citrate and uranyl acetate. Film 226/84

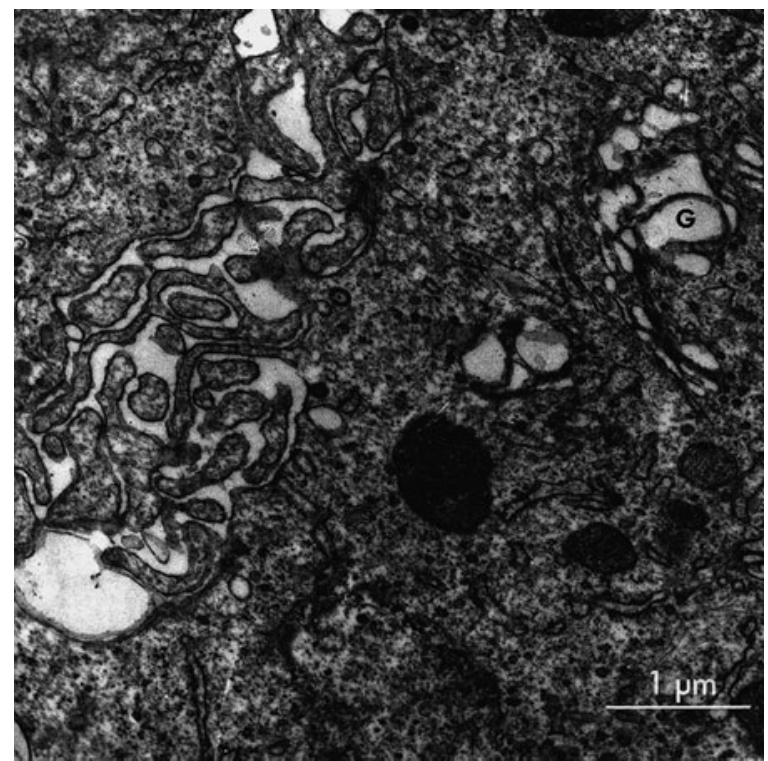




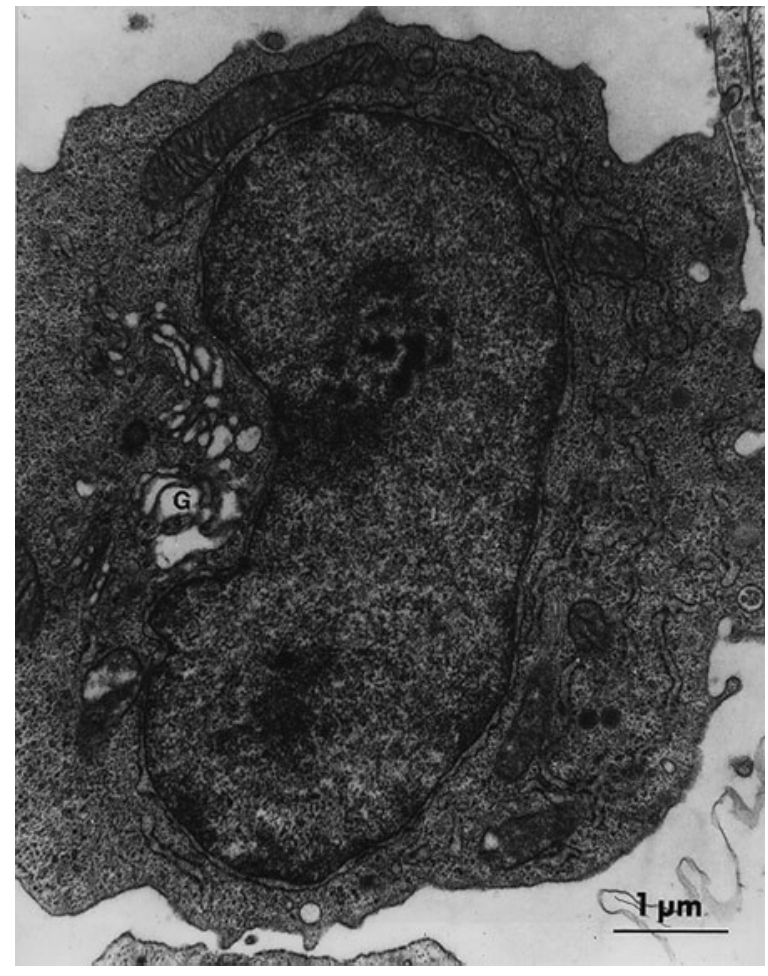

Fig. 185. Dilated Golgi cisternae (G) in a lymph node macrophage (block 4841) from a male Sprague-Dawley rat (Charles River, France) 16 weeks after an activation of the mononuclear phagocyte system by intravenous injection of $0.05 \mathrm{ml}$ complete Freund's adjuvant on two consecutive days. Under pentobarbital anaesthesia $(30 \mathrm{mg} / \mathrm{kg})$, the animal was perfused from the abdominal aorta with $2.5 \%$ glutaraldehyde in $0.1 \mathrm{M}$ sodium cacodylate buffer ( $\mathrm{pH} 7.4$ ). Postfixation with $1 \%$ osmium tetroxide in sodium cacodylate buffer. Embedded in Epon 812 and sectioned at $50 \mathrm{~nm}$. Lead citrate and uranyl acetate. Film 1289/79

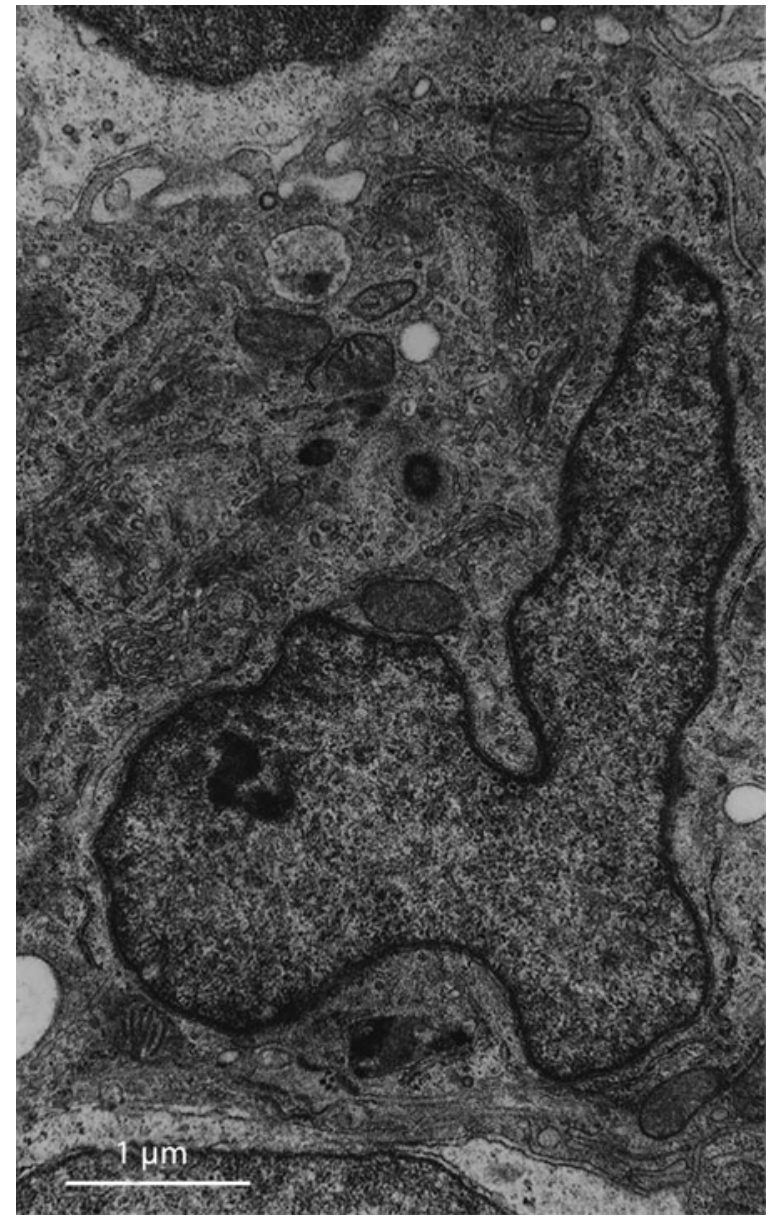

Fig. 186. An epithelioid-like mononuclear cell from a lymph node (block 4841) from a male Sprague-Dawley rat (Charles River, France) 16 weeks after an activation of the mononuclear phagocyte system by intravenous injection of $0.05 \mathrm{ml}$ complete Freund's adjuvant on two consecutive days. Under pentobarbital anaesthesia $(30 \mathrm{mg} / \mathrm{kg})$, the animal was perfused from the abdominal aorta with $2.5 \%$ glutaraldehyde in $0.1 \mathrm{M}$ sodium cacodylate buffer $(\mathrm{pH} 7.4)$. Postfixation with $1 \%$ osmium tetroxide in sodium cacodylate buffer. Embedded in Epon 812 and sectioned at $50 \mathrm{~nm}$. Lead citrate and uranyl acetate. Film 1290/79 


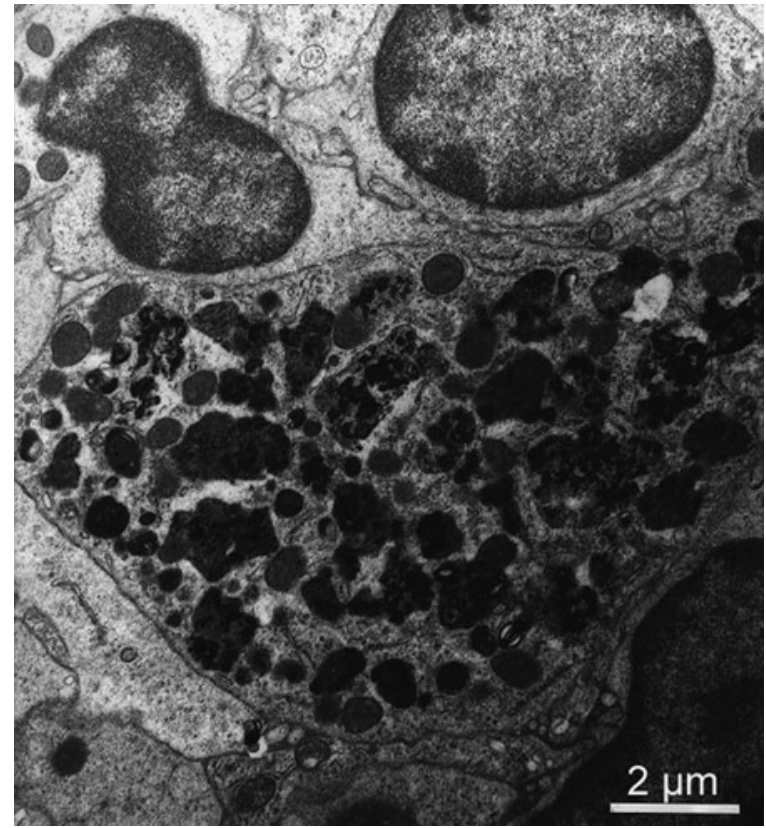

Fig. 187. Pleomorphic inclusions in a lymph node macrophage (block 4837) activated 16 weeks before by two portions of $0.05 \mathrm{ml}$ complete Freund's adjuvant given intravenously at an interval of $24 \mathrm{~h}$. Male Sprague-Dawley rat (Charles River, France) perfused under pentobarbital anaesthesia $(30 \mathrm{mg} / \mathrm{kg})$ from the abdominal aorta with $2.5 \%$ glutaraldehyde in $0.1 \mathrm{M}$ sodium cacodylate buffer ( $\mathrm{pH}$ 7.4). Postfixation with $1 \%$ osmium tetroxide in cacodylate buffer. Embedded in Epon 812 and sectioned at $50 \mathrm{~nm}$. Lead citrate and uranyl acetate. Film $219 / 84$

Fig. 189. Granules of different sizes and micropinocytotic coated pits in the wall of a tubular invagination. Lymph node macrophage (block 4841) 16 weeks after activation of the mononuclear phagocyte system by intravenous injection of $0.05 \mathrm{ml}$ complete Freund's adjuvant on two consecutive days. Male Sprague-Dawley rat (Charles River, France) perfused under pentobarbital anaesthesia $(30 \mathrm{mg} / \mathrm{kg})$ from the abdominal aorta with $2.5 \%$ glutaraldehyde in $0.1 \mathrm{M}$ sodium cacodylate buffer ( $\mathrm{pH}$ 7.4). Postfixation with $1 \%$ osmium tetroxide in sodium cacodylate buffer. Embedded in Epon 812 and sectioned at $50 \mathrm{~nm}$. Lead citrate and uranyl acetate. Film 231/84

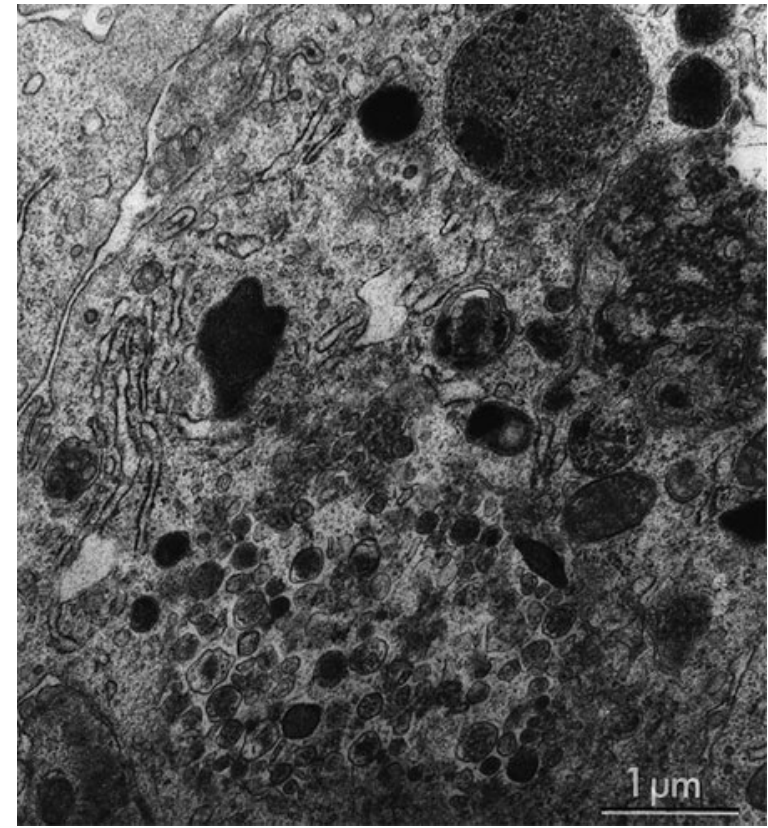

Fig. 188. Tubular invaginations in a splenic macrophage (block 4836) activated 16 weeks before by two portions of $0.05 \mathrm{ml}$ complete Freund's adjuvant given intravenously at an interval of $24 \mathrm{~h}$. Male Sprague-Dawley rat (Charles River, France) perfused under pentobarbital anaesthesia $(30 \mathrm{mg} / \mathrm{kg})$ from the abdominal aorta with $2.5 \%$ glutaraldehyde in $0.1 \mathrm{M}$ sodium cacodylate buffer ( $\mathrm{pH} 7.4$ ). Postfixation with $1 \%$ osmium tetroxide in cacodylate buffer. Embedded in Epon 812 and sectioned at $50 \mathrm{~nm}$. Lead citrate and uranyl acetate. Film $214 / 84$

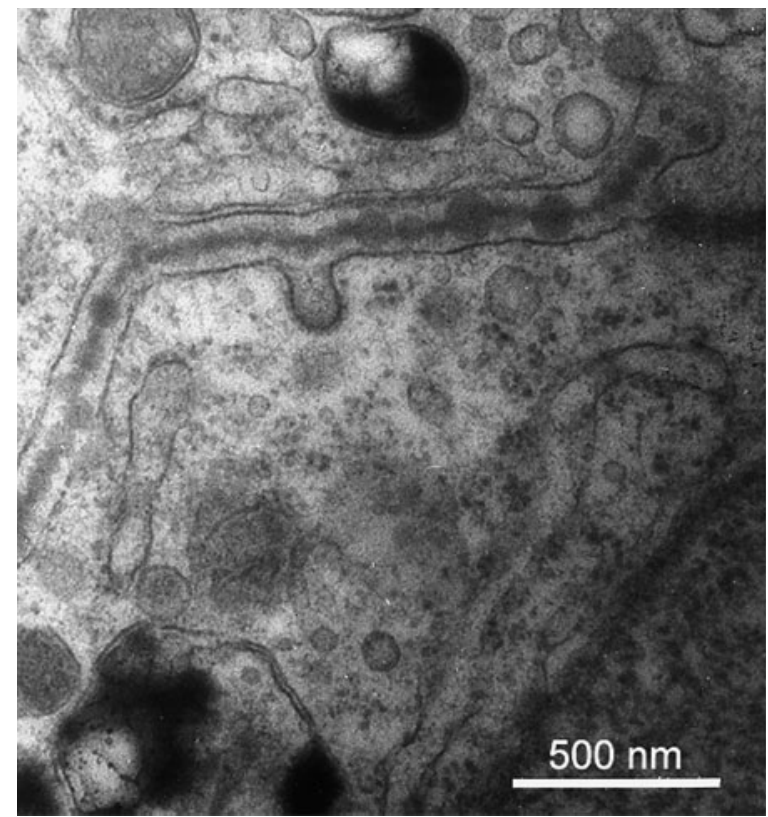




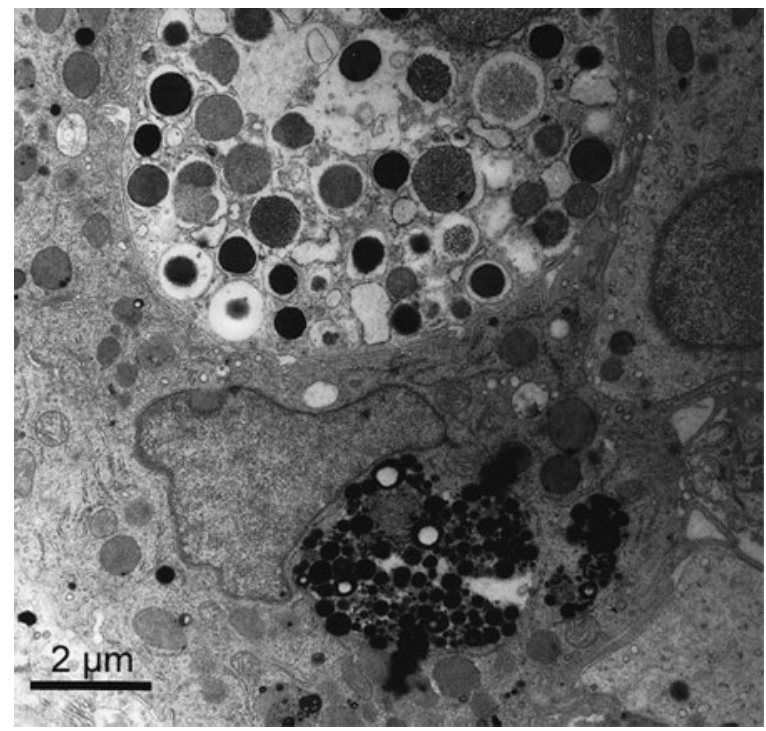

Fig. 190. Intimate contact between a mononuclear phagocyte (bottom) activated by complete Freund's adjuvant and a mast cell (top) in a lymph node (block 4841) from a male SpragueDawley rat (Charles River, France) 16 weeks after activation of the mononuclear phagocyte system by intravenous injection of $0.05 \mathrm{ml}$ complete Freund's adjuvant on two consecutive days. Under pentobarbital anaesthesia $(30 \mathrm{mg} / \mathrm{kg})$ the animal was perfused from the abdominal aorta with $2.5 \%$ glutaraldehyde in $0.1 \mathrm{M}$ sodium cacodylate buffer ( $\mathrm{pH} 7.4$ ). Postfixation with $1 \%$ osmium tetroxide in sodium cacodylate buffer. Embedded in Epon 812 and sectioned at $50 \mathrm{~nm}$. Lead citrate and uranyl acetate. Film 229/84

\subsection{3 \\ Prospective Pulmonary Fibrosis-Inducing Potency of Macrophages}

The prospective pulmonary fibrosis-inducing potency of the mononuclear phagocyte system of active coal workers and retired coal miners has been studied and discussed as to the usefulness of biological markers as representative signals in a continuum of events between causal exposure and resultant disease (Schulte 1989). Fowle and Sexton (1992) defined a biomarker as "a measurement of environmental pollutants or their biological consequences after the contaminants have crossed one of the body's boundaries and entered human tissues or fluids, and which serves as an indicator of exposure, effect, and/or susceptibility.

Asbestos or silica inhalation studies in rats showed that the antioxidant response and, more specifically, mRNA levels of Mn superoxide dismutase and heme oxygenase, correlated well with the inflammatory response in broncho-alveolar lavage fluid (JANSSEN et al. 1992). The upregulation of ornithine decarboxylase (Marsh and Mossman 1991) and c-fos/c-jun protooncogenes (HeInTz et al. 1993), need further human studies.

Tumour necrosis factor- $\alpha$ levels were significantly different between active coal miners from three French mining regions (Nord-Pas de Calais, Lorraine and Provence). However, after correlation for age and region, TNF was found not to be related to dust exposure (Porcher et al. 1994). In 66 Belgian coal miners exposed to dust at the coal face at least 12 yrs blood mononuclear phagocytes spontaneously and stimulated with coal mine dust, silica or lipopolysaccharide released TNF at a higher rate than blood monocytes from 12 non-exposed controls (BoRm et al. 1988). The greatest discriminator between controls and cases with coal workers' pneumoconiosis was coal mine dust-induced TNF release.

In the rat, asbestos fibres and silica particles stimulated alveolar macrophages to release TNF (DUBors et al. 1989). 